\title{
A Selenourea-Thiourea Brønsted Acid Catalyst Facilitates Asymmetric Conjugate Additions of Amines to $\alpha, \beta$-Unsaturated Esters
}

\author{
Yingfu Lin, ${ }^{\dagger+}$ William J. Hirschi, ${ }^{\S}$ Anuj Kunadia, ${ }^{*}$ Anirudra Paul, ${ }^{\dagger,}$ Ion Ghiviriga, ${ }^{\|}$Khalil A. Abboud, \\ Rachael W. Karugu, Mathew J. Vetticatt, ${ }^{*}{ }^{\S}$ Jennifer S. Hirschi, ${ }^{*},{ }^{\S}$ and Daniel Seidel ${ }^{*, \dagger, t}$ \\ ${ }^{\dagger}$ Center for Heterocyclic Compounds, Department of Chemistry, University of Florida, \\ Gainesville, Florida 32611, United States \\ ${ }^{*}$ Department of Chemistry and Chemical Biology, Rutgers, The State University of New \\ Jersey, Piscataway, New Jersey 08854, United States \\ ${ }^{\S}$ Department of Chemistry, Binghamton University, Binghamton, New York 13902, United \\ States \\ "Center for NMR Spectroscopy, Department of Chemistry, University of Florida, \\ Gainesville, Florida 32611, United States \\ ${ }^{I}$ Center for X-ray Crystallography, Department of Chemistry, University of Florida, \\ Gainesville, Florida 32611, United States \\ *Correspondence to: jhirschi@binghamton.edu, vetticatt@binghamton.edu, seidel@chem.ufl.edu

\section{Supporting Information}

\section{Table of Contents}

General Information

Reaction Optimization for the Conjugate Addition of Amines

Reaction Optimization for the Kinetic Resolution of Cyclic Amines.............................................................. S-7

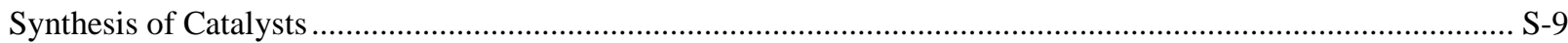

Preparation and Characterization of Products From the Conjugate Addition of Amines .................................. S-25

Preparation and Characterization of Products From the Kinetic Resolution of Cyclic Amines ......................... S-50

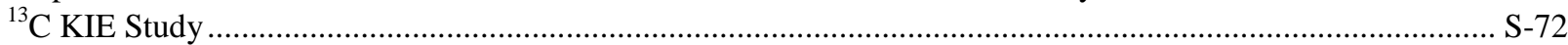

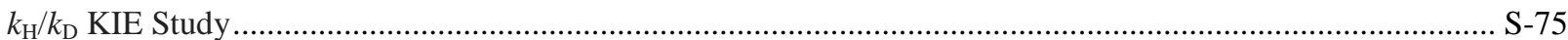

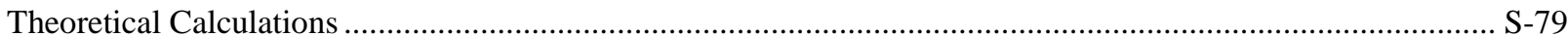

References .

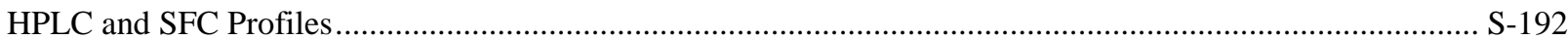

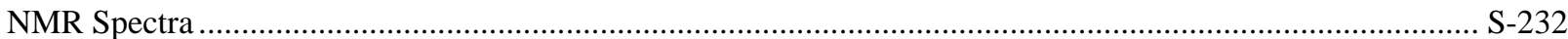


General Information: Reagents and solvents were purchased from commercial sources and were purified by distillation or recrystallization prior to use. Anhydrous toluene, dichloromethane, tetrahydrofuran (THF) and diethyl ether were dried using a JC Meyer solvent system. Reactions were run under a nitrogen atmosphere. Purification of reaction products was carried out by flash column chromatography using Sorbent Technologies Standard Grade silica gel (60 $\AA, 230-400$ mesh). Analytical thin layer chromatography was performed on EM Reagent $0.25 \mathrm{~mm}$ silica gel $60 \mathrm{~F}_{254}$ plates. Visualization was accomplished with UV light, and/or potassium permanganate stain, Dragendorff-Munier stain, followed by heating. Proton nuclear magnetic resonance spectra $\left({ }^{1} \mathrm{H}-\mathrm{NMR}\right)$ were recorded on Varian VNMRS-500 MHz, Varian VNMRS-400 MHz, Varian VNMRS-300 MHz, Bruker Avance 600 $\mathrm{MHz}$ and Bruker Avance $400 \mathrm{MHz}$ instruments and are reported in ppm using solvent as an internal standard $\left(\mathrm{CDCl}_{3}\right.$ at $\left.7.26 \mathrm{ppm}\right)$. Data are reported as app $=$ apparent, $\mathrm{s}=$ singlet, $\mathrm{d}=$ doublet, $\mathrm{t}=$ triplet, $\mathrm{dd}=$ doublet of doublets, $d d d=$ doublet of doublet of doublets, $\mathrm{m}=$ multiplet, comp $=$ complex, $\mathrm{br}=$ broad; integration; coupling constant(s) in Hz. Proton-decoupled carbon nuclear magnetic resonance spectra $\left({ }^{13} \mathrm{C}-\mathrm{NMR}\right)$ spectra were recorded on Varian VNMRS-500 MHz, Varian VNMRS-300 MHz, Bruker Avance $600 \mathrm{MHz}$ and Bruker Avance $400 \mathrm{MHz}$ instruments and are reported in ppm using solvent as an internal standard $\left(\mathrm{CDCl}_{3}\right.$ at $\left.77.16 \mathrm{ppm}\right) .{ }^{77} \mathrm{Se} \mathrm{NMR}$ spectra were recorded on a Bruker Avance $600 \mathrm{MHz}$ instrument, and chemical shifts are reported in ppm on the $\mathrm{Me}_{2} \mathrm{Se}$ scale, using $\mathrm{PhSeSePh}\left(463 \mathrm{ppm}\right.$, in $\left.\mathrm{CDCl}_{3}\right)$ as an external standard. ${ }^{14} \mathrm{~N}$ NMR spectra were recorded on a Bruker Avance $600 \mathrm{MHz}$ instrument, and chemical shifts were reported in ppm using nitromethane $\left(\delta \mathrm{N}=0 \mathrm{ppm}\right.$, in $\left.\mathrm{CDCl}_{3}\right)$ resonance as the external standard. HPLC analysis was carried out on an Agilent 1100 series instrument with auto sampler and multiple wavelength detectors. SFC analysis was carried out on an Agilent 1260 Infinity II instrument with auto sampler and multiple wavelength detectors. Optical rotations were measured using a $1 \mathrm{~mL}$ cell with a 1 $\mathrm{dm}$ path length on a Jasco P-2000 polarimeter at $589 \mathrm{~nm}$ and at $22{ }^{\circ} \mathrm{C}$. High resolution mass spectra (HRMS) were obtained from the Mass Spectrometry Core Laboratory of the University of Florida (Agilent 6200 ESI-TOF coupled to an IonSense DART ET-100 ionization source for DART-TOF analysis) and are reported as m/z (relative ratio). Accurate mass data (ESI) were obtained from an Agilent 1260 Infinity II LC/MSD instrument, using MassWorks 5.0 from CERNO bioscience. ${ }^{1}$ Benzyl crotonate, ${ }^{2}$ benzyl 2-pentenoate, ${ }^{3}$ were prepared according to literature procedures and their published characterization data matched with our own in all respects. Benzyl acrylate and amines shown in Scheme 1 were obtained from commercial sources. Cyclic 2-arylamines shown in Scheme 2 were prepared according to reported procedures by our group. ${ }^{4}$ Catalysts $1 \mathbf{a},{ }^{5} \mathbf{1 b},{ }^{6} \mathbf{1 c},{ }^{7} \mathbf{1 d},{ }^{8} \mathbf{1 e},{ }^{9} \mathbf{1 f},{ }^{10} \mathbf{1 j},{ }^{11} \mathbf{1 l},{ }^{11}$ and $\mathbf{1 m}{ }^{11}$ were prepared according to literature procedures and their published characterization data matched with our own in all respects. 


\section{Reaction Optimization for the Conjugate Addition of Amines}

Evaluation of Other Catalysts

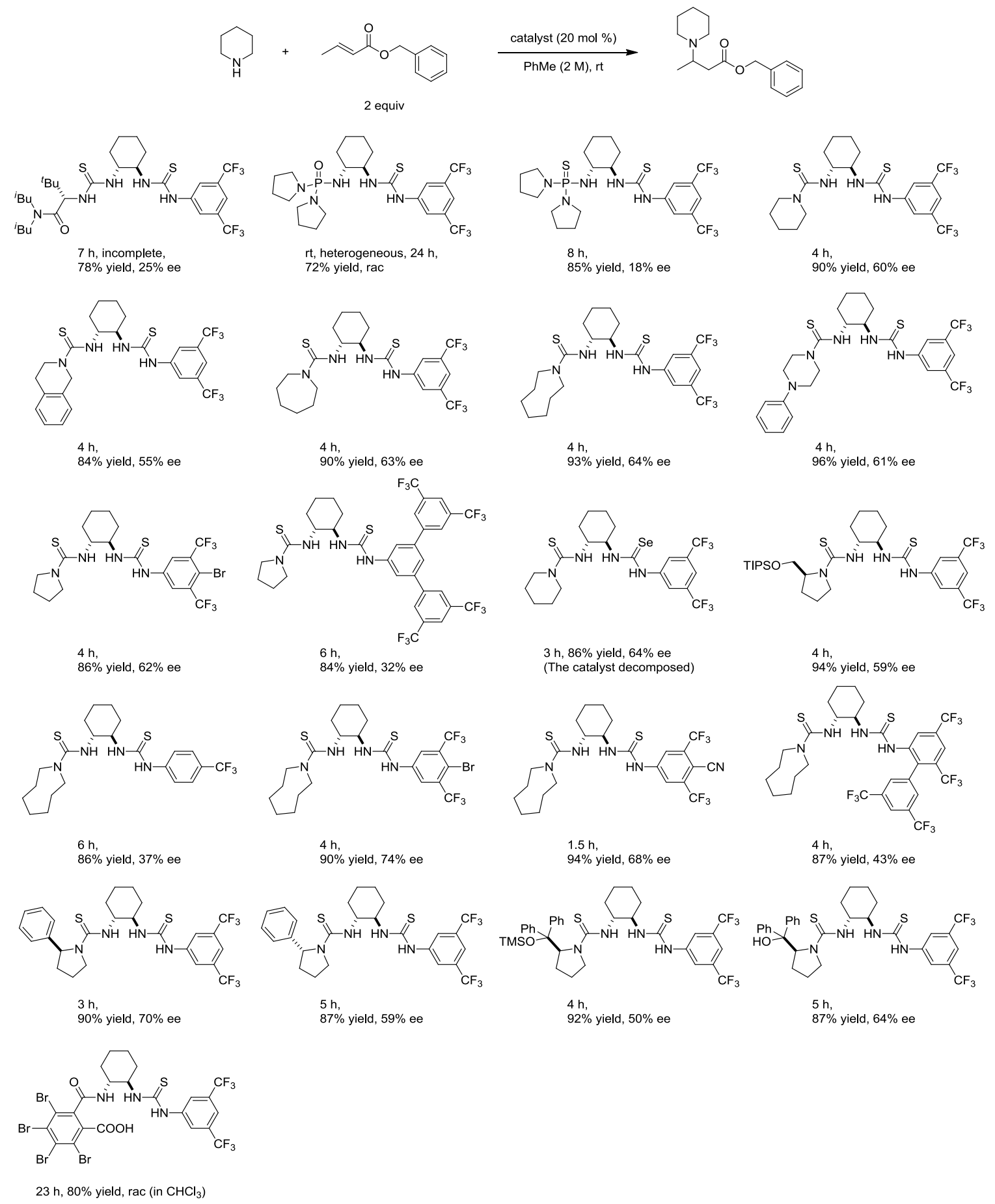




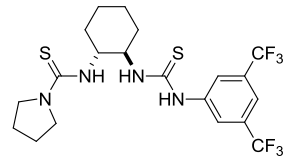

$29 \mathrm{~h}$, incomplete $67 \%$ yield, $65 \%$ ee

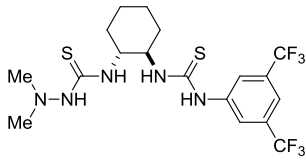

$26 \mathrm{~h}$, incomplete,<smiles>O=C(Nc1cc(C(F)(F)F)cc(C(F)(F)F)c1)NC1c2ccccc2C[C@H]1OC(=S)N1CCCC1</smiles>

$\mathrm{rt}, 48 \mathrm{~h}$, incomplete
$61 \%$ yield, $-5 \%$ ee<smiles>O=[N+]([O-])c1ccc(NC(=S)NC(Cc2ccccc2)Cc2ccccc2)cc1</smiles>

$24 \mathrm{~h}$, incomplete $38 \%$ yield, $-9 \%$ ee

yeterogeneous, 43 h, incomplete $48 \mathrm{~h}$, incomplete
$50 \%$ yield, $73 \%$ ee

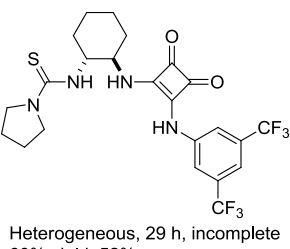
$60 \%$ yield, $52 \%$ ee

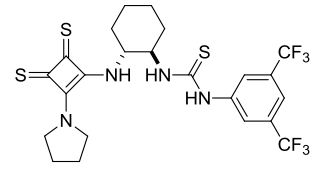

$26 \mathrm{~h}$, incomplete

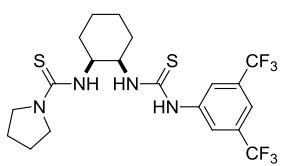

$24 \mathrm{~h}$, incomplete

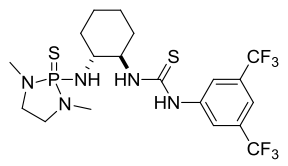

$44 \mathrm{~h}$, incomplete $47 \%$ yield, $10 \%$ ee

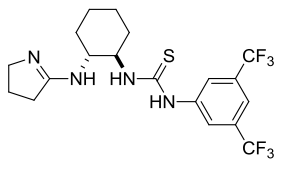

$50 \mathrm{~h}$, incomplete

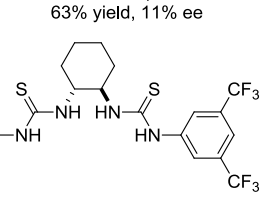

$47 \mathrm{~h}$, incomplete
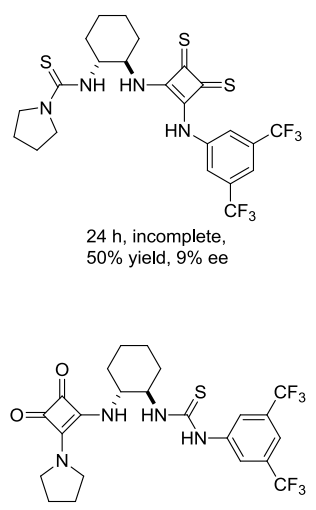

$29 \mathrm{~h}$, incomplete

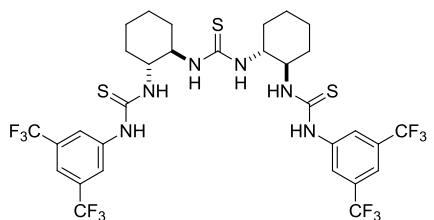

heterogeneous, $42 \mathrm{~h}$.
$88 \%$ yield, $43 \%$ ee

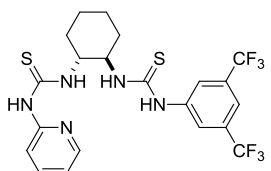

$48 \mathrm{~h}$, incomplete

$69 \%$ yield, $26 \%$ ee

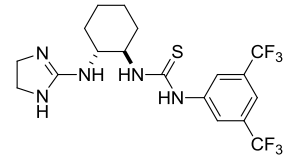

$50 \mathrm{~h}$, incomplete,

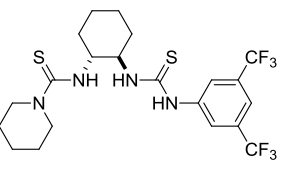

$26 \mathrm{~h}$,
$77 \%$ yield, $75 \%$ ee

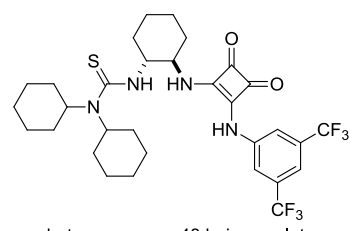

heterogeneous, $48 \mathrm{~h}$, incomplete $81 \%$ yield, $67 \%$ ee

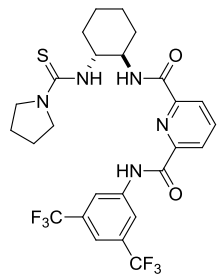

$24 \mathrm{~h}$, incomplete $23 \%$ yield, rac

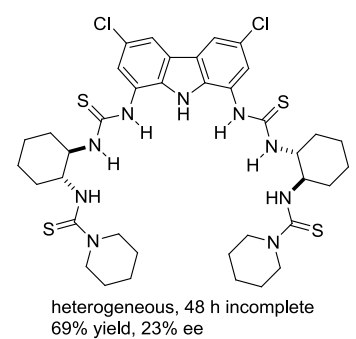

$69 \%$ yield, $23 \%$ ee

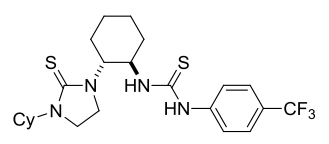

heterogeneous, $43 \mathrm{~h}$, incomplete $30 \%$ yield, rac

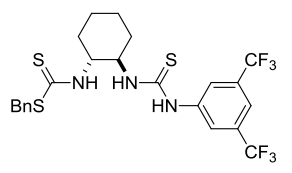

catalyst decomposed

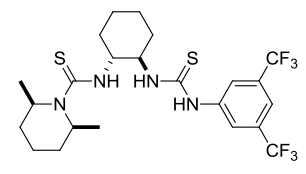

$26 \mathrm{~h}$,

$80 \%$ yield, $73 \%$ ee 


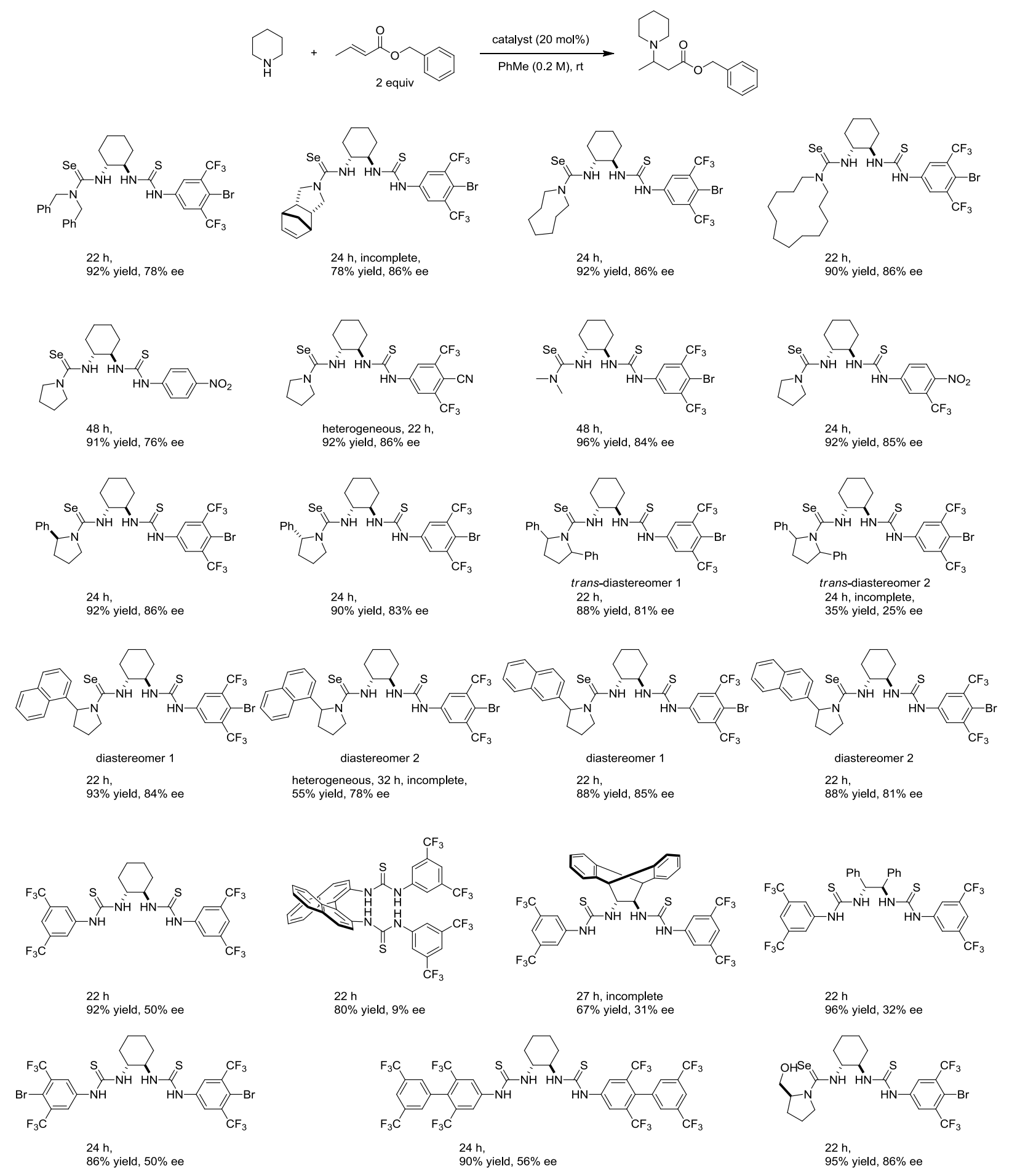


Evaluation of Other Conjugate Acceptors

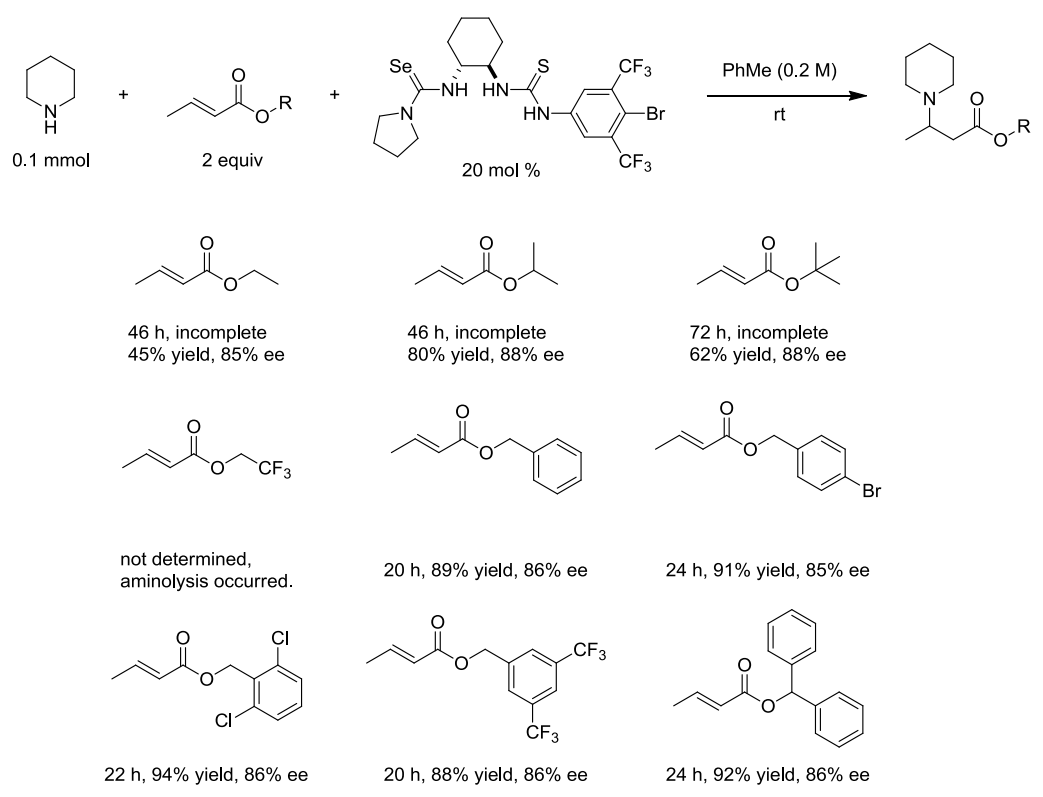




\section{Reaction Optimization for the Kinetic Resolution of Cyclic Amines}

Evaluation of Other Resolving Reagents
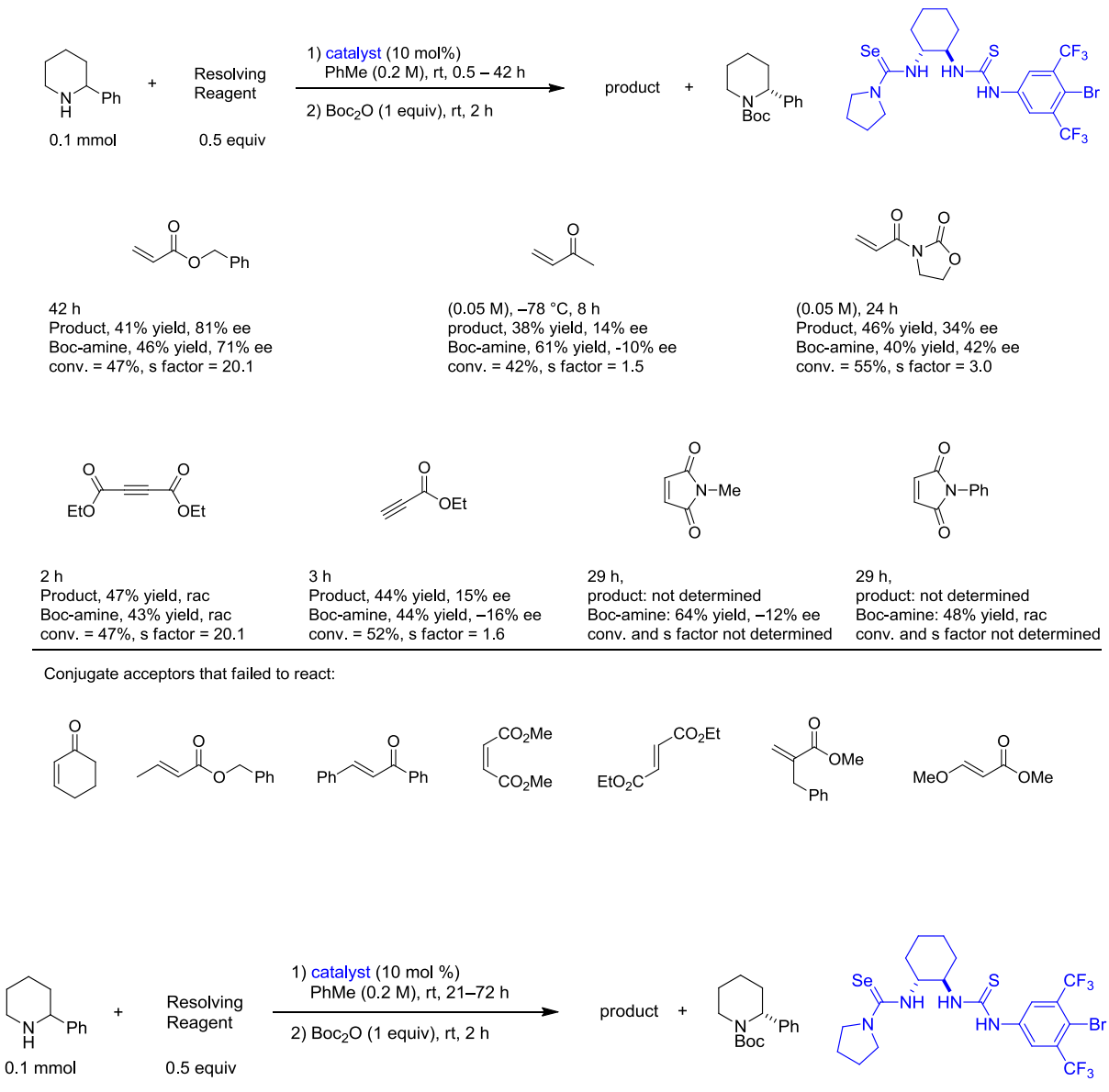

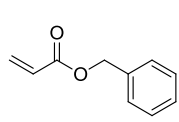

$42 \mathrm{~h}$

Product, $41 \%$ yield, $81 \%$ ee Boc-amine, $46 \%$ yield, $71 \%$ ee conv. $=47 \%$, s factor $=20.1$

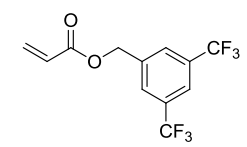

$48 \mathrm{~h}$

Product, $47 \%$ yield, $84 \%$ ee Boc-amine, $47 \%$ yield, $73 \%$ e conv. $=47 \%$, s factor $=25$

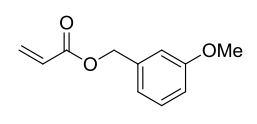

$48 \mathrm{~h}$

Product, $47 \%$ yield, $81 \%$ ee Boc-amine, $45 \%$ yield, $76 \%$ e conv. $=48 \%$, s factor $=21.7$

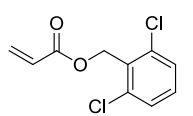

$48 \mathrm{~h}$

Product, $43 \%$ yield, $80 \%$ ee Boc-amine, $48 \%$ yield, $70 \%$ ee conv. $=47 \%, \mathrm{~s}$ factor $=18.7$
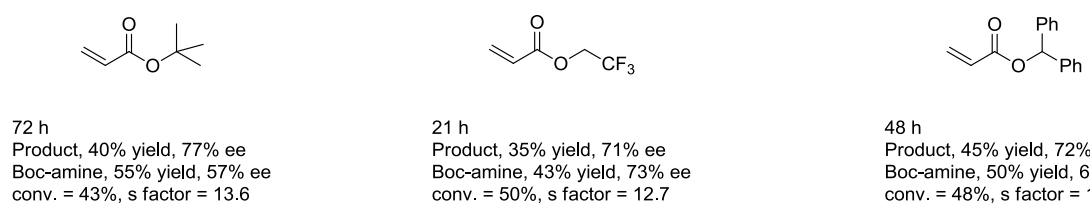

Product, $40 \%$ yield, $77 \%$ ee conv. $=43 \%$, s factor $=13.6$

Boc-amine, $43 \%$ yield, $73 \%$ ee conv. $=50 \%, \mathrm{~s}$ factor $=12.7$

$48 \mathrm{~h}$

Product, $45 \%$ yield, $72 \%$ ee Boc-amine, $50 \%$ yield, $66 \%$ ee conv. $=48 \%, \mathrm{~s}$ factor $=12$ 


\section{Evaluation of Other Solvents}

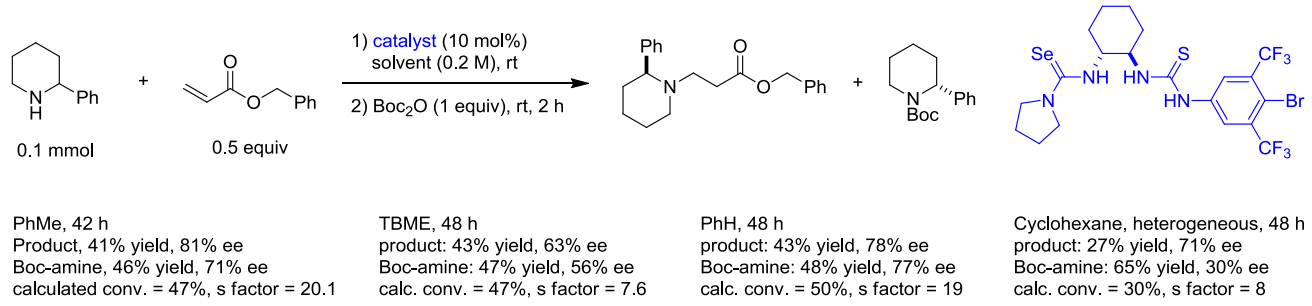

Evaluation of Other Catalysts

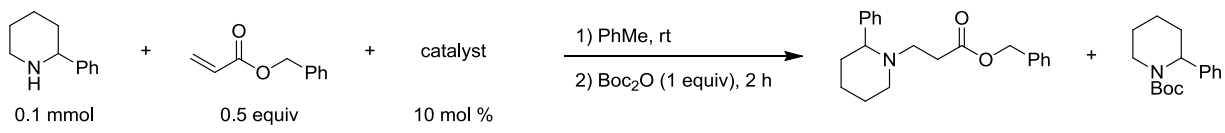

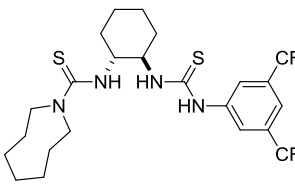

(0.5 M), rt, $22 \mathrm{~h}$

Product, $44 \%$ yield, $60 \%$ ee Boc-amine, $40 \%$ yield, $60 \%$ e conv. $=50 \%$, s factor $=7.2$

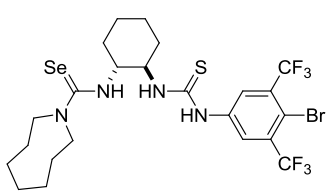

(0.2 M), rt, $42 \mathrm{~h}$ Product, $45 \%$ yield, $81 \%$ ee Boc-amine, $51 \%$ yield, $71 \%$ ee calculated conv. $=47 \%$, s factor $=20.1$

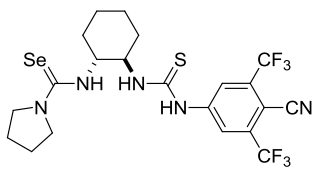

(0.2 M), rt, $48 \mathrm{~h}$ (Heterogeneous) Product, $42 \%$ yield, $73 \%$ ee Boc-amine, $49 \%$ yield, $65 \%$ ee calculated conv. $=47 \%$, s factor $=12.4$

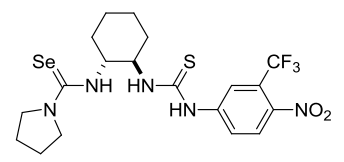

$(0.2 \mathrm{M}), \mathrm{rt}, 48 \mathrm{~h}$

Boc-amine: $46 \%$ yield, $81 \%$ ee; Product: $17 \mathrm{mg}, 53 \%$ yield, $79 \%$ ee Calc. conv. $=51 \%$, s factor $=21$.

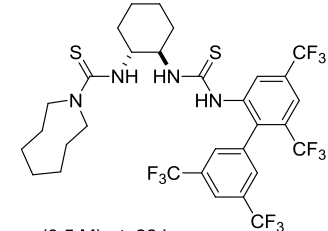

(0.5 M), rt, $22 \mathrm{~h}$.

Product, $44 \%$ yield, $69 \%$ ee;

Boc-amine, $57 \%$ yield, $55 \%$ ee.

Calc. conv. $=44 \%, \mathrm{~s}$ factor $=9.4$

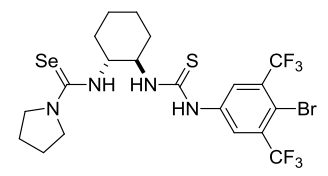

(0.2 M), rt, $42 \mathrm{~h}$

Product, $41 \%$ yield, $81 \%$ ee

Boc-amine, $46 \%$ yield, $71 \%$ e

calculated conv. $=47 \%$, s factor $=20.1$
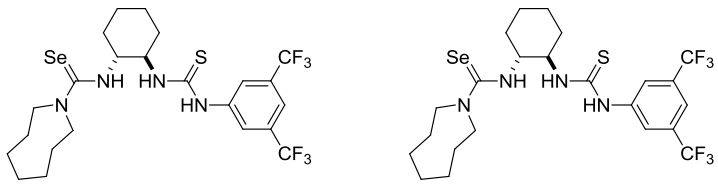

$(0.5 \mathrm{M}), \mathrm{rt}, 24 \mathrm{~h}$

Product, $42 \%$ yield, $75 \%$ ee

calculated conv $=46 \%$, s factor $=13.6$

(0.2 M), rt, $42 \mathrm{~h}$

Product, $42 \%$ yield, $80 \%$ ee

Boc-amine, $50 \%$ yield, $68 \%$ ee

calculated conv. $=46 \%, \mathrm{~s}$ factor $=18.2$
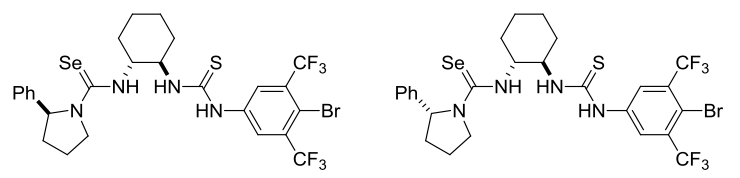

(0.2 M), rt, $42 \mathrm{~h}$

Product, $46 \%$ yield, $70 \%$ ee

Product, $41 \%$ yield, $82 \%$ ee

calculated conv. $=45 \%$, s factor $=10.0$

Boc-amine, $53 \%$ yield, $70 \%$ ee

calculated conv. $=46 \%, \mathrm{~s}$ factor $=21.0$

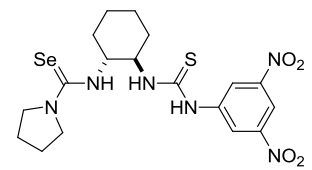

(0.2 M), rt, $48 \mathrm{~h}$

Product, $46 \%$ yield, $77 \%$ e

Boc-amine, $47 \%$ yield, $73 \%$ ee

calculated conv. $=49 \%$, s factor $=16.6$

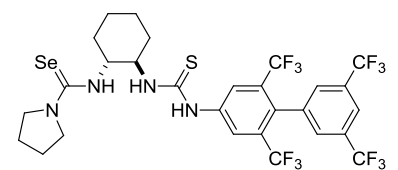

(0.2 M), rt, $48 \mathrm{~h}$

Product, $46 \%$ yield, $78 \%$ ee

Boc-amine, $47 \%$ yield, $89 \%$ ee

calc. conv. $=53 \%$, s factor $=24$
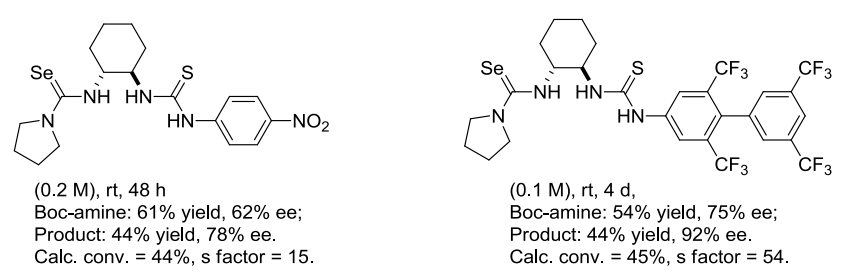


\section{Synthesis of Catalysts}

Scheme for the synthesis of catalyst $\mathbf{1 g}$ :

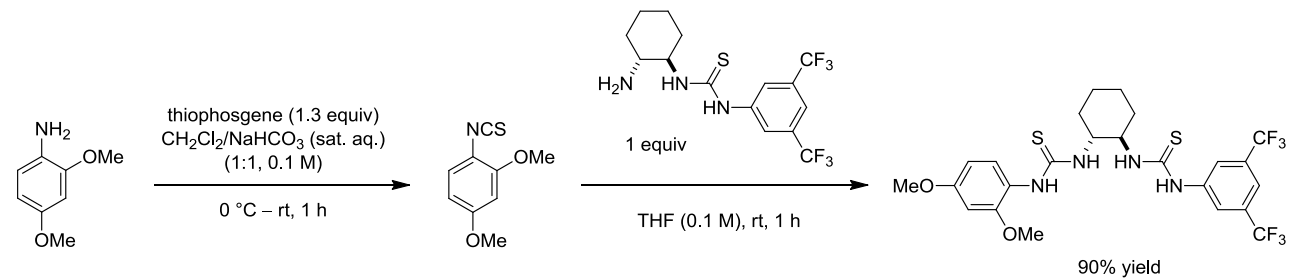

1-((1R,2R)-2-(3-(3,5-Bis(trifluoromethyl)phenyl)thioureido)cyclohexyl)-3-(2,4-dimethoxyphenyl)thiourea:

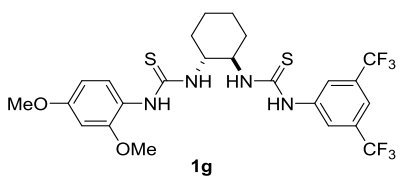

2,4-Dimethoxyaniline (153 mg, $1 \mathrm{mmol}, 1$ equiv) was added to a mixture of $\mathrm{CH}_{2} \mathrm{Cl}_{2}(5 \mathrm{~mL})$ and saturated aqueous $\mathrm{NaHCO}_{3}(5 \mathrm{~mL})$. After the biphasic mixture was cooled to $0{ }^{\circ} \mathrm{C}$, thiophosgene $(100 \mu \mathrm{L}, 1.3 \mathrm{mmol}, 1.3$ equiv) was added to the organic phase and the resulting mixture was slowly warmed to room temperature and stirred until the disappearance of the starting material $(1 \mathrm{~h})$ as judged by TLC. The reaction mixture was then extracted with $\mathrm{CH}_{2} \mathrm{Cl}_{2}$ $(3 \times 10 \mathrm{~mL})$ and the combined organic phases were dried over $\mathrm{Na}_{2} \mathrm{SO}_{4}$, concentrated, and directly used in the next step. To a solution of the crude 1-isothiocyanato-2,4-dimethoxybenzene in THF (10 mL, $0.1 \mathrm{M})$ was added amino(thio)urea ${ }^{12,13}$ (385 mg, $1 \mathrm{mmol}, 1$ equiv) and the solution was stirred at room temperature under nitrogen for 1 $\mathrm{h}$ before evaporation of the solvent. The residue was purified via flash chromatography on silica gel (eluent: EtOAc/Hexanes (30:70)), affording the desired product $\mathbf{1 g}(522 \mathrm{mg})$ as an off-white solid in $90 \%$ yield over 2 steps.

\section{Characterization data:}

$\mathbf{R}_{\mathbf{f}}=0.24$ in hexanes/EtOAc 70:30 v/v.

$[\alpha]_{\mathbf{D}}^{22}=-14.9\left(\mathrm{c} 0.5, \mathrm{CHCl}_{3}\right)$

${ }^{1}$ H-NMR $\left(600 \mathrm{MHz}, \mathrm{CDCl}_{3}\right): \delta=8.65(\mathrm{~s}, 1 \mathrm{H}), 8.02(\mathrm{~s}, 2 \mathrm{H}), 7.86(\mathrm{~s}, 1 \mathrm{H}), 7.61(\mathrm{~s}, 1 \mathrm{H}), 7.33(\mathrm{~s}, 1 \mathrm{H}), 7.11(\mathrm{~d}, J=8.5$ $\mathrm{Hz}, 1 \mathrm{H}), 6.50-6.35$ (comp, $3 \mathrm{H}), 4.41-4.21$ (comp, 2H), 3.78 (s, 3H), $3.66(\mathrm{~s}, 3 \mathrm{H}), 2.31-2.21(\mathrm{~m}, 1 \mathrm{H}), 2.19-2.10$ (m, 1H), 1.82-1.69 (comp, 2H), 1.44-1.15 (comp, 4H).

${ }^{13}$ C-NMR $\left(150 \mathrm{MHz}, \mathrm{CDCl}_{3}\right): \delta=181.0,180.4,160.7,155.0,140.3,132.0(\mathrm{q}, J=34.0 \mathrm{~Hz}), 128.1,123.6,123.3$ (q, $J=272.8 \mathrm{~Hz}), 118.4,116.7,104.9,100.0,59.3,55.7(3), 55.6(6), 32.1,32.0,24.8,24.6$.

Accurate Mass (ESI): Calculated for $\mathrm{C}_{24} \mathrm{H}_{26} \mathrm{~F}_{6} \mathrm{~N}_{4} \mathrm{O}_{2} \mathrm{~S}_{2}[\mathrm{M}-\mathrm{H}]^{-}: 579.1318$, Found: 579.1489 , Spectral Accuracy: $98.6 \%$. 
Synthesis of thiourea catalysts $\mathbf{1 h}, \mathbf{1 i}$ and $\mathbf{1 k}$ :
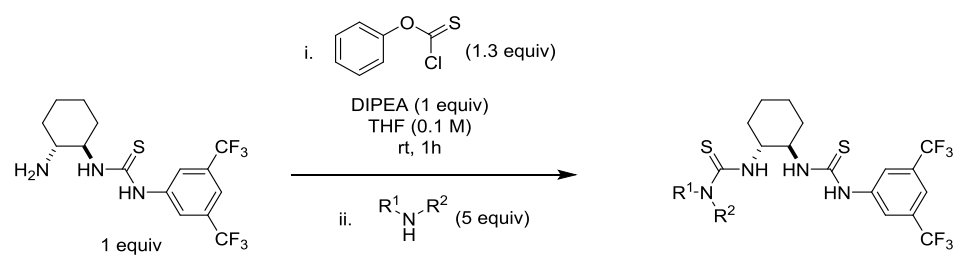

\section{General Procedure A:}

To a solution of amino(thio)urea ${ }^{12,13}(150 \mathrm{mg}, 0.39 \mathrm{mmol}, 1$ equiv) in anhydrous THF $(3.9 \mathrm{~mL}, 0.1 \mathrm{M})$ was added $o$ phenyl chlorothionoformate $(70 \mu \mathrm{L}, 0.51 \mathrm{mmol}, 1.3$ equiv) and $N, N$-diisopropylethylamine $(68 \mu \mathrm{L}, 0.39 \mathrm{mmol}, 1$ equiv). The resulting solution was stirred at room temperature for $1 \mathrm{~h}$, followed by addition of the amine (1.95 mmol, 5 equiv). The resulting mixture was stirred at the specified temperature for the indicated time. Subsequently, saturated aqueous $\mathrm{NaHCO}_{3}(10 \mathrm{~mL})$ was added and the mixture was extracted with EtOAc $(3 \times 10 \mathrm{~mL})$. The combined organic layers were washed with brine $(10 \mathrm{~mL})$, dried over $\mathrm{Na}_{2} \mathrm{SO}_{4}$ and filtered. The filtrate was then concentrated under reduced pressure, and the residue was purified by flash chromatography on silica gel. 
3-((1R,2R)-2-(3-(3,5-Bis(trifluoromethyl)phenyl)thioureido)cyclohexyl)-1,1-dimethylthiourea:

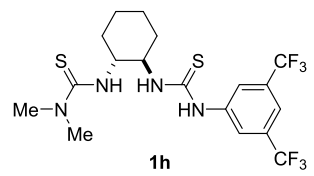

Following general procedure A, dimethylamine hydrochloride (159 mg, $1.95 \mathrm{mmol}, 5$ equiv) and $N, N$ diisopropylethylamine ( $340 \mu \mathrm{L}, 1.95 \mathrm{mmol}, 5$ equiv) were used instead of the free amine for the second step. The reaction mixture was heated und reflux for 4 hours and $\mathbf{1 h}$ was isolated as a white solid in $63 \%$ yield (116 $\mathrm{mg}$ ). EtOAc/hexanes (25:75) was used as the eluent for silica gel chromatography.

\section{Characterization data:}

$\mathbf{R}_{\mathbf{f}}=0.32$ in hexanes/EtOAc $70: 30 \mathrm{v} / \mathrm{v}$.

$[\alpha]_{\mathrm{D}}^{22}=+27.9\left(\mathrm{c} 0.5, \mathrm{CHCl}_{3}\right)$

${ }^{1}$ H-NMR $\left(600 \mathrm{MHz}, \mathrm{CDCl}_{3}\right): \delta=9.42(\mathrm{~s}, 1 \mathrm{H}), 8.23(\mathrm{~d}, J=8.5 \mathrm{~Hz}, 1 \mathrm{H}), 8.19(\mathrm{~s}, 2 \mathrm{H}), 7.58(\mathrm{~s}, 1 \mathrm{H}), 7.16(\mathrm{~d}, J=6.2$ $\mathrm{Hz}, 1 \mathrm{H}), 4.73-4.60(\mathrm{~m}, 1 \mathrm{H}), 4.12-4.00(\mathrm{~m}, 1 \mathrm{H}), 3.25(\mathrm{~s}, 6 \mathrm{H}), 2.62(\operatorname{app~d}, J=11.3 \mathrm{~Hz}, 1 \mathrm{H}), 2.23(\operatorname{app~d}, J=11.7 \mathrm{~Hz}$, $1 \mathrm{H}), 1.90(\operatorname{app~d}, J=13.0 \mathrm{~Hz}, 1 \mathrm{H}), 1.84(\mathrm{~d}, J=12.5 \mathrm{~Hz}, 1 \mathrm{H}), 1.72-1.59(\mathrm{~m}, 1 \mathrm{H}), 1.51-1.39(\mathrm{~m}, 1 \mathrm{H}), 1.38-1.21$ (comp, 2H).

${ }^{13}$ C-NMR $\left(150 \mathrm{MHz}, \mathrm{CDCl}_{3}\right): \delta=181.5,179.3,140.6,131.9\left(\mathrm{q}, J_{C-F}=33.5 \mathrm{~Hz}\right), 125.6,123.2\left(\mathrm{q}, J_{C-F}=272.9 \mathrm{~Hz}\right)$, 117.9 (comp), 62.8, 56.7, 41.2, 32.7, 32.6, 25.1, 24.8.

Accurate Mass (ESI): Calculated for $\mathrm{C}_{18} \mathrm{H}_{22} \mathrm{~F}_{6} \mathrm{~N}_{4} \mathrm{~S}_{2}[\mathrm{M}+\mathrm{H}]^{+}$: 473.1263, Found: 473.1246, Spectral Accuracy: $99.6 \%$. 


\section{3-((1R,2R)-2-(3-(3,5-Bis(trifluoromethyl)phenyl)thioureido)cyclohexyl)-1,1-diisobutylthiourea:}

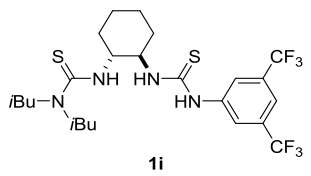

Following general procedure $\mathrm{A}$, the reaction mixture containing diisobutylamine ( $341 \mu \mathrm{L}, 1.95 \mathrm{mmol}, 5$ equiv) was heated under reflux for 4 hours and $\mathbf{1 i}$ was isolated as a white solid in $80 \%$ yield $(174 \mathrm{mg})$. EtOAc/hexanes (15:85) was used as the eluent for silica gel chromatography.

\section{Characterization data:}

$\mathbf{R}_{\mathbf{f}}=0.58$ in hexanes/EtOAc 70:30 v/v.

$[\alpha]_{\mathrm{D}}^{22}=+13.8\left(\mathrm{c} 0.5, \mathrm{CHCl}_{3}\right)$.

${ }^{1}$ H-NMR $\left(600 \mathrm{MHz}, \mathrm{CDCl}_{3}\right): \delta=8.72(\mathrm{~s}, 1 \mathrm{H}), 8.20(\mathrm{~d}, J=8.1 \mathrm{~Hz}, 1 \mathrm{H}), 7.94(\mathrm{~s}, 2 \mathrm{H}), 7.62(\mathrm{~s}, 1 \mathrm{H}), 6.34(\mathrm{~d}, J=7.4$ $\mathrm{Hz}, 1 \mathrm{H}), 4.57-4.48(\mathrm{~m}, 1 \mathrm{H}), 4.44-4.34(\mathrm{~m}, 1 \mathrm{H}), 3.63-3.46(\mathrm{~m}, 2 \mathrm{H}), 3.39-3.22(\mathrm{~m}, 2 \mathrm{H}), 2.45-2.35(\mathrm{~m}, 1 \mathrm{H}), 2.31-$ $2.21(\mathrm{~m}, 1 \mathrm{H}), 2.09-2.00$ (comp, 2H), 1.90-1.77 (comp, 2H), 1.57-1.47 (m, 1H), 1.47-1.22 (comp, 3H), 0.79 (d, $J=$ $6.7 \mathrm{~Hz}, 12 \mathrm{H})$.

${ }^{13}$ C-NMR $\left(150 \mathrm{MHz}, \mathrm{CDCl}_{3}\right): \delta=181.3,180.2,140.2,132.1\left(\mathrm{q}, J_{C-F}=33.7 \mathrm{~Hz}\right), 123.9,123.2\left(\mathrm{q}, J_{C-F}=272.7 \mathrm{~Hz}\right)$, $120.9,118.6,60.5,58.8,32.5,32.4,27.3,24.9(4), 24.8(9), 20.0(2), 19.9(5)$.

Accurate Mass (ESI): Calculated for $\mathrm{C}_{24} \mathrm{H}_{34} \mathrm{~F}_{6} \mathrm{~N}_{4} \mathrm{~S}_{2}[\mathrm{M}-\mathrm{H}]^{-}:$555.2045, Found: 555.2200, Spectral Accuracy: $99.0 \%$. 
1-((1R,2R)-2-(3-(3,5-Bis(trifluoromethyl)phenyl)thioureido)cyclohexyl)-3-methylthiourea:

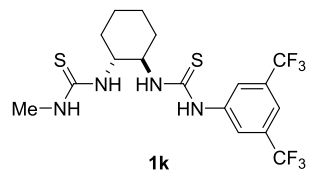

Following general procedure $\mathrm{A}$, the reaction mixture containing methylamine $(0.975 \mathrm{~mL}, 2 \mathrm{M}$ in methanol, 1.95 mmol, 5 equiv) was stirred at room temperature for 12 hours and $\mathbf{1 k}$ was isolated as a white solid in $70 \%$ yield (125 $\mathrm{mg}$ ). EtOAc/hexanes (30:70) was used as the eluent for silica gel chromatography.

\section{Characterization data:}

$\mathbf{R}_{\mathbf{f}}=0.21$ in hexanes/EtOAc $70: 30 \mathrm{v} / \mathrm{v}$.

$[\alpha]_{\mathrm{D}}^{22}=+37.9\left(\mathrm{c} 0.5, \mathrm{CHCl}_{3}\right)$.

${ }^{1}$ H-NMR $\left(400 \mathrm{MHz}, \mathrm{CDCl}_{3}\right): \delta=9.05(\mathrm{~s}, 1 \mathrm{H}), 8.03(\mathrm{~s}, 2 \mathrm{H}), 7.86(\mathrm{~s}, 1 \mathrm{H}), 7.61(\mathrm{~s}, 1 \mathrm{H}), 7.13(\mathrm{~s}, 1 \mathrm{H}), 6.31(\mathrm{~s}, 1 \mathrm{H})$, 4.65-4.33 (m, 1H), 4.25-4.00 (m, $1 \mathrm{H}), 2.91$ (s, 3H), 2.45-2.13 (comp, 2H), 1.92-1.70 (comp, 2H), 1.56-1.17 (comp, 4H).

${ }^{13}$ C-NMR $\left(100 \mathrm{MHz}, \mathrm{CDCl}_{3}\right): \delta=181.3,180.1,140.0,132.1\left(\mathrm{q}, J_{C-F}=33.6 \mathrm{~Hz}\right), 123,4,123.1\left(\mathrm{q}, J_{C-F}=272.9 \mathrm{~Hz}\right)$, 118.6, 61.6, 57.6, 32.4, 32.3, 30.7, 24.8, 24.7.

Accurate Mass (ESI): Calculated for $\mathrm{C}_{17} \mathrm{H}_{20} \mathrm{~F}_{6} \mathrm{~N}_{4} \mathrm{~S}_{2}[\mathrm{M}-\mathrm{H}]^{-}$: 457.0950, Found: 457.1221, Spectral Accuracy: $99.2 \%$. 
General Scheme for the synthesis of selenourea-thiourea catalysts $1 \mathbf{n}-\mathbf{1 q}$ :
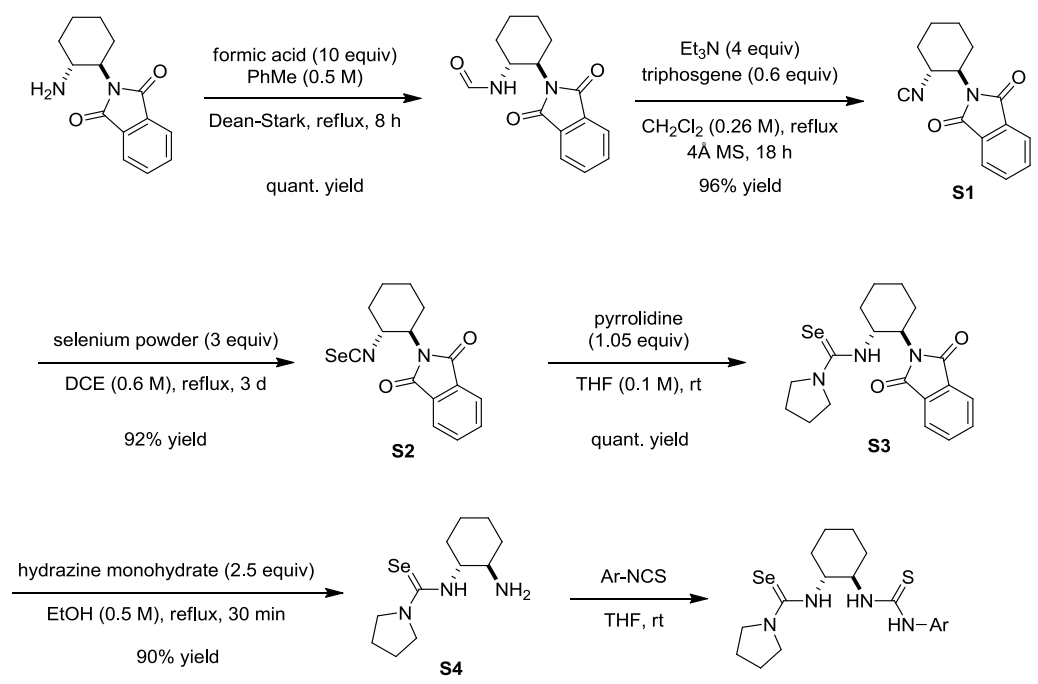


\section{2-((1R,2R)-2-Isocyanocyclohexyl)isoindoline-1,3-dione:}

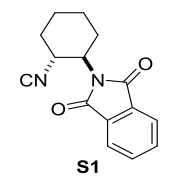

A solution of 2-((1R,2R)-2-aminocyclohexyl)isoindoline-1,3-dione ${ }^{14}(2.443 \mathrm{~g}, 10 \mathrm{mmol}, 1$ equiv) and $88 \%$ formic acid $(4.3 \mathrm{ml}, 100 \mathrm{mmol}, 10$ equiv) in toluene $(20 \mathrm{~mL}, 0.5 \mathrm{M})$ was heated under reflux with a Dean-Stark trap for $8 \mathrm{~h}$. The solvent was removed under reduced pressure to give crude product which was judged to be of sufficient purity to be used in the next step.

A solution of triphosgene ( $1.78 \mathrm{~g}, 6 \mathrm{mmol}, 0.6$ equiv) in anhydrous dichloromethane ( $13 \mathrm{~mL})$ was added dropwise under $\mathrm{N}_{2}$ over a period of 1 hour to a refluxing mixture of the formamide $\left(2.72 \mathrm{~g}, 10 \mathrm{mmol}, 1\right.$ equiv), $\mathrm{Et}_{3} \mathrm{~N}(5.58 \mathrm{~mL}$, $40 \mathrm{mmol}, 4$ equiv), and $4 \AA$ molecular sieves $(5.1 \mathrm{~g})$ in anhydrous dichloromethane $(26 \mathrm{~mL})$. After completion of the addition, the resulting mixture was heated under reflux for 18 hours. The mixture was then allowed to cool to room temperature and was filtered. The filtrate was collected and concentrated under reduced pressure. The resulting residue was purified by flash chromatography on silica gel (eluent: EtOAc/Hexanes $=15: 85$ ) to afford the title compound $\mathbf{S 1}$ as a white solid in $96 \%$ yield over 2 steps $(2.44 \mathrm{~g})$.

\section{Characterization data:}

$\mathbf{R}_{\mathbf{f}}=0.53$ in hexanes $/ \mathrm{Et}_{2} \mathrm{O} 80: 20 \mathrm{v} / \mathrm{v}$.

$[\alpha]_{\mathbf{D}}^{22}=-3.0\left(\mathrm{c} 0.5, \mathrm{CHCl}_{3}\right)$

${ }^{1}$ H-NMR $\left(500 \mathrm{MHz}, \mathrm{CDCl}_{3}\right): \delta=7.87-7.81(\mathrm{~m}, 2 \mathrm{H}), 7.76-7.70(\mathrm{~m}, 2 \mathrm{H}), 4.39($ app td, $J=11.5,4.0 \mathrm{~Hz}, 1 \mathrm{H}), 4.21$ (ddd, $J=12.2,10.8,3.9 \mathrm{~Hz}, 1 \mathrm{H}), 2.44-2.29(\mathrm{~m}, 1 \mathrm{H}), 2.16-2.05(\mathrm{~m}, 1 \mathrm{H}), 1.88-1.78(\mathrm{comp}, 3 \mathrm{H}), 1.74-1.62(\mathrm{~m}, 1 \mathrm{H})$, $1.48-1.29$ (comp, 2H).

${ }^{13}$ C-NMR $\left(125 \mathrm{MHz}, \mathrm{CDCl}_{3}\right): \delta=168.02,156.4\left(\mathrm{t}, J_{C-N}=5.2 \mathrm{~Hz}\right), 134.3,131.7,123.6,54.3,52.0\left(\mathrm{t}, J_{C-N}=6.4 \mathrm{~Hz}\right)$, $33.4,28.7,24.8,23.8$.

Accurate Mass (ESI): Calculated for $\mathrm{C}_{15} \mathrm{H}_{14} \mathrm{~N}_{2} \mathrm{O}_{2}[\mathrm{M}+\mathrm{Na}]^{+}$: 277.0947, Found: 277.0760, Spectral Accuracy: $99.2 \%$. 


\section{2-((1R,2R)-2-Isoselenocyanatocyclohexyl)isoindoline-1,3-dione:}

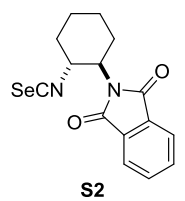

To a solution of 2-((1R,2R)-2-isocyanocyclohexyl)isoindoline-1,3-dione (S1) (2.40 g, $9.44 \mathrm{mmol}, 1$ equiv) in 1,2dichloroethane ( $16 \mathrm{~mL}, 0.6 \mathrm{M})$ was added black selenium powder $(2.24 \mathrm{~g}, 18.3 \mathrm{mmol}, 3$ equiv) at room temperature. The resulting mixture was heated under reflux for $3 \mathrm{~d}$. The mixture was then allowed to cool to room temperature and was filtered. The filtrate was collected and concentrated under reduced pressure. The resulting residue was purified by flash chromatography on silica gel (eluent: EtOAc/Hexanes $=15: 85$ ) to afford $\mathbf{S} 2$ as a white solid in $92 \%$ yield $(2.90 \mathrm{~g})$.

\section{Characterization data:}

$\mathbf{R}_{\mathbf{f}}=0.61$ in hexanes $\left./ \mathrm{Et}_{2} \mathrm{O} 80: 20 \mathrm{v} / \mathrm{v}\right)$.

$[\alpha]_{\mathrm{D}}^{22}=-10.4\left(\mathrm{c} 0.5, \mathrm{CHCl}_{3}\right)$

${ }^{1}$ H-NMR $\left(600 \mathrm{MHz}, \mathrm{CDCl}_{3}\right): \delta=7.89-7.85(\mathrm{~m}, 2 \mathrm{H}), 7.77-7.73(\mathrm{~m}, 2 \mathrm{H}), 4.61($ app td, $J=11.3,4.2 \mathrm{~Hz}, 1 \mathrm{H}), 4.21$ (ddd, $J=12.5,10.7,3.8 \mathrm{~Hz}, 1 \mathrm{H}), 2.37-2.30(\mathrm{~m}, 1 \mathrm{H}), 2.20-2.11(\mathrm{~m}, 1 \mathrm{H}), 1.89-1.81$ (comp, 3H), 1.73-1.63 (m, 1H), $1.46-1.34$ (comp, 2H).

${ }^{13}$ C-NMR (150 MHz, $\left.\mathrm{CDCl}_{3}\right): \delta=168.1,134.4,131.7,127.0,123.6,57.4,54.5,33.4,29.0,24.9,24.0$.

${ }^{77}$ Se-NMR $\left(114 \mathrm{MHz}, \mathrm{CDCl}_{3}\right): \delta=-345.32$.

HRMS (DART): Calculated for $\mathrm{C}_{15} \mathrm{H}_{14} \mathrm{~N}_{2} \mathrm{O}_{2} \mathrm{Se}\left[\mathrm{M}+\mathrm{NH}_{4}\right]^{+}: 352.0559$, Found: 352.0558 . 
Synthesis of intermediate $\mathbf{S 4}$ from $\mathbf{S 2}$ :

To a solution of 2-((1R,2R)-2-isoselenocyanatocyclohexyl)isoindoline-1,3-dione (S2) (167 mg, $0.5 \mathrm{mmol}, 1$ equiv) in anhydrous THF ( $5 \mathrm{~mL}, 0.1 \mathrm{M})$ was added pyrrolidine $(44 \mu \mathrm{L}, 0.525 \mathrm{mmol}, 1.05$ equiv). The resulting solution was then stirred at room temperature for 30 minutes before removing the solvent under reduced pressure to afford the crude selenourea $\mathbf{S 3}$, which was used directly for the next step without further purification.

A solution of the crude selenourea $\mathbf{S 3}$ and hydrazine monohydrate $(61 \mu \mathrm{L}, 1.25 \mathrm{mmol}, 2.5$ equiv) in ethanol (1 $\mathrm{mL}$, $0.5 \mathrm{M}$ ) was heated under reflux for 30 minutes. The mixture was then allowed to cool to room temperature prior to addition of diethyl ether $(10 \mathrm{~mL})$, resulting in the precipitation of phthaloyl hydrazide. The white solid was filtered and washed with diethyl ether $(2 \times 5 \mathrm{~mL})$. The resulting filtrate was evaporated to afford crude intermediate $\mathbf{S 4}$ as a light yellow solid, which was used in the following step without further purification.

Synthesis of selenourea-thiourea catalysts $\mathbf{1 n}-\mathbf{1 q}$ :

\section{General Procedure B:}

To a solution of the crude amino-selenourea $\mathbf{S 4}$ in anhydrous THF ( $5 \mathrm{~mL}, 0.1 \mathrm{M}$ ) was added aryl isothiocyanate ( $0.55 \mathrm{mmol}, 1.1$ equiv) at room temperature, and the resulting mixture was stirred at the same temperature for the indicated time. After evaporation of solvent, the resulting residue was then purified by flash chromatography on silica gel. 
$N$-((1R,2R)-2-(3-(3,5-Bis(trifluoromethyl)phenyl)thioureido)cyclohexyl)pyrrolidine-1-carboselenoamide:

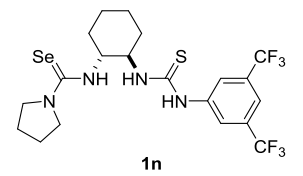

3,5-Bis(trifluoromethyl)phenyl isothiocyanate $(101 \mu \mathrm{L}, 0.55 \mathrm{mmol}, 1.1$ equiv) and $\mathbf{S 4}$ were treated according to general procedure B for 2 hours and $\mathbf{1 n}$ was isolated as an off-white solid in $87 \%$ yield $(237 \mathrm{mg})$. EtOAc/hexanes (30:70) was used as the eluent for silica gel chromatography.

\section{Characterization data:}

$\mathbf{R}_{\mathbf{f}}=0.34$ in hexanes/EtOAc $70: 30 \mathrm{v} / \mathrm{v}$.

$[\boldsymbol{\alpha}]_{\mathrm{D}}^{22}=+24.2\left(\mathrm{c} 0.5, \mathrm{CHCl}_{3}\right)$.

${ }^{1}$ H-NMR $\left(600 \mathrm{MHz}, \mathrm{CDCl}_{3}\right): \delta=9.54(\mathrm{~s}, 1 \mathrm{H}), 8.36(\mathrm{~d}, J=8.0 \mathrm{~Hz}, 1 \mathrm{H}), 8.25(\mathrm{~s}, 2 \mathrm{H}), 7.58(\mathrm{~s}, 1 \mathrm{H}), 7.15(\mathrm{~d}, J=5.5$ $\mathrm{Hz}, 1 \mathrm{H}), 4.74-4.63(\mathrm{~m}, 1 \mathrm{H}), 4.22-4.12(\mathrm{~m}, 1 \mathrm{H}), 4.06-3.94(\mathrm{~m}, 1 \mathrm{H}), 3.74-3.62(\mathrm{~m}, 1 \mathrm{H}), 3.57-3.47(\mathrm{~m}, 1 \mathrm{H}), 3.36-$ $3.24(\mathrm{~m}, 1 \mathrm{H}), 2.66(\operatorname{app~d}, J=11.5 \mathrm{~Hz}, 1 \mathrm{H}), 2.22(\operatorname{app~d}, J=11.9 \mathrm{~Hz}, 1 \mathrm{H}), 2.15-2.06(\mathrm{~m}, 1 \mathrm{H}), 2.06-1.96(\mathrm{~m}, 1 \mathrm{H})$, 1.95-1.75 (comp, 4H), 1.71-1.62 (m, 1H), 1.50-1.39 (m, 1H), 1.39-1.21 (comp, 2H).

${ }^{13}$ C-NMR $\left(150 \mathrm{MHz}, \mathrm{CDCl}_{3}\right): \delta=181.4,173.2,140.8,131.9\left(\mathrm{q}, J_{C-F}=33.6 \mathrm{~Hz}\right), 123.3\left(\mathrm{q}, J_{C-F}=272.7 \mathrm{~Hz}\right), 122.3$, 117.8, 64.4, 57.0, 55.4, 48.8, 33.1, 32.7, 26.1, 25.1, 24.8, 24.4 .

${ }^{77}$ Se-NMR $\left(114 \mathrm{MHz}, \mathrm{CDCl}_{3}\right): \delta=187.19$.

HRMS (DART): Calculated for $\mathrm{C}_{20} \mathrm{H}_{24} \mathrm{~F}_{6} \mathrm{~N}_{4} \mathrm{SSe}[\mathrm{M}+\mathrm{H}]^{+}:$547.0864, Found: 547.0881. 
$N$-((1R,2R)-2-(3-(4-Bromo-3,5-bis(trifluoromethyl)phenyl)thioureido)cyclohexyl)pyrrolidine-1carboselenoamide:

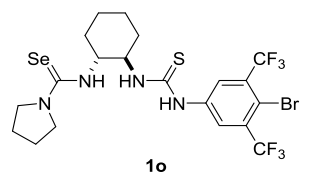

4-Bromo-3,5-bis(trifluoromethyl)phenyl isothiocyanate (193 mg, $0.55 \mathrm{mmol}, 1.1$ equiv) and $\mathbf{S 4}$ were treated according to general procedure B for 2 hours and 10 was isolated as an off-white solid in $88 \%$ yield (274 mg). EtOAc/hexanes (30:70) was used as the eluent for silica gel chromatography.

\section{Characterization data:}

$\mathbf{R}_{\mathbf{f}}=0.29$ in hexanes/EtOAc $70: 30 \mathrm{v} / \mathrm{v}$.

$[\alpha]_{\mathrm{D}}^{22}=+14.5\left(\mathrm{c} 0.5, \mathrm{CHCl}_{3}\right)$

${ }^{1} \mathbf{H}-\mathbf{N M R}\left(600 \mathrm{MHz}, \mathrm{CDCl}_{3}\right): \delta=9.72(\mathrm{~s}, 1 \mathrm{H}), 8.49(\mathrm{~d}, J=8.4 \mathrm{~Hz}, 1 \mathrm{H}), 8.37(\mathrm{~s}, 2 \mathrm{H}), 7.32(\mathrm{~d}, J=6.3 \mathrm{~Hz}, 1 \mathrm{H}), 4.76-$ $4.62(\mathrm{~m}, 1 \mathrm{H}), 4.16-4.05(\mathrm{~m}, 1 \mathrm{H}), 4.04-3.92(\mathrm{~m}, 1 \mathrm{H}), 3.73-3.58(\mathrm{~m}, 1 \mathrm{H}), 3.57-3.45(\mathrm{~m}, 1 \mathrm{H}), 3.36-3.22(\mathrm{~m}, 1 \mathrm{H})$, $2.72(\operatorname{app~d}, J=11.7 \mathrm{~Hz}, 1 \mathrm{H}), 2.21(\operatorname{app~d}, J=11.7 \mathrm{~Hz}, 1 \mathrm{H}), 2.15-2.07(\mathrm{~m}, 1 \mathrm{H}), 2.06-1.99$ (m, $1 \mathrm{H}), 1.95-1.75$ (comp, 4H), 1.76-1.66 (m, 1H), 1.50-1.38 (m, 1H), 1.38-1.19 (comp, 2H).

${ }^{13}$ C-NMR $\left(150 \mathrm{MHz}, \mathrm{CDCl}_{3}\right): \delta=181.4,172.8,139.0,132.7\left(\mathrm{q}, J_{C-F}=31.2 \mathrm{~Hz}\right), 124.21\left(\mathrm{q}, J_{C-F}=5.6 \mathrm{~Hz}\right), 122.5(\mathrm{q}$, $J=274.4 \mathrm{~Hz}), 112.3,64.7,56.6,55.4,48.9,33.0,32.8,26.0,25.1,24.7,24.4$.

${ }^{77} \mathrm{Se}-\mathrm{NMR}\left(114 \mathrm{MHz}, \mathrm{CDCl}_{3}\right): \delta=185.91$.

HRMS (DART): Calculated for $\mathrm{C}_{20} \mathrm{H}_{23} \mathrm{BrF}_{6} \mathrm{~N}_{4} \mathrm{SSe}[\mathrm{M}+\mathrm{H}]^{+}:$624.9969, Found: ${ }^{79} \mathrm{Br} 624.9959,{ }^{81} \mathrm{Br} 626.9945$. 
$N$-((1R,2R)-2-(3-(3,5-Dinitrophenyl)thioureido)cyclohexyl)pyrrolidine-1-carboselenoamide:

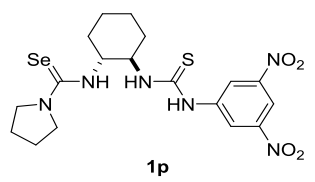

3,5-dinitrophenyl isothiocyanate (124 $\mathrm{mg}, 0.55 \mathrm{mmol}, 1.1$ equiv) and $\mathbf{S 4}$ were treated according to general procedure B for 4 hours and 1p was isolated as a yellow solid in 80\% yield (200 mg). EtOAc/hexanes (40:60) was used as the eluent for silica gel chromatography.

\section{Characterization data:}

$\mathbf{R}_{\mathbf{f}}=0.16$ in hexanes/EtOAc $70: 30 \mathrm{v} / \mathrm{v}$.

$[\alpha]_{\mathrm{D}}^{22}=+24.3\left(\mathrm{c} 0.5, \mathrm{CHCl}_{3}\right)$

${ }^{1} \mathbf{H}-\mathbf{N M R}\left(600 \mathrm{MHz}, \mathrm{CDCl}_{3}\right): \delta=9.94(\mathrm{~s}, 1 \mathrm{H}), 9.05(\mathrm{~d}, J=2.1 \mathrm{~Hz}, 2 \mathrm{H}), 8.68(\mathrm{t}, J=1.8 \mathrm{~Hz}, 1 \mathrm{H}), 8.55(\mathrm{~d}, J=8.3 \mathrm{~Hz}$, $1 \mathrm{H}), 7.08(\mathrm{~d}, J=6.6 \mathrm{~Hz}, 1 \mathrm{H}), 4.75-4.63(\mathrm{~m}, 1 \mathrm{H}), 4.25-4.15(\mathrm{~m}, 1 \mathrm{H}), 4.11-4.01(\mathrm{~m}, 1 \mathrm{H}), 3.84-3.71(\mathrm{~m}, 1 \mathrm{H}), 3.57-$ $3.45(\mathrm{~m}, 1 \mathrm{H}), 3.37-3.25(\mathrm{~m}, 1 \mathrm{H}), 2.66(\operatorname{app~d}, J=11.3 \mathrm{~Hz}, 1 \mathrm{H}), 2.25$ (app d, $J=11.9 \mathrm{~Hz}, 1 \mathrm{H}), 2.16-2.01$ (comp, 2H), 1.95-1.81 (comp, 4H), 1.75-1.64 (m, 1H), 1.52-1.40 (m, 1H), 1.38-1.25 (comp, 2H).

${ }^{13}$ C-NMR $\left(150 \mathrm{MHz} \mathrm{CDCl}_{3}\right): \delta=181.3,172.9,148.2,141.9,121.5,113.5,64.3,57.0,55.7,48.8,33.1,32.7,26.1$, $25.0,24.8,24.6$.

${ }^{77}$ Se-NMR $\left(114 \mathrm{MHz}, \mathrm{CDCl}_{3}\right): \delta=189.49$.

HRMS (DART): Calculated for $\mathrm{C}_{18} \mathrm{H}_{24} \mathrm{~N}_{6} \mathrm{O}_{4} \mathrm{SSe}[\mathrm{M}+\mathrm{H}]^{+}: 501.0818$, Found: 501.0794 . 
Scheme for the synthesis of intermediate S5:
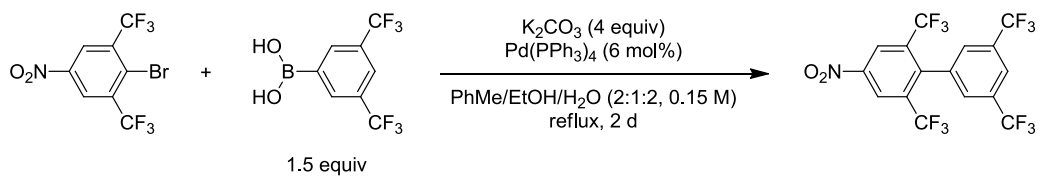

\section{4-Nitro-2,3',5',6-tetrakis(trifluoromethyl)-1,1'-biphenyl:}

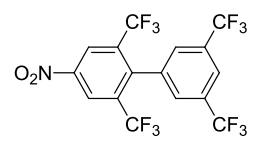

S5

Following a known procedure for a closely related product, ${ }^{15}$ to a solution of 2-bromo-5-nitro-1,3bis(trifluoromethyl)benzene $\left(0.676 \mathrm{~g}, 2 \mathrm{mmol}, 1\right.$ equiv), ${ }^{15}$ 3,5-bis(trifluoromethyl)phenylboronic acid $(0.774 \mathrm{~g}, 3$ mmol, 1.5 equiv), and $\mathrm{Pd}\left(\mathrm{PPh}_{3}\right)_{4}(0.139 \mathrm{~g}, 0.12 \mathrm{mmol}, 0.06$ equiv) in toluene $(5.33 \mathrm{~mL})$ and ethanol $(2.67 \mathrm{~mL})$, was added a solution of $\mathrm{K}_{2} \mathrm{CO}_{3}(1.106 \mathrm{~g}, 8 \mathrm{mmol}, 4$ equiv) in water $(5.33 \mathrm{~mL})$. The resulting mixture was heated at reflux for 2 days under $\mathrm{N}_{2}$. Subsequently, the mixture was extracted with EtOAc $(3 \times 10 \mathrm{~mL})$. The combined organic layers were washed with brine $(10 \mathrm{~mL})$, dried over anhydrous $\mathrm{Na}_{2} \mathrm{SO}_{4}$ and filtered. The filtrate was then concentrated under reduced pressure, and the residue was purified by flash chromatography on silica gel (eluent: $\mathrm{CH}_{2} \mathrm{Cl}_{2} /$ hexanes $\left.=10: 90-30: 70\right)$ to afford $\mathbf{S 5}$ as a yellow solid in $60 \%$ yield $(0.565 \mathrm{~g})$.

\section{Characterization data:}

$\mathbf{R}_{\mathbf{f}}=0.64$ in hexanes $/ \mathrm{Et}_{2} \mathrm{O} 80: 20 \mathrm{v} / \mathrm{v}$.

${ }^{1}$ H-NMR $\left(500 \mathrm{MHz}, \mathrm{CDCl}_{3}\right): \delta=8.88(\mathrm{~s}, 2 \mathrm{H}), 8.04(\mathrm{~s}, 1 \mathrm{H}), 7.75(\mathrm{~s}, 2 \mathrm{H})$.

${ }^{13}$ C-NMR $\left(125 \mathrm{MHz}, \mathrm{CDCl}_{3}\right): \delta=147.7,142.9,134.1,133.8\left(\mathrm{q}, J_{C-F}=31.9 \mathrm{~Hz}\right), 131.5\left(\mathrm{q}, J_{C-F}=34.1 \mathrm{~Hz}\right), 129.9$, $124.9\left(\mathrm{q}, J_{C-F}=5.6 \mathrm{~Hz}\right), 123.7$ (hept, $\left.J_{C-F}=3.6 \mathrm{~Hz}\right), 123.0\left(\mathrm{q}, J_{C-F}=272.9 \mathrm{~Hz}\right), 121.9\left(\mathrm{q}, J_{C-F}=275.3 \mathrm{~Hz}\right)$.

${ }^{19}$ F-NMR $\left(470 \mathrm{MHz}, \mathrm{CDCl}_{3}\right): \delta=-58.07,-63.27$.

${ }^{14} \mathrm{~N}-\mathrm{NMR}\left(43 \mathrm{MHz}, \mathrm{CDCl}_{3}\right): \delta=-16.55$.

HRMS (ESI-TOF): Calculated for $\mathrm{C}_{16} \mathrm{H}_{5} \mathrm{~F}_{12} \mathrm{NO}_{2}[\mathrm{M}+\mathrm{H}]^{+}$: 472.0207, Found: 472.0197. 
Scheme for the synthesis of intermediate $\mathbf{S 7}$ :

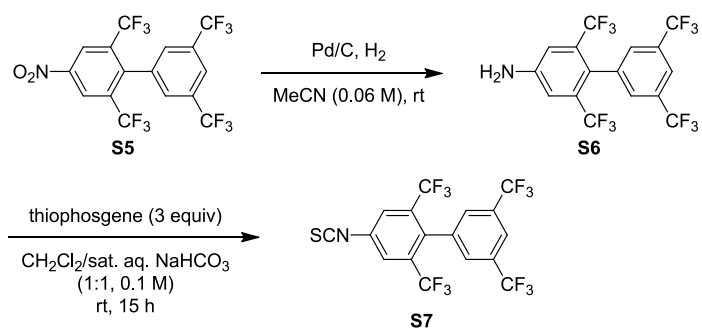

To a round-bottom flask equipped with a magnetic stirrer, 4-nitro-2,3',5',6-tetrakis(trifluoromethyl)-1,1'-biphenyl (S5) $(0.565 \mathrm{~g}, 1.2 \mathrm{mmol}, 1$ equiv) was added. The flask was purged with nitrogen before $10 \% \mathrm{Pd} / \mathrm{C}$ (64 mg, 0.06 mmol, 0.05 equiv) and $\mathrm{CH}_{3} \mathrm{CN}(20 \mathrm{~mL}, 0.06 \mathrm{M})$ were added. Subsequently, the flask was flushed with hydrogen gas and the reaction mixture was stirred under a hydrogen atmosphere for 12 hours. Subsequently, the catalyst was filtered off over Celite and the solvent was evaporated to yield crude product S6, which was used directly in the next step.

To a $25 \mathrm{~mL}$ round-bottom flask was added crude 2,3',5',6-tetrakis(trifluoromethyl)-[1,1'-biphenyl]-4-amine (S6) (1 equiv), $\mathrm{CH}_{2} \mathrm{Cl}_{2}(6 \mathrm{~mL})$ and saturated aqueous $\mathrm{NaHCO}_{3}(6 \mathrm{~mL})$. The resulting reaction mixture was cooled in an ice bath. Thiophosgene $(0.28 \mathrm{~mL}, 3.6 \mathrm{mmol}, 3$ equiv) was slowly added to the organic layer via syringe. The resulting mixture was then allowed to warm to room temperature and stirred until S6 was consumed as indicated by TLC (15 h). The layers were then separated and the aqueous layer was extracted with $\mathrm{CH}_{2} \mathrm{Cl}_{2}(3 \times 10 \mathrm{~mL})$. The combined organic layers were dried over anhydrous $\mathrm{Na}_{2} \mathrm{SO}_{4}$ and the solvent was removed under reduced pressure to afford crude product S7 (564 mg, 97\%) as a white solid, which was used directly in the next step without further purification. 
$N$-((1R,2R)-2-(3-(2,3',5',6-Tetrakis(trifluoromethyl)-[1,1'-biphenyl]-4-yl)thioureido)cyclohexyl)pyrrolidine-1carboselenoamide:

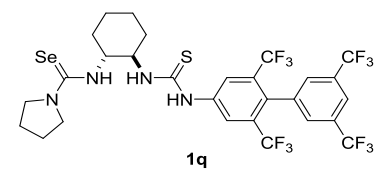

Compound S7 (266 mg, $0.55 \mathrm{mmol}, 1.1$ equiv) was treated according to general procedure B for 2 hours and 1q was isolated as an off-white solid in $88 \%$ yield (334 mg). EtOAc/hexanes (30:70) was used as the eluent for silica gel chromatography.

\section{Characterization data:}

$\mathbf{R}_{\mathbf{f}}=0.29$ in hexanes/EtOAc $70: 30 \mathrm{v} / \mathrm{v}$.

$[\alpha]_{\mathrm{D}}^{22}=+4.4\left(\mathrm{c} 0.5, \mathrm{CHCl}_{3}\right)$.

${ }^{1} \mathbf{H}-\mathbf{N M R}\left(600 \mathrm{MHz}, \mathrm{CDCl}_{3}\right): \delta=9.69(\mathrm{~s}, 1 \mathrm{H}), 8.54(\mathrm{~d}, J=8.3 \mathrm{~Hz}, 1 \mathrm{H}), 8.45(\mathrm{~s}, 2 \mathrm{H}), 7.95(\mathrm{~s}, 1 \mathrm{H}), 7.71(\mathrm{~s}, 2 \mathrm{H}), 7.20$ $(\mathrm{d}, J=6.5 \mathrm{~Hz}, 1 \mathrm{H}), 4.80-4.65(\mathrm{~m}, 1 \mathrm{H}), 4.27-4.14(\mathrm{~m}, 1 \mathrm{H}), 4.10-3.94(\mathrm{~m}, 1 \mathrm{H}), 3.79-3.64(\mathrm{~m}, 1 \mathrm{H}), 3.63-3.48(\mathrm{~m}$, 1H), 3.41-3.26 (m, 1H), 2.70 (app d, $J=11.4 \mathrm{~Hz}, 1 \mathrm{H}), 2.27$ (d, $J=11.3 \mathrm{~Hz}, 1 \mathrm{H}), 2.18-1.98$ (comp, 2H), $1.98-1.80$ (comp, 4H), 1.78-1.68 (m, 1H), 1.54-1.42 (m, 1H), 1.42-1.22 (comp, 2H).

${ }^{13}$ C-NMR $\left(150 \mathrm{MHz}, \mathrm{CDCl}_{3}\right): \delta=181.5,173.2,140.4,136.0,131.6(\mathrm{q}, J=30.2 \mathrm{~Hz}), 131.4,130.9$ (q, $\left.J=33.7 \mathrm{~Hz}\right)$, 130.7, $123.2(\mathrm{q}, J=272.6 \mathrm{~Hz}), 122.9(\mathrm{q}, J=274.8 \mathrm{~Hz}), 122.7$ (comp), 64.5, 57.1, 55.4, 48.8, 33.1, 32.8, 26.1, 25.1, $24.8,24.4$.

${ }^{77}$ Se-NMR $\left(114 \mathrm{MHz}, \mathrm{CDCl}_{3}\right): \delta=187.71$.

HRMS (DART): Calculated for $\mathrm{C}_{28} \mathrm{H}_{26} \mathrm{~F}_{12} \mathrm{~N}_{4} \mathrm{SSe}[\mathrm{M}+\mathrm{H}]^{+}$: 759.0925, Found: 759.0924 . 
Crystal structure of catalyst 1q:

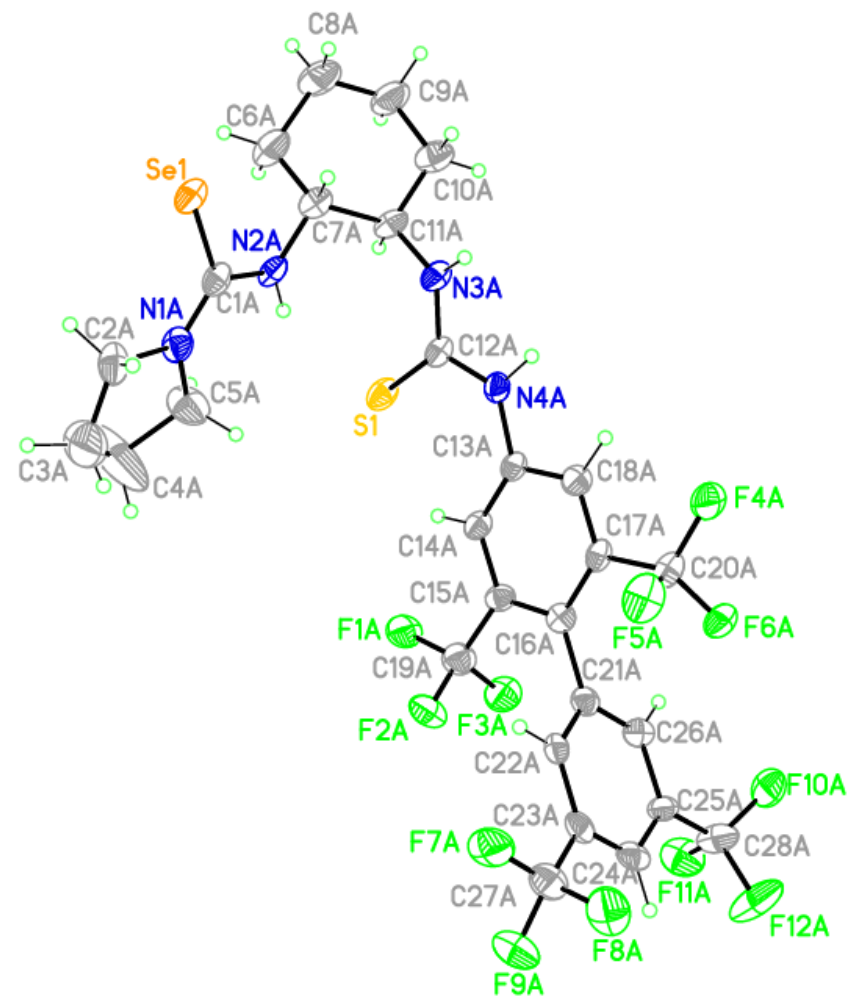

Compound 1q was crystallized from acetone/hexanes through slow evaporation at room temperature. The requisite CIF has been deposited with the CCDC (deposition \# 1938865). 


\section{Preparation and Characterization of Products From the Conjugate Addition of Amines}

\section{General Procedure C:}

Under $\mathrm{N}_{2}$, a vial was charged with $\alpha, \beta$-unsaturated ester ( 0.4 mmol, 2 equiv) and selenourea-thiourea catalyst $1 \mathbf{q}(15$ $\mathrm{mg}, 0.02 \mathrm{mmol}, 0.1$ equiv). Anhydrous toluene $(1 \mathrm{~mL}, 0.2 \mathrm{M})$ was added and the resulting mixture was cooled to the indicated temperature over $15 \mathrm{~min}$. The amine was then added $(0.2 \mathrm{mmol}, 1$ equiv) and the reaction mixture was stirred at the indicated temperature until the amine was judged to be consumed as indicated by TLC analysis. The reaction mixture was then directly purified by flash chromatography on silica gel. 
benzyl $(R)-3-($ piperidin-1-yl)butanoate:

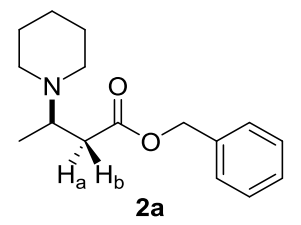

Following general procedure $\mathbf{C}$, the reaction was stirred at $-10^{\circ} \mathrm{C}$ for 3 days and $\mathbf{2 a}$ was isolated as a colorless oil in $90 \%$ yield $(47 \mathrm{mg})$.

\section{Characterization data:}

$\mathbf{R}_{\mathbf{f}}=0.26$ in $\mathrm{MeOH} / \mathrm{CH}_{2} \mathrm{Cl}_{2} 5: 95 \mathrm{v} / \mathrm{v}$.

$[\alpha]_{\mathbf{D}}^{22}=-3.8\left(\mathrm{c} 0.5, \mathrm{CHCl}_{3}, 93 \% e e\right)$.

${ }^{1}$ H-NMR $\left(600 \mathrm{MHz}, \mathrm{CDCl}_{3}\right): \delta=7.38-7.34(\mathrm{comp}, 4 \mathrm{H}), 7.34-7.30(\mathrm{~m}, 1 \mathrm{H}), 5.13(\mathrm{~d}, J=12.4 \mathrm{~Hz}, 1 \mathrm{H}), 5.11(\mathrm{~d}, J=$ $12.4 \mathrm{~Hz}, 1 \mathrm{H}), 3.15$ (app dt, $J=7.8,6.6 \mathrm{~Hz}, 1 \mathrm{H}), 2.63(\mathrm{dd}, J=14.3,6.3 \mathrm{~Hz}, 1 \mathrm{H}), 2.49-2.37$ (comp, 4H), $2.28(\mathrm{dd}, J=$ $14.3,8.2 \mathrm{~Hz}, 1 \mathrm{H}), 1.57-1.46$ (comp, 4H), 1.43-1.35 (comp, 2H), 1.03 (d, J=6.7 Hz, 3H).

${ }^{1} \mathbf{H}-\mathbf{N M R}\left(500 \mathrm{MHz}\right.$, toluene-d $\left.{ }_{8}\right): \delta=7.31-7.27(\mathrm{~m}, 2 \mathrm{H}), 7.22-7.17(\mathrm{~m}, 2 \mathrm{H}), 7.16-7.13(\mathrm{~m}, 1 \mathrm{H}), 5.10(\mathrm{~d}, J=12.4$ $\mathrm{Hz}, 1 \mathrm{H}), 5.06(\mathrm{~d}, J=12.4 \mathrm{~Hz}, 1 \mathrm{H}), 3.21(\mathrm{app} \mathrm{h}, J=6.9 \mathrm{~Hz}, 1 \mathrm{H}), 2.52\left(\mathrm{H}_{\mathrm{b}}, \mathrm{dd}, J=14.2,7.1 \mathrm{~Hz}, 1 \mathrm{H}\right), 2.40-2.32$ (comp, 2H), 2.31-2.23 (comp, 2H), $2.15\left(\mathrm{H}_{\mathrm{a}}\right.$, dd, $\left.J=14.2,7.7 \mathrm{~Hz}, 1 \mathrm{H}\right), 1.58-1.44$ (comp, 4H), 1.41-1.31 (comp, $2 \mathrm{H}), 0.95(\mathrm{~d}, J=6.6 \mathrm{~Hz}, 3 \mathrm{H})$.

${ }^{13}$ C-NMR $\left(150 \mathrm{MHz}, \mathrm{CDCl}_{3}\right): \delta=172.9,136.3,128.6,128.3,128.2,66.2,57.2,49.5,38.4,26.5,25.0,15.1$.

Accurate Mass (ESI): Calculated for $\mathrm{C}_{16} \mathrm{H}_{24} \mathrm{NO}_{2}[\mathrm{M}+\mathrm{H}]^{+}:$262.1802, Found: 262.1679, Spectral Accuracy: $99.0 \%$.

HPLC: Daicel Chiralcel OB-H, $n$-hexane $/ i-\mathrm{PrOH}=97 / 3$, Flow rate $=1 \mathrm{~mL} / \mathrm{min}, \mathrm{UV}=230 \mathrm{~nm}, \mathrm{t}_{\mathrm{R}}=5.0 \mathrm{~min}(\mathrm{major})$ and $t_{R}=6.4 \mathrm{~min}$.

The absolute configuration was assigned by analogy. 


\section{Benzyl (R)-3-(4-phenylpiperidin-1-yl)butanoate:}

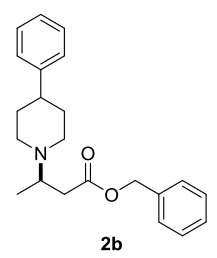

4-Phenylpiperidine ( $32 \mathrm{mg}, 0.2 \mathrm{mmol}, 1$ equiv) and benzyl crotonate (70 mg, $0.4 \mathrm{mmol}, 2$ equiv) were treated according to general procedure $\mathrm{C}$ at $-10{ }^{\circ} \mathrm{C}$ for 5 days. Purification by flash chromatography on silica gel eluting with $\mathrm{Et}_{2} \mathrm{O} / \mathrm{Hexanes} / \mathrm{Et}_{3} \mathrm{~N}$ (40:59:1) afforded $\mathbf{2 b}$ as a colorless oil in $80 \%$ yield (54 $\mathrm{mg}$ ).

\section{Characterization data:}

$\mathbf{R}_{\mathbf{f}}=0.29$ in $\mathrm{MeOH} / \mathrm{CH}_{2} \mathrm{Cl}_{2} 5: 95 \mathrm{v} / \mathrm{v}$.

$[\alpha]_{\mathrm{D}}^{22}=-2.3\left(\mathrm{c} 0.5, \mathrm{CHCl}_{3}, 89 \% e e\right)$.

${ }^{1}$ H-NMR (500 MHz, CDCl $): \delta=7.41-7.27$ (comp, 7H), 7.24-7.17 (comp, 3H), 5.15 (s, 2H), 3.31-3.21 (m, 1H), 2.94-2.85 (comp, 2H), 2.68 (dd, $J=14.3,6.5 \mathrm{~Hz}, 1 \mathrm{H}$ ), 2.44 (app tt, $J=12.6,3.9 \mathrm{~Hz}, 1 \mathrm{H}$ ), 2.40-2.25 (comp, 3H), 1.85-1.77 (comp, 2H), 1.75-7.62 (comp, 2H), 1.09 (d, $J=6.7 \mathrm{~Hz}, 3 \mathrm{H})$.

${ }^{13}$ C-NMR $\left(125 \mathrm{MHz}, \mathrm{CDCl}_{3}\right): \delta=172.7,146.6,136.3,128.7,128.5,128.4,128.3,127.0,126.2,66.3,56.9,50.2$, $48.3,43.2,38.6,34.1,33.8,15.1$.

Accurate Mass (ESI): Calculated for $\mathrm{C}_{22} \mathrm{H}_{27} \mathrm{NO}_{2}[\mathrm{M}+\mathrm{H}]^{+}: 338.2115$, Found: 338.2214, Spectral Accuracy: $98.1 \%$.

HPLC: Daicel Chiralcel OB-H, $n$-hexane $/ i$-PrOH $=97 / 3$, Flow rate $=1 \mathrm{~mL} / \mathrm{min}, \mathrm{UV}=230 \mathrm{~nm}, \mathrm{t}_{\mathrm{R}}=8.1 \mathrm{~min}$ and $\mathrm{t}_{\mathrm{R}}$ $=10.6 \min$ (major).

The absolute configuration was assigned by analogy. 


\section{Benzyl (R)-3-(4-(4-bromophenyl)piperidin-1-yl)butanoate:}

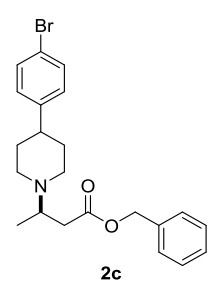

4-(4-Bromophenyl)piperidine ( $48 \mathrm{mg}, 0.2 \mathrm{mmol}, 1$ equiv) and benzyl crotonate (70 mg, $0.4 \mathrm{mmol}, 2$ equiv) were treated according to general procedure $\mathrm{C}$ at $-10{ }^{\circ} \mathrm{C}$ for 7 days. Purification by flash chromatography on silica gel eluting with $\mathrm{Et}_{2} \mathrm{O} / \mathrm{Hexanes} / \mathrm{Et}_{3} \mathrm{~N}$ (30:69:1) afforded $\mathbf{2 c}$ as a colorless oil in $93 \%$ yield $(77 \mathrm{mg})$.

\section{Characterization data:}

$\mathbf{R}_{\mathbf{f}}=0.26$ in $\mathrm{MeOH} / \mathrm{CH}_{2} \mathrm{Cl}_{2} 5: 95 \mathrm{v} / \mathrm{v}$.

$[\boldsymbol{\alpha}]_{\mathrm{D}}^{22}=+2.3\left(\mathrm{c} 0.5, \mathrm{CHCl}_{3}, 90 \% e e\right)$

${ }^{1}$ H-NMR (500 MHz, $\mathrm{CDCl}_{3}$ ): $\delta=7.43-7.29$ (comp, 7H), 7.06 (d, $J=8.3 \mathrm{~Hz}, 2 \mathrm{H}$ ), 5.15 (s, 2H), 3.24 (app dt, $J=7.8$, $6.6 \mathrm{~Hz}, 1 \mathrm{H}), 2.92-2.82(\mathrm{comp}, 2 \mathrm{H}), 2.65(\mathrm{dd}, J=14.3,6.7 \mathrm{~Hz}, 1 \mathrm{H}), 2.43-2.29$ (comp, 3H), 2.26 (app td, $J=11.5$, $2.2 \mathrm{~Hz}, 1 \mathrm{H}), 1.81-1.73$ (comp, 2H), 1.67-1.56 (comp, 2H), 1.07 (d, J = 6.7 Hz, 3H).

${ }^{13}$ C-NMR $\left(125 \mathrm{MHz}, \mathrm{CDCl}_{3}\right): \delta=172.7,145.6,136.3,131.5,128.8,128.7,128.4,128.3,119.8,66.3,56.9,50.2$, 48.1, 42.6, 38.7, 34.0, 33.7, 15.0.

Accurate Mass (ESI): Calculated for $\mathrm{C}_{22} \mathrm{H}_{26} \mathrm{BrNO}_{2}[\mathrm{M}+\mathrm{H}]^{+}:{ }^{79} \mathrm{Br}, 416.1220$, Found: $416.1245,{ }^{81} \mathrm{Br}$, Found: 418.1193, Spectral Accuracy: 98.7\%.

HPLC: Daicel Chiralpak AD-H, $n$-hexane $/ \mathrm{i}-\mathrm{PrOH}=95 / 5$, Flow rate $=1 \mathrm{~mL} / \mathrm{min}, \mathrm{UV}=254 \mathrm{~nm}, \mathrm{t}_{\mathrm{R}}=6.1$ min (major) and $t_{R}=6.9 \mathrm{~min}$.

The absolute configuration was assigned by analogy. 


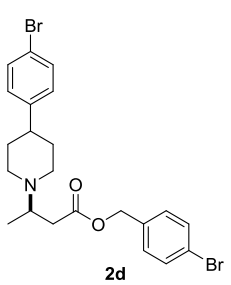

4-(4-Bromophenyl)piperidine ( $48 \mathrm{mg}, 0.2 \mathrm{mmol}, 1$ equiv) and 4-bromobenzyl crotonate (102 mg, $0.4 \mathrm{mmol}, 2$ equiv) were treated according to general procedure $\mathrm{C}$ at $-10{ }^{\circ} \mathrm{C}$ for 6 days. Purification by flash chromatography on silica gel eluting with $\mathrm{Et}_{2} \mathrm{O} / \mathrm{Hexanes} / \mathrm{Et}_{3} \mathrm{~N}$ (30:69:1) afforded 2d as a colorless oil in 85\% yield (84 mg).

\section{Characterization data:}

$\mathbf{R}_{\mathbf{f}}=0.29$ in $\mathrm{MeOH} / \mathrm{CH}_{2} \mathrm{Cl}_{2} 5: 95 \mathrm{v} / \mathrm{v}$.

$[\alpha]_{\mathrm{D}}^{22}=-0.82\left(\mathrm{c} 0.5, \mathrm{CHCl}_{3}, 89 \% e e\right)$.

${ }^{1}$ H-NMR $\left(500 \mathrm{MHz}, \mathrm{CDCl}_{3}\right): \delta=7.48(\mathrm{~d}, J=8.3 \mathrm{~Hz}, 2 \mathrm{H}), 7.41(\mathrm{~d}, J=8.3 \mathrm{~Hz}, 2 \mathrm{H}), 7.25(\mathrm{~d}, J=8.3 \mathrm{~Hz}, 2 \mathrm{H}), 7.06(\mathrm{~d}$, $J=8.4 \mathrm{~Hz}, 2 \mathrm{H}), 5.09(\mathrm{~s}, 2 \mathrm{H}), 3.29-3.12(\mathrm{~m}, 1 \mathrm{H}), 2.93-2.80(\mathrm{comp}, 2 \mathrm{H}), 2.64(\mathrm{dd}, J=14.3,6.8 \mathrm{~Hz}, 1 \mathrm{H}), 2.44-2.29$ (comp, 3H), 2.29-2.21 (m, 1H), 1.82-1.71 (comp, 2H), 1.67-1.51 (comp, 2H), 1.06 (d, $J=6.6 \mathrm{~Hz}, 3 \mathrm{H})$.

${ }^{13}$ C-NMR $\left(125 \mathrm{MHz}, \mathrm{CDCl}_{3}\right): \delta=172.5,145.5,135.3,131.8,131.6,130.1,128.7,122.3,119.8,65.4,57.0,50.4$, $47.8,42.6,38.7,33.9,33.7,14.8$.

Accurate Mass (ESI): Calculated for $\mathrm{C}_{22} \mathrm{H}_{25} \mathrm{Br}_{2} \mathrm{NO}_{2}[\mathrm{M}+\mathrm{H}]+{ }^{+}:{ }^{79} \mathrm{Br},{ }^{79} \mathrm{Br}, 494.0325$, Found: $494.0387,{ }^{79} \mathrm{Br},{ }^{81} \mathrm{Br}$, Found: 496.0419, ${ }^{81} \mathrm{Br},{ }^{81} \mathrm{Br}$, Found: 498.0340, Spectral Accuracy: $98.5 \%$.

HPLC: Daicel Chiralpak AS-H, $n$-hexane $/ i$-PrOH $=97 / 3$, Flow rate $=1 \mathrm{~mL} / \mathrm{min}, \mathrm{UV}=230 \mathrm{~nm}, \mathrm{t}_{\mathrm{R}}=5.8 \mathrm{~min}$ and $\mathrm{t}_{\mathrm{R}}$ $=6.7 \mathrm{~min}$ (major).

The absolute configuration of $\mathbf{2} \mathbf{d}$ was assigned by X-ray crystallography: 
Crystal structure of compound $\mathbf{2 d}$ :

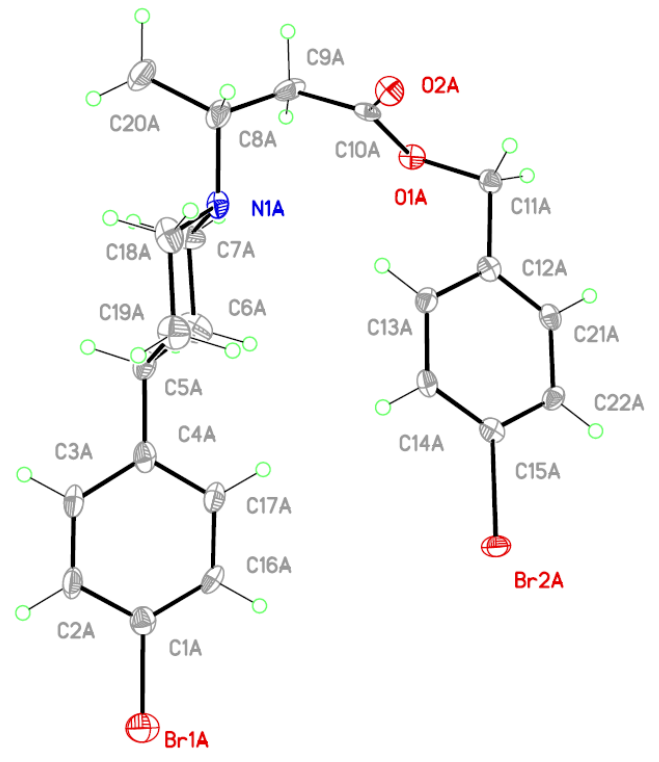

Compound 2d was crystallized from $\mathrm{MeCN}$ through slow evaporation at $4{ }^{\circ} \mathrm{C}$. The requisite $\mathrm{CIF}$ has been deposited with the CCDC (deposition \# 1938864). 


\section{Benzyl (R)-3-(4-benzylpiperidin-1-yl)butanoate:}

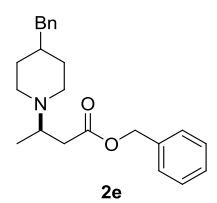

4-Benzylpiperidine ( $35 \mathrm{mg}, 0.2 \mathrm{mmol}, 1$ equiv) and benzyl crotonate $(70 \mathrm{mg}, 0.4 \mathrm{mmol}, 2$ equiv) were treated according to general procedure $\mathrm{C}$ at $-10{ }^{\circ} \mathrm{C}$ for 5 days. Purification by flash chromatography on silica gel eluting with $\mathrm{Et}_{2} \mathrm{O} / \mathrm{Hexanes} / \mathrm{Et}_{3} \mathrm{~N}$ (30:69:1) afforded $2 \mathbf{e}$ as a colorless oil in $94 \%$ yield (66 mg).

\section{Characterization data:}

$\mathbf{R}_{\mathbf{f}}=0.26$ in $\mathrm{MeOH} / \mathrm{CH}_{2} \mathrm{Cl}_{2} 5: 95 \mathrm{v} / \mathrm{v}$.

$[\boldsymbol{\alpha}]_{\mathrm{D}}^{22}=-2.8\left(\mathrm{c} 0.5, \mathrm{CHCl}_{3}, 92 \% e e\right)$.

${ }^{1}$ H-NMR $\left(500 \mathrm{MHz}, \mathrm{CDCl}_{3}\right.$ ): $\delta=7.39-7.30$ (comp, 5H), 7.27 (app t, $\left.J=7.4 \mathrm{~Hz}, 2 \mathrm{H}\right), 7.18(\mathrm{t}, J=7.4 \mathrm{~Hz}, 1 \mathrm{H}$ ), 7.13 (d, $J=7.0 \mathrm{~Hz}, 2 \mathrm{H}), 5.12$ (s, 2H), 3.17 (app dq, $J=8.2,6.5 \mathrm{~Hz}, 1 \mathrm{H}$ ), 2.78-2.69 (comp, 2H), 2.61 (dd, $J=14.3,6.4$ $\mathrm{Hz}, 1 \mathrm{H}), 2.50(\mathrm{~d}, J=7.2 \mathrm{~Hz}, 2 \mathrm{H}), 2.28(\mathrm{dd}, J=14.3,8.0 \mathrm{~Hz}, 1 \mathrm{H}), 2.15$ (app td, $J=11.6,2.5 \mathrm{~Hz}, 1 \mathrm{H}), 2.09$ (app td, $J$ $=11.5,2.5 \mathrm{~Hz}, 1 \mathrm{H}), 1.67-1.56(\mathrm{comp}, 2 \mathrm{H}), 1.46(\mathrm{app} t \mathrm{tt}, J=11.1,7.4,3.8 \mathrm{~Hz}, 1 \mathrm{H}), 1.26-1.12(\mathrm{comp}, 2 \mathrm{H}), 1.02(\mathrm{~d}$, $J=6.6 \mathrm{~Hz}, 3 \mathrm{H})$.

${ }^{13}$ C-NMR $\left(125 \mathrm{MHz}, \mathrm{CDCl}_{3}\right): \delta=172.8,140.9,136.3,129.2,128.6,128.4,128.3,128.3,125.9,66.2,56.9,49.6$, $48.0,43.4,38.5,38.5,32.8,32.6,15.1$.

Accurate Mass (ESI): Calculated for $\mathrm{C}_{23} \mathrm{H}_{29} \mathrm{NO}_{2}[\mathrm{M}+\mathrm{H}]^{+}:$352.2271, Found: 352.2322, Spectral Accuracy: 99.2\%.

HPLC: Daicel Chiralcel OJ-H, $n$-hexane $/ \mathrm{i}-\mathrm{PrOH}=90 / 10$, Flow rate $=1 \mathrm{~mL} / \mathrm{min}, \mathrm{UV}=230 \mathrm{~nm}, \mathrm{t}_{\mathrm{R}}=11.2 \mathrm{~min}$ and $\mathrm{t}_{\mathrm{R}}=17.4$ min (major).

The absolute configuration was assigned by analogy. 


\section{Benzyl (R)-3-(4-hydroxypiperidin-1-yl)butanoate:}

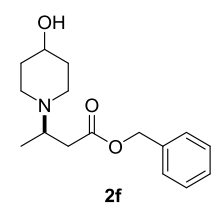

4-Hydroxypiperidine (20 mg, $0.2 \mathrm{mmol}, 1$ equiv) and benzyl crotonate $(70 \mathrm{mg}, 0.4 \mathrm{mmol}, 2$ equiv) were treated according to general procedure $\mathrm{C}$ at $-10{ }^{\circ} \mathrm{C}$ for 6 days. Purification by flash chromatography on silica gel eluting with EtOAc/Hexanes (40:60) followed by $\mathrm{MeOH} / \mathrm{CH}_{2} \mathrm{Cl}_{2}$ (10:90) afforded $\mathbf{2 f}$ as a colorless oil in $45 \%$ yield (24 $\mathrm{mg}$ ).

\section{Characterization data:}

$\mathbf{R}_{\mathbf{f}}=0.15$ in $\mathrm{MeOH} / \mathrm{CH}_{2} \mathrm{Cl}_{2} 5: 95 \mathrm{v} / \mathrm{v}$.

$[\boldsymbol{\alpha}]_{\mathrm{D}}^{22}=-3.6\left(\mathrm{c} 0.5, \mathrm{CHCl}_{3}, 92 \% e e\right)$.

${ }^{1}$ H-NMR (400 MHz, $\mathrm{CDCl}_{3}$ ): $\delta=7.42-7.28$ (comp, 5H), 5.12 (s, 2H), 3.62 (app tt, $J=8.8,4.2 \mathrm{~Hz}, 1 \mathrm{H}$ ), 3.21 (app hept, $J=6.8 \mathrm{~Hz}, 1 \mathrm{H}), 2.78-2.65$ (comp, $2 \mathrm{H}), 2.60$ (dd, $J=14.3,6.8 \mathrm{~Hz}, 1 \mathrm{H}), 2.37-2.18$ (comp, 3H), 1.91-1.69 (comp, 3H), 1.56-1.40 (comp, 2H), 1.03 (d, $J=6.7 \mathrm{~Hz}, 3 \mathrm{H})$.

${ }^{13}$ C-NMR $\left(100 \mathrm{MHz}, \mathrm{CDCl}_{3}\right): \delta=172.6,136.2,128.7,128.4,128.3,68.4,66.3,56.7,46.6,45.2,38.8,35.0,34.9$, 14.9.

Accurate Mass (ESI): Calculated for $\mathrm{C}_{16} \mathrm{H}_{23} \mathrm{NO}_{3}[\mathrm{M}+\mathrm{H}]^{+}:$278.1751, Found: 278.1655, Spectral Accuracy: 98.8\%.

HPLC: Daicel Chiralcel OB-H, $n$-hexane $/ i-\mathrm{PrOH}=97 / 3$, Flow rate $=1 \mathrm{~mL} / \mathrm{min}, \mathrm{UV}=230 \mathrm{~nm}, \mathrm{t}_{\mathrm{R}}=29.6 \mathrm{~min}$ (major) and $\mathrm{t}_{\mathrm{R}}=36.6 \mathrm{~min}$.

The absolute configuration was assigned by analogy. 


\section{Benzyl (R)-3-(4-(benzyloxy)piperidin-1-yl)butanoate:}

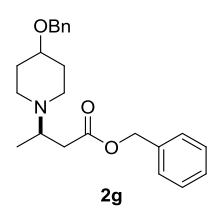

4-(Benzyloxy)piperidine (38 mg, $0.2 \mathrm{mmol}, 1$ equiv) and benzyl crotonate $(70 \mathrm{mg}, 0.4 \mathrm{mmol}, 2$ equiv) were treated according to general procedure $\mathrm{C}$ at $-10{ }^{\circ} \mathrm{C}$ for 6 days. Purification by flash chromatography on silica gel eluting with $\mathrm{Et}_{2} \mathrm{O} / \mathrm{Hexanes} / \mathrm{Et}_{3} \mathrm{~N}$ (40:59:1) afforded $\mathbf{2 g}$ as a colorless oil in $91 \%$ yield $(67 \mathrm{mg})$.

\section{Characterization data:}

$\mathbf{R}_{\mathbf{f}}=0.29 \mathrm{in} \mathrm{MeOH} / \mathrm{CH}_{2} \mathrm{Cl}_{2} 5: 95 \mathrm{v} / \mathrm{v}$.

$[\alpha]_{\mathbf{D}}^{22}=-6.8\left(\mathrm{c} 0.5, \mathrm{CHCl}_{3}, 90 \% e e\right)$.

${ }^{1} \mathbf{H}-\mathbf{N M R}\left(600 \mathrm{MHz}, \mathrm{CDCl}_{3}\right): \delta=7.38-7.30$ (comp, 9H), 7.29-7.25 (m, 1H), $5.14(\mathrm{~d}, J=12.4 \mathrm{~Hz}, 1 \mathrm{H}), 5.11(\mathrm{~d}, J=$ $12.4 \mathrm{~Hz}, 1 \mathrm{H}), 4.53$ (s, 2H), 3.36 (app tt, $J=8.5,4.0 \mathrm{~Hz}, 1 \mathrm{H}), 3.21$ (app h, $J=6.8 \mathrm{~Hz}, 1 \mathrm{H}$ ), 2.79-2.69 (comp, 2H), 2.60 (dd, $J=14.3,6.8 \mathrm{~Hz}, 1 \mathrm{H}), 2.34-2.26$ (comp, 2H), 2.26-2.20 (m, 1H), 1.92-1.84 (comp, 2H), 1.66-1.55 (comp, $2 \mathrm{H}), 1.03(\mathrm{~d}, J=6.7 \mathrm{~Hz}, 3 \mathrm{H})$.

${ }^{13}$ C-NMR $\left(150 \mathrm{MHz}, \mathrm{CDCl}_{3}\right): \delta=172.6,139.2,136.3,128.7,128.5,128.3,128.3,127.6,127.5,74.9,69.7,66.3$, $56.7,46.7,45.5,38.9,31.9,31.8,15.0$

Accurate Mass (ESI): Calculated for $\mathrm{C}_{23} \mathrm{H}_{29} \mathrm{NO}_{3}[\mathrm{M}+\mathrm{H}]^{+}:$368.2220, Found: 368.2262, Spectral Accuracy: $98.6 \%$.

The ee was determined after LAH reduction.

The absolute configuration was assigned by analogy. 
Due to poor separation of the enantiomers of $\mathbf{2} \mathbf{g}$ on various HPLC and SFC columns, this product was transformed to compound 2 ga to determine the ee value.

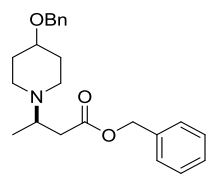

$2 \mathrm{~g}$

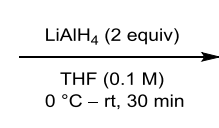

$0^{\circ} \mathrm{C}-\mathrm{rt}, 30 \mathrm{~min}$

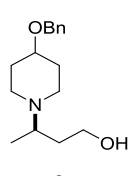

2ga

(R)-3-(4-(Benzyloxy)piperidin-1-yl)butan-1-ol:

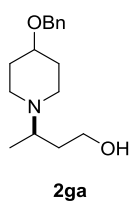

To a solution of compound $\mathbf{2 g}$ ( $37 \mathrm{mg}, 0.1 \mathrm{mmol}, 1$ equiv) in THF ( $1 \mathrm{~mL}, 0.1 \mathrm{M})$ cooled in an ice bath, $\mathrm{LiAlH}_{4}(7.6$ $\mathrm{mg}, 0.2 \mathrm{mmol}, 2$ equiv) was added and the resulting mixture was warmed to room temperature and stirred for 30 minutes before quenching with $0.2 \mathrm{~mL}$ of ammonium hydroxide solution $\left(28 \% \mathrm{NH}_{3}\right.$ in $\left.\mathrm{H}_{2} \mathrm{O}\right)$. After filtration over Celite, the filtrate was concentrated in vacuo, and the residue was purified by flash chromatography on silica gel (eluent: $\left.\mathrm{MeOH} / \mathrm{CH}_{2} \mathrm{Cl}_{2}=5: 95\right)$ to afford the title compound 2ga as a colorless oil in $90 \%$ yield $(23.7 \mathrm{mg}$ ).

\section{Characterization data:}

$\mathbf{R}_{\mathbf{f}}=0.2 \mathrm{in} \mathrm{MeOH} / \mathrm{CH}_{2} \mathrm{Cl}_{2} 10: 90 \mathrm{v} / \mathrm{v}$.

$[\alpha]_{\mathrm{D}}^{22}=-6.5\left(\mathrm{c} 0.5, \mathrm{CHCl}_{3}, 90 \% e e\right)$.

${ }^{1}$ H-NMR (600 MHz, $\left.\mathrm{CDCl}_{3}\right): \delta=7.35-7.30$ (comp, 4H), 7.29-7.25 (m, 1H), 4.52 (s, 2H), 3.84-3.76 (comp, 2H), 3.57-3.48 (m, 1H), 3.17-3.08 (m, 1H), 3.08-3.00 (m, 1H), 2.86-2.79 (m, 1H), 2.79-2.70 (m, 1H), 2.57-2.40 (m, $1 \mathrm{H}), 2.16-2.07$ (m, 1H), 2.07-1.91 (comp,2H), 1.88-1.67 (comp, 2H), 1.48-1.38 (m, 1H), 1.07 (d, J=6.7 Hz, 3H).

${ }^{13}$ C-NMR $\left(150 \mathrm{MHz}, \mathrm{CDCl}_{3}\right): \delta=138.7,128.5,127.7,127.5,72.5,70.0,62.4,61.3,44.9,34.0,30.3,13.3$.

Accurate Mass (ESI): Calculated for $\mathrm{C}_{16} \mathrm{H}_{25} \mathrm{NO}_{2}[\mathrm{M}+\mathrm{H}]^{+}:$264.1958, Found: 264.1904, Spectral Accuracy: $98.5 \%$.

HPLC: Daicel Chiralpak AS-H, $n$-hexane $/ i$-PrOH $=90 / 10$, Flow rate $=1 \mathrm{~mL} / \mathrm{min}, \mathrm{UV}=230 \mathrm{~nm}, \mathrm{t}_{\mathrm{R}}=7.9 \mathrm{~min}$ and $\mathrm{t}_{\mathrm{R}}$ $=10.8 \min$ (major). 


\section{Benzyl (R)-3-(4-methylpiperidin-1-yl)butanoate:}

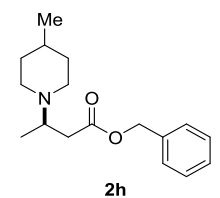

2h

4-Methylpiperidine ( $24 \mu \mathrm{L}, 0.2 \mathrm{mmol}, 1$ equiv) and benzyl crotonate $(70 \mathrm{mg}, 0.4 \mathrm{mmol}, 2$ equiv) were treated according to general procedure $\mathrm{C}$ at $-10{ }^{\circ} \mathrm{C}$ for 3 days. Purification by flash chromatography on silica gel eluting with $\mathrm{Et}_{2} \mathrm{O} / \mathrm{Hexanes} / \mathrm{Et}_{3} \mathrm{~N}$ (30:69:1) afforded $\mathbf{2 h}$ as a colorless oil in $93 \%$ yield (51 $\mathrm{mg}$ ).

\section{Characterization data:}

$\mathbf{R}_{\mathbf{f}}=0.21 \mathrm{in} \mathrm{MeOH} / \mathrm{CH}_{2} \mathrm{Cl}_{2} 5: 95 \mathrm{v} / \mathrm{v}$.

$[\boldsymbol{\alpha}]_{\mathrm{D}}^{22}=-3.6\left(\mathrm{c} 0.5, \mathrm{CHCl}_{3}, 93 \% e e\right)$.

${ }^{1}$ H-NMR (600 MHz, CDCl $): \delta=7.39-7.29$ (comp, 5H), 5.12 (s, 2H), 3.18 (app hept, $J=6.8 \mathrm{~Hz}, 1 \mathrm{H}$ ), 2.77-2.69 (comp, 2H), $2.63(\mathrm{dd}, J=14.3,6.3 \mathrm{~Hz}, 1 \mathrm{H}), 2.28(\mathrm{dd}, J=14.3,8.1 \mathrm{~Hz}, 1 \mathrm{H}), 2.21-2.09$ (comp, 2H), 1.62-1.54 (comp, 2H), 1.36-1.23 (m, 1H), 1.19-1.08 (comp, 2H), 1.04 (d, $J=6.7 \mathrm{~Hz}, 3 \mathrm{H}), 0.89$ (d, $J=6.5 \mathrm{~Hz}, 3 \mathrm{H})$.

${ }^{13} \mathrm{C}-\mathrm{NMR}\left(150 \mathrm{MHz}, \mathrm{CDCl}_{3}\right): \delta=172.8,136.3,128.6,128.3,128.2,66.2,56.9,49.6,48.1,38.5,34.9,34.7,31.3$, 22.1, 15.2.

Accurate Mass (ESI): Calculated for $\mathrm{C}_{17} \mathrm{H}_{25} \mathrm{NO}_{2}[\mathrm{M}+\mathrm{H}]^{+}:$276.1958, Found: 276.1877, Spectral Accuracy: 99.4\%.

The ee was determined after LAH reduction followed by acylation.

The absolute configuration was assigned by analogy. 
Due to poor separation of the enantiomers of $\mathbf{2 h}$ on various HPLC and SFC columns, this product was transformed to compound $\mathbf{2 h a}$ to determine the ee value.
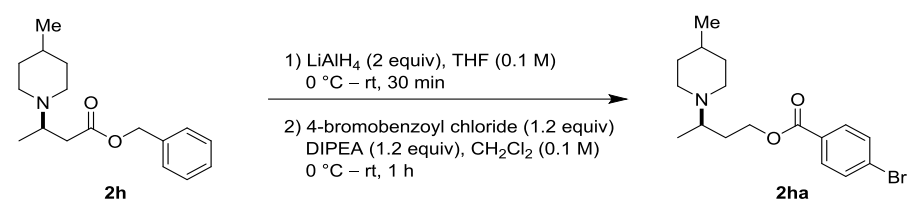

(R)-3-(4-Methylpiperidin-1-yl)butyl 4-bromobenzoate:

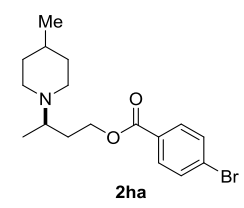

To a solution of compound $\mathbf{2 h}\left(28 \mathrm{mg}, 0.1 \mathrm{mmol}, 1\right.$ equiv) in THF $(1 \mathrm{~mL}, 0.1 \mathrm{M})$ cooled in an ice bath, $\mathrm{LiAlH}_{4}(7.6$ $\mathrm{mg}, 0.2 \mathrm{mmol}, 2$ equiv) was added and the resulting mixture was warmed to room temperature and stirred for 30 minutes before quenching with $0.2 \mathrm{~mL}$ of ammonium hydroxide solution $\left(28 \% \mathrm{NH}_{3}\right.$ in $\left.\mathrm{H}_{2} \mathrm{O}\right)$. The mixture was then filtered over Celite and concentrated in vacuo to afford crude intermediate, which was used directly for the next step. To a solution of the crude aminoalcohol in anhydrous $\mathrm{CH}_{2} \mathrm{Cl}_{2}(1 \mathrm{~mL}, 0.1 \mathrm{M})$ at $0{ }^{\circ} \mathrm{C}$, DIPEA $(21 \mu \mathrm{L}, 0.12 \mathrm{mmol}, 1.2$ equiv) and 4-bromobenzoyl chloride ( $26 \mathrm{mg}, 0.12 \mathrm{mmol}, 1.2$ equiv) were added and the mixture was warmed to room temperature and stirred for 1 hour. The crude mixture was purified by flash chromatography on silica gel (eluent: $\mathrm{MeOH} / \mathrm{CH}_{2} \mathrm{Cl}_{2}=5: 95$ ) to afford compound $\mathbf{2 h a}$ as a colorless oil in $90 \%$ yield (32 $\mathrm{mg}$ ).

\section{Characterization data:}

$\mathbf{R}_{\mathbf{f}}=0.37$ in $\mathrm{MeOH} / \mathrm{CH}_{2} \mathrm{Cl}_{2} 10: 90 \mathrm{v} / \mathrm{v}$.

$[\boldsymbol{\alpha}]_{\mathbf{D}}^{22}=-8.3\left(\mathrm{c} 0.5, \mathrm{CHCl}_{3}, 93 \% e e\right)$.

${ }^{1}$ H-NMR $\left(600 \mathrm{MHz}, \mathrm{CDCl}_{3}\right): \delta=7.89(\mathrm{~d}, J=8.5 \mathrm{~Hz}, 1 \mathrm{H}), 7.57(\mathrm{~d}, J=8.5 \mathrm{~Hz}, 1 \mathrm{H}), 4.43-4.31$ (comp, 2H), 2.872.68 (comp, 3H), 2.38-2.28 (m, 1H), 2.15 (app t, $J=11.2 \mathrm{~Hz}, 1 \mathrm{H}), 2.09-1.99(\mathrm{~m}, 1 \mathrm{H}), 1.70$ (app dq, $J=13.9,6.9$ $\mathrm{Hz}, 1 \mathrm{H}), 1.66-1.56$ (comp, 2H), 1.38-1.13 (comp, 3H), 1.04 (d, $J=6.6 \mathrm{~Hz}, 3 \mathrm{H}), 0.91$ (d, $J=6.4 \mathrm{~Hz}, 3 \mathrm{H})$.

${ }^{13} \mathrm{C}-\mathrm{NMR}\left(150 \mathrm{MHz}, \mathrm{CDCl}_{3}\right): \delta=166.1,131.8,131.2,129.5,128.1,63.8,56.6,50.9,46.7,34.8,34.6,32.6,31.3$, 22.1, 14.1.

Accurate Mass (ESI): Calculated for $\mathrm{C}_{17} \mathrm{H}_{24} \mathrm{BrNO}_{2}[\mathrm{M}+\mathrm{H}]^{+}:{ }^{79} \mathrm{Br}, 354.1063$, Found: $354.1022,{ }^{81} \mathrm{Br}$, Found: 356.1065, Spectral Accuracy: 97.9\%.

HPLC: Daicel Chiralpak AD-H, $n$-hexane $/ i-\mathrm{PrOH}=97 / 3$, Flow rate $=1 \mathrm{~mL} / \mathrm{min}, \mathrm{UV}=280 \mathrm{~nm}, \mathrm{t}_{\mathrm{R}}=4.6 \mathrm{~min}$ and $\mathrm{t}_{\mathrm{R}}$ $=5.1 \mathrm{~min}$ (major). 


\section{Benzyl (R)-3-(4-(methyl(phenyl)amino)piperidin-1-yl)butanoate:}

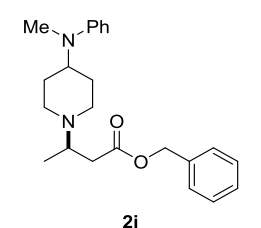

2i

4-( $N$-Methyl- $N$-phenylamino)-piperidine (38 $\mathrm{mg}, 0.2 \mathrm{mmol}, 1$ equiv) and benzyl crotonate $(70 \mathrm{mg}, 0.4 \mathrm{mmol}, 2$ equiv) were treated according to general procedure $\mathrm{C}$ at $-10{ }^{\circ} \mathrm{C}$ for 7 days. Purification by flash chromatography on silica gel eluting with $\mathrm{Et}_{2} \mathrm{O} / \mathrm{Hexanes} / \mathrm{Et}_{3} \mathrm{~N}$ (40:59:1) afforded $\mathbf{2 i}$ as a colorless oil in $75 \%$ yield $(55 \mathrm{mg})$.

\section{Characterization data:}

$\mathbf{R}_{\mathbf{f}}=0.24 \mathrm{in} \mathrm{MeOH} / \mathrm{CH}_{2} \mathrm{Cl}_{2} 5: 95 \mathrm{v} / \mathrm{v}$.

$[\boldsymbol{\alpha}]_{\mathbf{D}}^{22}=-2.4\left(\mathrm{c} 0.5, \mathrm{CHCl}_{3}, 88 \% e e\right)$.

${ }^{1} \mathbf{H}-\mathrm{NMR}\left(600 \mathrm{MHz}, \mathrm{CDCl}_{3}\right): \delta=7.42-7.36$ (comp, 4H), 7.35-7.31 (m, 1H), 7.23 (app t, $\left.J=7.7 \mathrm{~Hz}, 2 \mathrm{H}\right), 6.79$ (d, $J$ $=8.4 \mathrm{~Hz}, 2 \mathrm{H}), 6.71(\mathrm{t}, J=7.2 \mathrm{~Hz}, 1 \mathrm{H}), 5.17(\mathrm{~d}, J=12.5 \mathrm{~Hz}, 1 \mathrm{H}), 5.14(\mathrm{~d}, J=12.4 \mathrm{~Hz}, 1 \mathrm{H}), 3.58-3.46(\mathrm{~m}, 1 \mathrm{H})$, 3.30-3.21 (m, 1H), 2.87 (app t, $J=10.7 \mathrm{~Hz}, 2 \mathrm{H}), 2.76$ (s, 3H), 2.63 (dd, $J=14.3,6.7 \mathrm{~Hz}, 1 \mathrm{H}), 2.40-2.30$ (comp, 2H), 2.26 (app td, $J=10.9,4.0 \mathrm{~Hz}, 1 \mathrm{H}), 1.75-1.64$ (comp, 4H), 1.07 (d, $J=6.6 \mathrm{~Hz}, 3 \mathrm{H})$.

${ }^{13} \mathrm{C}-\mathrm{NMR}\left(150 \mathrm{MHz}, \mathrm{CDCl}_{3}\right): \delta=172.5,150.2,136.2,129.2,128.6,128.3,128.3,116.8,113.5,66.3,57.2,56.7$, $49.6,47.3,38.8,31.5,29.6,29.4,15.0$.

Accurate Mass (ESI): Calculated for $\mathrm{C}_{23} \mathrm{H}_{30} \mathrm{~N}_{2} \mathrm{O}_{2}[\mathrm{M}+\mathrm{H}]^{+}: 367.2380$, Found: 367.2363, Spectral Accuracy: $99.5 \%$.

HPLC: Daicel Chiralpak AS-H, $n$-hexane $/ i$-PrOH $=90 / 10$, Flow rate $=1 \mathrm{~mL} / \mathrm{min}, \mathrm{UV}=254 \mathrm{~nm}, \mathrm{t}_{\mathrm{R}}=4.7 \mathrm{~min}$ and $\mathrm{t}_{\mathrm{R}}$ $=5.4 \min$ (major).

The absolute configuration was assigned by analogy. 


\section{Benzyl (R)-3-(4-methylpiperazin-1-yl)butanoate:}

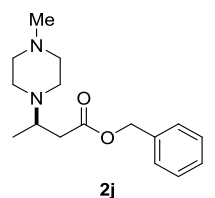

2j

1-Methylpiperazine ( $22 \mu \mathrm{L}, 0.2 \mathrm{mmol}, 1$ equiv) and benzyl crotonate $(70 \mathrm{mg}, 0.4 \mathrm{mmol}, 2$ equiv) were treated according to general procedure $\mathrm{C}$ at $-10{ }^{\circ} \mathrm{C}$ for 7 days. Purification by flash chromatography on silica gel eluting with $\mathrm{MeOH} / \mathrm{CH}_{2} \mathrm{Cl}_{2}(20: 80)$ afforded $\mathbf{2} \mathbf{j}$ as a colorless solid in $94 \%$ yield $(52 \mathrm{mg})$.

\section{Characterization data:}

$\mathbf{R}_{\mathbf{f}}=0.31$ in $\mathrm{MeOH} / \mathrm{CH}_{2} \mathrm{Cl}_{2} 10: 90 \mathrm{v} / \mathrm{v}$.

$[\alpha]_{\mathbf{D}}^{22}=+2.9\left(\mathrm{c} 0.5, \mathrm{CHCl}_{3}, 93 \% e e\right)$

${ }^{1}$ H-NMR $\left(500 \mathrm{MHz}, \mathrm{CDCl}_{3}\right): \delta=7.36-7.27(\mathrm{comp}, 5 \mathrm{H}), 5.11(\mathrm{~d}, J=12.3 \mathrm{~Hz}, 1 \mathrm{H}), 5.08(\mathrm{~d}, J=12.3 \mathrm{~Hz}, 1 \mathrm{H}), 3.21-$ $3.12(\mathrm{~m}, 1 \mathrm{H}), 2.66-2.59$ (comp, 2H), 2.58-2.37 (comp, 7H), 2.34-2.26 (comp, 4H), 1.01 (d, $J=6.6 \mathrm{~Hz}, 3 \mathrm{H})$.

${ }^{13}$ C-NMR (125 MHz, $\left.\mathrm{CDCl}_{3}\right): \delta=172.1,136.2,128.6,128.5,128.3,66.1,56.5,55.0,47.1,45.4,38.8,14.5$.

Accurate Mass (ESI): Calculated for $\mathrm{C}_{16} \mathrm{H}_{24} \mathrm{~N}_{2} \mathrm{O}_{2}[\mathrm{M}+\mathrm{H}]^{+}:$: 277.1911, Found: 277.1823, Spectral Accuracy: 98.1\%.

HPLC: Daicel Chiralpak AD-H, $n$-hexane $/ i-\mathrm{PrOH} /$ diethylamine $=95 / 5 / 0.05$, Flow rate $=1 \mathrm{~mL} / \mathrm{min}, \mathrm{UV}=254 \mathrm{~nm}$, $\mathrm{t}_{\mathrm{R}}=5.8$ min (major) and $\mathrm{t}_{\mathrm{R}}=6.4$ min.

The absolute configuration was assigned by analogy. 


\section{Benzyl (R)-3-morpholinobutanoate:}

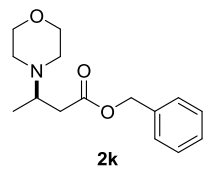

Morpholine (18 $\mu \mathrm{L}, 0.2 \mathrm{mmol}, 1$ equiv) and benzyl crotonate ( $70 \mathrm{mg}, 0.4 \mathrm{mmol}, 2$ equiv) were treated according to general procedure $\mathrm{C}$ at room temperature for 7 days. Purification by flash chromatography on silica gel eluting with $\mathrm{Et}_{2} \mathrm{O} /$ Hexanes/Et $3 \mathrm{~N}$ (40:59:1) afforded $\mathbf{2 k}$ as a colorless solid in $70 \%$ yield (37 $\left.\mathrm{mg}\right)$.

\section{Characterization data:}

$\mathbf{R}_{\mathbf{f}}=0.21$ in $\mathrm{MeOH} / \mathrm{CH}_{2} \mathrm{Cl}_{2} 5: 95 \mathrm{v} / \mathrm{v}$.

$[\alpha]_{\mathbf{D}}^{22}=-4.5\left(\mathrm{c} 0.5, \mathrm{CHCl}_{3}, 85 \% e e\right)$.

${ }^{1} \mathbf{H}-\mathrm{NMR}\left(500 \mathrm{MHz}, \mathrm{CDCl}_{3}\right): \delta=7.39-7.29(\mathrm{comp}, 5 \mathrm{H}), 5.15(\mathrm{~d}, J=12.3 \mathrm{~Hz}, 1 \mathrm{H}), 5.12(\mathrm{~d}, J=12.3 \mathrm{~Hz}, 1 \mathrm{H}), 3.67-$ 3.56 (comp, 4H), 3.19-3.08 (m, 1H), 2.60 (dd, $J=14.4,7.2 \mathrm{~Hz}, 1 \mathrm{H}), 2.55-2.49$ (comp, 2H), 2.48-2.42 (comp, 2H), $2.31(\mathrm{dd}, J=14.4,7.5 \mathrm{~Hz}, 1 \mathrm{H}), 1.04(\mathrm{~d}, J=6.6 \mathrm{~Hz}, 3 \mathrm{H})$.

${ }^{13}$ C-NMR $\left(125 \mathrm{MHz}, \mathrm{CDCl}_{3}\right): \delta=172.4,136.2,128.7,128.4,128.4,67.4,66.3,56.9,48.7,38.8,14.6$.

Accurate Mass (ESI): Calculated for $\mathrm{C}_{15} \mathrm{H}_{21} \mathrm{NO}_{3}[\mathrm{M}+\mathrm{H}]^{+}:$264.1594, Found: 264.1411, Spectral Accuracy: $98.9 \%$.

HPLC: Daicel Chiralpak AS-H, $n$-hexane $/ i-\mathrm{PrOH}=99 / 1$, Flow rate $=1 \mathrm{~mL} / \mathrm{min}, \mathrm{UV}=210 \mathrm{~nm}, \mathrm{t}_{\mathrm{R}}=12.7$ min and $\mathrm{t}_{\mathrm{R}}$ $=15.6 \mathrm{~min}$ (major).

The absolute configuration was assigned by analogy. 


\section{Benzyl (R)-3-(3,4-dihydroisoquinolin-2(1H)-yl)butanoate:}

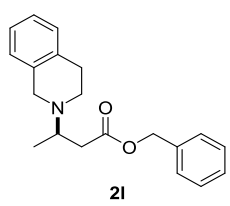

1,2,3,4-Tetrahydroisoquinoline ( $25 \mu \mathrm{L}, 0.2 \mathrm{mmol}, 1$ equiv) and benzyl crotonate ( $70 \mathrm{mg}, 0.4 \mathrm{mmol}, 2$ equiv) were treated according to general procedure $\mathrm{C}$ at $-10{ }^{\circ} \mathrm{C}$ for 7 days. Purification by flash chromatography on silica gel eluting with $\mathrm{Et}_{2} \mathrm{O} / \mathrm{Hexanes} / \mathrm{Et}_{3} \mathrm{~N}$ (25:74:1) afforded $2 \mathbf{l}$ as a colorless oil in $66 \%$ yield (41 $\left.\mathrm{mg}\right)$.

\section{Characterization data:}

$\mathbf{R}_{\mathbf{f}}=0.44$ in $\mathrm{MeOH} / \mathrm{CH}_{2} \mathrm{Cl}_{2}$ 5:95 v/v.

$[\boldsymbol{\alpha}]_{\mathrm{D}}^{22}=-3.9\left(\mathrm{c} 0.5, \mathrm{CHCl}_{3}, 85 \% e e\right)$.

${ }^{1} \mathbf{H}$-NMR (500 MHz, $\mathrm{CDCl}_{3}$ ): $\delta=7.33-7.29$ (comp, 2H), 7.28-7.22 (comp, 3H), 7.14-7.06 (comp, 3H), 7.03-6.98 $(\mathrm{m}, 1 \mathrm{H}), 5.14(\mathrm{~d}, J=12.4 \mathrm{~Hz}, 1 \mathrm{H}), 5.10(\mathrm{~d}, J=12.4 \mathrm{~Hz}, 1 \mathrm{H}), 3.77(\mathrm{~d}, J=14.6 \mathrm{~Hz}, 1 \mathrm{H}), 3.71(\mathrm{~d}, J=14.6 \mathrm{~Hz}, 1 \mathrm{H})$, 3.46-3.37 (m, 1H), 2.87-2.78 (comp, 3H), 2.76-2.68 (comp, 2H), 2.42 (dd, $J=14.4,7.7 \mathrm{~Hz}, 1 \mathrm{H}), 1.14$ (d, $J=6.7$ $\mathrm{Hz}, 3 \mathrm{H})$.

${ }^{13}$ C-NMR $\left(125 \mathrm{MHz}, \mathrm{CDCl}_{3}\right): \delta=172.5,136.2,135.4,134.8,128.9,128.6,128.2,126.8,126.0,125.6,66.3,56.4$, $51.5,45.7,38.8,30.0,14.6$.

Accurate Mass (ESI): Calculated for $\mathrm{C}_{20} \mathrm{H}_{23} \mathrm{NO}_{2}[\mathrm{M}+\mathrm{H}]^{+}: 310.1802$, Found: 310.1792, Spectral Accuracy: 99.3\%.

HPLC: Daicel Chiralcel OJ-H, $n$-hexane $/ i-\mathrm{PrOH}=90 / 10$, Flow rate $=1 \mathrm{~mL} / \mathrm{min}, \mathrm{UV}=254 \mathrm{~nm}, \mathrm{t}_{\mathrm{R}}=16.2 \mathrm{~min}$ (major) and $t_{\mathrm{R}}=22.4 \mathrm{~min}$.

The absolute configuration was assigned by analogy. 


\section{Benzyl (R)-3-(pyrrolidin-1-yl)butanoate:}

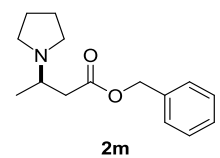

Pyrrolidine ( $17 \mu \mathrm{L}, 0.2 \mathrm{mmol}, 1$ equiv) and benzyl crotonate $(70 \mathrm{mg}, 0.4 \mathrm{mmol}, 2$ equiv) were treated according to general procedure $\mathrm{C}$ at $-10{ }^{\circ} \mathrm{C}$ in toluene $(8 \mathrm{~mL}, 0.025 \mathrm{M})$ for 4 days. Purification by flash chromatography on silica gel eluting with $\mathrm{Et}_{2} \mathrm{O} / \mathrm{Hexanes} / \mathrm{Et}_{3} \mathrm{~N}$ (45:54:1) afforded $\mathbf{2 m}$ as a colorless oil in $80 \%$ yield $(40 \mathrm{mg})$.

\section{Characterization data:}

$\mathbf{R}_{\mathbf{f}}=0.37$ in $\mathrm{MeOH} / \mathrm{CH}_{2} \mathrm{Cl}_{2} 10: 90 \mathrm{v} / \mathrm{v}$.

$[\boldsymbol{\alpha}]_{\mathbf{D}}^{22}=+5.2\left(\mathrm{c} 0.5, \mathrm{CHCl}_{3}, 93 \% e e\right)$.

${ }^{1}$ H-NMR $\left(600 \mathrm{MHz}, \mathrm{CDCl}_{3}\right): \delta=7.38-7.34(\mathrm{comp}, 4 \mathrm{H}), 7.34-7.30(\mathrm{~m}, 1 \mathrm{H}), 5.13(\mathrm{~s}, 2 \mathrm{H}), 2.92(\mathrm{dqd}, J=8.6,6.4$, $4.8 \mathrm{~Hz}, 1 \mathrm{H}), 2.70(\mathrm{dd}, J=14.7,4.8 \mathrm{~Hz}, 1 \mathrm{H}), 2.61-2.52(\mathrm{comp}, 4 \mathrm{H}), 2.35(\mathrm{dd}, J=14.7,8.6 \mathrm{~Hz}, 1 \mathrm{H}), 1.78-1.72$ (comp, 4H), $1.14(\mathrm{~d}, J=6.4 \mathrm{~Hz}, 3 \mathrm{H})$.

${ }^{13}$ C-NMR $\left(150 \mathrm{MHz}, \mathrm{CDCl}_{3}\right): \delta=172.5,136.2,128.7,128.3,128.3,66.3,55.5,50.7,40.6,23.6,18.6$.

Accurate Mass (ESI): Calculated for $\mathrm{C}_{15} \mathrm{H}_{21} \mathrm{NO}_{2}[\mathrm{M}+\mathrm{H}]^{+}: 248.1645$, Found: 248.1537, Spectral Accuracy: 98.9\%.

The ee was determined after LAH reduction followed by acylation.

The absolute configuration was assigned by analogy. 
Due to poor separation of the enantiomers of $\mathbf{2 m}$ on various HPLC and SFC columns, this product was transformed to compound $2 \mathrm{ma}$ to determine the ee value.

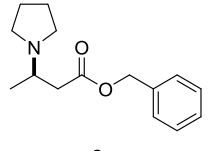

$2 m$

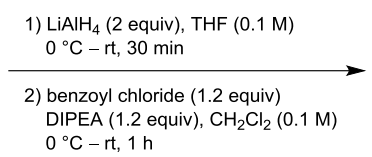

$0{ }^{\circ} \mathrm{C}-\mathrm{rt}, 1 \mathrm{~h}$

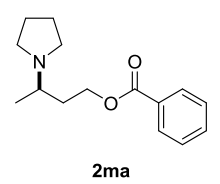

\section{(R)-3-(Pyrrolidin-1-yl)butyl benzoate:}

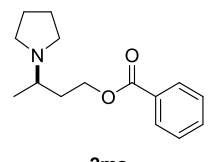

$2 \mathrm{ma}$

To a solution of compound $\mathbf{2 m}(25 \mathrm{mg}, 0.1 \mathrm{mmol}, 1$ equiv) in THF ( $1 \mathrm{~mL}, 0.1 \mathrm{M})$ cooled in an ice bath, $\mathrm{LiAlH}_{4}(7.6$ $\mathrm{mg}, 0.2 \mathrm{mmol}, 2$ equiv) was added and the resulting mixture was warmed to room temperature and stirred for 30 minutes before quenching with $0.2 \mathrm{~mL}$ of ammonium hydroxide solution $\left(28 \% \mathrm{NH}_{3}\right.$ in $\left.\mathrm{H}_{2} \mathrm{O}\right)$. The mixture was then

filtered over Celite and concentrated in vacuo to afford crude intermediate, which was used directly for the next step. To the solution of the crude aminoalcohol in $\mathrm{CH}_{2} \mathrm{Cl}_{2}(1 \mathrm{~mL}, 0.1 \mathrm{M})$ cooled in an ice bath, DIPEA (21 $\mu \mathrm{L}, 0.12$ mmol, 1.2 equiv) and benzoyl chloride ( $14 \mu \mathrm{L}, 0.12 \mathrm{mmol}, 1.2$ equiv) were added and the mixture was warmed to room temperature and stirred for 1 hour. The crude mixture was purified by flash chromatography on silica gel (eluent: $\left.\mathrm{MeOH} / \mathrm{CH}_{2} \mathrm{Cl}_{2}=5: 95\right)$ to afford the title compound $2 \mathrm{ma}$ as a colorless solid in $90 \%$ yield $(22.5 \mathrm{mg}$ ).

\section{Characterization data:}

$\mathbf{R}_{\mathbf{f}}=0.23$ in $\mathrm{MeOH} / \mathrm{CH}_{2} \mathrm{Cl}_{2} 10: 90 \mathrm{v} / \mathrm{v}$.

$[\boldsymbol{\alpha}]_{\mathrm{D}}^{22}=-5.4\left(\mathrm{c} 0.5, \mathrm{CHCl}_{3}, 93 \% e e\right)$.

${ }^{1}$ H-NMR $\left(600 \mathrm{MHz}, \mathrm{CDCl}_{3}\right): \delta=7.99(\mathrm{~d}, J=7.3 \mathrm{~Hz}, 2 \mathrm{H}), 7.57(\mathrm{t}, J=7.4 \mathrm{~Hz}, 1 \mathrm{H}), 7.44($ app t, $J=7.8 \mathrm{~Hz}, 2 \mathrm{H})$, 4.46 (app dt, $J=11.2,5.5 \mathrm{~Hz}, 1 \mathrm{H}$ ), 4.39 (ddd, $J=11.6,9.0,5.0 \mathrm{~Hz}, 1 \mathrm{H}), 3.60-2.88$ (comp, 5H), 2.54-2.44 (m, 1H), $2.21-2.01$ (comp, $5 \mathrm{H}), 1.51(\mathrm{~d}, J=6.5 \mathrm{~Hz}, 3 \mathrm{H})$.

${ }^{13} \mathrm{C}-\mathrm{NMR}\left(150 \mathrm{MHz}, \mathrm{CDCl}_{3}\right): \delta=166.4,133.5,129.7,129.6,128.7,61.1,58.2,50.9,31.8,23.7,15.7$.

Accurate Mass (ESI): Calculated for $\mathrm{C}_{15} \mathrm{H}_{21} \mathrm{NO}_{2}[\mathrm{M}+\mathrm{H}]^{+}$: 248.1645, Found: 248.1538, Spectral Accuracy: $98.5 \%$.

HPLC: Daicel Chiralcel OB-H, $n$-hexane $/ i$-PrOH/diethylamine $=99 / 1 / 0.05$, Flow rate $=1 \mathrm{~mL} / \mathrm{min}, \mathrm{UV}=280 \mathrm{~nm}$, $\mathrm{t}_{\mathrm{R}}=6.1$ min and $\mathrm{t}_{\mathrm{R}}=6.9$ min (major). 


\section{Benzyl (3R)-3-(hexahydrocyclopenta[c]pyrrol-2(1H)-yl)butanoate:}

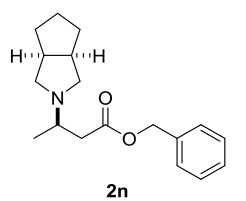

cis-7-Azabicyclo[3.3.0]octane ( $22 \mathrm{mg}, 0.2 \mathrm{mmol}, 1$ equiv) and benzyl crotonate (70 $\mathrm{mg}, 0.4 \mathrm{mmol}, 2$ equiv) were treated according to general procedure $\mathrm{C}$ at $-10{ }^{\circ} \mathrm{C}$ in toluene $(8 \mathrm{~mL}, 0.025 \mathrm{M})$ for 7 days. Purification by flash chromatography on silica gel eluting with $\mathrm{Et}_{2} \mathrm{O} / \mathrm{Hexanes} / \mathrm{Et}_{3} \mathrm{~N}$ (40:59:1) afforded $\mathbf{2 n}$ as a colorless oil in $72 \%$ yield (41 mg).

\section{Characterization data:}

$\mathbf{R}_{\mathbf{f}}=0.21 \mathrm{in} \mathrm{MeOH} / \mathrm{CH}_{2} \mathrm{Cl}_{2} 5: 95 \mathrm{v} / \mathrm{v}$.

$[\boldsymbol{\alpha}]_{\mathbf{D}}^{22}=+1.1\left(\mathrm{c} 0.5, \mathrm{CHCl}_{3}, 93 \% e e\right)$.

${ }^{1}$ H-NMR (500 MHz, $\mathrm{CDCl}_{3}$ ): $\delta=7.37-7.29$ (comp, 5H), 5.12 (s, 2H), 2.90-2.78 (comp, 3H), 2.65 (dd, $J=14.6,5.3$ $\mathrm{Hz}, 1 \mathrm{H}$ ), 2.58-2.49 (comp, 2H), 2.32 (dd, $J=14.6,8.2 \mathrm{~Hz}, 1 \mathrm{H}$ ), 2.08-2.01 (comp, 2H), 1.66-1.51 (comp, 3H), $1.49-1.41(\mathrm{~m}, 1 \mathrm{H}), 1.39-1.31$ (comp, 2H), 1.09 (d, $J=6.4 \mathrm{~Hz}, 3 \mathrm{H})$.

${ }^{13} \mathrm{C}-\mathrm{NMR}\left(125 \mathrm{MHz}, \mathrm{CDCl}_{3}\right): \delta=172.5,136.2,128.6,128.4,128.3,66.3,58.5,57.9,55.1,42.2,42.1,40.6,32.9$, $32.8,25.9,18.2$.

Accurate Mass (ESI): Calculated for $\mathrm{C}_{18} \mathrm{H}_{25} \mathrm{NO}_{2}[\mathrm{M}+\mathrm{H}]^{+}:$288.1958, Found: 288.1862, Spectral Accuracy: $98.7 \%$.

HPLC: Daicel Chiralpak AY-H, $n$-hexane $/ \mathrm{i}-\mathrm{PrOH}=99 / 1$, Flow rate $=1 \mathrm{~mL} / \mathrm{min}, \mathrm{UV}=230 \mathrm{~nm}, \mathrm{t}_{\mathrm{R}}=8.1$ min and $\mathrm{t}_{\mathrm{R}}$ $=8.4 \min$ (major).

The absolute configuration was assigned by analogy. 


\section{Benzyl (R)-3-(azepan-1-yl)butanoate:}

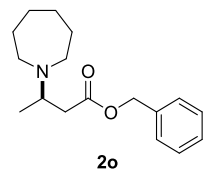

Azepane ( $23 \mu \mathrm{L}, 0.2 \mathrm{mmol}, 1$ equiv) and benzyl crotonate ( $70 \mathrm{mg}, 0.4 \mathrm{mmol}, 2$ equiv) were treated according to general procedure $\mathrm{C}$ at $-10{ }^{\circ} \mathrm{C}$ for 7 days. Purification by flash chromatography on silica gel eluting with $\mathrm{Et}_{2} \mathrm{O} / \mathrm{Hexanes} / \mathrm{Et}_{3} \mathrm{~N}$ (30:69:1) afforded $2 \mathrm{o}$ as a colorless oil in $85 \%$ yield $(47 \mathrm{mg})$.

\section{Characterization data:}

$\mathbf{R}_{\mathbf{f}}=0.18$ in $\mathrm{MeOH} / \mathrm{CH}_{2} \mathrm{Cl}_{2} 5: 95 \mathrm{v} / \mathrm{v}$.

$[\boldsymbol{\alpha}]_{\mathrm{D}}^{22}=+2.4\left(\mathrm{c} 0.5, \mathrm{CHCl}_{3}, 83 \% e e\right)$.

${ }^{1}$ H-NMR (500 MHz, $\mathrm{CDCl}_{3}$ ): $\delta=7.40-7.29$ (comp, 5H), 5.17-5.07 (comp, 2H), 3.32-3.21 (m, 1H), 2.66-2.48 (comp, 5H), 2.27 (dd, $J=14.1,7.6 \mathrm{~Hz}, 1 \mathrm{H}), 1.62-1.47$ (comp, 8H), 1.01 (d, $J=6.6 \mathrm{~Hz}, 3 \mathrm{H})$.

${ }^{13}$ C-NMR (125 MHz, $\left.\mathrm{CDCl}_{3}\right): \delta=172.8,136.3,128.6,128.4,128.3,66.2,58.1,51.1,39.5,29.8,27.0,15.3$.

Accurate Mass (ESI): Calculated for $\mathrm{C}_{17} \mathrm{H}_{25} \mathrm{NO}_{2}[\mathrm{M}+\mathrm{H}]^{+}$: 276.1958, Found: 276.1885, Spectral Accuracy: $98.6 \%$.

HPLC: Daicel Chiralpak AY-H, $n$-hexane $/ \mathrm{i}-\mathrm{PrOH}=99 / 1$, Flow rate $=1 \mathrm{~mL} / \mathrm{min}, \mathrm{UV}=230 \mathrm{~nm}, \mathrm{t}_{\mathrm{R}}=6.9 \mathrm{~min}$ and $\mathrm{t}_{\mathrm{R}}$ $=7.3 \mathrm{~min}$ (major).

The absolute configuration was assigned by analogy. 


\section{Benzyl (R)-3-(azocan-1-yl)butanoate:}

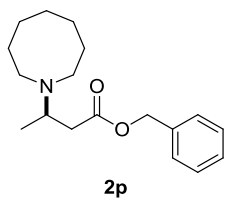

Azocane ( $25 \mu \mathrm{L}, 0.2 \mathrm{mmol}, 1$ equiv) and benzyl crotonate $(70 \mathrm{mg}, 0.4 \mathrm{mmol}, 2$ equiv) were treated according to general procedure $\mathrm{C}$ at $-10{ }^{\circ} \mathrm{C}$ for 7 days. Purification by flash chromatography on silica gel eluting with $\mathrm{Et}_{2} \mathrm{O} /$ Hexanes (5:95) followed by $\mathrm{Et}_{2} \mathrm{O} /$ Hexanes/Et ${ }_{3} \mathrm{~N}$ (20:79:1) afforded $\mathbf{2 p}$ as a colorless oil in $82 \%$ yield (47 $\mathrm{mg}$ ).

\section{Characterization data:}

$\mathbf{R}_{\mathbf{f}}=0.32$ in $\mathrm{MeOH} / \mathrm{CH}_{2} \mathrm{Cl}_{2} 5: 95 \mathrm{v} / \mathrm{v}$.

$[\boldsymbol{\alpha}]_{\mathrm{D}}^{22}=-4.8\left(\mathrm{c} 0.5, \mathrm{CHCl}_{3}, 81 \% e e\right)$.

${ }^{1}$ H-NMR $\left(500 \mathrm{MHz}, \mathrm{CDCl}_{3}\right): \delta=7.40-7.29(\mathrm{comp}, 5 \mathrm{H}), 5.13(\mathrm{~d}, J=12.4 \mathrm{~Hz}, 1 \mathrm{H}), 5.10(\mathrm{~d}, J=12.4 \mathrm{~Hz}, 1 \mathrm{H}), 3.32-$ 3.20 (m, 1H), 2.59-2.51 (comp, 3H), 2.51-2.43 (comp, 2H), 2.27 (dd, $J=14.3,7.0 \mathrm{~Hz}, 1 \mathrm{H}), 1.63-1.45$ (comp, 10H), $0.99(\mathrm{~d}, J=6.7 \mathrm{~Hz}, 3 \mathrm{H})$.

${ }^{13}$ C-NMR $\left(125 \mathrm{MHz}, \mathrm{CDCl}_{3}\right): \delta=172.9,136.2,128.6,128.5,128.3,66.3,57.5,49.8,39.5,28.6,27.8,25.8,15.0$.

Accurate Mass (ESI): Calculated for $\mathrm{C}_{18} \mathrm{H}_{27} \mathrm{NO}_{2}[\mathrm{M}+\mathrm{H}]^{+}:$290.2115, Found: 290.2065, Spectral Accuracy: $98.6 \%$.

HPLC: Daicel Chiralcel OJ-H, $n$-hexane $/ i$-PrOH $=99 / 1$, Flow rate $=1 \mathrm{~mL} / \mathrm{min}, \mathrm{UV}=230 \mathrm{~nm}, \mathrm{t}_{\mathrm{R}}=6.6$ min (major) and $t_{R}=7.2 \mathrm{~min}$.

The absolute configuration was assigned by analogy. 


\section{Benzyl (R)-3-(3-((tert-butoxycarbonyl)(methyl)amino)azetidin-1-yl)butanoate:}

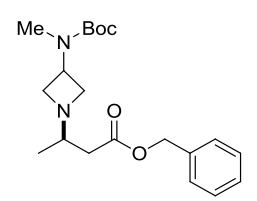

$2 q$

tert-Butyl azetidin-3-yl(methyl)carbamate $(37 \mathrm{mg}, 0.2 \mathrm{mmol}, 1$ equiv) and benzyl crotonate $(70 \mathrm{mg}, 0.4 \mathrm{mmol}, 2$ equiv) were treated according to general procedure $\mathrm{C}$ at $-10{ }^{\circ} \mathrm{C}$ in toluene $(8 \mathrm{~mL}, 0.025 \mathrm{M})$ for 7 days. Purification by flash chromatography on silica gel eluting with EtOAc/Hexanes (40:60) followed by $\mathrm{MeOH} / \mathrm{CH}_{2} \mathrm{Cl}_{2}(10: 90)$ afforded $\mathbf{2 q}$ as a colorless oil in $92 \%$ yield $(67 \mathrm{mg})$.

\section{Characterization data:}

$\mathbf{R}_{\mathbf{f}}=0.16$ in $\mathrm{MeOH} / \mathrm{CH}_{2} \mathrm{Cl}_{2} 5: 95 \mathrm{v} / \mathrm{v}$.

$[\boldsymbol{\alpha}]_{\mathbf{D}}^{22}=+5.8\left(\mathrm{c} 0.5, \mathrm{CHCl}_{3}, 86 \% e e\right)$

${ }^{1}$ H-NMR (500 MHz, CDCl $)$ ): $\delta=7.40-7.29$ (comp, 5H), 5.12 (s, 2H), 4.68-4.14 (m, 1H), 3.58-3.47 (comp, 2H), 3.03-3.89 (comp, 2H), 2.79 (s, 3H), 2.73-2.63 (m, 1H), 2.46 (dd, $J=15.0,4.3 \mathrm{~Hz}, 1 \mathrm{H}), 2.19$ (dd, $J=14.9,8.6 \mathrm{~Hz}$, $1 \mathrm{H}), 1.43(\mathrm{~s}, 9 \mathrm{H}), 1.00(\mathrm{~d}, J=6.1 \mathrm{~Hz}, 3 \mathrm{H})$.

${ }^{13}$ C-NMR $\left(125 \mathrm{MHz}, \mathrm{CDCl}_{3}\right): \delta=171.8,163.2,155.7,136.0,128.7,128.4,80.0,66.4,59.7,57.8,45.8,39.5,30.5$, 28.6, 17.5.

Accurate Mass (ESI): Calculated for $\mathrm{C}_{20} \mathrm{H}_{30} \mathrm{~N}_{2} \mathrm{O}_{4}[\mathrm{M}+\mathrm{H}]^{+}: 363.2278$, Found: 363.2309, Spectral Accuracy: 98.7\%.

HPLC: Daicel Chiralpak AY-H, $n$-hexane $/ i-\mathrm{PrOH}=90 / 10$, Flow rate $=1 \mathrm{~mL} / \mathrm{min}, \mathrm{UV}=254 \mathrm{~nm}, \mathrm{t}_{\mathrm{R}}=9.7 \mathrm{~min}$ and $\mathrm{t}_{\mathrm{R}}=10.2 \min$ (major).

The absolute configuration was assigned by analogy. 


\section{Benzyl (R)-3-((4-methoxybenzyl)amino)butanoate:}

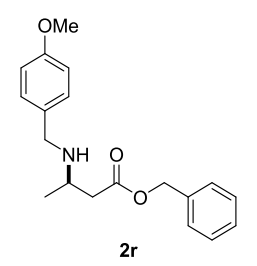

4-Methoxybenzylamine ( $26 \mu \mathrm{L}, 0.2 \mathrm{mmol}, 1$ equiv) and benzyl crotonate ( $70 \mathrm{mg}, 0.4 \mathrm{mmol}, 2$ equiv) were treated according to general procedure $\mathrm{C}$ at room temperature for 67 hours. Purification by flash chromatography on silica gel eluting with EtOAc/Hexanes (40:60) followed by $\mathrm{MeOH} / \mathrm{CH}_{2} \mathrm{Cl}_{2}(10: 90)$ afforded $2 \mathbf{r}$ as a colorless oil in $78 \%$ yield (49 mg).

\section{Characterization data:}

$\mathbf{R}_{\mathbf{f}}=0.16$ in $\mathrm{MeOH} / \mathrm{CH}_{2} \mathrm{Cl}_{2} 5: 95 \mathrm{v} / \mathrm{v}$.

$[\alpha]_{\mathrm{D}}^{22}=+4.2\left(\mathrm{c} 0.5, \mathrm{CHCl}_{3}, 78 \% e e\right)$.

${ }^{1}$ H-NMR $\left(500 \mathrm{MHz}, \mathrm{CDCl}_{3}\right): \delta=7.40-7.29(\mathrm{comp}, 5 \mathrm{H}), 7.20(\mathrm{~d}, J=7.6 \mathrm{~Hz}, 2 \mathrm{H}), 6.84(\mathrm{~d}, J=7.3 \mathrm{~Hz}, 2 \mathrm{H}), 5.12(\mathrm{~s}$, 2H), 3.83-3.73 (comp, 4H), $3.68(\mathrm{~d}, J=12.7 \mathrm{~Hz}, 1 \mathrm{H}), 3.23-3.13(\mathrm{~m}, 1 \mathrm{H}), 2.55(\mathrm{dd}, J=15.2,6.9 \mathrm{~Hz}, 1 \mathrm{H}), 2.44(\mathrm{dd}$, $J=15.1,5.7 \mathrm{~Hz}, 1 \mathrm{H}), 1.75(\mathrm{br} \mathrm{s}, 1 \mathrm{H}), 1.15(\mathrm{~d}, J=6.2 \mathrm{~Hz}, 3 \mathrm{H})$.

${ }^{13}$ C-NMR $\left(125 \mathrm{MHz}, \mathrm{CDCl}_{3}\right): \delta=172.3,158.7,136.0,132.5,129.4,128.7,128.4,114.0,66.4,55.4,50.7,49.8,41.8$, 20.6 .

Accurate Mass (ESI): Calculated for $\mathrm{C}_{19} \mathrm{H}_{23} \mathrm{NO}_{3}[\mathrm{M}+\mathrm{H}]^{+}:$314.1751, Found: 314.1724, Spectral Accuracy: $99.5 \%$.

HPLC: Daicel Chiralcel OB-H, $n$-hexane $/ i-\mathrm{PrOH}=90 / 10$, Flow rate $=1 \mathrm{~mL} / \mathrm{min}, \mathrm{UV}=230 \mathrm{~nm}, \mathrm{t}_{\mathrm{R}}=17.6 \mathrm{~min}$ (major) and $t_{R}=21.3 \mathrm{~min}$.

The absolute configuration was assigned by analogy. 


\section{Benzyl (R)-3-((4-methoxybenzyl)amino)pentanoate:}

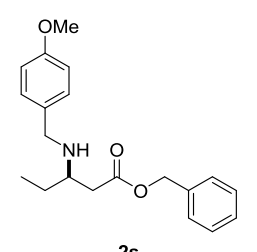

$2 s$

4-Methoxybenzylamine ( $26 \mu \mathrm{L}, 0.2 \mathrm{mmol}, 1$ equiv) and benzyl (E)-pent-2-enoate ( $76 \mathrm{mg}, 0.4 \mathrm{mmol}, 2$ equiv) were treated according to general procedure $\mathrm{C}$ at room temperature for 7 days. Purification by flash chromatography on silica gel eluting with EtOAc/Hexanes (40:60) followed by $\mathrm{MeOH} / \mathrm{CH}_{2} \mathrm{Cl}_{2}$ (10:90) afforded $2 \mathbf{s}$ as a colorless oil in $66 \%$ yield $(43 \mathrm{mg})$.

\section{Characterization data:}

$\mathbf{R}_{\mathbf{f}}=0.24 \mathrm{in} \mathrm{MeOH} / \mathrm{CH}_{2} \mathrm{Cl}_{2} 5: 95 \mathrm{v} / \mathrm{v}$.

$[\alpha]_{\mathrm{D}}^{22}=+1.8\left(\mathrm{c} 0.5, \mathrm{CHCl}_{3}, 82 \% e e\right)$

${ }^{1}$ H-NMR $\left(500 \mathrm{MHz}, \mathrm{CDCl}_{3}\right): \delta=7.38-7.29(\mathrm{comp}, 5 \mathrm{H}), 7.20(\mathrm{~d}, J=8.6 \mathrm{~Hz}, 2 \mathrm{H}), 6.83(\mathrm{~d}, J=8.6 \mathrm{~Hz}, 2 \mathrm{H}), 5.14(\mathrm{~d}$, $J=12.4 \mathrm{~Hz}, 1 \mathrm{H}), 5.11(\mathrm{~d}, J=12.4 \mathrm{~Hz}, 1 \mathrm{H}), 3.79(\mathrm{~s}, 3 \mathrm{H}), 3.71(\mathrm{~d}, J=12.8 \mathrm{~Hz}, 1 \mathrm{H}), 3.68(\mathrm{~d}, J=12.8 \mathrm{~Hz}, 1 \mathrm{H}), 3.03-$ $2.95(\mathrm{~m}, 1 \mathrm{H}), 2.55-2.45$ (comp, 2H), 1.62-1.43 (comp, 3H), 0.91 (t, $J=7.4 \mathrm{~Hz}, 3 \mathrm{H})$.

${ }^{13}$ C-NMR $\left(125 \mathrm{MHz}, \mathrm{CDCl}_{3}\right): \delta=172.6,158.7,136.1,132.8,129.4,128.7,128.4,128.3,113.9,66.3,55.6,55.4$, $50.5,39.1,27.0,10.1$.

Accurate Mass (ESI): Calculated for $\mathrm{C}_{20} \mathrm{H}_{25} \mathrm{NO}_{3}[\mathrm{M}+\mathrm{H}]^{+}$: 328.1907, Found: 328.1994, Spectral Accuracy: $99.2 \%$.

HPLC: Daicel Chiralcel OD-H, $n$-hexane $/ i-\mathrm{PrOH}=90 / 10$, Flow rate $=1 \mathrm{~mL} / \mathrm{min}, \mathrm{UV}=280 \mathrm{~nm}, \mathrm{t}_{\mathrm{R}}=7.2 \mathrm{~min}$ (major) and $t_{R}=8.7 \mathrm{~min}$.

The absolute configuration was assigned by analogy. 


\section{Benzyl (R)-3-(pyrrolidin-1-yl)pentanoate:}

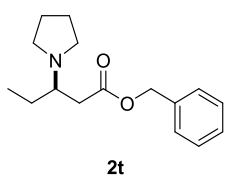

Pyrrolidine ( $17 \mu \mathrm{L}, 0.2 \mathrm{mmol}, 1$ equiv) and benzyl $(E)$-pent-2-enoate $(76 \mathrm{mg}, 0.4 \mathrm{mmol}, 2$ equiv) were treated according to general procedure $\mathrm{C}$ at $-10{ }^{\circ} \mathrm{C}$ for 6 days. Purification by flash chromatography on silica gel eluting with $\mathrm{Et}_{2} \mathrm{O} / \mathrm{Hexanes}_{\mathrm{Et}} \mathrm{N}$ (40:59:1) afforded $\mathbf{2 t}$ as a colorless oil in $92 \%$ yield $(48 \mathrm{mg})$.

\section{Characterization data:}

$\mathbf{R}_{\mathbf{f}}=0.4$ in $\mathrm{MeOH} / \mathrm{CH}_{2} \mathrm{Cl}_{2} 10: 90 \mathrm{v} / \mathrm{v}$.

$[\alpha]_{\mathrm{D}}^{22}=-5.0\left(\mathrm{c} 0.5, \mathrm{CHCl}_{3}, 67 \% e e\right)$.

${ }^{1}$ H-NMR $\left(600 \mathrm{MHz}, \mathrm{CDCl}_{3}\right): \delta=7.38-7.34$ (comp, 4H), 7.33-7.30 (m, 1H), 5.13 (s, 2H), 2.87 (app p, $J=6.7 \mathrm{~Hz}$, 1H), 2.63-2.51 (comp, 5H), 2.43 (dd, $J=14.8,7.0 \mathrm{~Hz}, 1 \mathrm{H}$ ), 1.77-1.67 (comp, 4H), 1.59 (dqd, $J=14.9,7.5,4.9 \mathrm{~Hz}$, 1H), 1.50 (app dp, $J=14.5,7.3 \mathrm{~Hz}, 1 \mathrm{H}), 0.91(\mathrm{t}, J=7.4 \mathrm{~Hz}, 3 \mathrm{H})$.

${ }^{13}$ C-NMR $\left(150 \mathrm{MHz}, \mathrm{CDCl}_{3}\right): \delta=173.0,136.2,128.6,128.3,128.3,66.3,60.6,50.0,36.8,25.4,23.6,10.2$.

Accurate Mass (ESI): Calculated for $\mathrm{C}_{16} \mathrm{H}_{23} \mathrm{NO}_{2}[\mathrm{M}+\mathrm{H}]^{+}:$262.1802, Found: 262.1806, Spectral Accuracy: 98.2\%.

HPLC: Daicel Chiralcel OB-H, $n$-hexane $/ i-\mathrm{PrOH}=99 / 1$, Flow rate $=1 \mathrm{~mL} / \mathrm{min}, \mathrm{UV}=230 \mathrm{~nm}, \mathrm{t}_{\mathrm{R}}=9.1 \mathrm{~min}$ and $\mathrm{t}_{\mathrm{R}}$ $=11.9$ min (major).

The absolute configuration was assigned by analogy. 


\section{Preparation and Characterization of Products From the Kinetic Resolution of Cyclic Amines}

\section{General Procedure D:}

Under $\mathrm{N}_{2}$, a vial was charged with the cyclic amine ( $0.2 \mathrm{mmol}, 1$ equiv) and selenourea-thiourea catalyst $1 \mathbf{q}(15 \mathrm{mg}$, $0.02 \mathrm{mmol}, 0.1$ equiv). Anhydrous toluene $(2 \mathrm{~mL}, 0.1 \mathrm{M})$ was added and the resulting mixture was stirred for $5 \mathrm{~min}$. Benzyl acrylate was then added $(15.8 \mu \mathrm{L}, 0.1 \mathrm{mmol}, 0.5$ equiv) and the reaction mixture was stirred at room temperature until the disappearance of benzyl acrylate as judged by TLC analysis. Subsequently, $\mathrm{Boc}_{2} \mathrm{O}(44 \mathrm{mg}, 0.2$ mmol, 1 equiv) was added and the resulting mixture was stirred for another 2 hours before direct purification by flash chromatography on silica gel.

The conversion, $\boldsymbol{C}_{\boldsymbol{H P L C}}$, for each catalytic reaction was calculated using the following equation: ${ }^{16}$

$$
c_{H P L C}=\frac{e e_{S M}}{e e_{P}+e e_{S M}}
$$

Where $\boldsymbol{e} \boldsymbol{e}_{\boldsymbol{P}}$ is the enantiomeric excess of the conjugate adduct and $\boldsymbol{e} \boldsymbol{e}_{S M}$ is the enantiomeric excess of the recovered $N$-Boc-amine.

The s-factor was calculated using the calculated conversion and ee from either the product, $\boldsymbol{e}_{\boldsymbol{P}}$, or recovered starting material, $\boldsymbol{e} e_{S M}$, following the equation: ${ }^{16}$

$$
\begin{aligned}
& s=\frac{\ln \left[1-c_{H P L C} *\left(1+e e_{P}\right)\right]}{\ln \left[1-c_{H P L C} *\left(1-e e_{P}\right)\right]} \\
& s=\frac{\ln \left[\left(1-c_{H P L C}\right)\left(1-e e_{S M}\right)\right]}{\ln \left[\left(1-c_{H P L C}\right)\left(1+e e_{S M}\right)\right]}
\end{aligned}
$$




\section{Benzyl (S)-3-(2-phenylpiperidin-1-yl)propanoate (4a) and tert-Butyl (R)-2-phenylpiperidine-1-carboxylate}

(5a):

2-Phenylpiperidine ( $32 \mathrm{mg}, 0.2 \mathrm{mmol}, 1$ equiv) was treated according to general procedure D. The reaction time for the conjugate addition step was 4 days. Purification by gradient flash chromatography on silica gel (eluent: $\mathrm{Et}_{2} \mathrm{O} /$ hexanes (5:95) $-\mathrm{Et}_{2} \mathrm{O} /$ hexanes (30:70)) sequentially afforded the less polar product $\mathbf{5 a}$ as a colorless solid in $54 \%$ yield $(28 \mathrm{mg})$, and the more polar product $4 \mathbf{a}$ as a colorless oil in $44 \%$ yield $(28 \mathrm{mg}$ ).

\section{Characterization data of $4 a$ :}

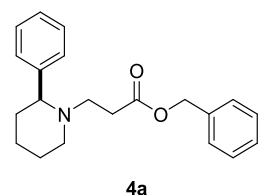

$\mathbf{R}_{\mathbf{f}}=0.32$ in $\mathrm{Et}_{2} \mathrm{O} /$ Hexanes $30: 70 \mathrm{v} / \mathrm{v}$.

$[\alpha]_{\mathrm{D}}^{22}=-24.4\left(\mathrm{c} 0.5, \mathrm{CHCl}_{3}, 92 \% e e\right)$.

${ }^{1}$ H-NMR $\left(600 \mathrm{MHz}, \mathrm{CDCl}_{3}\right): \delta=7.38-7.25(\mathrm{comp}, 9 \mathrm{H}), 7.25-7.20(\mathrm{~m}, 1 \mathrm{H}), 5.09(\mathrm{~d}, J=12.5 \mathrm{~Hz}, 1 \mathrm{H}), 5.03(\mathrm{~d}, J=$ $12.5 \mathrm{~Hz}, 1 \mathrm{H}), 3.11(\operatorname{app} \mathrm{d}, J=11.4 \mathrm{~Hz}, 1 \mathrm{H}), 3.04(\mathrm{dd}, J=11.1,2.5 \mathrm{~Hz}, 1 \mathrm{H}), 2.85$ (ddd, $J=12.9,8.8,7.2 \mathrm{~Hz}, 1 \mathrm{H})$, 2.46 (ddd, $J=15.4,8.5,7.2 \mathrm{~Hz}, 1 \mathrm{H}), 2.39$ (ddd, $J=14.8,8.8,5.0 \mathrm{~Hz}, 1 \mathrm{H}), 2.31$ (ddd, $J=13.3,8.5,5.0 \mathrm{~Hz}, 1 \mathrm{H}$ ), 2.11 (app td, $J=11.8,2.8 \mathrm{~Hz}, 1 \mathrm{H}), 1.77(\mathrm{~d}, J=12.8 \mathrm{~Hz}, 1 \mathrm{H}), 1.74-1.67$ (comp, 2H), 1.63 (app qt, $J=12.9,4.0 \mathrm{~Hz}$, $1 \mathrm{H}), 1.58-1.48(\mathrm{~m}, 1 \mathrm{H}), 1.34$ (app qt, $J=12.9,4.0 \mathrm{~Hz}, 1 \mathrm{H})$.

${ }^{13} \mathrm{C}-\mathrm{NMR}\left(150 \mathrm{MHz}, \mathrm{CDCl}_{3}\right): \delta=172.7,144.8,136.2,128.6,128.5,128.2,127.6,127.1,68.7,66.1,53.2,50.7,36.8$, $31.8,26.2,25.2$.

Accurate Mass (ESI): Calculated for $\mathrm{C}_{21} \mathrm{H}_{25} \mathrm{NO}_{2}[\mathrm{M}+\mathrm{H}]^{+}: 324.1958$, Found: 324.1956, Spectral Accuracy: $98.1 \%$.

HPLC: Daicel Chiralpak AD-H, $n$-hexane $i-\mathrm{PrOH}=99 / 1$, Flow rate $=1 \mathrm{~mL} / \mathrm{min}, \mathrm{UV}=230 \mathrm{~nm}, \mathrm{t}_{\mathrm{R}}=7.5 \mathrm{~min}$ (major) and $t_{\mathrm{R}}=8.9 \mathrm{~min}$.

The absolute configuration was assigned by the absolute configuration of $\mathbf{5 a}$. 


\section{Characterization data of 5a:}

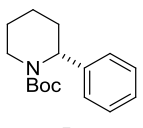

$5 a$

$\mathbf{R}_{\mathbf{f}}=0.42$ in $\mathrm{Et}_{2} \mathrm{O} /$ Hexanes $20: 80 \mathrm{v} / \mathrm{v}$.

$[\alpha]_{\mathrm{D}}^{22}=+20.5\left(\mathrm{c} 0.5, \mathrm{CHCl}_{3}, 76 \% e e\right)$.

${ }^{1}$ H-NMR $\left(600 \mathrm{MHz}, \mathrm{CDCl}_{3}\right) \delta=7.37-7.31$ (comp, 2H), 7.25-7.19 (comp, 3H), 5.46-5.37 (m, $\left.1 \mathrm{H}\right), 4.05$ (app d, J = $13.4 \mathrm{~Hz}, 1 \mathrm{H}), 2.80-2.37$ (m, 1H), 2.31 (app dd, $J=13.9,3.0 \mathrm{~Hz}, 1 \mathrm{H}), 1.88$ (app tdd, $J=13.5,5.5,3.7 \mathrm{~Hz}, 1 \mathrm{H}), 1.61$ (app dt, $J=12.1,2.8 \mathrm{~Hz}, 1 \mathrm{H}), 1.58-1.49$ (comp, 2H), $1.46(\mathrm{~s}, 9 \mathrm{H}), 1.44-1.38(\mathrm{~m}, 1 \mathrm{H})$.

${ }^{13}$ C-NMR $\left(150 \mathrm{MHz}, \mathrm{CDCl}_{3}\right) \delta=155.8,140.6,128.6,126.6,126.4,79.7,53.4,40.2,28.6,28.2,25.6,19.5$.

Accurate Mass (ESI): Calculated for $\mathrm{C}_{16} \mathrm{H}_{23} \mathrm{NO}_{2}[\mathrm{M}+\mathrm{Na}]^{+}:$284.1621, Found: 284.1466, Spectral Accuracy: $99.5 \%$.

HPLC: Daicel Chiralcel OJ-H, $n$-hexane $/ i$-PrOH $=99 / 1$, Flow rate $=0.5 \mathrm{~mL} / \mathrm{min}, \mathrm{UV}=230 \mathrm{~nm}, \mathrm{t}_{\mathrm{R}}=12.5 \mathrm{~min}$ (major) and $t_{\mathrm{R}}=14.0 \mathrm{~min}$.

The absolute configuration of $\mathbf{5 a}\left([\boldsymbol{\alpha}]_{\mathbf{D}}^{\mathbf{2 2}}=+20.5\left(\mathrm{c} 0.5, \mathrm{CHCl}_{3}, 76 \% e e\right)\right)$ was assigned by comparison with the compound reported in the literature ${ }^{17}\left([\boldsymbol{\alpha}]_{\mathrm{D}}^{22}=+76.2\left(\mathrm{c} 1, \mathrm{CHCl}_{3}, 92 \%\right.\right.$ ee $\left.)\right)$.

Calculated conversion $=45 \% ; s$ factor $=55$. 


\section{Benzyl (S)-3-(2-(4-methoxyphenyl)piperidin-1-yl)propanoate (4b) and tert-Butyl $\quad(R)$-2-(4- methoxyphenyl)piperidine-1-carboxylate (5b)}

2-(4-Methoxyphenyl)piperidine ( $38 \mathrm{mg}, 0.2 \mathrm{mmol}, 1$ equiv) was treated according to general procedure D. The reaction time for the conjugate addition step was 3 days. Purification by gradient flash chromatography on silica gel (eluent: $\mathrm{Et}_{2} \mathrm{O} /$ hexanes (5:95) $-\mathrm{Et}_{2} \mathrm{O} /$ hexanes (20:80), then $\mathrm{Et}_{2} \mathrm{O} /$ hexanes/i $\mathrm{PrNH}_{2}(25: 74: 1)$ ) sequentially afforded the less polar product $\mathbf{5 b}$ as a white solid in $49 \%$ yield $(28.7 \mathrm{mg})$, and the more polar product $\mathbf{4 b}$ as a colorless oil in $43 \%$ yield (30 mg).

\section{Characterization data of $4 \mathrm{~b}$ :}

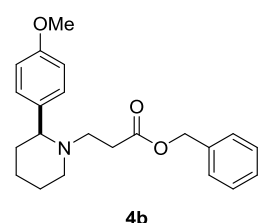

$\mathbf{R}_{\mathbf{f}}=0.18$ in $\mathrm{Et}_{2} \mathrm{O} / \mathrm{Hexanes} 30: 70 \mathrm{v} / \mathrm{v}$.

$[\boldsymbol{\alpha}]_{\mathbf{D}}^{22}=-18.1\left(\mathrm{c} 0.5, \mathrm{CHCl}_{3}, 89 \% e e\right)$.

${ }^{1}$ H-NMR (500 MHz, $\left.\mathrm{CDCl}_{3}\right): \delta=7.38-7.27(\mathrm{comp}, 5 \mathrm{H}), 7.19(\mathrm{~d}, J=8.5 \mathrm{~Hz}, 2 \mathrm{H}), 6.81(\mathrm{~d}, J=8.7 \mathrm{~Hz}, 2 \mathrm{H}), 5.08(\mathrm{~d}$, $J=12.5 \mathrm{~Hz}, 1 \mathrm{H}), 5.03(\mathrm{~d}, J=12.5 \mathrm{~Hz}, 1 \mathrm{H}), 3.78(\mathrm{~s}, 3 \mathrm{H}), 3.10(\operatorname{app~d}, J=11.4 \mathrm{~Hz}, 1 \mathrm{H}), 2.99(\mathrm{dd}, J=11.0,2.8 \mathrm{~Hz}$, $1 \mathrm{H}$ ), 2.85 (ddd, $J=12.8,8.8,7.1 \mathrm{~Hz}, 1 \mathrm{H}$ ), 2.49-2.34 (comp, 2H), 2.29 (ddd, $J=13.2,8.5,4.9 \mathrm{~Hz}, 1 \mathrm{H}$ ), 2.09 (app td, $J=11.7,2.9 \mathrm{~Hz}, 1 \mathrm{H}), 1.81-1.56$ (comp, 4H), 1.51 (app tdd, $J=13.0,10.9,3.7 \mathrm{~Hz}, 1 \mathrm{H}), 1.32$ (app qt, $J=12.9,4.1$ $\mathrm{Hz}, 1 \mathrm{H})$.

${ }^{13} \mathbf{C}$-NMR $\left(125 \mathrm{MHz}, \mathrm{CDCl}_{3}\right): \delta=172.7,158.7,136.9,136.2,128.6,128.6,128.2,128.2,113.9,67.9,66.1,55.3$, 53.3, 50.6, 36.8, 31.7, 26.2, 25.2 .

Accurate Mass (ESI): Calculated for $\mathrm{C}_{22} \mathrm{H}_{27} \mathrm{NO}_{3}[\mathrm{M}+\mathrm{H}]^{+}$: 354.2064, Found: 354.2092, Spectral Accuracy: $99.5 \%$.

HPLC: Daicel Chiralcel OJ-H, $n$-hexane $/ i-P r O H=90 / 10$, Flow rate $=1 \mathrm{~mL} / \mathrm{min}, \mathrm{UV}=280 \mathrm{~nm}, \mathrm{t}_{\mathrm{R}}=12.0 \mathrm{~min}$ (major) and $t_{\mathrm{R}}=15.5 \mathrm{~min}$.

The absolute configuration was assigned by analogy. 


\section{Characterization data of $5 \mathbf{b}$ :}

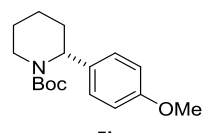

5b

$\mathbf{R}_{\mathbf{f}}=0.28$ in $\mathrm{Et}_{2} \mathrm{O} /$ Hexanes $\left.20: 80 \mathrm{v} / \mathrm{v}\right)$.

$[\alpha]_{\mathrm{D}}^{22}=+29.6\left(\mathrm{c} 0.5, \mathrm{CHCl}_{3}, 85 \% e e\right)$.

${ }^{1}$ H-NMR $\left(500 \mathrm{MHz}, \mathrm{CDCl}_{3}\right): \delta=7.13(\mathrm{~d}, J=8.4 \mathrm{~Hz}, 2 \mathrm{H}), 6.87(\mathrm{~d}, J=8.7 \mathrm{~Hz}, 2 \mathrm{H}), 5.40-5.34(\mathrm{~m}, 1 \mathrm{H}), 4.02(\mathrm{~d}, J=$ $13.9 \mathrm{~Hz}, 1 \mathrm{H}), 3.80(\mathrm{~s}, 3 \mathrm{H}), 2.73(\mathrm{ddd}, J=13.5,11.7,3.7 \mathrm{~Hz}, 1 \mathrm{H}), 2.26(\operatorname{app~d}, J=14.2 \mathrm{~Hz}, 1 \mathrm{H}), 1.85$ (app tt, $J=$ 14.0, $4.5 \mathrm{~Hz}, 1 \mathrm{H}), 1.63-1.38$ (comp, $13 \mathrm{H})$;

${ }^{13}$ C-NMR $\left(125 \mathrm{MHz}, \mathrm{CDCl}_{3}\right): \delta=158.2,155.7,132.4,127.8,114.0,79.6,55.4,52.8,40.0,28.6,28.2,25.7,19.5$;

Accurate Mass (ESI): Calculated for $\mathrm{C}_{17} \mathrm{H}_{25} \mathrm{NO}_{3}[\mathrm{M}+\mathrm{Na}]^{+}$: 314.1727, Found: 314.1602, Spectral Accuracy: $99.4 \%$.

SFC: Daicel Chiralcel OJ-H, column temperature $=40{ }^{\circ} \mathrm{C}, \mathrm{CO}_{2} / \mathrm{MeOH}=90 / 10$, Flow rate $=2 \mathrm{~mL} / \mathrm{min}$, UV $=280$ $\mathrm{nm}, \mathrm{t}_{\mathrm{R}}=2.1 \mathrm{~min}$ (major) and $\mathrm{t}_{\mathrm{R}}=2.4 \mathrm{~min}$.

The absolute configuration was assigned by analogy.

Calculated conversion $=49 \% ; s$ factor $=47$. 


\section{Benzyl (S)-3-(2-(4-bromophenyl)piperidin-1-yl)propanoate $\quad(4 c)$ and tert-Butyl $\quad(R)-2-(4-$ bromophenyl)piperidine-1-carboxylate (5c)}

2-(4-Bromophenyl)piperidine ( $48 \mathrm{mg}, 0.2 \mathrm{mmol}, 1$ equiv) was treated according to general procedure D. The reaction time for the conjugate addition step was 4 days. Purification by gradient flash chromatography on silica gel (eluent: $\mathrm{Et}_{2} \mathrm{O} /$ hexanes $(5: 95)-\mathrm{Et}_{2} \mathrm{O} /$ hexanes (30:70)) sequentially afforded the less polar product $\mathbf{5 c}$ as a colorless oil in $61 \%$ yield $(41.2 \mathrm{mg}$ ), and the more polar product $\mathbf{4 c}$ as a colorless oil in $38 \%$ yield ( $30.3 \mathrm{mg}$ ).

\section{Characterization data of $4 \mathrm{c}$ :}

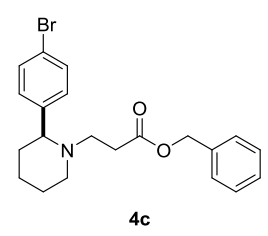

$\mathbf{R}_{\mathbf{f}}=0.35$ in $\mathrm{Et}_{2} \mathrm{O} / \mathrm{Hexanes} 30: 70 \mathrm{v} / \mathrm{v}$.

$[\boldsymbol{\alpha}]_{\mathbf{D}}^{22}=+18.2\left(\mathrm{c} 0.5, \mathrm{CHCl}_{3}, 88 \% e e\right)$.

${ }^{1}$ H-NMR $\left(500 \mathrm{MHz}, \mathrm{CDCl}_{3}\right): \delta=7.40-7.28(\mathrm{comp}, 7 \mathrm{H}), 7.15(\mathrm{~d}, J=7.9 \mathrm{~Hz}, 2 \mathrm{H}), 5.09(\mathrm{~d}, J=12.4 \mathrm{~Hz}, 1 \mathrm{H}), 5.04(\mathrm{~d}$, $J=12.4 \mathrm{~Hz}, 1 \mathrm{H}), 3.11(\operatorname{app~d}, J=11.1 \mathrm{~Hz}, 1 \mathrm{H}), 3.00(\mathrm{dd}, J=11.1,2.8 \mathrm{~Hz}, 1 \mathrm{H}), 2.81(\mathrm{dddd}, J=13.1,8.7,7.3,1.3$ $\mathrm{Hz}, 1 \mathrm{H}$ ), 2.49-2.32 (comp, 2H), 2.26 (dddd, $J=13.2,8.4,4.9,1.2 \mathrm{~Hz}, 1 \mathrm{H}), 2.11-2.01$ (m, 1H), 1.76 (app d, $J=12.6$ $\mathrm{Hz}, 1 \mathrm{H}), 1.72-1.53$ (comp, 3H), 1.50-1.39 (m, 1H), 1.32 (app tdd, $J=12.9,8.3,6.2 \mathrm{~Hz}, 1 \mathrm{H}$ ).

${ }^{13}$ C-NMR $\left(125 \mathrm{MHz}, \mathrm{CDCl}_{3}\right): \delta=172.5,144.0,136.1,131.7,129.3,128.7,128.3,128.3,120.6,68.1,66.2,53.1$, $50.8,36.9,31.9,26.0,25.0$.

Accurate Mass (ESI): Calculated for $\left.\mathrm{C}_{21} \mathrm{H}_{24} \mathrm{BrNO}_{2}[\mathrm{M}+\mathrm{H}]\right]^{+}:{ }^{79} \mathrm{Br}$, 402.1063, Found: $402.1021,{ }^{81} \mathrm{Br}$, Found: 404.1078, Spectral Accuracy: 98.1\%.

SFC: Daicel Chiralcel OD-H, column temperature $=40{ }^{\circ} \mathrm{C}, \mathrm{CO}_{2} / \mathrm{MeOH}=90 / 10$, Flow rate $=1 \mathrm{~mL} / \mathrm{min}, \mathrm{UV}=210$ $\mathrm{nm}, \mathrm{t}_{\mathrm{R}}=13.6 \mathrm{~min}$ (major) and $\mathrm{t}_{\mathrm{R}}=14.4 \mathrm{~min}$.

The absolute configuration was assigned by analogy. 


\section{Characterization data of $5 \mathrm{c}$ :}

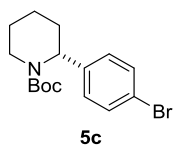

$\mathbf{R}_{\mathbf{f}}=0.42$ in $\mathrm{Et}_{2} \mathrm{O} /$ Hexanes $20: 80 \mathrm{v} / \mathrm{v}$.

$[\alpha]_{\mathrm{D}}^{22}=+24.0\left(\mathrm{c} 0.5, \mathrm{CHCl}_{3}, 54 \% e e\right)$.

${ }^{1}$ H-NMR $\left(500 \mathrm{MHz}, \mathrm{CDCl}_{3}\right): \delta=7.45(\mathrm{~d}, J=8.4 \mathrm{~Hz}, 2 \mathrm{H}), 7.08(\mathrm{~d}, J=8.5 \mathrm{~Hz}, 2 \mathrm{H}), 5.38-5.29(\mathrm{~m}, 1 \mathrm{H}), 4.03$ (app d, $J=12.8 \mathrm{~Hz}, 1 \mathrm{H}), 2.72$ (app td, $J=12.9,3.4 \mathrm{~Hz}, 1 \mathrm{H}), 2.23($ app d, $J=15.3 \mathrm{~Hz}, 1 \mathrm{H}), 1.88$ (app tt, $J=13.9,4.6 \mathrm{~Hz}$, 1H), 1.64-1.52 (comp, 2H), 1.50 (app dt, $J=12.4,4.3 \mathrm{~Hz}, 1 \mathrm{H}), 1.45$ (s, 9H), 1.42-1.30 (m, 1H).

${ }^{13}$ C-NMR $\left(125 \mathrm{MHz}, \mathrm{CDCl}_{3}\right): \delta=155.7,139.8,131.7,128.5,120.4,79.9,53.0,40.2,28.6,28.2,25.5,19.4$.

Accurate Mass (ESI): Calculated for $\left.\mathrm{C}_{16} \mathrm{H}_{22} \mathrm{BrNO}_{2}[\mathrm{M}+\mathrm{Na}]\right]^{+}:{ }^{79} \mathrm{Br}, 362.0726$, Found: $362.0555,{ }^{81} \mathrm{Br}$, Found: 364.0551, Spectral Accuracy: $98.1 \%$.

SFC: Daicel Chiralcel OJ-H, column temperature $=40{ }^{\circ} \mathrm{C}, \mathrm{CO}_{2} / \mathrm{MeOH}=90 / 10$, Flow rate $=2 \mathrm{~mL} / \mathrm{min}, \mathrm{UV}=210$ $\mathrm{nm}, \mathrm{t}_{\mathrm{R}}=2.2 \min$ (major) and $\mathrm{t}_{\mathrm{R}}=2.4 \mathrm{~min}$.

The absolute configuration was assigned by analogy.

Calculated conversion $=38 \% ; s$ factor $=27$. 


\section{Benzyl (S)-3-(2-(4-chlorophenyl)piperidin-1-yl)propanoate $\quad(4 d) \quad$ and $\quad$ tert-Butyl $\quad(R)-2-(4-$ chlorophenyl)piperidine-1-carboxylate (5d)}

2-(4-Chlorophenyl)piperidine (39 mg, $0.2 \mathrm{mmol}, 1$ equiv) was treated according to general procedure D. The reaction time for the conjugate addition step was 5 days. Purification by gradient flash chromatography on silica gel (eluent: $\mathrm{Et}_{2} \mathrm{O} /$ hexanes $(5: 95)-\mathrm{Et}_{2} \mathrm{O} /$ hexanes $(30: 70)$ ) sequentially afforded the less polar product $\mathbf{5 d}$ as a colorless oil in $57 \%$ yield $(33.7 \mathrm{mg}$ ), and the more polar product $\mathbf{4 d}$ as a colorless oil in $41 \%$ yield $(29 \mathrm{mg}$ ).

\section{Characterization data of $\mathbf{4 d}$ :}

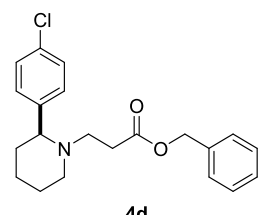

$\mathbf{R}_{\mathbf{f}}=0.38$ in $\mathrm{Et}_{2} \mathrm{O} / \mathrm{Hexanes} 30: 70 \mathrm{v} / \mathrm{v}$.

$[\boldsymbol{\alpha}]_{\mathbf{D}}^{22}=-26.1\left(\mathrm{c} 0.5, \mathrm{CHCl}_{3}, 87 \% e e\right)$.

${ }^{1}$ H-NMR $\left(500 \mathrm{MHz}, \mathrm{CDCl}_{3}\right): \delta=7.39-7.27$ (comp, 5H), 7.25-7.17 (comp, 4H), $5.09(\mathrm{~d}, J=12.4 \mathrm{~Hz}, 1 \mathrm{H}), 5.03(\mathrm{~d}$, $J=12.4 \mathrm{~Hz}, 1 \mathrm{H}), 3.11(\operatorname{app~d}, J=11.4 \mathrm{~Hz}, 1 \mathrm{H}), 3.01(\mathrm{dd}, J=11.1,2.8 \mathrm{~Hz}, 1 \mathrm{H}), 2.81(\mathrm{ddd}, J=12.9,8.7,7.4 \mathrm{~Hz}$, $1 \mathrm{H}), 2.45(\operatorname{app} \mathrm{dt}, J=15.5,7.8 \mathrm{~Hz}, 1 \mathrm{H}), 2.38(\mathrm{ddd}, J=14.9,8.7,4.9 \mathrm{~Hz}, 1 \mathrm{H}), 2.26$ (ddd, $J=13.1,8.3,4.9 \mathrm{~Hz}, 1 \mathrm{H}$ ), 2.07 (app td, $J=11.7,2.8 \mathrm{~Hz}, 1 \mathrm{H}), 1.80-1.74$ (m, 1H), 1.72-1.54 (comp, 3H), 1.45 (app tdd, $J=13.0,11.0,3.7 \mathrm{~Hz}$, $1 \mathrm{H}), 1.32$ (app qt, $J=13.2,3.9 \mathrm{~Hz}, 1 \mathrm{H}$ ).

${ }^{13}$ C-NMR $\left(125 \mathrm{MHz}, \mathrm{CDCl}_{3}\right): \delta=172.5,143.5,136.1,132.5,128.9,128.7,128.6,128.3,128.3,68.0,66.2,53.1$, 50.7, 36.9, 31.9, 26.1, 25.0.

Accurate Mass (ESI): Calculated for $\mathrm{C}_{21} \mathrm{H}_{24} \mathrm{ClNO}_{2}[\mathrm{M}+\mathrm{H}]^{+}: 358.1568$, Found: 358.1493 , Spectral Accuracy: $97.6 \%$.

SFC: Daicel Chiralcel OD-H, column temperature $=40{ }^{\circ} \mathrm{C}, \mathrm{CO}_{2} / \mathrm{MeOH}=95 / 5$, Flow rate $=2 \mathrm{~mL} / \mathrm{min}, \mathrm{UV}=210$ $\mathrm{nm}, \mathrm{t}_{\mathrm{R}}=7.7$ min (major) and $\mathrm{t}_{\mathrm{R}}=8.1 \mathrm{~min}$.

The absolute configuration was assigned by analogy. 


\section{Characterization data of $5 \mathrm{~d}$ :}

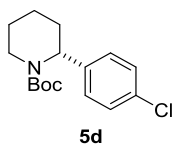

$\mathbf{R}_{\mathbf{f}}=0.42$ in $\mathrm{Et}_{2} \mathrm{O} / \mathrm{Hexanes} 20: 80 \mathrm{v} / \mathrm{v}$.

$[\boldsymbol{\alpha}]_{\mathbf{D}}^{22}=+30.2\left(\mathrm{c} 0.5, \mathrm{CHCl}_{3}, 61 \% e e\right)$.

${ }^{1} \mathbf{H}-\mathbf{N M R}\left(500 \mathrm{MHz}, \mathrm{CDCl}_{3}\right): \delta=7.30(\mathrm{~d}, J=8.5 \mathrm{~Hz}, 2 \mathrm{H}), 7.14(\mathrm{~d}, J=7.9 \mathrm{~Hz}, 2 \mathrm{H}), 5.41-5.32(\mathrm{~m}, 1 \mathrm{H}), 4.13-3.97$ (m, $1 \mathrm{H}), 2.72$ (ddd, $J=13.5,12.2,3.6 \mathrm{~Hz}, 1 \mathrm{H}), 2.40-2.15(\mathrm{~m}, 1 \mathrm{H}), 1.88$ (app tdd, $J=13.8,5.5,3.8 \mathrm{~Hz}, 1 \mathrm{H}), 1.64-$ 1.47 (comp, 3H), 1.45 (s, 9H), 1.37 (app qt, $J=13.1,4.1 \mathrm{~Hz}, 1 \mathrm{H}$ ).

${ }^{13} \mathbf{C}-\mathrm{NMR}\left(125 \mathrm{MHz}, \mathrm{CDCl}_{3}\right): \delta=155.7,139.2,132.3,128.8,128.1,79.88,53.0,40.2,28.6,28.3,25.5,19.4$.

Accurate Mass (ESI): Calculated for $\mathrm{C}_{16} \mathrm{H}_{22} \mathrm{ClNO}_{2}[\mathrm{M}+\mathrm{Na}]^{+}$. Theoretical: 318.1231 . Found: 318.1098 . Spectral Accuracy: $96.4 \%$.

SFC: Daicel Chiralcel OJ-H, column temperature $=40^{\circ} \mathrm{C}, \mathrm{CO}_{2} / \mathrm{MeOH}=95 / 5$, Flow rate $=2 \mathrm{~mL} / \mathrm{min}, \mathrm{UV}=210 \mathrm{~nm}$, $\mathrm{t}_{\mathrm{R}}=2.3 \min$ (major) and $\mathrm{t}_{\mathrm{R}}=2.6$ min.

The absolute configuration was assigned by analogy.

Calculated conversion $=41 \% ; s$ factor $=27$. 


\section{Benzyl (S)-3-(2-(thiophen-2-yl)piperidin-1-yl)propanoate (4e) and tert-Butyl (R)-2-(thiophen-2-yl)piperidine- 1-carboxylate (5e)}

2-(Thiophen-2-yl)piperidine ( $33.5 \mathrm{mg}, 0.2 \mathrm{mmol}, 1$ equiv) was treated according to general procedure D. The reaction time for the conjugate addition step was 5 days. Purification by gradient flash chromatography on silica gel (eluent: $\mathrm{Et}_{2} \mathrm{O} /$ hexanes $(5: 95)-\mathrm{Et}_{2} \mathrm{O} /$ hexanes $(30: 70)$ ) sequentially afforded the less polar product $\mathbf{5 e}$ as a colorless oil in $52 \%$ yield $(27.6 \mathrm{mg})$, and the more polar product $4 \mathbf{e}$ as a colorless oil in $43 \%$ yield $(28.5 \mathrm{mg})$.

\section{Characterization data of $4 \mathrm{e}$ :}

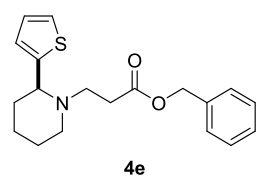

$\mathbf{R}_{\mathbf{f}}=0.35$ in $\mathrm{Et}_{2} \mathrm{O} /$ Hexanes $30: 70 \mathrm{v} / \mathrm{v}$.

$[\alpha]_{\mathrm{D}}^{22}=-25.3\left(\mathrm{c} 0.5, \mathrm{CHCl}_{3}, 91 \% e e\right)$.

${ }^{1} \mathbf{H}-\mathrm{NMR}\left(500 \mathrm{MHz}, \mathrm{CDCl}_{3}\right.$ ): $\delta=7.38-7.29$ (comp, 5H), 7.19 (ddd, $J=4.6,1.8,0.6 \mathrm{~Hz}, 1 \mathrm{H}$ ), 6.92-6.87 (comp, 2H), $5.09(\mathrm{~d}, J=12.5 \mathrm{~Hz}, 1 \mathrm{H}), 5.05(\mathrm{~d}, J=12.4 \mathrm{~Hz}, 1 \mathrm{H}), 3.47$ (dd, $J=10.6,3.0 \mathrm{~Hz}, 1 \mathrm{H}), 3.07$ (app dtd, $J=11.7,3.8,1.4$ $\mathrm{Hz}, 1 \mathrm{H}), 3.01-2.91(\mathrm{~m}, 1 \mathrm{H}), 2.50-2.38(\mathrm{comp}, 3 \mathrm{H}) 2.15(\mathrm{td}, J=11.3,3.8 \mathrm{~Hz}, 1 \mathrm{H}), 1.89-1.82(\mathrm{~m}, 1 \mathrm{H}), 1.82-1.74(\mathrm{~m}$, 1H), 1.71-1.58 (comp, 3H), 1.41-1.29 (m, 1H).

${ }^{13}$ C-NMR (125 MHz, $\left.\mathrm{CDCl}_{3}\right): \delta=172.6,148.7,136.2,128.6,128.2,126.1,124.5,124.3,66.2,63.0,53.0,50.6,37.4$, $31.9,25.8,24.7$.

Accurate Mass (ESI): Calculated for $\mathrm{C}_{19} \mathrm{H}_{23} \mathrm{NO}_{2} \mathrm{~S}[\mathrm{M}+\mathrm{H}]^{+}:$330.1522, Found: 330.1513, Spectral Accuracy: $98.8 \%$.

SFC: Daicel Chiralcel OD-H, column temperature $=40^{\circ} \mathrm{C}, \mathrm{CO}_{2} / \mathrm{MeOH}=95 / 5$, Flow rate $=2 \mathrm{~mL} / \mathrm{min}, \mathrm{UV}=230$ $\mathrm{nm}, \mathrm{t}_{\mathrm{R}}=8.0 \mathrm{~min}$ (major) and $\mathrm{t}_{\mathrm{R}}=8.7 \mathrm{~min}$.

The absolute configuration was assigned by analogy. 


\section{Characterization data of $5 \mathrm{e}$ :}

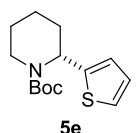

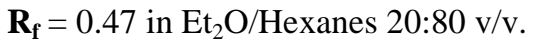

$[\alpha]_{\mathrm{D}}^{22}=+27.5\left(\mathrm{c} 0.5, \mathrm{CHCl}_{3}, 69 \% e e\right)$.

${ }^{1}$ H-NMR $\left(500 \mathrm{MHz}, \mathrm{CDCl}_{3}\right): \delta=7.20(\mathrm{dt}, J=5.1,0.9 \mathrm{~Hz}, 1 \mathrm{H}), 6.96(\mathrm{dd}, J=5.1,3.5 \mathrm{~Hz}, 1 \mathrm{H}), 6.82(\mathrm{dt}, J=3.5,1.3$ $\mathrm{Hz}, 1 \mathrm{H}), 5.59$ (app d, $J=3.5 \mathrm{~Hz}, 1 \mathrm{H}), 4.01$ (app d, $J=11.8 \mathrm{~Hz}, 1 \mathrm{H}), 2.84$ (app td, $J=13.2,2.9 \mathrm{~Hz}, 1 \mathrm{H}$ ), 2.17 (app ddt, $J=14.2,3.6,1.7 \mathrm{~Hz}, 1 \mathrm{H}$ ), 1.91 (app ddt, $J=13.9,12.4,5.3 \mathrm{~Hz}, 1 \mathrm{H}$ ), 1.69-1.55 (comp, 3H), 1.52-1.43 (comp, $10 \mathrm{H})$.

${ }^{13}$ C-NMR (125 MHz, $\left.\mathrm{CDCl}_{3}\right): \delta=155.1,145.6,126.9,124.4,124.3,80.0,50.9,39.9,29.6,28.6,25.5,19.7$.

Accurate Mass (ESI): Calculated for $\mathrm{C}_{14} \mathrm{H}_{21} \mathrm{NO}_{2} \mathrm{~S}[\mathrm{M}+\mathrm{Na}]^{+}$: 290.1185, Found: 290.1138, Spectral Accuracy: $98.3 \%$.

SFC: Daicel Chiralcel OJ-H, column temperature $=40^{\circ} \mathrm{C}, \mathrm{CO}_{2} / \mathrm{MeOH}=95 / 5$, Flow rate $=2 \mathrm{~mL} / \mathrm{min}, \mathrm{UV}=230 \mathrm{~nm}$, $t_{R}=2.2 \min$ (major) and $t_{R}=2.4$ min.

The absolute configuration was assigned by analogy.

Calculated conversion $=43 \% ; s$ factor $=44$. 


\section{Benzyl (S)-3-(2-(o-tolyl)piperidin-1-yl)propanoate (4f) and tert-Butyl (R)-2-(o-tolyl)piperidine-1-carboxylate}

(5f):

2-(o-Tolyl)piperidine ( $35 \mathrm{mg}, 0.2 \mathrm{mmol}, 1$ equiv) was treated according to general procedure $\mathrm{D}$. The reaction time for the conjugate addition step was 4 days. Purification by gradient flash chromatography on silica gel (eluent: $\mathrm{Et}_{2} \mathrm{O} /$ hexanes (5:95) $-\mathrm{Et}_{2} \mathrm{O} /$ hexanes (30:70)) sequentially afforded the less polar product $\mathbf{5 f}$ as a colorless oil in $50 \%$ yield $(27.6 \mathrm{mg})$, and the more polar product $\mathbf{4 f}$ as a colorless oil in $44 \%$ yield $(29.5 \mathrm{mg})$.

\section{Characterization data of $4 f$ :}

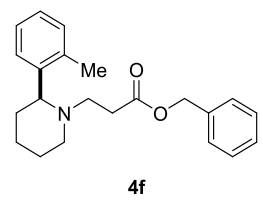

$\mathbf{R}_{\mathbf{f}}=0.41$ in $\mathrm{Et}_{2} \mathrm{O} /$ Hexanes $30: 70 \mathrm{v} / \mathrm{v}$.

$[\alpha]_{\mathrm{D}}^{22}=-32.0\left(\mathrm{c} 0.5, \mathrm{CHCl}_{3}, 84 \% e e\right)$.

${ }^{1}$ H-NMR $\left(500 \mathrm{MHz}, \mathrm{CDCl}_{3}\right): \delta=7.42(\mathrm{~d}, J=7.6 \mathrm{~Hz}, 1 \mathrm{H}), 7.38-7.27$ (comp, 5H), 7.18-7.11 (m, 1H), 7.11-7.07 (comp, 2H), 5.09 (d, $J=12.5 \mathrm{~Hz}, 1 \mathrm{H}), 5.03(\mathrm{~d}, J=12.5 \mathrm{~Hz}, 1 \mathrm{H}), 3.30(\operatorname{app~d}, J=10.1 \mathrm{~Hz}, 1 \mathrm{H}), 3.16(\operatorname{app} \mathrm{d}, J=11.0$ $\mathrm{Hz}, 1 \mathrm{H}), 2.84(\mathrm{ddd}, J=12.3,9.1,7.4 \mathrm{~Hz}, 1 \mathrm{H}), 2.46(\mathrm{ddd}, J=15.4,8.5,7.1 \mathrm{~Hz}, 1 \mathrm{H}), 2.42-2.35(\mathrm{~m}, 1 \mathrm{H}), 2.34(\mathrm{~s}, 3 \mathrm{H})$, 2.26 (ddd, $J=13.1,8.3,5.1 \mathrm{~Hz}, 1 \mathrm{H}), 2.09$ (app td, $J=11.8,3.0 \mathrm{~Hz}, 1 \mathrm{H}), 1.78($ app d, $J=12.6 \mathrm{~Hz}, 1 \mathrm{H}), 1.73-1.57$ (comp, 3H), 1.46 (dt, $J=16.4,13.1 \mathrm{~Hz}, 1 \mathrm{H}), 1.34$ (qt, $J=13.2,4.1 \mathrm{~Hz}, 1 \mathrm{H})$.

${ }^{13}$ C-NMR (125 MHz, $\left.\mathrm{CDCl}_{3}\right): \delta=172.7,142.7,136.2,135.0,130.4,128.6,128.2(2), 128.2(1), 127.3,126.4,126.4$, $66.1,64.0,53.5,50.6,35.3,32.2,26.2,25.3,19.6$.

Accurate Mass (ESI): Calculated for $\mathrm{C}_{22} \mathrm{H}_{27} \mathrm{NO}_{2}[\mathrm{M}+\mathrm{H}]^{+}:$338.2115, Found: 338.2109, Spectral Accuracy: 98.5\%.

SFC: Daicel Chiralcel OD-H, column temperature $=40{ }^{\circ} \mathrm{C}, \mathrm{CO}_{2} / \mathrm{MeOH}=90 / 10$, Flow rate $=1 \mathrm{~mL} / \mathrm{min}, \mathrm{UV}=210$ $\mathrm{nm}, \mathrm{t}_{\mathrm{R}}=9.9 \min$ (major) and $\mathrm{t}_{\mathrm{R}}=10.5 \mathrm{~min}$.

The absolute configuration was assigned by analogy. 


\section{Characterization data of $5 f$ :}

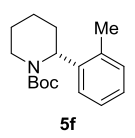

$\mathbf{R}_{\mathbf{f}}=0.39$ in $\mathrm{Et}_{2} \mathrm{O} /$ Hexanes 20:80 v/v.

$[\alpha]_{\mathrm{D}}^{22}=+16.6\left(\mathrm{c} 0.5, \mathrm{CHCl}_{3}, 72 \% e e\right)$.

${ }^{1}$ H-NMR $\left(500 \mathrm{MHz}, \mathrm{CDCl}_{3}\right): \delta=7.22(\mathrm{~d}, J=6.2 \mathrm{~Hz}, 1 \mathrm{H}), 7.16-7.08(\mathrm{comp}, 3 \mathrm{H}), 5.21(\mathrm{dd}, J=6.4,4.5 \mathrm{~Hz}, 1 \mathrm{H})$, 4.05 (ddd, $J=13.9,5.5,2.7 \mathrm{~Hz}, 1 \mathrm{H}), 3.23$ (ddd, $J=13.6,11.5,4.8 \mathrm{~Hz}, 1 \mathrm{H}), 2.32(\mathrm{~s}, 3 \mathrm{H}), 1.95-1.86(\mathrm{~m}, 1 \mathrm{H}), 1.86-$ $1.79(\mathrm{~m}, 1 \mathrm{H}), 1.79-1.71(\mathrm{~m}, 1 \mathrm{H}), 1.68-1.54(\mathrm{comp}, 3 \mathrm{H}), 1.27$ (s, 9H).

${ }^{13}$ C-NMR $\left(125 \mathrm{MHz}, \mathrm{CDCl}_{3}\right): \delta=155.8,142.7,135.1,130.8,126.4,125.8,125.6,79.5,52.9,41.5,28.8,28.4,24.4$, $19.5,19.0$.

Accurate Mass (ESI): Calculated for $\mathrm{C}_{17} \mathrm{H}_{25} \mathrm{NO}_{2}[\mathrm{M}+\mathrm{Na}]^{+}$: 298.1778, Found: 298.1616, Spectral Accuracy: $99.1 \%$.

SFC: Daicel Chiralcel OD-H, column temperature $=40^{\circ} \mathrm{C}, \mathrm{CO}_{2} / \mathrm{MeOH}=98 / 2$, Flow rate $=2 \mathrm{~mL} / \mathrm{min}, \mathrm{UV}=210$ $\mathrm{nm}, \mathrm{t}_{\mathrm{R}}=4.6 \mathrm{~min}$ (major) and $\mathrm{t}_{\mathrm{R}}=4.9 \mathrm{~min}$.

The absolute configuration was assigned by analogy.

Calculated conversion $=46 \% ; s$ factor $=25$. 


\section{Benzyl (S)-3-(2-(naphthalen-2-yl)piperidin-1-yl)propanoate $(4 \mathrm{~g})$ and tert-Butyl $(R)$-2-(naphthalen-2- yl)piperidine-1-carboxylate $(5 \mathrm{~g})$ :}

2-(Naphthalen-2-yl)piperidine ( $42 \mathrm{mg}, 0.2 \mathrm{mmol}, 1$ equiv) was treated according to general procedure D. The reaction time for the conjugate addition step was 3.5 days. Purification by gradient flash chromatography on silica gel (eluent: $\mathrm{Et}_{2} \mathrm{O} /$ hexanes $(5: 95)-\mathrm{Et}_{2} \mathrm{O} /$ hexanes (30:70)) sequentially afforded the less polar product $\mathbf{5 g}$ as a white solid in $46 \%$ yield $(28.2 \mathrm{mg}$ ), and the more polar product $4 \mathrm{~g}$ as a colorless oil in $46 \%$ yield (34 $\mathrm{mg}$ ).

\section{Characterization data of $\mathbf{4 g}$ :}

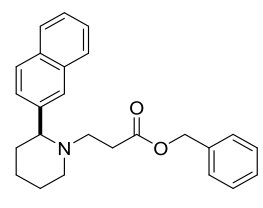

$4 g$

$\mathbf{R}_{\mathbf{f}}=0.29$ in $\mathrm{Et}_{2} \mathrm{O} / \mathrm{Hexanes} 30: 70 \mathrm{v} / \mathrm{v}$.

$[\alpha]_{\mathbf{D}}^{22}=-14.5\left(\mathrm{c} 0.5, \mathrm{CHCl}_{3}, 88 \% e e\right)$.

${ }^{1}$ H-NMR $\left(500 \mathrm{MHz}, \mathrm{CDCl}_{3}\right.$ ): $\delta=7.83-7.75$ (comp, 3H), 7.72 (s, 1H), 7.52-7.42 (comp, 3H), 7.31-7.26 (comp, 3H), 7.26-7.21 (comp, 2H), $5.06(\mathrm{~d}, J=12.4 \mathrm{~Hz}, 1 \mathrm{H}), 5.00(\mathrm{~d}, J=12.5 \mathrm{~Hz}, 1 \mathrm{H}), 3.24(\mathrm{dd}, J=11.1,2.8 \mathrm{~Hz}, 1 \mathrm{H}), 3.16$ (app d, $J=11.6 \mathrm{~Hz}, 1 \mathrm{H}), 2.90($ app dt, $J=13.0,7.7 \mathrm{~Hz}, 1 \mathrm{H}), 2.49(\operatorname{app~dt}, J=14.8,7.5 \mathrm{~Hz}, 1 \mathrm{H}), 2.45-2.40(\mathrm{~m}, 1 \mathrm{H})$, 2.38-2.31 (m, 1H), 2.17 (app td, $J=11.6,3.0 \mathrm{~Hz}, 1 \mathrm{H}), 1.82$ (app d, $J=13.3 \mathrm{~Hz}, 1 \mathrm{H}), 1.78-1.59$ (comp, 4H), 1.40 (app qt, $J=12.7,4.2 \mathrm{~Hz}, 1 \mathrm{H}$ ).

${ }^{13} \mathbf{C}-\mathrm{NMR}\left(125 \mathrm{MHz}, \mathrm{CDCl}_{3}\right): \delta=172.6,142.4,136.1,133.6,133.0,128.6,128.3,128.2,127.8,127.8,126.2,126.0$, $125.8,125.5,68.7,66.1,53.2,50.9,36.7,31.8,26.2,25.2$.

Accurate Mass (ESI): Calculated for $\mathrm{C}_{25} \mathrm{H}_{27} \mathrm{NO}_{2}[\mathrm{M}+\mathrm{H}]^{+}$: 374.2115, Found: 374.2059, Spectral Accuracy: 97.8\%.

SFC: Daicel Chiralcel OD-H, column temperature $=40{ }^{\circ} \mathrm{C}, \mathrm{CO}_{2} / \mathrm{MeOH}=90 / 10$, Flow rate $=2 \mathrm{~mL} / \mathrm{min}, \mathrm{UV}=210$ $\mathrm{nm}, \mathrm{t}_{\mathrm{R}}=9.7 \mathrm{~min}$ (major) and $\mathrm{t}_{\mathrm{R}}=10.6 \mathrm{~min}$.

The absolute configuration was assigned by analogy. 


\section{Characterization data of $5 \mathrm{~g}$ :}

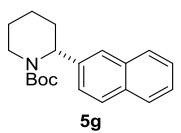

$\mathbf{R}_{\mathbf{f}}=0.36$ in $\mathrm{Et}_{2} \mathrm{O} / \mathrm{Hexanes} 20: 80 \mathrm{v} / \mathrm{v}$.

$[\alpha]_{\mathrm{D}}^{22}=+48.5\left(\mathrm{c} 0.5, \mathrm{CHCl}_{3}, 80 \% e e\right)$.

${ }^{1}$ H-NMR (500 MHz, CDCl $)$ ): $\delta=7.86-7.78$ (comp, 3H), 7.65 (s, 1H), 7.51-7.43 (comp, 2H), 7.37 (d, $J=8.6 \mathrm{~Hz}$, $1 \mathrm{H}), 5.63-5.52(\mathrm{~m}, 1 \mathrm{H}), 4.11(\mathrm{app} \mathrm{d}, J=13.6 \mathrm{~Hz}, 1 \mathrm{H}), 2.84(\mathrm{ddd}, J=13.4,10.6,4.8 \mathrm{~Hz}, 1 \mathrm{H}), 2.45$ (app ddt, $J=$ 14.4, 4.5, $2.7 \mathrm{~Hz}, 1 \mathrm{H}), 1.97$ (app tt, $J=13.2,4.3 \mathrm{~Hz}, 1 \mathrm{H}), 1.70-1.62(\mathrm{~m}, 1 \mathrm{H}), 1.61-1.51$ (comp, 3H), 1.49 (s, 9H).

${ }^{13}$ C-NMR $\left(125 \mathrm{MHz}, \mathrm{CDCl}_{3}\right): \delta=155.9,138.1,133.6,132.3,128.4,128.0,127.6,126.2,125.8,125.3,125.1,79.8$, 53.6, 40.4, 28.6, 28.3, 25.6, 19.6.

Accurate Mass (ESI): Calculated for $\mathrm{C}_{20} \mathrm{H}_{25} \mathrm{NO}_{2}[\mathrm{M}+\mathrm{Na}]^{+}: 334.1778$, Found: 334.1731, Spectral Accuracy: 99.0\%.

SFC: Daicel Chiralcel OD-H, column temperature $=40{ }^{\circ} \mathrm{C}, \mathrm{CO}_{2} / \mathrm{MeOH}=90 / 10$, Flow rate $=2 \mathrm{~mL} / \mathrm{min}, \mathrm{UV}=210$ $\mathrm{nm}, \mathrm{t}_{\mathrm{R}}=4.0 \mathrm{~min}$ (major) and $\mathrm{t}_{\mathrm{R}}=4.9 \mathrm{~min}$.

The absolute configuration was assigned by analogy.

Calculated conversion $=48 \% ; s$ factor $=39$. 


\section{Benzyl (S)-3-(2-(3-methoxyphenyl)piperidin-1-yl)propanoate $\quad(4 \mathrm{~h}) \quad$ and $\quad$ tert-Butyl $\quad(R)-2-(3-$ methoxyphenyl)piperidine-1-carboxylate (5h):}

2-(3-Methoxyphenyl)piperidine (38 mg, $0.2 \mathrm{mmol}, 1$ equiv) was treated according to general procedure D. The reaction time for the conjugate addition step was 3 days. Purification by gradient flash chromatography on silica gel (eluent: $\mathrm{Et}_{2} \mathrm{O} /$ hexanes $(5: 95)-\mathrm{Et}_{2} \mathrm{O} /$ hexanes $(30: 70)$ ) sequentially afforded the less polar product $\mathbf{5 h}$ as a colorless oil in $51 \%$ yield $(29.8 \mathrm{mg})$, and the more polar product $\mathbf{4 h}$ as a colorless oil in $44 \%$ yield $(31.3 \mathrm{mg})$.

\section{Characterization data of $4 \mathrm{~h}$ :}

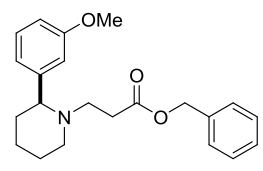

$4 h$

$\mathbf{R}_{\mathbf{f}}=0.24$ in $\mathrm{Et}_{2} \mathrm{O} /$ Hexanes $30: 70 \mathrm{v} / \mathrm{v}$.

$[\alpha]_{\mathrm{D}}^{22}=-25.9\left(\mathrm{c} 0.5, \mathrm{CHCl}_{3}, 89 \% e e\right)$.

${ }^{1}$ H-NMR (500 MHz, $\mathrm{CDCl}_{3}$ ): $\delta=7.38-7.28$ (comp, 5H), 7.22-7.15 (m, 1H), 6.91-6.84 (comp, 2H), 6.79-6.74 (m, $1 \mathrm{H}), 5.10(\mathrm{~d}, J=12.5 \mathrm{~Hz}, 1 \mathrm{H}), 5.03(\mathrm{~d}, J=12.5 \mathrm{~Hz}, 1 \mathrm{H}), 3.78(\mathrm{~s}, 3 \mathrm{H}), 3.11(\operatorname{app~d}, J=11.4 \mathrm{~Hz}, 1 \mathrm{H}), 3.02(\mathrm{dd}, J=$ 11.1, $2.8 \mathrm{~Hz}, 1 \mathrm{H}), 2.88(\mathrm{ddd}, J=12.8,8.6,7.2 \mathrm{~Hz}, 1 \mathrm{H}), 2.47(\mathrm{ddd}, J=15.4,8.4,7.2 \mathrm{~Hz}, 1 \mathrm{H}), 2.43-2.36(\mathrm{~m}, 1 \mathrm{H})$, 2.32 (ddd, $J=13.2,8.4,5.0 \mathrm{~Hz}, 1 \mathrm{H}), 2.09$ (app td, $J=11.7,2.9 \mathrm{~Hz}, 1 \mathrm{H}), 1.77($ app d, $J=12.3 \mathrm{~Hz}, 1 \mathrm{H}), 1.73-1.59$ (comp, 3H), 1.53 (app tdd, $J=14.7,8.7,3.3 \mathrm{~Hz}, 1 \mathrm{H}$ ), 1.33 (app qt, $J=12.9,4.0 \mathrm{~Hz}, 1 \mathrm{H}$ ).

${ }^{13}$ C-NMR $\left(125 \mathrm{MHz}, \mathrm{CDCl}_{3}\right): \delta=172.7,159.9,146.6,136.2,129.4,128.6,128.2,128.1,120.0,112.8,112.8,68.8$, $66.1,55.3,53.2,50.8,36.7,31.8,26.1,25.1$.

Accurate Mass (ESI): Calculated for $\mathrm{C}_{22} \mathrm{H}_{27} \mathrm{NO}_{3}[\mathrm{M}+\mathrm{H}]^{+}:$354.2064, Found: 354.2146, Spectral Accuracy: $99.1 \%$.

SFC: Daicel Chiralcel OD-H, column temperature $=40^{\circ} \mathrm{C}, \mathrm{CO}_{2} / \mathrm{MeOH}=90 / 10$, Flow rate $=2 \mathrm{~mL} / \mathrm{min}, \mathrm{UV}=210$ $\mathrm{nm}, \mathrm{t}_{\mathrm{R}}=5.1 \mathrm{~min}$ (major) and $\mathrm{t}_{\mathrm{R}}=5.6 \mathrm{~min}$.

The absolute configuration was assigned by analogy. 


\section{Characterization data of $5 \mathrm{~h}$ :}

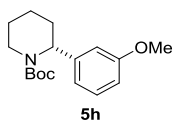

$\mathbf{R}_{\mathbf{f}}=0.33$ in $\mathrm{Et}_{2} \mathrm{O} /$ Hexanes $20: 80 \mathrm{v} / \mathrm{v}$.

$[\alpha]_{\mathbf{D}}^{22}=+27.5\left(\mathrm{c} 0.5, \mathrm{CHCl}_{3}, 76 \% e e\right)$.

${ }^{1} \mathbf{H}-\mathbf{N M R}\left(500 \mathrm{MHz}, \mathrm{CDCl}_{3}\right): \delta=7.28-7.23(\mathrm{~m}, 1 \mathrm{H}), 6.83-6.79(\mathrm{~m}, 1 \mathrm{H}), 6.78-6.74(\mathrm{comp}, 2 \mathrm{H}), 5.41-5.35(\mathrm{~m}, 1 \mathrm{H})$, 4.04 (app d, $J=13.4 \mathrm{~Hz}, 1 \mathrm{H}), 3.79$ (s, 3H), 2.77 (ddd, $J=13.5,11.9,3.7 \mathrm{~Hz}, 1 \mathrm{H}), 2.27$ (app ddt, $J=15.5,5.5,2.5$ $\mathrm{Hz}, 1 \mathrm{H}), 1.86$ (dddd, $J=13.9,12.6,5.5,3.7 \mathrm{~Hz}, 1 \mathrm{H}), 1.63-1.48$ (comp, 3H), 1.46 (s, 9H), 1.45-1.38 (m, 1H).

${ }^{13}$ C-NMR $\left(125 \mathrm{MHz}, \mathrm{CDCl}_{3}\right): \delta=160.1,155.7,142.4,129.6,119.0,112.8,111.5,79.7,55.3,53.4,40.3,28.6,28.3$, $25.6,19.6$.

Accurate Mass (ESI): Calculated for $\mathrm{C}_{17} \mathrm{H}_{25} \mathrm{NO}_{3}[\mathrm{M}+\mathrm{Na}]^{+}: 314.1727$, Found: 314.1759 , Spectral Accuracy: $98.9 \%$.

SFC: Daicel Chiralcel OJ-H, column temperature $=40{ }^{\circ} \mathrm{C}, \mathrm{CO}_{2} / \mathrm{MeOH}=98 / 2$, Flow rate $=2 \mathrm{~mL} / \mathrm{min}, \mathrm{UV}=210 \mathrm{~nm}$, $\mathrm{t}_{\mathrm{R}}=2.6 \mathrm{~min}$ (major) and $\mathrm{t}_{\mathrm{R}}=3.0 \mathrm{~min}$.

The absolute configuration was assigned by analogy.

Calculated conversion $=46 \% ; s$ factor $=40$. 


\section{Benzyl (R)-3-(4-methyl-2-phenylpiperazin-1-yl)propanoate $\quad(4 i)$ and tert-Butyl (S)-4-methyl-2- phenylpiperazine-1-carboxylate (5i):}

1-Methyl-3-phenylpiperazine ( $35 \mathrm{mg}, 0.2 \mathrm{mmol}, 1$ equiv) was treated according to general procedure D. The reaction time for the conjugate addition step was 7 days. Purification by gradient flash chromatography on silica gel (eluent: $\mathrm{Et}_{2} \mathrm{O} /$ hexanes $/ i \mathrm{PrNH}_{2}$ (49:50:1), then EtOAc/i $\mathrm{PrNH}_{2}$ (99:1)) sequentially afforded the less polar product 5i as a colorless oil in $51 \%$ yield $(28 \mathrm{mg})$, and the more polar product $4 \mathbf{i}$ as a colorless oil in $41 \%$ yield $(27.5 \mathrm{mg})$.

\section{Characterization data of $4 i$ :}

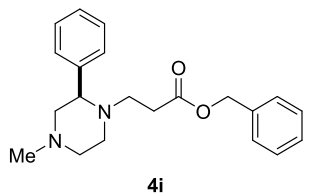

$\mathbf{R}_{\mathbf{f}}=0.13$ in $\mathrm{MeOH} / \mathrm{CH}_{2} \mathrm{Cl}_{2}$ 5:95 v/v.

$[\alpha]_{\mathbf{D}}^{22}=-16.3\left(\mathrm{c} 0.5, \mathrm{CHCl}_{3}, 92 \% e e\right)$.

${ }^{1}$ H-NMR $\left(500 \mathrm{MHz}, \mathrm{CDCl}_{3}\right): \delta=7.40-7.24(\mathrm{comp}, 10 \mathrm{H}), 5.13(\mathrm{~d}, J=12.4 \mathrm{~Hz}, 1 \mathrm{H}), 5.05(\mathrm{~d}, J=12.4 \mathrm{~Hz}, 1 \mathrm{H}), 3.34$ $(\mathrm{dd}, J=10.4,3.0 \mathrm{~Hz}, 1 \mathrm{H}), 3.07$ (app dt, $J=11.2,2.7 \mathrm{~Hz}, 1 \mathrm{H}), 2.92($ app dt, $J=12.8,8.0 \mathrm{~Hz}, 1 \mathrm{H}), 2.84($ app dq, $J=$ 11.1, $2.5 \mathrm{~Hz}, 1 \mathrm{H}), 2.75$ (app dt, $J=11.4,2.6 \mathrm{~Hz}, 1 \mathrm{H}), 2.50$ (app dt, $J=15.5,7.9 \mathrm{~Hz}, 1 \mathrm{H}), 2.44$ (app dd, $J=8.5,4.8$ $\mathrm{Hz}, 1 \mathrm{H}), 2.40$ (app td, $J=11.1,10.0,3.5 \mathrm{~Hz}, 1 \mathrm{H}), 2.33(\mathrm{ddd}, J=12.9,8.1,4.8 \mathrm{~Hz}, 1 \mathrm{H}), 2.28(\mathrm{~s}, 3 \mathrm{H}), 2.24(\operatorname{app} \mathrm{td}, J$ $=11.4,2.9 \mathrm{~Hz}, 1 \mathrm{H}), 2.04(\operatorname{app~dd}, J=11.4,10.3 \mathrm{~Hz}, 1 \mathrm{H})$.

${ }^{13}$ C-NMR $\left(125 \mathrm{MHz}, \mathrm{CDCl}_{3}\right): \delta=172.4,141.4,136.1,128.6,128.5,128.2(9), 128.2(7), 128.1,127.7,67.2,66.2$, 64.0, 55.3, 51.7, 50.0, 45.9, 32.0.

Accurate Mass (ESI): Calculated for $\mathrm{C}_{21} \mathrm{H}_{26} \mathrm{~N}_{2} \mathrm{O}_{2}[\mathrm{M}+\mathrm{H}]^{+}:$339.2067, Found: 339.2101 , Spectral Accuracy: $98.3 \%$.

SFC: Daicel Chiralcel OD-H, column temperature $=40{ }^{\circ} \mathrm{C}, \mathrm{CO}_{2} / \mathrm{MeOH}=90 / 10$, Flow rate $=2 \mathrm{~mL} / \mathrm{min}, \mathrm{UV}=210$ $\mathrm{nm}, \mathrm{t}_{\mathrm{R}}=4.6 \mathrm{~min}$ (major) and $\mathrm{t}_{\mathrm{R}}=5.9 \mathrm{~min}$.

The absolute configuration was assigned by analogy. 


\section{Characterization data of $5 i$ :}

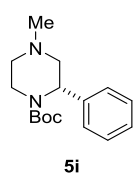

$\mathbf{R}_{\mathbf{f}}=0.37$ in EtOAc/Hexanes 30:70 v/v.

$[\alpha]_{\mathrm{D}}^{22}=+10.3\left(\mathrm{c} 0.5, \mathrm{CHCl}_{3}, 75 \% e e\right)$.

${ }^{1}$ H-NMR (500 MHz, CDCl $)_{3}$ : $\delta=7.49-7.39(\mathrm{~m}, 2 \mathrm{H}), 7.36-7.28(\mathrm{~m}, 2 \mathrm{H}), 7.26-7.19(\mathrm{~m}, 1 \mathrm{H}), 5.41-5.10(\mathrm{~m}, 1 \mathrm{H})$, 3.93 (app d, $J=13.4 \mathrm{~Hz}, 1 \mathrm{H}$ ), 3.29 (app d, $J=11.8 \mathrm{~Hz}, 1 \mathrm{H}$ ), 3.03 (ddd, $J=13.4,12.2,3.3 \mathrm{~Hz}, 1 \mathrm{H}$ ), 2.74 (app ddt, $J$ $=11.1,3.5,1.9 \mathrm{~Hz}, 1 \mathrm{H}), 2.38(\mathrm{dd}, J=11.9,4.3 \mathrm{~Hz}, 1 \mathrm{H}), 2.28(\mathrm{~s}, 3 \mathrm{H}), 2.06(\operatorname{app} \mathrm{td}, J=11.8,3.3 \mathrm{~Hz}, 1 \mathrm{H}), 1.47(\mathrm{~s}$, 9H).

${ }^{13}$ C-NMR (125 MHz, $\left.\mathrm{CDCl}_{3}\right): \delta=155.2,140.5,128.5,127.3,126.9,80.1,57.7,55.3,53.4,46.6,40.1,28.6$.

Accurate Mass (ESI): Calculated for $\mathrm{C}_{16} \mathrm{H}_{24} \mathrm{~N}_{2} \mathrm{O}_{2}[\mathrm{M}+\mathrm{H}]^{+}:$277.1911, Found: 277.1823, Spectral Accuracy: 98.3\%.

SFC: Daicel Chiralcel OD-H, column temperature $=40^{\circ} \mathrm{C}, \mathrm{CO}_{2} / \mathrm{MeOH}=97 / 3$, Flow rate $=2 \mathrm{~mL} / \mathrm{min}, \mathrm{UV}=210$ $\mathrm{nm}, \mathrm{t}_{\mathrm{R}}=3.4$ min and $\mathrm{t}_{\mathrm{R}}=3.7$ min (major).

The absolute configuration was assigned by analogy.

Calculated conversion $=45 \% ; s$ factor $=54$. 
rac-trans-4-Benzyl-2-phenylpiperidine: ${ }^{4}$

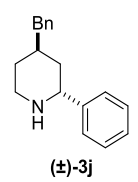

Following a known procedure, ${ }^{4}$ to a solution of 4-benzyl piperidine $(525.8 \mathrm{mg}, 3 \mathrm{mmol}, 1$ equiv) in anhydrous ether $(6 \mathrm{~mL})$ cooled to $-78^{\circ} \mathrm{C}$ was slowly added $n$-BuLi in hexanes $(3 \mathrm{mmol}, 1$ equiv) under the protection of nitrogen, and the resulting solution was stirred at the same temperature for $10 \mathrm{~min}$. To this was then added via cannula a solution of trifluoroacetophenone $(522.3 \mathrm{mg}, 3 \mathrm{mmol}, 1$ equiv) in anhydrous ether $(3 \mathrm{~mL})$. The resulting mixture was stirred at $-78{ }^{\circ} \mathrm{C}$ for $10 \mathrm{~min}$, followed by the addition of phenyl lithium solution (4.5 mmol, $\left.1.5 \mathrm{equiv}\right)$ in one portion and the reaction mixture was stirred at that temperature for 4 hours. The reaction was then quenched via the addition of methanol $(1.5 \mathrm{~mL})$. The resulting mixture was diluted with ether $(20 \mathrm{~mL})$ and washed with water $(20$ $\mathrm{mL})$. The aqueous layer was then extracted with ether $(3 \times 20 \mathrm{~mL})$ and the combined organic layers washed with brine $(30 \mathrm{~mL})$ and dried over anhydrous $\mathrm{Na}_{2} \mathrm{SO}_{4}$. Solvent was then removed under reduced pressure and the residue purified by silica gel chromatography (eluent: $\mathrm{MeOH} / \mathrm{EtOAc} / \mathrm{PrNH}_{2}(1: 98: 1-9: 90: 1)$ ) to yield compound ( \pm )-3j as a colorless oil in $48 \%$ yield (360 mg).

\section{Characterization data:}

$\mathbf{R}_{\mathbf{f}}=0.42$ in $\mathrm{MeOH} /$ isopropyl amine/EtOAc 9:1:90 v/v.

${ }^{1}$ H-NMR $\left(300 \mathrm{MHz}, \mathrm{CDCl}_{3}\right): \delta=7.44-7.18(\mathrm{comp}, 10 \mathrm{H}), 4.05(\mathrm{dd}, J=10.2,3.1 \mathrm{~Hz}, 1 \mathrm{H}), 3.13(\mathrm{app} \mathrm{td}, J=11.6,3.0$ $\mathrm{Hz}, 1 \mathrm{H}), 2.99$ (app dt, $J=12.1,4.3 \mathrm{~Hz}, 1 \mathrm{H}), 2.87$ (d, $J=8.0 \mathrm{~Hz}, 2 \mathrm{H}), 2.31-2.16$ (m, 1H), 1.96-1.65 (comp, 4H), $1.62-1.46(\mathrm{~m}, 1 \mathrm{H})$.

${ }^{13}$ C-NMR $\left(75 \mathrm{MHz}, \mathrm{CDCl}_{3}\right): \delta=145.1,141.2,129.1,128.4(3), 128.3(7), 126.9,126.7,125.9,55.9,42.1,38.7,38.0$, $34.2,29.8$.

Accurate Mass (ESI): Calculated for $\mathrm{C}_{18} \mathrm{H}_{21} \mathrm{~N}[\mathrm{M}+\mathrm{H}]^{+}:$252.1747, Found: 252.1905, Spectral Accuracy: $97.6 \%$. 


\section{Benzyl 3-((2S,4S)-4-benzyl-2-phenylpiperidin-1-yl)propanoate $(4 \mathbf{j})$ and tert-Butyl $(2 R, 4 R)-4-b e n z y l-2-$ phenylpiperidine-1-carboxylate $(\mathbf{5 j})$ :}

( \pm )-3j (50 mg, $0.2 \mathrm{mmol}, 1$ equiv) was treated according to general procedure D. The reaction time for the conjugate addition step was 4 days. Purification by gradient flash chromatography on silica gel (eluent: $\mathrm{Et}_{2} \mathrm{O} /$ hexanes (5:95) $-\mathrm{Et}_{2} \mathrm{O} /$ hexanes (30:70)) sequentially afforded the less polar product $\mathbf{5} \mathbf{j}$ as a colorless oil in $51 \%$ yield $(35.7 \mathrm{mg})$, and the more polar product $\mathbf{4} \mathbf{j}$ as a colorless oil in $47 \%$ yield $(39 \mathrm{mg})$.

\section{Characterization data of $\mathbf{4 j}$ :}

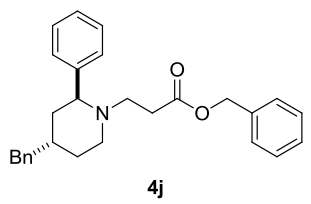

$\mathbf{R}_{\mathbf{f}}=0.35$ in $\mathrm{Et}_{2} \mathrm{O} / \mathrm{Hexanes} 30: 70 \mathrm{v} / \mathrm{v}$.

$[\alpha]_{\mathrm{D}}^{22}=-11.5\left(\mathrm{c} 0.5, \mathrm{CHCl}_{3}, 81 \% e e\right)$.

${ }^{1}$ H-NMR (500 MHz, $\mathrm{CDCl}_{3}$ ): $\delta=7.39-7.26(\mathrm{comp}, 11 \mathrm{H}), 7.24-7.16(\mathrm{comp}, 4 \mathrm{H}), 5.12(\mathrm{~d}, J=12.4 \mathrm{~Hz}, 1 \mathrm{H}), 5.06(\mathrm{~d}$, $J=12.4 \mathrm{~Hz}, 1 \mathrm{H}), 3.49$ (dd, $J=10.7,3.1 \mathrm{~Hz}, 1 \mathrm{H}), 2.96-2.87$ (comp, 2H), 2.78 (d, $J=8.0 \mathrm{~Hz}, 2 \mathrm{H}), 2.56-2.40$ (comp, 4H), 2.16-2.07 (m, 1H), 1.89-1.74 (comp, 2H), 1.64-1.49 (comp, 2H).

${ }^{13} \mathrm{C}-\mathrm{NMR}\left(125 \mathrm{MHz}, \mathrm{CDCl}_{3}\right): \delta=172.7,144.6,141.2,136.2,129.2,128.6,128.5,128.4,128.3,128.2,127.6,127.0$, 126.0, 66.2, 62.8, 50.7, 47.3, 39.3, 38.1, 33.8, 32.4, 29.0.

Accurate Mass (ESI): Calculated for $\mathrm{C}_{28} \mathrm{H}_{31} \mathrm{NO}_{2}[\mathrm{M}+\mathrm{H}]^{+}$: 414.2428, Found: 414.2344, Spectral Accuracy: 98.7\%.

HPLC: REGIS $(S, S)$-Whelk-O1, $n$-hexane $/ i-\mathrm{PrOH}=90 / 10$, Flow rate $=0.1 \mathrm{~mL} / \mathrm{min}, \mathrm{UV}=230 \mathrm{~nm}, \mathrm{t}_{\mathrm{R}}=111.1 \mathrm{~min}$ (major) and $\mathrm{t}_{\mathrm{R}}=117.5 \mathrm{~min}$.

The absolute configuration was assigned by analogy. 


\section{Characterization data of $\mathbf{5 j}$ :}

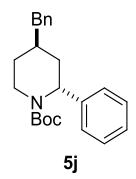

$\mathbf{R}_{\mathbf{f}}=0.42$ in $\mathrm{Et}_{2} \mathrm{O} / \mathrm{Hexanes} 20: 80 \mathrm{v} / \mathrm{v}$.

$[\alpha]_{\mathrm{D}}^{22}=+24.3\left(\mathrm{c} 0.5, \mathrm{CHCl}_{3}, 73 \% e e\right)$.

${ }^{1}$ H-NMR (500 MHz, CDCl $)$ ): $\delta=7.34-7.26$ (comp, 4H), 7.24-7.17 (comp, 2H), 7.13-7.08 (comp, 4H), 5.63-5.32 (m, 1H), 4.26-3.88 (m, 1H), 2.71 (app td, $J=13.3,3.0 \mathrm{~Hz}, 1 \mathrm{H}), 2.54$ (d, $J=7.2 \mathrm{~Hz}, 2 \mathrm{H}), 2.32$ (app d, $J=13.7 \mathrm{~Hz}$, 1H), 1.84-1.72 (m, 1H), 1.61-1.35 (comp, 11H), 1.28-1.14 (m, 1H).

${ }^{13}$ C-NMR $\left(125 \mathrm{MHz}, \mathrm{CDCl}_{3}\right): \delta=155.7,140.3,140.1,129.2,128.7,128.4,126.5,126.5,126.1,79.8,53.4,43.4$, $39.8,34.5,32.8,32.2,28.6$.

Accurate Mass (ESI): Calculated for $\mathrm{C}_{23} \mathrm{H}_{29} \mathrm{NO}_{2}[\mathrm{M}+\mathrm{Na}]^{+}$: 374.2091, Found: 374.1980, Spectral Accuracy: $99.0 \%$.

SFC: Daicel Chiralcel OJ-H, column temperature $=40{ }^{\circ} \mathrm{C}, \mathrm{CO}_{2} / \mathrm{MeOH}=90 / 10$, Flow rate $=2 \mathrm{~mL} / \mathrm{min}, \mathrm{UV}=210$ $\mathrm{nm}, \mathrm{t}_{\mathrm{R}}=2.4 \mathrm{~min}$ (major) and $\mathrm{t}_{\mathrm{R}}=2.8 \mathrm{~min}$.

The absolute configuration was assigned by analogy.

Calculated conversion $=48 \% ; s$ factor $=21$. 


\section{${ }^{13}$ C KIE Study}

Procedure for ${ }^{13} \mathrm{C}$ KIE determination by analysis of benzyl crotonate:

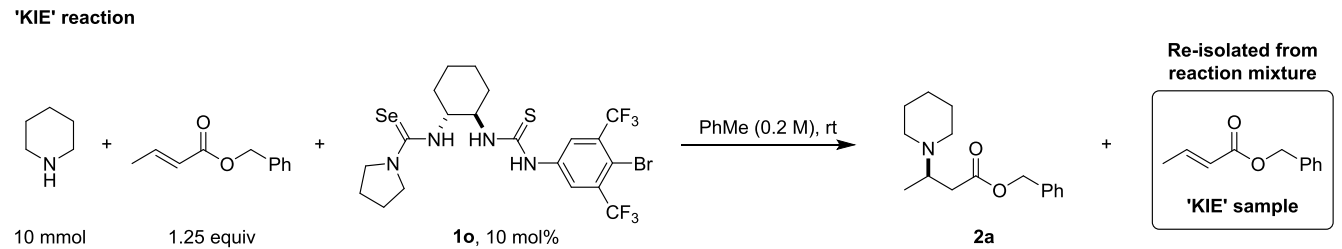

Under $\mathrm{N}_{2}$, the catalyst $1 \mathrm{o}(0.624 \mathrm{~g}, 1.0 \mathrm{mmol}, 10 \mathrm{~mol} \%)$ was added to a solution of benzyl crotonate $(2.200 \mathrm{~g}, 12.5$ mmol, 1.25 equiv) in anhydrous toluene $(50 \mathrm{~mL}, 0.2 \mathrm{M})$ at room temperature followed by the addition of piperidine (0.988 mL, $10.0 \mathrm{mmol}, 1$ equiv).

This reaction was conducted twice.

The first reaction mixture was stirred at room temperature for $88 \mathrm{~h}$ before direct purification by flash column chromatography on silica gel to sequentially afford benzyl crotonate (eluent: $\mathrm{Et}_{2} \mathrm{O} / \mathrm{Hexanes}=5: 95 ; 0.507 \mathrm{~g}, 23 \%$ yield), 2a (eluent: $\mathrm{Et}_{2} \mathrm{O} / \mathrm{Hexanes} / \mathrm{Et}_{3} \mathrm{~N}=40: 59: 1 ; 2.316 \mathrm{~g}, 89 \%$ yield, $85 \%$ ee), and catalyst 10 (eluent: EtOAc/Hexanes $=30: 70 ; 0.599 \mathrm{~g}, 96 \%$ recovered). The exact conversion $(74.4 \%)$ was determined by ${ }^{1} \mathrm{H}$ NMR analysis of the crude reaction mixture, with 1,3,5-trimethoxybenzene as internal standard.

The second reaction mixture was stirred at room temperature for $89 \mathrm{~h}$ before direct purification by flash column chromatography on silica gel to sequentially afford benzyl crotonate (eluent: $\mathrm{Et}_{2} \mathrm{O} / \mathrm{Hexanes}=5: 95 ; 0.486 \mathrm{~g}, 22 \%$ yield), 2a (eluent: $\mathrm{Et}_{2} \mathrm{O} / \mathrm{Hexanes} / \mathrm{Et}_{3} \mathrm{~N}=40: 59: 1 ; 2.347 \mathrm{~g}, 90 \%$ yield, $85 \%$ ee), and catalyst 1o (eluent: EtOAc/Hexanes $=30: 70 ; 0.602 \mathrm{~g}, 96 \%$ recovered). The exact conversion $(79.9 \%)$ was determined by ${ }^{1} \mathrm{H}$ NMR analysis of the crude reaction mixture, with 1,3,5-trimethoxybenzene as internal standard. 
Determination of Experimental Kinetic Isotope Effects

Table S 1. NMR integrations for KIEs of benzyl crotonate.

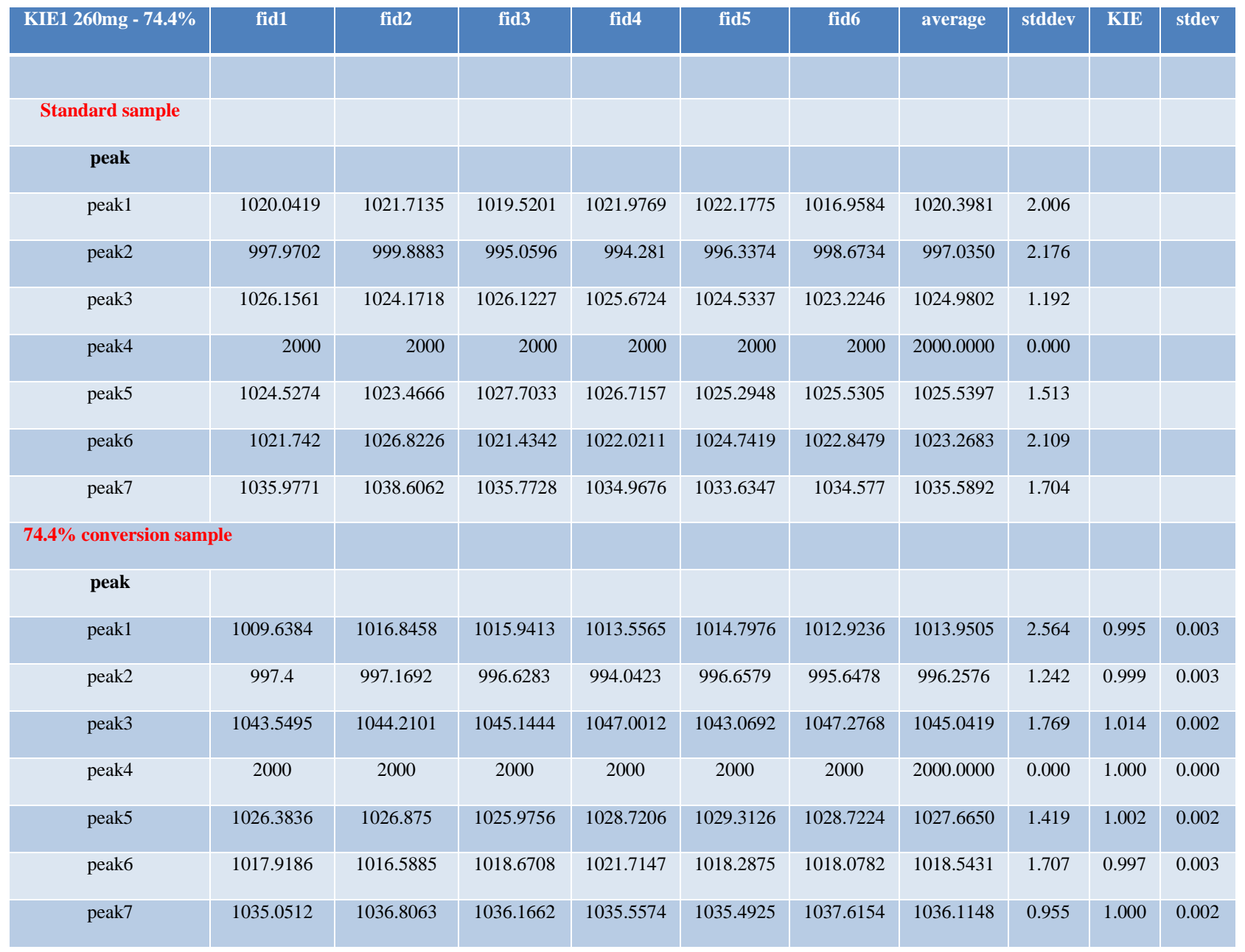




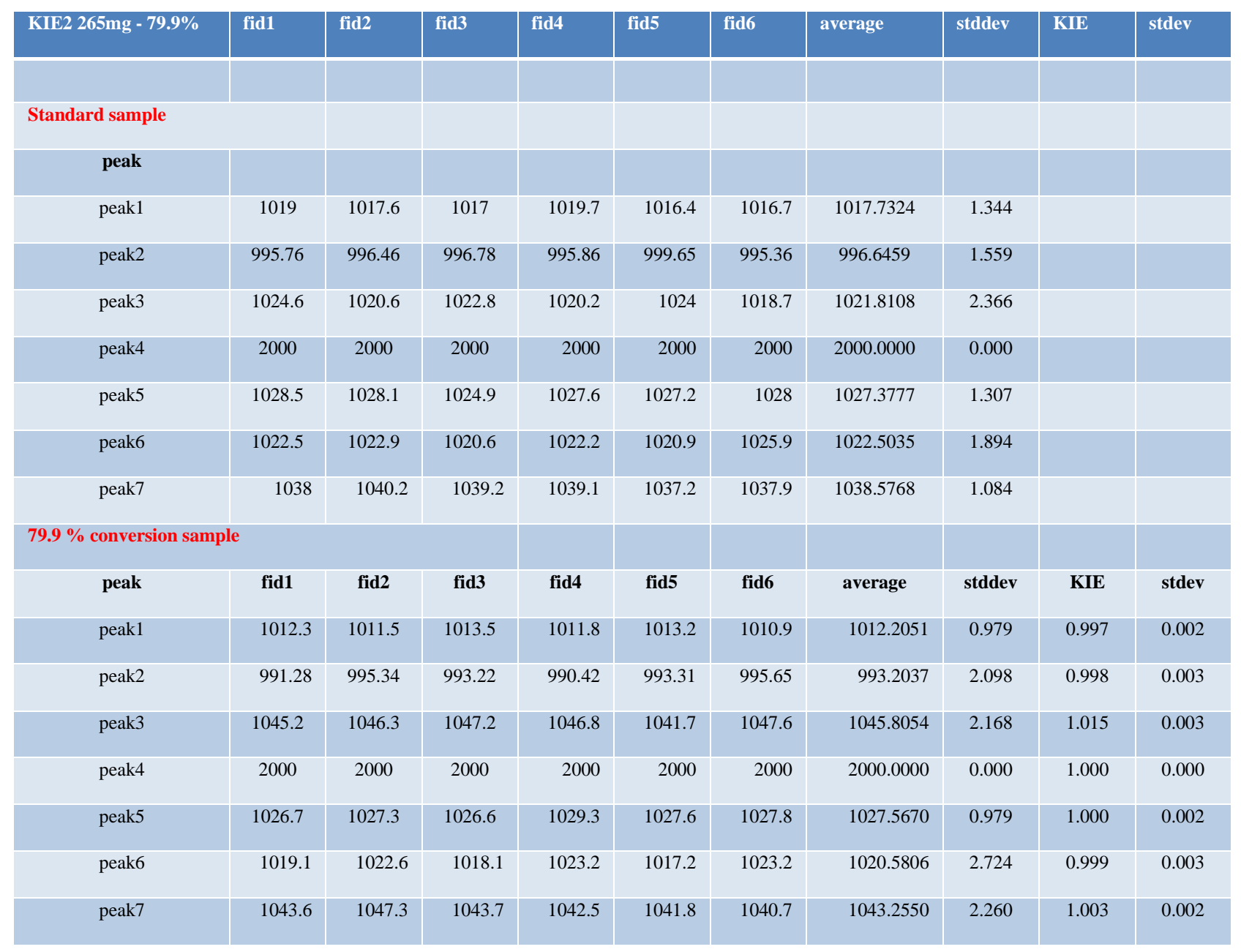




\section{$k_{\mathrm{H}} / k_{\mathrm{D}}$ KIE Study}

Reaction rates were compared for NH piperidine and ND piperidine. ${ }^{18} \mathrm{~A} k_{\mathrm{H}} / k_{\mathrm{D}}$ value of 1.86 was observed in the first two hours of the reaction, consistent with the proposed mechanism. For $\alpha$-deuterated product, only one product diastereomer was obtained, consistent with the proposed transition state. We believe that the value of 1.86 is an under-estimate of the actual $k_{\mathrm{H}} / k_{\mathrm{D}}$ for this reaction for two key reasons - (1) the kinetic experiment for the determination of $k_{\mathrm{D}}$ was carried out using $90 \%$ deuterated piperidine. This could lead to an increase initial rate for the " $k_{\mathrm{D}}$ reaction" leading to a lower value for $k_{\mathrm{H}} / k_{\mathrm{D}}$, and (2) the kinetic experiment for determination of $k_{\mathrm{D}}$ was carried out using protio-selenothiourea catalyst (without deuterating the thiourea NHs). Even though some of thiourea NHs presumably exchange to deuterium during the reaction, the high catalyst loading and $90 \%$ deuterated piperidine probably result in a significant amount of protio-selenothiourea catalyst still being present. Since proton transfer is proposed to proceed from the thiourea NHs, we expect our rate from the " $k_{\mathrm{D}}$ reaction" to be significantly enhanced (compared to a $100 \%$ deuterated catalyst) resulting in a diminished $k_{\mathrm{H}} / k_{\mathrm{D}}$ ratio.
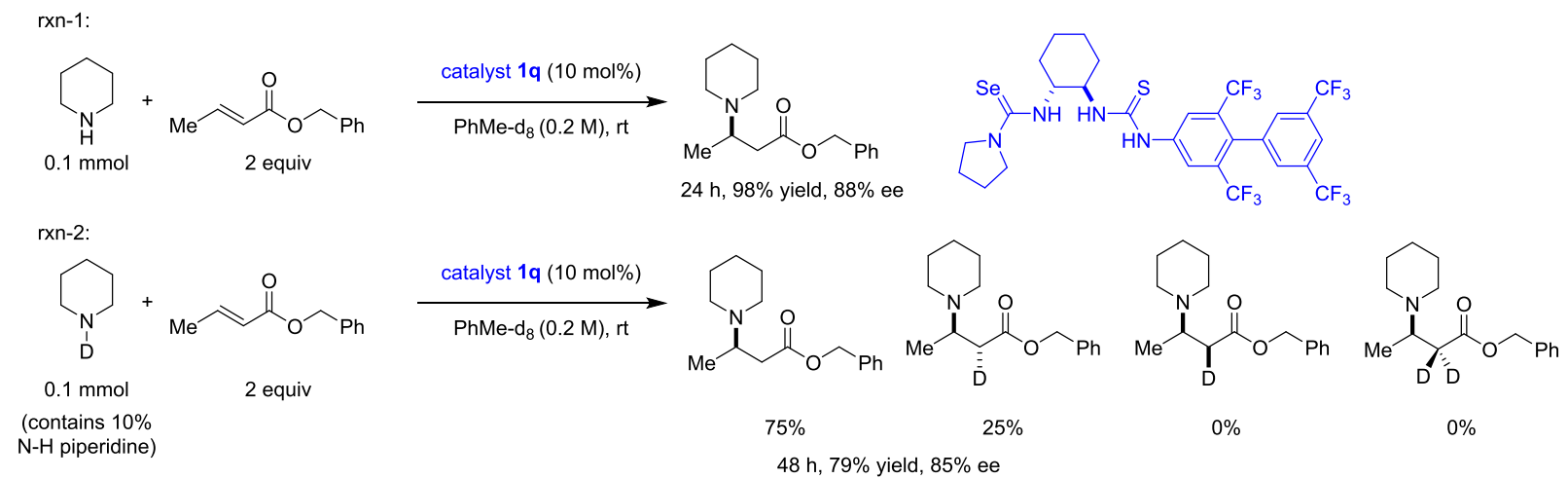

In reaction 2, no deuterium incorporation was observed in the recovered benzyl crotonate (based on the ${ }^{1} \mathrm{H}$ NMR's of both crude and purified benzyl crotonate).

In the crude ${ }^{1} \mathrm{H}$ NMR of reaction 2, all three NHs of the catalyst were invisible due to H-D exchange. In comparison, all catalyst $\mathrm{NH}$ signals were observed in the crude ${ }^{1} \mathrm{H}$ NMR of reaction 1.

Reaction conversion was monitored by ${ }^{1} \mathrm{H}$ NMR in toluene- $\mathrm{d}_{8}$ and calculated based on the ratio of the piperidine peak (all $4 \alpha$-protons) and product peaks (all $4 \alpha$-ring-protons).

The two $\alpha$-diastereotopic protons to carbonyl group were assigned by Excitation-Sculptured Indirect-Detection Experiment (EXSIDE). ${ }^{19}$

Observed diastereomer in reaction 2 matches the prediction of the computation model. 
Conversion of the Two Reactions with $N$-H Piperidine (rxn-1) and $N$-D Piperidine (rxn-2)

\begin{tabular}{|c|c|c|c|c|c|c|c|c|c|}
\hline $\begin{array}{c}\text { time } \\
\text { (min) }\end{array}$ & 5 & 10 & 15 & 20 & 25 & 30 & 35 & 40 & 45 \\
\hline $\begin{array}{c}\text { rxn-1 } \\
\text { (conv.) }\end{array}$ & $5.4 \%$ & $8.1 \%$ & $10.9 \%$ & $13.4 \%$ & $16.0 \%$ & $18.6 \%$ & $20.8 \%$ & $22.8 \%$ & $24.8 \%$ \\
\hline $\begin{array}{c}\text { rxn-2 } \\
\text { (conv.) }\end{array}$ & $3.5 \%$ & $5.1 \%$ & $6.2 \%$ & $7.6 \%$ & $8.5 \%$ & $9.9 \%$ & $11.0 \%$ & $12.2 \%$ & $13.3 \%$ \\
\hline
\end{tabular}

\begin{tabular}{|c|c|c|c|c|c|c|c|c|c|}
\hline $\begin{array}{c}\text { time } \\
\text { (min) }\end{array}$ & 50 & 55 & 60 & 65 & 70 & 75 & 80 & 85 & 90 \\
\hline $\begin{array}{c}\text { rxn-1 } \\
\text { (conv.) }\end{array}$ & $26.9 \%$ & $28.8 \%$ & $31.0 \%$ & $32.8 \%$ & $34.5 \%$ & $36.3 \%$ & $38.0 \%$ & $39.4 \%$ & $40.9 \%$ \\
\hline $\begin{array}{c}\text { rxn-2 } \\
\text { (conv.) }\end{array}$ & $14.4 \%$ & $15.4 \%$ & $16.5 \%$ & $17.4 \%$ & $18.4 \%$ & $19.2 \%$ & $20.3 \%$ & $21.1 \%$ & $22.0 \%$ \\
\hline
\end{tabular}

\begin{tabular}{|c|c|c|c|c|c|c|c|c|c|}
\hline $\begin{array}{c}\text { time } \\
(\mathrm{min})\end{array}$ & 95 & 100 & 105 & 110 & 115 & 120 & 240 & 480 & 720 \\
\hline $\begin{array}{c}\text { rxn-1 } \\
\text { (conv.) }\end{array}$ & $42.4 \%$ & $44.0 \%$ & $45.1 \%$ & $46.6 \%$ & $47.7 \%$ & $49.0 \%$ & $69.7 \%$ & $88.5 \%$ & $95.2 \%$ \\
\hline $\begin{array}{c}\text { rxn-2 } \\
\text { (conv.) }\end{array}$ & $23.0 \%$ & $23.7 \%$ & $24.6 \%$ & $25.5 \%$ & $26.2 \%$ & $26.7 \%$ & $42.1 \%$ & $60.9 \%$ & $70.5 \%$ \\
\hline
\end{tabular}

\begin{tabular}{|c|c|c|c|c|c|c|c|c|c|}
\hline $\begin{array}{c}\text { time } \\
\text { (min) }\end{array}$ & 1080 & 1440 & 1800 & 2160 & 2880 & & & & \\
\hline $\begin{array}{c}\text { rxn-1 } \\
\text { (conv.) }\end{array}$ & $98.0 \%$ & $99.0 \%$ & & & & & & & \\
\hline $\begin{array}{c}\text { rxn-2 } \\
\text { (conv. })\end{array}$ & $78.6 \%$ & $83.2 \%$ & $88.3 \%$ & $91.5 \%$ & $95.2 \%$ & & & & \\
\hline
\end{tabular}


Reaction Rate Plot within the First Two Hours

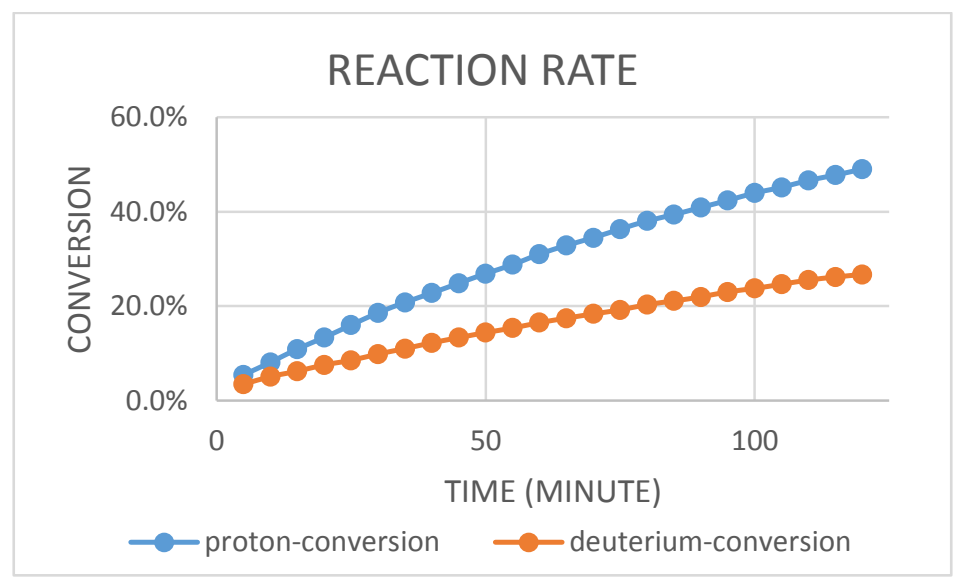

$\mathrm{k}_{\mathrm{H}} / \mathrm{k}_{\mathrm{D}}=1.861$

Reaction Rate Plot within the First Four Hours

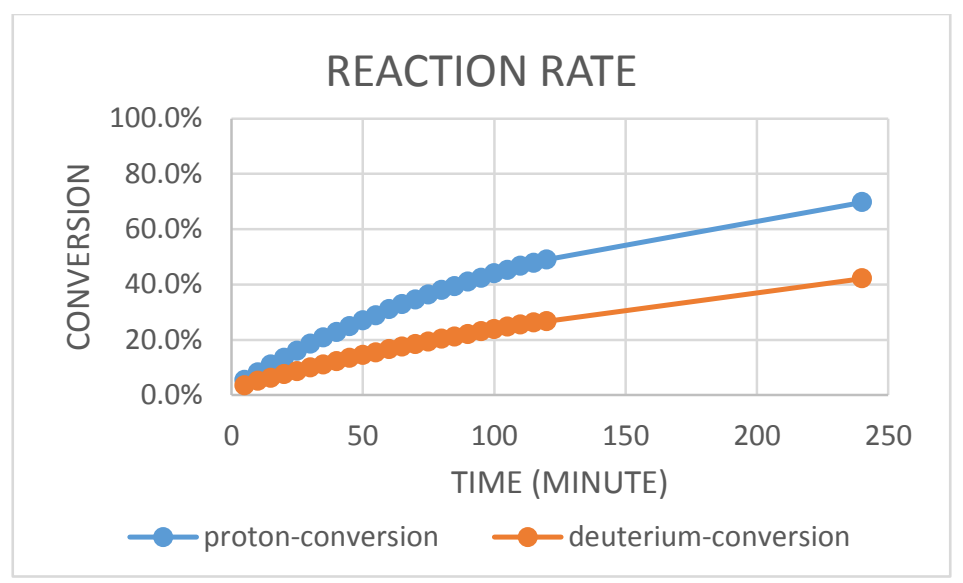

Reaction Rate Plot

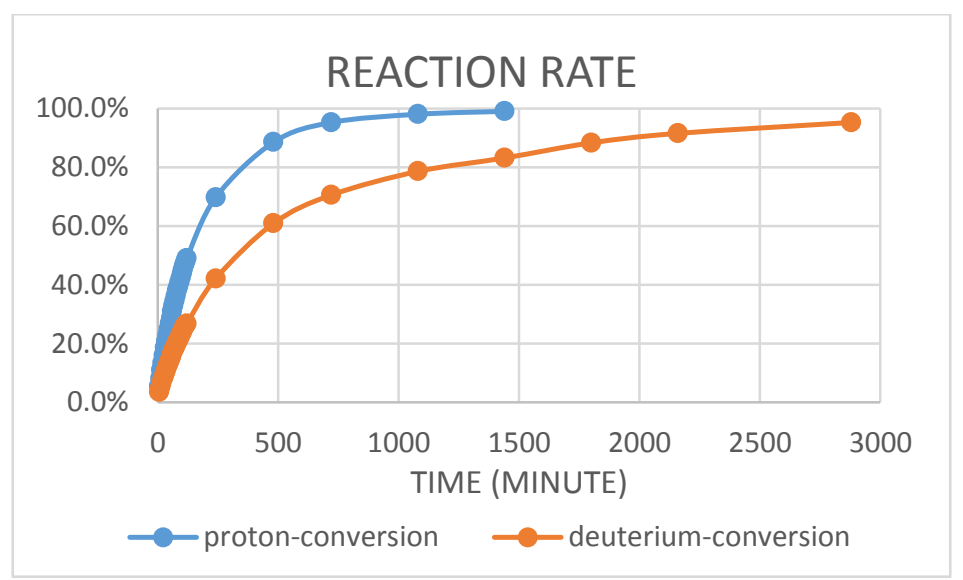


The two $\alpha$-diastereotopic protons to carbonyl group were assigned by Excitation-Sculptured Indirect-Detection Experiment (EXSIDE) $)^{19}$ :

$\underline{\mathrm{H}_{a}}:$ pro- $R ; \mathrm{H}_{\mathrm{b}} \underline{\underline{p}} \underline{\text { pro-S}}$.

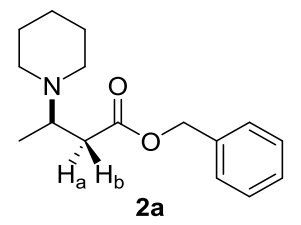

${ }^{1}$ H-NMR $\left(500 \mathrm{MHz}\right.$, toluene- $\left.\mathrm{d}_{8}\right): \delta=7.31-7.27(\mathrm{~m}, 2 \mathrm{H}), 7.22-7.17(\mathrm{~m}, 2 \mathrm{H}), 7.16-7.13(\mathrm{~m}, 1 \mathrm{H}), 5.10(\mathrm{~d}, J=12.4$ $\mathrm{Hz}, 1 \mathrm{H}), 5.06(\mathrm{~d}, J=12.4 \mathrm{~Hz}, 1 \mathrm{H}), 3.21(\mathrm{app} \mathrm{h}, J=6.9 \mathrm{~Hz}, 1 \mathrm{H}), 2.52\left(\mathrm{H}_{\mathrm{b}}, \mathrm{dd}, J=14.2,7.1 \mathrm{~Hz}, 1 \mathrm{H}\right), 2.40-2.32$ (comp, 2H), 2.31-2.23 (comp, 2H), $2.15\left(\mathrm{H}_{\mathrm{a}}\right.$, dd, $\left.J=14.2,7.7 \mathrm{~Hz}, 1 \mathrm{H}\right), 1.58-1.44$ (comp, 4H), 1.41-1.31 (comp, $2 \mathrm{H}), 0.95(\mathrm{~d}, J=6.6 \mathrm{~Hz}, 3 \mathrm{H})$.

Three possible conformers,

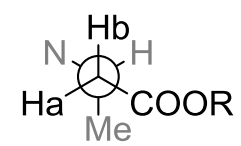

ge

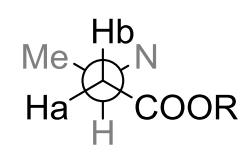

anti

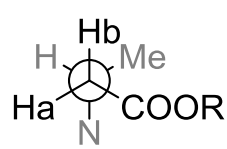

$g z$

Prediction of coupling constants for the three conformers based on Murata's method ${ }^{19 a}$,

\begin{tabular}{|c|l|l|c|}
\hline conformer & $g e$ & $a n t i$ & $g z$ \\
\hline${ }^{3} J_{\mathrm{Ha}-\mathrm{H}}$ & large & small & small \\
\hline${ }^{3} J_{\mathrm{Hb}-\mathrm{H}}$ & small & large & small \\
\hline${ }^{3} J_{\mathrm{Ha}-\mathrm{Me}}$ & small & small & large \\
\hline${ }^{3} J_{\mathrm{Hb}-\mathrm{Me}}$ & large & small & small \\
\hline${ }^{3} J_{\mathrm{H}-\mathrm{CO}}$ & small & small & large \\
\hline${ }^{2} J_{\mathrm{Ha}-\mathrm{C}(\mathrm{N})}$ & large & small & small \\
\hline${ }^{2} J_{\mathrm{Hb}-\mathrm{C}(\mathrm{N})}$ & large & large & large \\
\hline
\end{tabular}

Observed coupling constant,

\begin{tabular}{|c|c|c|}
\hline $2.15-3.21$ & 6.54 & medium \\
\hline $2.52-3.21$ & 7.04 & medium \\
\hline $2.15-14.0$ & 2.0 & small \\
\hline $2.52-14.0$ & 4.1 & medium \\
\hline $3.21-171.4$ & 2.8 & small \\
\hline $2.15-57.1$ & 3.8 & medium \\
\hline $2.52-57.1$ & 4.4 & large \\
\hline
\end{tabular}

Coupling constants $\mathrm{H}_{\mathrm{a}}-\mathrm{H}, \mathrm{H}_{\mathrm{b}}-\mathrm{H}$ and $\mathrm{H}-\mathrm{CO}$ indicate that the conformer equilibrium is primarily a mixture of $g e$ and anti. The other coupling constants indicate that chemical shift $2.15 \mathrm{ppm}$ is pro- $R$ and $2.52 \mathrm{ppm}$ is pro-S. 


\section{Theoretical Calculations}

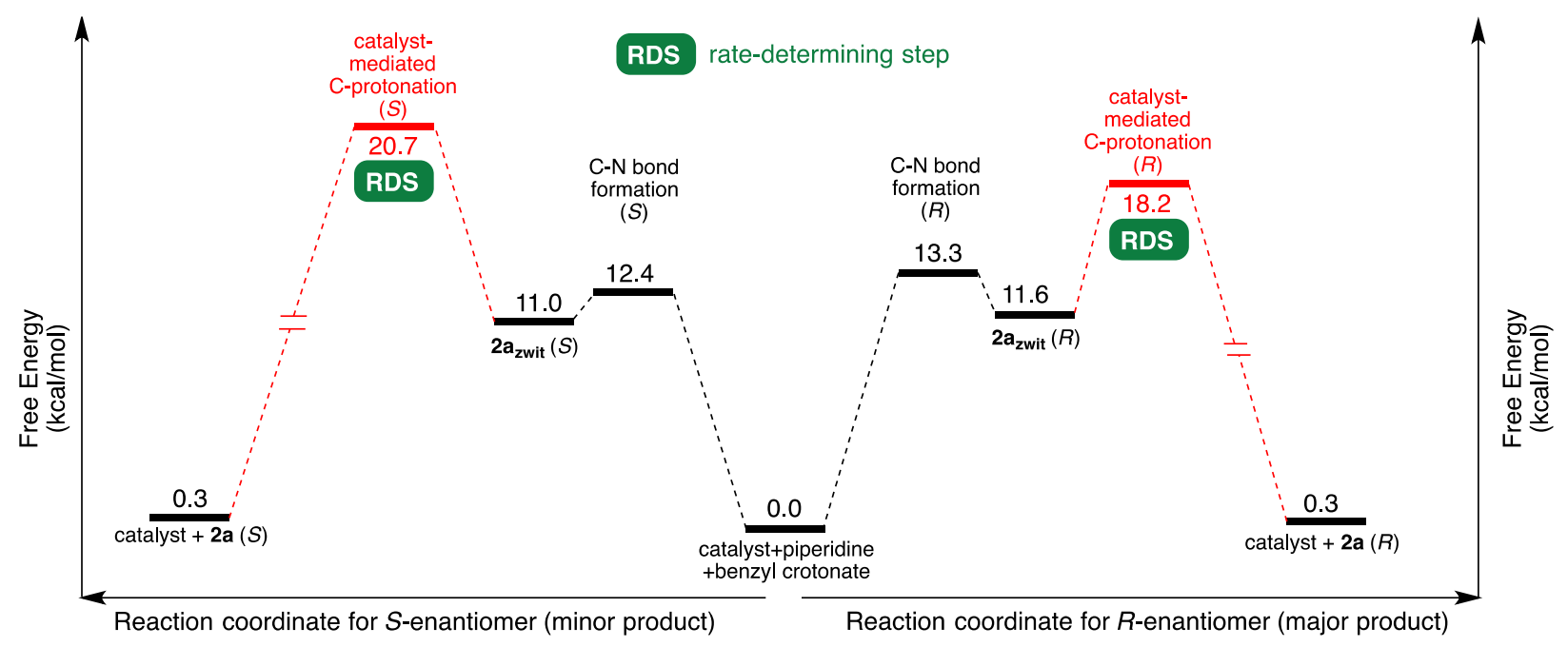

Figure S1. Computed reaction coordinate diagram depicting the proposed pathway leading to $(R)$ - and (S)enantiomers of $2 a$. Free Energies calculated in Gaussian 09 (RRHO rigid rotor harmonic oscillator approximation). 
Table of Free Energies calculated using qRRHO approximation at 263.15 and 298.15K

\begin{tabular}{|c|c|}
\hline Transition Structure/Method & $\begin{array}{l}\text { Free Energy barrier in } \\
\text { kcal/mol }\end{array}$ \\
\hline \multicolumn{2}{|l|}{ C-N bond formation } \\
\hline S-enantiomer $(-10 \mathrm{C})$ & 13.5 \\
\hline R-enantiomer $(-10 \mathrm{C})$ & 14.2 \\
\hline \multicolumn{2}{|c|}{ Direct C-Protonation from selenothiourea catalyst } \\
\hline S-enantiomer $(-10 \mathrm{C})$ & 20.1 \\
\hline R-enantiomer $(-10 \mathrm{C})$ & 18.3 \\
\hline S-enantiomer (room temp) & 23.9 \\
\hline R-enantiomer (room temp) & 22.0 \\
\hline \multicolumn{2}{|l|}{ Intermediate Zwitterion } \\
\hline S-enantiomer $(-10 \mathrm{C})$ & 11.7 \\
\hline R-enantiomer $(-10 \mathrm{C})$ & 11.6 \\
\hline \multicolumn{2}{|c|}{ Intramolecular Proton Transfer ( $\mathrm{N}$ to $\mathrm{O}$ ) } \\
\hline S-enantiomer $(-10 \mathrm{C})$ & 18.4 \\
\hline R-enantiomer $(-10 \mathrm{C})$ & 18.1 \\
\hline \multicolumn{2}{|l|}{ Tautomerization step } \\
\hline S-enantiomer $(-10 \mathrm{C})$ & 55.5 \\
\hline R-enantiomer $(-10 \mathrm{C})$ & 48.7 \\
\hline \multicolumn{2}{|c|}{ Direct C-Protonation from sulfamidethiourea catalyst } \\
\hline S-enantiomer $(-10 \mathrm{C})$ & 20.4 \\
\hline R-enantiomer $(-10 \mathrm{C})$ & 19.6 \\
\hline S-enantiomer (room temp) & 24.2 \\
\hline R-enantiomer (room temp) & 23.4 \\
\hline \multicolumn{2}{|c|}{ Direct C-Protonation from selenothiourea catalyst $\beta$-ethyl substituent } \\
\hline S-enantiomer (room temp) & 24.7 \\
\hline R-enantiomer (room temp) & 23.3 \\
\hline
\end{tabular}


The difference in ee, at room temperature, upon going from oxygen (53\% for catalyst 1m) to sulfur (59\% for catalyst 11) to selenium ( $71 \%$ for catalyst $1 \mathbf{n}$ ) corresponds to a small difference of $0.4 \mathrm{kcal} / \mathrm{mol}$ in Gibbs Free Energy. We calculated the sulfurcat- $\boldsymbol{R}$ - $\mathbf{T S} \mathbf{S}_{\mathbf{C} \text {-prot }}$ and sulfurcat-S-TS $\mathbf{S}_{\text {-prot }}$ for the catalyst where the Se is replaced by $\mathrm{S}$ in the catalyst 1q. Gratifyingly, our computations revealed that the relative free energies of these transition structures $\left(\Delta \Delta \mathrm{G}^{\sharp}\right)$ is $0.8 \mathrm{kcal} / \mathrm{mol}$ (59\% ee @ room temperature) favoring the $R$-enantiomer. This is in good agreement with the experimental $62 \%$ ee observed for this reaction using the sulfur analogue of catalyst 10 at room temperature (We do not have experimental data for the sulfur analogue of our computational model catalyst 1q. However, based on the similarity in ee of at room temperature for $\mathbf{1 0}$ and $\mathbf{1 q}$, the assumption is that ee for the sulfur analogue of $\mathbf{1 q}$ will be very similar to the sulfur analogue of $\mathbf{1 0}$. We chose to model catalyst $\mathbf{1 q}$ with both $\mathrm{Se}$ and $\mathrm{S}$ so as to minimize any other computational artifacts that might affect the computed energetics). The corresponding $\Delta \Delta \mathrm{G}^{\ddagger}$ for the parent Secatalyst 1q is $1.9 \mathrm{kcal} / \mathrm{mol}$ (92\% ee at room temperature), which is in excellent agreement with the experimental $88 \%$ ee at room temperature. (Note: The computational results here are used to predict experimental results at room temperature. The computational discussion in the manuscript is for the reaction at $263 \mathrm{~K}$, the temperature of the optimized reaction.)

To analyze the origin of change in $\Delta \Delta \mathrm{G}^{\ddagger}$ upon switching from Se to $\mathrm{S}$, we compared the key transition state stabilization interactions in the $R$ - and $S$ - transition structures for both cataysts. We also computed the absolute Gibbs free energy barriers $\left(\Delta \mathrm{G}^{*}\right)$ for each of the four transition structures at room temperature. The results are shown in the Figure S2. The first key result is that selenothiourea catalyst 1q has a significantly lower $\Delta \mathrm{G}^{\sharp}$ for the formation of the major enantiomer $(22.0 \mathrm{kcal} / \mathrm{mol})$ of product $(R)$ compared to its sulfur analogue $(23.4 \mathrm{kcal} / \mathrm{mol})$. In comparison, the $\Delta \mathrm{G}^{\ddagger}$ for minor enantiomer of product $(S)$ are much closer $(23.9$ versus $24.2 \mathrm{kcal} / \mathrm{mol}$ for $\mathbf{1 q}$ versus the sulfur analogue). These results suggest that the decrease in ee upon going from the Se to the $\mathrm{S}$ catalyst occurs due to destabilization of the transition structure for the major enantiomer in the sulfur analogue of catalyst 1q (sulfurcat- $\left.\boldsymbol{R}-\mathbf{T S}_{\mathbf{C} \text {-prot }}\right)$ relative to the corresponding transition structure for catalyst $\mathbf{1 q}\left(\boldsymbol{R}\right.$-TS $\left.\mathbf{T S}_{\mathbf{C} \text {-prot }}\right)$. Careful analysis of the key stabilizing interactions in these transition structures reveal that the distance of the $\mathrm{CH}^{\cdots}$ Se interaction between the $\alpha-\mathrm{CH}$ group of piperidine and the selenium Lewis base $(2.76 \AA$ ) becomes slightly shorter when Se is replaced by $\mathrm{S}(2.67 \AA)$. While this small change is not expected to make a significant difference in the relative energies, we observe an important manifestation of this change in the interaction between the two arms of the catalyst (intramolecular H-bonding contact between the thiourea $\mathrm{S}$ and the selenourea/thiourea $\mathrm{NH}$ ). This $\mathrm{NH}^{*} \mathrm{~S}$ changes from $2.52 \AA$ in $\boldsymbol{R}$ - $\mathbf{T S} \mathbf{C}_{\text {-prot }}$ to $2.60 \AA$ in sulfurcat- $\boldsymbol{R}$ - $\mathbf{T S} \mathbf{S}_{\text {C-prot }}$. We believe that the weaker interaction in sulfurcat- $\boldsymbol{R}$ - $\mathbf{T S}_{\mathbf{C} \text {-prot }}$ is at least partially responsible for the $1.4 \mathrm{kcal} / \mathrm{mol}$ increase in $\Delta \mathrm{G}^{\ddagger}$ of this transition structure. In support of this hypothesis, we observe that this distance remains almost identical in the two transition structures for the minor enantiomer $-2.42 \AA$ in $S$ - SS $_{\mathbf{C} \text {-prot }}$ and $2.44 \AA$ in in sulfurcat-S-TS $\mathbf{S}_{\mathbf{C} \text {-prot }}$ resulting in a much smaller increase in the $\Delta \mathrm{G}^{\ddagger}(0.3 \mathrm{kcal} / \mathrm{mol})$ upon going from the Se to the $S$ catalyst. 

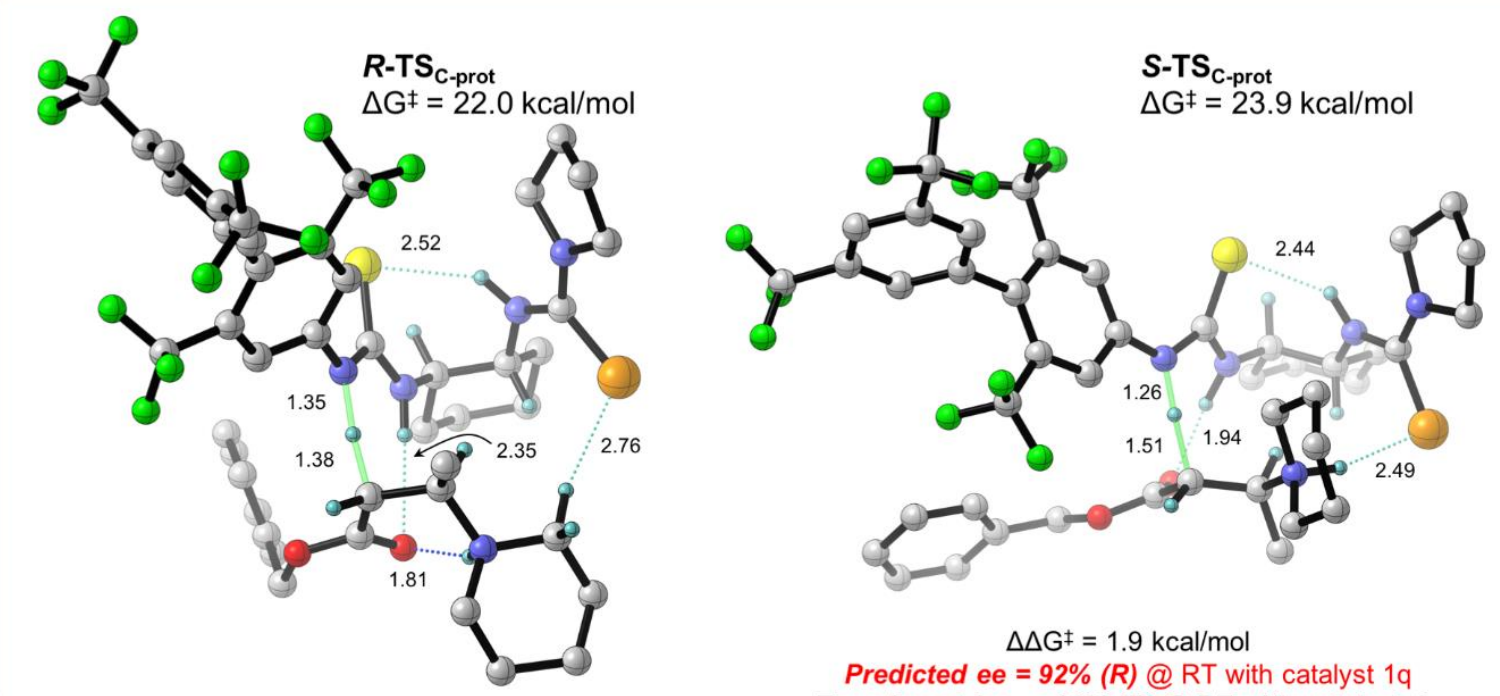

Predicted ee $=92 \%(R) @$ RT with catalyst 1q

Experimental ee $=88 \%(R) @ \mathrm{RT}$ with catalyst 1q
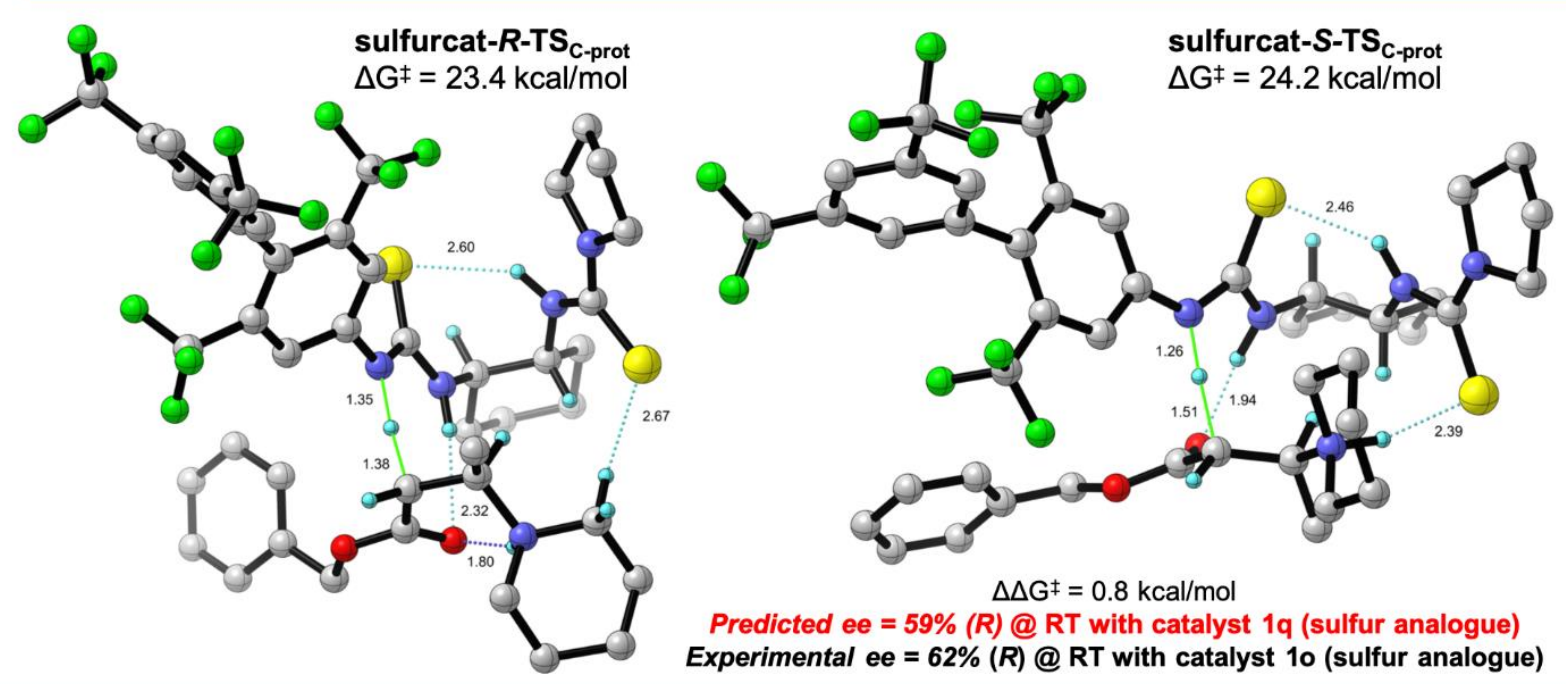

Predicted ee $=59 \%(R) @$ RT with catalyst 1q (sulfur analogue)

Experimental ee $=62 \%(R) @$ RT with catalyst 10 (sulfur analogue)

Figure S2. Comparion of free energy barriers, key interactions, and transition structures for the rate-determining step for both enantiomers of the reaction catalyzed by $1 q$ and its sulfur analogue.

To identify any other differences in the transition structures, further analysis was carried out by overlaying the two pairs of transition structures $\boldsymbol{R}$-TS $\mathbf{S}_{\mathrm{C} \text {-prot }} /$ sulfurcat- $\boldsymbol{R}$ - $\mathbf{T S} \mathbf{S}_{\mathrm{C} \text {-prot }}$ and $\boldsymbol{S}$-TS $\mathbf{T S}_{\mathrm{C} \text {-prot }} /$ sulfurcat- $\boldsymbol{S}$-TS $\mathbf{S}_{\mathrm{C} \text {-prot }}$. As can be seen in the figure above (Figure S3), there is very little difference in the transition structures with the two catalyst. The biggest visible difference being the orientation of the benzyl portion of benzyl crotonate in the overlay of the $R$ enantiomer transition structures (highlighted in yellow in Figure S3) - this difference might also contribute to the $1.4 \mathrm{kcal} / \mathrm{mol}$ increase in $\Delta \mathrm{G}^{\ddagger}$ for the sulfur analogue of $\mathbf{1 q}$, due to diminished $\mathrm{CH}-\mathrm{pi}$ interactions with the cyclohexane ring $\mathrm{CHs}$ of the catalyst. 

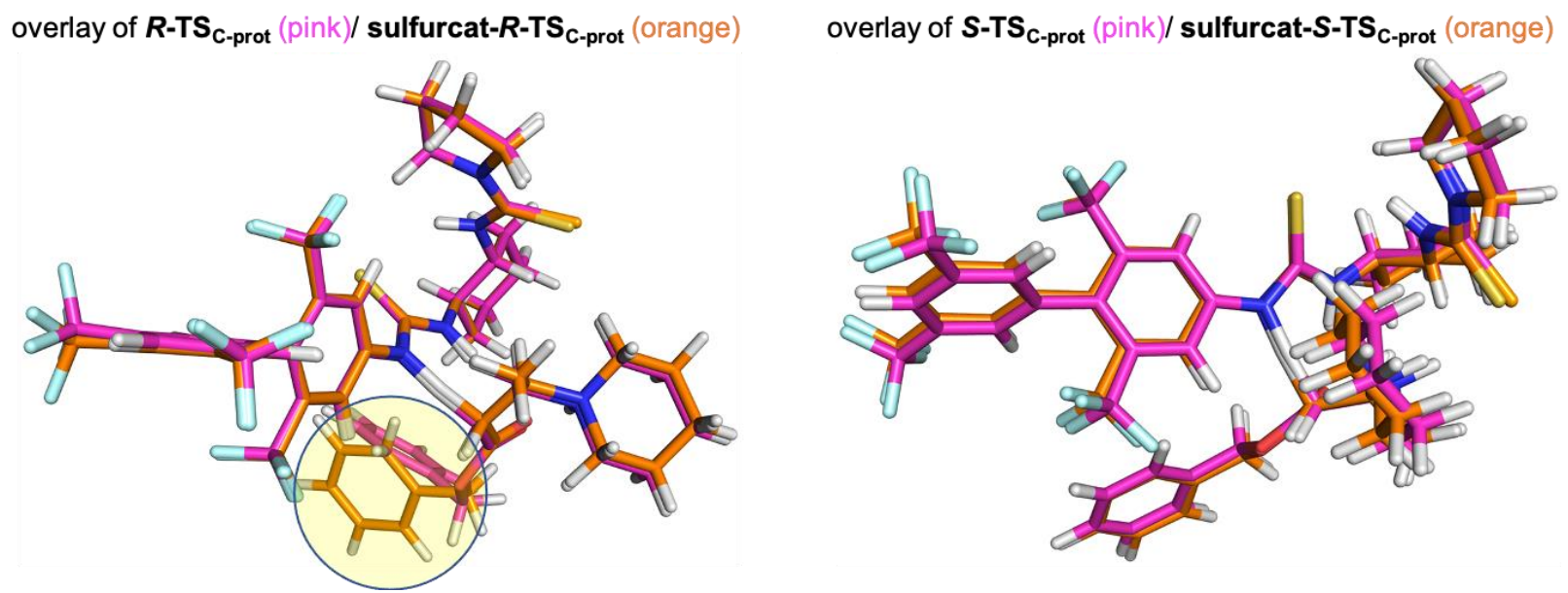

Figure S3. Overlay of $(R)$ - and $(S)$ - transition structures of catalysts $1 \mathrm{q}$ and its sulfur analogue.
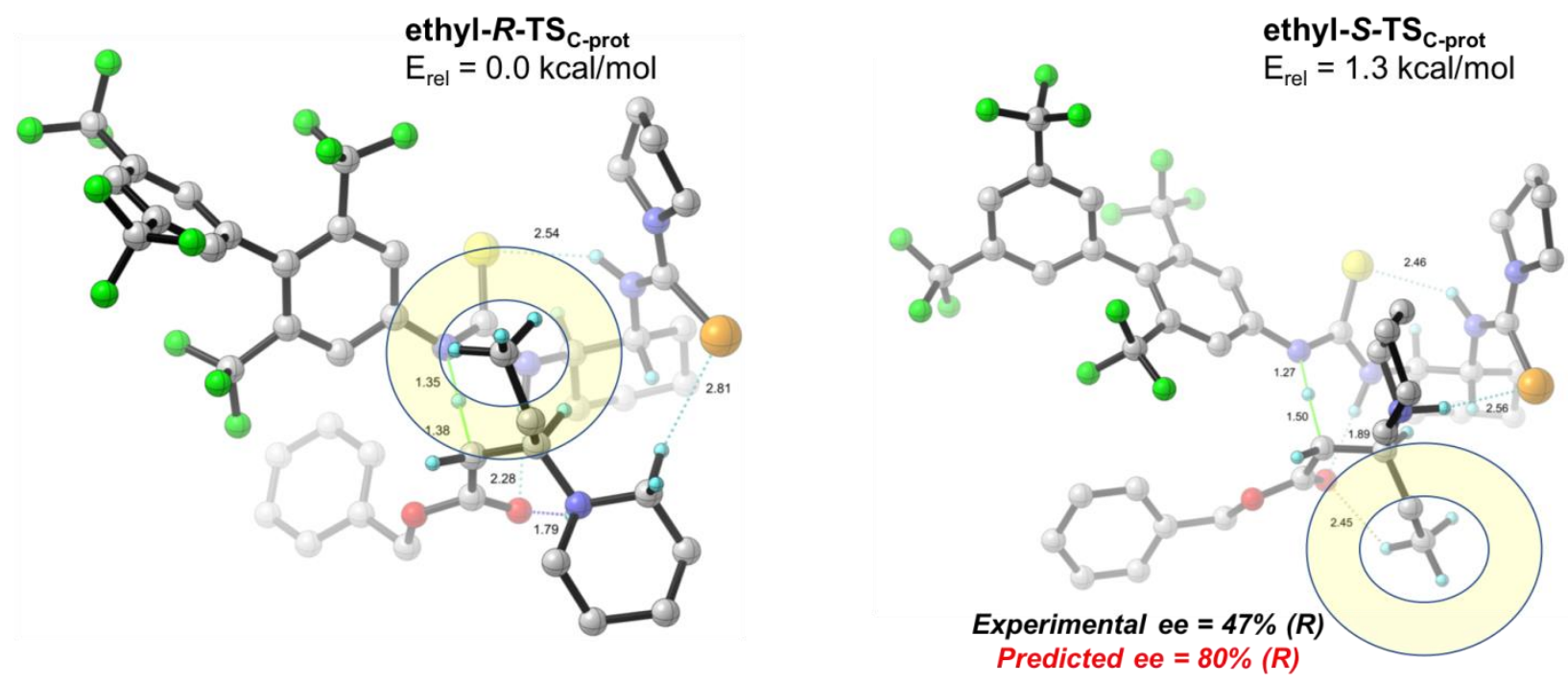

Figure S4. Comparison of lowest energy transition structues for direct C-protonation of the $\beta$-ethyl ester

We recalculated TSs corresponding to $\boldsymbol{R}$ - $\mathbf{T S}_{\mathbf{C} \text {-prot }}$ and $\boldsymbol{S}$-TS $\mathbf{S}_{\mathbf{C} \text {-prot }}$ with an ethyl substituent at the $\beta$-position. By sequentially replacing each of the three hydrogens of the $\beta$-methyl group of the crotonate ester, three distinct transition structure searches were initiated for each of the enantiomeric transition states with the $\beta$-ethyl substituent. Comparison of the lowest energy transition structures ethyl- $\boldsymbol{R}$ - $\mathbf{T S} \mathbf{S}_{\mathrm{C} \text {-prot }}$ and ethyl-S-TS $\mathbf{S}_{\mathbf{C} \text {-prot }}$ with the $\beta$-ethyl substituent revealed that the energy difference decreases to $1.3 \mathrm{kcal} / \mathrm{mol}$. This corresponds to a predicted ee of $80 \%$ at room temperature, which is an overprediction of the experimental ee of $47 \%$ ee observed for this reaction. However, it is important to note that there is a decrease in predicted ee from $92 \%$ to $80 \%$ ee upon changing the $\beta$ substituent from methyl to ethyl - which is in qualitative agreement with the experimental change from $88 \%$ to $47 \%$ ee.

Careful analysis of the two transition structures with the $\beta$-ethyl substituent revealed a weak intramolecular $\mathrm{CH}^{\cdots} \mathrm{O}$ interaction between the enolate oxygen and the $\mathrm{CH}_{3}$ group (highlighted in yellow, structure on right in Figure S4) of the ethyl substituent $(2.45 \AA)$ in ethyl-S $-\mathbf{T S}_{\mathrm{C} \text {-prot }}$. This $\mathrm{CH}^{\cdots} \mathrm{O}$ interaction is absent in ethyl- $\boldsymbol{R}$ - $\mathbf{T S} \mathbf{C}_{\mathrm{C}-\text { prot }}$ since that enolate oxygen is engaged in an intramolecular $\mathrm{H}$-bond with the $\mathrm{NH}^{+}$group, thereby forcing the ethyl group into the 
space between the two arms of the catalyst (highlighted in yellow, structure on left in Figure S4). We believe that this selective stabilization of the minor enantiomer contributes to part of the decreased ee observed with the $\beta$-ethyl group.

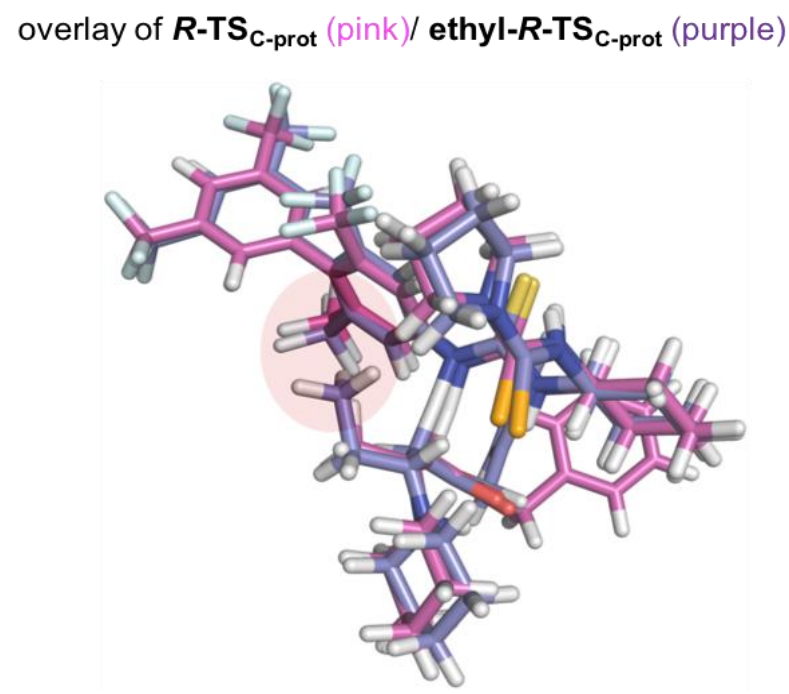

overlay of $\boldsymbol{S}$-TS $\mathbf{S}_{\text {C-prot }}\left(\right.$ pink)/ ethyl-S-TS $\mathbf{S}_{\text {C-prot }}$ (purple)

Figure S5. Overlay of $(R)$ - and $(S)$ - transition structures for direct C-protonation of $\beta$-ethyl versus $\beta$-methyl ester substrates.

To identify any other differences in the transition structures, further analysis was carried out by overlaying the two

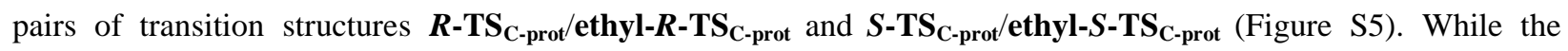
overlay of the minor enantiomer (right panel) shows virtually identical transition structures for both substrates (ethyl and methyl), the overlay of the major enantiomer transition structure reveals a significant deviation for the two substrates. We believe that this is caused by the extra ethyl group occupying the space between the catalyst arms leading to unfavorable steric interactions between the $\beta$-substituent and the two arms of the catalyst (highlighted in red on the left panel). This unfavorable steric interaction is absent in ethyl-S-TS $\mathbf{S}_{\mathbf{C}-\text { prot }}$ since this group is pointed away from the two arms of the catalyst (highlighted in green on the right panel). Therefore, the stabilizing weak $\mathrm{CH}^{\cdots} \mathrm{O}$ in ethyl-S-TS $\mathbf{S}_{\mathbf{C} \text {-prot }}$ and the unfavorable steric interactions in ethyl- $\boldsymbol{R}-\mathbf{T S} \mathbf{S}_{\mathrm{C} \text {-prot }}$ are responsible for the lower $\Delta \Delta \mathrm{G}^{\ddagger}$ for the $\beta$-ethyl ester compared to the $\beta$-methyl ester. 
S-sulfurcat-newbasis.log

Description: Direct C-Protonation catalyzed by

Sulfamidethiourea, S-enantiomer

Free Energy $\quad=-4124.088213$

Zero-point Energy $=-4123.981227$

Potential Energy $=-4126.25290049$

Nimag $=1(-1326.0223 \mathrm{~cm}-1)$

Charge $=0$ Multiplicity $=1$

C $-7.25648 \quad 1.52673-3.06613$

C $-6.08351 \quad 1.99589-3.93647$

C $-4.78027 \quad 2.04817-3.12804$

C $-4.450060 .69942-2.45893$

C $-5.641590 .21726-1.59380$

C $-6.943670 .17172-2.41867$

H $-3.937652 .33033-3.76976$

H $-5.96106 \quad 1.30630-4.78402$

H $-6.290652 .98438-4.36387$

H $-7.452712 .27162-2.28152$

H $-8.173751 .45147-3.66265$

H -4.25790 -0.04985 -3.23541

H $-5.780110 .91185-0.75892$

H $-6.83663-0.59542-3.19933$

H $-7.76090-0.14741-1.76192$

H $-4.855442 .82295-2.35231$

N $-3.22295 \quad 0.85461-1.68154$

N -5.33978 -1.10909-1.04190

H $-3.042631 .80455-1.34044$

C -2.20689-0.04299-1.54630

$\begin{array}{llll}\text { N } & -1.22341 & 0.42234 & -0.72707\end{array}$

S -2.28787 - $1.60682-2.26200$ $\begin{array}{llll}\text { H } & -1.60475 & 1.14871 & 0.23438\end{array}$

C $0.09683-0.00198-0.65917$

C $0.76720-0.76278-1.63557$

$\begin{array}{lllll}\text { C } & 0.85988 & 0.44597 & 0.43645\end{array}$

C $2.11718-1.07822-1.49762$

H $\quad 0.22878-1.11000-2.50383$

C 2.213280 .144640 .56068

H 0.387941 .063291 .18899

C $2.88438-0.64075-0.39817$

H -4.64565 -1.64191-1.57080

C $-5.87221-1.659840 .07303$

N -5.73663 -3.00027 0.20059

C $-5.24855-3.88654-0.88100$

C $-6.27414-3.773751 .33616$

C $-5.71744-5.28457-0.45349$

H -5.67087 -3.57358 -1.84207

C $-5.75411-5.193191 .07939$

H -5.94190 -3.35159 2.28655

H -6.72363 -5.47713 -0.84374

H $-6.38969-5.953281 .54251$

H -7.37047 -3.72822 1.32922

H -4.74270 -5.30472 1.48916

H -4.15590 -3.82600 -0.95922

H -5.05593 -6.07111 -0.82696

C 2.951940 .713771 .75028

C $2.75150-1.92304-2.58275$

F 3.875621 .636771 .37334

F 2.120201 .341162 .61379

F $3.60748-0.231332 .45619$

F 1.91950 -2.12817-3.62335

F 3.87194-1.35481-3.08198

F $3.10324-3.14730-2.11708$ 

C $4.33615-0.98889-0.25631$
H $\quad 0.403137 .50365-0.39140$
C $4.72067-2.246820 .21956$
C 3.445496 .336490 .59047
C $5.33007-0.06290-0.59623$
H $3.85467 \quad 4.28521 \quad 1.13725$
C $\quad 6.07200-2.571540 .35409$
H 2.719518 .281490 .00332
H $3.96362-2.974210 .48934$
H $4.46134 \quad 6.678320 .77017$
C $6.67902-0.39143-0.46066$
C $-2.77411-0.758031 .95114$
H $5.049350 .91351-0.97307$
C $-3.42690 \quad 0.59130 \quad 3.93652$
C $7.05824-1.647220 .01452$
H $-4.64621 \quad 0.00397 \quad 2.34488$
H $8.10679-1.901140 .11719$
C $-2.94615-2.045592 .75488$
C $6.46104-3.951770 .81437$
H -1.74947 -0.38884 2.01078
C $7.739410 .63250-0.77061$
H $\quad-3.02397-0.90158 \quad 0.89991$
F $6.49778-4.82818-0.21431$
C $-3.60478-0.688034 .75608$
F $5.58261-4.443231 .71678$
H -2.410720 .985714 .02826$
F $7.68395-3.964621 .38826$
H -4.126641 .360984 .26113$
F $8.860920 .05729-1.25811$
C $-2.70003-1.817374 .25045$
F $7.31721 \quad 1.53853-1.67764$
H $-2.24687-2.783862 .34638$
F $8.10211 \quad 1.32024 \quad 0.33681$
H $-3.95646-2.441512 .59746$
C -2.180772 .045991 .30168$
H $-3.39136-0.447145 .80386$
H -1.551512 .113592 .19255$
H -4.65665 -1.00219 4.71205
C $-2.06199 \quad 3.23093 \quad 0.47684$
H $-2.88761-2.736984 .81549$
O $-2.80410 \quad 3.52550-0.47160$
H -1.64712 -1.55057 4.41685
C $-3.615051 .61821 \quad 1.58656$
N -3.66738 $0.34796 \quad 2.46850$
O $\quad-0.94338 \quad 3.950890 .75647$
C $-4.52712 \quad 2.698552 .18028$
C $-0.583564 .98405-0.17943$
H -4.090513 .180503 .06094$
H - $1.301605 .80856-0.10764$
H $-4.64720 \quad 3.46747 \quad 1.41255$
H $-0.661264 .57805-1.19497$
H $\quad-5.524612 .31325 \quad 2.42651$
C 0.818625 .452850 .12912
$\begin{array}{llll}H & -4.08488 & 1.27008 & 0.66538\end{array}$
C $1.804834 .55808 \quad 0.56324$
S -6.66359-0.72595 1.29126
C $1.162246 .79572-0.06490$
C 3.108164 .996580 .79590
R-sulfurcat-newbasis.log
H $\quad \begin{array}{llll}1.54707 & 3.51871 & 0.73457\end{array}$
Description: Direct C-Protonation catalyzed by

C $\quad 2.468697 .235320 .15836$ 


\begin{tabular}{|c|c|}
\hline & C $0.999441 .55516-0.01470$ \\
\hline$=-4124.101258$ & C $2.31823-0.81446-0.61116$ \\
\hline Zero-point Energy $=-4123.990342$ & H $\quad 0.38122-1.57910-1.12231$ \\
\hline Potential Energy $\quad=-4126.25357549$ & C 2.383361 .464790 .15266 \\
\hline Nimag $\quad=1(-1287.5409 \mathrm{~cm}-1)$ & H $\quad 0.48802 \quad 2.48421 \quad 0.20646$ \\
\hline & C $3.081550 .27953-0.14679$ \\
\hline Charge $=0$ Multiplicity $=1$ & H $-2.98398-2.29441-2.62054$ \\
\hline C $-7.14057-1.04096-2.70585$ & C $-3.34566-3.47597-1.00270$ \\
\hline C $-6.66932 \quad 0.39233-2.99214$ & $\mathrm{~N}-2.25047-4.21924-1.28670$ \\
\hline C $-5.50717 \quad 0.79736-2.07269$ & C $-1.58648-4.24943-2.60817$ \\
\hline C $-4.33727-0.19759-2.17025$ & C $-1.67074-5.20632-0.36033$ \\
\hline C $-4.82310-1.63509-1.85886$ & C $-0.69498-5.49784-2.54168$ \\
\hline C $-5.97613-2.03863-2.79545$ & H $-2.33803-4.30440-3.40431$ \\
\hline H $-5.14128 \quad 1.79880-2.32795$ & C $-0.35913-5.60985-1.04670$ \\
\hline H $-6.342740 .46478-4.03953$ & H $-1.52939-4.762480 .62775$ \\
\hline H $-7.499681 .09967-2.87464$ & Н $-1.25688-6.37972-2.87200$ \\
\hline H -7.57851 -1.08608 -1.69787 & H -0.03182 -6.61132 -0.75195 \\
\hline H - $-7.93578-1.32863-3.40467$ & H - $-2.35378-6.05743-0.24169$ \\
\hline H -3.93193 -0.17141 -3.18633 & H $\quad 0.43864-4.90367-0.78993$ \\
\hline H -5.18092 -1.67098 -0.82482 & H -0.99582 -3.33794 -2.76439 \\
\hline H -5.60071 -2.08127 -3.82828 & H $\quad 0.18924-5.40256-3.17817$ \\
\hline H -6.30652 -3.05018 -2.53290 & C $3.121252 .68277 \quad 0.66225$ \\
\hline H $-5.85758 \quad 0.84330-1.03072$ & C $2.98049-2.13742-0.92193$ \\
\hline N $-3.25405 \quad 0.20206-1.28173$ & F $3.993843 .16813-0.25056$ \\
\hline $\mathrm{N}-3.70253-2.57086-1.95194$ & F $2.279493 .69233 \quad 0.97255$ \\
\hline H $-3.543680 .46750-0.34739$ & F $3.83195 \quad 2.41088 \quad 1.78185$ \\
\hline C $-1.910610 .15430-1.54539$ & F $2.10527-3.03524-1.43748$ \\
\hline $\mathrm{N}-1.139610 .57640-0.52308$ & F $3.98255-2.01571-1.81642$ \\
\hline S - $-1.32943-0.34618-3.08730$ & F $3.50733-2.711120 .18841$ \\
\hline H -1.65140 0.956760 .66349 & C $4.56997 \quad 0.183720 .01694$ \\
\hline $\begin{array}{llll}\text { C } & 0.25222 & 0.46760 & -0.48557\end{array}$ & C $5.12547-0.370221 .17495$ \\
\hline C $\quad 0.93875-0.72402-0.76971$ & C $5.425230 .64257-0.99096$ \\
\hline
\end{tabular}



C $6.51036-0.465081 .32067$
H -2.05114 4.24797 -3.31614
H $4.47496-0.738301 .95995$
H -3.87469 $7.87862-1.89447$
C $6.809510 .54372-0.84288$
H -2.64461 $6.64080-3.66845$
H $\quad 5.00838 \quad 1.06717-1.89714$
C $-4.14732-1.661593 .60572$
C $7.35977-0.010160 .31261$
C -3.497810 .491144 .66412$
H $8.43438-0.099750 .41924$
H $-4.30982 \quad 0.241302 .78051$
C $7.09063-1.012232 .59806$
C -5.51815 -1.59909 4.28129
C $7.71504 \quad 1.08762-1.91724$
H $\quad-3.41979-2.182734 .23529$
F $8.30231-1.575002 .39961$
H $-4.18881-2.160562 .63282$
F $6.29085-1.950033 .15215$
C $-4.86155 \quad 0.59241 \quad 5.35020$
F $7.25439-0.040193 .52500$
H -2.77963 -0.04698 5.28998
F $7.164190 .96894-3.14406$
H $-3.08390 \quad 1.473364 .42605$
F $7.975182 .40094-1.72708$
C -5.47148 -0.79679 5.58965
F $8.905990 .44969-1.93954$
H $-5.85710-2.625354 .46028$
C $-2.14518 \quad 1.200241 .92588$
H $-6.24119-1.148813 .58736$
H -1.351601 .763972 .41807$
H $\quad-4.73481 \quad 1.135096 .29381$
C -3.322651 .988161 .62830$
H $-5.53243 \quad 1.197084 .72480$
$\begin{array}{llll}\text { O } & -4.47010 & 1.50705 & 1.50436\end{array}$
H $-6.47711-0.700126 .01308$
C $-2.37145-0.205792 .45519$
H -4.86646 -1.33811 6.33053
H $-2.67171-0.87423 \quad 1.63874$
N -3.61945 -0.26913 3.37479
O $\quad-3.05463 \quad 3.28872 \quad 1.40204$
C -1.12795-0.78218 3.12433
C -4.109564 .138420 .89039$
H $-0.78874-0.166473 .96424$
H -4.341384 .866201 .67482$
H $-0.32379-0.796602 .38274$
H $-4.99388 \quad 3.518740 .71994$
H -1.27661 -1.80996 3.46888
C $-3.65617 \quad 4.83665-0.36997$
S $-4.22242-3.711100 .45490$
C -2.96033 $4.14593-1.37143$
C -3.97566 $6.18416-0.56903$
C $-2.596364 .79470-2.55168$
D3gen-sulfur-cat2.log
H -2.68554 $3.10559-1.22571$
Description: Sulfamidethiourea catalyst
C $-3.620306 .83136-1.75432$
Free Energy $\quad=-3295.709311$
H $-4.50488 \quad 6.73273 \quad 0.20756$
Zero-point Energy $=-3295.623867$
C $-2.92900 \quad 6.13755-2.74835$ 


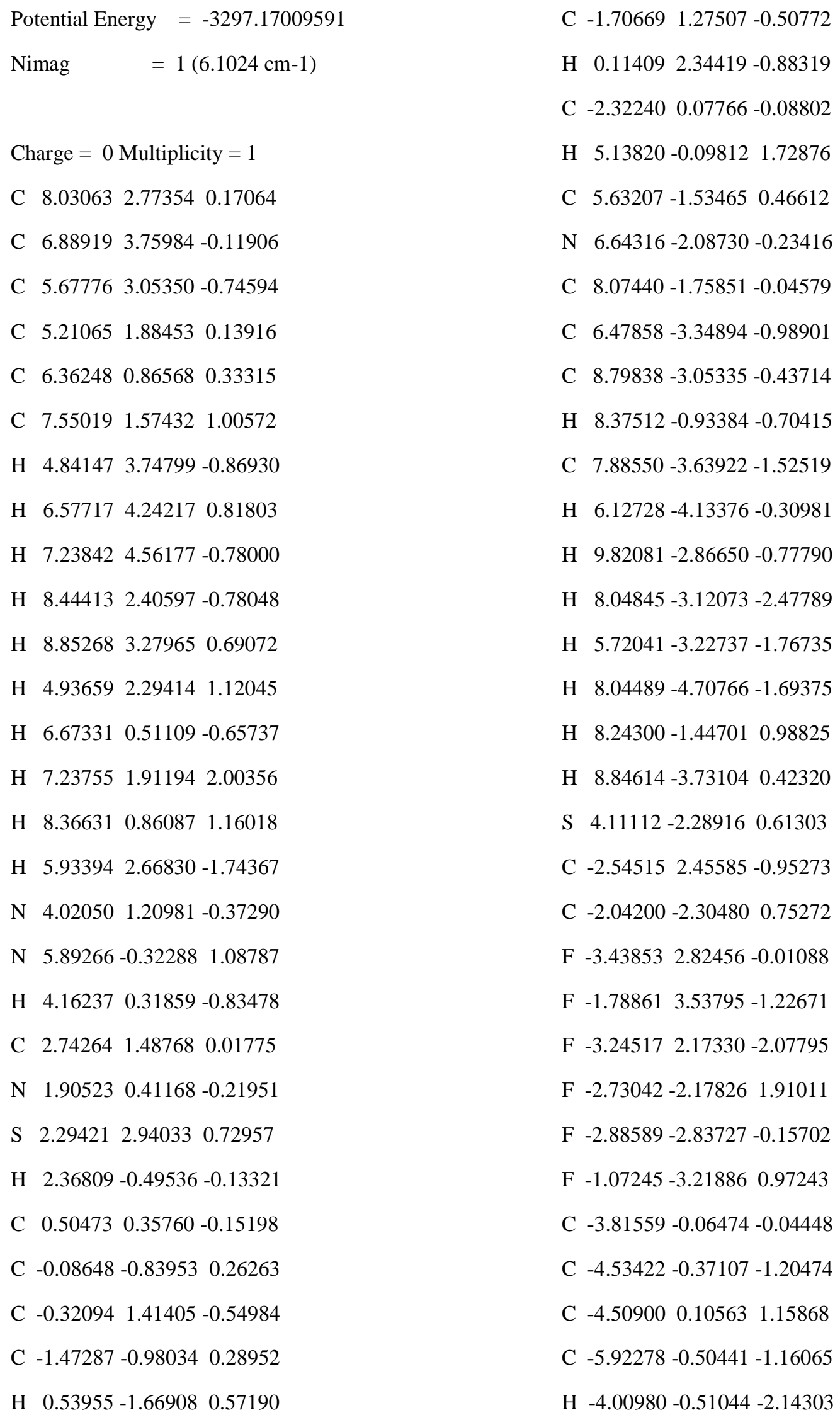




$$
\begin{array}{llll}
\text { C } & -5.89752 & -0.02819 & 1.19775 \\
\text { H } & -3.96554 & 0.34859 & 2.06454 \\
\text { C } & -6.61173 & -0.33370 & 0.03953 \\
\text { H } & -7.68996 & -0.43720 & 0.07195 \\
\text { C } & -6.68651 & -0.77544 & -2.43067 \\
\text { C } & -6.62383 & 0.09804 & 2.51184 \\
\text { F } & -7.00877 & 0.37140 & -3.07008 \\
\text { F } & -7.84260 & -1.43107 & -2.18966 \\
\text { F } & -5.96662 & -1.52056 & -3.29748 \\
\text { F } & -5.99983 & 0.95443 & 3.34848 \\
\hline \text { F } & -7.88953 & 0.53896 & 2.34308 \\
\text { F } & -6.70242 & -1.09142 & 3.15016
\end{array}
$$

D3gen-lowestR-ethyl2.log

Description: Direct C-Protonation catalyzed by selenothiourea, R-enantiomer, $\beta$-ethyl ester

$$
\begin{aligned}
& \text { Free Energy = }-6164.125965 \\
& \text { Zero-point Energy }=-6164.013205 \\
& \text { Potential Energy }=-6168.91615639 \\
& \text { Nimag = } 1(-1322.8018 \mathrm{~cm}-1)
\end{aligned}
$$$$
\text { Charge }=0 \text { Multiplicity }=1
$$$$
\text { C }-6.950620 .02053-3.05507
$$$$
\text { C }-6.046381 .12073-3.62830
$$$$
\text { C }-4.851541 .40534-2.70505
$$$$
\text { C }-4.044550 .12911-2.40577
$$$$
\text { C }-4.96651-0.96299-1.81714
$$$$
\text { C }-6.14945-1.25473-2.75813
$$$$
\text { H }-4.182392 .14711-3.15734
$$$$
\text { H } \quad-5.673460 .80721-4.61385
$$

H -6.61717 2.04372 -3.78938

H $-7.419510 .37873-2.12672$

H $-7.76840-0.20287-3.75109$

H $-3.60823-0.24196-3.33850$

H $-5.36353-0.61452-0.85757$

H -5.76292 -1.67972 -3.69568

H -6.78477 -2.01658 -2.29322

H -5.21077 1.83816-1.75930

N -2.93038 $0.43475-1.51450$

N -4.18530 -2.17095 -1.54793

H $\quad-3.119191 .14541-0.81595$

C - $1.64058-0.02685-1.61820$

N $-0.818820 .46776-0.67350$

S - $1.20662-1.10478-2.89150$

H $-1.30030 \quad 1.07055 \quad 0.43303$

C $\quad 0.55158 \quad 0.21470-0.55804$

C $1.15633-1.04392-0.70850$

C $\quad 1.36627 \quad 1.28344-0.15270$

C $2.52203-1.21406-0.49133$

H $\quad 0.55315-1.88777-1.00519$

C $2.73224 \quad 1.111710 .07473$

H $\quad 0.920772 .26435-0.03761$

C $3.35139-0.14236-0.09652$

H $-3.33423-2.25723-2.10627$

C $-4.35443-3.00718-0.50012$

N -3.56040 -4.09730 -0.47033

C $-2.68815-4.51301-1.59086$

C $-3.55993-5.089680 .61916$

C $-2.31926-5.96357-1.25083$

H $-3.22828-4.42312-2.54048$

C $-2.36093-5.98621 \quad 0.28440$

H $\quad-3.47935-4.589931 .58665$ 


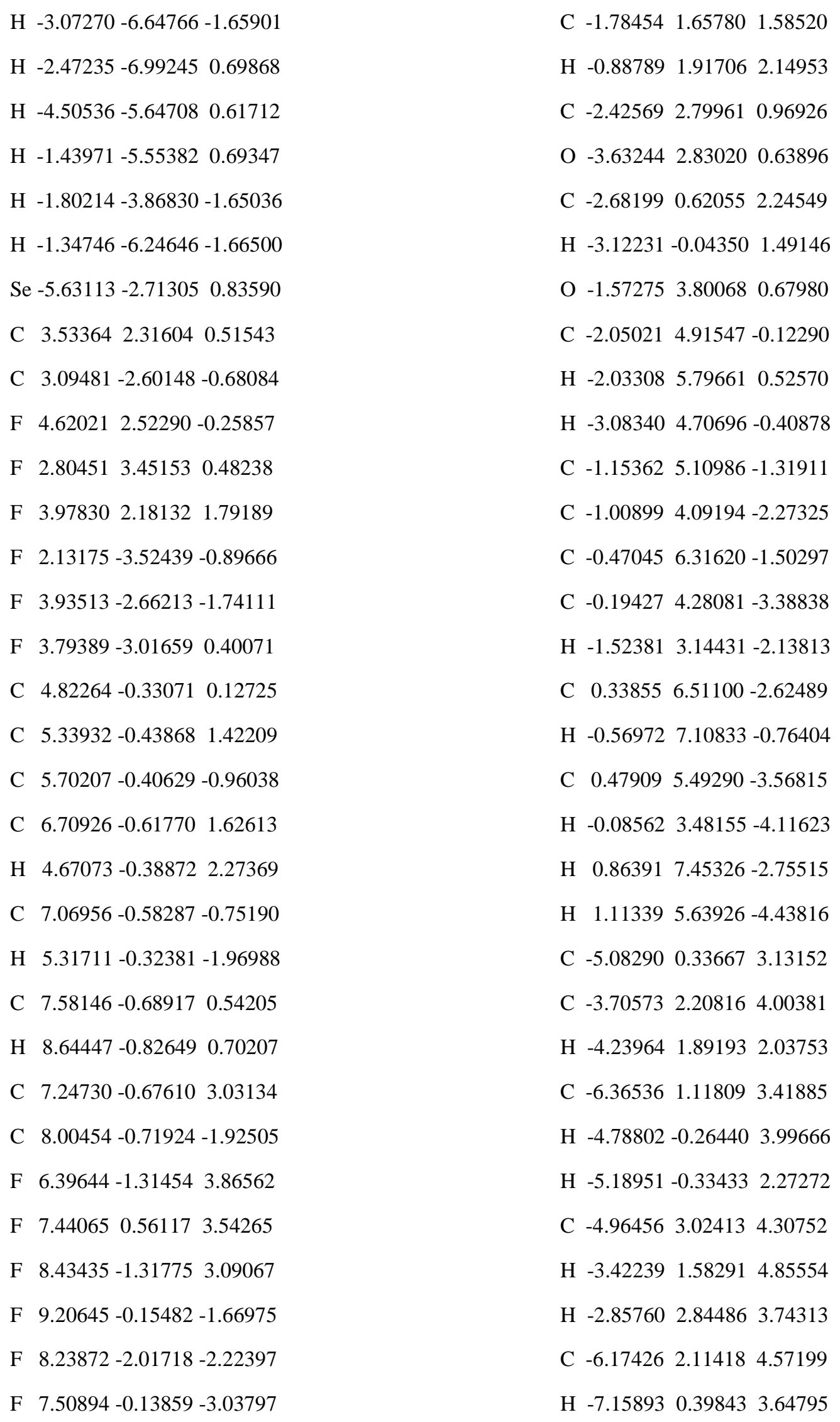
C $-1.78454 \quad 1.65780 \quad 1.58520$
H -0.887891 .917062 .14953$
C -2.425692 .799610 .96926$
O $-3.632442 .83020 \quad 0.63896$
C $-2.68199 \quad 0.62055 \quad 2.24549$
H $-3.12231-0.043501 .49146$
O $\quad-1.57275 \quad 3.80068 \quad 0.67980$
C -2.05021 $4.91547-0.12290$
H $-2.03308 \quad 5.796610 .52570$
H -3.08340 $4.70696-0.40878$
C -1.15362 $5.10986-1.31911$
C -1.00899 $4.09194-2.27325$
C $-0.47045 \quad 6.31620-1.50297$
C $-0.19427 \quad 4.28081-3.38838$
H $-1.523813 .14431-2.13813$
C $0.338556 .51100-2.62489$
H -0.56972 $7.10833-0.76404$
C $0.479095 .49290-3.56815$
H $-0.085623 .48155-4.11623$
H $\quad 0.863917 .45326-2.75515$
Н $1.113395 .63926-4.43816$
C $-5.08290 \quad 0.33667 \quad 3.13152$
C -3.705732 .208164 .00381$
H -4.239641 .891932 .03753$
C -6.365361 .118093 .41885$
H $-4.78802-0.264403 .99666$
H $-5.18951-0.334332 .27272$
C -4.964563 .024134 .30752$
H $\quad-3.422391 .582914 .85554$
H $-2.85760 \quad 2.844863 .74313$
C -6.174262 .114184 .57199$

H $-7.15893 \quad 0.39843 \quad 3.64795$ 


\begin{tabular}{|c|c|c|c|c|c|}
\hline Н -6.67842 & 1.648212 .50868 & $\mathrm{H}$ & 5.94102 & 2.81888 & 4.59279 \\
\hline H -4.76024 & 3.667945 .17050 & $\mathrm{H}$ & 7.14130 & 2.13442 & 2.52093 \\
\hline H -5.17141 & $3.68903 \quad 3.45784$ & $\mathrm{H}$ & 7.82761 & 1.28512 & 3.90229 \\
\hline Н -7.07782 & 2.718384 .70816 & $\mathrm{H}$ & 3.90096 & -0.17397 & 3.35923 \\
\hline H -6.01802 & 1.562575 .50970 & $\mathrm{H}$ & 5.49295 & 0.80655 & 0.93402 \\
\hline N -3.95295 & 1.289832 .84101 & $\mathrm{H}$ & 6.48494 & -0.74210 & 3.37755 \\
\hline C -1.95462 & -0.236353 .28831 & $\mathrm{H}$ & 7.44211 & -0.27766 & 1.96656 \\
\hline H - -2.68318 & -0.839633 .84260 & $\mathrm{H}$ & 4.55288 & 2.70791 & 2.54672 \\
\hline C -0.92746 & -1.182072 .65150 & $\mathrm{~N}$ & 2.92160 & 0.77544 & 1.79406 \\
\hline H -0.45123 & -1.794083 .42445 & $\mathrm{~N}$ & 5.03090 & -1.21136 & 1.18472 \\
\hline H - 1.40922 & -1.856941 .93536 & $\mathrm{H}$ & 2.75322 & 1.73952 & 1.48341 \\
\hline Н -0.14179 & $\begin{array}{lll}-0.63630 & 2.12248\end{array}$ & $\mathrm{C}$ & 1.88956 & -0.09665 & 1.62619 \\
\hline H -1.45179 & $0.41447 \quad 4.01537$ & $\mathrm{~N}$ & 0.92648 & 0.40735 & 0.80564 \\
\hline \multirow{4}{*}{\multicolumn{2}{|c|}{$\begin{array}{l}\text { D3gen-lowestS-ethyl2.log } \\
\text { Description: Direct C-Protonation catalyzed by } \\
\text { selenothiourea, S-enantiomer, } \beta \text {-ethyl ester }\end{array}$}} & $\mathrm{S}$ & 1.92980 & -1.68141 & 2.30103 \\
\hline & & $\mathrm{H}$ & 1.33472 & 1.15464 & -0.14240 \\
\hline & & $\mathrm{C}$ & -0.39384 & -0.00813 & 0.70741 \\
\hline & & $\mathrm{C}$ & -1.09890 & -0.72001 & 1.69644 \\
\hline \multicolumn{2}{|l|}{ Free Energy } & $\mathrm{C}$ & -1.11994 & 0.39539 & -0.42908 \\
\hline \multicolumn{2}{|c|}{ Zero-point Energy $=-6164.003501$} & $\mathrm{C}$ & -2.44554 & -1.03475 & 1.53230 \\
\hline \multicolumn{2}{|c|}{ Potential Energy $=-6168.91437312$} & $\mathrm{H}$ & -0.58929 & -1.02880 & 2.59628 \\
\hline \multirow[t]{2}{*}{ Nimag } & \multirow[t]{2}{*}{$=1(-1326.9848 \mathrm{~cm}-1)$} & $\mathrm{C}$ & -2.47105 & 0.09372 & -0.58198 \\
\hline & & $\mathrm{H}$ & -0.62175 & 0.97445 & -1.19505 \\
\hline \multicolumn{2}{|c|}{ Charge $=0$ Multiplicity $=1$} & $\mathrm{C}$ & -3.17588 & -0.64358 & 0.39014 \\
\hline C 6.92341 & 1.377913 .28861 & $\mathrm{H}$ & 4.31475 & -1.73951 & 1.69073 \\
\hline C 5.73567 & 1.840414 .14208 & $\mathrm{C}$ & 5.58027 & -1.75690 & 0.08063 \\
\hline C 4.45207 & 1.919383 .30549 & $\mathrm{~N}$ & 5.43452 & -3.08873 & -0.07039 \\
\hline C 4.12347 & 0.587212 .60246 & $\mathrm{C}$ & 4.89569 & -3.97954 & 0.98508 \\
\hline C 5.33090 & 0.106391 .76020 & $\mathrm{C}$ & 5.97244 & -3.86208 & -1.20733 \\
\hline C 6.61408 & 0.036752 .61189 & $\mathrm{C}$ & 5.33373 & -5.38320 & 0.54549 \\
\hline Н 3.59704 & 2.198483 .93187 & $\mathrm{H}$ & 5.30391 & -3.69230 & 1.96020 \\
\hline Н 5.58912 & 1.135944 .97349 & $\mathrm{C}$ & 5.40516 & -5.26876 & -0.98444 \\
\hline & & $\mathrm{H}$ & 5.67437 & -3.41276 & -2.15593 \\
\hline
\end{tabular}



H $\quad 6.32531-5.61110 \quad 0.95361$
H $6.02799-6.03963-1.44691$
H $7.06904-3.84960-1.17455$
H $4.39989-5.34337-1.41703$
H $3.80387-3.887581 .03782$
H $4.64173-6.155430 .89262$
Se $6.48192-0.73238-1.21265$
C $-3.167830 .61154-1.81927$
C $-3.11814-1.823982 .63605$
F $-4.070851 .58183-1.51640$
F $-2.30040 \quad 1.16136-2.70007$
F -3.83745 -0.35337 -2.48313
F -2.32354 -1.97305 3.71491
F -4.25598 -1.23346 3.06454
F -3.45230 -3.07138 2.22193
C $-4.62490-0.989660 .21872$
C -5.00443 -2.27144-0.19334
C $-5.62140-0.036990 .46437$
C $-6.35313-2.59308-0.35847$
H -4.24520 -3.01974-0.39000
C $-6.96773-0.36277 \quad 0.29893$
H $-5.34506 \quad 0.958670 .79088$
C -7.34195 -1.64221 -0.11295
H -8.38838 $-1.89381-0.23901$
C $-6.73541-3.99561-0.75247$
$\begin{array}{llll}\text { C } & -8.02653 & 0.68838 & 0.50615\end{array}$
F $\quad-6.77225-4.82150 \quad 0.31709$
F -5.85026 -4.52590 -1.62610
F -7.95524 -4.04188 -1.33078
F $\quad-9.182020 .151850 .95647$
F $-7.63810 \quad 1.627301 .39486$
F $-8.31710 \quad 1.33273-0.64775$
C $1.913762 .09086-1.16504$
H $1.278742 .21050-2.04622$
C $1.758983 .22146-0.26793$
$\begin{array}{llll}\text { O } & 2.43099 & 3.44601 & 0.74863\end{array}$
C $3.336321 .67148-1.51238$
O $\quad 0.661793 .96529-0.56856$
C $\quad 0.23251 \quad 4.923610 .41893$
H $\quad 0.933715 .765200 .43931$
H $\quad 0.27045 \quad 4.447721 .40553$
C -1.16227 5.383190 .06917
C -2.12976 $4.47461-0.37986$
C $-1.51966 \quad 6.726110 .23165$
C $-3.427084 .90090-0.66106$
H -1.86136 $3.43285-0.52199$
C -2.82176 $7.15369-0.03804$
H -0.774947 .443640 .56979$
C $-3.77843 \quad 6.24211-0.48695$
H -4.15814 4.18104-1.01756
H $-3.08396 \quad 8.200260 .09344$
H $-4.790116 .57439-0.70410$
C $2.53027-0.75520-1.74198$
C $2.920250 .56751-3.80281$
H $4.320940 .05097-2.33118$
C $2.68376-2.05369-2.53287$
H $1.48980-0.43160-1.71652$
H $2.88176-0.86340-0.71677$
C $3.07841-0.71833-4.61603$
H $1.878800 .90049-3.78882$
H $3.532521 .36791-4.21576$
C $2.28340-1.87581-4.00153$
H $2.06298-2.81173-2.04224$
H $3.72295-2.39720-2.46867$ 

H $2.74903-0.50969-5.64044$
H $-4.99379-5.06527-2.75344$
H $4.14333-0.98267-4.67385$
H -5.89140 -3.99422 -3.82324
H $2.46107-2.79989-4.56259$
H -6.65849 -2.63605 -1.89264
H 1.20764 -1.66302 -4.07106
H -7.06347 -4.32535 -1.60808
N $3.32759 \quad 0.36887-2.36336$
H -2.90980 -4.08765 -1.46861
C $4.229112 .70257-2.24621$
H -4.56745 - $1.62819-0.69865$
H $5.034992 .17403-2.77660$
H -5.07241 -4.59447-0.13404
H $3.86757 \quad 1.35350-0.61217$
H -6.05548 -3.24247 0.43919
H $3.636023 .23286-3.00154$
H -4.41368 -2.08832 -3.23599
C $4.891833 .72161-1.30880$
N -2.14154 -2.20729-1.80816
H $4.161844 .31527-0.75717$
N $\quad-3.43867-2.599350 .73869$
H $5.532773 .21571-0.57790$
H -2.31839-1.25866 -2.16444
H $5.523214 .40074-1.89214$
C $-0.89528-2.44974-1.33794$
N -0.06207 -1.37546 -1.54766
S - $-0.45756-3.89929-0.55614$
H -0.56111 -0.52892 - 1.84803
D3genbenattackrbenbenturn-ethyl.log
C $1.28568-1.15301-1.25070$
Description: C-N bond formation catalyzed by selenothiourea, R-enantiomer, $\beta$-ethyl ester
C $2.23798-2.13403-0.94760$
C $1.707710 .18464-1.30128$
Free Energy $\quad=-6164.140353$
C $3.55646-1.76958-0.66869$
Zero-point Energy $=-6164.027161$
H $1.95002-3.17328-0.92540$
Potential Energy $\quad=-6168.92614620$
C $3.019150 .54331-1.01339$
Nimag = $1(-100.8604 \mathrm{~cm}-1)$
H $\quad 0.99047 \quad 0.95242-1.56287$
Charge $=0$ Multiplicity $=1$
C $3.98687-0.42814-0.68227$
C $-6.22209-3.62803-1.70194$
H $-2.60161-3.180550 .74471$
C $-5.32608-4.02546-2.88373$
C $-3.63167-1.73681 \quad 1.76008$
C $-4.09524-3.11207-2.98820$
N -2.81571 -1.86099 2.82348
C $-3.29595-3.08416-1.67300$
C $-1.85153-2.968423 .00137$
C -4.21193 -2.64643 -0.50468
C -2.83453-0.95834 3.99110
C $-5.42664-3.58540-0.38937$
C - $1.44663-2.867674 .47826$
H -3.43271 -3.44276 -3.79604
H - $2.32600-3.926272 .75820$
C $-1.58309-1.367624 .77819$ 


\begin{tabular}{|c|c|c|c|}
\hline H - $-2.82082 \quad 0.08272 \quad 3.66415$ & H 9.07075 & 0.96313 & 0.46966 \\
\hline H -2.14487-3.44303 5.09765 & C 7.59058 & 0.48975 & 2.69792 \\
\hline H - $1.68016-1.148225 .84528$ & C 8.59089 & 0.89902 & -2.20292 \\
\hline Н $-3.75559-1.115764 .56679$ & F 7.34882 & 1.72782 & 3.18714 \\
\hline H $-0.70847-0.82428 \quad 4.40123$ & F 8.91408 & 0.26164 & 2.84097 \\
\hline Н $-0.99091-2.842002 .33195$ & F 6.93607 & -0.38218 & 3.49452 \\
\hline H $-0.44038-3.258284 .65368$ & F 9.55850 & 1.76234 & -1.82414 \\
\hline Se -4.99427-0.44969 1.74825 & F 9.20904 & -0.23401 & -2.60632 \\
\hline O $-1.969930 .56904-2.24356$ & F 7.97806 & 1.42100 & -3.28694 \\
\hline C $-2.85115 \quad 1.38727-1.86932$ & C -5.86344 & 3.26645 & -3.13850 \\
\hline C $-4.10061 \quad 1.58786-2.47119$ & H -6.30492 & 2.48930 & -3.77487 \\
\hline H $-4.31603 \quad 0.99976-3.35754$ & H -6.69569 & 3.83446 & -2.70150 \\
\hline C $-5.006812 .62059-2.05302$ & C -6.61294 & 3.07261 & 0.09512 \\
\hline H $-4.529103 .36980-1.41947$ & C -7.22173 & 1.18744 & -1.39650 \\
\hline O - $-2.61142 \quad 2.20035-0.77723$ & H -5.49704 & 1.37899 & -0.30472 \\
\hline C $-1.325352 .10312-0.15989$ & C -7.49805 & 2.50352 & 1.20988 \\
\hline C $3.372162 .01446-1.05522$ & H -7.17386 & 3.79143 & -0.51269 \\
\hline C $4.52874-2.88339-0.33593$ & H -5.73424 & 3.58210 & 0.50163 \\
\hline F $4.215962 .30523-2.07115$ & C -8.10529 & 0.57797 & -0.30332 \\
\hline F $2.274792 .79271-1.23079$ & H -7.81392 & 1.84180 & -2.04383 \\
\hline F 3.961852 .428820 .08496 & H -6.74988 & 0.42096 & -2.01861 \\
\hline F $3.98734-4.10337-0.52352$ & C -8.64966 & 1.66503 & 0.63559 \\
\hline F $5.64451-2.82563-1.09476$ & H -7.88123 & 3.33771 & 1.81000 \\
\hline F $4.92543-2.826400 .95906$ & H -6.88460 & 1.88663 & 1.87918 \\
\hline C $5.40151-0.04579-0.36277$ & H -8.92584 & 0.03139 & -0.78419 \\
\hline $\begin{array}{llll}\text { C } & 5.84083 & 0.00537 & 0.96481\end{array}$ & H -7.51881 & -0.15082 & 20.26940 \\
\hline C $6.30130 \quad 0.27071-1.38668$ & H -9.22598 & 1.20944 & 1.44841 \\
\hline $\begin{array}{llll}\text { C } & 7.15483 & 0.37041 & 1.26078\end{array}$ & H -9.34063 & 2.31929 & 0.08365 \\
\hline Н $5.15800-0.244931 .76829$ & N -6.11703 & 2.01353 & -0.83077 \\
\hline C $7.616390 .62844-1.08611$ & C -1.32255 & 2.91667 & 1.11197 \\
\hline Н $5.97455 \quad 0.24147-2.41967$ & C -0.25942 & 3.78334 & 1.38691 \\
\hline $\begin{array}{llll}\text { C } & 8.05005 & 0.68227 & 0.23810\end{array}$ & C -2.35430 & 2.78190 & 2.05251 \\
\hline
\end{tabular}


C $-0.22403 \quad 4.50828 \quad 2.58112$

H $\quad 0.550793 .882650 .66795$

C -2.322523 .512243 .24094$

H $\quad-3.173332 .09473 \quad 1.85449$

C -1.258044 .378593 .50872$

H $\quad 0.60883 \quad 5.17720 \quad 2.78130$

H -3.128923 .402103 .96222$

H -1.23493 4.946054 .43546

H $-1.10667 \quad 1.049600 .05150$

H $-0.553312 .47138-0.84571$

C $-5.022434 .21278-4.00841$

H $-4.589995 .02185-3.40800$

H $-4.198843 .67267-4.48387$

H -5.63982 $4.66736-4.79110$

D3genamineadds-ethyl.log

Description: C-N bond formation catalyzed by selenothiourea, R-enantiomer, $\beta$-ethyl ester

Free Energy $\quad=-6164.143135$

Zero-point Energy = -6164.029464

Potential Energy $\quad=-6168.92827131$

Nimag = $\quad 1(-195.3959 \mathrm{~cm}-1)$

Charge $=0$ Multiplicity $=1$

C $-6.78078-1.62674-3.54856$

C $-5.81777-1.20891-4.66849$

C $-4.58177-0.49131-4.10589$

C $-3.86301-1.33989-3.04019$

C $-4.84816-1.71114-1.90526$

C $-6.06241-2.46416-2.48142$

H $-3.87014-0.25751-4.90600$
H -5.49484 -2.10168 -5.22294

H $-6.32515-0.55868-5.39164$

H -7.20678-0.72934-3.07666

H $-7.62485-2.19240-3.96126$

H $\quad-3.49797-2.26143-3.50491$

H -5.20055 -0.79393-1.42041

H $\quad-5.71605-3.41200-2.91854$

H -6.74112 -2.71048 -1.65888

H $-4.883200 .46754-3.65826$

N -2.69127 -0.62629 -2.55016

N $-4.15499-2.49896-0.87991$

$\begin{array}{llll}\text { H } & -2.80321 & 0.36707 & -2.31372\end{array}$

C $-1.45786-1.14891-2.35775$

N $-0.55139-0.19039-1.97490$

S -1.10396 -2.80667 -2.53697

H $\quad-0.97220 \quad 0.73558-1.80874$

C $0.76945-0.32201-1.52381$

C $1.196900 .57964-0.54079$

C $1.68994-1.24633-2.02956$

C $\quad 2.50102 \quad 0.54497-0.05253$

H $\quad 0.49410 \quad 1.30279-0.14434$

C $2.99439-1.28039-1.53589$

H $1.39000-1.93246-2.80636$

C $3.43796-0.39178-0.53527$

H $\quad-3.32377-2.99020-1.20757$

C $-4.49284-2.57140 \quad 0.42390$

N $-3.74707-3.37561 \quad 1.20799$

C $-2.61563-4.19170 \quad 0.71589$

C $-4.03170-3.642442 .63119$

C $-2.02596-4.810621 .98972$

H $\quad-2.98688-4.96302 \quad 0.02511$

C $-3.24045-4.923572 .92261$ 


\begin{tabular}{|c|c|c|c|c|}
\hline H -3.68399-2.80201 & 3.24644 & Н -5.91172 & 4.98917 & 3.73511 \\
\hline H -1.53619-5.76777 & 1.79024 & H -7.08693 & 3.72743 & 3.37335 \\
\hline H -3.84124 -5.80224 & 2.65833 & O -2.63343 & 3.61914 & 0.00975 \\
\hline H -5.10751 -3.73960 & 2.78565 & C -2.38884 & 4.60546 & -1.00007 \\
\hline H - $-2.96853-5.00832$ & 3.97871 & C 3.93531 & -2.31390 & -2.12040 \\
\hline H - $-1.89026-3.57868$ & 0.17187 & C 2.87603 & 1.53414 & 1.02927 \\
\hline H - $-1.27743-4.13474$ & 2.41984 & F 4.26451 & $-3.25933-$ & -1.20647 \\
\hline Se -5.94994-1.61981 & 1.12757 & F 3.38912 & $-2.96016-$ & -3.16958 \\
\hline O -2.250472 .04311 & -1.58151 & F 5.08976 & $-1.76273-$ & -2.55346 \\
\hline C -2.507442 .29613 & -0.37418 & F 3.44452 & 0.93643 & 2.09663 \\
\hline C $-2.66608 \quad 1.35572$ & 0.65567 & F 3.75267 & 2.46759 & 0.58311 \\
\hline H - -2.578760 .31321 & 0.36364 & F 1.79795 & 2.21191 & 1.49176 \\
\hline C $-2.95032 \quad 1.66247$ & 2.01939 & C 4.84270 & -0.43449 & -0.01099 \\
\hline H -2.827962 .71323 & 2.27612 & C 5.78491 & $0.51220-$ & -0.42842 \\
\hline C -2.379030 .72620 & 3.07924 & C 5.22867 & -1.41344 & 0.91126 \\
\hline H - -2.737461 .00607 & 4.07562 & C 7.08800 & 0.47936 & 0.07015 \\
\hline H - $-2.72340-0.29924$ & 2.88743 & H 5.49905 & 1.28177 & -1.13602 \\
\hline $\mathrm{N}-4.770341 .52041$ & 2.27801 & C 6.53156 & -1.43931 & 1.41050 \\
\hline H -4.922510 .56482 & 1.91195 & H 4.50849 & -2.14930 & 1.24924 \\
\hline C $-5.31972 \quad 1.56466$ & 3.66376 & C 7.46819 & -0.49419 & 0.99333 \\
\hline H -4.773410 .84355 & 4.27355 & H 8.47521 & -0.50668 & 1.39335 \\
\hline H -6.363241 .22100 & 3.61901 & C 8.10498 & 1.47173 & -0.43119 \\
\hline C -5.511082 .44862 & 1.37881 & C 6.94056 & -2.52593 & 2.37069 \\
\hline H -5.04171 2.37803 & 0.39607 & F 8.74158 & $1.01442-$ & -1.53291 \\
\hline H -6.539402 .07393 & 1.28788 & F 9.05424 & 1.72651 & 0.49554 \\
\hline C -5.50116 3.88560 & 1.90480 & F 7.53392 & $2.64875-$ & -0.76623 \\
\hline H -6.120684 .49868 & 1.23943 & F 5.91008 & -2.91609 & 3.15288 \\
\hline H -4.482944 .28522 & 1.83720 & F 7.37833 & -3.62581 & 1.71843 \\
\hline C -5.258382 .97689 & 4.24685 & F 7.94150 & -2.12473 & 3.18429 \\
\hline H -5.685772 .95943 & 5.25633 & H -2.87925 & 5.50598 & -0.61267 \\
\hline H -4.210633 .28856 & 4.35238 & H -2.88252 & 4.31207 & -1.93057 \\
\hline C -6.014283 .96814 & 3.34971 & C -0.91963 & 4.87672 & -1.25774 \\
\hline
\end{tabular}


C $0.066594 .56511-0.31618$

C $-0.541025 .49715-2.45469$

C $1.408384 .86055-0.57045$

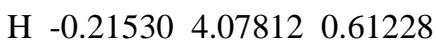

C $0.795875 .80697-2.70452$

H -1.29760 $5.73323-3.20056$

C $1.776155 .48662-1.76247$

H $2.16567 \quad 4.58983 \quad 0.15872$

H $1.073326 .28636-3.63980$
H $2.819765 .71682-1.95951$

C -0.843050 .757623 .09309$

H -0.431590 .388242 .15064$

H $-0.46497 \quad 1.77548 \quad 3.24165$

H -0.457040 .135123 .90850$ 
C-N Bond Formation Transition Structures

$\mathrm{R}-\mathrm{TS}_{\mathrm{C}-\mathrm{N}}$ (figure 3)

Filename: RTSscis-2.log

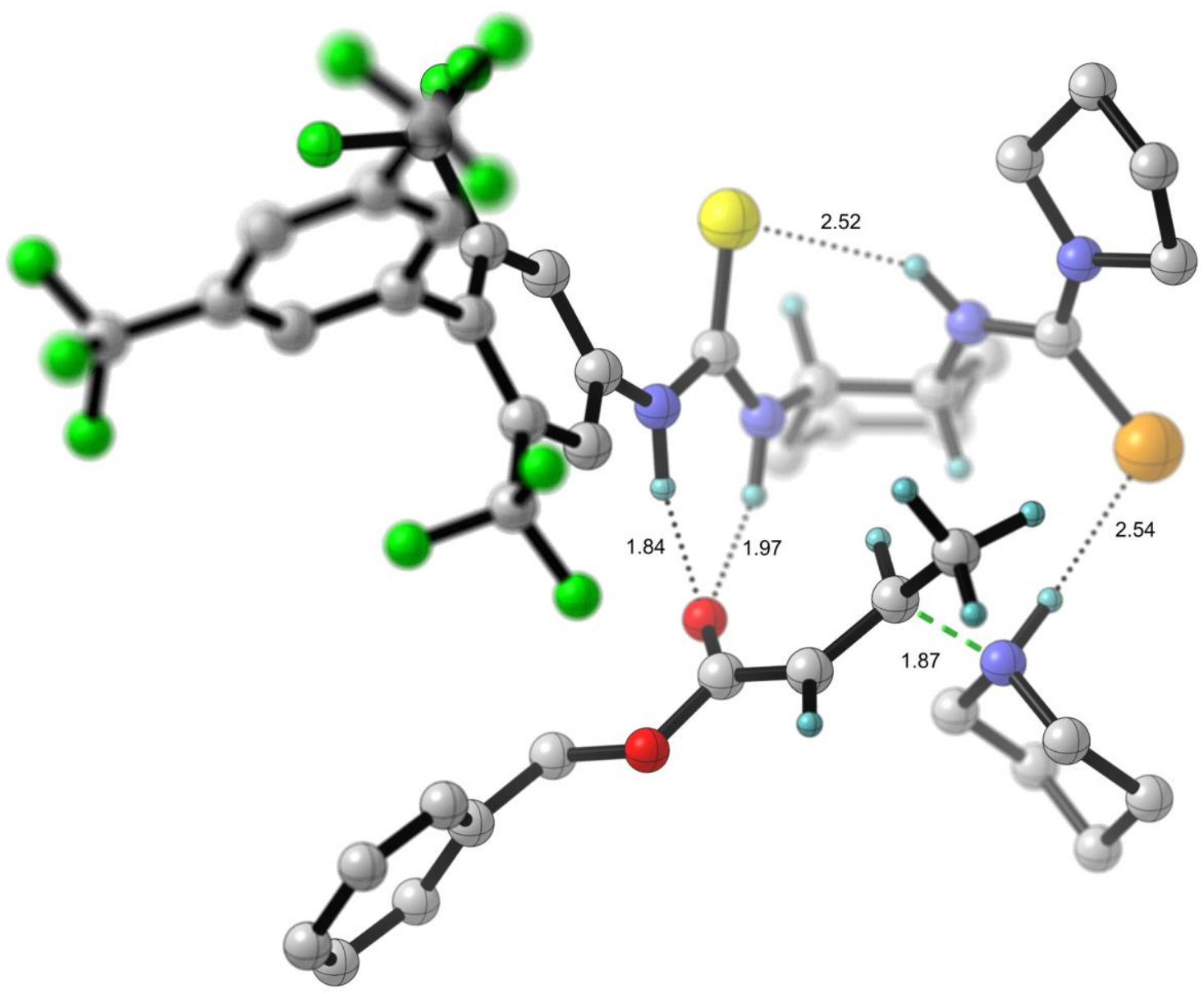

Free Energy $\quad=-6124.859755$

Zero-point Energy = -6124.747867

Potential Energy = -6129.59801403

Nimag $\quad=1(-170.3568 \mathrm{~cm}-1)$

Charge $=0$ Multiplicity $=1$

C $4.07639-0.614792 .78630$

C $6.97933-0.50228 \quad 3.43212$

C $5.14343-1.129661 .79248$

C $5.927550 .04545 \quad 4.40653$

C $6.39313-1.614662 .55161$

C $4.66345 \quad 0.505623 .66499$

H 3.894980 .834794 .37375 


\begin{tabular}{|c|c|c|}
\hline $\mathrm{H}$ & $5.65616-0.738695 .12757$ & H $3.88936-4.750710 .85160$ \\
\hline $\mathrm{H}$ & 6.337220 .878964 .98981 & C $3.59706-5.13896-2.45691$ \\
\hline $\mathrm{H}$ & $\begin{array}{lll}7.34639 & 0.31260 & 2.79123\end{array}$ & H $4.58264-3.21677-2.93144$ \\
\hline $\mathrm{H}$ & $7.84904-0.881193 .98210$ & H $4.21210-6.43506-0.82433$ \\
\hline $\mathrm{H}$ & $3.76751-1.44528 \quad 3.42937$ & H $3.91788-5.87459-3.19991$ \\
\hline $\mathrm{H}$ & $5.43675-0.31028 \quad 1.12636$ & Н $5.67590-4.44723-2.29790$ \\
\hline $\mathrm{H}$ & $6.11463-2.477373 .17400$ & H $2.66214-4.68824-2.81126$ \\
\hline $\mathrm{H}$ & $7.12993-1.96277 \quad 1.82051$ & H $2.49749-4.046900 .01192$ \\
\hline $\mathrm{H}$ & $4.90348 \quad 1.373353 .03206$ & H $\quad 2.45249-6.27363-0.94106$ \\
\hline $\mathrm{N}$ & $2.87105-0.135422 .11724$ & Se $6.29684-1.55615-1.20759$ \\
\hline $\mathrm{N}$ & $4.57205-2.188570 .95618$ & $\begin{array}{llll}O & 2.08567 & 2.37226 & 0.83169\end{array}$ \\
\hline $\mathrm{H}$ & $2.883610 .81963 \quad 1.74882$ & C $\quad 1.734772 .81222-0.30001$ \\
\hline $\mathrm{C}$ & $1.69013-0.799072 .02823$ & C $1.998252 .24569-1.56240$ \\
\hline $\mathrm{N}$ & $0.70033-0.00748 \quad 1.50723$ & H $1.565362 .72368-2.43480$ \\
\hline S & $1.50432-2.430562 .47826$ & C $2.785141 .07300-1.69876$ \\
\hline $\mathrm{H}$ & $\begin{array}{lll}1.01957 & 0.93289 & 1.23455\end{array}$ & $\begin{array}{llll}\mathrm{H} & 2.97801 & 0.55690 & -0.75835\end{array}$ \\
\hline $\mathrm{C}$ & $-0.63418-0.288231 .17660$ & $\begin{array}{lllll}O & 0.96437 & 3.94164 & -0.36940\end{array}$ \\
\hline $\mathrm{C}$ & $-1.43961-1.216381 .84525$ & C $\quad 0.576344 .547620 .87269$ \\
\hline $\mathrm{C}$ & $\begin{array}{lll}-1.20234 & 0.47509 & 0.14758\end{array}$ & C $-3.08817 \quad 1.19364-1.31193$ \\
\hline $\mathrm{C}$ & $-2.77292-1.38637 \quad 1.47177$ & C $-3.58519-2.416842 .22953$ \\
\hline $\mathrm{H}$ & $-1.02881-1.800952 .65353$ & F -4.03997 $2.04162-0.85648$ \\
\hline $\mathrm{C}$ & $-2.540580 .31846-0.20500$ & F -2.12449 $1.96218-1.87059$ \\
\hline $\mathrm{H}$ & $-0.58558 \quad 1.18324-0.39562$ & F $-3.640140 .47174-2.30909$ \\
\hline $\mathrm{C}$ & $-3.36266-0.626990 .44176$ & F -2.89514 -2.94765 3.25899 \\
\hline $\mathrm{H}$ & $3.74640-2.64495 \quad 1.34690$ & F -4.71760 -1.88914 2.74303 \\
\hline $\mathrm{C}$ & $4.97096-2.51544-0.29171$ & F -3.95234 -3.44721 1.43095 \\
\hline $\mathrm{N}$ & $4.38682-3.59015-0.85526$ & 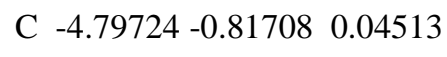 \\
\hline $\mathrm{C}$ & $3.48025-4.51212-0.13671$ & C $-5.16513-1.87514-0.79209$ \\
\hline $\mathrm{C}$ & $4.65497-4.05122-2.23130$ & $\begin{array}{llll}C & -5.78579 & 0.06138 & 0.50378\end{array}$ \\
\hline $\mathrm{C}$ & $3.39866-5.73770-1.05668$ & C $-6.49854-2.04762-1.16827$ \\
\hline
\end{tabular}




\begin{tabular}{|c|c|c|}
\hline H - $-4.41166-2.56907-1.14680$ & H 4.36336 & $3.28204-1.00437$ \\
\hline C $-7.11739-0.118180 .12943$ & H 4.87100 & 1.940290 .02181 \\
\hline H $-5.51496 \quad 0.88867 \quad 1.14913$ & C 6.74582 & $3.41515-2.52231$ \\
\hline C -7.48094 -1.17162-0.71009 & Н $\quad 6.36917$ & $2.84060-4.59418$ \\
\hline H -8.51551 -1.30700-1.00300 & Н 6.92443 & $1.52238-3.56453$ \\
\hline C $-6.86299-3.15252-2.12544$ & Н 6.74116 & $3.58367-0.34807$ \\
\hline $\begin{array}{llll}C & -8.18420 & 0.79461 & 0.67645\end{array}$ & Н 7.15073 & $1.97670-0.95000$ \\
\hline F $-8.15228-3.52976-1.98945$ & Н 7.80708 & $3.65339-2.65819$ \\
\hline F -6.09364 -4.24704 -1.94097 & Н 6.19069 & $4.35705-2.63829$ \\
\hline F $-6.69666-2.76799-3.41174$ & $\mathrm{~N} 4.58382$ & $1.47093-1.99233$ \\
\hline F -7.69124 2.010220 .99452 & C -0.50647 & 5.563170 .59971 \\
\hline F -9.18477 $0.97785-0.21290$ & C -0.43777 & 6.836831 .17365 \\
\hline F $-8.74370 \quad 0.28719 \quad 1.79798$ & C -1.61696 & $5.23370-0.19031$ \\
\hline C $2.47180 \quad 0.11775-2.83427$ & C -1.46186 & 7.765860 .97422 \\
\hline H $2.27591 \quad 0.64723-3.77240$ & H $\quad 0.42395$ & 7.105351 .78109 \\
\hline H $3.26663-0.61913-2.99752$ & C -2.63621 & $6.16236-0.39645$ \\
\hline H $\quad 1.55784-0.42928-2.57578$ & H -1.67691 & $4.25287-0.65233$ \\
\hline C $4.82537 \quad 1.99044-3.35915$ & C -2.56380 & 7.430470 .18736 \\
\hline C $5.035682 .41865-0.94689$ & H -1.39333 & 8.751361 .42749 \\
\hline H $5.064290 .56553-1.85819$ & H -3.49091 & $5.89445-1.01208$ \\
\hline C $\quad 6.282052 .41338-3.58751$ & H -3.36065 & 8.151830 .02663 \\
\hline H $4.148112 .84406-3.49007$ & H $\quad 0.22585$ & 3.768461 .55946 \\
\hline H $4.53434 \quad 1.21464-4.07111$ & H 1.44711 & 5.019081 .34371 \\
\hline C $6.497932 .84349-1.12030$ & & \\
\hline
\end{tabular}

File name: STSscis-eq.log 


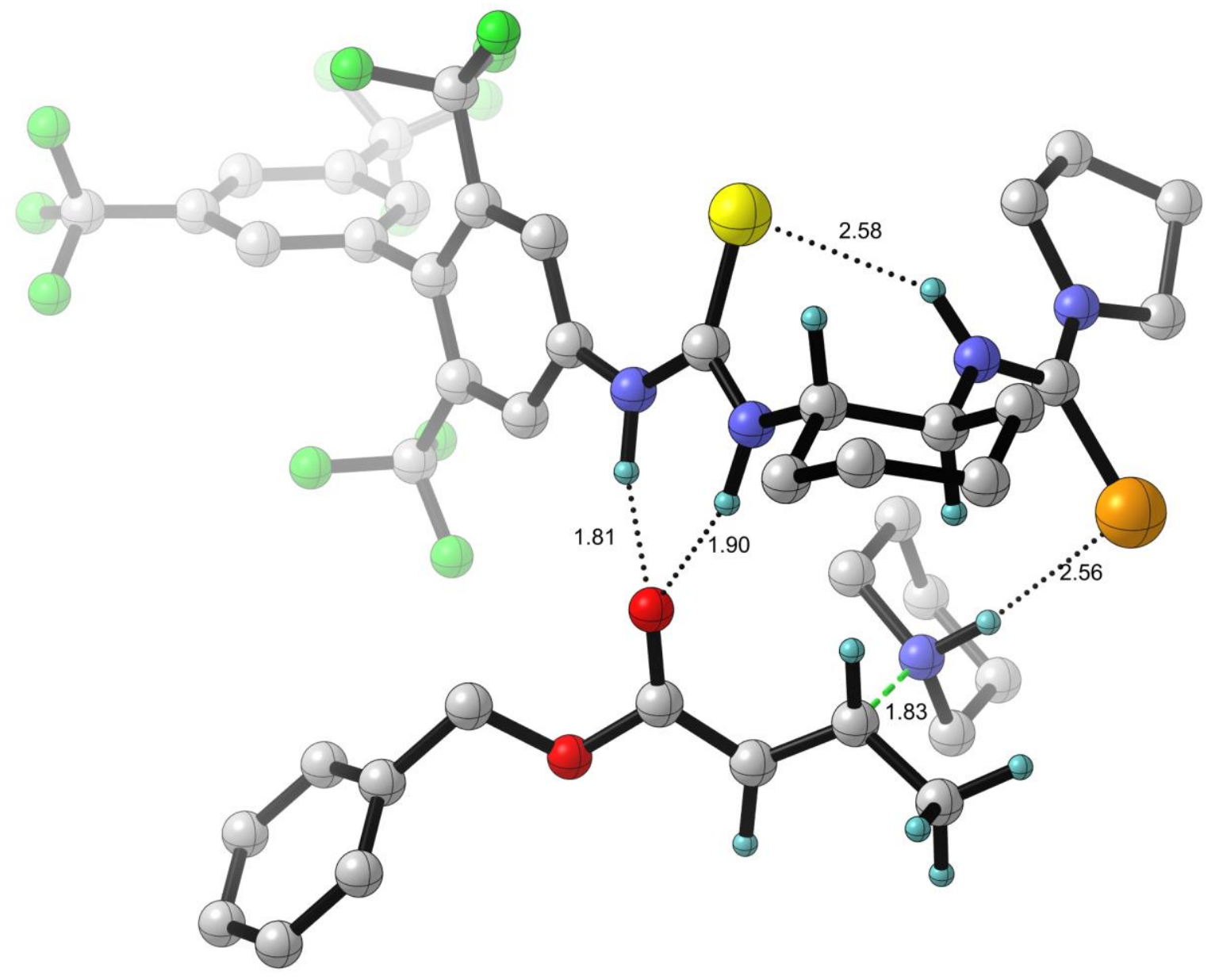

Free Energy $\quad=-6124.860505$

Zero-point Energy $=-6124.748101$

Potential Energy $\quad=-6129.59898426$

Nimag $=1(-169.3649 \mathrm{~cm}-1)$

Charge $=0$ Multiplicity $=1$

C $7.01388 \quad 0.241043 .45132$

C 5.907120 .801814 .35476

C $4.62557 \quad 1.088053 .55805$

C $4.13921-0.153522 .78626$

C $5.26169-0.68403 \quad 1.86373$

C $6.52806-0.995892 .68394$
H $3.82191 \quad 1.424164 .22329$

H 5.684160 .076805 .15052

H 6.242911 .719264 .85306

H 7.327881 .011962 .73284

H $7.90061-0.011894 .04482$

H $3.87607-0.938043 .50316$

H 5.508860 .080921 .11951 

H $\quad 6.30112-1.809603 .38807$
H $4.59281-6.05071-2.57540$
H $7.30250-1.359462 .00102$
H $\quad 2.90637-3.731690 .49881$
H 4.810131 .906062 .84598
H $2.62548-5.14165-1.41293$
N $2.91946 \quad 0.174202 .05340$
Se $6.51561-1.31079-1.04757$
N $4.79033-1.863871 .13141$
$\begin{array}{llll}\text { O } & 2.02162 & 2.46778 & 0.60695\end{array}$
H $2.87108 \quad 1.10157 \quad 1.61543$
C $2.232693 .33708-0.28791$
C $\quad 1.78483-0.568192 .02304$
C $3.323353 .40715-1.17019$
N $\quad 0.75616 \quad 0.099541 .40490$
H $3.382734 .26115-1.83619$
S $1.69664-2.148172 .65297$
C $4.384332 .46069-1.12028$
H 1.015111 .030721 .05052
H $4.36687 \quad 1.83123-0.22999$
C $-0.57485-0.24526 \quad 1.14244$
C $5.785262 .93840-1.45913$
C -1.246910 .576890 .22440$
H $6.148193 .54782-0.62398$
C -1.29164-1.28761 1.74313
H $\quad 6.491452 .11188-1.59788$
C -2.58875 $0.37656-0.08042$
H $5.794953 .57510-2.34986$
H $-0.706561 .38451-0.25493$
N $4.136701 .06708-2.27955$
C $-2.63258-1.496811 .41719$
H $4.887040 .39718-2.04114$
H $-0.80632-1.928752 .46199$
C $2.825430 .43587-1.99045$
C $-3.32226-0.676260 .50394$
H $2.850340 .09496-0.95274$
H $4.03394-2.374251 .58601$
C $4.251491 .50005-3.69343$
C $5.24737-2.28588-0.06674$
H $5.26277 \quad 1.88473-3.84566$
N $4.75411-3.45017-0.53470$
O $\quad 1.342154 .36442-0.44760$
C $3.79531-4.299350 .20590$
C $\quad 0.261814 .447790 .49756$
C $5.20553-4.10007-1.78142$
C -3.34207 -2.65306 2.09344
C $3.46941-5.43219-0.77615$
C $-3.244161 .33041-1.05518$
H $4.26907-4.680221 .12251$
F -3.71328 -3.59857 1.19758
C $4.75087-5.55504-1.61333$
F -2.56074 -3.26430 3.00592
H $4.72748-3.62258-2.64679$
F $-4.46086-2.252802 .73558$
H $3.19433-6.35527-0.25839$
F $-3.798560 .68879-2.10408$
H $5.51059-6.12286-1.06279$
F - $-4.220142 .05784-0.46661$
H $\quad 6.28405$-3.98137 -1.89499
F -2.35632 $2.21932-1.56430$ 

C $-4.76526-0.90830 \quad 0.16451$
C -1.78048 $5.65752-0.36762$
$\begin{array}{llll}\text { C } & -5.77342 & -0.17990 & 0.80347\end{array}$
C $-0.67482 \quad 8.13350 \quad 0.28671$
C -5.12181-1.85772 -0.80007
H 1.023617 .010790 .99215
C $-7.11503-0.401670 .48588$
C -2.49612 $6.83030-0.62061$
H $-5.512730 .56382 \quad 1.54815$
H -2.20921 $4.69355-0.63075$
C -6.46279-2.07078 - 1.11926
C -1.94486 $8.06982-0.29404$
H -4.35210 -2.43560 - 1.29848
H -0.241019 .096460 .54344$
C $-7.46704-1.34608-0.47657$
H $-3.482596 .77394-1.07318$
H -8.50865 - $1.51560-0.72239$
H $-2.50113 \quad 8.98289-0.48934$
C $-8.18493 \quad 0.34903 \quad 1.23597$
C $3.945070 .37342-4.68771$
C -6.82857 -3.04358 -2.20994
H $3.99095 \quad 0.78263-5.70444$
F -8.45389 -0.23191 2.42660
H $4.72898-0.39309-4.61724$
F -9.344490 .396100 .54589$
C $2.49447-0.71348-2.94799$
F -7.81052 $1.62051 \quad 1.49554$
H $3.19611-1.54100-2.77664$
F -6.90365 -2.43043 -3.41337
H $1.49197-1.08886-2.70835$
F -5.91677 -4.03257-2.32770
C $2.57530-0.25891-4.41076$
F -8.02987-3.61820 -1.98562
H $2.40122-1.10420-5.08669$
H $\quad 0.671324 .441461 .51394$
H $1.782960 .47625-4.61126$
H $-0.39067 \quad 3.57370 \quad 0.40063$
H $3.548132 .33327-3.81530$
C $-0.51113 \quad 5.71183 \quad 0.21856$
H $\quad 2.08148 \quad 1.23683-2.06289$
C $0.03492 \quad 6.961280 .54210$

RTSscis-4.log

Description: C-C bond formation, $\mathrm{R}$ enantiomer, s-cis 


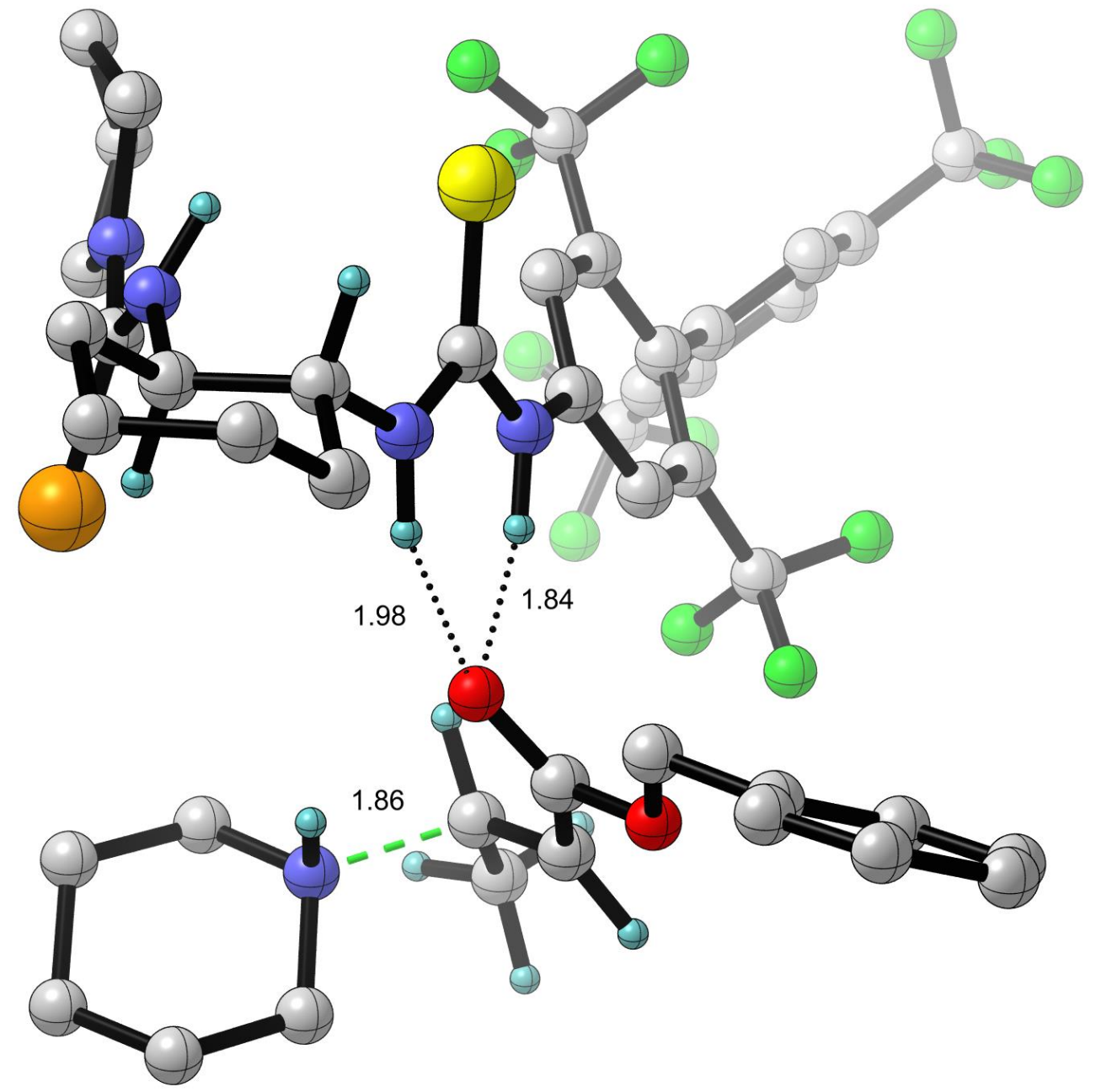

Free Energy $\quad=-6124.854500$

Zero-point Energy $=-6124.741688$

Potential Energy $\quad=-6129.58930488$

Nimag $\quad=1(-188.1427 \mathrm{~cm}-1)$

Charge $=0$ Multiplicity $=1$

C $-7.09090-2.59470-1.77302$

C $-6.87910-1.32366-2.60878$

C $-5.67129-0.51700-2.10878$

C $-4.39538-1.37517-2.07606$

C $-4.61767-2.64283-1.20524$
C $-5.81781-3.45236-1.72678$

H $-5.496470 .35626-2.74836$

H -6.71655 - $1.60089-3.66020$

H -7.77862 -0.69636-2.58714

H - $7.37343-2.31382-0.74813$

H -7.92418 -3.18199 -2.17741 


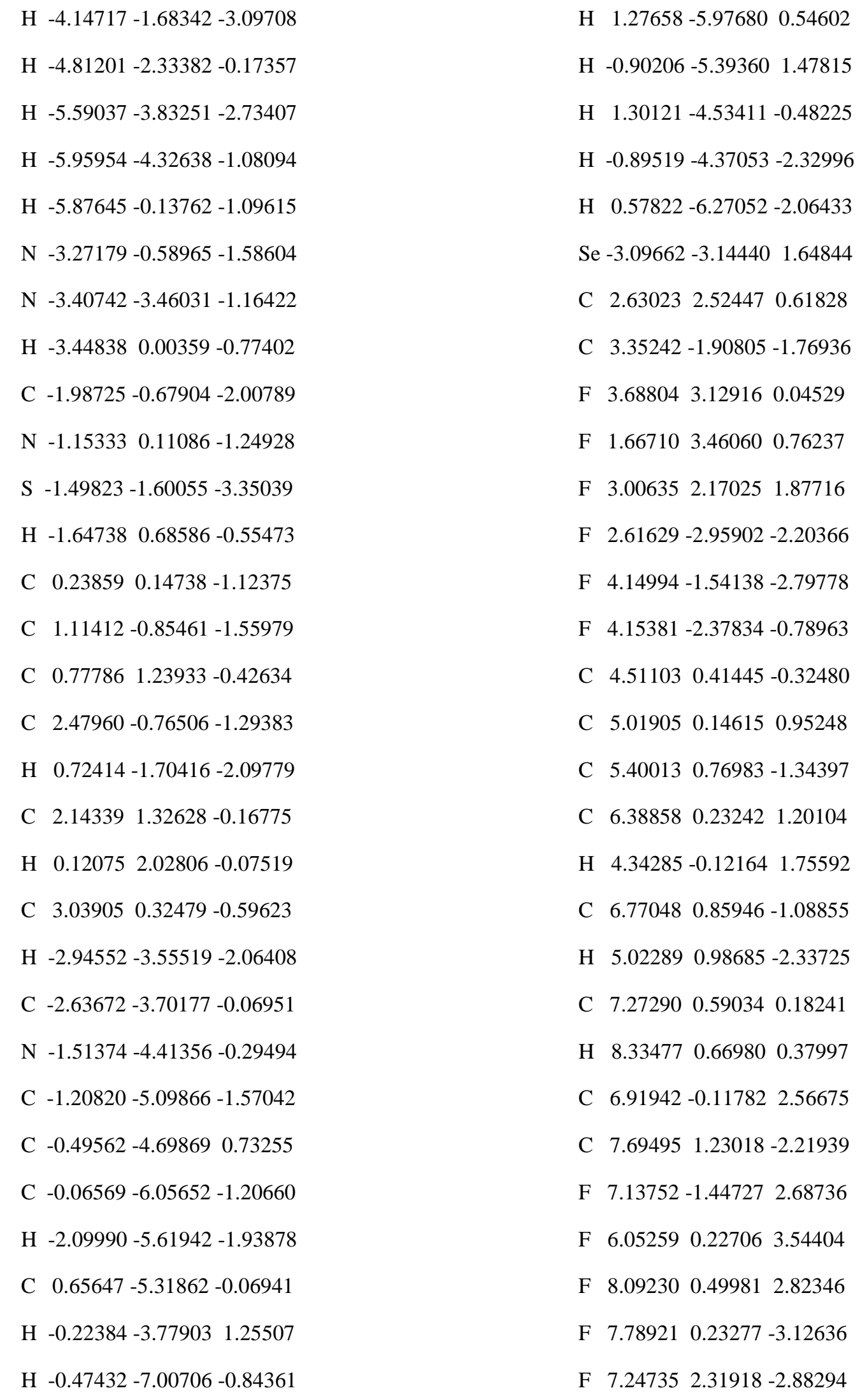



F $8.944591 .50059-1.78731$
C $-2.12335-0.316024 .27001$
C -1.90754 2.951841 .99646
C -3.333141 .814464 .57738$
H -1.863303 .985932 .31818$
H $\quad-3.118090 .753622 .82253$
C -2.718972 .602790 .90564$
C $-3.31984-1.10050 \quad 4.82601$
$\begin{array}{llll}\text { O } & -2.94903 & 1.41675 & 0.52435\end{array}$
H -1.41906 -0.06823 5.07249
C $-1.25512 \quad 1.927292 .73782$
H $-1.59743-0.898303 .50713$
H $-0.96095 \quad 1.048252 .16241$
C -4.562321 .079985 .12298$
$\begin{array}{llll}\text { O } & -3.29349 & 3.65848 & 0.25886\end{array}$
H -2.661912 .094755 .39845$
C $-4.13423 \quad 3.35756-0.86181$
H $-3.599662 .72328 \quad 4.02924$
H $-5.010582 .78762-0.52988$
C $-4.15659-0.244615 .78844$
H $-3.584202 .71894-1.56475$
H -2.94925 -2.00456 5.32309
C $-4.552964 .64943-1.52244$
H -3.94054-1.43819 3.98552
C -3.66513 $5.72697-1.63477$
H $-5.08424 \quad 1.733465 .83249$
C $-5.829814 .76220-2.08366$
H $\quad-5.26247 \quad 0.883864 .29859$
C $-4.049596 .89243-2.29780$
H $-5.04621-0.797606 .11063$
H -2.67853 $5.65113-1.18847$
H -3.57218 -0.03055 6.69530
C $-6.212965 .92469-2.75556$
N -2.55156 0.964363 .65137
H $-6.531493 .93538-1.99372$
C -0.220912 .337963 .76265$
C $-5.322966 .99420-2.86367$
H $\quad 0.68978 \quad 2.637913 .23284$
H $-3.352697 .72307-2.37381$
H -0.556363 .194414 .35688$
H $-7.208515 .99669-3.18562$
H $\quad 0.05035 \quad 1.521924 .43963$

H -5.62014 7.90239 -3.38122

RTSscis-ax.log

Description: C-C bond formation, R enantiomer, s-cis, axial conformation 


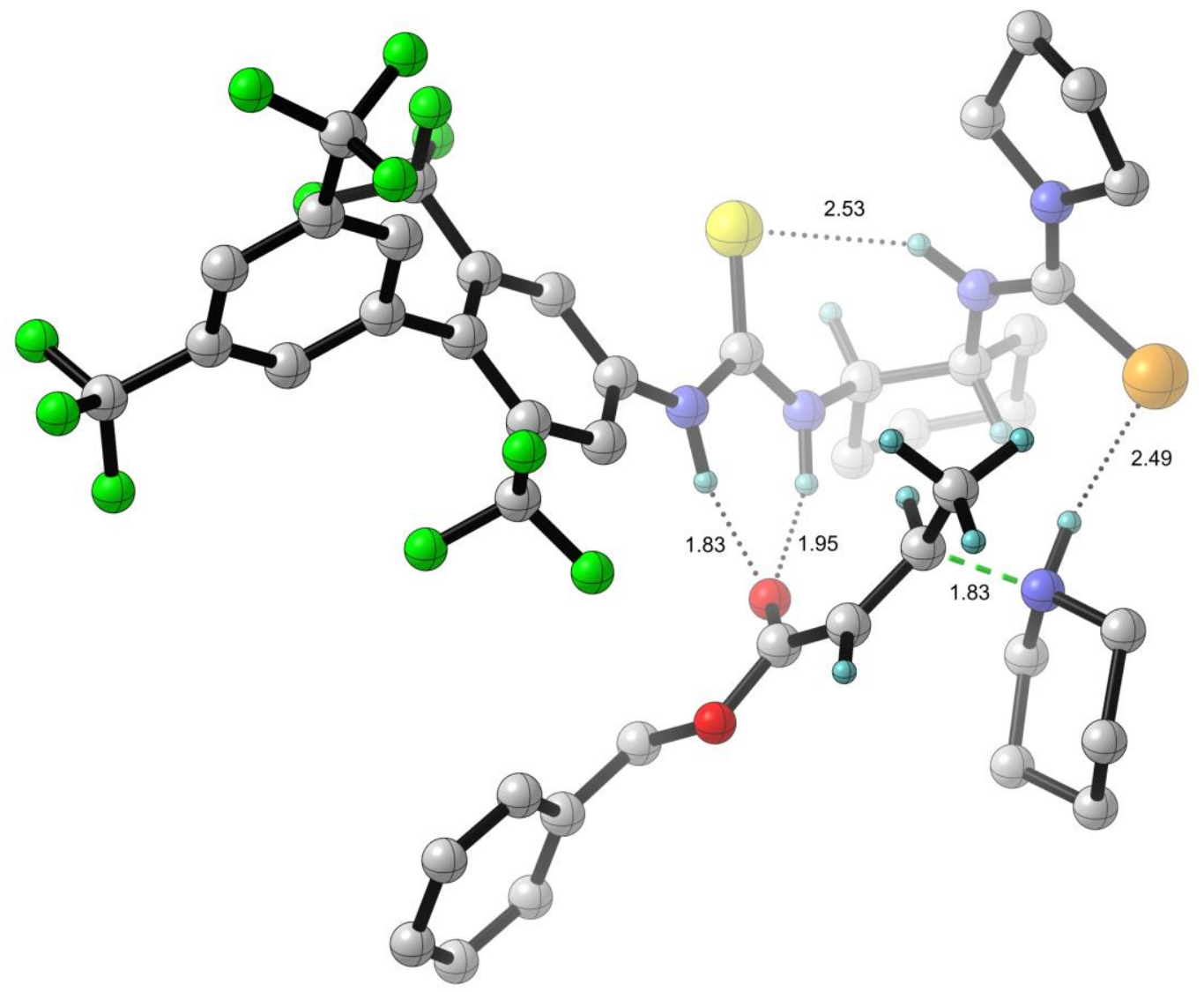

Free Energy $\quad=-6124.852112$

Zero-point Energy = -6124.740672

Potential Energy $\quad=-6129.59188062$

Nimag $=1(-193.7995 \mathrm{~cm}-1)$

Charge $=0$ Multiplicity $=1$

C $7.07116-0.535113 .39763$

C $\quad 6.02438-0.00453 \quad 4.38701$

C $4.74701 \quad 0.446173 .66255$

C $4.16149-0.675912 .78511$

C $5.22276-1.176731 .77870$

C $6.48778-1.650392 .51873$

H $3.98344 \quad 0.76397 \quad 4.38170$

H $5.77003-0.795555 .10680$
H 6.431850 .830124 .97018

H $7.42058 \quad 0.287152 .75638$

H $7.95183-0.906103 .93546$

H $3.86398-1.511013 .42763$

H $5.49737-0.352331 .11123$

H $6.22887-2.519943 .14000$

H $7.21942-1.985611 .77622$

H 4.970541 .318943 .03073

N $2.94962-0.203612 .12463$ 

N $4.65158-2.24055 \quad 0.94827$
$\begin{array}{llll}\text { O } & 2.17081 & 2.28949 & 0.84771\end{array}$
H 2.959620 .748311 .74822
C $1.787672 .77586-0.25829$
C $1.77338-0.874352 .03323$
C $2.021712 .27678-1.54743$
N $0.78284-0.08732 \quad 1.50638$
H $1.581802 .80744-2.38542$
S $\quad 1.59473-2.505712 .48829$
C $2.824951 .11994-1.76769$
H $\quad \begin{array}{llll}1.10245 & 0.85481 & 1.23834\end{array}$
H $3.01103 \quad 0.55884-0.85071$
C $-0.55177-0.36268 \quad 1.17521$
O $1.016553 .91069-0.25416$
C -1.35444 -1.31168 1.81776
C 0.647154 .449161 .02316
$\begin{array}{llll}\text { C } & -1.12455 & 0.43030 & 0.17068\end{array}$
C $-3.015221 .19326-1.25835$
C $-2.68948-1.469841 .44469$
C $-3.49850-2.522862 .17438$
H -0.94004-1.92074 2.60589
F -3.95836 2.03351 -0.77103
C $-2.46388 \quad 0.28414-0.18102$
F -2.05204 $1.97207-1.80298$
H $-0.51017 \quad 1.15458-0.35410$
F -3.57986 $0.50314-2.27088$
C $-3.28397-0.679690 .44093$
F -2.80615 -3.08103 3.18770
H $3.84185-2.712181 .35403$
F -4.63150 -2.01249 2.70377
C $5.01408-2.54234-0.31618$
F -3.86438 -3.53141 1.34752
N $4.44181-3.62752-0.87068$
C $-4.72093-0.85572 \quad 0.04657$
C $3.59622-4.58749-0.12743$
C -5.09706 -1.89191-0.81402
C $4.66789-4.06392-2.26245$
C $-5.70413 \quad 0.01492 \quad 0.53121$
C $3.52820-5.80871-1.05380$
C $-6.43306-2.05058-1.18732$
H $4.04965-4.816050 .84369$
H $-4.34809-2.57989-1.18927$
C $3.64550-5.19168-2.45511$
C -7.03817-0.150520.15912
H 4.53148 -3.22625 -2.94923
H -5.427110 .825401 .19498$
H $4.37838-6.47326-0.86013$
C -7.40989-1.18217-0.70356
H $3.96488-5.90799-3.21733$
H -8.44634 -1.30671 -0.99453
H $5.69993-4.41745-2.37744$
C $-6.80573-3.13244-2.16734$
H $2.67967-4.77626-2.76710$
C -8.098370 .753500 .73286$
H $2.60259-4.160420 .05862$
F -8.09870 -3.50044 -2.04174
H $2.61007-6.38395-0.90567$
F -6.04693 -4.23755 -2.00285
Se $6.28125-1.53714-1.26731$
F -6.63256 - $2.72434-3.44550$ 


\begin{tabular}{|c|c|c|c|}
\hline F - -7.59950 & $1.96097 \quad 1.07205$ & H -1.62864 & $4.22872-0.47859$ \\
\hline F -9.10510 & $0.95782-0.14489$ & C -2.50227 & 7.361290 .52824 \\
\hline F -8.65086 & 0.224791 .84805 & $\mathrm{H}-1.31288$ & 8.620721 .81343 \\
\hline C 2.45922 & $0.21227-2.92781$ & H -3.44794 & $5.88507-0.73040$ \\
\hline H 2.29032 & $0.77615-3.85160$ & H -3.30116 & 8.089370 .41477 \\
\hline H 3.20988 & $-0.56438-3.11328$ & H 0.30462 & 3.635821 .67361 \\
\hline H 1.51676 & $-0.28757-2.67839$ & Н 1.52379 & 4.895961 .50727 \\
\hline C 5.06343 & $1.72733-3.44006$ & C 4.95422 & $3.90048-1.48830$ \\
\hline C 5.14407 & $2.43970-1.06360$ & Н 3.89561 & $4.16488-1.41728$ \\
\hline H 4.99632 & $0.53135-1.78724$ & H 5.49505 & $4.52994-0.77106$ \\
\hline H 4.58793 & $1.00048-4.10094$ & C 4.78559 & $3.16403-3.88007$ \\
\hline H 4.64348 & $2.25014-0.11113$ & H 3.70377 & $3.34127-3.91302$ \\
\hline N 4.60226 & $1.45275-2.04863$ & Н 5.16518 & $3.29203-4.90089$ \\
\hline C -0.43963 & 5.476830 .81845 & C 5.44935 & $4.15566-2.91685$ \\
\hline C -0.36194 & 6.721001 .45264 & H 5.23080 & $5.18751-3.21466$ \\
\hline C -1.56199 & 5.185870 .02996 & H 6.54165 & $4.03421-2.96630$ \\
\hline C -1.38855 & $7.65837 \quad 1.31387$ & H 6.21293 & $2.21705-0.94059$ \\
\hline H $\quad 0.50887$ & 6.959782 .05955 & Н 6.14569 & $1.53321-3.47184$ \\
\hline-2.58397 & $6.12298-0.11536$ & & \\
\hline
\end{tabular}

STSstrans-eq.log

Description: C-C bond formation, $\mathrm{S}$ enantiomer, s-trans, equatorial conformation 


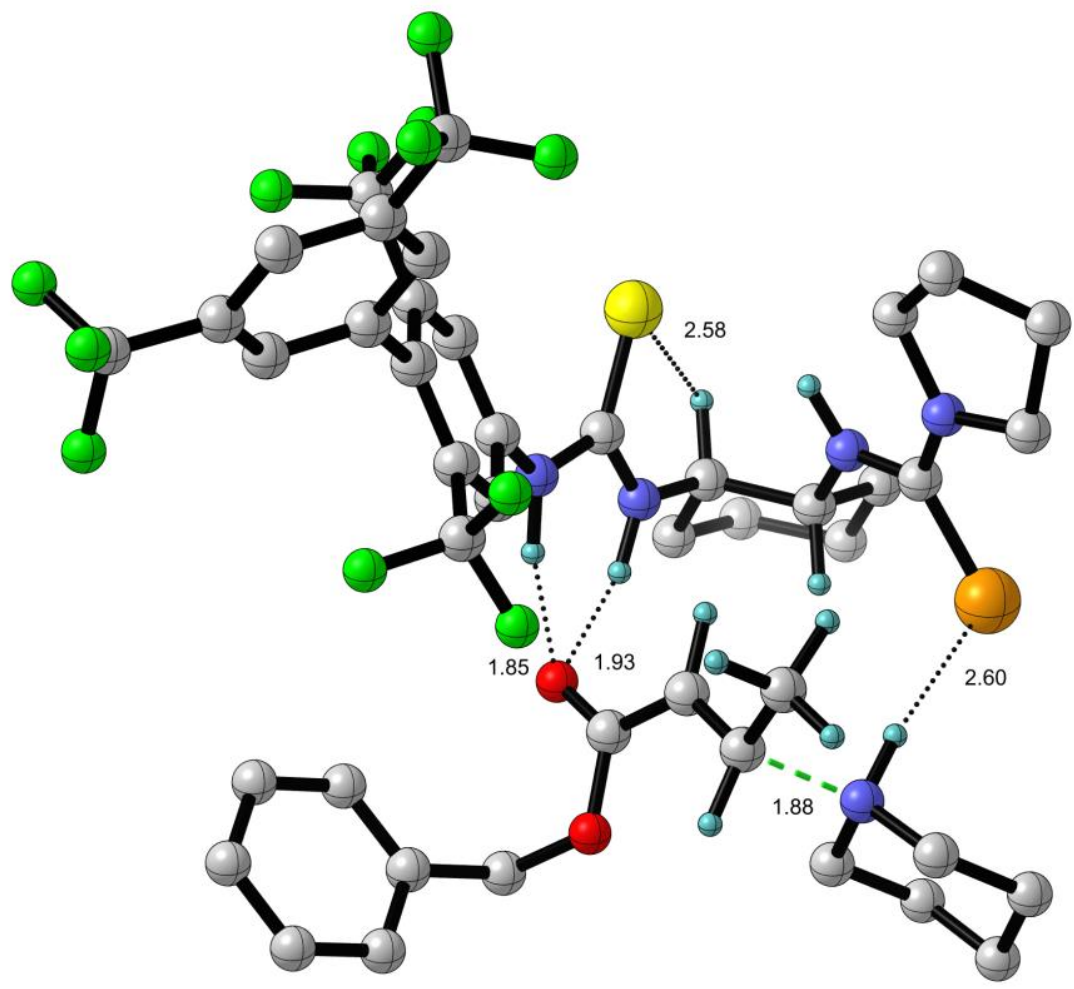

Free Energy $\quad=-6124.856750$

Zero-point Energy $=-6124.746196$

Potential Energy $\quad=-6129.59618904$

Nimag $=1(-187.9547 \mathrm{~cm}-1)$

Charge $=0$ Multiplicity $=1$

H $7.70283-2.128473 .64528$

C $6.83446-1.559543 .29176$

H $3.53098-2.096493 .58103$

C $5.99287-1.073224 .47929$

H $5.07603-0.77981 \quad 1.28224$

C $4.72646-0.344984 .00496$

H $5.67571-3.341462 .84516$

C $3.88742-1.210233 .04608$

H $6.58882-2.719941 .46378$

C $4.75109-1.663401 .84310$

H $5.00787 \quad 0.586913 .49185$

C $5.99854-2.421642 .33611$

N $2.70601-0.467812 .62672$

H $4.09941-0.059124 .85729$

N $\quad 3.94850-2.481790 .92743$

H $5.70408-1.934755 .09825$

H $2.83942 \quad 0.510202 .34515$

H $6.58096-0.408585 .12391$

C $\quad 1.44894-0.95338 \quad 2.51487$

H $7.23067-0.692582 .74320$

$\begin{array}{llll}\mathrm{N} & 0.55783 & 0.01647 & 2.12127\end{array}$ 

S $\quad 1.03540-2.580352 .80514$
C $2.55797 \quad 1.70763-2.01562$
H 1.001260 .916661 .88769
H $\quad 2.50537 \quad 2.75898-2.29757$
C $-0.75347-0.139791 .63892$
C $1.798930 .78261-2.94895$
C -1.111530 .591560 .50126$
H $\quad 0.72776 \quad 0.93700-2.77823$
C -1.71809-0.93966 2.25877
H $\quad 1.991150 .97759-4.00722$
C -2.39902 $0.50885-0.02522$
H $2.02178-0.26918-2.73515$
H $\quad-0.36409 \quad 1.20368 \quad 0.00809$
N $4.34684 \quad 1.46325-2.54625$
C $-3.00603-1.02212 \quad 1.72823$
H $\quad 4.529020 .49719-2.22870$
H -1.46481 -1.49144 3.15131
C $4.630791 .56734-4.00036$
C $-3.38247-0.30340 \quad 0.57481$
H $4.02236 \quad 0.82475-4.52020$
H $3.14477-2.940011 .35534$
C $5.204462 .37614-1.74754$
C $4.15716-2.62247-0.39835$
H $4.956752 .20534-0.69905$
N $3.33640-3.45983-1.06430$
O $2.77120 \quad 3.70237-0.03754$
C $2.25990-4.24693-0.42384$
C 2.680174 .765570 .93840
C $3.48314-3.80159-2.49252$
C -4.00275-1.91504 2.43723
C $1.54970-4.92682-1.60136$
C $-2.69828 \quad 1.29234-1.28470$
H $2.69856-4.98415 \quad 0.26481$
F -4.34891 -2.97873 1.67248
C $2.66819-5.09240-2.64042$
F -3.50965 -2.40784 3.59085
H $3.07884-2.99277-3.11526$
F $-5.14378-1.259512 .74327$
H $1.08177-5.87037-1.30712$
F -3.20003 $0.50702-2.26267$
H $3.29207-5.95916-2.39065$
F -3.59418 2.28185 -1.06915
H $4.53933-3.91268-2.74357$
F - $1.586751 .87910-1.78892$
H $\quad 2.29542-5.22951-3.65957$
C $-4.77040-0.391830 .01206$
H $\quad 1.58934-3.605340 .15609$
C -5.698220 .627250 .25309$
H $\quad 0.76300-4.26995-1.99091$
C $-5.15376-1.48620-0.77059$
Se $5.53568-1.71427-1.28981$
$\begin{array}{llll}\text { C } & -6.98568 & 0.55140 & -0.28017\end{array}$
$\begin{array}{llll}\text { O } & 2.29945 & 2.21275 & 1.61763\end{array}$
H $-5.41256 \quad 1.486180 .84944$
C 2.492872 .428020 .38871
C $-6.44083-1.55538-1.30588$
C $2.433641 .45359-0.62525$
H -4.44283 - 2.27906 -0.97230
H $2.266920 .43247-0.29582$
C -7.36365 -0.53841 -1.06357 


\begin{tabular}{|c|c|c|c|}
\hline H - $-8.35770-0.58692-1.49185$ & H $\quad 0.61045$ & 4.04554 & 2.55623 \\
\hline C $-7.98652 \quad 1.63221 \quad 0.03670$ & C -1.28802 & 6.46583 & 1.09113 \\
\hline C $-6.84795-2.76125-2.11212$ & $\mathrm{H}-0.60550$ & 7.78559 & -0.47328 \\
\hline F $-8.63199 \quad 1.38391 \quad 1.19859$ & H -1.67299 & 5.02535 & 2.64953 \\
\hline F $-8.929801 .73990-0.92390$ & H -2.28645 & 6.89350 & 1.12803 \\
\hline F -7.39444 2.839240 .16617 & C 6.11680 & 1.35876 & -4.32871 \\
\hline$F-5.80997-3.26513-2.81533$ & H 6.38345 & 0.31511 & -4.11354 \\
\hline F -7.30647 -3.75629 -1.32052 & Н 6.26199 & 1.50929 & -5.40568 \\
\hline F -7.83212 -2.46872 -2.98995 & C 6.69675 & 2.16278 & -2.01797 \\
\hline Н 3.404665 .509140 .59462 & Н 7.26836 & 2.89029 & -1.42861 \\
\hline H $2.98794 \quad 4.37949 \quad 1.91150$ & Н 6.98648 & 1.16494 & -1.66242 \\
\hline C 1.294015 .363801 .00135 & C 7.01161 & 2.30071 & -3.51355 \\
\hline $\begin{array}{llll}\text { C } & 0.93790 & 6.41731 & 0.14981\end{array}$ & H 8.06832 & 2.08333 & -3.70695 \\
\hline C $\quad 0.339594 .86744 \quad 1.90016$ & Н 6.83763 & 3.33941 & -3.83088 \\
\hline C $-0.344356 .96641 \quad 0.19161$ & H 4.90484 & 3.40045 & -1.99921 \\
\hline H $1.67286 \quad 6.81182-0.54887$ & H 4.29981 & 2.56372 & -4.32142 \\
\hline C $\quad-0.94288 \quad 5.41585 \quad 1.94532$ & & & \\
\hline
\end{tabular}

strans-D3genamineadds

Description: C-C bond formation, S enantiomer, s-trans 


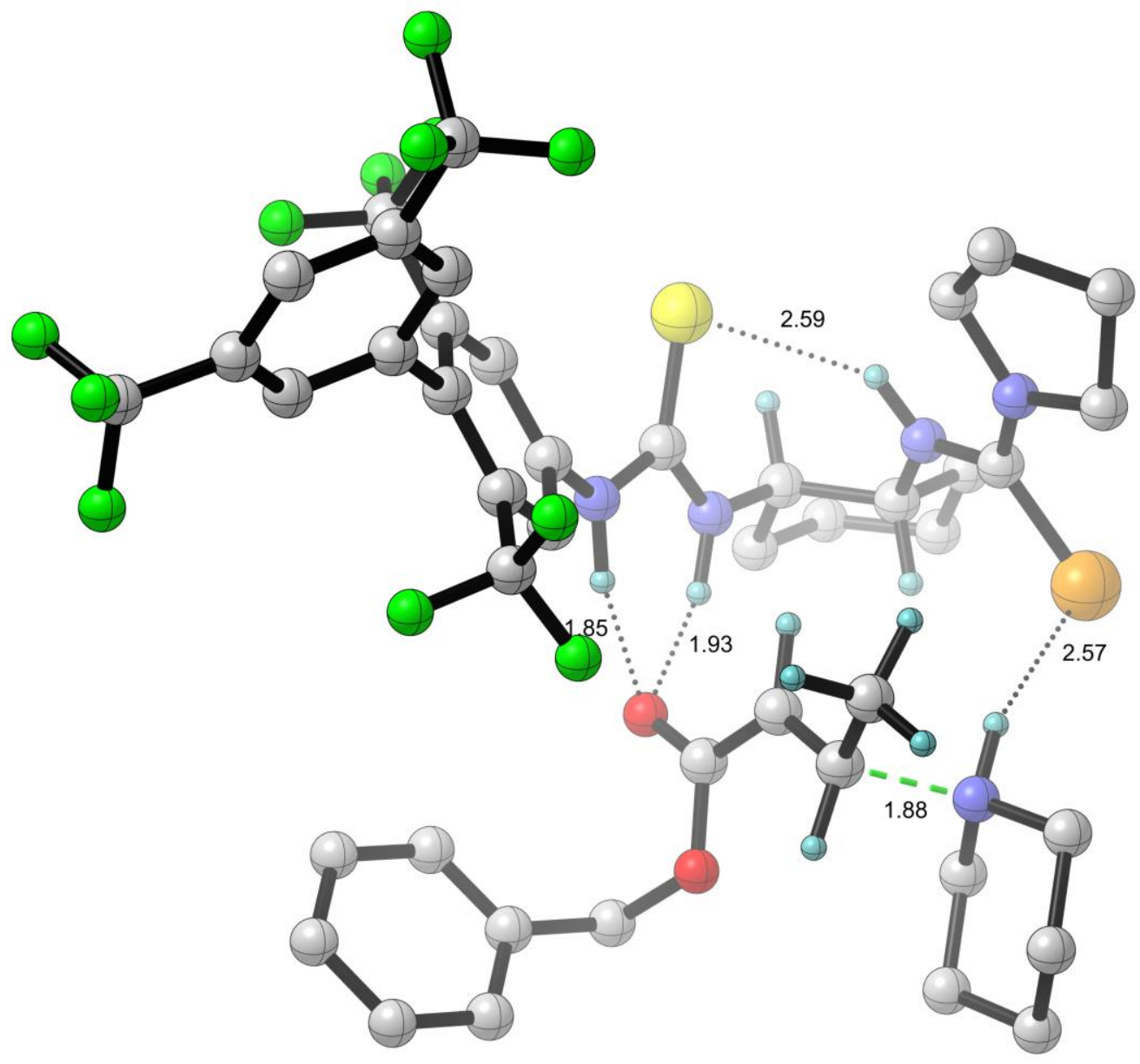

Free Energy $\quad=-6124.855605$

Zero-point Energy $=-6124.744491$

Potential Energy $\quad=-6129.59543834$

Nimag $=1(-216.8129 \mathrm{~cm}-1)$

Charge $=0$ Multiplicity $=1$

C -6.85857-2.13476-3.15015

C $-6.02605-1.73926-4.37760$

C $-4.79674-0.91026-3.97709$

C $-3.93182-1.64014-2.93243$

C $-4.78580-1.99801-1.69093$
C -5.99686-2.85512-2.10394

H $-4.17575-0.68743-4.85235$

H -5.69345 -2.64758 -4.90032

H -6.63635 - $1.17339-5.09209$

H -7.29719-1.23316 -2.69832

H $-7.69817-2.77459-3.44765$ 


\begin{tabular}{|c|c|c|}
\hline H $-3.54456-2.56442-3.37289$ & H $-3.10658-5.68888$ & 3.03386 \\
\hline H - $-5.14952-1.07485-1.22606$ & H $-4.47479-3.69208$ & 3.12882 \\
\hline H -5.63265 -3.80967 -2.51103 & H $-2.15344-4.74418$ & 4.18879 \\
\hline H $-6.58233-3.08299-1.20750$ & H $-1.55031-3.58791$ & 0.18851 \\
\hline H -5.12206 $0.05616-3.56353$ & H $-0.68351-3.91915$ & 2.39679 \\
\hline $\mathrm{N}-2.77453-0.82495-2.58768$ & Se $-5.57742-1.72687$ & 1.42459 \\
\hline$N-3.95615-2.68010-0.69154$ & $\begin{array}{lll}\mathrm{O} & -2.44030 & 1.93053\end{array}$ & -1.76869 \\
\hline H - $-2.938890 .16612-2.37825$ & C $-2.62528 \quad 2.25181$ & -0.56224 \\
\hline C $-1.50445-1.26490-2.43546$ & C -2.498741 .38844 & 0.54080 \\
\hline N $-0.63873-0.24186-2.12886$ & H -2.287600 .34836 & 0.31076 \\
\hline S - $-1.04973-2.90060-2.58119$ & C -2.608151 .77979 & 1.90224 \\
\hline H $-1.106320 .66169-1.96478$ & H -2.607482 .85461 & 2.06416 \\
\hline C $\quad 0.68141-0.32141-1.65329$ & C -1.770801 .01543 & 2.91054 \\
\hline C $\quad 1.03923 \quad 0.52771-0.60034$ & H $-0.71573 \quad 1.20489$ & 2.68492 \\
\hline C $1.65581-1.15976-2.20376$ & H $-1.94535 \quad 1.32825$ & 3.94375 \\
\hline C $2.332640 .51755-0.08168$ & H $-1.93661-0.06575$ & 2.83442 \\
\hline $\begin{array}{lllll}\text { H } & 0.28803 & 1.17652 & -0.16327\end{array}$ & $\mathrm{~N}-4.36278 \quad 1.44411$ & 2.48134 \\
\hline C $2.94886-1.17037-1.67990$ & H -4.467540 .46956 & 2.15481 \\
\hline H $\quad 1.40560-1.79930-3.03650$ & C $-4.67257 \quad 1.47116$ & 3.93746 \\
\hline C $3.32294-0.33874-0.60432$ & Н -3.947180 .83937 & 4.45378 \\
\hline H $-3.13825-3.15786-1.06794$ & H -5.660501 .00901 & 4.08097 \\
\hline C $-4.15718-2.670010 .64253$ & C -5.336412 .25815 & 1.70332 \\
\hline $\begin{array}{llll}\mathrm{N} & -3.29827 & -3.38307 & 1.39911\end{array}$ & H -5.027182 .21021 & 0.65792 \\
\hline C $-2.18147-4.18508 \quad 0.85387$ & H $-6.31999 \quad 1.77527$ & 1.78496 \\
\hline C $-3.42670-3.550952 .85922$ & C -5.401053 .70309 & 2.20282 \\
\hline C $-1.43051-4.665602 .10198$ & H -6.184104 .22634 & 1.64116 \\
\hline H $\quad-2.57967-5.028770 .27088$ & H -4.457894 .20944 & 1.96601 \\
\hline C $-2.53545-4.761703 .16400$ & C -4.67966 2.89957 & 4.48395 \\
\hline H - $-3.07349-2.646593 .37240$ & H -4.93216 2.86743 & 5.55039 \\
\hline H $-0.90786-5.610091 .92694$ & H -3.671793 .33010 & 4.41037 \\
\hline
\end{tabular}




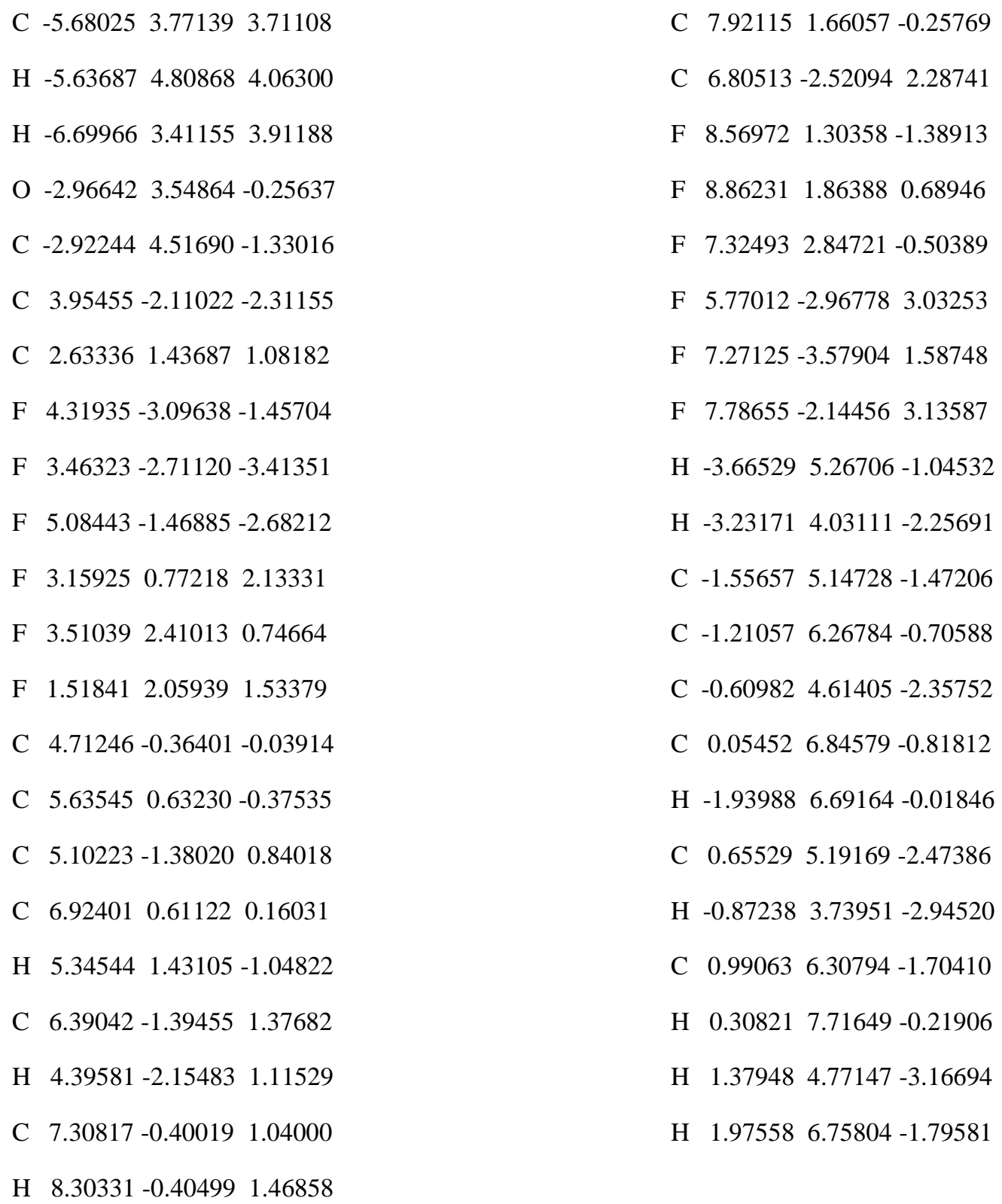

strans-D3genbattackrbenbenturn

Description: $\mathrm{C}-\mathrm{C}$ bond formation, $\mathrm{R}$ enantiomer, s-trans 


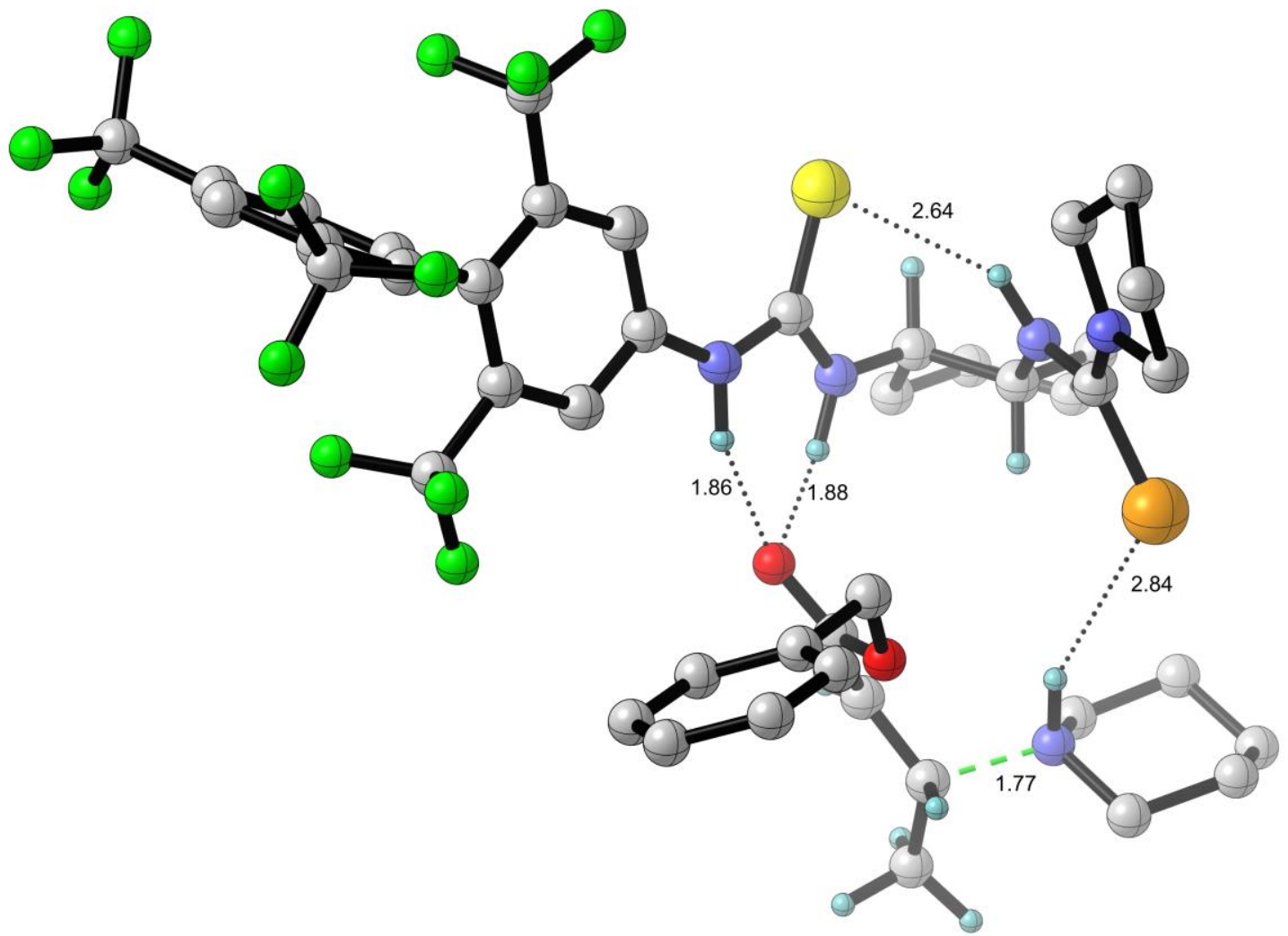

Free Energy $\quad=-6124.850841$

Zero-point Energy $=-6124.740435$

Potential Energy $\quad=-6129.59473581$

Nimag $=1(-171.4417 \mathrm{~cm}-1)$

Charge $=0$ Multiplicity $=1$

C $-6.35449-3.31669-1.93636$

C $-5.34200-3.64577-3.04293$

C $-4.11823-2.71909-2.98055$

C -3.45312 -2.75645 -1.59279

C $-4.48181-2.37904-0.50181$

C -5.69221 -3.32927-0.55104

H $\quad-3.37637-3.00468-3.73488$
H $-5.00993-4.68784-2.93227$

H -5.81368 -3.57188 -4.03052

H -6.78624 -2.32173 -2.12141

H -7.19013 -4.02678 -1.95892

H -3.08871 -3.77058 -1.40195

H -4.82711 -1.35513 -0.68148

H -5.35438 -4.34682 -0.30762

H $-6.40352-3.029150 .22596$ 


\begin{tabular}{|c|c|}
\hline H $-4.42140-1.68566-3.20728$ & $\mathrm{H}-1.23368-3.234464 .95936$ \\
\hline $\mathrm{N}-2.28857-1.88344-1.56838$ & Se -5.47856 -0.27098 1.75418 \\
\hline $\mathrm{N}-3.83618-2.387550 .81182$ & $\begin{array}{llll}O & -2.03180 & 0.91594 & -1.86465\end{array}$ \\
\hline H -2.41603 -0.91947-1.90106 & C $-2.988351 .67439-1.55299$ \\
\hline C $-1.08048-2.18241-1.03292$ & $\begin{array}{llll}C & -4.09173 & 1.97980 & -2.36102\end{array}$ \\
\hline N $-0.20432-1.12855-1.14830$ & H $-4.10726 \quad 1.54765-3.35585$ \\
\hline S - $-0.74165-3.67720-0.28849$ & C $-5.095362 .92782-1.97236$ \\
\hline H - $-0.65104-0.26372-1.47182$ & H $-4.773653 .59779-1.17446$ \\
\hline C $\quad 1.16774-0.99362-0.90687$ & O $\quad-3.01554 \quad 2.30039-0.32121$ \\
\hline C $2.02448-1.98825-0.41958$ & C -1.98712 2.011620 .63544 \\
\hline C $1.716530 .26436-1.20485$ & C $3.577301 .90815-1.36925$ \\
\hline C $3.37896-1.71529-0.22309$ & C $4.23986-2.838970 .31818$ \\
\hline H $\quad 1.63257-2.96577-0.18687$ & F $\quad 4.64456 \quad 1.85786-2.19442$ \\
\hline C $3.068300 .52712-1.01519$ & F $2.634822 .64898-1.99563$ \\
\hline H $1.07415 \quad 1.04469-1.59542$ & F $3.953852 .60369-0.27158$ \\
\hline C $3.94317-0.45757-0.51001$ & F $3.50980-3.920700 .65762$ \\
\hline H -3.00535 -2.97324 0.87757 & F $\quad 5.15274-3.25368-0.59281$ \\
\hline C $-4.13884-1.575641 .84877$ & F $4.92348-2.463831 .42176$ \\
\hline N -3.44096 -1.76218 2.98589 & C $5.39733-0.17232-0.27766$ \\
\hline C $-2.49213-2.87572 \quad 3.20013$ & C 5.802530 .543490 .85639 \\
\hline C $-3.58896-0.928694 .19302$ & C $6.36810-0.61665-1.17978$ \\
\hline C $-2.22491-2.844854 .71146$ & $\begin{array}{llll}\text { C } & 7.15348 & 0.80101 & 1.08319\end{array}$ \\
\hline H -2.93666 -3.82188 2.86978 & $\begin{array}{llll}\text { H } & 5.06147 & 0.89318 & 1.56515\end{array}$ \\
\hline C -2.40910 -1.36298 5.07175 & C $7.72094-0.35433-0.94909$ \\
\hline H $\quad-3.573320 .129193 .92425$ & H $6.06977-1.16614-2.06541$ \\
\hline H -2.96851 -3.45675 5.23562 & C $\quad 8.121150 .352100 .18254$ \\
\hline H -2.60633 -1.19885 6.13494 & $\begin{array}{llll}\mathrm{H} & 9.17147 & 0.55010 & 0.36150\end{array}$ \\
\hline H - $-4.55617-1.132324 .67062$ & C $7.58225 \quad 1.612642 .27747$ \\
\hline H - $-1.51060-0.794144 .80365$ & C $8.74704-0.89258-1.91195$ \\
\hline H - $-1.57385-2.717102 .61967$ & F $8.73761 \quad 1.14882 \quad 2.80385$ \\
\hline
\end{tabular}


F $6.65379 \quad 1.60105 \quad 3.25726$

F 7.795862 .906601 .94693

F $8.97969-2.20784-1.70412$

F $8.33898-0.76556-3.19392$

F $9.93209-0.25569-1.79573$

C -5.79916 $3.66877-3.09762$

H $-6.090072 .99440-3.90990$

H $-6.683664 .22589-2.76940$

H $-5.092654 .39365-3.51531$

C $-7.18042 \quad 3.05621-0.18945$

C -7.21686 $1.24241-1.87099$

H $-5.80401 \quad 1.53417-0.41091$

C $-8.201262 .30558 \quad 0.67568$

H -7.69113 $3.74636-0.86989$

H $-6.482583 .63544 \quad 0.42283$

C $-8.216700 .44564-1.02875$

H $-7.74415 \quad 1.88246-2.58650$

H $\quad-6.53140 \quad 0.59521-2.42634$

C $-9.08580 \quad 1.38342-0.17699$

H -8.808133 .041921 .21632$

Protonation Transition Structures

R-TS C-prot (figure 6)

File name: lowestR-newbasis-tight.log
H -7.669951 .714311 .43194$
H -8.83777 -0.16043 -1.69967
H -7.66787 -0.24304-0.37446
H -9.752520 .800890 .46848$
H -9.72804 $1.99033-0.83226$
N -6.36885 $2.13260-1.03241$
C -1.001323 .149950 .77347$
C $-0.230003 .56528-0.32186$
C -0.821603 .787662 .00540$
C $0.706824 .58854-0.18218$
H $-0.372233 .08290-1.28513$
C $\quad 0.115724 .81392 \quad 2.14894$
H $-1.41933 \quad 3.477712 .86027$
C 0.882865 .215281 .05508
H $1.309324 .88715-1.03498$
H $\quad 0.24423 \quad 5.298603 .11338$
H 1.615846 .010211 .16403
H -2.502941 .833561 .58403$
H -1.479501 .090610 .33797$ 


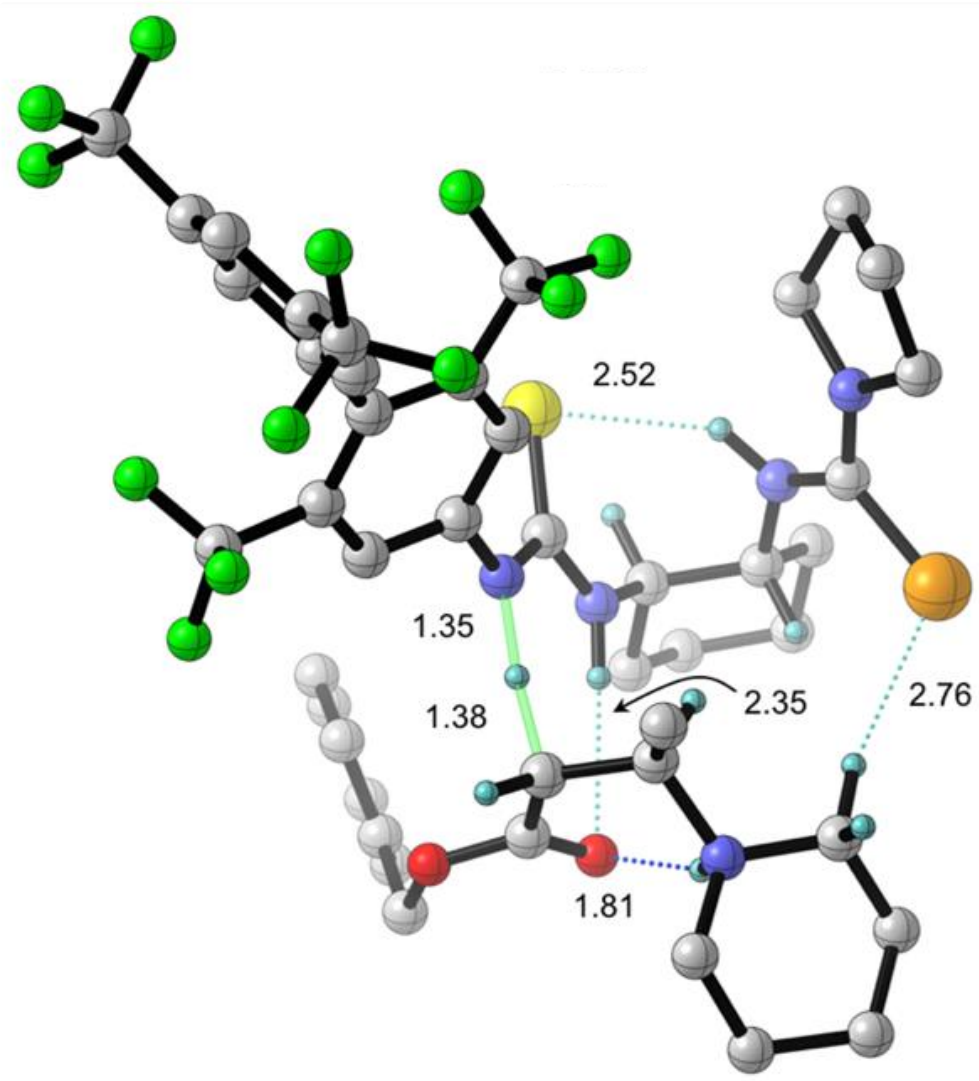

Free Energy $\quad=-6124.841972$

Zero-point Energy = -6124.731986

Potential Energy $\quad=-6129.58919642$

Nimag $=1(-1279.1890 \mathrm{~cm}-1)$

H $6.16366-0.58107-3.94231$

H $7.28657-1.27482-2.77760$

Charge $=0$ Multiplicity $=1$

H $7.341480 .85741-1.50115$

C $6.930390 .85818-2.52142$

H $7.744201 .17515-3.18538$

C $6.46256-0.55834-2.88428$

H $3.728080 .02249-3.11867$

C $5.27589-1.00203-2.01553$

H $4.929731 .40888-0.66400$

C $4.11177 \quad 0.00058-2.09375$

H $5.419651 .94916-3.63560$

C $4.595161 .41996-1.70678$

H $6.09507 \quad 2.85958-2.28118$

C $5.77047 \quad 1.86165-2.59697$

H $5.60048-1.09984-0.96882$

H $4.91597-1.98802-2.32749$

N $3.00994-0.43524-1.24266$ 

N $3.47798 \quad 2.36322-1.78475$
C $-3.46210-2.873700 .52061$
H $3.28817-0.86854-0.36989$
C $-3.133462 .00747-0.82969$
C $1.67466-0.37450-1.52948$
F -4.34982 -3.28284 - 0.41440
N $\quad 0.87999-0.87889-0.56186$
F $-2.65923-3.928550 .78349$
S $1.125110 .25751-3.03650$
F -4.16453 -2.62880 1.65142
H $\quad 1.37859-1.329300 .60786$
F -2.22061 2.89787 -1.29009
C $-0.50579-0.71811-0.51724$
F -4.12917 $1.96824-1.73858$
C $-1.148010 .51197-0.74018$
F -3.65153 2.542060 .30374
C -1.29729-1.79629-0.09891
C -4.81529-0.29428 -0.01639
C $-2.523450 .64633-0.58206$
C $-5.35952 \quad 0.21515 \quad 1.16757$
H $-0.559031 .36334-1.04652$
C -5.67885-0.66200-1.05352
C -2.67750 -1.66200 0.06995
C $-6.74090 \quad 0.35228 \quad 1.31049$
H $-0.82445-2.753790 .08288$
H -4.702010 .511291 .97694$
C $-3.33042-0.43931-0.17535$
C -7.06001-0.51765 -0.90923
H $2.751062 .09346-2.45044$
H -5.27134 -1.06431-1.97432
C $3.164803 .30529-0.86675$
C $-7.59883-0.011050 .27246$
N $2.089724 .07172-1.14259$
H -8.670820 .099780 .38278$
C $1.400164 .08480-2.45309$
C -7.308840 .839592 .61726$
C $1.541755 .09236-0.23085$
C -7.96115 -0.86219-2.06645
C $0.529075 .34740-2.39809$
F -6.49556 1.739943 .21241
H $2.139144 .10843-3.26250$
F -7.47712 -0.17600 3.49548
C $0.221525 .49416-0.90040$
F $-8.51539 \quad 1.424372 .45514$
H 1.419784 .677090 .77162
F -7.53070 - $1.96208-2.72109$
H $\quad 1.097526 .21417-2.75604$
F -9.23007 -1.09496 -1.66621
H $-0.08997 \quad 6.50471-0.62013$
F -8.00832 $0.14024-2.97290$
H $2.238175 .93711-0.15260$
C $1.85833-1.626151 .86304$
H $-0.57760 \quad 4.80077-0.61430$
H $\quad 1.03600-2.158302 .34347$
H $\quad 0.79357 \quad 3.17975-2.57856$
C $3.00055-2.466701 .57343$
H $-0.36725 \quad 5.25128-3.01724$
O $4.17203-2.050571 .46139$
Se $4.16598 \quad 3.587810 .68899$
C $2.14561-0.240792 .41975$ 

H $\quad 2.47355 \quad 0.42800 \quad 1.61462$
C $\quad \begin{array}{llll}5.34440 & 0.98999 & 4.26343\end{array}$
O $2.67865-3.761331 .36216$
H $3.26791 \quad 1.644394 .25986$
C $3.72134-4.645890 .90155$
H 4.020881 .654732 .65073
H $\quad 3.41122-5.62723 \quad 1.27511$
C $4.61360-1.20175$ 5.28415
H $\quad 4.66752-4.369401 .37329$
H $2.55587-0.482325 .25404$
C $3.86099-4.66057-0.60614$
H $2.79684-1.992594 .35337$
C $2.78776-4.34380-1.44643$
C 5.280140 .156405 .55104
C $5.07955-5.05166-1.17445$
H $5.72202 \quad 1.998304 .46554$
C $2.93349-4.41280-2.83388$
H 6.044320 .532823 .55037
H $1.84236-4.02642-1.01807$
H $4.47299-1.761556 .21573$
C $5.22222-5.13393-2.56018$
H $5.25498-1.817234 .63859$
H $5.92332-5.29044-0.53006$
H 6.285190 .008975 .96112
C $4.14862-4.81236-3.39430$
H $4.70453 \quad 0.701776 .31207$
H $2.09615-4.15023-3.47424$
N $3.39406-0.247103 .33919$
H $6.17394-5.43839-2.98765$
C $\quad 0.92748 \quad 0.37461 \quad 3.10065$
Н $4.25984-4.86731-4.47373$
H $\quad 0.122390 .43175 \quad 2.36210$
C 3.970671 .119823 .60541
H $\quad 0.56709-0.240103 .93251$
C $3.24935-1.032754 .61139$
H 1.117661 .390533 .45938
H $4.06549-0.770422 .73485$

\section{S-TS C-prot $_{\text {(figure 6) }}$}

File name: lowestS-newbasistight.log 


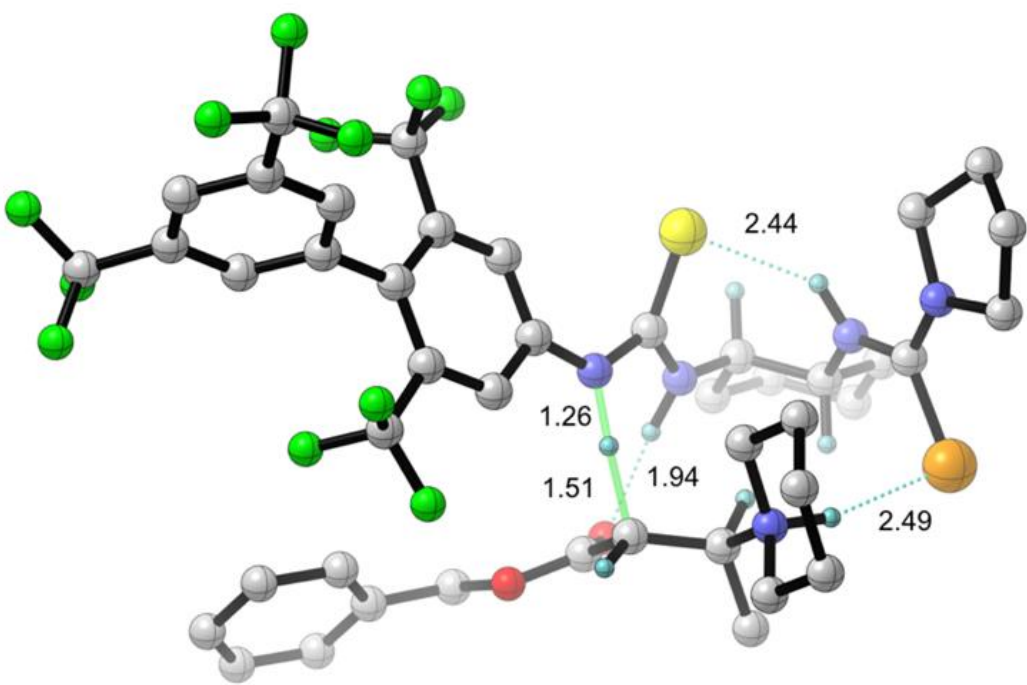

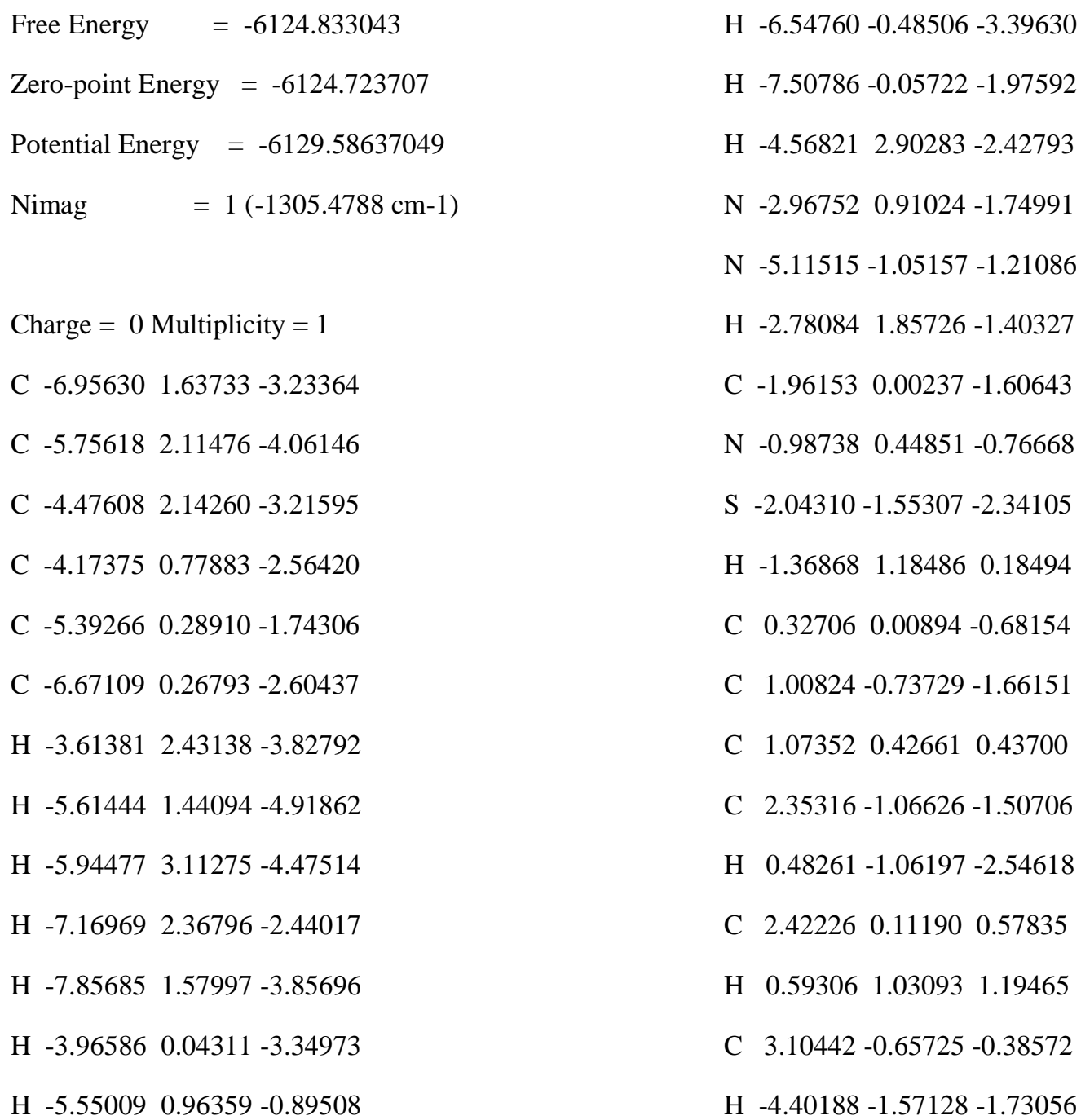




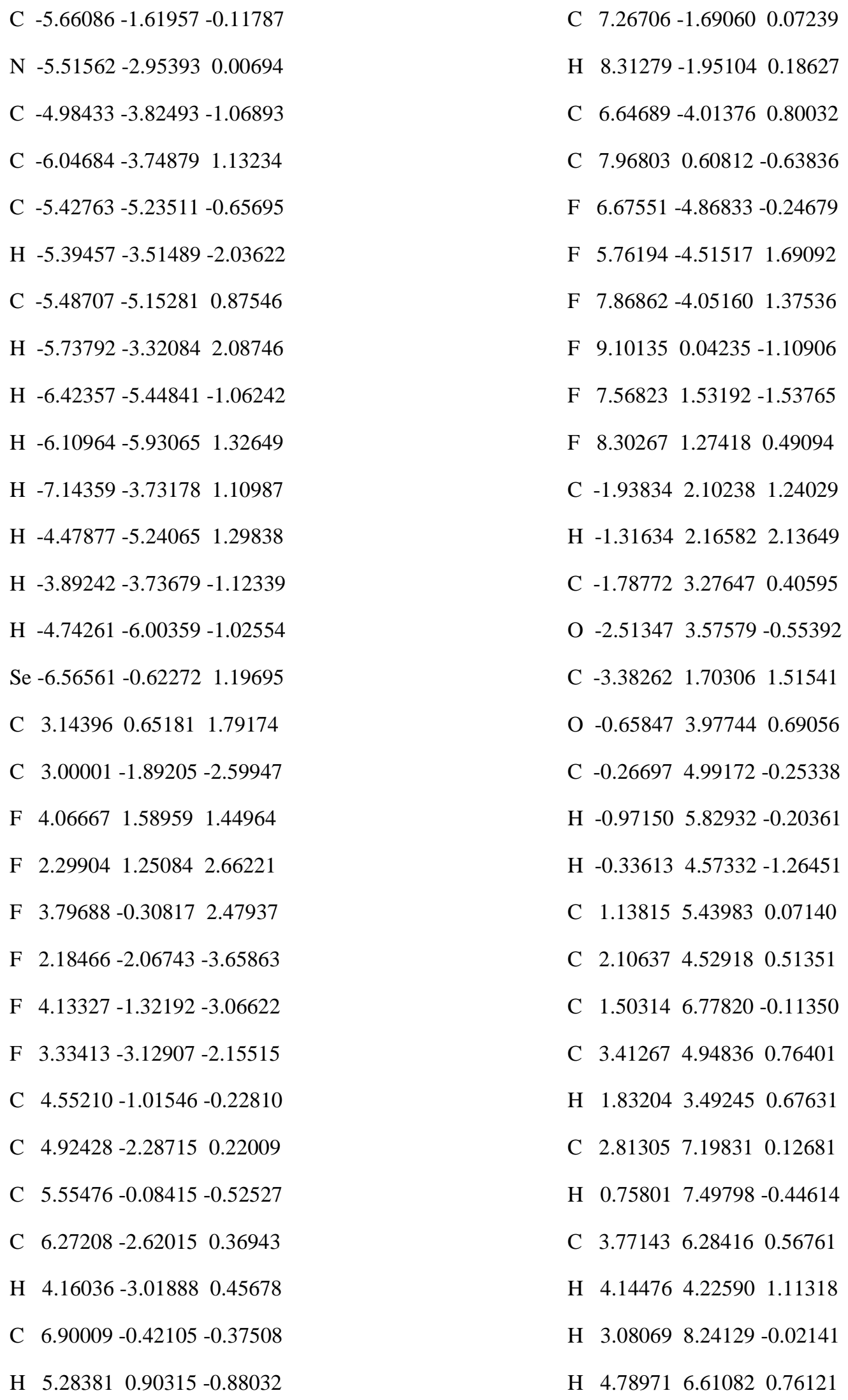



C $-2.59097-0.68343 \quad 1.91571$
H $-3.81567-2.329192 .57786$
C $-3.22865 \quad 0.70333 \quad 3.87970$
H -3.22326 -0.31173 5.76012
$\begin{array}{llll}\text { H } & -4.45020 & 0.11838 & 2.28724\end{array}$
H $-4.49384-0.855704 .66856$
C $-2.79608-1.957032 .73411$
H - $-2.76185-2.623924 .80321$
H -1.55909-0.33511 1.97706
H -1.49468 -1.46853 4.39664
H -2.83724 -0.83436 0.86455
$\begin{array}{llll}\text { N } & -3.46441 & 0.44622 & 2.41350\end{array}$
C $-3.43634-0.561754 .71441$
C -4.281392 .806832 .08582$
H -2.205691 .079323 .97366$
H $\quad-3.846223 .291262 .96581$
H $\quad-3.915591 .490414 .18979$
H $-4.37907 \quad 3.56846 \quad 1.30769$
C $-2.55210-1.715514 .22797$
H $\quad-5.288092 .44140 \quad 2.32430$
H $-2.11257-2.716892 .33867$
H -3.850941 .350270 .59462$

pxferra.log

Description: intramolecular proton transfer, $\mathrm{R}$ conformation 


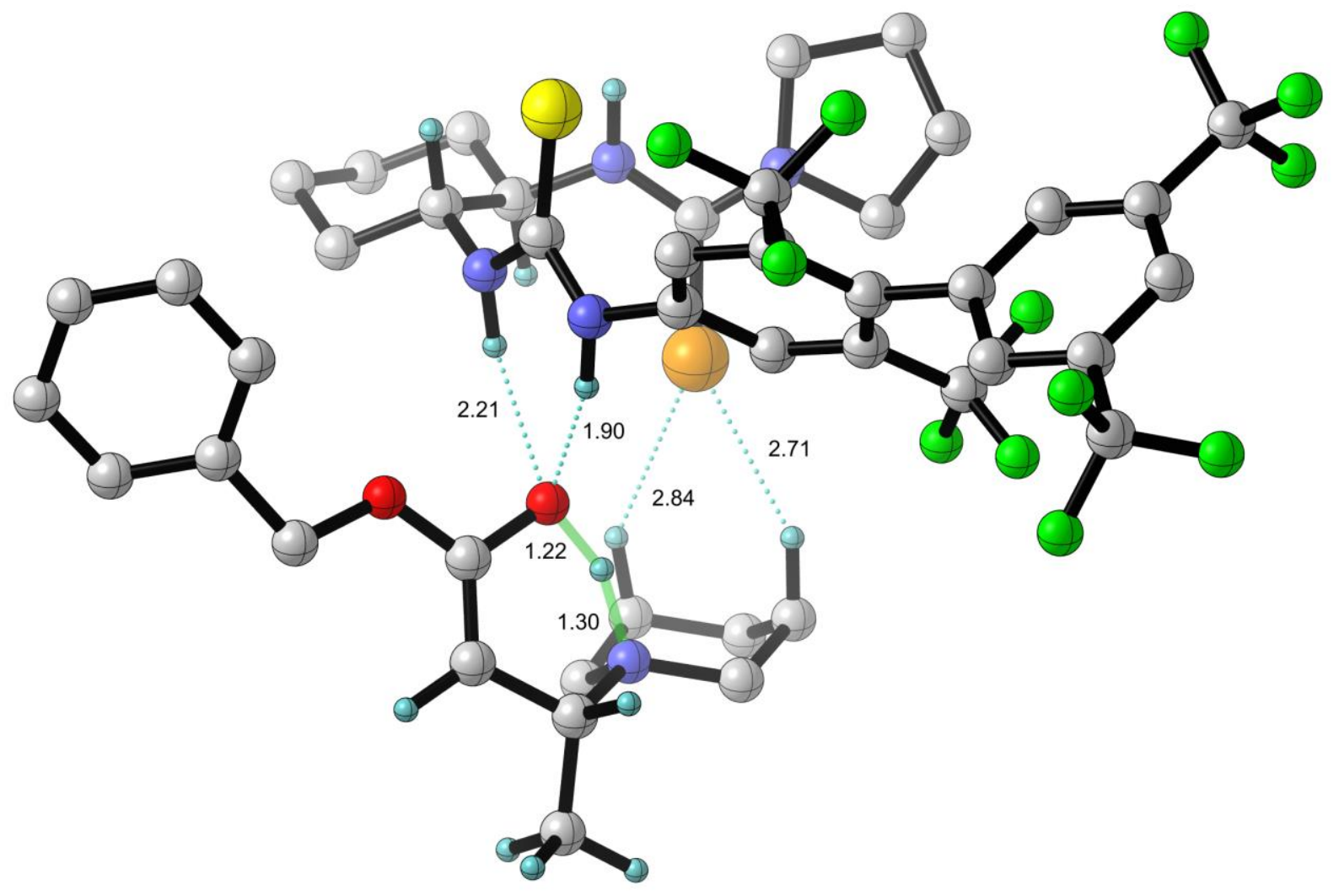
Free Energy $\quad=-6125.355768$
H $6.95230 \quad 2.77917-1.32923$
Zero-point Energy $=-6125.246575$
H $7.290453 .31731-2.97096$
Potential Energy $\quad=-6126.14338158$
H $3.884990 .94456-3.21057$
Nimag
$=1(-1071.5544 \mathrm{~cm}-1)$
H $4.452232 .45081-0.60261$
H $4.869513 .33621-3.51236$
Charge $=0$ Multiplicity $=1$
H $5.15807 \quad 4.32126-2.07683$
C $6.601692 .71176-2.36919$
H $5.912260 .33894-0.99125$
C $6.639061 .24551-2.82410$
N $3.256680 .15626-1.42222$
C $5.625280 .38844-2.05216$
N $2.84403 \quad 3.02566-1.73543$
C $4.205600 .96837-2.16401$
H $3.459150 .03907-0.42890$
C $4.180532 .43886-1.66299$
C $1.98457-0.10033-1.80068$
C $5.182463 .29351-2.45750$
N $1.25684-0.68056-0.78298$
H $5.61884-0.64204-2.42297$
$\begin{array}{llll}\text { S } & 1.34653 & 0.22190 & -3.33927\end{array}$
H $\quad 6.41270 \quad 1.19253-3.89897$
H $1.72497-0.665860 .13125$
H $7.647860 .83384-2.69831$
C $-0.15185-0.67914-0.70226$ 

C $-0.96363-1.40522-1.57380$
C -2.69066 $0.95871 \quad 1.57444$
$\begin{array}{llll}\text { C } & -0.73885 & 0.06161 & 0.32272\end{array}$
C $-3.18374-2.20716-2.37542$
C $-2.34978-1.38891-1.41254$
F -3.565600 .312852 .37216$
H - $-0.51289-1.97394-2.37466$
F $-1.71970 \quad 1.45373 \quad 2.37657$
$\begin{array}{llll}\text { C } & -2.12373 & 0.07638 & 0.48635\end{array}$
F -3.34249 2.032551 .05529
H $-0.09403 \quad 0.677040 .94139$
F -2.43631 - $2.74823-3.35731$
C -2.96526 -0.65083 -0.37877
F -3.80752 -3.23519-1.74756
H $\quad 2.46400 \quad 3.08772-2.67285$
F -4.14488 - $1.46746-2.97073$
C $1.958003 .13062-0.70852$
C $-4.45670-0.63368-0.20906$
N $\quad 0.77558 \quad 3.70383-1.00435$
C $-5.21443 \quad 0.44017-0.68734$
C $0.399454 .19393-2.34481$
C $-5.11005-1.689860 .43477$
C $-0.280223 .96693-0.00943$
C $-6.599770 .45740-0.52048$
C $-0.909024 .96471-2.10777$
H $-4.72434 \quad 1.26177-1.19668$
H $1.189834 .83398-2.75606$
C $-6.49547-1.66900 \quad 0.59813$
C $-1.50828 \quad 4.28463-0.86719$
H $-4.53647-2.528660 .81187$
H -0.411973 .106790 .64606$
C -7.24804 -0.59578 0.12272
H $\quad-0.68755 \quad 6.01642-1.89138$
H $-8.32391-0.580990 .25120$
H -2.22915 $4.91375-0.33767$
C $-7.389491 .65401-0.98263$
H 0.016364 .813840 .62389
C $-7.18848-2.84355 \quad 1.23839$
H -2.01631 $3.35454-1.14574$
F -6.84760 2.21624 -2.08518
H $\quad 0.25421 \quad 3.34155-3.02307$
F -7.43279 $2.61606-0.03224$
H -1.56572 $4.93420-2.98184$
F -8.66759 $1.33036-1.27452$
Se 2.327392 .509550 .99583
F -7.50598 -3.79035 0.32714
O $4.19125-2.297740 .61162$
F -6.40924 -3.43740 2.16885
C $3.59409-1.882891 .75883$
F -8.33924 -2.47691 1.84393
C $3.64642-2.501212 .96693$
C $3.27442-2.493085 .46526$
H $\quad 4.22428-3.399173 .13654$
H $\quad 4.34620-2.364105 .64588$
C $2.85174-1.918744 .11045$
H $\quad 2.72117-2.04730 \quad 6.29823$
H $1.77896-2.127983 .97713$
H $3.06686-3.568445 .46937$
O $2.95870-0.731691 .58032$
C 1.779050 .256764 .75332 


$\begin{array}{llllllllll}\text { C } & 4.21840 & 0.18269 & 4.42033 & & \text { N } & 2.90287 & -0.39527 & 4.02884 \\ \text { H } & 2.82483 & -0.33744 & 2.72928 & \text { C } & 4.76482 & -3.60726 & 0.58754 \\ \text { C } & 1.77216 & 1.77756 & 4.57007 & \text { H } & 5.63929 & -3.64447 & 1.25156 \\ \text { H } & 1.85347 & 0.00640 & 5.82198 & \text { H } & 4.02403 & -4.32154 & 0.97591 \\ \text { H } & 0.84816 & -0.17827 & 4.37473 & \text { C } & 5.15980 & -3.94799 & -0.82915 \\ \text { C } & 4.25626 & 1.68823 & 4.15369 & \text { C } & 4.34365 & -3.58453 & -1.90823 \\ \text { H } & 4.39256 & -0.01078 & 5.48834 & \text { C } & 6.32958 & -4.67426 & -1.07448 \\ \text { H } & 4.98497 & -0.34901 & 3.85042 & \text { C } & 4.69736 & -3.94129 & -3.20959 \\ \text { C } & 3.13086 & 2.40324 & 4.91359 & \text { H } & 3.44165 & -3.00944 & -1.72562 \\ \text { H } & 0.98029 & 2.19365 & 5.20489 & \text { C } & 6.67784 & -5.04223 & -2.37554 \\ \text { H } & 1.50790 & 2.02630 & 3.53576 & \text { H } & 6.97513 & -4.95250 & -0.24386 \\ \text { H } & 5.23729 & 2.07198 & 4.45958 & \text { C } & 5.86288 & -4.67447 & -3.44673 \\ \text { H } & 4.15877 & 1.87763 & 3.07720 & \text { H } & 4.05921 & -3.64606 & -4.03811 \\ \text { H } & 3.12231 & 3.47042 & 4.66565 & \text { H } & 7.58989 & -5.60670 & -2.55112 \\ \text { H } 3.31377 & 2.32542 & 5.99573 & \text { H } & 6.13527 & -4.95378 & -4.46106\end{array}$

pxfersa.log

Description: intramolecular proton transfer, $\mathrm{S}$ conformation 


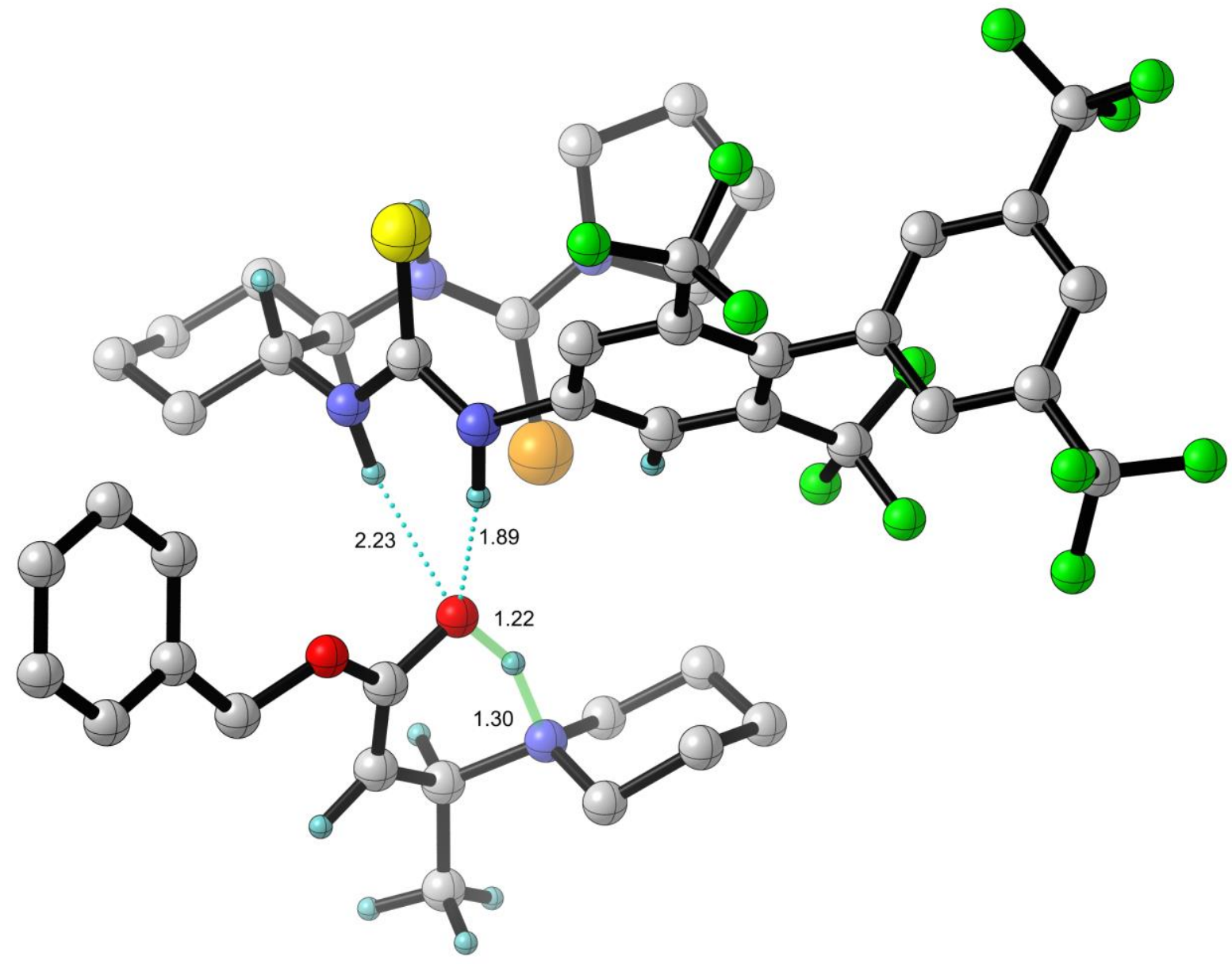

Free Energy $\quad=-6125.355380$

H $6.527962 .44638-3.33026$

Zero-point Energy $=-6125.245115$

H $7.757761 .75889-2.27590$

Potential Energy $\quad=-6126.14143309$

H $7.025853 .20276-0.39915$

Nimag

$=1(-1039.1865 \mathrm{~cm}-1)$

H $7.37178 \quad 4.20718-1.80281$

H $4.003001 .98707-2.77809$

Charge $=0$ Multiplicity $=1$

H 4.528822 .651230 .16781

C $6.684973 .44439-1.41622$

H $4.953514 .36488-2.34185$

C $6.745192 .18020-2.28579$

H $5.218934 .88047-0.67548$

C $5.734541 .12399-1.81435$

H $6.014370 .76661-0.81188$

C $4.308841 .69858-1.76733$

N $3.356950 .69172-1.32374$

C $4.262002 .95126-0.85066$

N $2.917723 .51684-0.76097$

C $5.259344 .01309-1.34454$

H $3.500910 .34658-0.37392$

H $5.745620 .25019-2.47558$

C $2.085420 .57004-1.78769$ 

N $\quad 1.34913-0.29759-1.02037$
H $\quad 5.43165-3.121912 .23256$
S $1.47604 \quad 1.36635-3.15542$
C $4.54494-1.524403 .53100$
H $1.82416-0.64132-0.17687$
O $2.97366-1.091151 .25254$
C $-0.05793-0.33596-0.94314$
C $-2.57527 \quad 0.290221 .81552$
C $-0.87555-0.71143-2.00872$
C $-3.10341-1.18609-3.02280$
C $-0.63676-0.014640 .28448$
F -3.14593 -0.76204 2.44658
C -2.25957-0.77108 - 1.83597
F -1.607210 .755272 .64551$
H $-0.43126-0.95767-2.96254$
F -3.51036 1.261491 .73222
C $-2.01767-0.086360 .46193$
F -2.34736 - $1.56541-4.07184$
$\begin{array}{lllll}\mathrm{H} & 0.01130 & 0.36077 & 1.06873\end{array}$
F -3.91680 -2.22460 - 2.72632
C -2.86524-0.46727 -0.59783
F -3.89458 -0.17401 -3.45379
H $2.526433 .79950-1.65291$
C $-4.35325-0.53600-0.41377$
C 2.028073 .303480 .24841
C $-5.17147 \quad 0.51051-0.85137$
N $\quad 0.835513 .915170 .11963$
C $-4.94220-1.642710 .20652$
C $0.469804 .79927-1.00436$
C $-6.553120 .45207-0.66552$
C -0.238603 .835591 .12652$
H $-4.73024 \quad 1.37005-1.34276$
C $-0.830295 .47222-0.54025$
C $-6.32529-1.701110 .38173$
H $1.269975 .52415-1.19621$
H $-4.32002-2.458850 .55570$
C $-1.45179 \quad 4.43378 \quad 0.40607$
C $-7.13801-0.65372-0.05028$
H $\quad-0.385092 .80487 \quad 1.44892$
H -8.21140 -0.698840.09062
H -0.598196 .392460 .00869$
C $-7.40622 \quad 1.62372-1.07658$
H $\quad-2.17357 \quad 4.86657 \quad 1.10444$
C $-6.94743-2.932680 .98669$
H $\quad 0.04861 \quad 4.414422 .01410$
F -6.92448 $2.22865-2.18366$
H -1.96570 $3.65389-0.16804$
F -7.45483 $2.56491-0.10508$
H $\quad 0.31712 \quad 4.20148-1.91391$
F -8.67941 $1.25433-1.33240$
H -1.47700 $5.73801-1.38127$
F -7.19367 -3.87551 0.04913
Se 2.400962 .232651 .70957
F -6.14005 -3.49816 1.91055
O $3.97092-2.58976-0.04050$
F -8.12496 -2.65626 1.58803
C $3.92201-2.017371 .18855$
C $5.18173-2.219904 .73724$
C $4.69884-2.327362 .25949$
H $6.25667-2.324934 .55521$ 

H $5.05843-1.646155 .66143$
C $2.21971-2.329194 .09129$
H $4.77388-3.22430 \quad 4.88910$
C $2.85563-0.015754 .62915$
H $2.85147-0.934732 .45282$
C $0.74095-1.943784 .03814$
C $5.04407-3.48786-0.31908$
H $2.48309-2.661865 .10540$
H $5.99210-3.00381-0.03979$
H $2.45242-3.140783 .39721$
H $\quad 4.94429-4.39137 \quad 0.29859$
C 1.380660 .400544 .69824
C $5.03291-3.84579-1.78695$
H $3.22012-0.282115 .63211$
C $4.54830-2.95375-2.75070$
H 3.464330 .815664 .25896
C $5.56255-5.07364-2.19953$
C $\quad 0.44850-0.781264 .99511$
C $4.59623-3.28830-4.10485$
H $\quad 0.13493-2.82224 \quad 4.28995$
H $4.11915-2.00838-2.43525$
H $\quad 0.48183-1.658743 .01023$
C $5.61979-5.40340-3.55407$
H 1.284131 .174045 .46991
H $5.93073-5.77897-1.45691$
H 1.098080 .875413 .75240
C $5.13531-4.51040-4.51132$
H - $-0.59596-0.464474 .90226$
Н $4.20732-2.59127-4.84244$
H $\quad 0.59108-1.121656 .03134$
H $\quad 6.03246-6.36139-3.85950$
H $5.01545-0.53543 \quad 3.42054$
H $5.17120-4.76833-5.56642$
N $3.07371-1.170023 .71699$

pxferrc.log

Description: intramolecular proton transfer, $\mathrm{R}$ conformation 


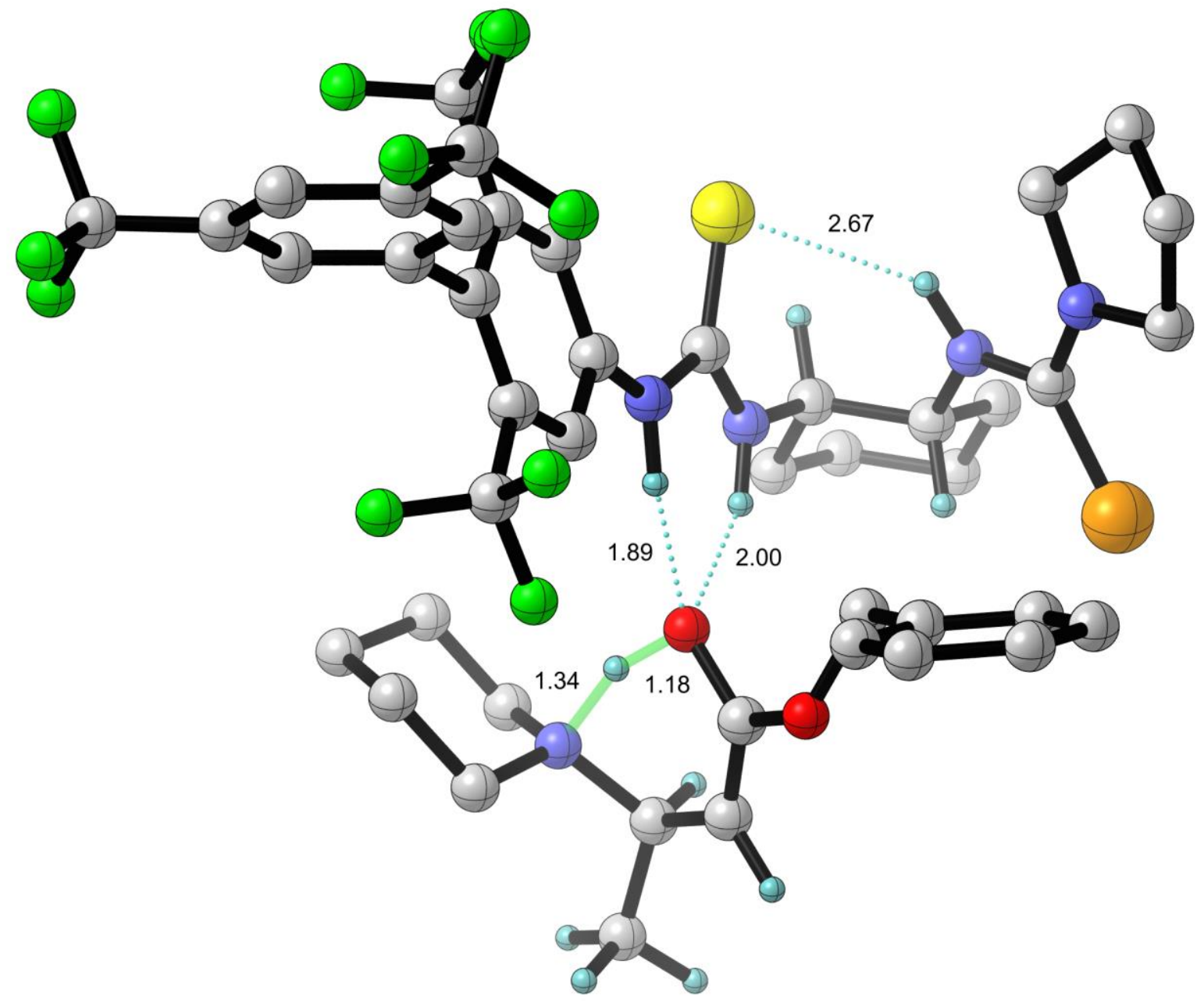
Free Energy $\quad=-6125.353910$
C -6.53669- $-0.66399-2.45046$
Zero-point Energy $=-6125.243362$
H $-4.590132 .73181-2.98764$
Potential Energy $=-6126.14043506$
H -6.36749 1.51114 -4.22498
Nimag
$=1(-848.3624 \mathrm{~cm}-1)$
H $-7.099642 .70328-3.15684$
H -7.61132 $0.93956-1.47655$
Charge $=0$ Multiplicity $=1$
H $\quad-8.28737 \quad 0.47848$-3.03485
C $-7.343690 .63940-2.49982$
H $-4.095810 .36056-3.43999$
C $-6.53678 \quad 1.76172-3.16794$
H -5.38000 -0.18490 -0.70649
C $-5.18057 \quad 1.96318-2.47456$
H -6.36299-1.03732 -3.47017
C $-4.36148 \quad 0.66059-2.42149$
H -7.07616 -1.44345 -1.90164
C -5.18163-0.46002-1.75001
H $\quad-5.350252 .31890-1.44629$ 

N $-3.105820 .89489-1.71391$
Se $-5.60944-2.381620 .73296$
N -4.40485 -1.69674 -1.71063
O -2.476112 .078760 .91753$
H $-3.186371 .31731-0.78651$
C -2.97076 1.945552 .17060
C $-1.880840 .43386-2.07074$
C -3.686112 .939232 .74549$
N $-0.921770 .80393-1.15182$
H -4.055672 .818453 .75557$
S - $1.59989-0.48492-3.46725$
C -3.987334 .204801 .98003$
H -1.30309 $1.25051-0.31333$
H $-4.79664 \quad 4.027521 .25361$
C $\quad 0.449830 .53947-1.05003$
$\begin{array}{llll}\text { O } & -2.63739 & 0.83970 & 2.86788\end{array}$
C $\quad 1.289680 .13858-2.09531$
C -2.49177-0.41727 2.16295
$\begin{array}{llll}\text { C } & 1.01667 & 0.74828 & 0.21657\end{array}$
C 2.898180 .779211 .84416
C $2.65124-0.06031-1.86060$
C $3.50718-0.48859-3.03625$
H $\quad 0.88132-0.02418-3.08080$
F $3.87798 \quad 1.708381 .86991$
$\begin{array}{llll}\text { C } & 2.37345 & 0.54844 & 0.44328\end{array}$
F $\quad 1.92622 \quad 1.214582 .68188$
H $\quad 0.383091 .067631 .03624$
F $3.40198-0.349952 .38794$
C $3.234240 .13523-0.59371$
F $2.77629-0.68869-4.15003$
H -3.67352 -1.78640 -2.41119
F $4.439170 .44612-3.34060$
C -4.50332 -2.62343-0.72495
F $4.16735-1.64009-2.78292$
N -3.76516 -3.74232 -0.87062
C $4.70138-0.07435-0.35600$
C $-3.00695-4.09365-2.08581$
C $5.17531-1.288140 .14889$
C $-3.73571-4.821260 .13302$
C $5.614290 .95192-0.62520$
C -2.76108 -5.60143 - 1.93623
C $6.54002-1.471490 .38208$
H -3.58955 -3.85188 -2.98218
H $\quad 4.47822-2.08827 \quad 0.37183$
C -2.68519-5.79761-0.41428
C $6.975130 .76624-0.38673$
H -3.47668 -4.41806 1.11383
H $\quad 5.26013 \quad 1.90000-1.01267$
H -3.61106 -6.15799 -2.34827
C $7.44557-0.446340 .11874$
H -2.88160 -6.82789-0.10400
H $8.50172-0.584740 .31812$
H $-4.73337-5.269940 .21717$
C $7.03163-2.805890 .88035$
H - $1.68925-5.52245-0.04715$
C $7.95748 \quad 1.86025-0.71529$
H - $2.06608-3.52839-2.13456$
F $7.16359-3.69382-0.13102$
H - $1.85930-5.92607-2.46330$
F $6.17429-3.350681 .77098$ 
F $8.23663-2.706001 .48136$

F $8.51363 \quad 1.68007-1.93444$

F $7.37223 \quad 3.07744-0.72224$

F $\quad 8.97211 \quad 1.89988 \quad 0.17634$

C $-4.42428 \quad 5.357412 .88831$

H $-3.682885 .56607 \quad 3.66579$

H $-4.61409 \quad 6.27710 \quad 2.32449$

H $-5.35630 \quad 5.076983 .38997$

C $-3.128165 .35332-0.09530$

C -1.621235 .069871 .81784$

H $-2.52985 \quad 3.24903 \quad 0.78750$

C -1.94055 5.41885-1.06234

H $\quad-3.41153 \quad 6.36698 \quad 0.22649$

H $-3.999534 .90468-0.58364$

C $-0.393725 .10509 \quad 0.90447$

H $-1.85076 \quad 6.08378 \quad 2.17584$

H -1.45369 4.432322 .68976

C $-0.673415 .92816-0.36015$

H $-2.20605 \quad 6.06581-1.90678$
H -1.75984 $4.41696-1.47522$

H $\quad 0.452625 .522391 .46231$

H -0.118124 .078040 .63068$

H $\quad 0.18245 \quad 5.88873-1.04353$

H $-0.80936 \quad 6.98380-0.08411$

N $-2.79764 \quad 4.52700 \quad 1.08902$

C -1.97169-1.43807 3.14412

C $-0.73178-1.255563 .77186$

C $-2.71745-2.588373 .42481$

C $-0.24966-2.205454 .67084$

H $-0.14147-0.367673 .55882$

C $-2.23052-3.542654 .32206$

H $-3.66955-2.736012 .92006$

C $-0.99898-3.35223 \quad 4.94891$

H $\quad 0.71282-2.05370 \quad 5.15260$

H $-2.81917-4.431814 .53339$

H $-0.62148-4.092785 .64942$

H $-3.45807-0.730621 .75002$

H $-1.78649-0.284991 .33541$

D3genpxferra2.log

Description: intramolecular proton transfer, $\mathrm{R}$ conformation 


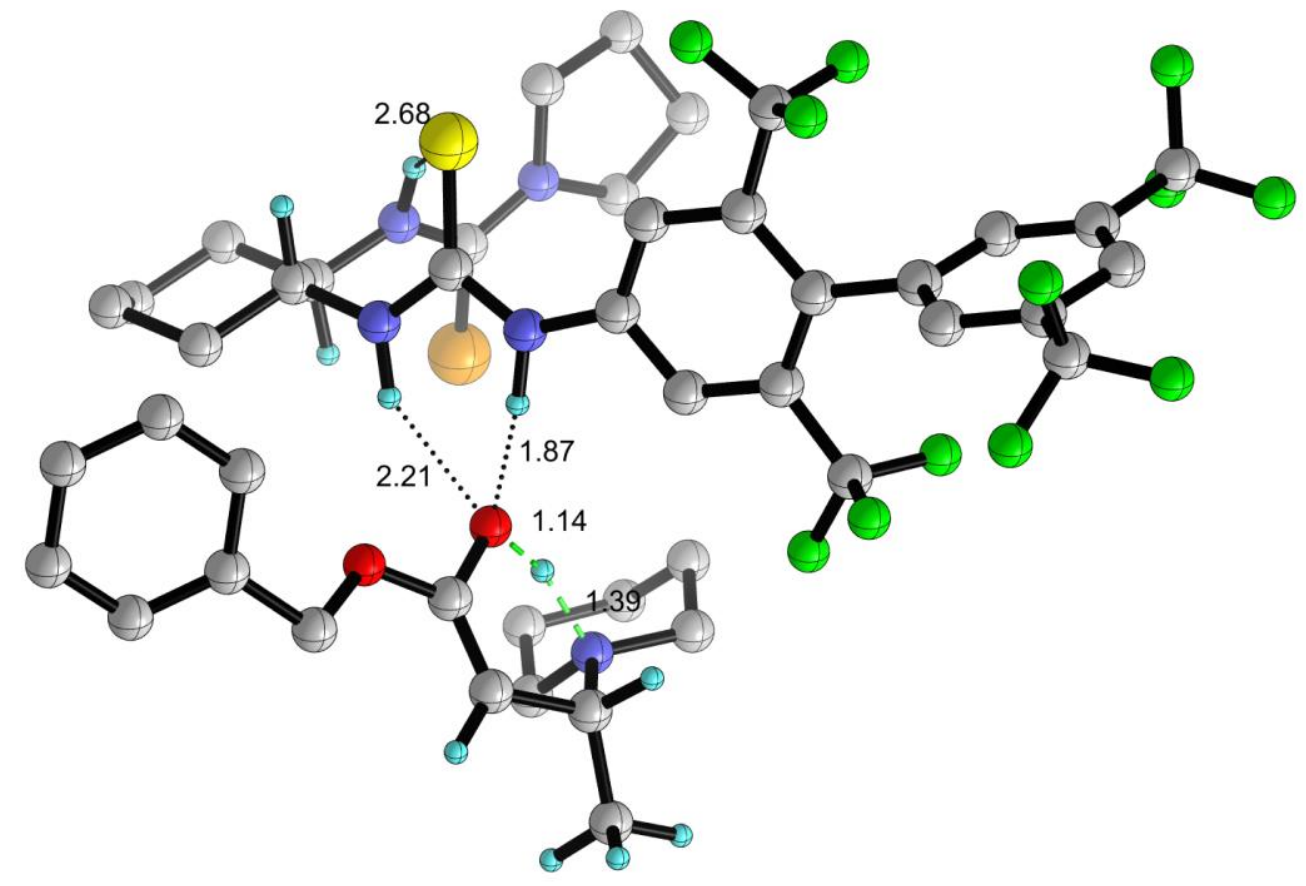

Free Energy $\quad=-6124.854740$

Zero-point Energy $=-6124.745481$

Potential Energy $\quad=-6129.58873955$

Nimag $=1(-1033.5157 \mathrm{~cm}-1)$
Charge $=0$ Multiplicity $=1$
H -4.11715 -0.42396 -3.10317
C -7.31217-0.60667-2.04370
H -5.18819-1.15238 -0.32410
C $-6.695490 .62727-2.71725$
H -6.13295 -2.11435 -3.07030
C $-5.35670 \quad 1.01280-2.06941$
H -6.74702 -2.64175 -1.49878
C $-4.36895-0.16794-2.06932$
H -5.52936 $1.33787-1.03264$
C $-4.99528-1.39865-1.37390$
N $-3.121330 .22535-1.42106$
C -6.32865 -1.78549 -2.03906
N -4.04804 -2.51285 -1.37580
H $-4.898751 .86085-2.59072$
H $\quad-3.21727 \quad 0.69789-0.52323$
H $-6.533350 .41713-3.78445$
C -1.86681 -0.07491-1.83307
H $-7.385491 .47855-2.66866$
N $-0.922010 .39686-0.94951$
H -7.58505 -0.35993-1.00748
S - $-1.53242-0.93454-3.26047$
H -8.24086 - $0.89453-2.55133$
H $-1.321190 .78573-0.08867$ 

$\begin{array}{llll}\text { C } & 0.47024 & 0.28387 & -0.89484\end{array}$
$\begin{array}{llll}\text { O } & -2.31751 & 1.28793 & 1.41048\end{array}$
C $1.31675-0.07434-1.95121$
C 2.966060 .899151 .90056
C 1.053940 .600460 .34195
C $3.55086-0.54045-2.93892$
C $2.69769-0.13204-1.75420$
F $3.69738 \quad 2.03558 \quad 1.88152$
H $\quad 0.89785-0.30902-2.91721$
F $\quad 1.96579 \quad 1.103852 .79941$
$\begin{array}{llll}\text { C } & 2.42920 & 0.54581 & 0.53129\end{array}$
F $3.74834-0.072862 .41002$
H $\quad 0.41326 \quad 0.88728 \quad 1.16661$
F $2.82715-0.65417-4.06992$
C $3.297050 .17091-0.51576$
F $4.532890 .35297-3.18594$
H -3.39073 -2.51913 -2.15109
F $4.14621-1.73983-2.73164$
C -3.84144-3.37684-0.35018
C $4.782140 .10051-0.31646$
N - $2.93733-4.35525-0.57255$
C $5.41295-1.13030-0.10529$
C $-2.30948-4.62586-1.88190$
C $5.557871 .26486-0.33168$
C $-2.54061-5.34788 \quad 0.44077$
C $6.79367-1.192860 .08943$
C -1.67636-6.01360 - 1.70652
H $4.82468-2.04047-0.08311$
H -3.06453 -4.60650 -2.67692
C $6.938621 .19718-0.14020$
C - $1.34752-6.06251-0.20695$
H $\quad 5.08247 \quad 2.22691-0.48500$
H -2.29835 -4.84961 1.38156
C $7.56423-0.030880 .07198$
H -2.40635 -6.79192 -1.95846
H $8.63407-0.08017 \quad 0.23543$
H -1.22115 -7.08013 0.17385
C $7.45498-2.534830 .26959$
H -3.37490 -6.03279 0.63982
C $7.759182 .45864-0.21710$
H -0.42173 -5.50998 -0.00495
F $6.67369-3.384520 .97235$
H -1.55551 -3.86196 -2.11486
F $8.63390-2.431340 .91963$
H -0.80294 -6.15042 -2.35056
F $7.70740-3.12580-0.91934$
Se -4.75298 -3.25004 1.26793
F $8.079002 .76284-1.49487$
$\begin{array}{llll}\text { O } & -3.05109 & 3.16627 & 0.50543\end{array}$
F 7.089513 .519960 .28316
C $-2.499992 .60357 \quad 1.60713$
F $8.91862 \quad 2.34698 \quad 0.46591$
C $-2.18193 \quad 3.20283 \quad 2.77800$
C -1.559263 .096815 .22866$
H -2.384914 .250232 .95606$
H $-2.58862 \quad 3.31527 \quad 5.53000$
C -1.500162 .396183 .86675$
H -1.082572 .501626 .01442$
H -0.438792 .252813 .61413$
H -1.022684 .049575 .16373$ 


$\begin{array}{llllllll}\text { C }-1.12530 & -0.02312 & 4.42631 & \text { N }-2.05444 & 0.98566 & 3.86121 \\ \text { C }-3.41142 & 0.86549 & 4.45549 & \text { C }-3.18832 & 4.59310 & 0.47951 \\ \text { H }-2.19112 & 0.90489 & 2.48001 & \text { H }-3.91049 & 4.90815 & 1.24392 \\ \text { C }-1.65268 & -1.44296 & 4.19025 & \text { H }-2.21624 & 5.04003 & 0.73268 \\ \text { H }-0.99508 & 0.15604 & 5.50535 & \text { C }-3.64555 & 5.01415 & -0.89488 \\ \text { H }-0.14917 & 0.11410 & 3.94974 & \text { C }-3.02070 & 4.50423 & -2.04139 \\ \text { C }-3.99512 & -0.52695 & 4.20465 & \text { C }-4.67485 & 5.94954 & -1.03979 \\ \text { H }-3.35294 & 1.05663 & 5.53737 & \text { C }-3.42332 & 4.92337 & -3.30916 \\ \text { H }-4.03524 & 1.64525 & 4.00869 & \text { H }-2.22745 & 3.76975 & -1.93696 \\ \text { C }-3.06887 & -1.61981 & 4.75335 & \text { C }-5.07198 & 6.37827 & -2.30858 \\ \text { H }-0.95663 & -2.15688 & 4.64720 & \text { H }-5.17157 & 6.34436 & -0.15622 \\ \text { H }-1.66403 & -1.65103 & 3.11215 & \text { C }-4.44817 & 5.86447 & -3.44576 \\ \text { H }-4.98427 & -0.58351 & 4.67503 & \text { H }-2.93394 & 4.51826 & -4.19075 \\ \text { H }-4.14533 & -0.68339 & 3.12898 & \text { H }-5.87321 & 7.10579 & -2.40633 \\ \text { H }-3.45712 & -2.60898 & 4.48835 & \text { H }-4.75865 & 6.19259 & -4.43401 \\ \text { H }-3.03772 & -1.56324 & 5.85170 & & & \end{array}$

lowestR-newbasis.log

Description: catalyst mediated C-protonation, R conformation 


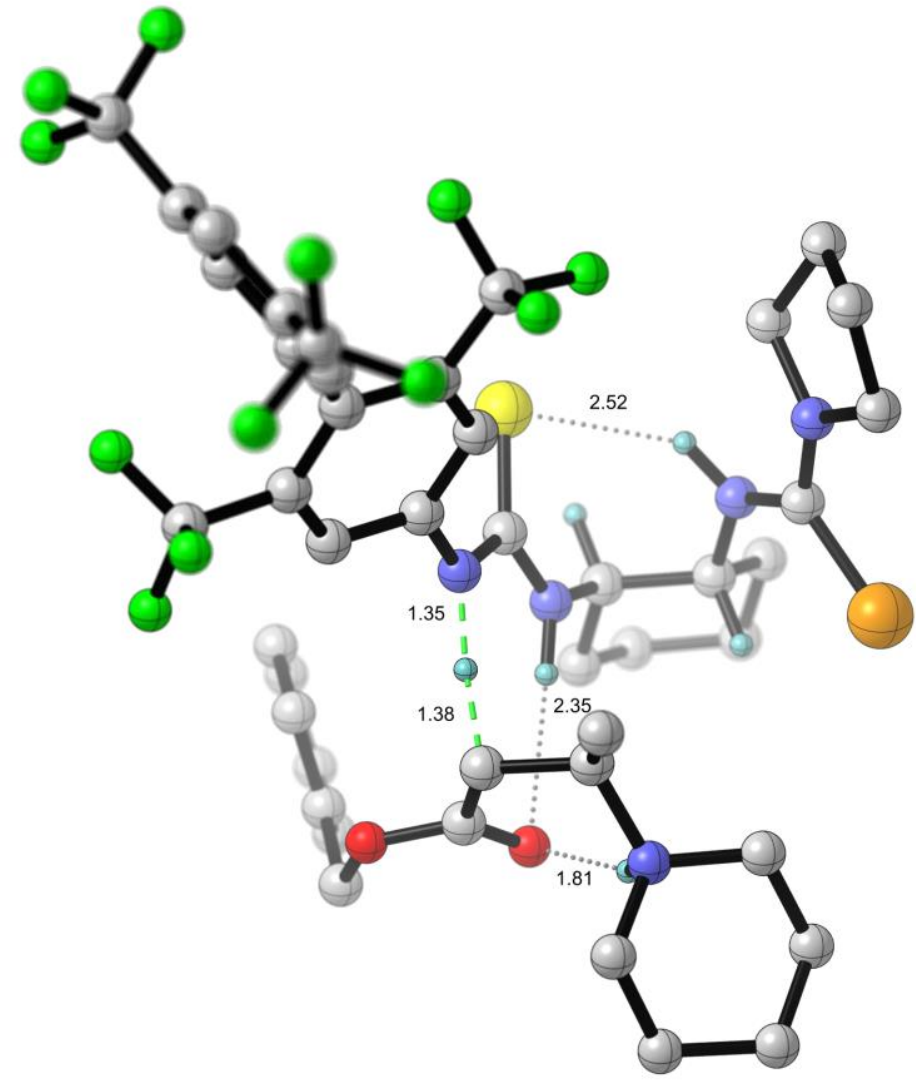

Free Energy = -6124.841866

Zero-point Energy $=-6124.731990$

Potential Energy $\quad=-6125.62908034$

Nimag $=1(-1308.3606 \mathrm{~cm}-1)$

Charge $=0$ Multiplicity $=1$

C -6.93119-0.85615 -2.52177

C $-6.463230 .56068-2.88326$

C $-5.276231 .00331-2.01442$

C $-4.112330 .00054-2.09390$

C $-4.59592-1.41909-1.70812$

C $-5.77143-1.85973-2.59854$

H $-4.916191 .98951-2.32560$

H $-6.164690 .58453-3.94136$

H -7.28712 $1.27716-2.77553$
H $-7.34208-0.85637-1.50143$

H -7.74515 -1.17231 -3.18592

H -3.72898 -0.02048 -3.11895

H -4.93029-1.40892-0.66527

H -5.42081 -1.94624 -3.63733

H -6.09613 -2.85794 -2.28372

H -5.60048 $1.10026-0.96753$

N -3.01015 $0.43532-1.24280$

N -3.47891 -2.36246-1.78711

H $\quad-3.28800 \quad 0.86673-0.36897$ 


\begin{tabular}{|c|c|c|}
\hline C $-1.674990 .37498-1.53024$ & F 4.34758 & $3.28487-0.41689$ \\
\hline $\mathrm{N}-0.88004 \quad 0.87870-0.56252$ & F 2.65858 & 3.927930 .78468 \\
\hline S - $-1.12589-0.25568-3.03797$ & F 4.16649 & 2.628601 .64860 \\
\hline H - $-1.37826 \quad 1.32916 \quad 0.60741$ & F 2.22111 & $-2.89691-1.29415$ \\
\hline C $\quad 0.505760 .71821-0.51853$ & F 4.13045 & $-1.96743-1.73958$ \\
\hline C $\quad 1.14811-0.51172-0.74198$ & F 3.65013 & -2.542650 .30173 \\
\hline C $\quad 1.29722 \quad 1.79639-0.10013$ & C 4.81533 & $30.29472-0.01788$ \\
\hline C $2.52359-0.64599-0.58401$ & C 5.35931 & $1-0.21654 \quad 1.16543$ \\
\hline H $\quad 0.55916-1.36304-1.04855$ & C 5.67918 & $80.66464-1.05401$ \\
\hline C $2.67745 \quad 1.662190 .06863$ & С 6.74069 & $9-0.35311 \quad 1.30877$ \\
\hline $\begin{array}{llll}\text { H } & 0.82427 & 2.75377 & 0.08197\end{array}$ & Н 4.70162 & $2-0.514391 .97401$ \\
\hline C $3.330480 .43959-0.17698$ & C 7.06035 & $50.52080-0.90929$ \\
\hline H - $-2.75245-2.09263-2.45325$ & Н 5.27193 & $31.06836-1.97431$ \\
\hline C $-3.16524-3.30488-0.86966$ & C 7.59891 & $\begin{array}{lll}1 & 0.01255 & 0.27179\end{array}$ \\
\hline $\mathrm{N}-2.09034-4.07125-1.14637$ & Н 8.67092 & $2-0.097850 .38243$ \\
\hline C $-1.40126-4.08359-2.45713$ & C 7.30835 & $5-0.842362 .61494$ \\
\hline C $-1.54181-5.09216-0.23528$ & C 7.96190 & $0.86760-2.06550$ \\
\hline C $-0.52997-5.34609-2.40303$ & F 6.49503 & -1.743753 .20847 \\
\hline H $-2.14052-4.10697-3.26629$ & F 7.47626 & 0.171893 .49477 \\
\hline C $-0.22177-5.49341-0.90553$ & F 8.51502 & -1.426722 .45224 \\
\hline H - $-1.41953-4.677280 .76731$ & F 7.53157 & $1.96854-2.71847$ \\
\hline H - $-1.09847-6.21278-2.76108$ & F 9.23061 & $1.09987-1.66430$ \\
\hline H $\quad 0.09007-6.50400-0.62580$ & F 8.00962 & $-0.13331-2.97361$ \\
\hline H - $-2.23803-5.93708-0.15712$ & C -1.85696 & $\begin{array}{lll}6 & 1.62487 & 1.86319\end{array}$ \\
\hline H $\quad 0.57733-4.79996-0.61949$ & H -1.03492 & $\begin{array}{lll}2 & 2.15824 & 2.34278\end{array}$ \\
\hline H $-0.79486-3.17837-2.58237$ & C -3.00127 & $\begin{array}{lll}7 & 2.46346 & 1.57610\end{array}$ \\
\hline H $\quad 0.36606-5.24961-3.02254$ & O -4.17207 & $\begin{array}{lll}7 & 2.04505 & 1.46546\end{array}$ \\
\hline Se $-4.16568-3.587920 .68650$ & C -2.14073 & $\begin{array}{lll}3 & 0.23860 & 2.41951\end{array}$ \\
\hline C 3.461872 .874010 .51929 & H -2.46863 & $3-0.430331 .61449$ \\
\hline C $3.13361-2.00707-0.83198$ & O -2.68236 & $\begin{array}{lll}6 & 3.75903 & 1.36594\end{array}$ \\
\hline
\end{tabular}




\begin{tabular}{llllllll} 
C -3.72782 & 4.64224 & 0.90902 & H -3.25615 & -1.64961 & 4.26068 \\
H -3.42012 & 5.62326 & 1.28544 & H -4.01200 & -1.66074 & 2.65279 \\
H -4.67284 & 4.36162 & 1.38063 & C -4.60578 & 1.19352 & 5.28832 \\
C -3.86871 & 4.66130 & -0.59849 & H -2.54683 & 0.47783 & 5.25459 \\
C -2.79504 & 4.35099 & -1.44063 & H -2.79197 & 1.98808 & 4.35503 \\
C -5.08912 & 5.04985 & -1.16459 & C -5.26931 & -0.16598 & 5.55585 \\
C -2.94216 & 4.42382 & -2.82773 & H -5.70915 & -2.00851 & 4.47057 \\
H -1.84817 & 4.03574 & -1.01395 & H -6.03603 & -0.54348 & 3.55634 \\
C -5.23321 & 5.13594 & -2.54994 & H -4.46468 & 1.75323 & 6.21988 \\
H -5.93319 & 5.28358 & -0.51877 & H -5.24936 & 1.80804 & 4.64406 \\
C -4.15917 & 4.82079 & -3.38592 & H -6.27402 & -0.02060 & 5.96748 \\
H -2.10440 & 4.16638 & -3.46962 & H -4.69150 & -0.71043 & 6.31588 \\
H -6.18635 & 5.43835 & -2.97569 & N -3.38766 & 0.24195 & 3.34098 \\
H -4.27146 & 4.87875 & -4.46509 & C -0.92034 & -0.37484 & 3.09816 \\
C -3.96110 & -1.12620 & 3.60765 & H -0.11625 & -0.42984 & 2.35839 \\
C -3.24232 & 1.02729 & 4.61330 & H -0.55997 & 0.23999 & 3.92994 \\
H -4.06115 & 0.76419 & 2.73803 & H -1.10800 & -1.39139 & 3.45644 \\
C -5.33396 & -0.99937 & 4.26810 & & & & \\
\hline
\end{tabular}

lowestS-newbasis.log

Description: catalyst mediated C-protonation, S conformation 


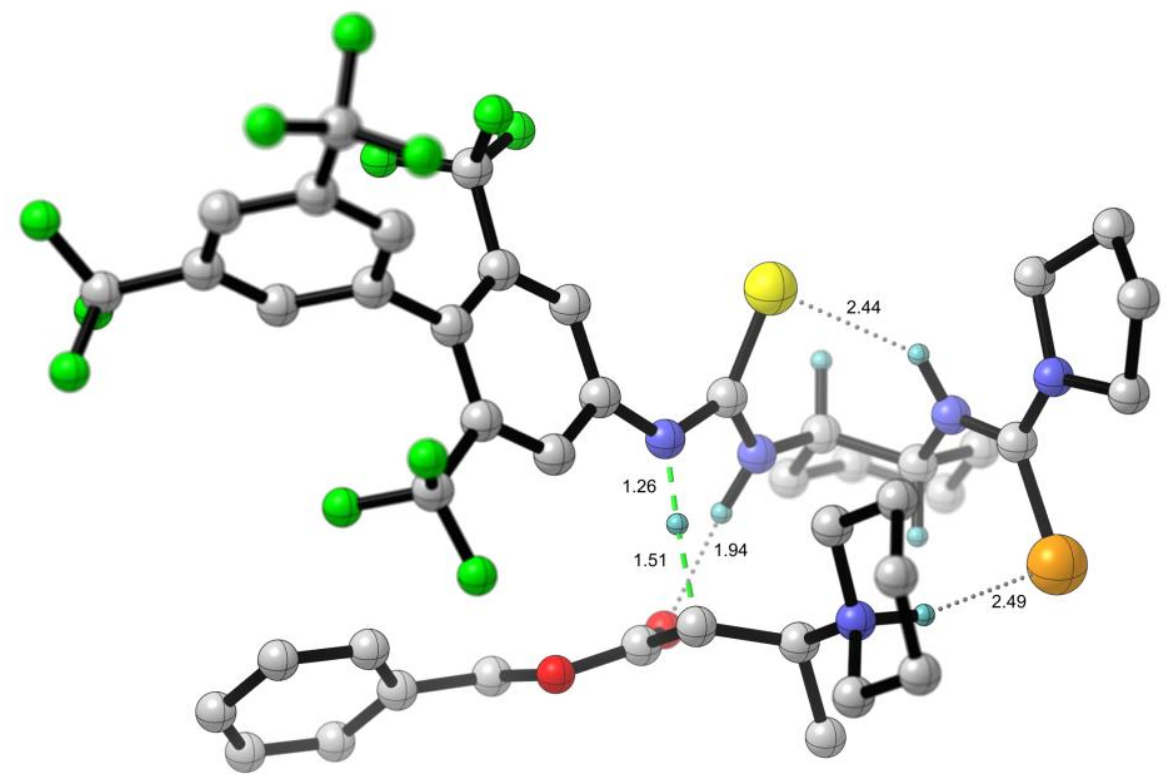

B3LYP-D3(BJ)/6-311++G** PCM(toluene) Potential Energy = -6129.58635541

Free Energy = $\quad-6124.832752$

Zero-point Energy $=-6124.723703$

Potential Energy = -6125.62119908

Nimag $=1(-1308.9123 \mathrm{~cm}-1)$

Charge $=0$ Multiplicity $=1$

C $-6.956361 .63842-3.23307$

C $-5.756252 .11579-4.06093$

C $-4.476062 .14326-3.21555$

C $-4.173890 .77930-2.56410$

C $-5.392790 .28962-1.74292$

C $-6.67133 \quad 0.26884-2.60410$

H $-3.613802 .43201-3.82756$

H $-5.61471 \quad 1.44209-4.91822$

H $-5.944703 .11389-4.47442$

H $-7.169532 .36893-2.43943$
H $-7.856991 .58134-3.85630$

H $-3.966240 .04371-3.34981$

H $\quad-5.550020 .96397-0.89479$

H -6.54806 -0.48402 -3.39619

H -7.50808 -0.05628 -1.97561

H $-4.56798 \quad 2.90335-2.42736$

$\begin{array}{llll}\text { N } & -2.96751 & 0.91034 & -1.74995\end{array}$

N -5.11547-1.05122 -1.21102

H $-2.780541 .85728-1.40328$

C $-1.961630 .00225-1.60684$

$\begin{array}{llll}\text { N } & -0.98719 & 0.44823 & -0.76737\end{array}$ 

S - $2.04384-1.55306-2.34167$
F $3.79732-0.301552 .47843$
H -1.368381 .184430 .18446$
F $2.18282-2.07562-3.65514$
$\begin{array}{llll}\text { C } & 0.32727 & 0.00864 & -0.68229\end{array}$
F $4.13186-1.32883-3.06581$
C $1.00778-0.74014-1.66078$
F $3.33307-3.13357-2.14965$
$\begin{array}{llll}\text { C } & 1.07450 & 0.42897 & 0.43477\end{array}$
C $4.55234-1.01572-0.22853$
C $2.35273-1.06898-1.50622$
C $4.92424-2.286320 .22296$
H $\quad 0.48163-1.06694-2.54436$
C $5.55522-0.08566-0.52885$
$\begin{array}{llll}\text { C } & 2.42324 & 0.11439 & 0.57615\end{array}$
C $6.27199-2.619490 .37241$
H $\quad 0.59460 \quad 1.03537 \quad 1.19110$
H 4.16016-3.01708 0.46212
C $3.10470-0.65740-0.38633$
C $6.90049-0.42270-0.37850$
H $-4.40228-1.57091-1.73085$
H $\quad 5.28448 \quad 0.90077-0.88647$
C -5.66121 -1.61935-0.11812
C $7.26718-1.691180 .07223$
N $\quad-5.51619-2.953770 .00641$
H $8.31287-1.951750 .18622$
C $-4.98524-3.82466-1.06972$
C $6.64654-4.012090 .80677$
C $-6.04749-3.748751 .13169$
C $7.968760 .60526-0.64516$
C $-5.42912-5.23478-0.65814$
F $6.67514-4.86922-0.23825$
H -5.39534 -3.51417-2.03692
F $5.76144-4.511201 .69851$
C $-5.48835-5.152930 .87429$
F $7.86821-4.048691 .38200$
H $-5.73819-3.321202 .08687$
F $9.101350 .03773-1.11550$
H -6.42520 -5.44750 - 1.06357
F $7.56871 \quad 1.52717-1.54626$
H -6.11120 -5.93063 1.32515
F $8.30476 \quad 1.273740 .48230$
H -7.14424 -3.73127 1.10948
C $-1.937912 .10157 \quad 1.24023$
H -4.48004 -5.24134 1.29707
H -1.315872 .164572 .13643$
H -3.89330 -3.73696-1.12413
C -1.787193 .276020 .40642$
H - -4.74448 -6.00344 -1.02706
O $-2.51288 \quad 3.57583-0.55334$
Se -6.56569 -0.62262 1.19697
C $-3.38221 \quad 1.70233 \quad 1.51534$
C 3.145730 .657181 .78779
$\begin{array}{lllll}\text { O } & -0.65791 & 3.97681 & 0.69141\end{array}$
C $2.99882-1.89764-2.59692$
C $-0.266594 .99173-0.25190$
F 4.069731 .592601 .44276
H $-0.971965 .82864-0.20250$
F $2.30167 \quad 1.260212 .65633$
H $-0.334534 .57355-1.26322$ 

C $\quad 1.13787 \quad 5.441130 .07402$
C $-3.43582-0.56328 \quad 4.71378$
C 2.106364 .531700 .51797
H $-2.20520 \quad 1.07797 \quad 3.97337$
C $1.502016 .77961-0.11191$
H $-3.91509 \quad 1.489014 .18971$
C 3.412154 .952120 .76912
C -2.55166 -1.71696 4.22696
H $\quad \begin{array}{llll}1.83268 & 3.49498 & 0.68181\end{array}$
H $-2.11238-2.71790 \quad 2.33737$
C $2.81137 \quad 7.200970 .12915$
H -3.81544 -2.33015 2.57682
H $0.756637 .49848-0.44598$
H $-3.22262-0.313535 .75954$
C $3.77008 \quad 6.28798 \quad 0.57167$
H $-4.49333-0.857194 .66797$
H 4.144504 .230511 .11950
H -2.76139-2.62550 4.80201
H $\quad 3.07833 \quad 8.24401-0.01987$
H -1.49422 -1.47004 4.39559
H $4.78795 \quad 6.615610 .76577$
N $-3.46404 \quad 0.44528 \quad 2.41313$
C $-2.59069-0.684301 .91497$
C -4.280842 .806042 .08610$
$\begin{array}{llll}\text { C } & -3.22817 & 0.70201 & 3.87938\end{array}$
H $-3.84558 \quad 3.290172 .96622$
H $-4.44987 \quad 0.117552 .28686$
H $\quad-4.37847 \quad 3.567901 .30820$
C $-2.79580-1.958092 .73307$
H -5.28756 2.44063 2.32451
H -1.55879-0.33603 1.97630
$\begin{array}{llll}\text { H } & -3.85065 & 1.34981 & 0.59451\end{array}$
H $\quad-2.83709-0.834950 .86380$

nhprot1.log

Description: catalyst mediated C-protonation, $\mathrm{R}$ conformation 


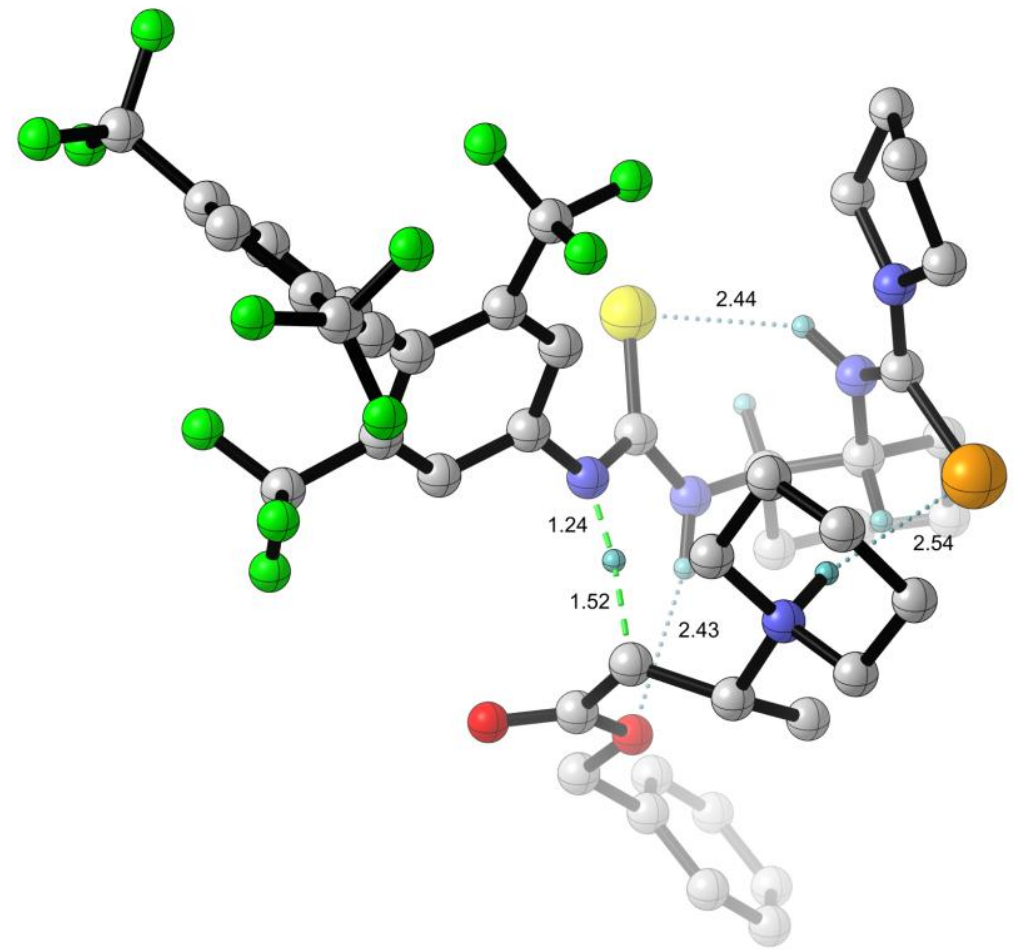

Free Energy $=-6125.326191$
Zero-point Energy $=-6125.217399$
Potential Energy $=-6126.11430610$
Nimag = $1(-1149.8553 \mathrm{~cm}-1)$

Charge $=0$ Multiplicity $=1$

H - $7.85259-1.95227-2.21998$

C -6.99199-1.40799-1.81248

H -4.01987 -0.38938 -3.14446

C -6.78351-0.08910 - 2.56953

H -4.63077-1.34284-0.29771

C $-5.552760 .66499-2.04657$

H -5.55829 -2.59736 -2.92369

C $-4.27720-0.19114-2.09858$

H -5.85402 -3.19719-1.28751

C $-4.49483-1.53731-1.36678$

H -5.73779 $0.98113-1.00977$

C -5.73601 -2.28530-1.88448

N $-3.164840 .55002-1.51593$

H $-5.38187 \quad 1.57987-2.62482$

N -3.29774 -2.37528 -1.49584

H -6.65520 -0.30012 -3.64097

H -3.38609 $1.12892-0.71092$

H $-7.66897 \quad 0.55143-2.47875$

C $-1.839160 .39339-1.81323$

H -7.22765 -1.19197-0.76048

N - $1.028960 .95707-0.89528$ 


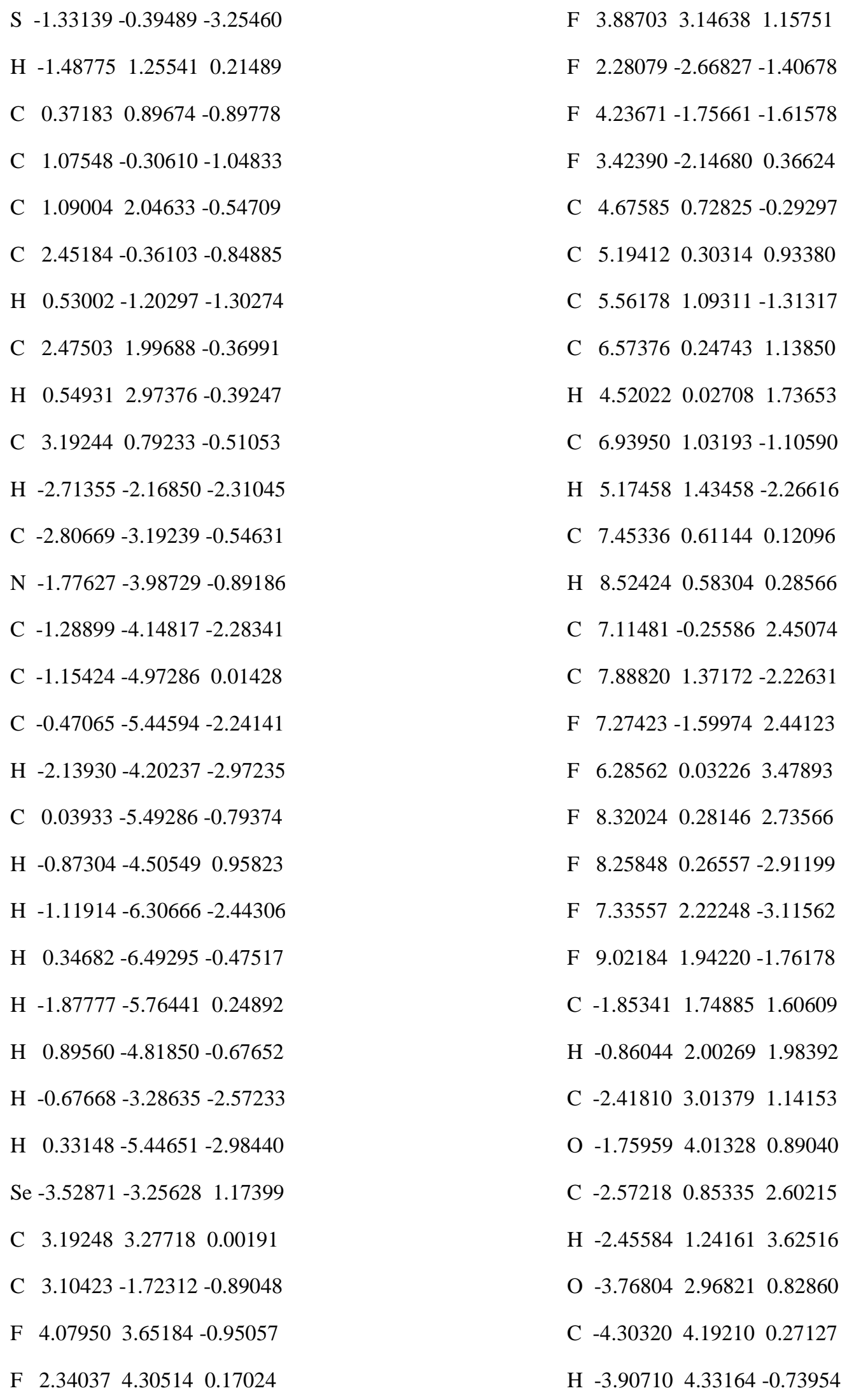

F $3.88703 \quad 3.146381 .15751$

F $2.28079-2.66827-1.40678$

F $4.23671-1.75661-1.61578$

F $3.42390-2.146800 .36624$

C $4.675850 .72825-0.29297$

C 5.194120 .303140 .93380

C $5.561781 .09311-1.31317$

C 6.573760 .247431 .13850

H $4.520220 .02708 \quad 1.73653$

C $6.939501 .03193-1.10590$

H $5.17458 \quad 1.43458-2.26616$

C 7.453360 .611440 .12096

H 8.524240 .583040 .28566

C $7.11481-0.255862 .45074$

C $7.888201 .37172-2.22631$

F $7.27423-1.599742 .44123$

F 6.285620 .032263 .47893

F 8.320240 .281462 .73566

F $8.25848 \quad 0.26557-2.91199$

F $7.33557 \quad 2.22248-3.11562$

F $9.021841 .94220-1.76178$

C -1.853411 .748851 .60609$

H -0.860442 .002691 .98392$

C $-2.418103 .01379 \quad 1.14153$

O -1.759594 .013280 .89040$

C $-2.57218 \quad 0.85335 \quad 2.60215$

H -2.455841 .241613 .62516$

$\begin{array}{llll}\text { O } & -3.76804 & 2.96821 & 0.82860\end{array}$

C $-4.30320 \quad 4.19210 \quad 0.27127$

H -3.90710 $4.33164-0.73954$ 


\begin{tabular}{|c|c|c|c|}
\hline H -3.94809 & 5.031290 .87688 & H $-0.28436-0.15054$ & 41.38814 \\
\hline C -5.80757 & 4.102630 .26593 & C $-1.46927-2.53785$ & 4.25460 \\
\hline C -6.51863 & 3.991891 .46962 & H $-1.57253-0.42846$ & 54.79898 \\
\hline C -6.52324 & $4.15814-0.93461$ & H -3.12713 -1.12906 & 54.31676 \\
\hline C -7.91069 & 3.927161 .47056 & C $\quad 0.01246-2.57870$ & 3.86148 \\
\hline H -5.97313 & 3.959892 .40981 & H $1.23096-1.94541$ & 2.15195 \\
\hline C -7.91939 & $4.10086-0.93782$ & H $-0.35216-2.59885$ & 1.73036 \\
\hline H -5.98361 & $4.25174-1.87416$ & H $-1.61559-2.85494$ & 5.29391 \\
\hline C -8.61580 & 3.982050 .26473 & H - $-2.03550-3.22597$ & 73.61676 \\
\hline H -8.44797 & 3.841732 .41153 & H $\quad 0.38023-3.61038$ & 3.88557 \\
\hline H -8.45993 & $4.15001-1.87941$ & H $\quad 0.61403-2.00957$ & 4.58453 \\
\hline H -9.70159 & 3.936960 .26532 & $\mathrm{~N}-1.85708-0.55400$ & 2.72708 \\
\hline C -0.38706 & $-0.56380 \quad 2.38652$ & C $-4.05516 \quad 0.57205$ & 2.35403 \\
\hline C -2.05562 & -1.131384 .11175 & H -4.622181 .49487 & 2.47104 \\
\hline H -2.33204 & -1.204262 .06018 & H -4.222530 .20505 & 1.33953 \\
\hline C 0.18032 & -1.979332 .46186 & H $-4.45740-0.17091$ & 13.04965 \\
\hline
\end{tabular}

H $0.10793 \quad 0.109493 .09450$

nhprot1b.log

Description: catalyst mediated C-protonation, R conformation 


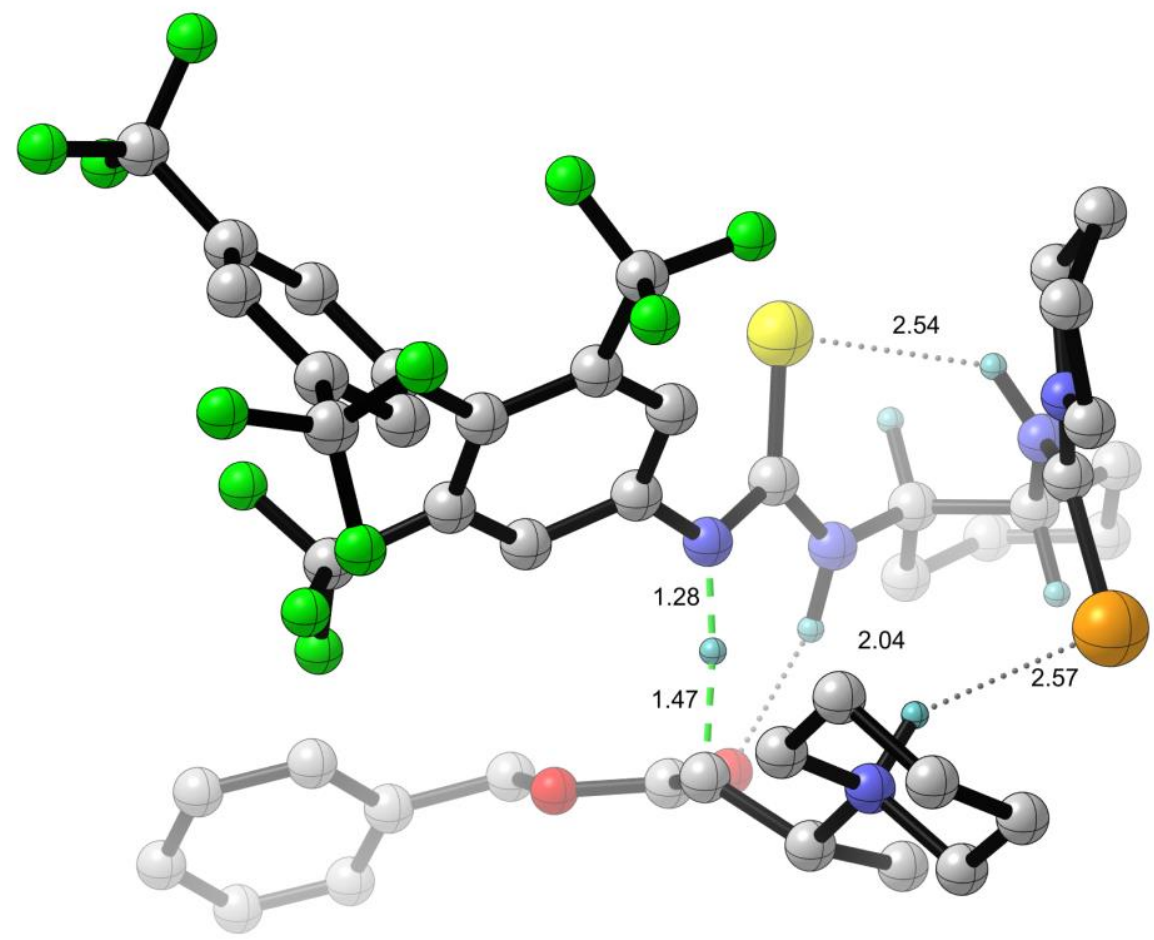

Free Energy $\quad=-6125.334231$

Zero-point Energy $=-6125.226067$

Potential Energy = -6126.12344939

Nimag $=1(-1327.1553 \mathrm{~cm}-1)$

Charge $=0$ Multiplicity $=1$

C $-7.65241-0.10565-2.48850$

C $-6.96732 \quad 1.18498-2.96255$

C $-5.72028 \quad 1.50329-2.12555$

C $-4.732590 .32523-2.12639$

C $-5.43238-0.96101-1.61550$

C $-6.67030-1.28700-2.46671$

H $-5.21102 \quad 2.39480-2.50811$

H $-6.67785 \quad 1.07628-4.01771$

H $-7.669202 .02664-2.91712$

H $-8.05500 \quad 0.04697-1.47663$

H -8.50827 -0.34318 -3.13216
H -4.38919 0.15026 -3.15229

H $-5.73869-0.81219-0.57593$

H $-6.35185-1.52398$-3.49261

H -7.15011 -2.18763 -2.06606

H $-6.01394 \quad 1.73238-1.09137$

N $-3.560810 .63959-1.32425$

N - $4.48609-2.08014-1.61428$

H -3.70506 $1.32724-0.58529$

C $-2.283110 .23801-1.57729$

N -1.40623 $0.60853-0.61519$

S -1.87295 -0.60212-3.02481

H -1.807941 .165910 .46192$ 

C $-0.038100 .33045-0.61957$
F $3.35080-2.60153-1.97224$
C $0.50565-0.93800-0.88158$
F $\quad 2.88576-3.13947 \quad 0.08359$
C $0.840961 .34640-0.21829$
C $4.24817-0.39176-0.30119$
C $1.87454-1.16734-0.77286$
C $4.80848-0.955210 .84905$
H - $-0.15280-1.73805-1.18427$
C $5.08940-0.05911-1.36876$
C $2.213911 .11799-0.11021$
C $6.18330-1.181750 .93024$
H $\quad 0.43548 \quad 2.32766-0.00413$
H $4.17149-1.209091 .68874$
C $2.77071-0.14416-0.39245$
C $6.46286-0.29025-1.28552$
H -3.85239-2.07045 -2.41586
H $\quad 4.670680 .38950-2.26261$
C $-4.04159-2.74783-0.52958$
C $7.01790-0.85247-0.13633$
N -3.12783 -3.70987 -0.75304
H $8.08628-1.01813-0.06790$
C $-2.75956-4.21189-2.09740$
C $6.75071-1.830402 .16516$
C $-2.50401-4.508530 .31493$
C $7.340240 .02505-2.46937$
C -2.09845 -5.56635 -1.81034
F $6.60798-3.175402 .13139$
H -3.65812 -4.29697-2.71854
F $6.12461-1.400913 .28465$
C $-1.46568-5.36128-0.42565$
F $8.06802-1.575342 .31166$
H $-2.06808-3.848991 .06853$
F $7.34974-0.99003-3.36322$
H -2.85917 -6.35484 -1.76802
F $6.91353 \quad 1.12401-3.12752$
H -1.25944 -6.29985 0.09687
F $8.62213 \quad 0.24025-2.10162$
H -3.26766 -5.11518 0.81722
C -2.156241 .917471 .67868$
H - $0.52194-4.81312-0.52197$
H -1.191802 .207282 .09939$
H -2.07009 -3.51248 -2.58469
C -2.722993 .051190 .95807$
H - $1.37235-5.83855-2.58130$
O
Se -4.68166 -2.43649 1.19653
C $-3.003621 .21102 \quad 2.72660$
C 3.097092 .272040 .30849
H $-2.77493 \quad 1.622243 .72053$
C $2.37867-2.56800-1.03908$
O $-1.81249 \quad 4.056380 .81003$
F $3.956962 .63716-0.66977$
C $-2.183805 .12400-0.09236$
F $2.37758 \quad 3.37373 \quad 0.62503$
H $\quad-3.203115 .448590 .13068$
F $3.84093 \quad 1.971191 .39791$
H -2.18398 $4.72332-1.11390$
F $1.39325-3.39147-1.47244$
C $-1.20057 \quad 6.257040 .06069$ 

C $\quad 0.18152 \quad 6.03345-0.00557$
C $-2.70710-2.346964 .37405$
C $-1.66509 \quad 7.564450 .23912$
H -2.91000 -0.25515 4.97271
C $1.075997 .09658 \quad 0.10571$
H $-4.24585-0.876493 .98196$
H $\quad 0.561275 .02454-0.13246$
C $-1.18173-2.45907 \quad 4.44658$
C $-0.77115 \quad 8.632610 .34026$
H $\quad 0.53428-1.821413 .25617$
H $-2.73544 \quad 7.74873 \quad 0.29954$
H $\quad-0.80952-2.44123 \quad 2.30889$
$\begin{array}{llll}\text { C } & 0.60251 & 8.40038 & 0.27527\end{array}$
H -3.17385-2.69075 5.30480
H $2.14488 \quad 6.90601 \quad 0.05822$
H -3.10340 -2.97124 3.56450
H -1.149239 .642420 .47670$
H $-0.88067-3.507764 .54497$
H $\quad \begin{array}{lll}1.30142 & 9.22853 & 0.35822\end{array}$
H -0.80815 -1.93686 5.33910
C -1.03114 -0.40238 2.97451
N $-2.53253-0.285602 .90880$
C $-3.16749-0.908884 .13193$
C $-4.52379 \quad 1.20982 \quad 2.52721$
H $\quad-2.88862-0.828202 .09069$
H $-4.837602 .17922 \quad 2.14275$
C $-0.55972-1.838983 .19152$
H $-4.83104 \quad 0.446681 .80452$
H $-0.713720 .24346 \quad 3.80153$
H $-5.049801 .02273 \quad 3.46784$
H $\quad-0.63537 \quad 0.007562 .04803$

nhprot1c.log

Description: catalyst mediated C-protonation, $\mathrm{R}$ conformation 


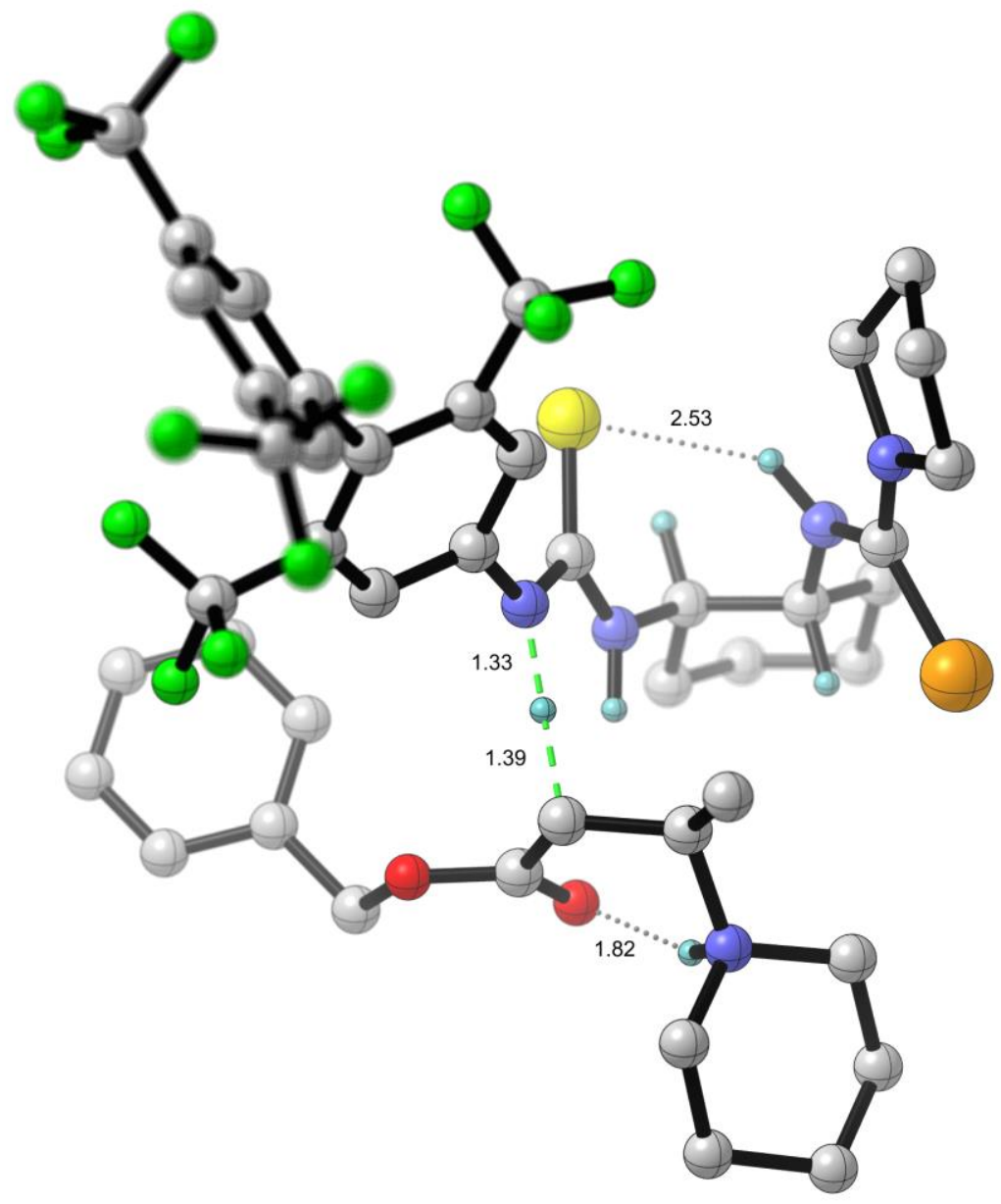

Free Energy $\quad=-6125.343851$

Zero-point Energy $=-6125.234801$

Potential Energy $\quad=-6126.13211573$

Nimag = $\quad 1(-1307.6671 \mathrm{~cm}-1)$

Charge $=0$ Multiplicity $=1$

C $-7.06578-1.00597-2.67713$

C $-6.551630 .28376-3.33338$

C $-5.35183 \quad 0.86546-2.57024$

C $-4.21555-0.16051-2.42580$

C $-4.74290-1.46035-1.77223$

C $-5.94305-2.04303-2.53678$

H $-4.961051 .75413-3.07916$
H $-6.25146 \quad 0.06916-4.36908$

H $-7.35255 \quad 1.03132-3.38948$

H -7.46968 -0.77171 -1.68119

H -7.89551 -1.42589-3.25885

H $-3.81373-0.39243-3.41699$

H -5.07115 - $1.23389-0.75174$

H -5.61385 -2.37639-3.53138

H -6.28996 -2.93051 -1.99479 


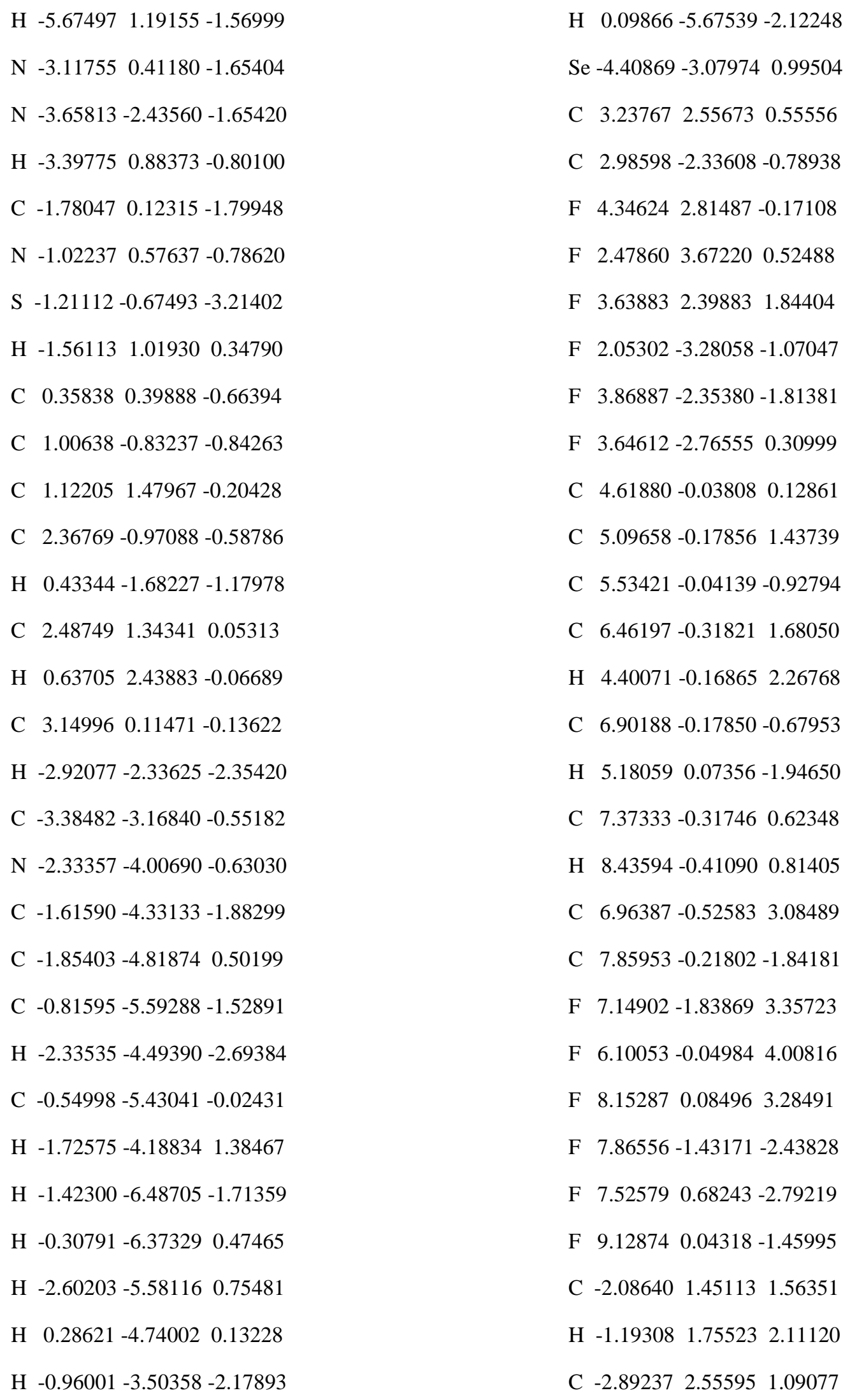




\begin{tabular}{|c|c|c|c|}
\hline O -4.12197 & 2.480990 .87849 & H $-4.48760 \quad 1.40472$ & 2.29976 \\
\hline C -2.83435 & 0.262632 .13250 & C -6.336850 .32005 & 3.86999 \\
\hline H -3.33028 & -0.311021 .34121 & H $-4.60157-0.98813$ & 4.04086 \\
\hline O -2.17265 & 3.659120 .80121 & H -5.24810 -0.89016 & 2.39824 \\
\hline C -2.82952 & 4.759980 .11604 & C -4.944322 .22243 & 4.77396 \\
\hline H -2.86665 & 5.595360 .82212 & H -3.255600 .85372 & 4.92532 \\
\hline H -3.85037 & $4.45065-0.11874$ & H - -2.935952 .27092 & 3.90714 \\
\hline C -2.04473 & $5.13138-1.11635$ & C $-6.04211 \quad 1.19720$ & 5.09519 \\
\hline C -1.86362 & $4.20089-2.14974$ & H - -7.04026 -0.48145 & 4.12162 \\
\hline C -1.49774 & $6.41115-1.25018$ & H -6.815790 .92102 & 3.08432 \\
\hline C -1.14485 & $4.54709-3.29227$ & H -4.655322 .78276 & 5.67033 \\
\hline H -2.27650 & $3.19971-2.05792$ & H -5.31586 2.95840 & 4.04772 \\
\hline C -0.78646 & $6.76348-2.39928$ & H -6.95242 1.70866 & 5.42654 \\
\hline H -1.62651 & $7.13695-0.45001$ & H -5.714710 .56133 & 5.92966 \\
\hline C -0.60687 & $5.83088-3.42070$ & $\mathrm{~N}-4.052000 .74628$ & 2.98185 \\
\hline H -1.00411 & $3.81258-4.08030$ & C $-1.95001-0.71757$ & 2.89294 \\
\hline H -0.36675 & $7.76176-2.49031$ & H $-1.10723-0.97356$ & 2.24339 \\
\hline H -0.04709 & $6.09981-4.31246$ & H $-1.53578-0.29635$ & 3.81541 \\
\hline C -5.06425 & -0.317503 .31074 & H - $-2.48500-1.64807$ & 3.10358 \\
\hline C -3.69689 & 1.546004 .20232 & & \\
\hline
\end{tabular}

nhprot2.log

Description: catalyst mediated C-protonation, S conformation 


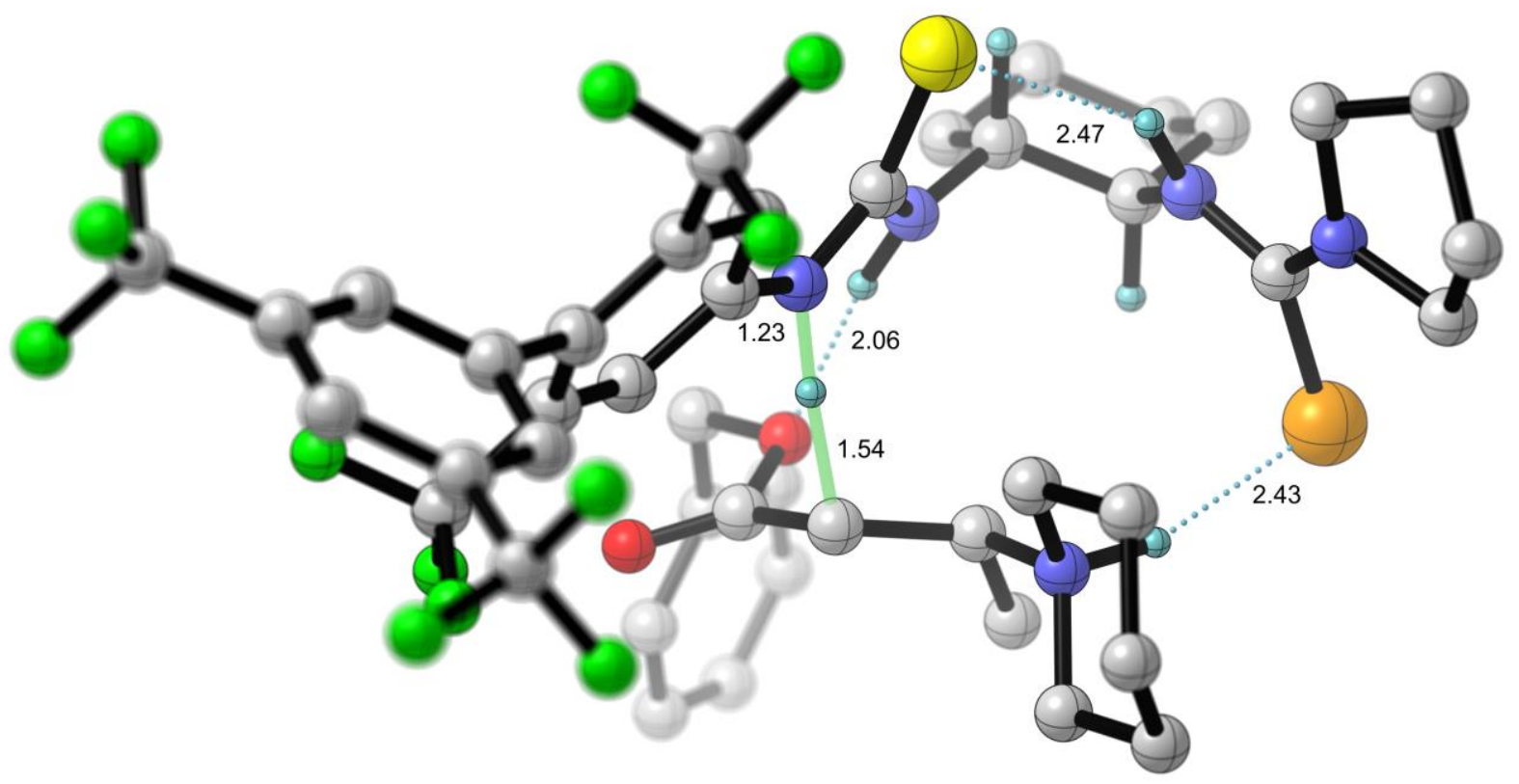

Free Energy $\quad=-6125.330878$

Zero-point Energy $=-6125.222096$

Potential Energy = -6126.12013347

Nimag $=1(-1122.7247 \mathrm{~cm}-1)$

Charge $=0$ Multiplicity $=1$

C $-7.00056-0.27474-2.66075$

C $-6.198750 .64890-3.58735$

C $-4.88085 \quad 1.08423-2.93143$

C $-4.01562-0.11620-2.50730$

C -4.83071-1.05991-1.59194

C $-6.16090-1.48699-2.24045$

H $-4.29581 \quad 1.71285-3.61295$

H $\quad-5.98147 \quad 0.12423-4.52870$

H $-6.78818 \quad 1.53495$ - 3.85288

H $-7.31140 \quad 0.28364-1.76599$

H -7.92033 -0.61033 -3.15467

H -3.70613 -0.66783 -3.40188
H $-5.06027-0.54686-0.65190$

H -5.94575 -2.11272 -3.11857

H -6.69990 -2.11069-1.51797

H $-5.102401 .69248-2.04215$

N $-2.803640 .36603-1.85309$

N - $-4.02588-2.24106-1.25662$

H -2.89172 $1.25142-1.35605$

C $-1.54524-0.16873-1.93587$

N $-0.673280 .48061-1.12692$

S -1.23910 -1.52045 -2.95365

H -1.14842 $1.01161-0.12072$

C $\quad 0.714870 .35261-1.01604$

C $1.49830-0.74092-1.42029$ 

C $\quad 1.368851 .42038-0.36885$
C $5.013110 .26383-0.27208$
C $2.87382-0.75791-1.18624$
C $5.52281-0.441150 .82262$
H $\quad 1.02922-1.56832-1.93077$
C $5.904070 .93485-1.11717$
C $2.740021 .39221-0.12995$
C $6.89626-0.474741 .06776$
H $\quad 0.79154 \quad 2.28627-0.06287$
H $4.84605-0.962051 .48989$
C $3.535610 .29929-0.53247$
C $7.276470 .89798-0.86976$
H -3.28324 -2.46108 -1.92519
H $\quad 5.525791 .48293-1.97218$
C $-4.16027-2.98822-0.14541$
C $7.780840 .19348 \quad 0.22315$
N -3.52758 -4.17677-0.11724
H 8.847540 .166600 .41336
C $-2.84028-4.77718-1.28349$
C $7.42849-1.287852 .21773$
C -3.63402 -5.13073 1.00412
C $8.22178 \quad 1.67305-1.75009$
C -2.67813 -6.25489-0.89655
F $7.67423-2.567591 .85383$
H $-3.44488-4.64101-2.18724$
F $6.55138-1.335153 .24586$
C $-2.62323-6.221810 .63885$
F $8.58822-0.785402 .69476$
H -3.43503 -4.62643 1.95104
F $9.421011 .05783-1.85616$
H -3.55277 -6.82579 -1.22891
F $7.73273 \quad 1.83177-2.99824$
H -2.87121 -7.18235 1.09901
F $8.458652 .90989-1.25675$
H -4.66020 -5.51614 1.06044
C $-1.77507 \quad 1.56814 \quad 1.16811$
H $-1.61950-5.936070 .97666$
H -1.00957 1.656731 .94116
H -1.87519 -4.28343-1.45095
C $-2.030812 .88140 \quad 0.62312$
H -1.79194 -6.70320 -1.35385
O -1.287763 .852130 .68765$
Se -5.16745 -2.41325 1.32038
C $-2.93826 \quad 0.65875 \quad 1.54611$
C 3.364462 .579940 .57036
O $-3.183832 .91209-0.17426$
C $3.64347-1.98196-1.63469$
C $-3.482874 .18103-0.81553$
F $4.235373 .23806-0.23231$
H $-3.94633 \quad 3.91158-1.76778$
F 2.444093 .477780 .96900
H -2.53912 4.69690 -0.99981
F $4.05337 \quad 2.20594 \quad 1.67524$
C -4.420735 .024310 .01426$
F $2.87878-2.83446-2.34687$
C $-3.93343 \quad 5.79322 \quad 1.08177$
F $4.70541-1.66615-2.40709$
C $-5.792125 .04803-0.26699$
F $4.12184-2.68612-0.57527$
C $-4.804786 .56491 \quad 1.84980$ 


$\begin{array}{llllllll}\text { H }-2.87024 & 5.77368 & 1.30198 & \text { H }-2.99345 & 0.04821 & 4.21963 \\ \text { C }-6.66520 & 5.82202 & 0.49952 & \text { C }-0.54854 & -2.38492 & 3.74189 \\ \text { H }-6.17855 & 4.46120 & -1.09806 & \text { H }-0.01883 & -2.99207 & 1.71332 \\ \text { C }-6.17187 & 6.58174 & 1.56104 & \text { H }-1.69487 & -3.31062 & 2.15406 \\ \text { H }-4.41558 & 7.15999 & 2.67193 & \text { H }-1.49126 & -1.47506 & 5.48864 \\ \text { H }-7.72669 & 5.83378 & 0.26605 & \text { H }-2.59842 & -2.36892 & 4.45012 \\ \text { H }-6.84823 & 7.18795 & 2.15805 & \text { H }-0.32670 & -3.34532 & 4.21993 \\ \text { C }-1.27405 & -1.27404 & 1.58484 & \text { H } & 0.35290 & -1.76449 & 3.84117 \\ \text { C }-2.11047 & -0.38856 & 3.75453 & \text { N }-2.42417 & -0.60158 & 2.29685 \\ \text { H }-3.22922 & -1.26948 & 2.22452 & \text { C }-4.08875 & 1.28768 & 2.34017 \\ \text { C }-0.87823 & -2.58643 & 2.25906 & \text { H }-3.74896 & 1.80149 & 3.24448 \\ \text { H }-0.44419 & -0.56700 & 1.57967 & \text { H }-4.55040 & 2.03912 & 1.69626 \\ \text { H }-1.59187 & -1.43132 & 0.55408 & \text { H }-4.85573 & 0.54516 & 2.59191 \\ \text { C }-1.72690 & -1.70003 & 4.44219 & \text { H }-3.37900 & 0.20501 & 0.65475 \\ \text { H }-1.29790 & 0.34204 & 3.80156 & & & & \end{array}$

nhprot2b.log

Description: catalyst mediated C-protonation, S conformation 


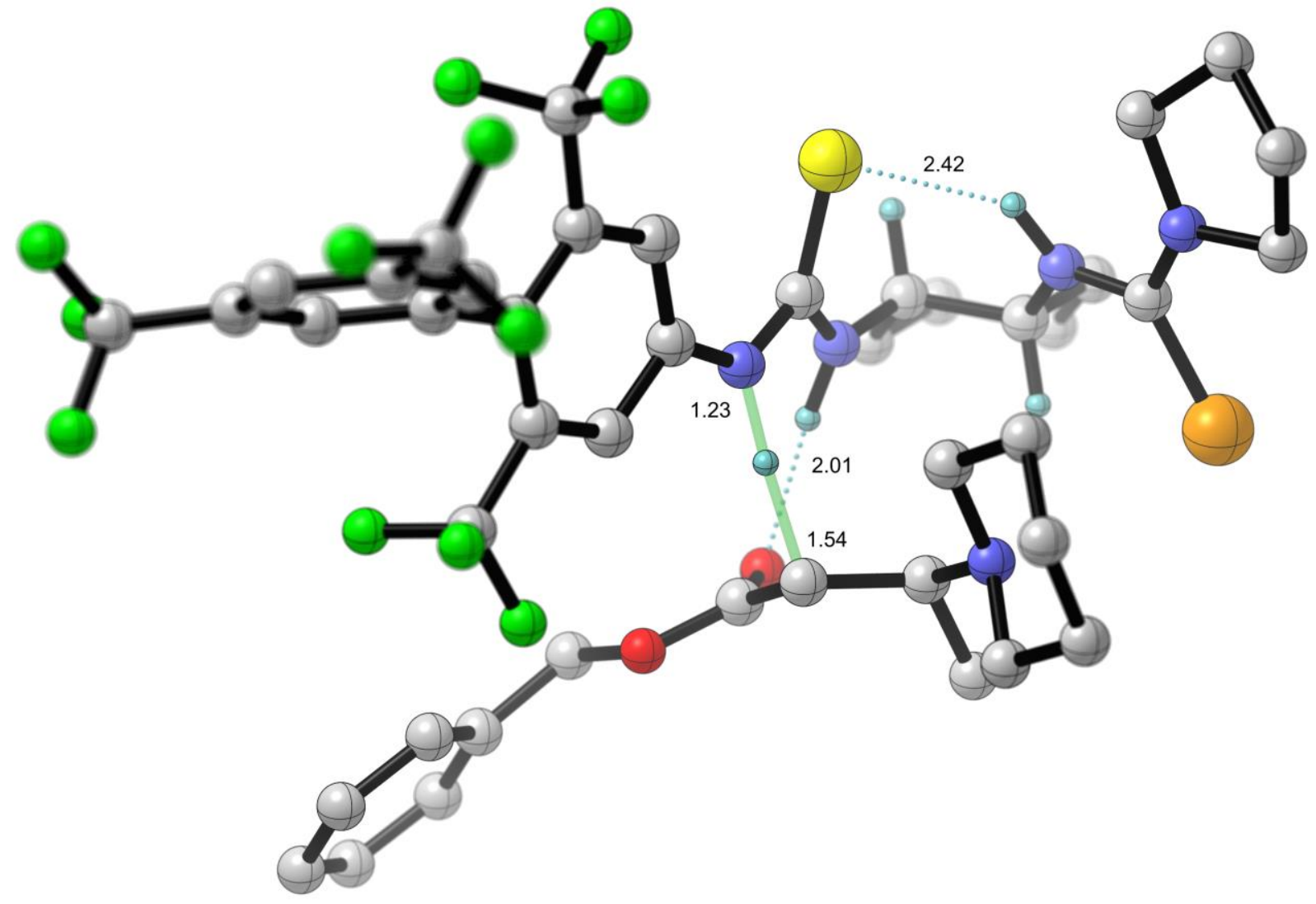

Potential Energy $\quad=-6126.11996439$

Charge $=0$ Multiplicity $=1$

C $-7.27337 \quad 1.45508-2.72382$

C $-6.223092 .04862-3.67012$

C $-4.845152 .11653-2.99836$

C $-4.375200 .74411-2.47823$

C -5.45163 $0.11911-1.55441$

C $-6.835100 .07160-2.23049$

H $-4.092082 .49784-3.69742$

H $-6.16028 \quad 1.43012-4.57723$

H $-6.520593 .05253-3.99642$

H $-7.410732 .12423-1.86233$

H $-8.24723 \quad 1.38314-3.22310$

H $-4.210890 .07195-3.32775$
H $-5.540430 .71661-0.64108$

H -6.79375 -0.62969 -3.07635

H -7.54685 -0.33496 -1.50283

H $-4.877752 .82275-2.15764$

N $-3.088360 .92355-1.80330$

N -5.03860 -1.23437-1.15655

H -2.94596 $1.84655-1.38208$

C $-2.036310 .05655-1.78352$

N $-1.01503 \quad 0.48888-0.98750$

S -2.11107-1.42111-2.66266

H -1.30536 $1.19495-0.01934$

C $\quad 0.304250 .04345-0.91863$

C $0.86233-1.09145-1.53307$ 

C $1.169080 .83204-0.13319$
C $4.51103-1.01279-0.34625$
C $2.20937-1.41298-1.35966$
C $4.86420-2.152350 .38108$
H $\quad 0.24175-1.70927-2.16407$
C $5.53121-0.21026-0.87811$
C $2.50303 \quad 0.491120 .06395$
C $6.20789-2.484760 .57402$
H $\quad \begin{array}{llll}0.78330 & 1.73333 & 0.32500\end{array}$
H $4.09052-2.786900 .79827$
C $3.07156-0.64831-0.54657$
C $6.86836-0.54725-0.68461$
H -4.34477 -1.66934-1.77124
H $\quad 5.278010 .67740-1.44480$
C -5.43470 - $1.88225-0.04567$
C $7.21675-1.687890 .04306$
N $-5.15048-3.197490 .03828$
H $8.25797-1.95077 \quad 0.18778$
C -4.60816 -3.99973-1.08228
C $6.53464-3.731831 .35227$
C $-5.58777-4.053151 .15800$
C $7.965260 .33799-1.21601$
C $-4.88314-5.45322-0.66670$
F $\quad 6.10866-4.84230 \quad 0.70965$
H -5.10950 -3.72592-2.01755
F $5.92802-3.729272 .56257$
C $-4.90187-5.391450 .86867$
F $7.85964-3.87202 \quad 1.56462$
H -5.31304 -3.60053 2.11203
F $8.96989-0.38918-1.75520$
H -5.86222 -5.77161 -1.04286
F $7.52082 \quad 1.18674-2.16570$
H -5.42882 -6.23229 1.32836
F $8.51362 \quad 1.08855-0.23281$
H $-6.68229-4.137031 .14974$
C -1.75205 2.023591 .19611
H $-3.87680-5.38250 \quad 1.25924$
H -1.00191 2.052961 .98861
H $-3.53729-3.79987-1.20781$
C -1.804473 .247750 .43881$
H -4.13220 -6.14146-1.06430
O $-2.698893 .55989-0.36426$
Se -6.33107 -1.01759 1.34539
C $-3.10528 \quad 1.47727 \quad 1.62556$
C 3.319851 .418050 .93883
$\begin{array}{llll}\text { O } & -0.68156 & 4.01253 & 0.57688\end{array}$
C $2.70209-2.65931-2.06822$
C $-0.564145 .13516-0.32057$
F $4.08064 \quad 2.267090 .20421$
H -1.51655 $5.66997-0.36047$
F $2.52872 \quad 2.19547 \quad 1.72045$
H $-0.368034 .75055-1.33038$
F 4.150390 .760531 .77326
C $\quad 0.55108 \quad 6.037850 .14757$
F $1.86512-3.04784-3.05034$
C 1.749945 .524120 .65901
F $3.91663-2.49404-2.63270$
C 0.408877 .424470 .02082
F $2.80407-3.70892-1.20897$
C 2.782706 .383991 .03265 

H $1.87296 \quad 4.452790 .77767$
H $\quad-3.26681 \quad 1.136094 .32895$
C $\quad 1.44542 \quad 8.285140 .38566$
C -1.69448 -1.96131 4.07103
H $-0.521607 .83461-0.36612$
H -1.36044 -2.83585 2.09939
C 2.636447 .766110 .89409
H $-3.05681-2.605392 .51137$
H 3.706125 .971141 .43039
H -2.31056 -0.68355 5.73165
H $\quad \begin{array}{llll}1.31773 & 9.35940 & 0.28038\end{array}$
H -3.64046 -1.26552 4.73292
H 3.444128 .433201 .18365
H -1.78354 -2.90840 4.61437
C -2.04198 -0.833141 .82781$
H - $-0.64569-1.644464 .15458$
C -2.568420 .411683 .91156$
N -2.94904 0.190002 .47142
H -3.90816 -0.22739 2.45077
C -4.039512 .462712 .33659$
C -2.06073 -2.15414 2.59500
H -3.576342 .934103 .20913$
H -1.04172 -0.39817 1.80350
H $-4.25928 \quad 3.25437 \quad 1.61597$
H -2.38234 -0.96016 0.80000
H -4.988741 .993082 .61941$
C -2.60504 -0.89947 4.69835
H $\quad-3.647351 .099770 .75664$
H $-1.56807 \quad 0.85433 \quad 3.91191$
nhprot2d.log
Description: catalyst mediated C-protonation, S conformation 


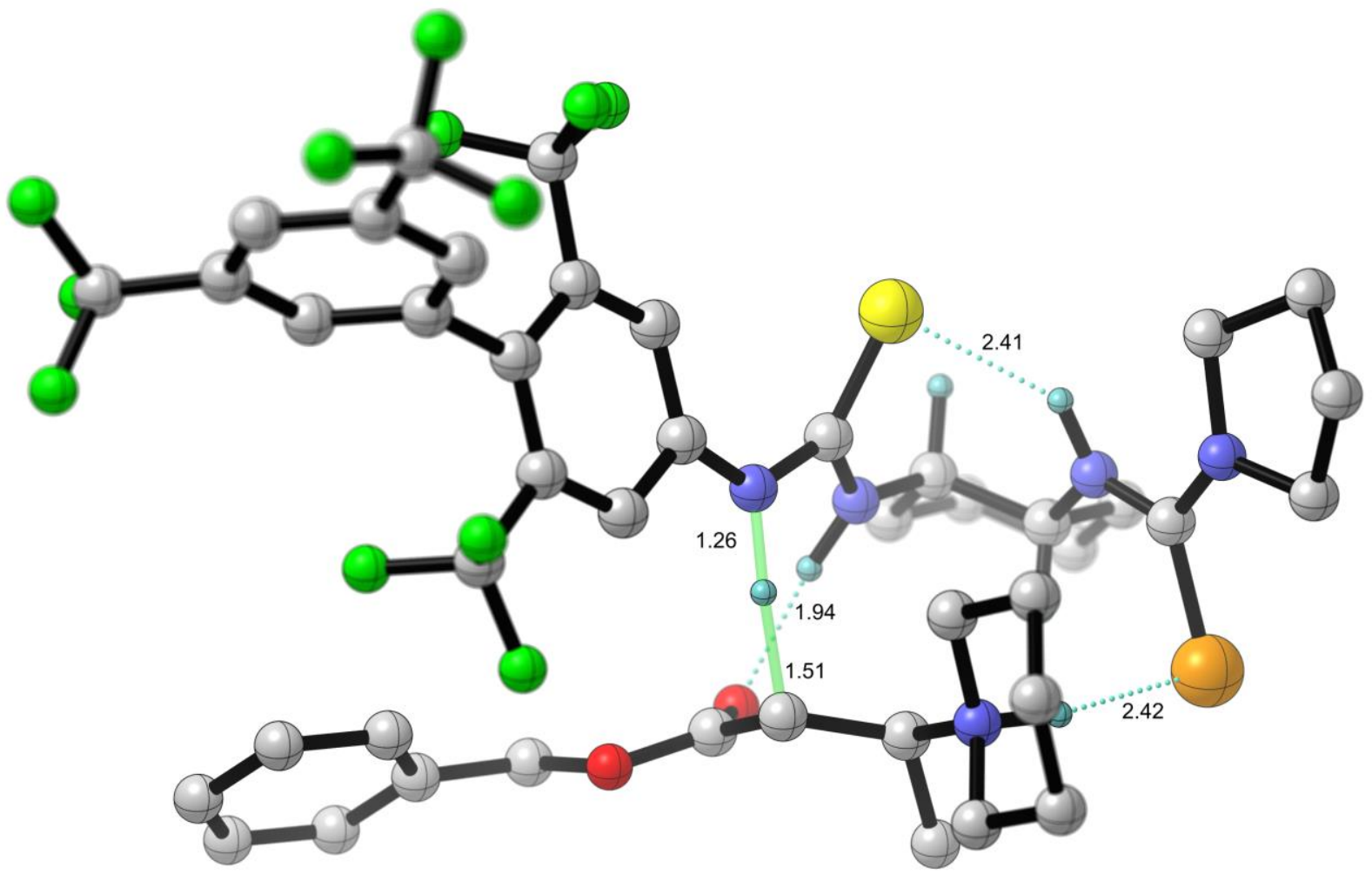

Free Energy $\quad=-6125.337701$

Zero-point Energy $=-6125.230106$

Potential Energy $\quad=-6126.12754804$

Nimag $=1(-1310.4672 \mathrm{~cm}-1)$

Charge $=0$ Multiplicity $=1$

C $-7.00773 \quad 1.61142-3.16527$

C $-5.84258 \quad 2.10161-4.03479$

C $-4.533292 .14227-3.23522$

C $-4.189590 .77941-2.60384$

C $-5.37473 \quad 0.26522-1.75265$

C $-6.689890 .24138-2.55450$

H $-3.69720 \quad 2.44814-3.87441$

H $-5.72464 \quad 1.43059-4.89775$

H $-6.055943 .09813-4.43987$

H $-7.19547 \quad 2.33716-2.36114$
H $-7.930641 .55174-3.75478$

H $\quad-3.988250 .05298$-3.39964

H $\quad-5.516310 .92181-0.88785$

H -6.60634 -0.51125 -3.35162

H $-7.48742-0.08651-1.87779$

H $-4.61000 \quad 2.89498-2.43840$

N $-2.965310 .92297-1.81858$

N $-5.06833-1.07780-1.24210$

H -2.79815 $1.86045-1.43830$

C -1.95556 $0.01957-1.68512$

N $-0.98260 \quad 0.46327-0.84014$ 

S - $2.03071-1.52616-2.43897$
F $3.77696-0.194792 .43424$
H -1.37606 1.159140 .13818
F $2.20722-2.11835-3.66971$
$\begin{array}{llll}\text { C } & 0.33417 & 0.03162 & -0.75356\end{array}$
F $4.15033-1.34698-3.09349$
C $1.01885-0.74058-1.71097$
F $3.35894-3.13404-2.13644$
$\begin{array}{llll}\text { C } & 1.08037 & 0.48507 & 0.35179\end{array}$
C $4.56147-0.97208-0.26193$
C $2.36549-1.05849-1.54535$
C $4.93021-2.22657 \quad 0.23433$
H $0.49534-1.09396-2.58605$
C $5.56656-0.05484-0.59140$
$\begin{array}{llll}\text { C } & 2.42861 & 0.17757 & 0.50518\end{array}$
C $6.27665-2.556600 .39867$
H $\quad 0.59732 \quad 1.112681 .08844$
H $\quad 4.16439-2.947620 .49638$
C $3.11421-0.61792-0.43478$
C $6.91066-0.38868-0.42631$
H -4.34653 - $1.58251-1.76673$
H $5.298250 .91922-0.98319$
C -5.58213 - $1.62844-0.12833$
C $7.27400-1.641140 .06878$
N $-5.39371-2.949900 .05538$
H $8.31903-1.899200 .19447$
C $-4.81680-3.85524-0.96448$
C $6.64938-3.93383 \quad 0.88041$
C -5.94888 -3.69916 1.19919
C $7.981720 .62731-0.72507$
C $-5.23101-5.25594-0.49053$
F $6.70214-4.82205-0.13788$
H -5.21507 -3.60743 - 1.95460
F $5.75064-4.411631 .77060$
C $-5.34857-5.09891 \quad 1.03371$
F $7.86053-3.947031 .47896$
H -5.69145 -3.20468 2.13752
F $9.112470 .04166-1.17820$
H -6.20481 -5.52236 -0.91737
F $7.584381 .52180-1.65473$
H -5.96903 -5.86954 1.49964
F 8.321251 .329940 .38072
H -7.04453 -3.70575 1.13606
C $-1.98025 \quad 2.00555 \quad 1.23411$
H $-4.35503-5.141741 .49671$
H -1.365852 .047372 .13663$
H -3.72734 -3.73633-1.00412
C $-1.872943 .22401 \quad 0.46154$
H - $-4.51133-6.02090-0.79463$
O $-2.600713 .54241-0.49040$
Se -6.51143 -0.62019 1.14098
C $-3.40446 \quad 1.51548 \quad 1.46989$
C 3.145900 .750901 .70597
O $-0.77557 \quad 3.957700 .79365$
C $3.01838-1.91048-2.61400$
C $-0.422595 .03542-0.09298$
F $4.08563 \quad 1.663461 .34419$
H -1.150545 .847800 .01173$
$\begin{array}{llll}\text { F } & 2.30060 & 1.39187 & 2.54670\end{array}$
H $-0.491024 .67543-1.12630$ 

C $\quad 0.97263 \quad 5.50756 \quad 0.24109$
C $-3.43162-0.77878 \quad 4.64450$
C 1.960724 .613440 .67043
H $-2.26430 \quad 0.938493 .97279$
C 1.308616 .855560 .07204
H $-4.00210 \quad 1.249924 .12423$
C 3.258705 .056910 .92168
C $-2.45751-1.870244 .18773$
H $1.708093 .57033 \quad 0.82415$
H -1.86877 -2.81643 2.31101
C 2.609377 .300400 .31452
H $\quad-3.60132-2.552702 .47877$
H $\quad 0.54778 \quad 7.56347-0.25072$
H $\quad-3.28517-0.533315 .70256$
C 3.588596 .401690 .74076
H -4.46716 -1.13090 4.54083
H 4.007154 .344621 .25716
H -2.63405 -2.79699 4.74458
H $2.85410 \quad 8.35070 \quad 0.17868$
H - $1.42626-1.559624 .40538$
H $\quad 4.60043 \quad 6.747610 .93482$
$\begin{array}{llll}\text { N } & -3.42740 & 0.25073 & 2.35984\end{array}$
C $-2.46833-0.816141 .88279$
C $-4.395292 .55232 \quad 2.01043$
C -3.259970 .506863 .83421$
H -4.050353 .031562 .93202$
H $-4.39067-0.13500 \quad 2.20255$
H $\quad-4.479883 .331541 .24855$
C -2.61914-2.10942 2.68241
H -5.391262 .118512 .15728$
H -1.46226 -0.40572 1.98171
H $\quad-3.82493 \quad 1.132940 .53875$
H -2.66716-0.97294 0.82198

nhprot2e.log

Description: catalyst mediated C-protonation, S conformation 


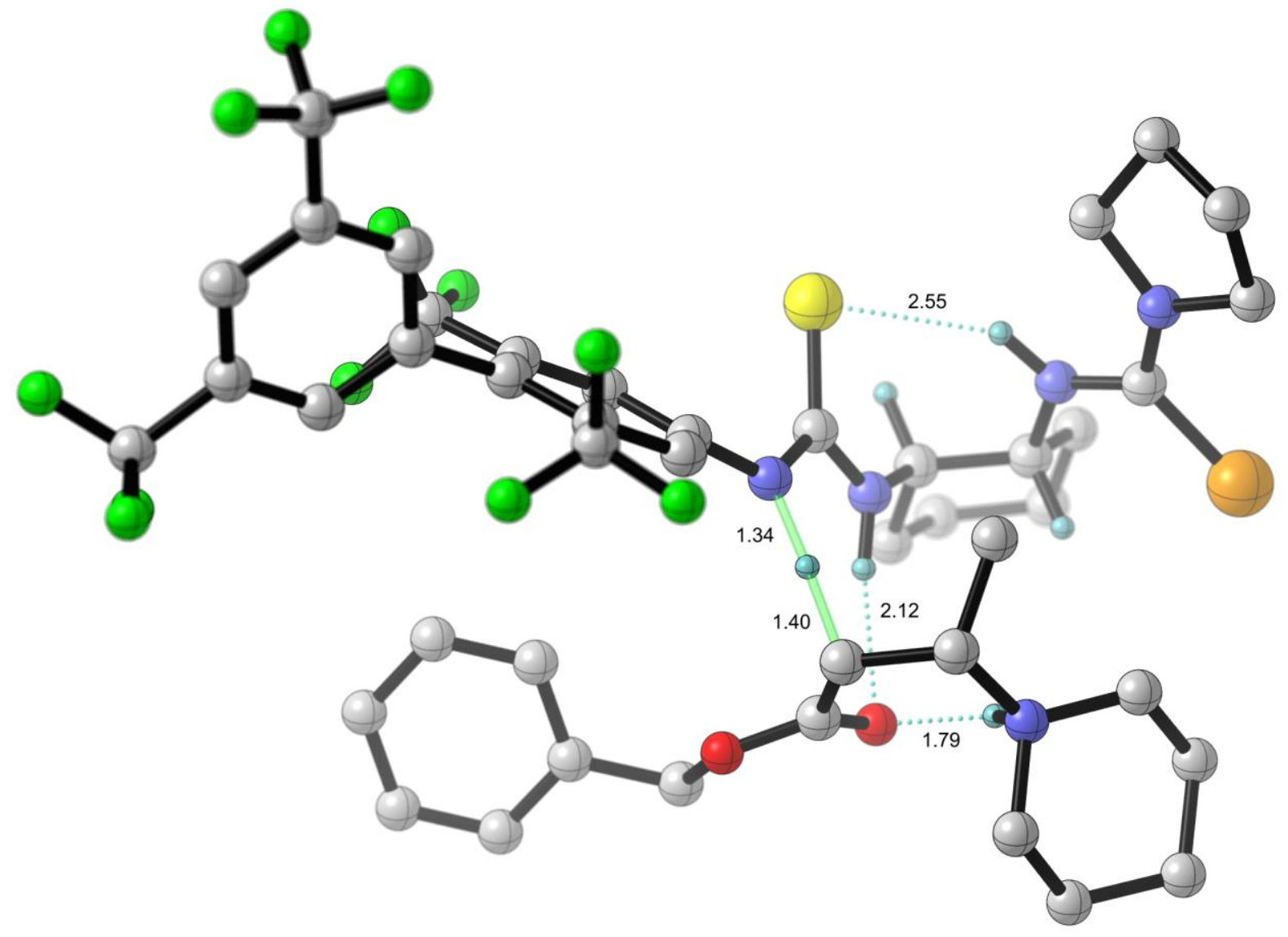

Free Energy $\quad=-6125.335309$

Zero-point Energy $=-6125.227541$

Potential Energy $\quad=-6126.12500306$

Nimag $=1(-1362.0571 \mathrm{~cm}-1)$

Charge $=0$ Multiplicity $=1$

C $6.44613 \quad 1.086703 .56990$

C $5.20244 \quad 1.870304 .01308$

C $4.12465 \quad 1.883602 .91787$

C $3.75001 \quad 0.461002 .46339$

C $5.01175-0.296372 .00535$

C $6.07444-0.331013 .11769$

H $3.21942 \quad 2.390093 .27350$

H 4.787301 .410234 .92116
H 5.469782 .900414 .27975

H 6.936311 .614992 .73906

H 7.178601 .042024 .38513

H $3.29897-0.078223 .30298$

H $5.44745 \quad 0.21243 \quad 1.13666$

H $5.68230-0.906243 .96870$

H $6.94934-0.865052 .73246$

H 4.489142 .453122 .04967

N $2.73277 \quad 0.52462 \quad 1.41558$ 

N $4.64693-1.643501 .56365$
C -3.09120 - $1.07651-2.42839$
H $2.80293 \quad 1.327900 .79692$
C $-3.68991 \quad 0.022272 .49845$
C $\quad 1.57522-0.20467 \quad 1.36738$
F -4.06918 -0.24169-2.84191
N $\quad 0.74766 \quad 0.149920 .35525$
F -2.12394 -1.04002 -3.37567
S $1.30293-1.502982 .45860$
F -3.60398 -2.33039 -2.45262
H $1.21648 \quad 0.73455-0.75626$
F -2.95497 $0.10806 \quad 3.62428$
C $-0.60415-0.17740 \quad 0.30087$
F $-4.36622 \quad 1.20271 \quad 2.38897$
C -1.46430 -0.04656 1.40534
F -4.61466 -0.93746 2.70216
C $-1.18278-0.52237-0.92832$
C $-4.91272-0.72960-0.10923$
C $-2.83742-0.22806 \quad 1.27434$
C $-5.48328-2.00537-0.16268$
H $-1.04809 \quad 0.208712 .37010$
C $-5.749390 .38977-0.19301$
C $-2.55926-0.70676-1.06183$
C -6.86487 -2.15766 -0.29276
H $-0.54929-0.64463-1.79683$
H -4.84764 -2.88154-0.10575
C $-3.42938-0.55901 \quad 0.03502$
C $-7.128530 .23265-0.32815$
H $3.74532-1.985611 .90335$
H $\quad-5.32295 \quad 1.38497-0.14849$
C $5.33287-2.349370 .64042$
C $-7.69465-1.04131-0.37630$
N $\quad 4.88756-3.59050 \quad 0.35990$
H -8.76681 -1.16246 -0.47789
C $3.80482-4.275381 .09453$
C $-7.46520-3.53912-0.27998$
C $5.50928-4.46152-0.65117$
C $-8.01402 \quad 1.44047-0.48624$
C $3.93495-5.739570 .65000$
F -7.67618 -3.97852 0.98198
H $3.93784-4.149282 .17502$
F -6.65776-4.44104 -0.87934
C $4.52514-5.63214-0.76522$
F -8.65791 -3.57432 -0.91413
H $5.65934-3.90505-1.57895$
F $-9.22361 \quad 1.250300 .08612$
H $4.63280-6.268841 .30940$
F -7.46857 2.543890 .06893
H $5.01499-6.55168-1.09834$
F $-8.23922 \quad 1.72409-1.79034$
H $6.50144-4.78201-0.30745$
C $1.67517 \quad 1.38855-1.90676$
H $3.73470-5.39244-1.48701$
H $\quad 0.77443 \quad 1.52129-2.50505$
H $2.82785-3.849360 .83159$
C $2.064032 .60473-1.23304$
H $2.97678-6.265590 .68446$
O $3.175002 .79726-0.68447$
Se $6.83483-1.66171-0.20690$
C $2.726590 .50961-2.61090$ 


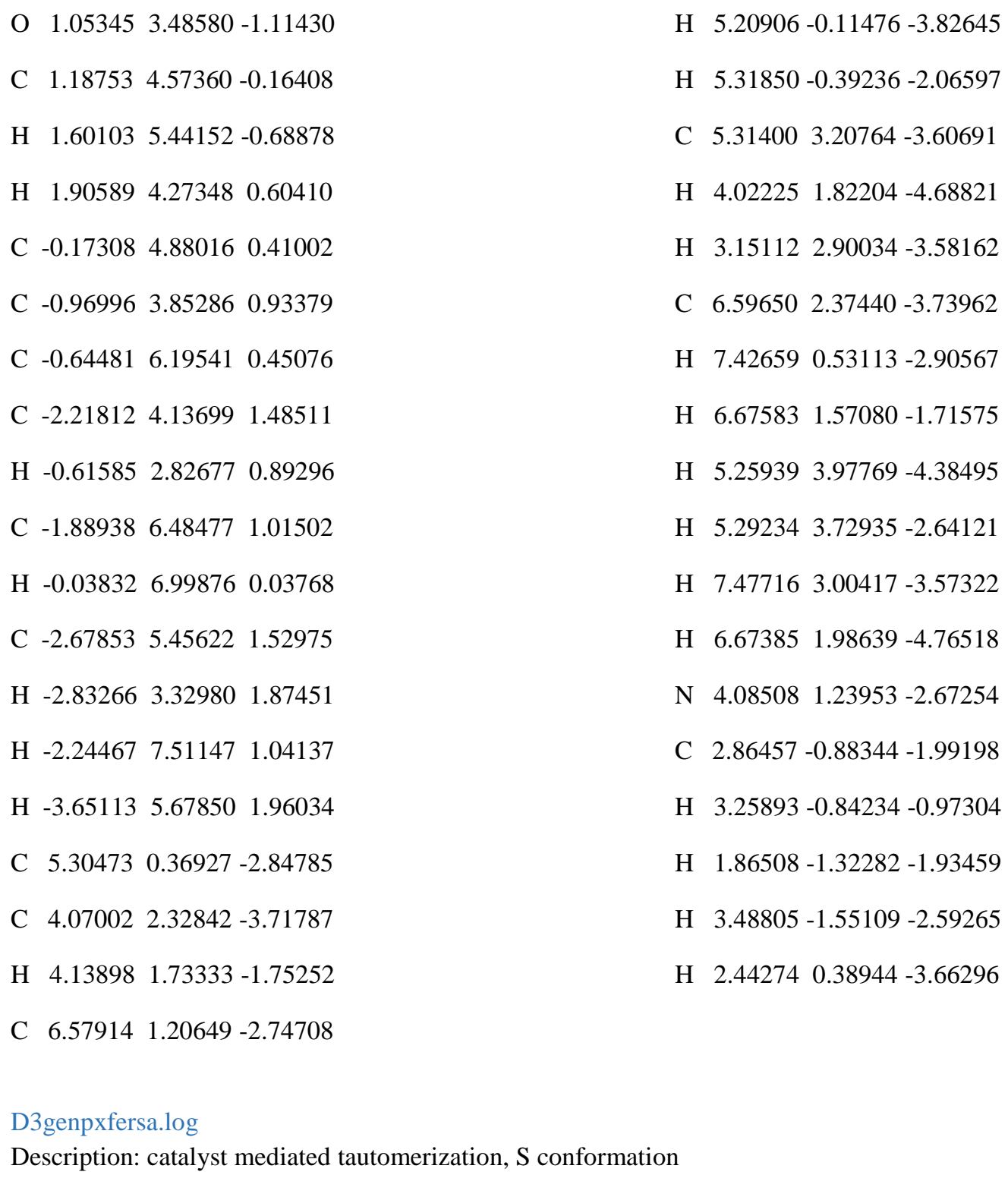




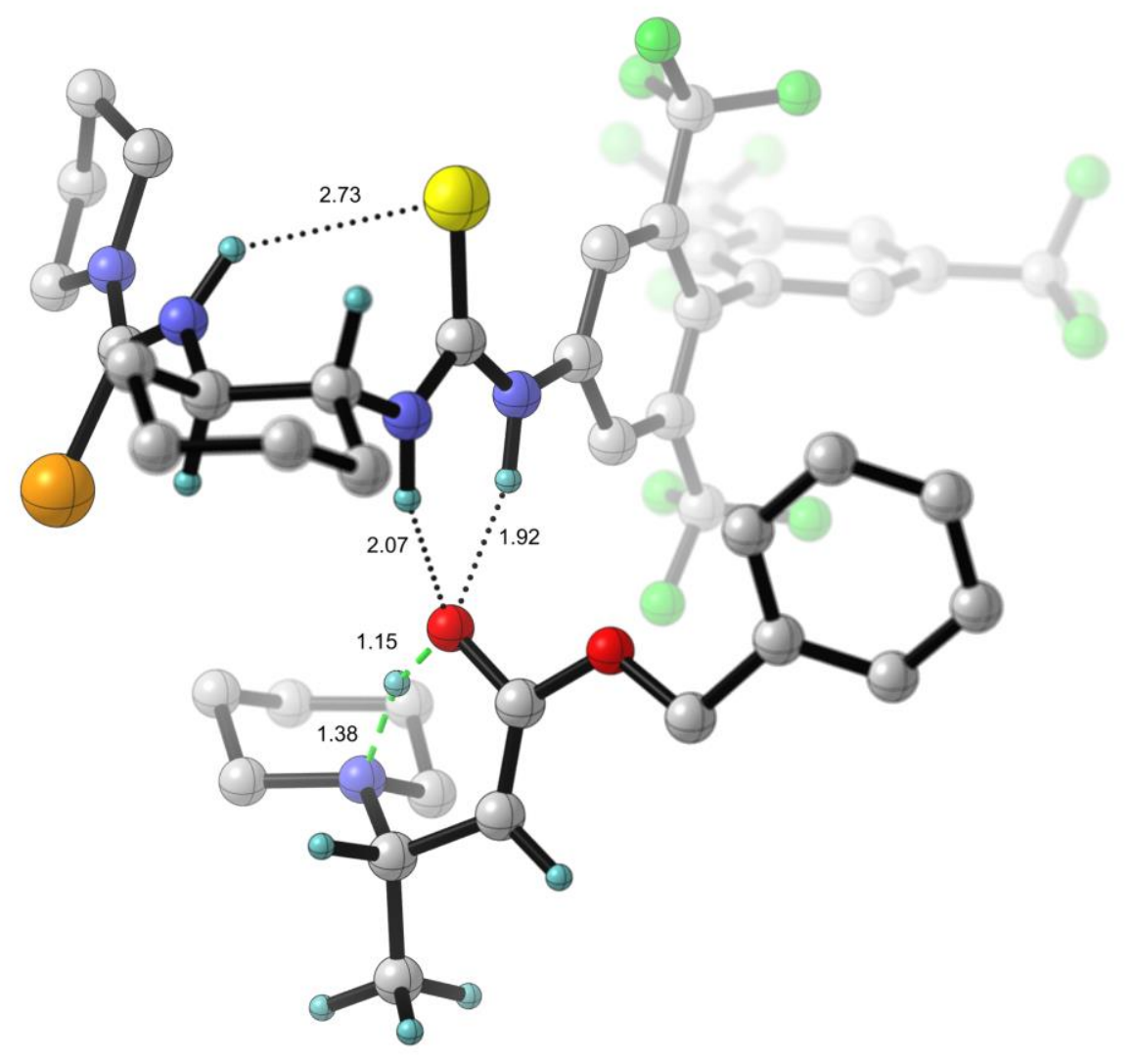

Free Energy = -6124.856406

Zero-point Energy $=-6124.744158$

Potential Energy $\quad=-6129.58841392$

Nimag = $1(-1039.1839 \mathrm{~cm}-1)$

Charge $=0$ Multiplicity $=1$

C $7.45640-0.14352-2.31917$

C $6.67892-1.35826-2.84621$

C $5.34746-1.53979-2.10073$

C $4.48833-0.26406-2.16093$

C $5.278400 .94177-1.60111$

C $6.599731 .12973-2.36893$

H $4.77612-2.37421-2.52418$

H $6.47585-1.22394-3.91842$

H $7.28093-2.27048-2.75415$
H $7.76539-0.32971-1.28046$

H $8.376800 .00011-2.89801$

H $4.21872-0.06604-3.20295$

H $5.511100 .75673-0.54722$

H $6.376201 .38984-3.41407$

H $7.137291 .97885-1.93260$

H $5.54850-1.79059-1.04833$

N $3.23558-0.46274-1.44121$

N $4.452702 .14609-1.63825$

H $3.31144-0.80840-0.48362$ 


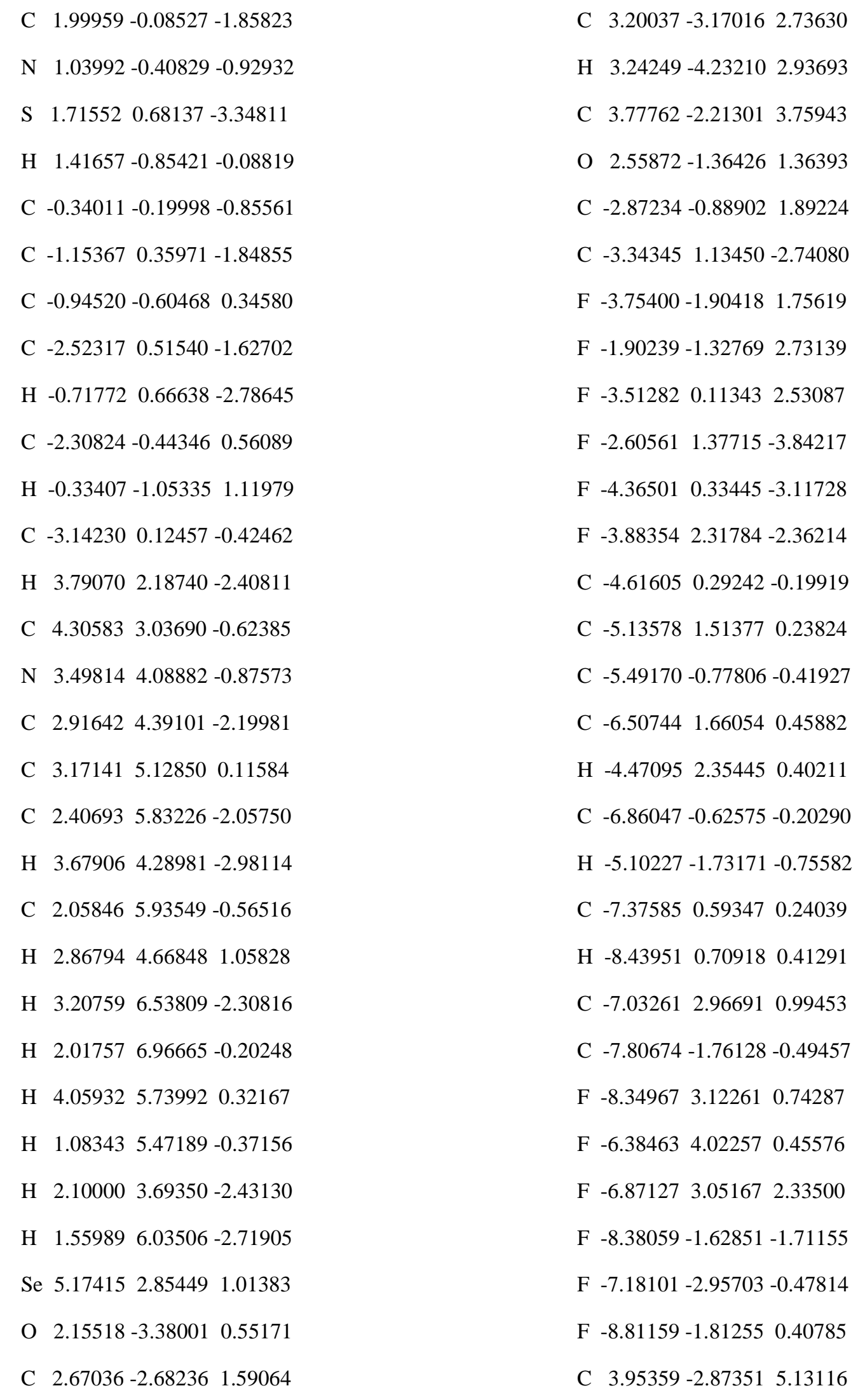



H $4.66777-3.699065 .04005$
C $1.62945-1.089574 .43891$
H $4.34956-2.174095 .87452$
C 3.691660 .238004 .28973
H $3.01307-3.289425 .50612$
C $\quad 0.769580 .157694 .21828$
H $2.71998-0.923912 .41660$
H $1.77376-1.251985 .51723$
C $2.30542-4.802870 .55598$
H $\quad 1.15040-1.981894 .02583$
H $3.36894-5.040920 .70597$
C 2.880211 .524984 .11146
H $\quad 1.74808-5.23081 \quad 1.39997$
H 3.931990 .084545 .35339
C $1.79867-5.35745-0.75380$
H 4.633760 .308633 .73711
C $1.17791-6.61100-0.78338$
C $\quad 1.48625 \quad 1.408714 .74174$
C $1.97739-4.65650-1.95307$
H -0.197660 .014214 .71342$
C $0.75076-7.16322-1.99238$
H $\quad 0.560890 .269373 .14641$
H $\quad 1.02275-7.15824 \quad 0.14415$
H 3.443462 .353884 .55571
C $1.54018-5.20281-3.16003$
H 2.801841 .748643 .04018
H $2.44118-3.67516-1.93490$
H 0.896142 .307964 .52996
C $0.92963-6.45907-3.18412$
H 1.578851 .342175 .83589
H $\quad 0.26842-8.13697-2.00015$
H $4.76682-1.863023 .42813$
H $1.67531-4.64445-4.08246$
N $2.95145-0.941963 .77545$
H $\quad 0.58972-6.88300-4.12509$
D3genpxferra2.log
Description: catalyst mediated tautomerization, S conformation 


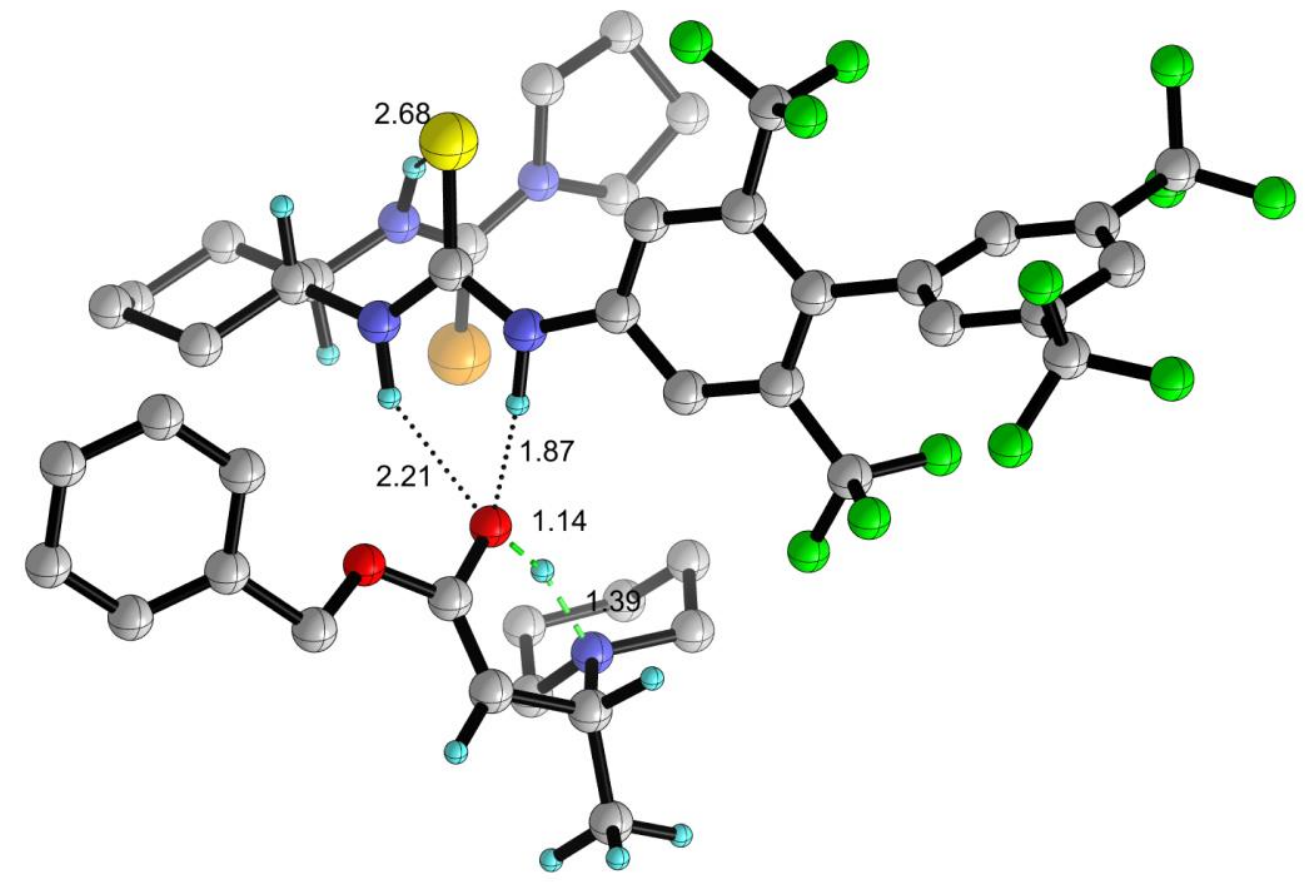

Free Energy $\quad=-6124.854740$

Zero-point Energy $=-6124.745481$

Potential Energy $\quad=-6129.58873955$

Nimag $=1(-1033.5157 \mathrm{~cm}-1)$
Charge $=0$ Multiplicity $=1$
H -4.11715 -0.42396 -3.10317
C -7.31217-0.60667-2.04370
H -5.18819-1.15238 -0.32410
C $-6.695490 .62727-2.71725$
H -6.13295 -2.11435 -3.07030
C $-5.35670 \quad 1.01280-2.06941$
H -6.74702 -2.64175 -1.49878
C $-4.36895-0.16794-2.06932$
H -5.52936 $1.33787-1.03264$
C $-4.99528-1.39865-1.37390$
N $-3.121330 .22535-1.42106$
C -6.32865 -1.78549 -2.03906
N -4.04804 -2.51285 -1.37580
H $-4.898751 .86085-2.59072$
H $\quad-3.21727 \quad 0.69789-0.52323$
H $-6.533350 .41713-3.78445$
C -1.86681 -0.07491-1.83307
H $-7.385491 .47855-2.66866$
N $-0.922010 .39686-0.94951$
H -7.58505 -0.35993-1.00748
S - $-1.53242-0.93454-3.26047$
H -8.24086 - $0.89453-2.55133$
H $-1.321190 .78573-0.08867$ 

$\begin{array}{llll}\text { C } & 0.47024 & 0.28387 & -0.89484\end{array}$
O $-2.31751 \quad 1.28793 \quad 1.41048$
C $1.31675-0.07434-1.95121$
C 2.966060 .899151 .90056
C 1.053940 .600460 .34195
C $3.55086-0.54045-2.93892$
C $2.69769-0.13204-1.75420$
F $3.69738 \quad 2.03558 \quad 1.88152$
H $\quad 0.89785-0.30902-2.91721$
F $\quad 1.96579 \quad 1.103852 .79941$
$\begin{array}{llll}\text { C } & 2.42920 & 0.54581 & 0.53129\end{array}$
F $3.74834-0.072862 .41002$
H $\quad 0.41326 \quad 0.88728 \quad 1.16661$
F $2.82715-0.65417-4.06992$
C $3.297050 .17091-0.51576$
F $4.532890 .35297-3.18594$
H -3.39073 -2.51913 -2.15109
F $4.14621-1.73983-2.73164$
C -3.84144-3.37684-0.35018
C $4.782140 .10051-0.31646$
N - $2.93733-4.35525-0.57255$
C $5.41295-1.13030-0.10529$
C $-2.30948-4.62586-1.88190$
C $5.557871 .26486-0.33168$
C $-2.54061-5.34788 \quad 0.44077$
C $6.79367-1.192860 .08943$
C -1.67636-6.01360 - 1.70652
H $4.82468-2.04047-0.08311$
H -3.06453 -4.60650 -2.67692
C $6.938621 .19718-0.14020$
C - $1.34752-6.06251-0.20695$
H $5.08247 \quad 2.22691-0.48500$
H -2.29835 -4.84961 1.38156
C $7.56423-0.030880 .07198$
H -2.40635 -6.79192 -1.95846
H $8.63407-0.08017 \quad 0.23543$
H -1.22115 -7.08013 0.17385
C $7.45498-2.534830 .26959$
H -3.37490 -6.03279 0.63982
C $7.759182 .45864-0.21710$
H -0.42173 -5.50998 -0.00495
F $6.67369-3.384520 .97235$
H -1.55551 -3.86196 -2.11486
F $8.63390-2.431340 .91963$
H -0.80294 -6.15042 -2.35056
F $7.70740-3.12580-0.91934$
Se -4.75298 -3.25004 1.26793
F $8.079002 .76284-1.49487$
$\begin{array}{llll}\text { O } & -3.05109 & 3.16627 & 0.50543\end{array}$
F 7.089513 .519960 .28316
C $-2.499992 .60357 \quad 1.60713$
F $8.91862 \quad 2.34698 \quad 0.46591$
C $-2.18193 \quad 3.20283 \quad 2.77800$
C -1.559263 .096815 .22866$
H -2.384914 .250232 .95606$
H $-2.58862 \quad 3.31527 \quad 5.53000$
C -1.500162 .396183 .86675$
H -1.082572 .501626 .01442$
H -0.438792 .252813 .61413$
H -1.022684 .049575 .16373$ 


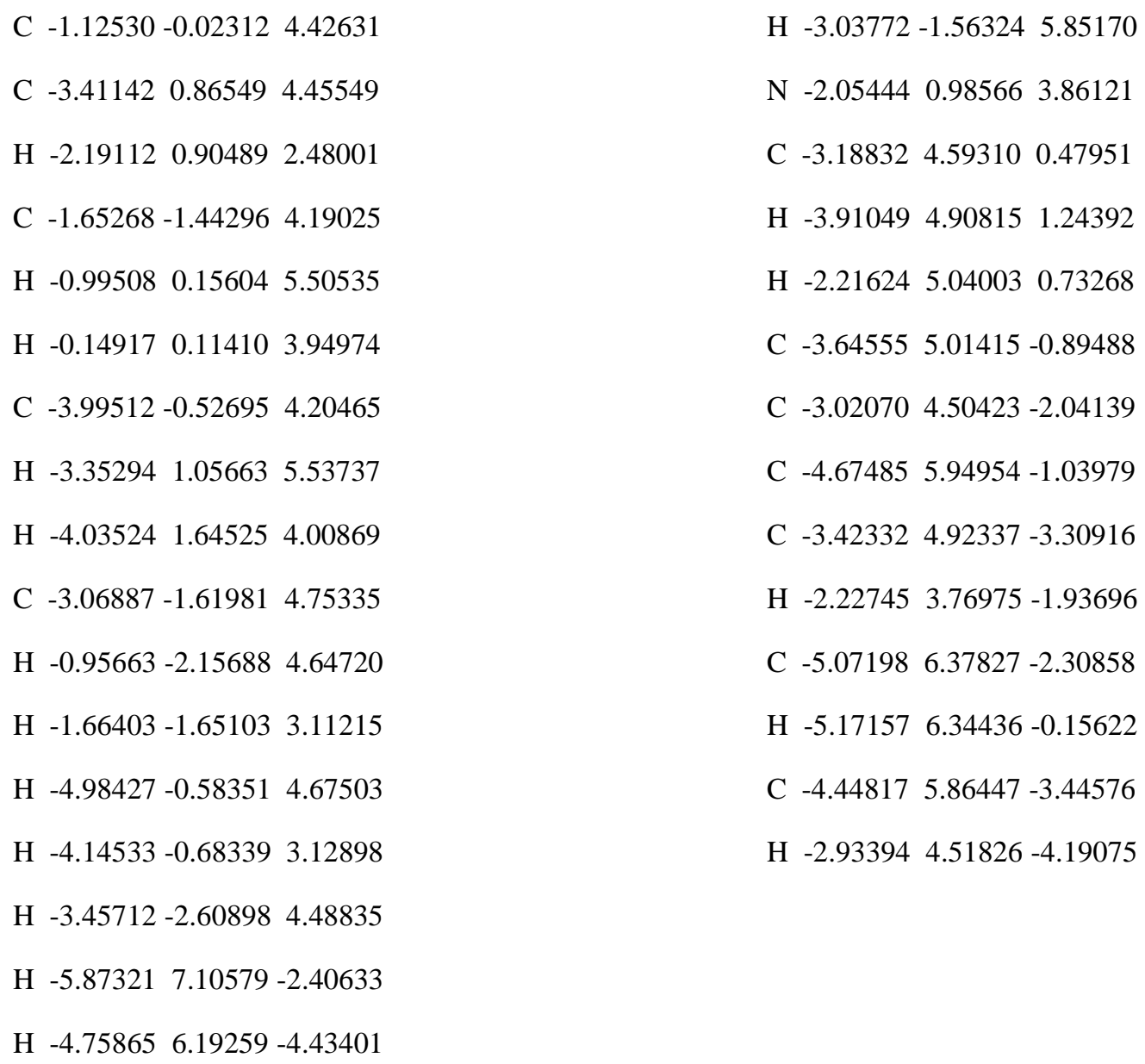




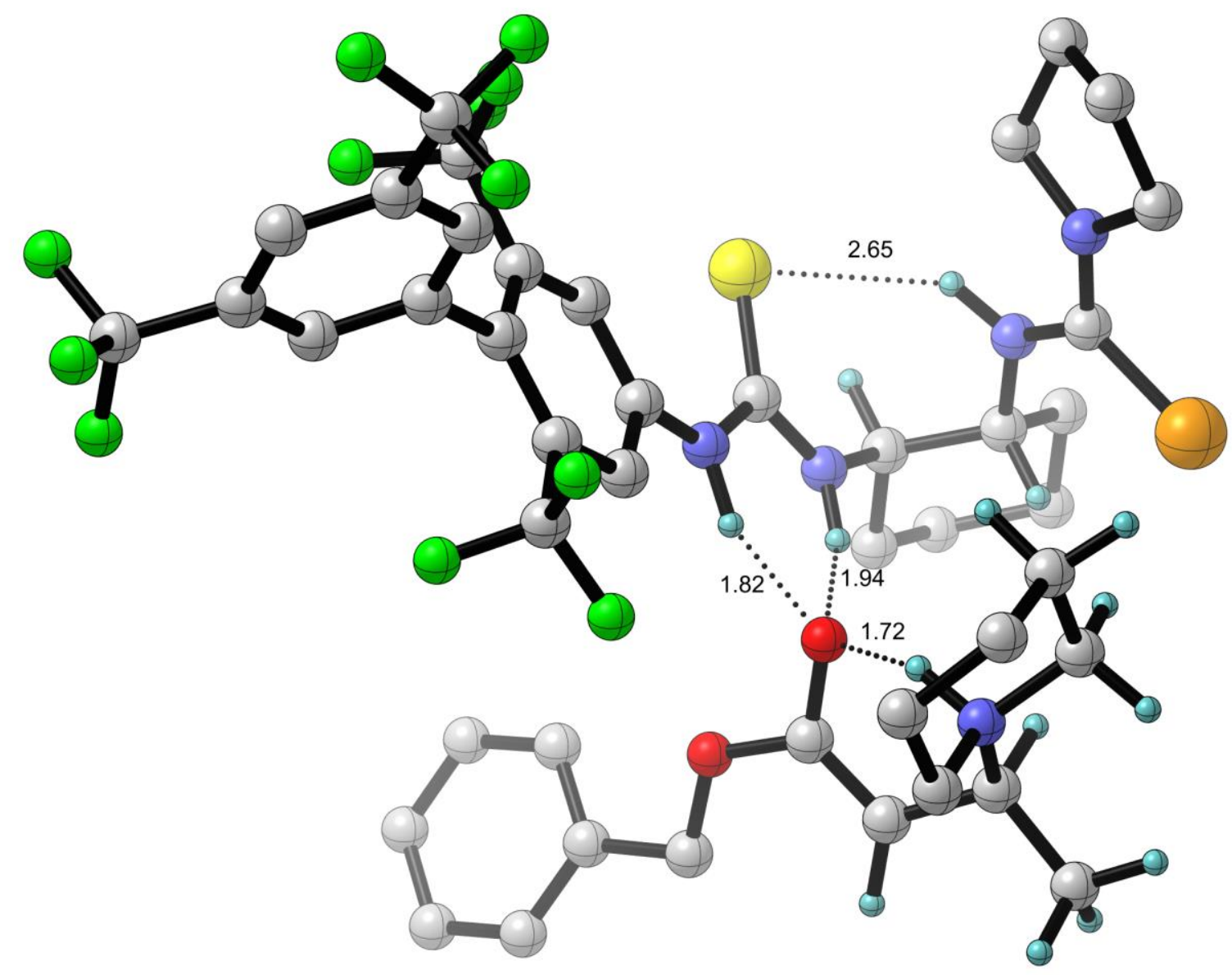

Free Energy $\quad=-6124.860472$

Zero-point Energy $=-6124.748078$

Potential Energy $\quad=-6129.59710113$

Nimag $\quad=1(5.6994 \mathrm{~cm}-1)$

Charge $=0$ Multiplicity $=1$

C $-7.28585-0.18633-2.39813$

C $-6.55202 \quad 1.01485-3.01203$

C $-5.21862 \quad 1.28127-2.29757$

C $-4.320520 .03057-2.28353$

C -5.06966 -1.15844 -1.63834

C $-6.39143-1.43415-2.37816$

H $-4.67483 \quad 2.10142-2.78070$
H $-6.35790 \quad 0.81828-4.07629$

H -7.18038 1.91297 -2.96999

H $-7.587880 .05677-1.36903$

H -8.20838 -0.39509-2.95337

H -4.05387 -0.22979 -3.31286

H $-5.29834-0.91249-0.59547$

H -6.16654 -1.75116 -3.40700

H -6.89883 -2.26997 -1.88440 


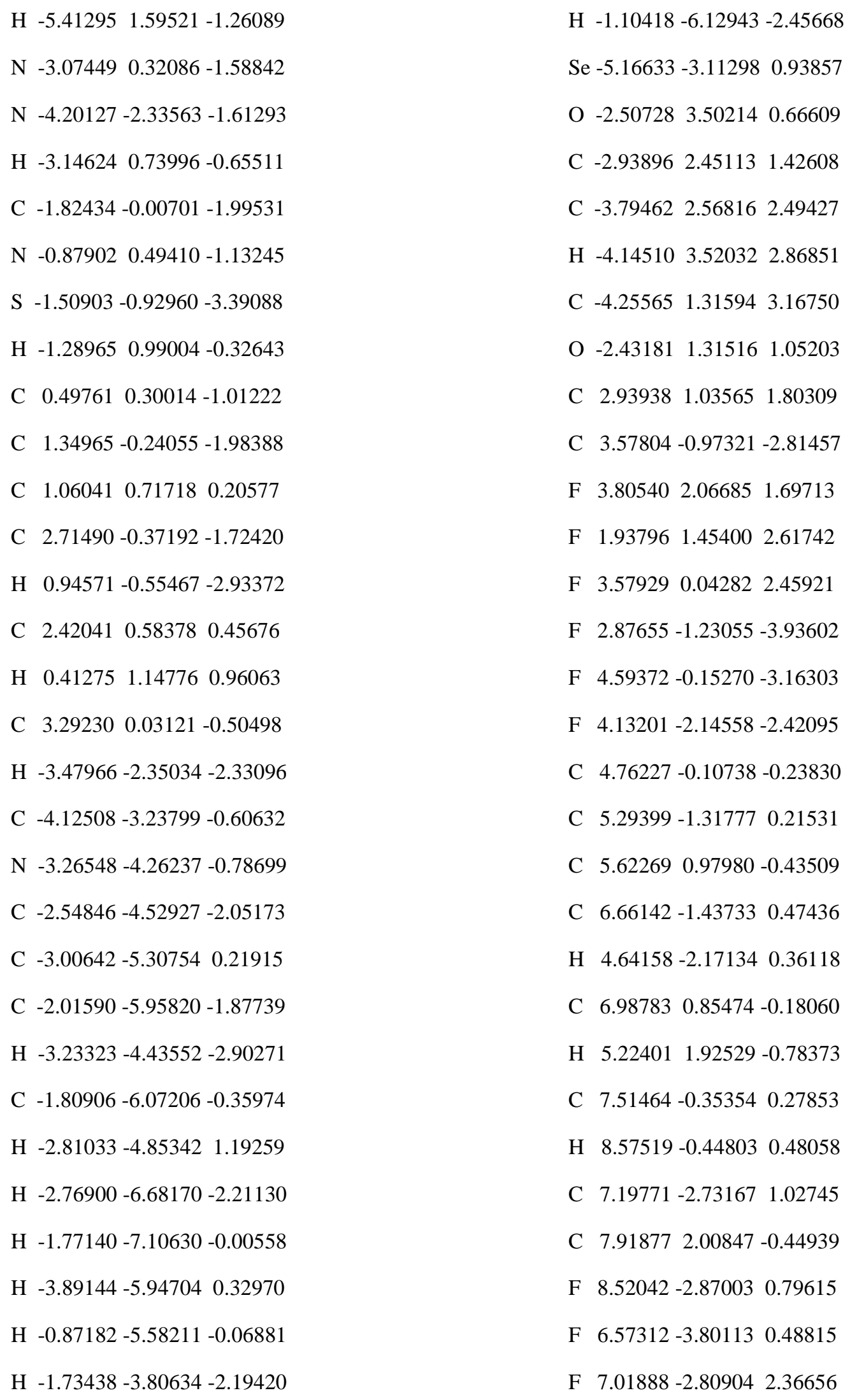




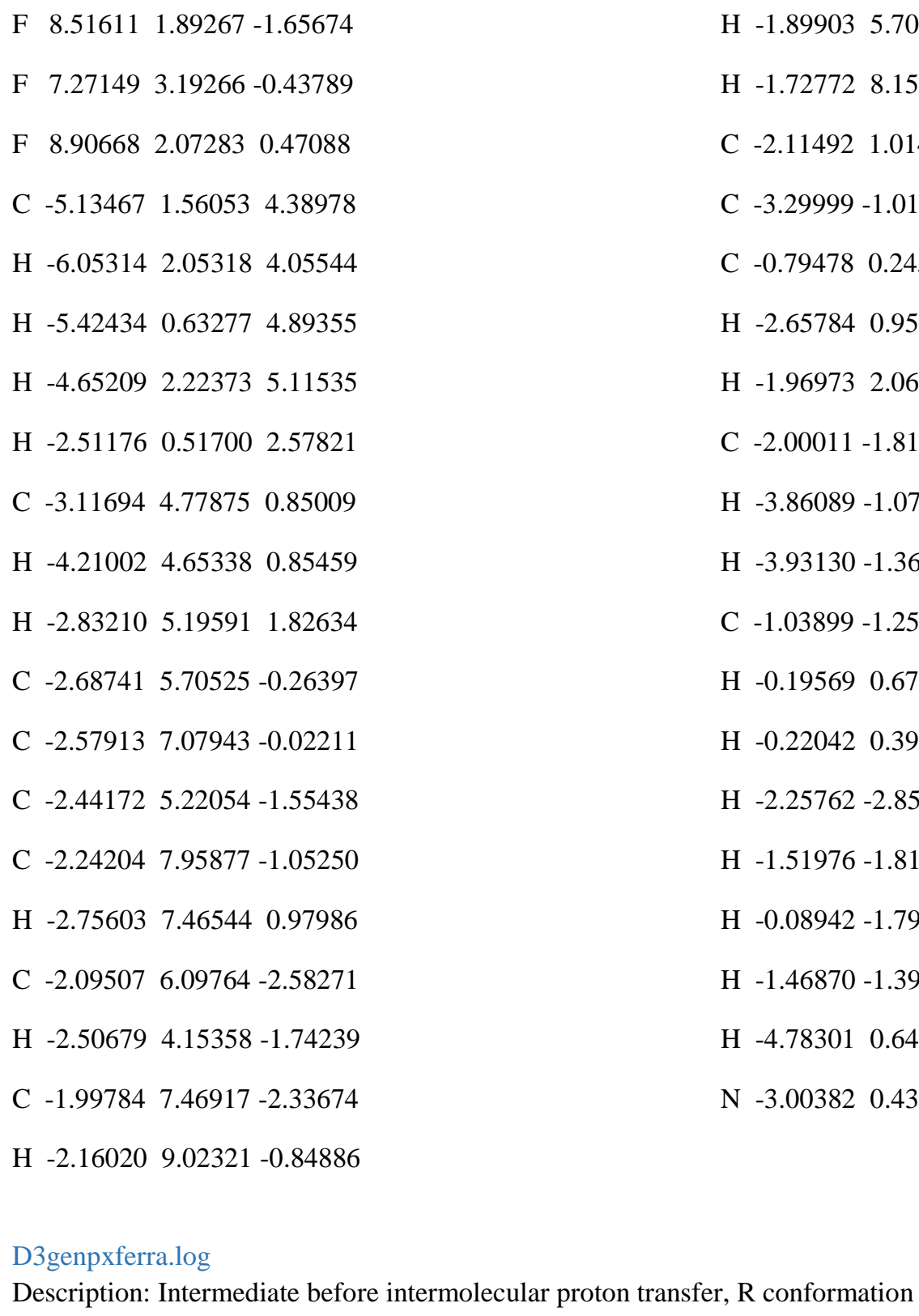




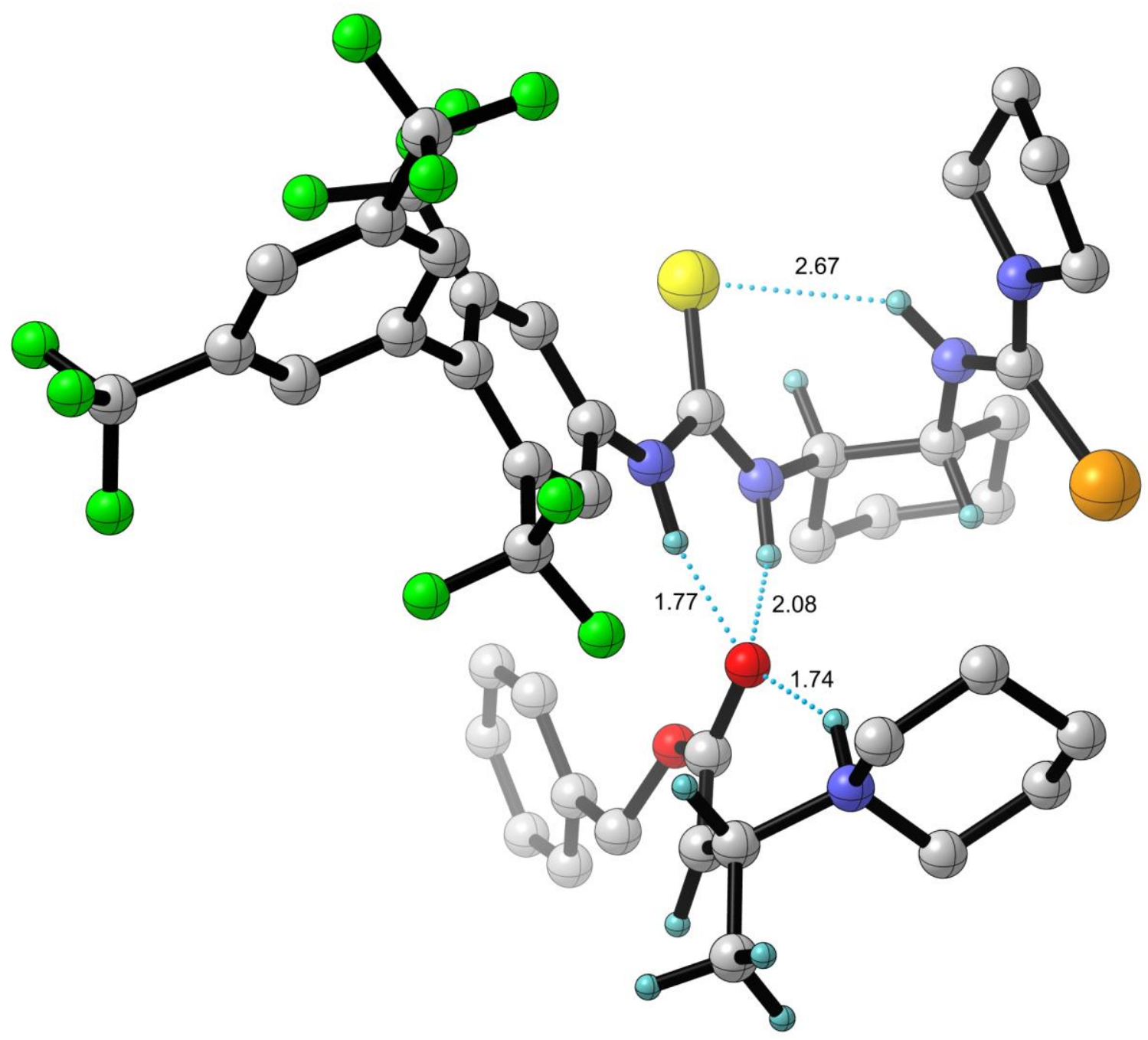

Free Energy $\quad=-6124.860043$

Zero-point Energy $=-6124.748505$

Potential Energy $\quad=-6129.59808328$

Nimag $\quad=1(5.9293 \mathrm{~cm}-1)$

Charge $=0$ Multiplicity $=1$

C -7.22244-1.02865 -2.05030

C $-6.70845 \quad 0.24434-2.73854$

C $-5.393020 .73252-2.11285$

C $-4.32123-0.37211-2.12008$
C $-4.84682-1.63720-1.40011$

C $-6.15318-2.13070-2.04741$

H -5.00792 $1.60742-2.64803$

H $\quad-6.546310 .03957-3.80679$

H -7.46012 $1.04125-2.68422$ 


\begin{tabular}{|c|c|}
\hline H - $-7.50226-0.79552-1.01274$ & H $-1.73308-4.73264 \quad 1.35848$ \\
\hline H - $-8.13198-1.39016-2.54551$ & $\mathrm{H}-1.88102-6.83281-1.88160$ \\
\hline H $-4.07450-0.62529-3.15626$ & $\begin{array}{llll}\mathrm{H} & -0.55981 & -6.93166 & 0.18786\end{array}$ \\
\hline H -5.04702 -1.39208 -0.35207 & H - $2.75853-6.022420 .73045$ \\
\hline H $-5.94409-2.45231-3.07825$ & H $\quad 0.10739-5.31758-0.10735$ \\
\hline H - $-6.50224-3.01209-1.49785$ & H - $1.26020-3.85923-2.21476$ \\
\hline H $-5.57136 \quad 1.05536-1.07686$ & H $-0.35362-6.09766-2.39487$ \\
\hline $\mathrm{N}-3.09355 \quad 0.11264-1.50345$ & Se $-4.31929-3.33849 \quad 1.31497$ \\
\hline $\mathrm{N}-3.81752-2.67684-1.39914$ & $\begin{array}{llll}O & -3.41592 & 3.01662 & 0.46759\end{array}$ \\
\hline H $-3.187260 .56698-0.59084$ & C -2.721602 .416851 .48483 \\
\hline C $-1.83114-0.10278-1.94155$ & C -2.500932 .995322 .71125 \\
\hline N $-0.911090 .46934-1.09472$ & H $-2.91823 \quad 3.950652 .99832$ \\
\hline S - $-1.45694-0.97835-3.35261$ & C -1.64891 2.260123 .69194 \\
\hline H - $-1.33822 \quad 0.86919-0.24300$ & H $-0.63843 \quad 2.07625 \quad 3.30085$ \\
\hline C $\quad 0.478370 .37730-1.01465$ & $\begin{array}{llll}\mathrm{O} & -2.32880 & 1.21866 & 1.18338\end{array}$ \\
\hline C $1.354410 .06674-2.06246$ & C 2.894200 .941641 .85793 \\
\hline $\begin{array}{llll}\text { C } & 1.02818 & 0.66798 & 0.24385\end{array}$ & C $3.61895-0.32169-3.01320$ \\
\hline C $2.731920 .03152-1.83625$ & F 3.585972 .101071 .91192 \\
\hline H $\quad 0.96087-0.14553-3.04450$ & F 1.866821 .070672 .74381 \\
\hline C 2.399190 .633090 .46330 & F $3.69688-0.026422 .34431$ \\
\hline $\begin{array}{lllll}\text { H } & 0.35574 & 0.91220 & 1.05675\end{array}$ & F $2.92508-0.40428-4.16527$ \\
\hline C $3.297750 .30937-0.57561$ & F $4.595370 .59164-3.20427$ \\
\hline H $-3.18256-2.64751-2.19371$ & F $\quad 4.22642-1.52085-2.83518$ \\
\hline C $-3.48380-3.46439-0.34807$ & C $4.779110 .26966-0.34434$ \\
\hline $\mathrm{N}-2.51430-4.37637-0.57457$ & C $5.44189-0.95470-0.17973$ \\
\hline C $-1.94058-4.66388-1.90632$ & C $5.51953 \quad 1.45285-0.28211$ \\
\hline C $-1.98942-5.289360 .45503$ & C $6.81605-0.986750 .04519$ \\
\hline C $-1.19810-5.99281-1.70772$ & H $\quad 4.88225-1.88078-0.22621$ \\
\hline H - $-2.73868-4.73569-2.65465$ & C $6.899431 .41520-0.06181$ \\
\hline C $-0.78350-5.94560-0.22956$ & H $5.020412 .40795-0.40063$ \\
\hline
\end{tabular}



C $7.55395 \quad 0.198210 .10380$
C -3.07506 -1.79154 4.90270
H $8.62277 \quad 0.17114 \quad 0.28391$
H $-0.96554-2.302534 .66232$
C $7.54244-2.292890 .23364$
H -1.79697 -1.87989 3.16467
C $7.676572 .70598-0.05414$
H -5.01962 -0.80047 4.89666
F $8.48073-2.47687-0.72168$
H -4.27635 -0.96265 3.30601
F $\quad 6.71039-3.353840 .19484$
H $-3.45608-2.800554 .71537$
F $8.18811-2.326821 .42189$
H -2.97051 -1.67799 5.99187
F $8.86787 \quad 2.574890 .56731$
N -2.17998 0.790413 .82196
F $7.927453 .14015-1.30944$
C $-3.75937 \quad 4.39751 \quad 0.58759$
F $\quad 6.99480 \quad 3.69061 \quad 0.57171$
H $-4.52642 \quad 4.529531 .36339$
C -1.53416 2.951395 .04706
H -2.868094 .957140 .90703$
H -2.515983 .171995 .47830$
C $-4.263594 .90237-0.74397$
H -0.951602 .370945 .76981$
C $-3.676314 .47691-1.94287$
H $-1.01833 \quad 3.90537 \quad 4.89945$
C $-5.296925 .84426-0.79046$
C $-1.18436-0.18898 \quad 4.36817$
C $-4.118304 .98628-3.16433$
C -3.510140 .668694 .50396$
H $-2.882803 .73665-1.91341$
H $-2.31345 \quad 0.580392 .79699$
C $-5.733486 .36204-2.01173$
C -1.70685 -1.62149 4.22802
H -5.76664 6.173820 .13406
H $-1.01317 \quad 0.066975 .41947$
C -5.14563 $5.93291-3.20246$
H $-0.24861-0.040133 .82211$
H $-3.65725 \quad 4.64527-4.08755$
C $-4.06405-0.749394 .36163$
H $-6.537977 .09250-2.03180$
H -3.356780 .925965 .55766$
H $\quad-5.48735 \quad 6.32999-4.15454$
H -4.159091 .419784 .04765$

RTSscis-2int.log

Description: Intermediate before intermolecular proton transfer, R conformation 


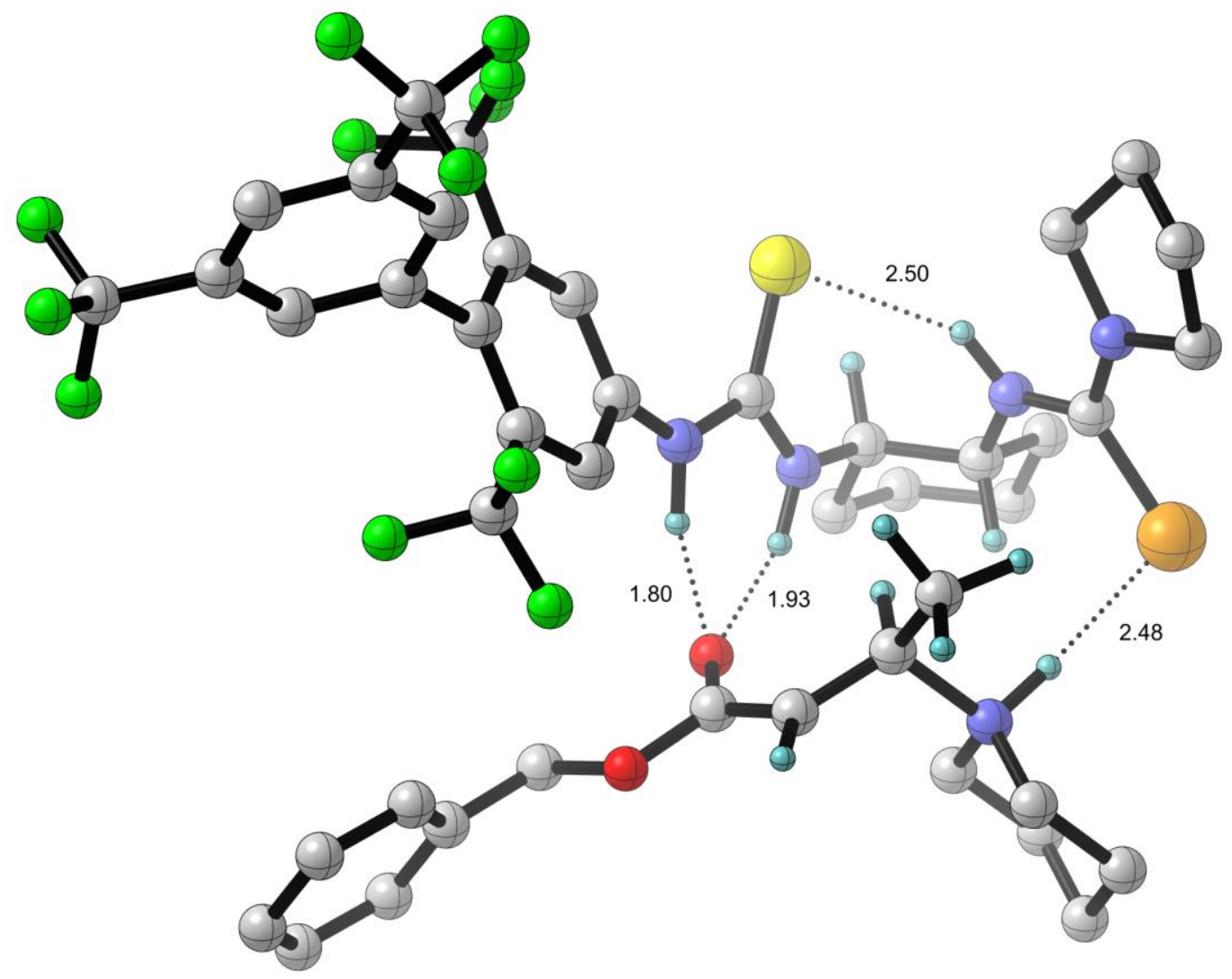

Free Energy $\quad=-6124.858074$

Zero-point Energy = -6124.747366

Potential Energy $\quad=-6129.60334013$

Nimag $\quad=1(7.3803 \mathrm{~cm}-1)$

Charge $=0$ Multiplicity $=1$

C $7.00709-0.448723 .41557$

C $5.96328 \quad 0.09873 \quad 4.39875$

C 4.694160 .561903 .66782

C $4.09827-0.556172 .79214$

C $5.15784-1.071301 .78981$

C $6.41331-1.559052 .53729$

H 3.931520 .890774 .38281

H $5.69658-0.686385 .12045$
H 6.379030 .930394 .98031

H 7.370460 .366952 .77346

H $7.88054-0.829763 .95809$

H $3.79485-1.387763 .43658$

H $5.44700-0.251801 .12225$

H $6.13916-2.42273 \quad 3.16013$

H 7.14449-1.90665 1.80005

H 4.929551 .430823 .03481

N $2.88959-0.075562 .13279$ 

N $4.57814-2.129680 .95732$
$\begin{array}{llll}\text { O } & 2.10237 & 2.38619 & 0.84284\end{array}$
H 2.894550 .880641 .76143
C $\quad 1.727602 .78626-0.30801$
C $1.70974-0.742012 .04504$
C $2.036602 .23287-1.54600$
$\begin{array}{llll}\mathrm{N} & 0.71701 & 0.04965 & 1.53433\end{array}$
H $\quad 1.57753 \quad 2.65336-2.43410$
S $\quad 1.53282-2.379542 .48463$
C $2.890871 .05457-1.65076$
H $1.03728 \quad 0.995311 .26439$
H $3.030020 .58548-0.67360$
C $-0.61418-0.24158 \quad 1.19946$
O $\quad 0.881173 .87190-0.38305$
C -1.41645 -1.16374 1.87996
C $\quad 0.502384 .48566 \quad 0.85287$
C $-1.181830 .50150 \quad 0.15519$
C $-3.064541 .17272-1.33167$
C $-2.74650-1.350401 .50327$
C $-3.55504-2.37292 \quad 2.27515$
H -1.00579-1.73074 2.70080
F -4.02514 2.02377 -0.90076
C $-2.516710 .32618-0.20276$
F -2.10420 $1.93400-1.90388$
H $-0.56780 \quad 1.20796-0.39382$
F $-3.607220 .42340-2.31459$
C $-3.33535-0.61395 \quad 0.45621$
F -2.86742 -2.87845 3.31905
H $\quad 3.75483-2.584491 .35770$
F -4.69545 -1.84589 2.77144
C $4.95985-2.45587-0.29450$
F -3.90839-3.42196 1.49435
N $4.37947-3.53623-0.84860$
C $-4.76632-0.82193 \quad 0.05553$
C $3.49208-4.46583-0.11434$
C -5.12266 -1.90072 -0.75999
C $4.63645-4.00223-2.22569$
$\begin{array}{llll}\text { C } & -5.76323 & 0.06018 & 0.48839\end{array}$
C $3.41434-5.69687-1.02703$
C -6.45257-2.09021-1.14016
H $3.91631-4.693370 .87006$
H -4.36264 - $2.59728-1.09487$
C $3.58854-5.10325-2.43256$
C $-7.09148-0.136620 .11067$
H 4.54645 -3.17326 -2.93001
H $\quad-5.50151 \quad 0.903701 .11624$
H $4.23853-6.38358-0.80098$
C -7.44338 -1.21082 -0.70719
H $3.90902-5.83879-3.17574$
H -8.47539-1.35939-1.00287
H $5.66091-4.38715-2.30143$
C $-6.80476-3.21678-2.07617$
H $2.64430-4.66508-2.77766$
C $-8.167810 .78033 \quad 0.63134$
H $2.50658-4.010210 .04362$
F -8.08919-3.60750 -1.93099
H $2.47591-6.24299-0.89744$
F -6.02175 -4.29848 -1.87398
Se $6.26581-1.48516-1.23503$
F -6.64588 -2.85351 -3.36968 


\begin{tabular}{|c|c|c|}
\hline F -7.68497 $2.00465 \quad 0.93037$ & H 6.78672 & $3.43442-0.45657$ \\
\hline F -9.16217 $0.94054-0.26955$ & H 7.08191 & $1.81204-1.08084$ \\
\hline F -8.73400 $0.29077 \quad 1.75759$ & Н 7.73669 & $3.45894-2.81867$ \\
\hline C $2.45534 \quad 0.00322-2.67158$ & H 6.16391 & $4.24924-2.71352$ \\
\hline H $\quad 2.191830 .44962-3.63610$ & $\mathrm{~N} \quad 4.45520$ & $1.42358-1.98914$ \\
\hline H $3.21695-0.77047-2.83204$ & C -0.56586 & 5.516640 .57473 \\
\hline H $1.55456-0.48397-2.28649$ & C -0.50238 & $6.77621 \quad 1.18004$ \\
\hline C $\quad 4.64128 \quad 1.95850-3.37391$ & C -1.65886 & $5.21608-0.25028$ \\
\hline C $4.999442 .37073-0.96333$ & C -1.51353 & 7.718580 .97735 \\
\hline H $4.975040 .52732-1.89140$ & H $\quad 0.34610$ & 7.023151 .81479 \\
\hline C $6.102652 .30719-3.66863$ & C -2.66554 & $6.15797-0.45934$ \\
\hline H $3.99410 \quad 2.83924-3.44547$ & H -1.71362 & $4.24675-0.73596$ \\
\hline H $4.27497 \quad 1.20374-4.07183$ & C -2.59824 & 7.411470 .15562 \\
\hline C $6.467992 .71223-1.21767$ & H -1.44829 & 8.692691 .45533 \\
\hline H $4.357903 .25517-1.00356$ & H -3.50668 & $5.91186-1.10233$ \\
\hline H $4.85434 \quad 1.90051 \quad 0.01111$ & H -3.38504 & $8.14327-0.00792$ \\
\hline C $6.672713 .27881-2.62830$ & H 0.13411 & 3.719131 .54620 \\
\hline H $6.15506 \quad 2.73393-4.67740$ & H 1.37535 & 4.948001 .32981 \\
\hline H $6.699671 .38515-3.68446$ & & \\
\hline $\begin{array}{l}\text { Starting Material } \\
\text { D3gen-pip.log } \\
\text { Description: Piperidine Starting Material }\end{array}$ & & \\
\hline Free Energy $\quad=-251.772747$ & & \\
\hline Zero-point Energy $=-251.744053$ & & \\
\hline Potential Energy $\quad=-252.003543164$ & & \\
\hline$=1(232.6306 \mathrm{~cm}-1)$ & & \\
\hline Charge $=0$ Multiplicity $=1$ & C 0.00832 & $-0.79160-1.21661$ \\
\hline C $\quad 0.008320 .74696 \quad 1.26588$ & C 0.00832 & -0.791601 .21661 \\
\hline C $-0.64000 \quad 1.330590 .00000$ & H -0.51413 & $1.09794-2.16615$ \\
\hline C $\quad 0.008320 .74696-1.26588$ & H -1.71264 & +1.085000 .00000 \\
\hline
\end{tabular}




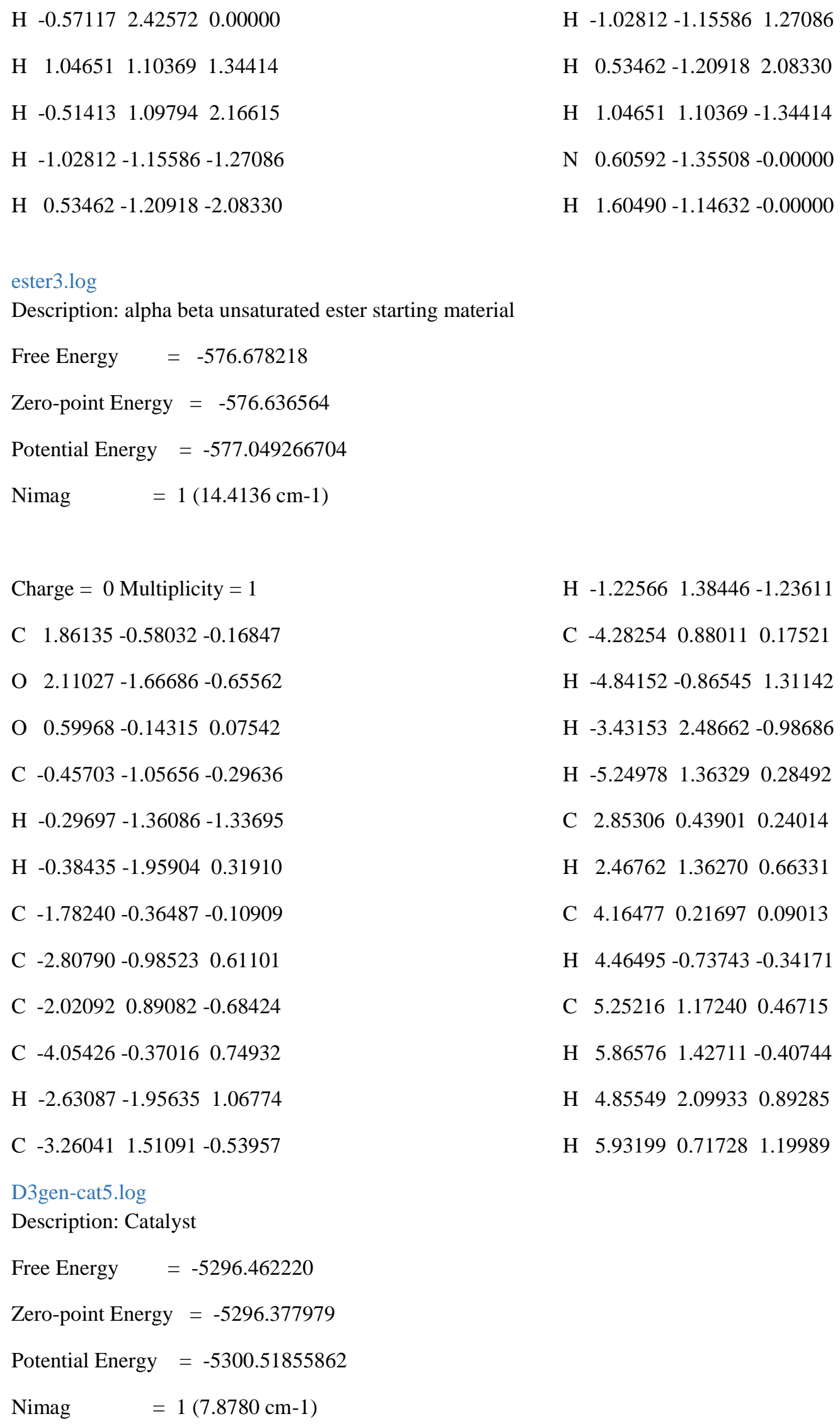




\begin{tabular}{|c|c|}
\hline Charge $=0$ Multiplicity $=1$ & C $\quad 1.38565-1.69331-0.12623$ \\
\hline C $-8.55641-1.37008-0.02812$ & H $-0.43007-2.83771-0.12417$ \\
\hline C $-7.91809-2.74551-0.27198$ & C $2.01586-0.45404-0.36798$ \\
\hline C $-6.56012-2.61867-0.97922$ & H $-5.30807 \quad 0.403191 .74487$ \\
\hline C $-5.61214-1.69857-0.19372$ & C $-4.49994 \quad 1.499340 .23622$ \\
\hline C $-6.25815-0.301860 .01456$ & N $-3.709142 .13837 \quad 1.11777$ \\
\hline C $-7.60825-0.43322 \quad 0.73760$ & C -2.789383 .240400 .76332 \\
\hline H - $-6.08575-3.59930-1.09509$ & C $-3.66373 \quad 1.834342 .56405$ \\
\hline H - -7.77766 -3.25874 0.69015 & C -2.430183 .854702 .12169 \\
\hline H $-8.59026-3.37565-0.86625$ & H -1.90499 2.840860 .25259 \\
\hline H -8.81238 -0.90977-0.99299 & C -2.469542 .651993 .07588 \\
\hline H $-9.49634-1.477060 .52614$ & H $-4.60183 \quad 2.148273 .04550$ \\
\hline H -5.40047 -2.14886 0.78090 & H -1.458044 .354622 .10029 \\
\hline H $-6.41787 \quad 0.16313-0.96286$ & H -1.551352 .061492 .98175 \\
\hline H $-7.44281-0.830311 .75145$ & H -3.525710 .758752 .72745 \\
\hline H $-8.05398 \quad 0.56190 \quad 0.85135$ & H $-2.58440 \quad 2.93048 \quad 4.12704$ \\
\hline H - $-6.70554-2.21217-1.99128$ & H $-3.281873 .93367 \quad 0.07987$ \\
\hline $\mathrm{N}-4.32608-1.55296-0.86713$ & H -3.184774 .595882 .41139 \\
\hline N -5.369440 .594740 .75269 & Se $-4.38808 \quad 1.85735-1.59985$ \\
\hline H - $-4.36906-0.97478-1.70405$ & $\begin{array}{llll}\text { C } & 2.18854 & -2.88770 & 0.34835\end{array}$ \\
\hline C $-3.11250-1.60064-0.25146$ & C $\quad 1.770281 .97624-1.09150$ \\
\hline N $-2.15785-0.91467-0.98122$ & F $2.65115-2.705851 .60815$ \\
\hline S - $-2.82716-2.376261 .21897$ & F $1.45197-4.016950 .36166$ \\
\hline H - $-2.54178-0.13860-1.52563$ & F $3.25920-3.12296-0.43941$ \\
\hline C $-0.78009-0.80519-0.73903$ & F $2.499772 .44012-0.05536$ \\
\hline C $-0.171910 .42547-1.00133$ & F $2.571891 .98118-2.18061$ \\
\hline C $\quad 0.01550-1.87236-0.31100$ & F $\quad 0.794852 .88603-1.32064$ \\
\hline C $\quad 1.19780 \quad 0.60225-0.81543$ & C $3.48975-0.26499-0.16298$ \\
\hline H $-0.778801 .25691-1.34210$ & C $4.36001-0.23428-1.25770$ \\
\hline
\end{tabular}



C $4.01435-0.109101 .12529$
C $5.73036-0.04887-1.06576$
H $3.96955-0.35988-2.26131$
C 5.384290 .077941 .31132
H $3.35180-0.128171 .98286$
C $\quad \begin{array}{lll}6.24971 & 0.10944 & 0.21783\end{array}$
H $7.31285 \quad 0.256020 .36454$
C $6.636840 .03172-2.26688$
Products
D3gen-prodSd
Description: $\mathrm{S}$ enantiomer of product
Free Energy $\quad=-828.433206$
Zero-point Energy $=-828.382594$
Potential Energy $\quad=-829.076202446$
Nimag $\quad=1(11.3102 \mathrm{~cm}-1)$

Charge $=0$ Multiplicity $=1$

C $\quad 0.351461 .01784-0.47853$

O $\quad 0.31813 \quad 2.17753-0.83741$

O $\quad 1.50225 \quad 0.34685-0.24273$

C $2.719631 .09451-0.48054$

H $2.703721 .45130-1.51694$

Н $2.72986 \quad 1.977590 .16546$

C $3.899140 .19654-0.21186$

C 4.948440 .636450 .60163

C $3.98253-1.07329-0.79984$

C $6.06735-0.17093 \quad 0.81965$

H 4.890461 .617101 .06850

C $5.09347-1.88475-0.57569$

H $3.16641-1.42704-1.42378$

C $\quad 6.14140-1.43414 \quad 0.23250$
C 5.935700 .190392 .70911

F $6.28745-0.86755-3.21244$

F $7.92679-0.19634-1.94101$

F $\quad 6.58150 \quad 1.25147-2.84708$

F $\quad 5.07854 \quad 0.83335 \quad 3.53237$

F $6.16426-1.026163 .25137$

F $7.10721 \quad 0.86174 \quad 2.73354$
H $\quad 6.87500 \quad 0.18532 \quad 1.45348$
H $5.14400-2.86925-1.03334$
H $7.00836-2.066390 .40442$
C $-0.864520 .14289-0.25403$
H $-0.57543-0.742940 .31906$
C $-2.02497 \quad 0.922350 .42545$
H $-2.11143 \quad 1.87210-0.11408$
H -1.18944 -0.20325 -1.24485
C $-3.928730 .36606-1.02328$
C $-3.43511-1.081040 .85258$
C $-5.41431-0.01325-0.98968$
H -3.41169-0.29019-1.75542
H $-3.812201 .39537-1.38202$
C $-4.90224-1.51550 \quad 0.96366$
H $-2.89303-1.81626 \quad 0.22240$ 


$$
\begin{aligned}
& \text { H }-2.96819-1.10377 \quad 1.84223 \\
& \text { C }-5.60576-1.41501-0.39636 \\
& \text { H } \quad-5.828800 .03977-2.00446 \\
& \text { H } \quad-5.94977 \quad 0.72348-0.37687 \\
& \text { H }-4.95124-2.540861 .35200 \\
& \text { H }-5.40655-0.864581 .68967 \\
& \text { H -6.67232-1.65355-0.30013 } \\
& \text { D3gen-prodRc } \\
& \text { Description: } \mathrm{R} \text { enantiomer of product } \\
& \text { Free Energy } \quad=\quad-828.447904 \\
& \text { Zero-point Energy = }-828.397806 \\
& \text { Potential Energy } \quad=-829.077335451 \\
& \text { Nimag } \quad=1(12.4335 \mathrm{~cm}-1)
\end{aligned}
$$

$$
\begin{aligned}
& \text { Charge }=0 \text { Multiplicity }=1 \\
& \text { C }-0.61493 \quad 1.06068 \quad 0.12242 \\
& \text { O } \quad-1.14652 \quad 2.07598 \quad 0.52428 \\
& \text { O }-1.16504 \quad 0.23198-0.79987 \\
& \text { C }-2.47437 \quad 0.60047-1.31779 \\
& \text { H }-2.547851 .68966-1.32540 \\
& \text { H }-2.469920 .22398-2.34329 \\
& \text { C }-3.59180-0.02245-0.51805 \\
& \text { C }-4.10557-1.27412-0.87888 \\
& \text { C }-4.124340 .63505 \quad 0.59969 \\
& \text { C }-5.13221-1.86201-0.13941 \\
& \text { H -3.69972 -1.78962-1.74666 } \\
& \text { C }-5.151100 .048621 .33976 \\
& \text { H } \quad-3.720151 .600910 .88752 \\
& \text { C }-5.65717-1.200170 .97209 \\
& \text { H }-5.52386-2.83263-0.43216
\end{aligned}
$$

H $\quad-5.17600-2.16057-1.08204$

C -1.687751 .259361 .88397$

H $\quad-2.52438 \quad 1.79212 \quad 2.34618$

H -1.477290 .364672 .48154$

H -0.801701 .902061 .92825$

$\begin{array}{llll}\mathrm{N} & -3.33880 & 0.27630 & 0.31202\end{array}$
H $\quad-5.55832 \quad 0.56827 \quad 2.20310$
H $\quad-6.45932-1.654421 .54787$
C $\quad 0.752830 .568540 .53308$
H $\quad 0.68614-0.50204 \quad 0.75492$
C $\quad 1.825370 .79371-0.58235$
Н $1.429640 .33371-1.49585$
H 1.031571 .102271 .44535
C 3.776030 .469990 .92572
C $3.05905-1.33285-0.52315$
C $5.22222-0.041340 .92324$
H $3.251920 .05078 \quad 1.80881$
H 3.770791 .558351 .03847
C $4.47597-1.91586-0.57936$
H $\quad 2.49280-1.84887 \quad 0.28028$
Н $2.53276-1.53626-1.46331$
C $5.26499-1.555950 .68578$ 
H $5.70177 \quad 0.21673 \quad 1.87599$

C $2.029302 .28700-0.86840$

H $\quad \begin{array}{llll}5.77502 & 0.47379 & 0.12694\end{array}$

H $1.096792 .74496-1.21393$

H $4.41827-3.00441-0.70602$

H 2.344762 .839900 .02324

H $4.98719-1.50898-1.46144$

H $2.792162 .41599-1.64217$

H $\quad 6.30128-1.90791 \quad 0.60970$

N $3.101950 .11675-0.32621$

H $4.81934-2.070991 .54987$

Intermediate enol

D3genpxferra2-enol.log

Description: Intermediate after intramolecular proton transfer, $\mathrm{R}$ conformation

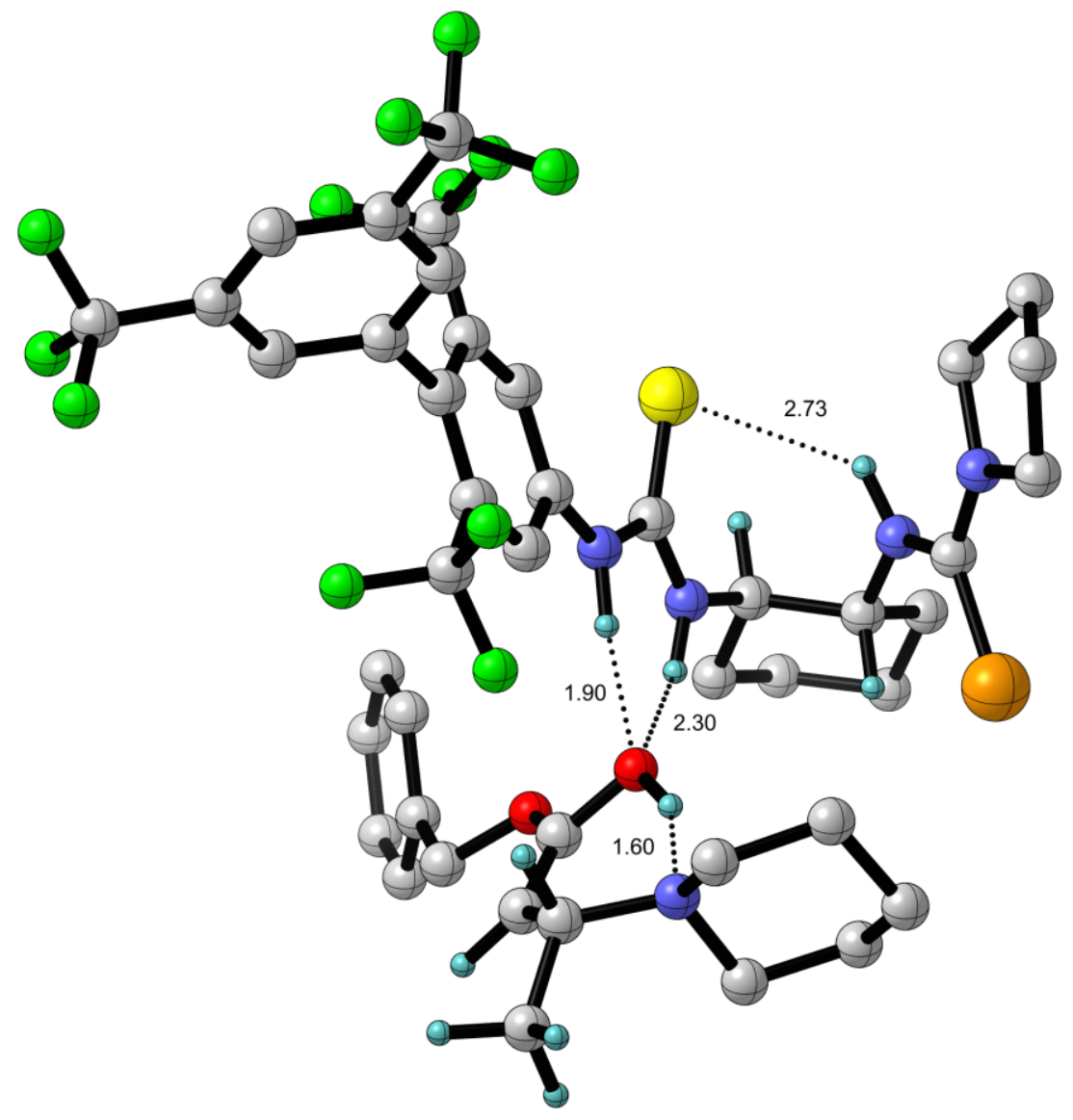

Free Energy $\quad=-6124.855931$

Zero-point Energy $=-6124.743154$

Potential Energy $\quad=-6129.58879505$

Nimag $\quad=1(6.2161 \mathrm{~cm}-1)$ 

Charge $=0$ Multiplicity $=1$
$\begin{array}{llll}\mathrm{H} & 0.35447 & 0.79350 & 1.14407\end{array}$
C $-7.34893-0.76225-2.13348$
C $3.244180 .14500-0.55500$
C $-6.739010 .45890-2.83613$
H -3.45954 -2.67085 -2.14981
C $-5.41600 \quad 0.88540-2.18092$
C $-3.75248-3.31889-0.23784$
C -4.41404-0.28156-2.13062
N -2.84008 -4.29788 -0.41849
C -5.03261 -1.49663-1.39934
C -2.32759-4.72471-1.73690
C -6.34884-1.92598-2.07215
C -2.32593-5.14755 0.66975
H $-4.962861 .72293-2.72298$
C -1.65730 -6.07574-1.45018
H $-6.556840 .21779-3.89348$
H -3.15188 -4.80872 -2.45508
H -7.44246 $1.30036-2.82508$
C -1.18694 -5.93147 0.00489
H -7.64392 -0.48608 -1.11094
H -2.00270 -4.52918 1.50962
H -8.26400 -1.08047-2.64765
H -2.39498 -6.88290 -1.53049
H -4.14997 -0.56939-3.15291
H $-1.00657-6.891610 .49692$
H -5.24353 -1.21695 -0.36200
H -3.12325 -5.80612 1.03716
H -6.13307 -2.28754-3.08851
H $-0.25617-5.352570 .04528$
H -6.76585 -2.76996 -1.51141
H -1.60956 -3.99136 -2.12808
H -5.60946 $1.23958-1.15733$
H -0.84646 -6.28907 -2.15260
N -3.17496 $0.14522-1.48731$
Se $-4.52610-3.013771 .42710$
N -4.07314 -2.59767 -1.34366
$\begin{array}{llll}\text { O } & -3.06988 & 3.08138 & 0.42331\end{array}$
H -3.28478 $0.64699-0.60864$
C -2.51436 2.58500 1.55157
C -1.91618 -0.17645 - 1.87080
C -2.16064 3.238462 .67684
N $-0.98046 \quad 0.29419-0.97629$
H -2.34483 4.29958 2.78307
S -1.56545 - $1.05746-3.28030$
C -1.46286 2.504983 .81009
H $-1.38348 \quad 0.67340-0.11559$
H -0.41495 2.32136 3.52903
C $0.414640 .20515-0.92299$
$\begin{array}{llll}\text { O } & -2.36025 & 1.24709 & 1.40999\end{array}$
C $1.26391-0.13012-1.98443$
C 2.909980 .862761 .86351
C 0.996060 .525570 .31370
C $3.50211-0.55109-2.98251$
C $2.64633-0.16316-1.79279$
F 3.628612 .007721 .83662
H $\quad 0.84642-0.36773-2.95024$
F 1.910791 .060292 .76360
C 2.373260 .499150 .49657
F $3.70532-0.098112 .37313$ 


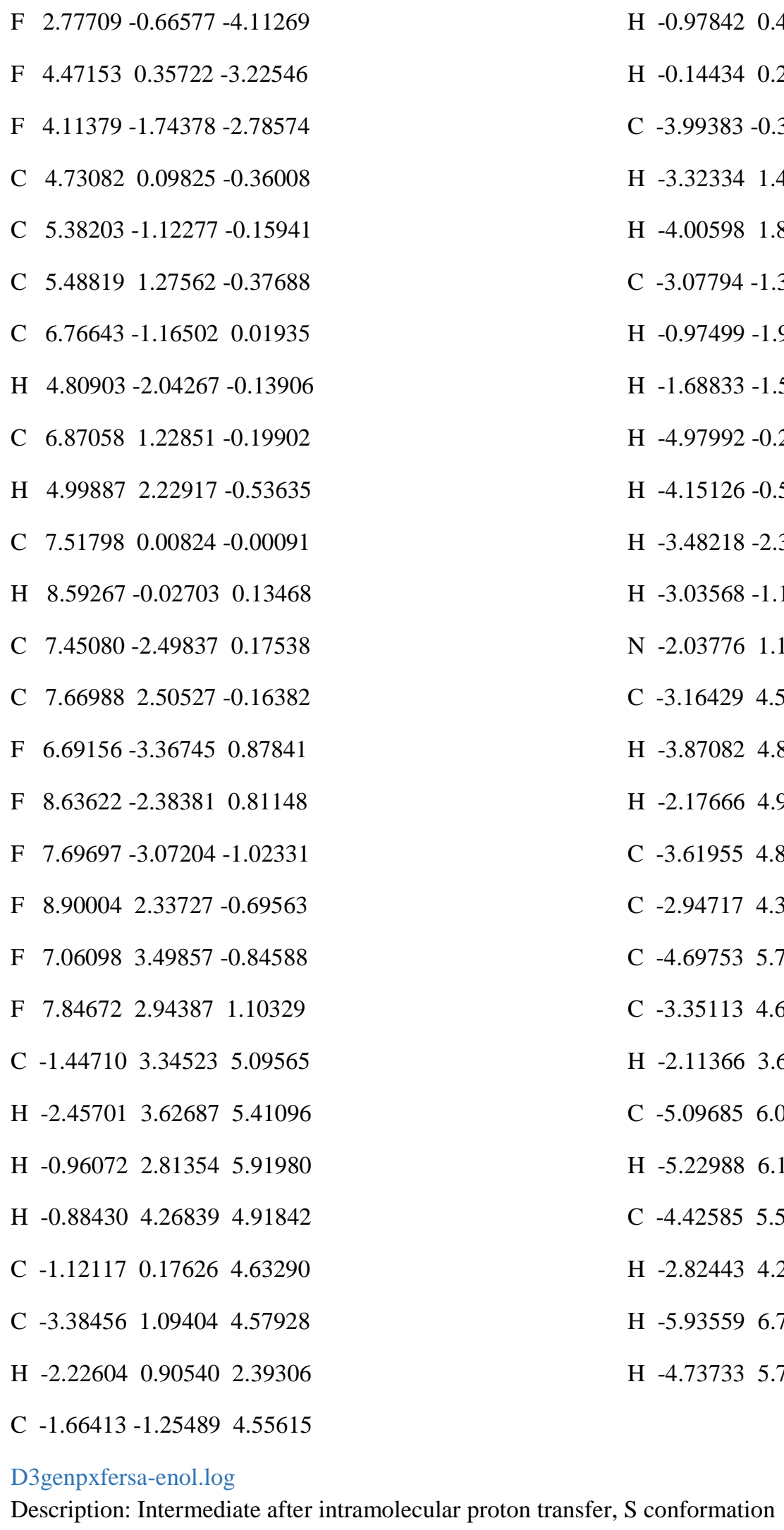




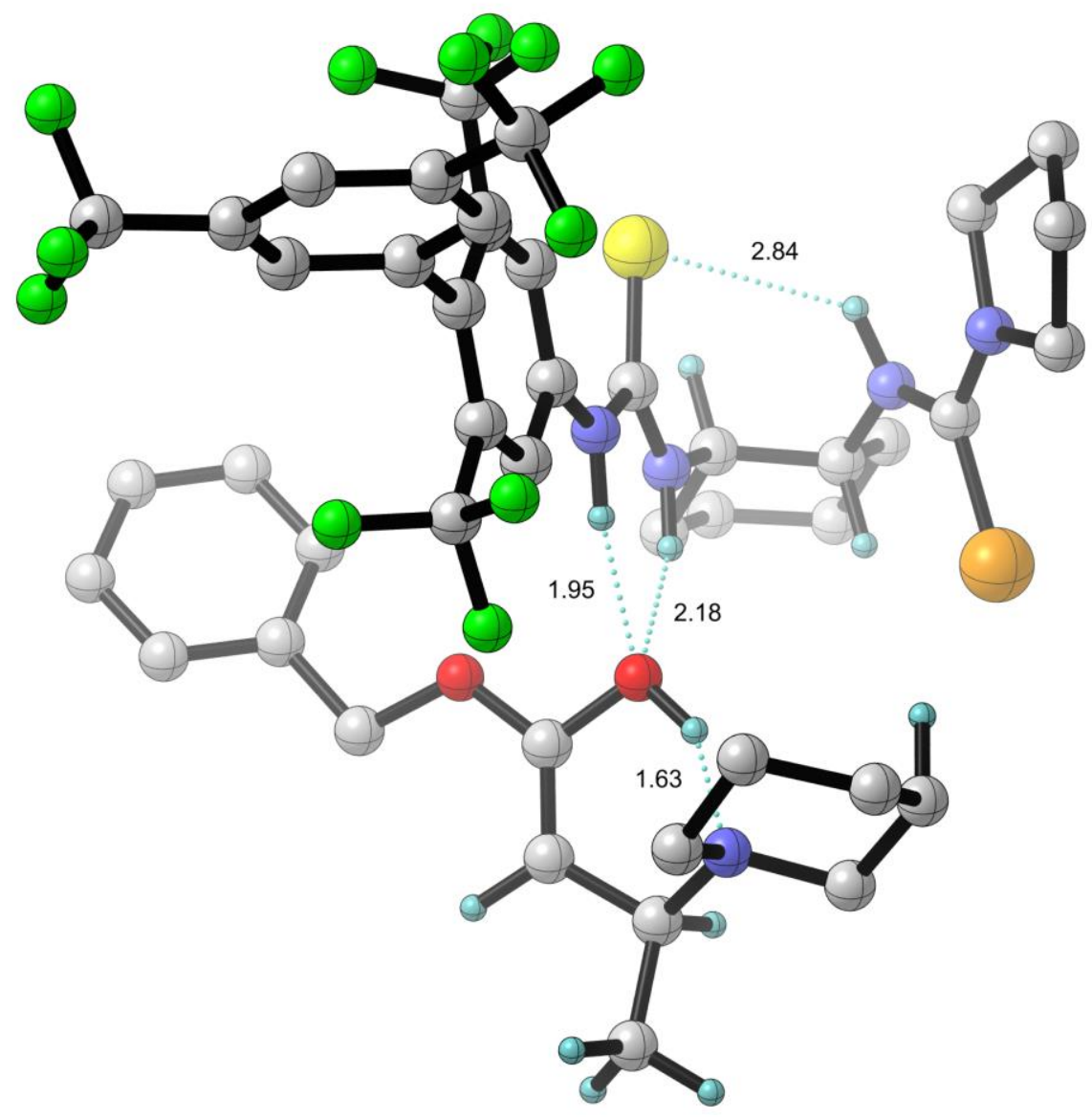

Free Energy $\quad=-6124.854120$

Zero-point Energy $=-6124.741726$

Potential Energy $\quad=-6129.58875706$

Nimag $\quad=1(6.4619 \mathrm{~cm}-1)$

Charge $=0$ Multiplicity $=1$

C $-7.56283-0.06329-2.42000$

C $-6.80260 \quad 1.16775-2.93521$

C $-5.49046 \quad 1.38322-2.16467$

C $-4.606820 .12450-2.20533$

C -5.38103-1.09437-1.64449

C -6.67826 -1.31904-2.44175

H $-4.93022 \quad 2.22786-2.58280$
H $-6.574831 .03504-4.00263$

H $\quad-7.428862 .06488-2.85861$

H $-7.899350 .12069-1.38958$

H -8.46630 -0.23037 -3.01887

H - $-4.32505-0.07729-3.24330$

H -5.63941 -0.90276 -0.59834

H -6.42844 -1.58134 -3.48073

H -7.20955 -2.17652 -2.01349 


\begin{tabular}{|c|c|}
\hline H $-5.71640 \quad 1.63449-1.11748$ & H $-1.53370-6.12810-2.56798$ \\
\hline N -3.36166 $0.34947-1.48088$ & Se $-4.79956-2.602911 .17294$ \\
\hline $\mathrm{N}-4.53491-2.28396-1.63918$ & O $-2.12174 \quad 3.26658 \quad 0.43310$ \\
\hline H $-3.453890 .69529-0.52711$ & C $-2.608572 .69819 \quad 1.55740$ \\
\hline C $-2.12013-0.02968-1.88431$ & C -2.983653 .294302 .70636 \\
\hline $\mathrm{N}-1.171250 .27708-0.93940$ & H $-2.90172 \quad 4.36745 \quad 2.81959$ \\
\hline S -1.81748 $-0.77539-3.37988$ & C -3.558102 .490613 .85929 \\
\hline H - $-1.551160 .71659-0.09939$ & $\begin{array}{llll}O & -2.64416 & 1.35424 & 1.38168\end{array}$ \\
\hline C $\quad 0.214350 .10127-0.86768$ & C $\quad 2.73657 \quad 0.86825 \quad 1.86861$ \\
\hline C $1.03836-0.44257-1.86039$ & C $3.24408-1.16927-2.75419$ \\
\hline $\begin{array}{llll}\text { C } & 0.81160 & 0.52271 & 0.33168\end{array}$ & F $3.600821 .89657 \quad 1.71828$ \\
\hline C $2.41177-0.56468-1.64141$ & F $\quad 1.76057 \quad 1.302272 .70325$ \\
\hline H $\quad 0.60757-0.76266-2.79615$ & F $3.39440-0.114942 .51868$ \\
\hline $\begin{array}{llll}\text { C } & 2.17888 & 0.39768 & 0.54310\end{array}$ & F $\quad 2.50890-1.43837-3.85128$ \\
\hline H $\quad 0.192050 .955591 .10776$ & F $4.24293-0.34517-3.13960$ \\
\hline C $3.02406-0.15374-0.44235$ & F $\quad 3.81639-2.33547-2.37008$ \\
\hline Н -3.96168 -2.39558 -2.46969 & C $4.50171-0.28690-0.21899$ \\
\hline C $-4.16815-2.98998-0.53488$ & C $5.04787-1.491900 .23112$ \\
\hline N -3.32627 -4.02144 -0.75245 & C $5.354220 .79916-0.45322$ \\
\hline C $-2.91796-4.49400-2.09199$ & C $6.42285-1.607600 .44980$ \\
\hline C $-2.78354-4.876830 .31779$ & H $4.40126-2.344450 .40619$ \\
\hline C $-2.28924-5.86815-1.82109$ & C $6.72620 \quad 0.67815-0.23781$ \\
\hline Н $-3.78934-4.55433-2.75480$ & H $\quad 4.94424 \quad 1.74059-0.79958$ \\
\hline C $-1.72306-5.72079-0.40104$ & C $7.26815-0.52506 \quad 0.21773$ \\
\hline H -2.38255 -4.26078 1.12497 & $\begin{array}{llll}\text { H } & 8.33437 & -0.61639 & 0.38920\end{array}$ \\
\hline H -3.06418 -6.64350 -1.84031 & C $6.97573-2.897750 .99693$ \\
\hline H - $-1.55394-6.679810 .09706$ & C $7.648051 .83007-0.54309$ \\
\hline Н $-3.58505-5.493560 .74399$ & F $8.29529-3.028820 .74444$ \\
\hline H - $-0.76779-5.18263-0.42878$ & F $\quad 6.34858-3.971150 .46852$ \\
\hline H - $-2.19413-3.79906-2.53914$ & F $\quad 6.81801-2.973692 .33822$ \\
\hline
\end{tabular}




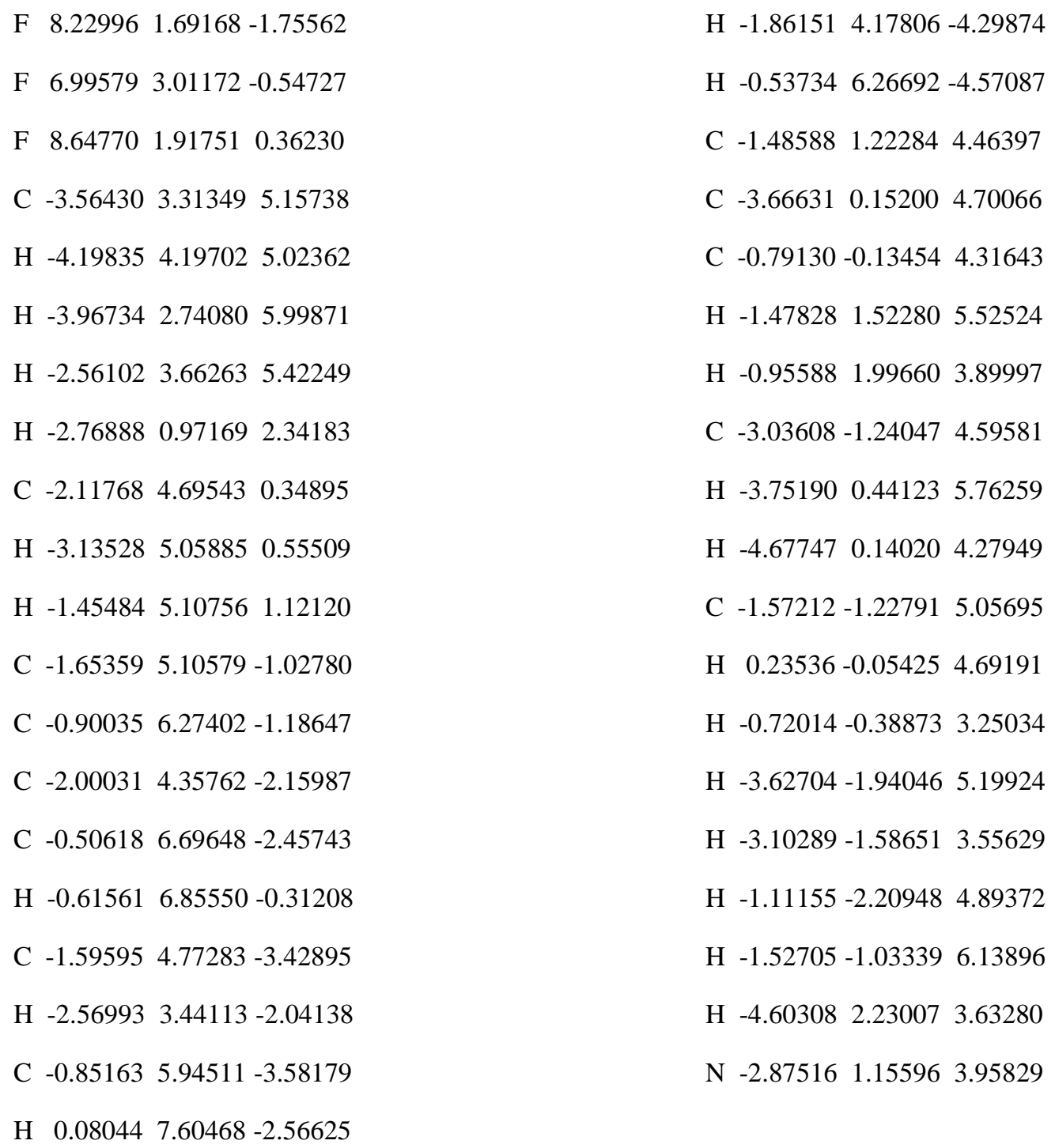




\section{References:}

1) a) Seven Golden Rules for Heuristic Filtering of Molecular Formulas Obtained by Accurate Mass Spectrometry. Kind, T.; Fiehn, O. BMC Bioinformatics 2007, 8, 105. b) The concept of spectral accuracy for MS. Wang, Y.; Gu, M. Anal. Chem. 2010, 82, 7055 .

2) Reductive Coupling of Acrylates with Ketones and Ketimines by a Nickel-Catalyzed Transfer-Hydrogenative Strategy. Buxton, C. S.; Blakemore, D. C.; Bower, J. F. Angew. Chem., Int. Ed. 2017, 56, 13824.

3) Stereoselective Synthesis of Polysubstituted Alkenes through a Phosphine-Mediated Three-Component System of Aldehydes, $\alpha$-Halo Carbonyl Compounds, and Terminal Alkenes. Liu, D.-N.; Tian, S.-K. Chem. Eur. J. 2009, 15, 4538 .

4) Direct $\alpha-\mathrm{C}-\mathrm{H}$ bond functionalization of unprotected cyclic amines. Chen, W.; Ma, L.; Paul, A.; Seidel, D. Nat. Chem. 2018, 10, 165.

5) Enantioselective Michael reaction of malonates to nitroolefins catalyzed by bifunctional organocatalysts. Okino, T.; Hoashi, Y.; Takemoto, Y. J. Am. Chem. Soc. 2003, 125, 12672.

6) Highly enantioselective conjugate addition of nitromethane to chalcones using bifunctional cinchona organocatalysts. Vakulya, B.; Varga, S.; Csámpai, A.; Soós, T. Org. Lett. 2005, 7, 1967.

7) Catalytic enantioselective Friedel-Crafts alkylation of indoles with nitroalkenes by using a simple thiourea organocatalyst. Herrera, R. P.; Sgarzani, V.; Bernardi, L.; Ricci, A. Angew. Chem. Int. Ed. 2005, 44, 6576.

8) Enantioselective Thiourea-Catalyzed Additions to Oxocarbenium Ions. Reisman, S. E.; Doyle, A. G.; Jacobsen, E. N. J. Am. Chem. Soc. 2008, 130, 7198.

9) Development of bis-thiourea-type organocatalyst for asymmetric Baylis-Hillman reaction. Sohtome, Y.; Tanatani, A.; Hashimoto, Y.; Nagasawa, K. Tetrahedron Lett. 2004, 45, 5589.

10) Merging Nucleophilic and Hydrogen Bonding Catalysis: An Anion Binding Approach to the Kinetic Resolution of Propargylic Amines. Klauber, E. G.; De, C. K.; Shah, T. K.; Seidel, D. J. Am. Chem. Soc. 2010, 132, 13624.

11) Direct Formation of Oxocarbenium Ions under Weakly Acidic Conditions: Catalytic Enantioselective OxaPictet-Spengler Reactions. Zhao, C.; Chen, S. B.; Seidel, D. J. Am. Chem. Soc. 2016, 138, 9053.

12) Enantioselective Catalytic $\alpha$-Alkylation of Aldehydes via an $S_{N} 1$ Pathway. Brown, A. R.; Kuo, W.-H.; Jacobsen, E. N. J. Am. Chem. Soc. 2010, 132, 9286.

13) Indium-Mediated Asymmetric Allylation of Acylhydrazones Using a Chiral Urea Catalyst. Tan, K. L.; Jacobsen, E. N. Angew. Chem. Int. Ed. 2007, 46, 1315.

14) Facile monoprotection of trans-1,2-diaminocyclohexane. Kaik, M.; Gawroński, J. Tetrahedron: Asymmetry 2003, 14, 1559.

15) Poly(arylene ether)s with Low Refractive Indices: Poly(biphenylene oxide)s with Trifluoromethyl Pendant Groups via a Meta-Activated Nitro Displacement Reaction. Kim, S. D.; Ka, D.; Chung, I. S.; Kim, S. Y. Macromolecules 2012, 45, 3023.

16) Kagan, H.B.; Fiaud, J.C. Kinetic Resolution. In Topics in Stereochemistry. Eliel, E. L., Wilen, S. H., Eds.; Wiley: New York, 1988, 18, 249.

17) Application of Catalytic Dynamic Resolution of $N$-Boc-2-lithiopiperidine to the Asymmetric Synthesis of 2-Aryl and 2-Vinyl Piperidines. Beng, T. K.; Gawley, R. E. Org. Lett. 2011, 13, 394.

18) Electronic Role of 3-Iminophosphine Ligands in Palladium-Catalyzed Intermolecular Hydroamination. Tafazolian, H.; Samblanet, D. C.; Schmidt, J. A. R. Organometallics 2015, 34, 1809. 
19) a) Stereochemical Determination of Acyclic Structures Based on Carbon-Proton Spin-Coupling Constants. A Method of Configuration Analysis for Natural Products. Matsumori, N.; Kaneno, D.; Murata, M.; Nakamura, H.; Tachibana, K. J. Org. Chem. 1999, 64, 866. b) Excitation-Sculptured Indirect-Detection Experiment (EXSIDE) for Long-Range CH Coupling-Constant Measurement. Krishnamurthy, V. V. J. Magn. Reson., Ser. A 1996, 121, 33. 


\section{HPLC and SFC Profiles}

\section{HPLC Profile of 2a}

Conditions: Daicel Chiralcel OB-H, $n$-hexane $/ i-\mathrm{PrOH}=97 / 3$, Flow rate $=1 \mathrm{~mL} / \mathrm{min}, \mathrm{UV}=230 \mathrm{~nm}, \mathrm{t}_{\mathrm{R}}=5.0 \mathrm{~min}$ (major) and $t_{R}=6.4$ min.

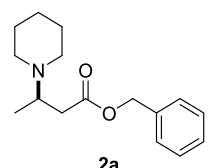

2a

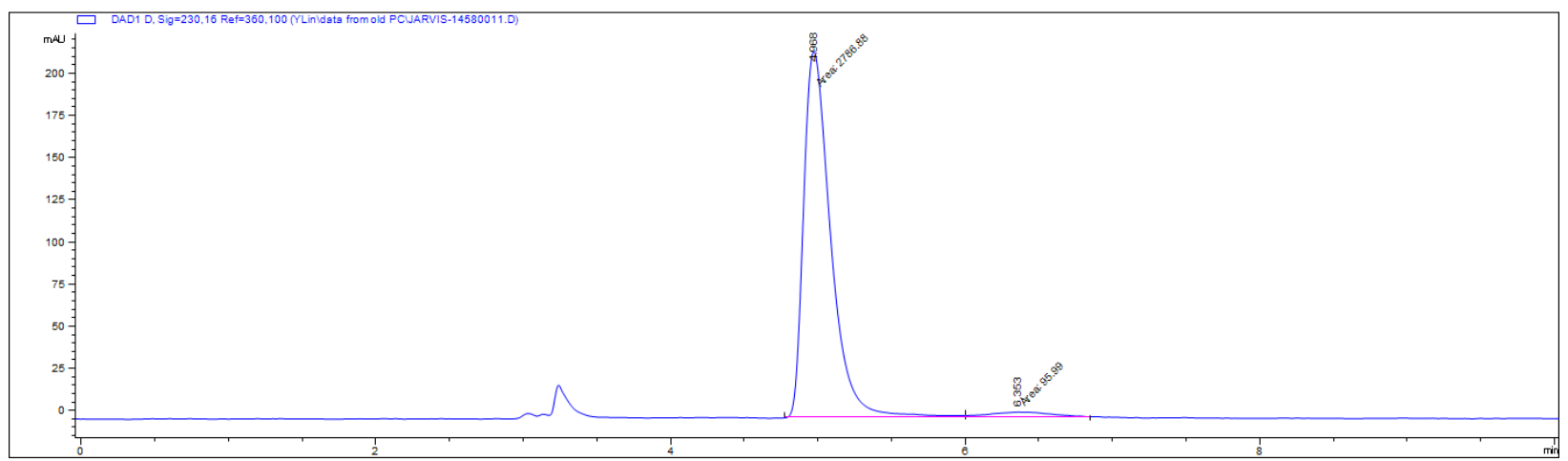

\begin{tabular}{|l|l|l|}
\hline Peak & Retention time $(\mathrm{min})$ & Area \% \\
\hline 1 & 4.968 & 96.670 \\
\hline 2 & 6.353 & 3.330 \\
\hline
\end{tabular}

Racemic sample

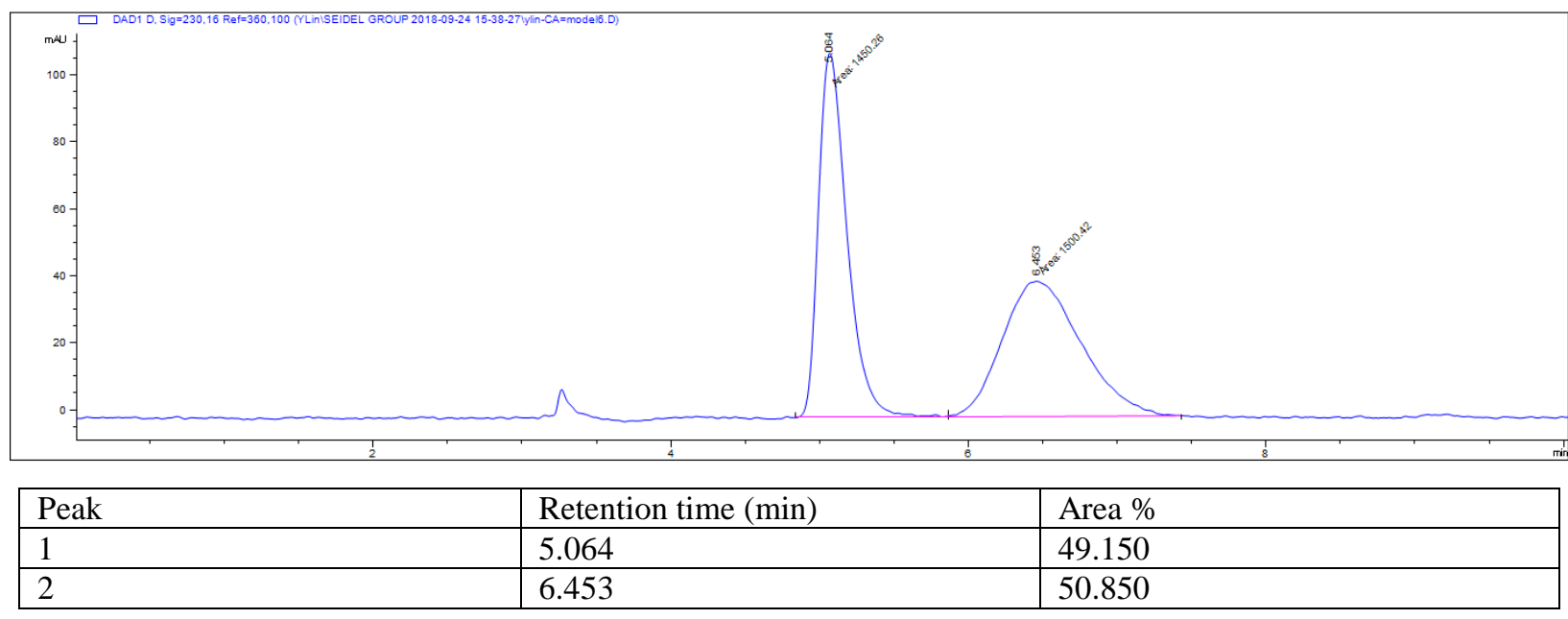




\section{HPLC Profile of $\mathbf{2} \mathbf{b}$}

Conditions: Daicel Chiralcel OB-H, $n$-hexane $/ i-\mathrm{PrOH}=97 / 3$, Flow rate $=1 \mathrm{~mL} / \mathrm{min}, \mathrm{UV}=230 \mathrm{~nm}, \mathrm{t}_{\mathrm{R}}=8.1 \mathrm{~min}$ and $t_{\mathrm{R}}=10.6 \min$ (major).
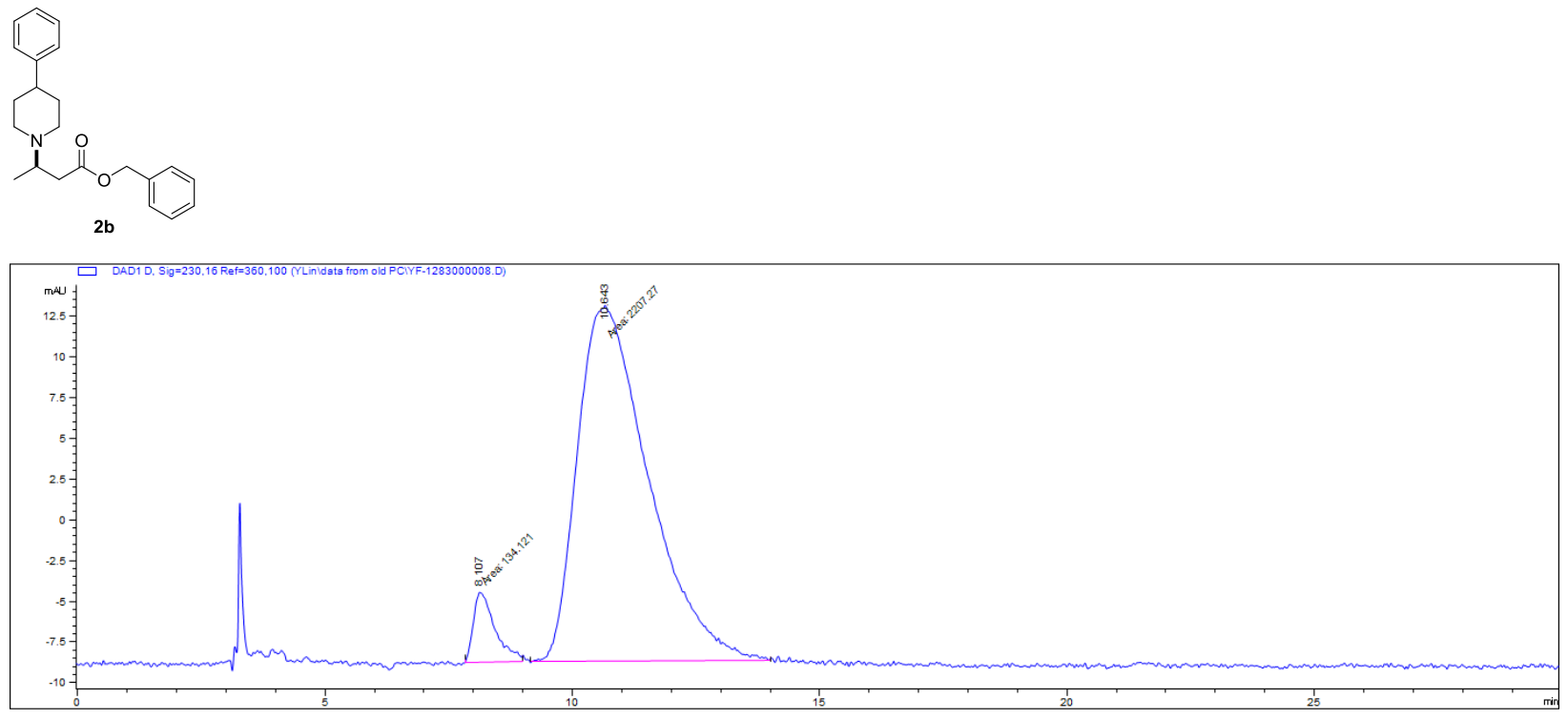

\begin{tabular}{|l|l|l|}
\hline Peak & Retention time (min) & Area \% \\
\hline 1 & 8.107 & 5.728 \\
\hline 2 & 10.643 & 94.272 \\
\hline
\end{tabular}

\section{Racemic sample}

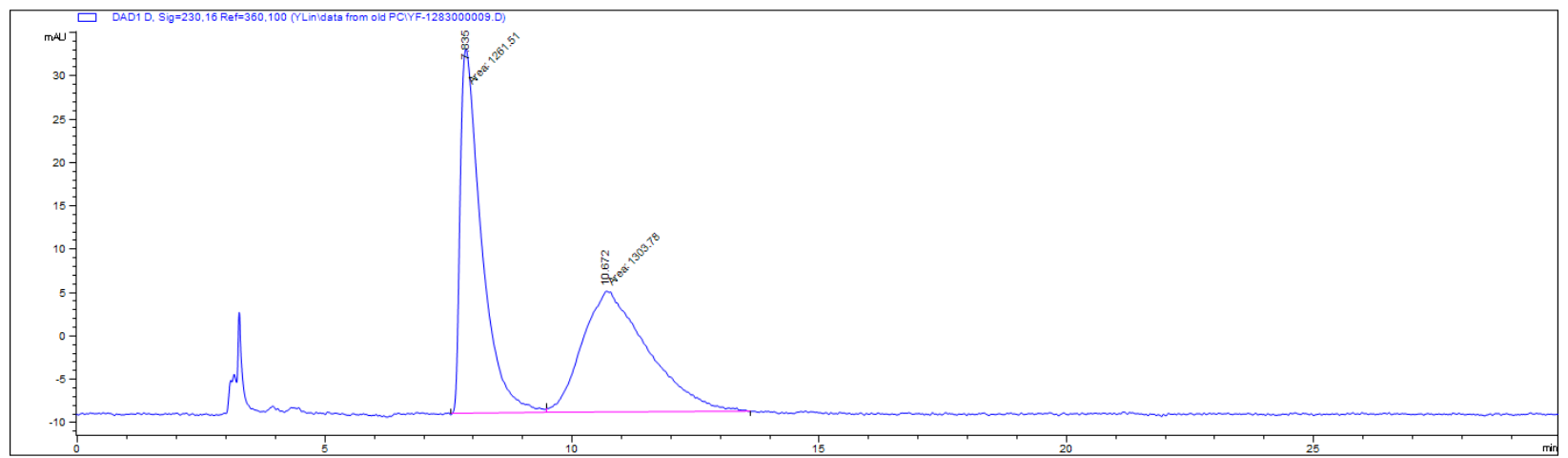

\begin{tabular}{|l|l|l|}
\hline Peak & Retention time (min) & Area \% \\
\hline 1 & 7.835 & 49.176 \\
\hline 2 & 10.672 & 50.824 \\
\hline
\end{tabular}


HPLC Profile of $\mathbf{2 c}$

Conditions: Daicel Chiralpak AD-H, $n$-hexane $/ i-\mathrm{PrOH}=95 / 5$, Flow rate $=1 \mathrm{~mL} / \mathrm{min}, \mathrm{UV}=254 \mathrm{~nm}, \mathrm{t}_{\mathrm{R}}=6.1 \mathrm{~min}$ (major) and $t_{R}=6.9$ min.
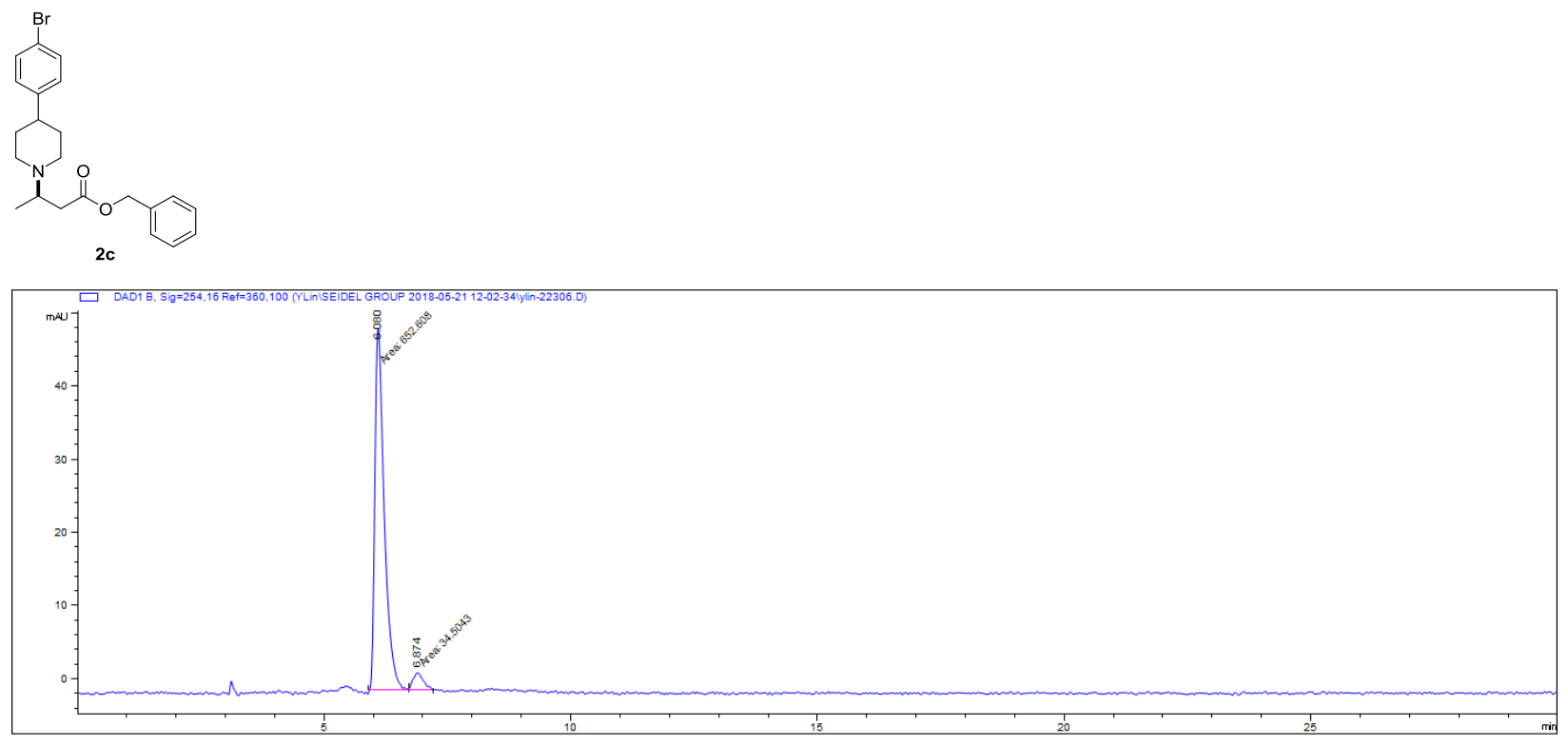

\begin{tabular}{|l|l|l|}
\hline Peak & Retention time $(\mathrm{min})$ & Area \% \\
\hline 1 & 6.080 & 94.978 \\
\hline 2 & 6.874 & 5.022 \\
\hline
\end{tabular}

\section{Racmic sample}

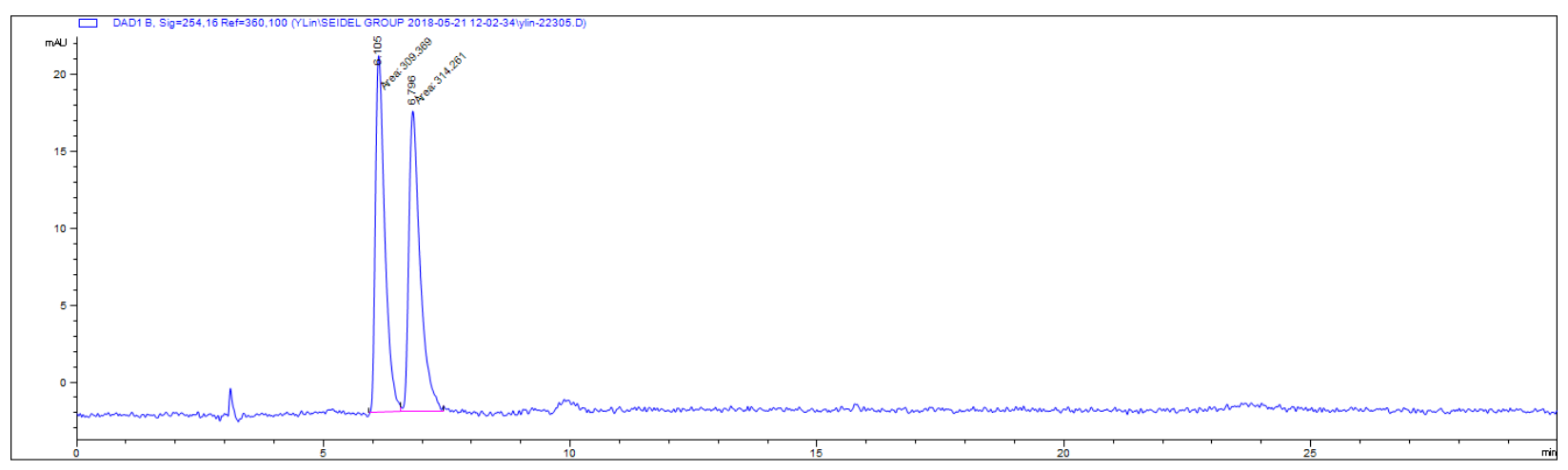

\begin{tabular}{|l|l|l|}
\hline Peak & Retention time $(\mathrm{min})$ & Area \% \\
\hline 1 & 6.105 & 49.608 \\
\hline 2 & 6.796 & 50.392 \\
\hline
\end{tabular}


HPLC Profile of 2d

Conditions: Daicel Chiralpak AS-H, $n$-hexane $/ i-\mathrm{PrOH}=97 / 3$, Flow rate $=1 \mathrm{~mL} / \mathrm{min}, \mathrm{UV}=230 \mathrm{~nm}, \mathrm{t}_{\mathrm{R}}=5.8 \mathrm{~min}$ and $t_{R}=6.7$ min (major).
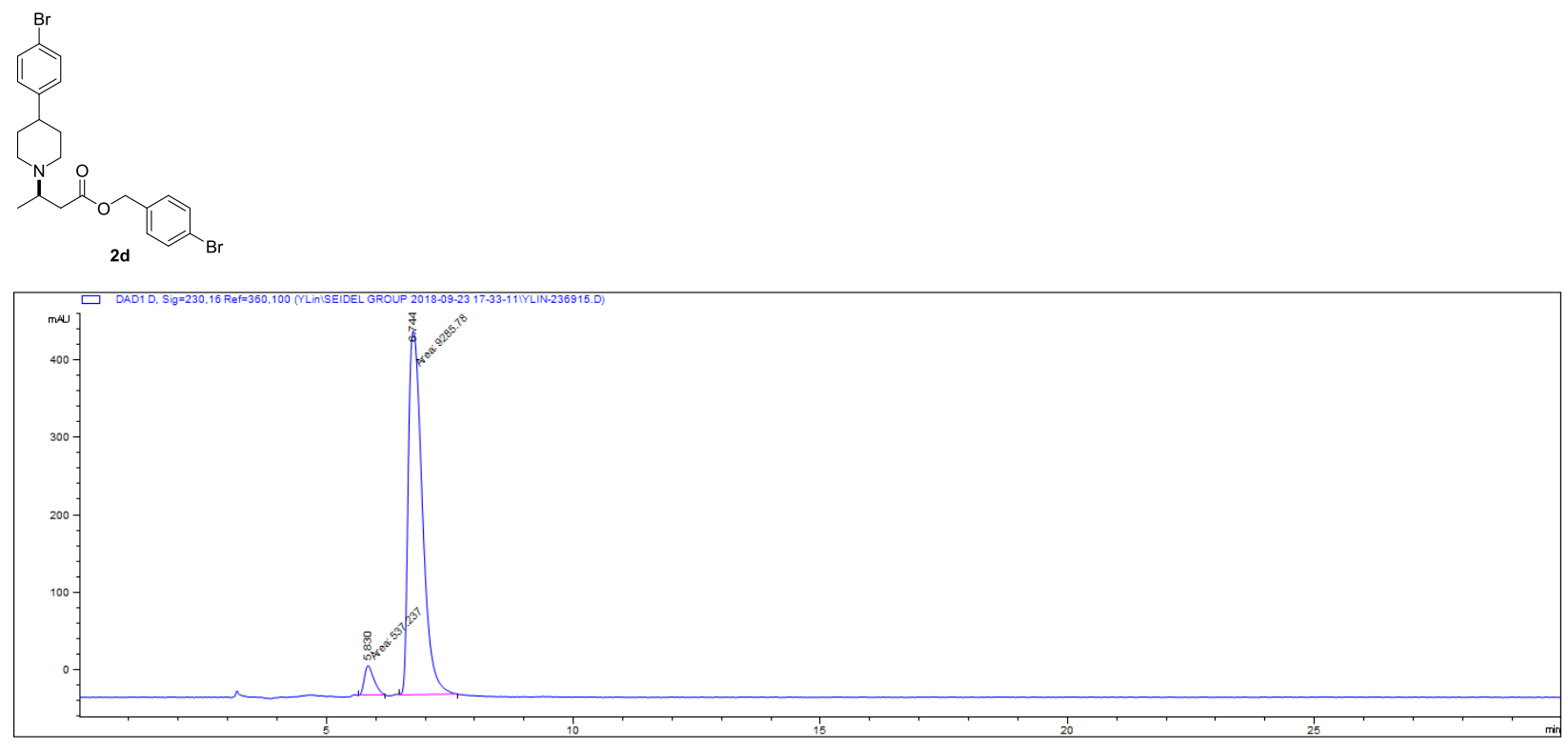

\begin{tabular}{|l|l|l|}
\hline Peak & Retention time $(\mathrm{min})$ & Area \% \\
\hline 1 & 5.830 & 5.469 \\
\hline 2 & 6.744 & 94.531 \\
\hline
\end{tabular}

Racemic sample

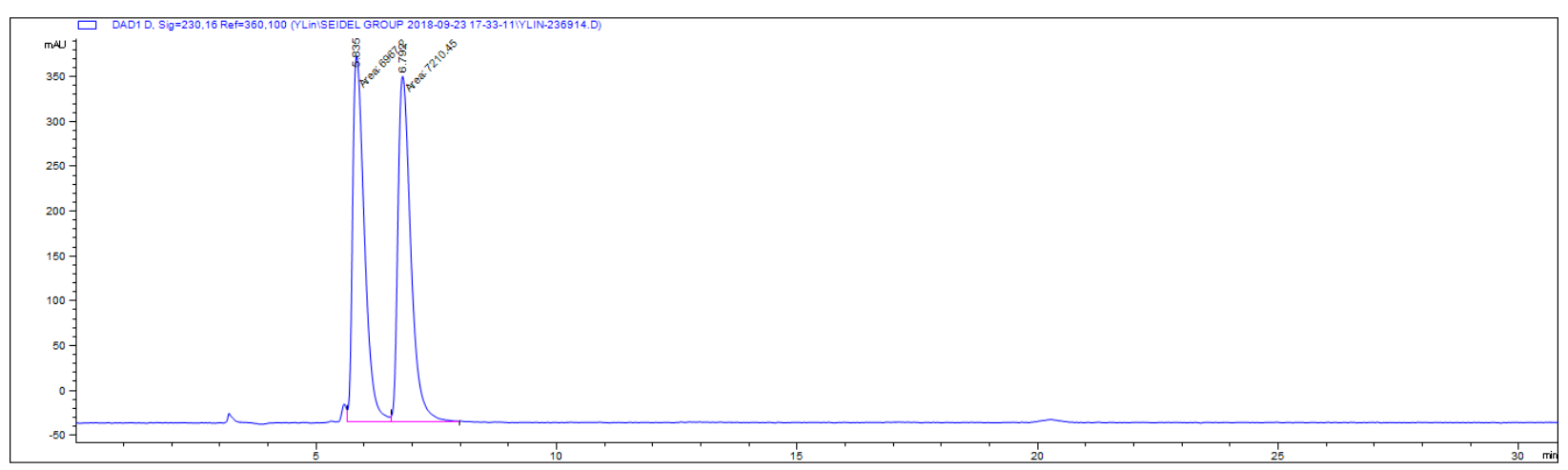

\begin{tabular}{|l|l|l|}
\hline Peak & Retention time $(\mathrm{min})$ & Area \% \\
\hline 1 & 5.835 & 49.142 \\
\hline 2 & 6.792 & 50.858 \\
\hline
\end{tabular}


HPLC Profile of $\mathbf{2 e}$

Conditions: Daicel Chiralcel OJ-H, $n$-hexane $/ i-\mathrm{PrOH}=90 / 10$, Flow rate $=1 \mathrm{~mL} / \mathrm{min}, \mathrm{UV}=230 \mathrm{~nm}, \mathrm{t}_{\mathrm{R}}=11.2 \mathrm{~min}$ and $t_{\mathrm{R}}=17.4 \min$ (major).
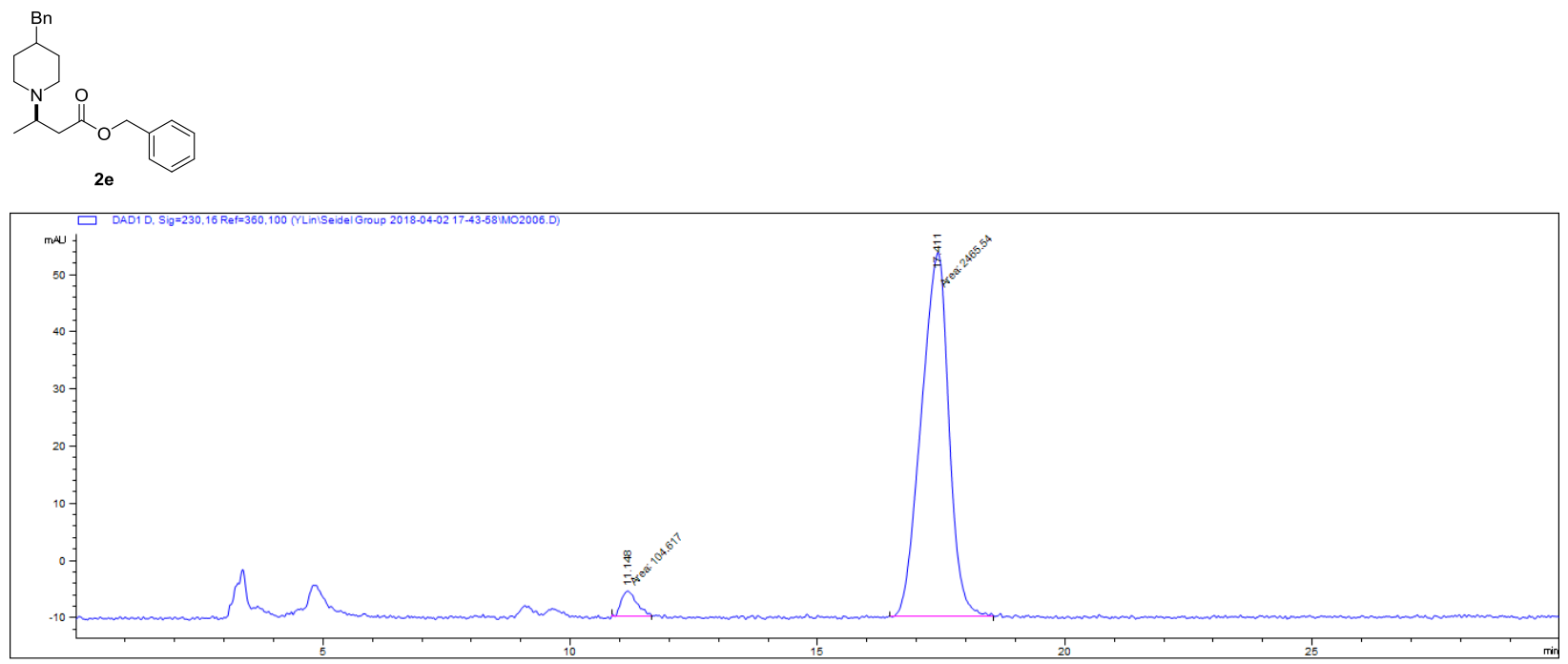

\begin{tabular}{|l|l|l|}
\hline Peak & Retention time $(\min )$ & Area \% \\
\hline 1 & 11.148 & 4.071 \\
\hline 2 & 17.411 & 95.929 \\
\hline
\end{tabular}

Racemic sample

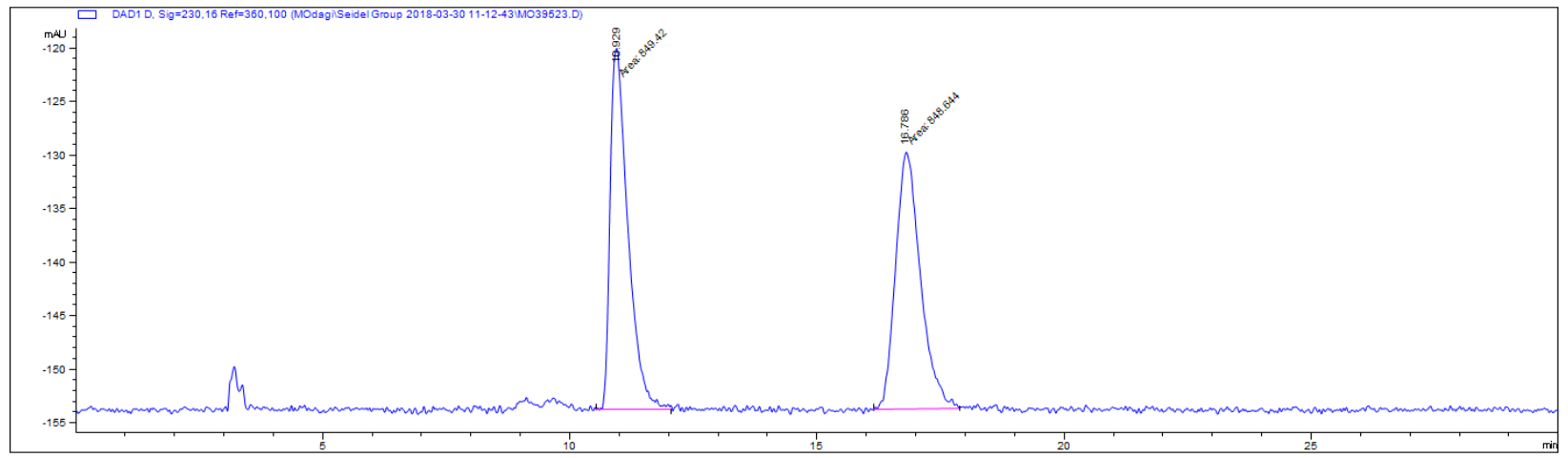

\begin{tabular}{|l|l|l|}
\hline Peak & Retention time $(\min )$ & Area \% \\
\hline 1 & 10.929 & 50.023 \\
\hline 2 & 16.786 & 49.977 \\
\hline
\end{tabular}


HPLC Profile of $\mathbf{2 f}$

Conditions: Daicel Chiralcel OB-H, $n$-hexane $/ \mathrm{i}-\mathrm{PrOH}=97 / 3$, Flow rate $=1 \mathrm{~mL} / \mathrm{min}, \mathrm{UV}=230 \mathrm{~nm}, \mathrm{t}_{\mathrm{R}}=29.6 \mathrm{~min}$ (major) and $t_{R}=36.6$ min.
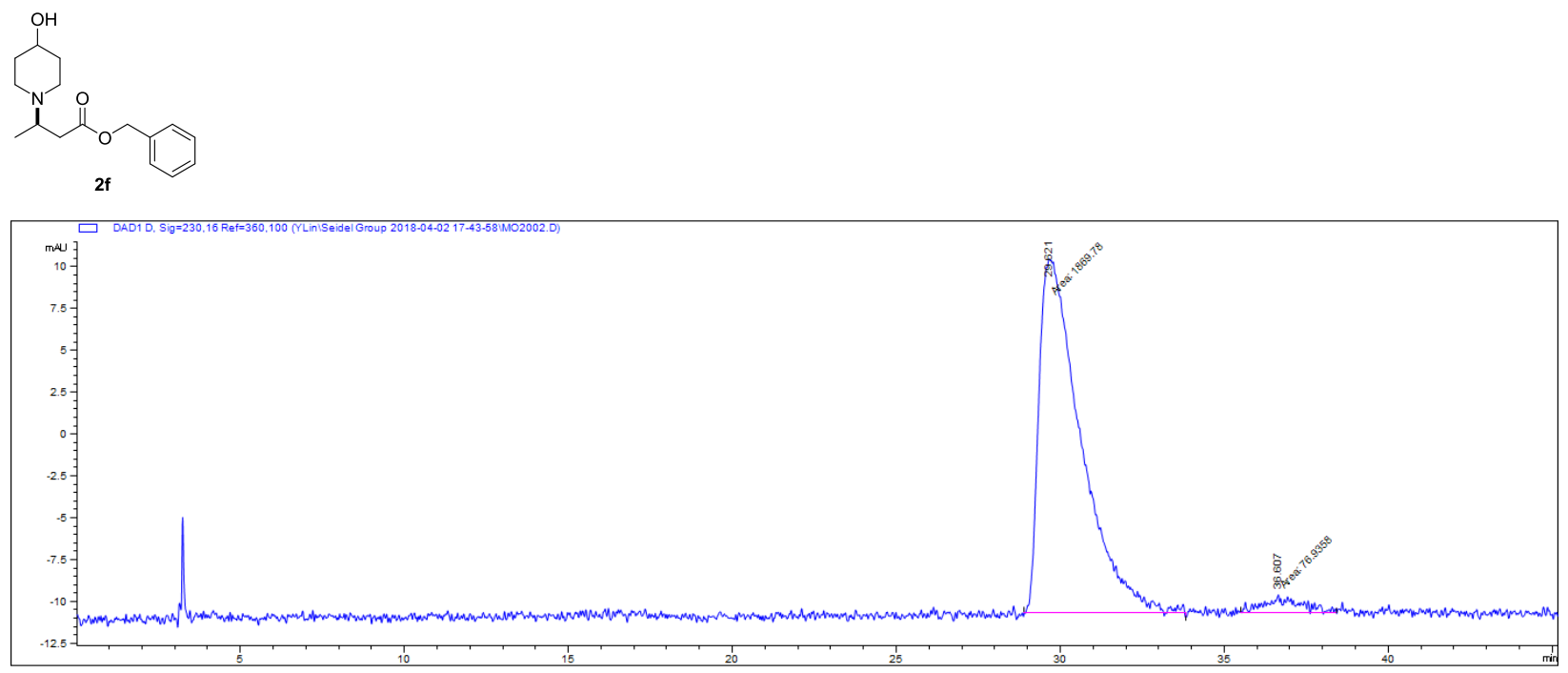

\begin{tabular}{|l|l|l|}
\hline Peak & Retention time $(\mathrm{min})$ & Area \% \\
\hline 1 & 29.621 & 96.048 \\
\hline 2 & 36.607 & 3.952 \\
\hline
\end{tabular}

Racemic sample

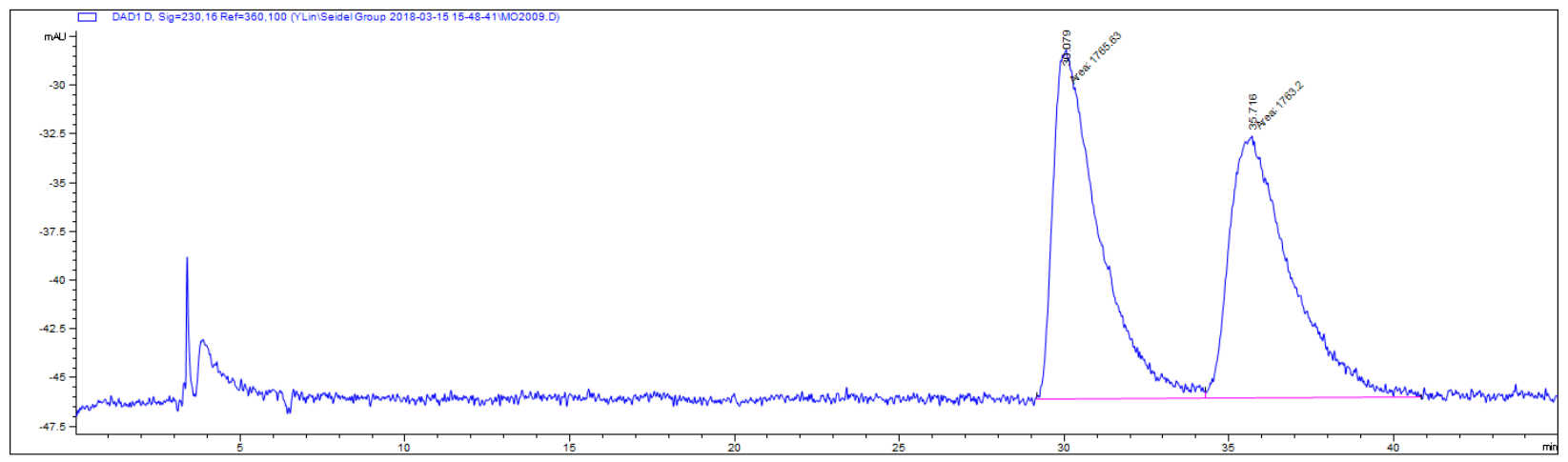

\begin{tabular}{|l|l|l|}
\hline Peak & Retention time $(\min )$ & Area \% \\
\hline 1 & 30.079 & 50.034 \\
\hline 2 & 35.716 & 49.966 \\
\hline
\end{tabular}




\section{HPLC Profile of 2ga}

Conditions: Daicel Chiralpak AS-H, $n$-hexane $/ i-\mathrm{PrOH}=90 / 10$, Flow rate $=1 \mathrm{~mL} / \mathrm{min}, \mathrm{UV}=230 \mathrm{~nm}, \mathrm{t}_{\mathrm{R}}=7.9 \mathrm{~min}$ and $t_{R}=10.8 \min$ (major).

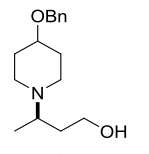

2ga

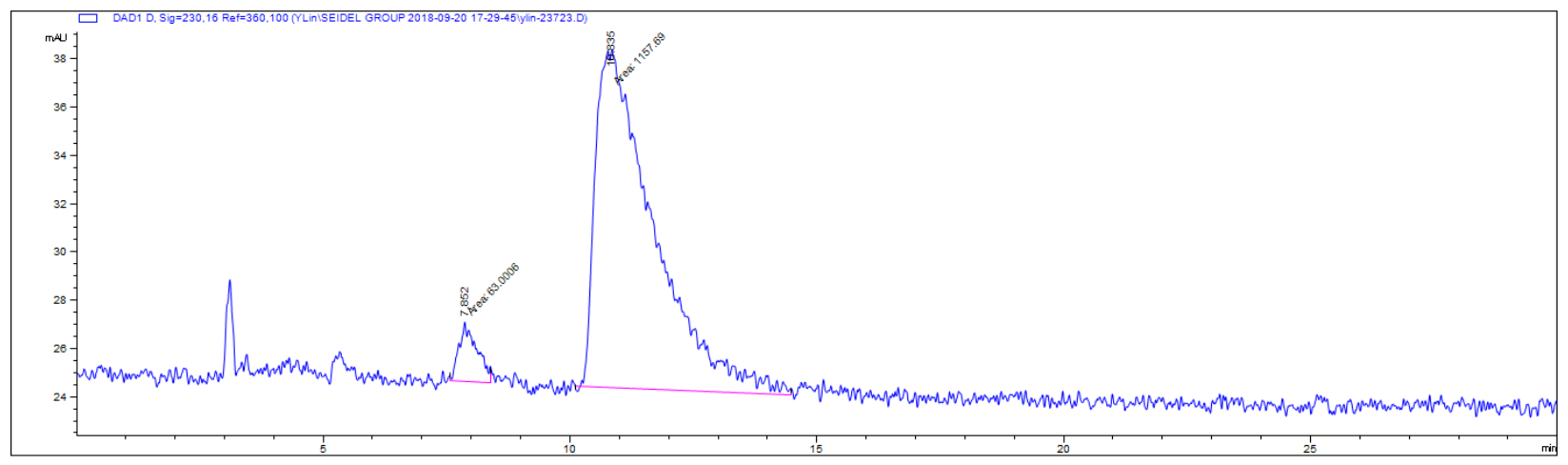

\begin{tabular}{|l|l|l|}
\hline Peak & Retention time $(\min )$ & Area \% \\
\hline 1 & 7.852 & 5.161 \\
\hline 2 & 10.835 & 94.839 \\
\hline
\end{tabular}

Racemic sample

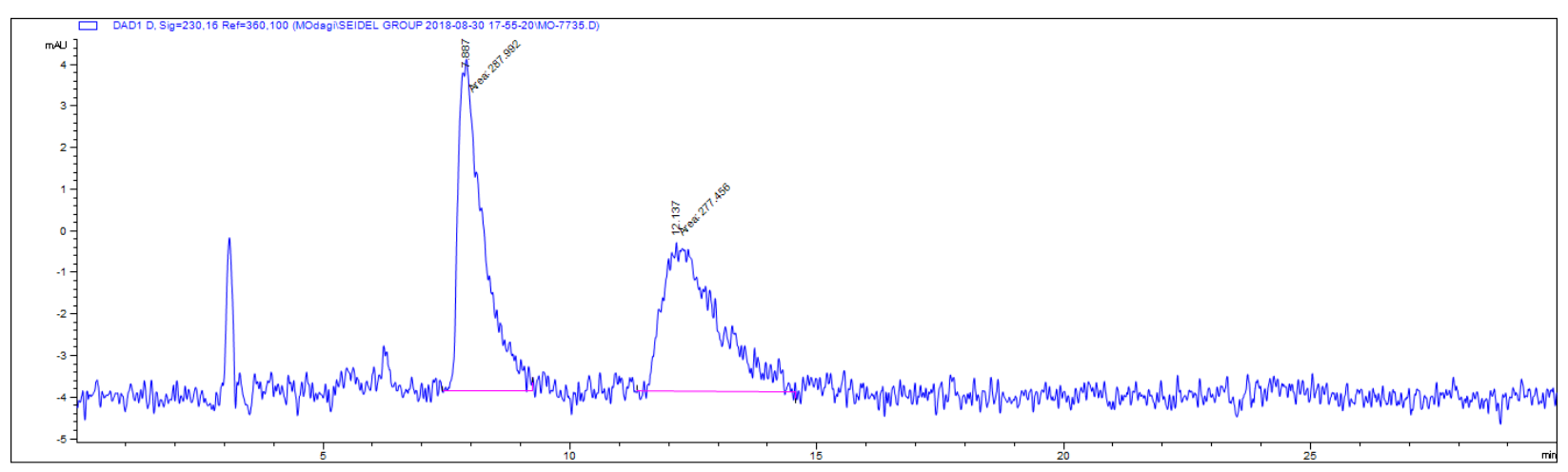

\begin{tabular}{|l|l|l|}
\hline Peak & Retention time $(\min )$ & Area \% \\
\hline 1 & 7.887 & 50.932 \\
\hline 2 & 12.137 & 49.068 \\
\hline
\end{tabular}




\section{HPLC Profile of $\mathbf{2 h a}$}

Conditions: Daicel Chiralpak AD-H, $n$-hexane $/ i-\mathrm{PrOH}=97 / 3$, Flow rate $=1 \mathrm{~mL} / \mathrm{min}, \mathrm{UV}=280 \mathrm{~nm}, \mathrm{t}_{\mathrm{R}}=4.6 \mathrm{~min}$ and $t_{R}=5.1$ min (major).
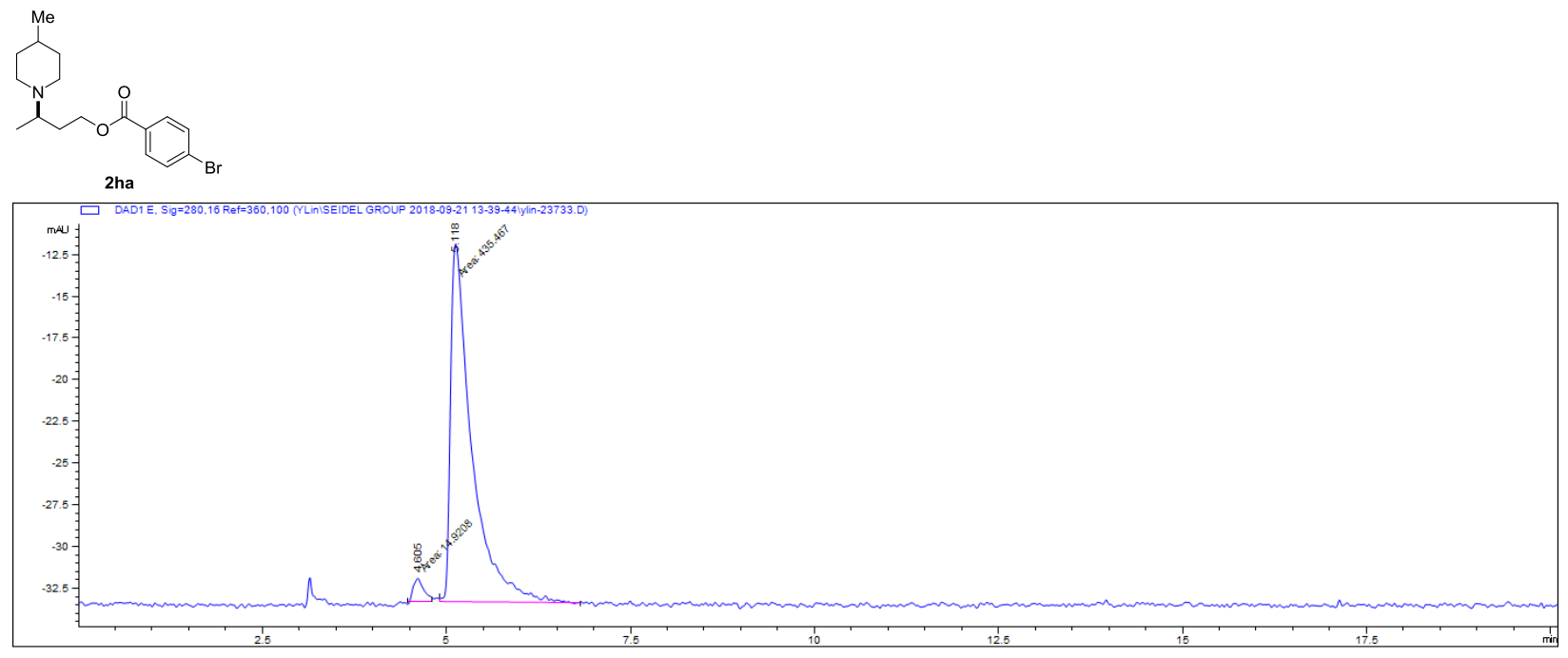

\begin{tabular}{|l|l|l|}
\hline Peak & Retention time $(\min )$ & Area \% \\
\hline 1 & 4.605 & 3.313 \\
\hline 2 & 5.118 & 96.687 \\
\hline
\end{tabular}

Racemic sample

\begin{tabular}{|l|l|l|}
\hline \\
\hline
\end{tabular}




\section{HPLC Profile of $\mathbf{2 i}$}

Conditions: Daicel Chiralpak AS-H, $n$-hexane $/ i-\mathrm{PrOH}=90 / 10$, Flow rate $=1 \mathrm{~mL} / \mathrm{min}, \mathrm{UV}=254 \mathrm{~nm}, \mathrm{t}_{\mathrm{R}}=4.7 \mathrm{~min}$ and $t_{R}=5.4 \min$ (major).
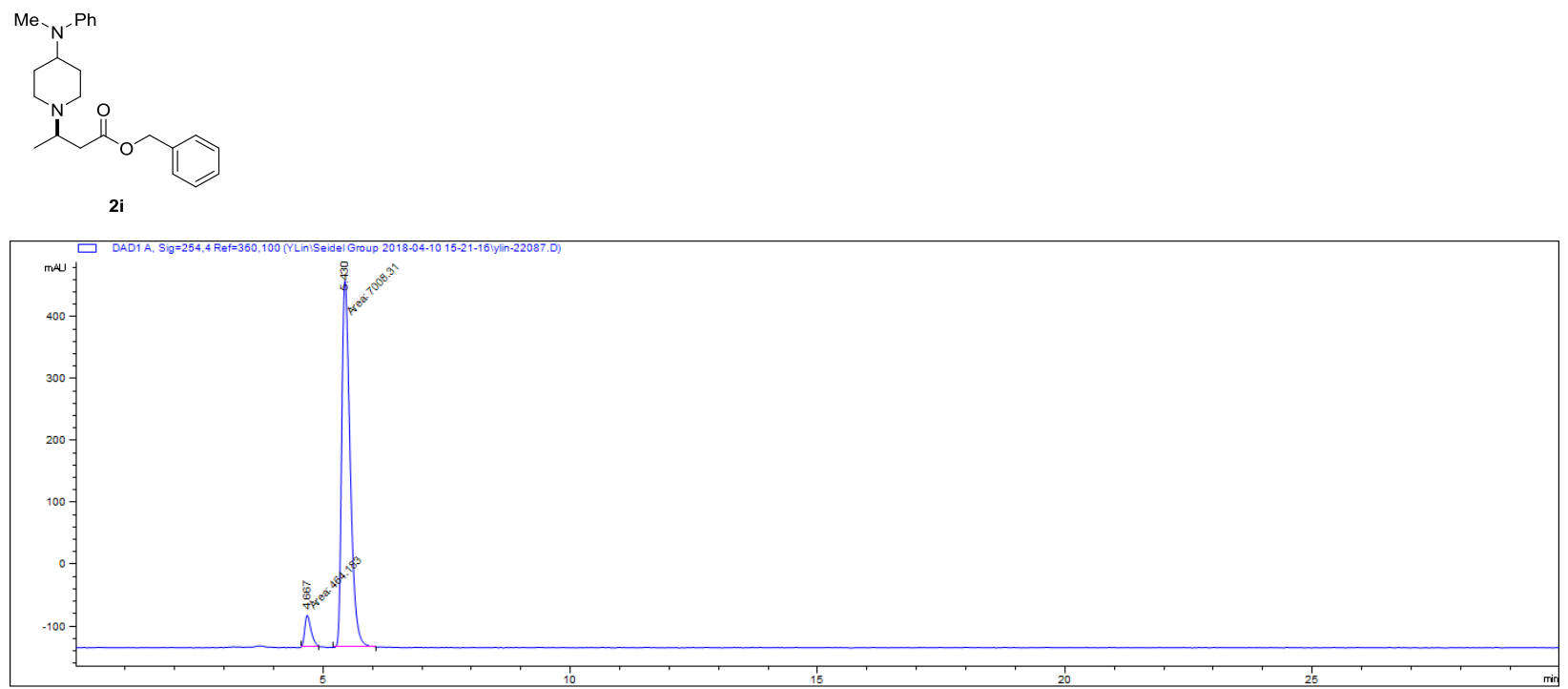

\begin{tabular}{|l|l|l|}
\hline Peak & Retention time (min) & Area \% \\
\hline 1 & 4.667 & 6.212 \\
\hline 2 & 5.430 & 93.788 \\
\hline
\end{tabular}

Racemic sample

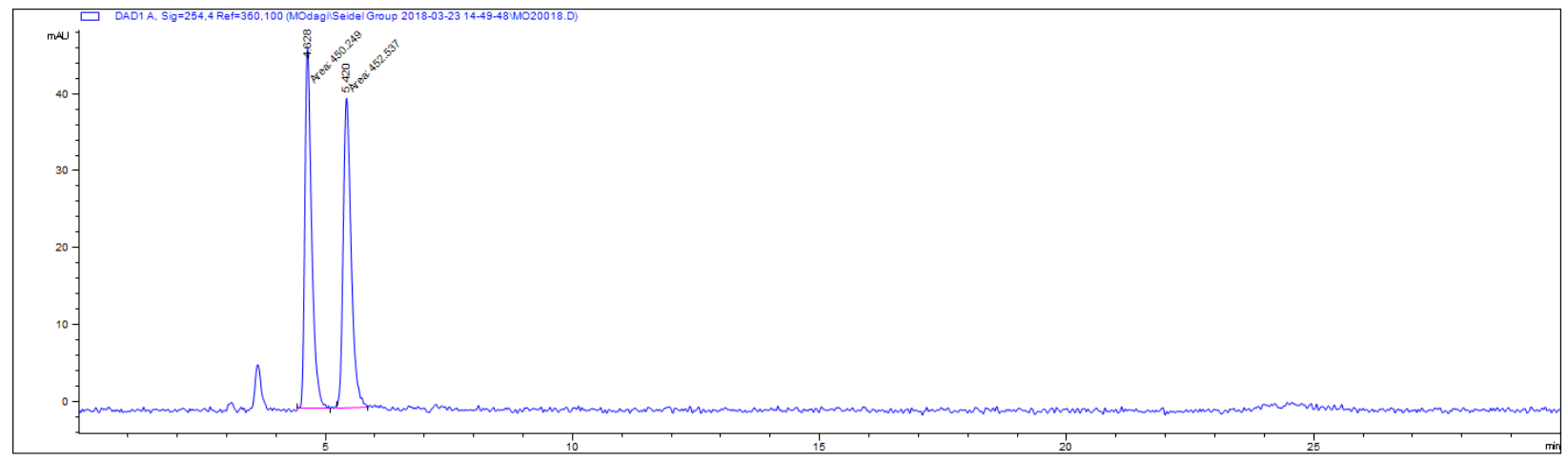

\begin{tabular}{|l|l|l|}
\hline Peak & Retention time (min) & Area \% \\
\hline 1 & 4.628 & 49.873 \\
\hline 2 & 5.420 & 50.127 \\
\hline
\end{tabular}




\section{HPLC Profile of $\mathbf{2 j}$}

Conditions: Daicel Chiralpak AD-H, $n$-hexane $/ i$-PrOH/diethylamine $=95 / 5 / 0.05$, Flow rate $=1 \mathrm{~mL} / \mathrm{min}, \mathrm{UV}=254$ $\mathrm{nm}, \mathrm{t}_{\mathrm{R}}=5.8 \mathrm{~min}$ (major) and $\mathrm{t}_{\mathrm{R}}=6.4 \mathrm{~min}$.
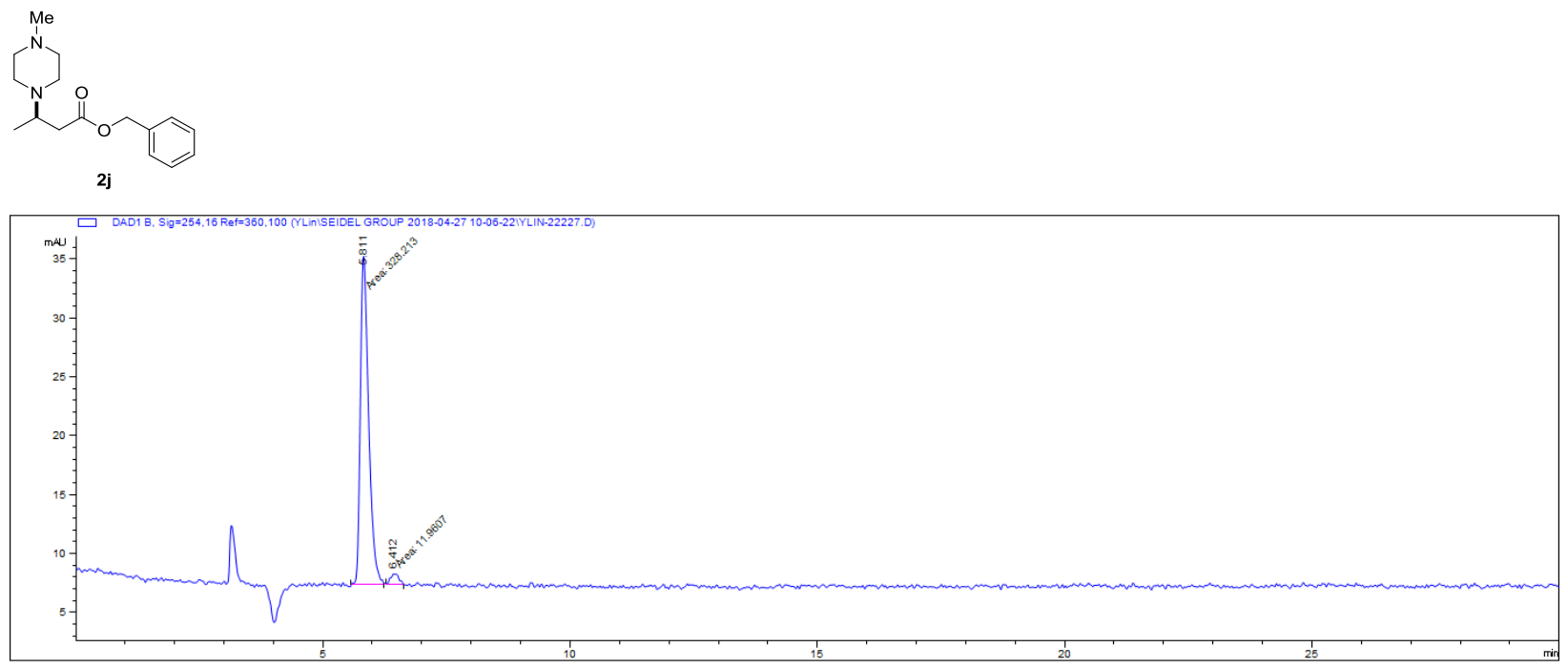

\begin{tabular}{|l|l|l|}
\hline Peak & Retention time $(\min )$ & Area \% \\
\hline 1 & 5.811 & 96.484 \\
\hline 2 & 6.412 & 3.516 \\
\hline
\end{tabular}

Racemic sample

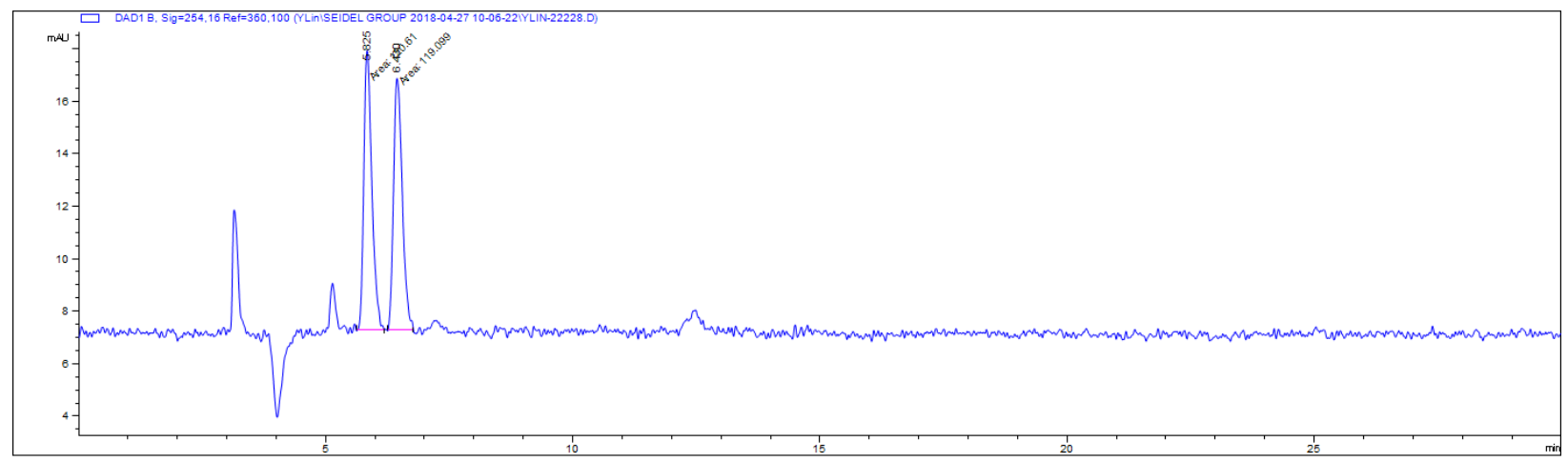

\begin{tabular}{|l|l|l|}
\hline Peak & Retention time (min) & Area \% \\
\hline 1 & 5.825 & 50.315 \\
\hline 2 & 6.430 & 49.685 \\
\hline
\end{tabular}


HPLC Profile of $\mathbf{2 k}$

Conditions: Daicel Chiralpak AS-H, $n$-hexane $/ i-\mathrm{PrOH}=99 / 1$, Flow rate $=1 \mathrm{~mL} / \mathrm{min}, \mathrm{UV}=210 \mathrm{~nm}, \mathrm{t}_{\mathrm{R}}=12.7 \mathrm{~min}$ and $t_{R}=15.6 \min$ (major).
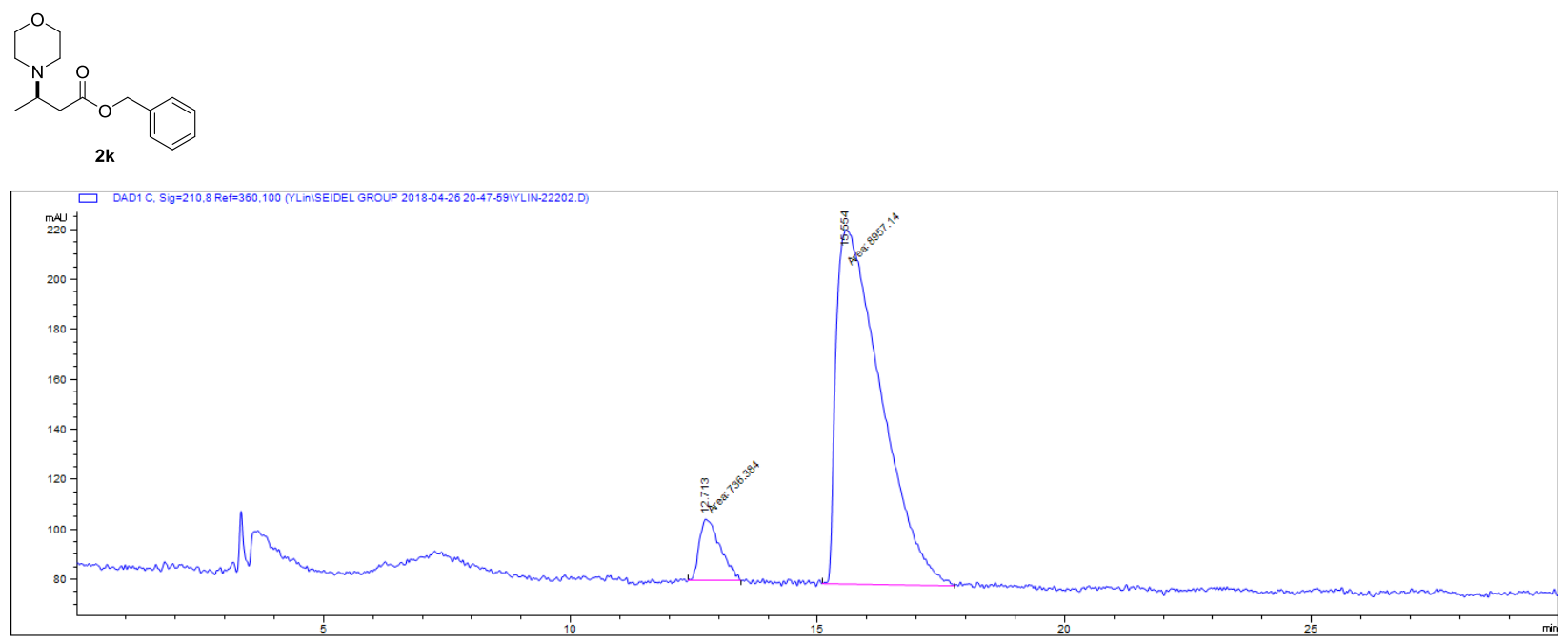

\begin{tabular}{|l|l|l|}
\hline Peak & Retention time $(\min )$ & Area \% \\
\hline 1 & 12.713 & 7.597 \\
\hline 2 & 15.554 & 92.403 \\
\hline
\end{tabular}

\section{Racemic sample}

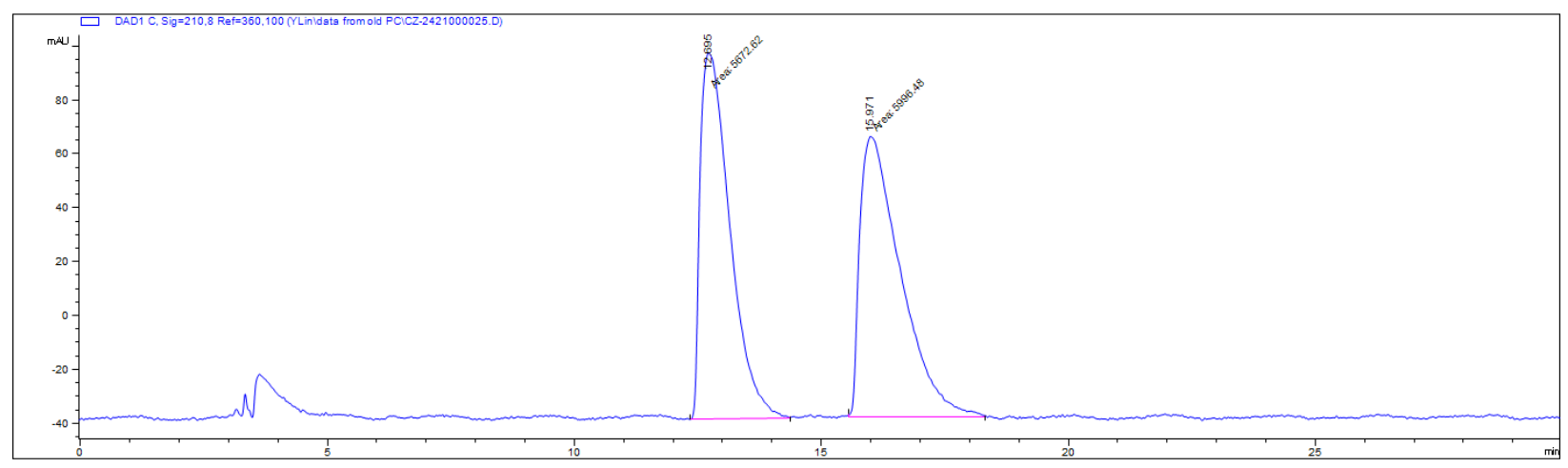

\begin{tabular}{|l|l|l|}
\hline Peak & Retention time $(\min )$ & Area \% \\
\hline 1 & 12.695 & 48.612 \\
\hline 2 & 15.971 & 51.388 \\
\hline
\end{tabular}




\section{HPLC Profile of 21}

Conditions: Daicel Chiralcel OJ-H, $n$-hexane $/ i-\mathrm{PrOH}=90 / 10$, Flow rate $=1 \mathrm{~mL} / \mathrm{min}, \mathrm{UV}=254 \mathrm{~nm}, \mathrm{t}_{\mathrm{R}}=16.2 \mathrm{~min}$ (major) and $t_{\mathrm{R}}=22.4 \mathrm{~min}$.
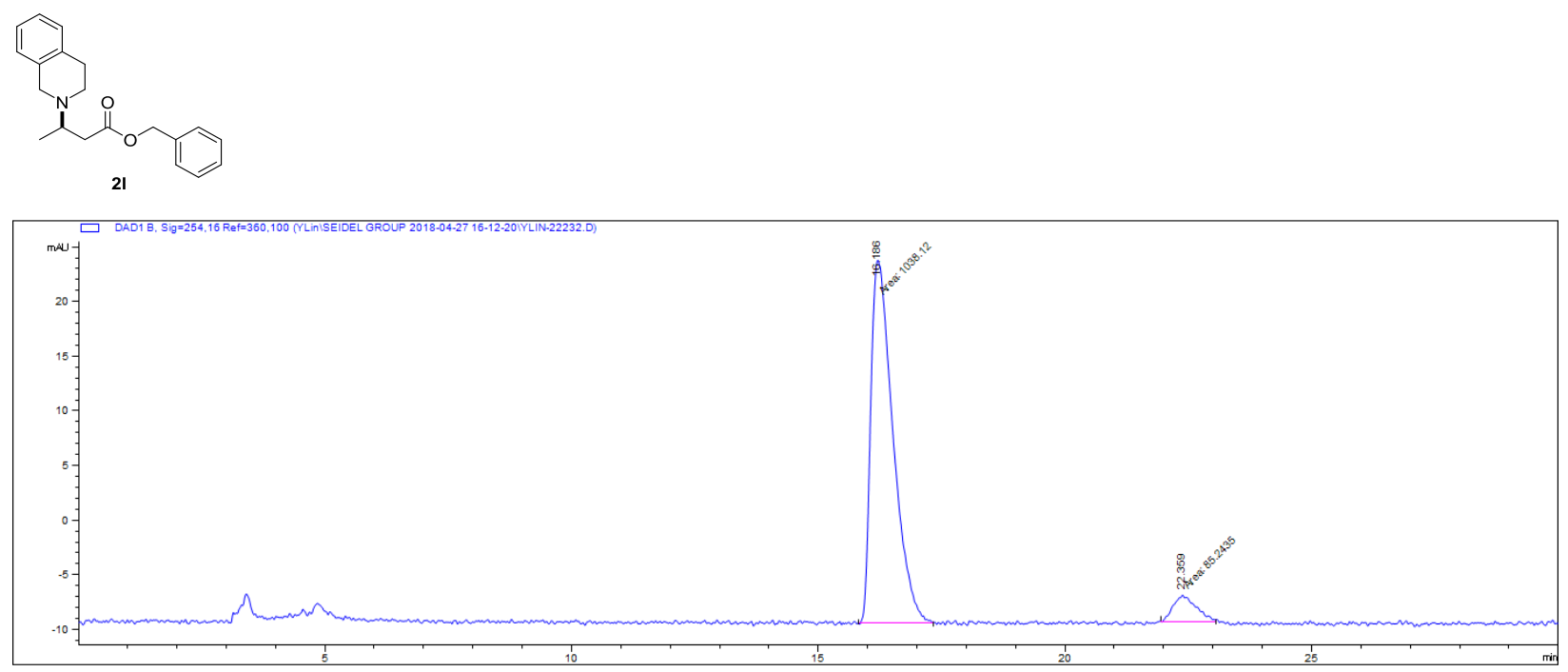

\begin{tabular}{|l|l|l|}
\hline Peak & Retention time $(\mathrm{min})$ & Area \% \\
\hline 1 & 16.186 & 92.412 \\
\hline 2 & 22.359 & 7.588 \\
\hline
\end{tabular}

Racemic sample

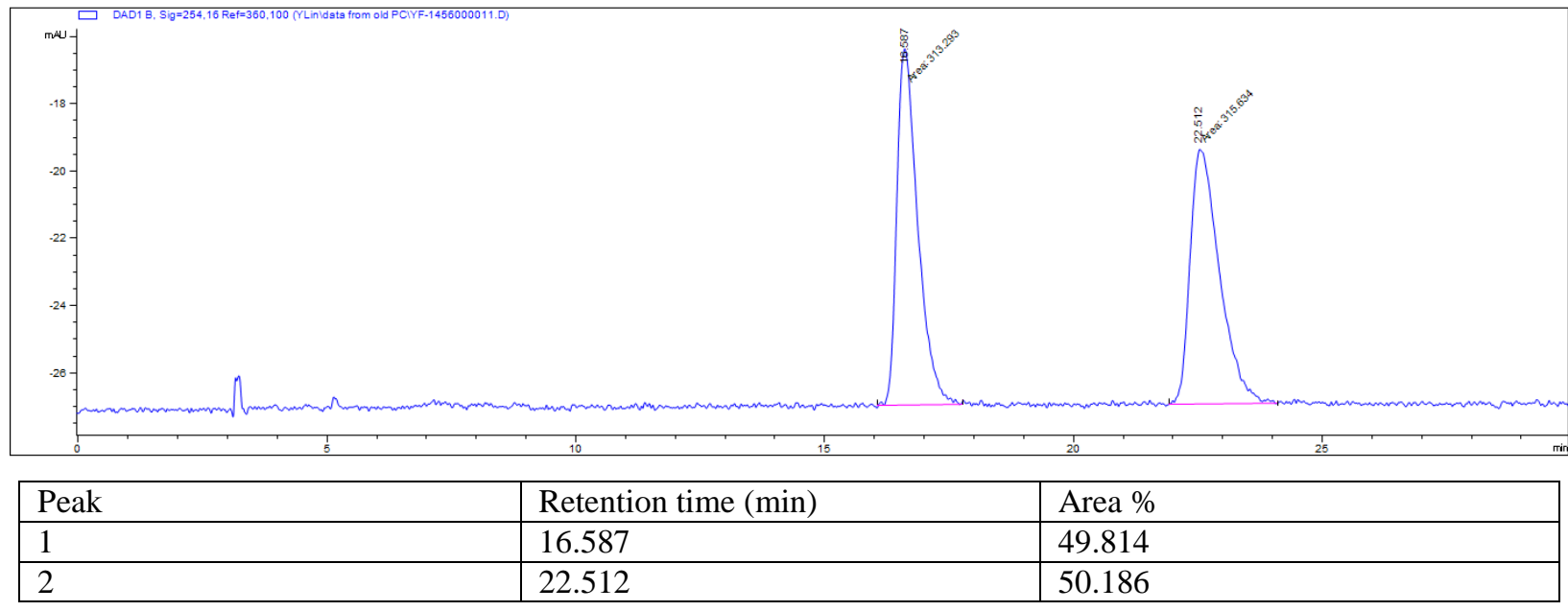




\section{HPLC Profile of $\mathbf{2 m a}$}

Conditions: Daicel Chiralcel OB-H, $n$-hexane $/ i$-PrOH/diethylamine $=99 / 1 / 0.05$, Flow rate $=1 \mathrm{~mL} / \mathrm{min}, \mathrm{UV}=280$ $\mathrm{nm}, \mathrm{t}_{\mathrm{R}}=6.1 \mathrm{~min}$ and $\mathrm{t}_{\mathrm{R}}=6.9 \min$ (major).
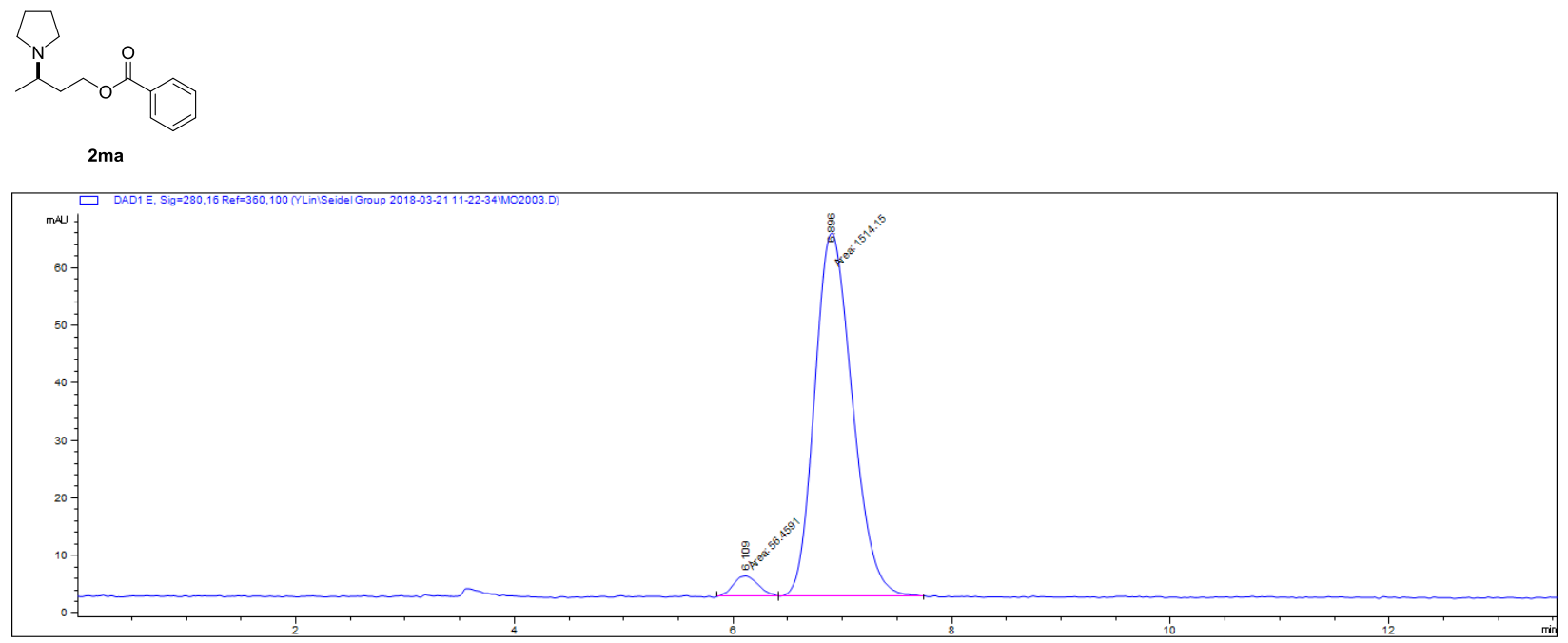

\begin{tabular}{|l|l|l|}
\hline Peak & Retention time $(\min )$ & Area \% \\
\hline 1 & 6.109 & 3.595 \\
\hline 2 & 6.896 & 96.405 \\
\hline
\end{tabular}

\section{Racemic sample}

\begin{tabular}{|l|l|l|l|}
\hline \\
\hline Peak
\end{tabular}




\section{HPLC Profile of 2n}

Conditions: Daicel Chiralpak AY-H, $n$-hexane $/ i-\mathrm{PrOH}=99 / 1$, Flow rate $=1 \mathrm{~mL} / \mathrm{min}, \mathrm{UV}=230 \mathrm{~nm}, \mathrm{t}_{\mathrm{R}}=8.1 \mathrm{~min}$ and $t_{R}=8.4$ min (major).
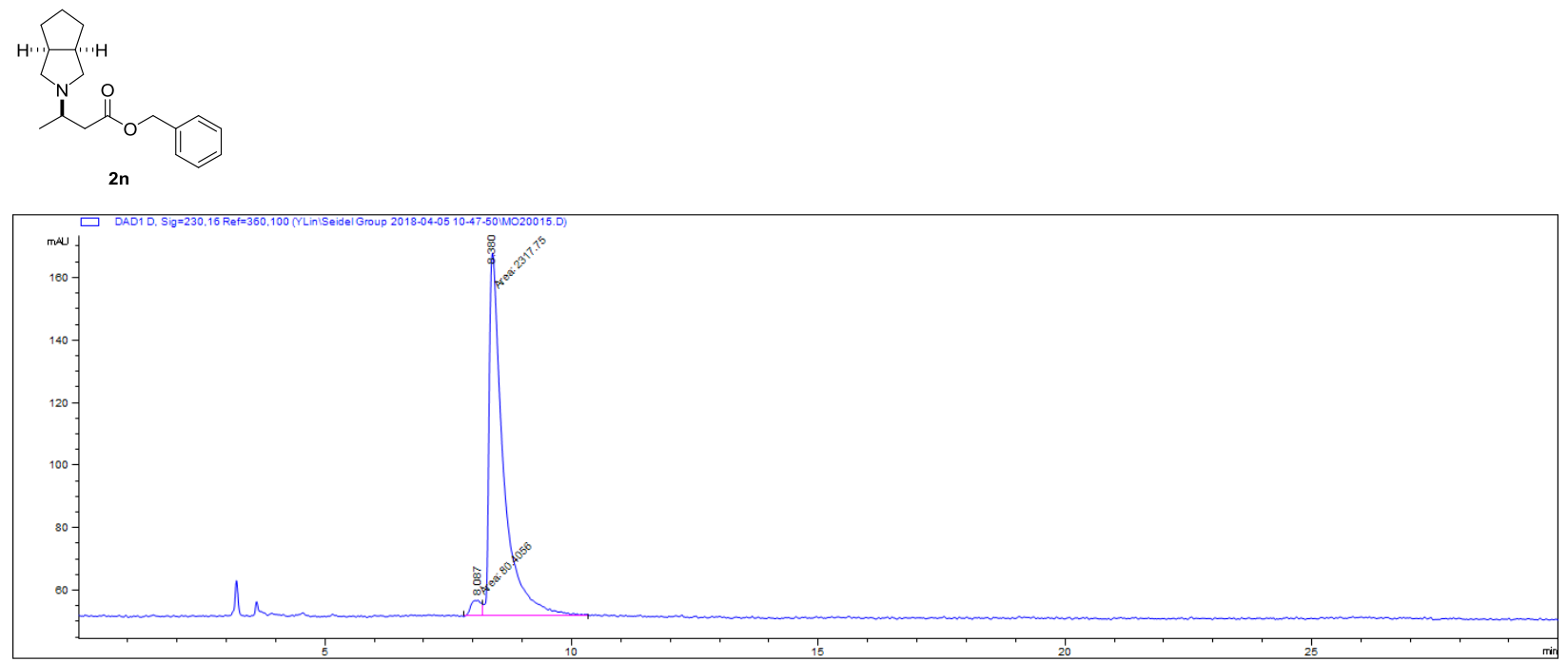

\begin{tabular}{|l|l|l|}
\hline Peak & Retention time $(\min )$ & Area \% \\
\hline 1 & 8.087 & 3.353 \\
\hline 2 & 8.380 & 96.647 \\
\hline
\end{tabular}

Racemic sample

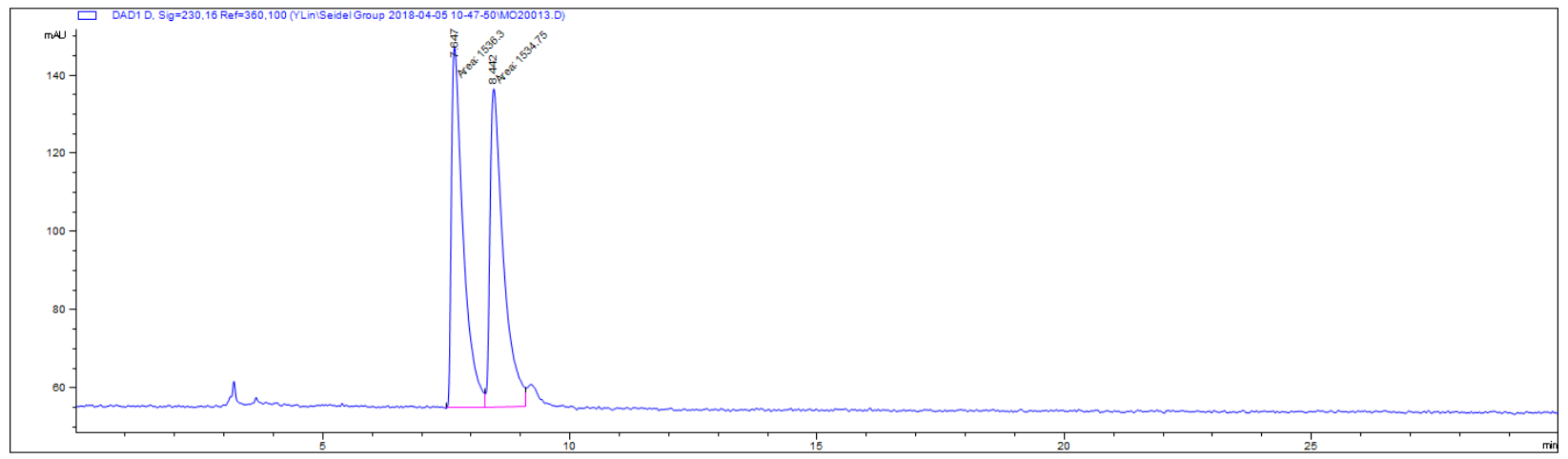

\begin{tabular}{|l|l|l|}
\hline Peak & Retention time (min) & Area \% \\
\hline 1 & 7.647 & 50.025 \\
\hline 2 & 8.442 & 49.975 \\
\hline
\end{tabular}


HPLC Profile of 20

Conditions: Daicel Chiralpak AY-H, $n$-hexane $/ i-\mathrm{PrOH}=99 / 1$, Flow rate $=1 \mathrm{~mL} / \mathrm{min}, \mathrm{UV}=230 \mathrm{~nm}, \mathrm{t}_{\mathrm{R}}=6.9 \mathrm{~min}$ and $t_{R}=7.3$ min (major).
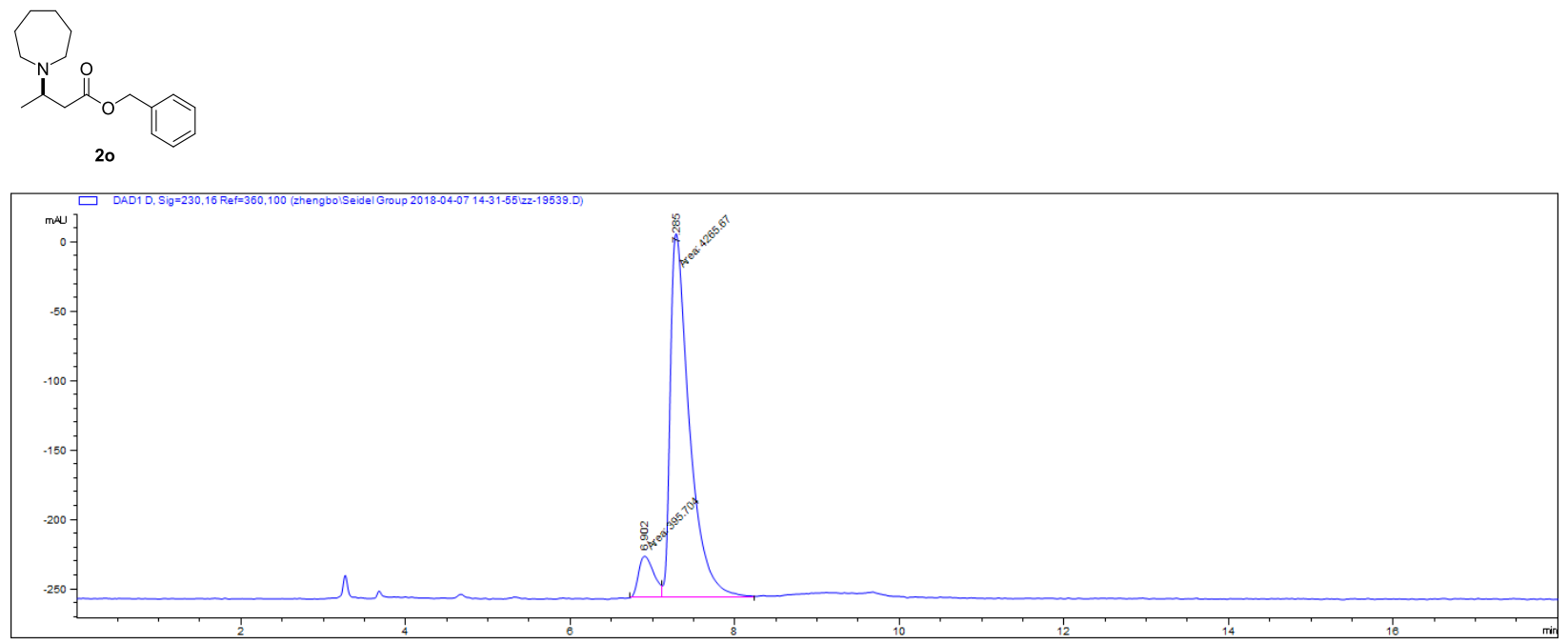

\begin{tabular}{|l|l|l|}
\hline Peak & Retention time (min) & Area \% \\
\hline 1 & 6.902 & 8.489 \\
\hline 2 & 7.285 & 91.511 \\
\hline
\end{tabular}

\section{Racemic sample}

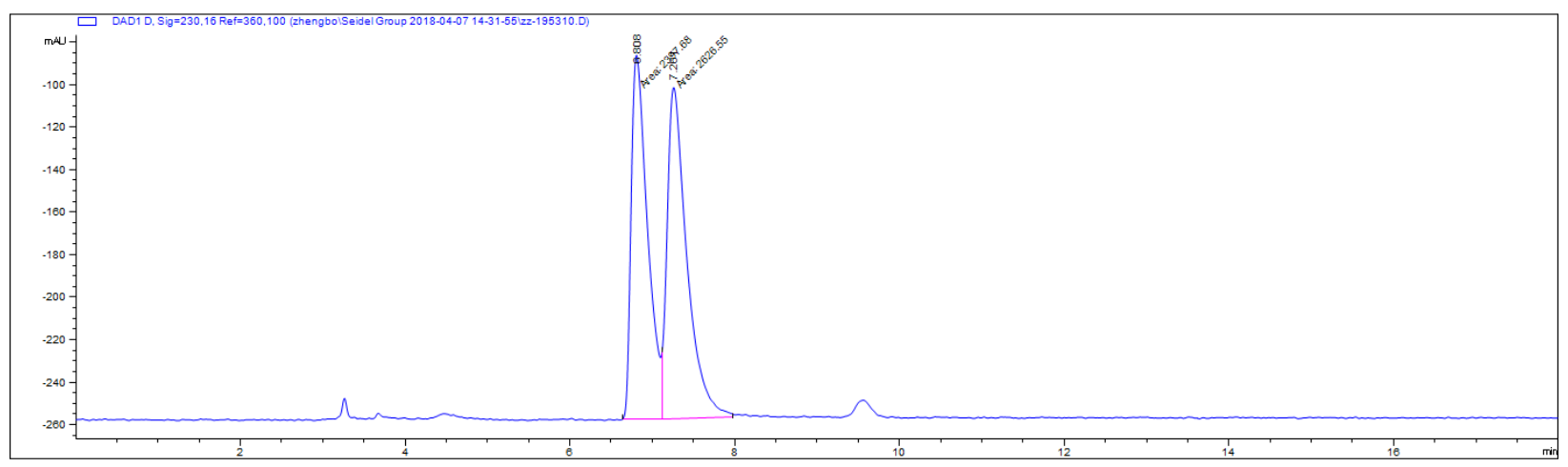

\begin{tabular}{|l|l|l|}
\hline Peak & Retention time $(\min )$ & Area \% \\
\hline 1 & 6.808 & 47.722 \\
\hline 2 & 7.269 & 52.278 \\
\hline
\end{tabular}




\section{HPLC Profile of $\mathbf{2 p}$}

Conditions: Daicel Chiralcel OJ-H, $n$-hexane $/ i-\mathrm{PrOH}=99 / 1$, Flow rate $=1 \mathrm{~mL} / \mathrm{min}, \mathrm{UV}=230 \mathrm{~nm}, \mathrm{t}_{\mathrm{R}}=6.6 \mathrm{~min}$ (major) and $t_{R}=7.2$ min.
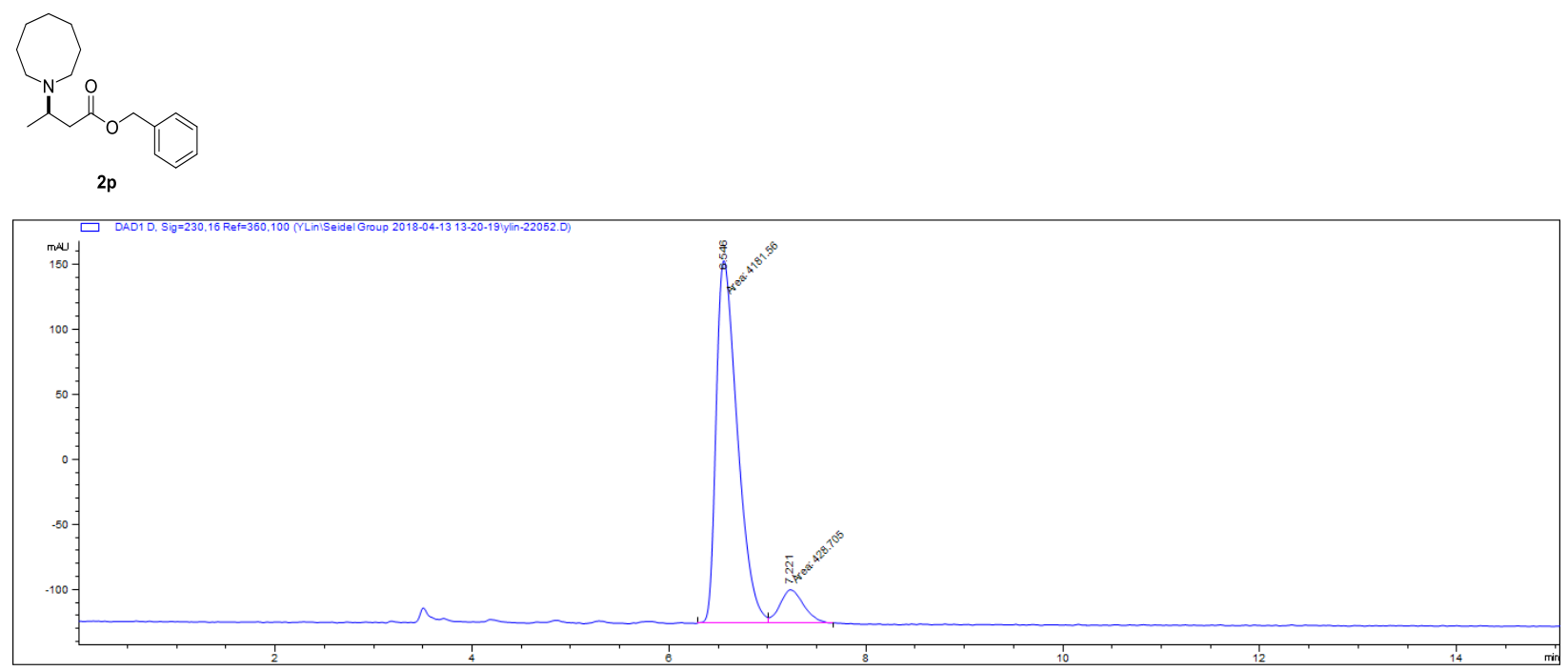

\begin{tabular}{|l|l|l|}
\hline Peak & Retention time $(\mathrm{min})$ & Area \% \\
\hline 1 & 6.546 & 90.740 \\
\hline 2 & 7.221 & 9.260 \\
\hline
\end{tabular}

Racemic sample

\begin{tabular}{|l|l|l|l|}
\hline \\
\hline Peak
\end{tabular}




\section{HPLC Profile of $\mathbf{2 q}$}

Conditions: Daicel Chiralpak AY-H, $n$-hexane $/ i-\mathrm{PrOH}=90 / 10$, Flow rate $=1 \mathrm{~mL} / \mathrm{min}, \mathrm{UV}=254 \mathrm{~nm}, \mathrm{t}_{\mathrm{R}}=9.7 \mathrm{~min}$ and $t_{R}=10.2 \min$ (major).

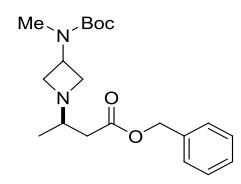

2q

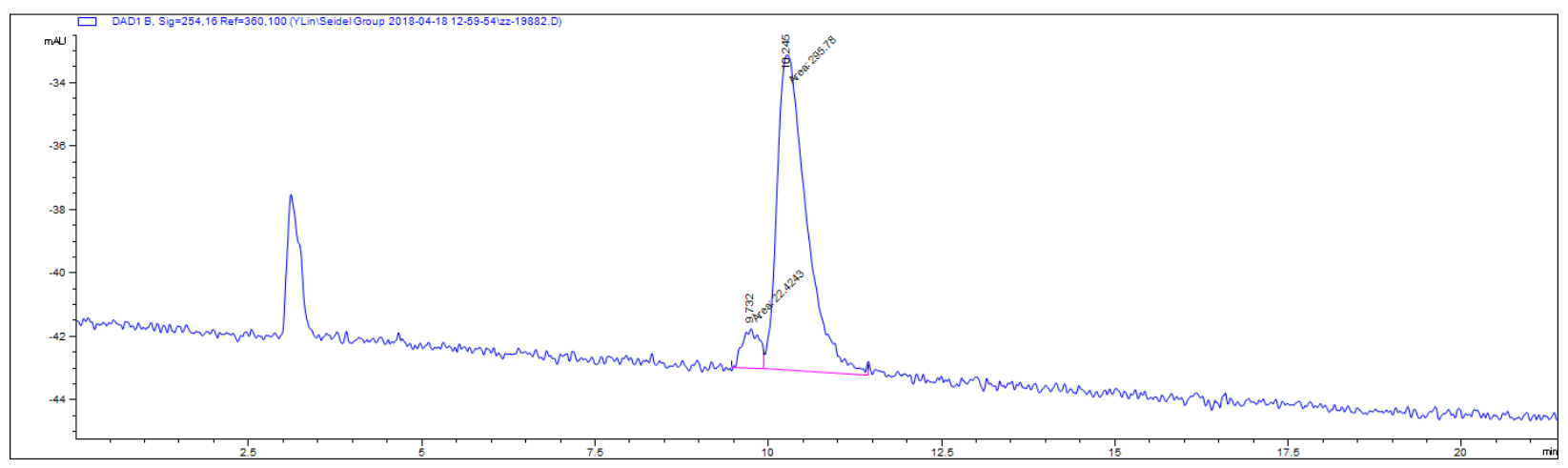

\begin{tabular}{|l|l|l|}
\hline Peak & Retention time $(\min )$ & Area \% \\
\hline 1 & 9.732 & 7.047 \\
\hline 2 & 10.245 & 92.953 \\
\hline
\end{tabular}

Racemic sample

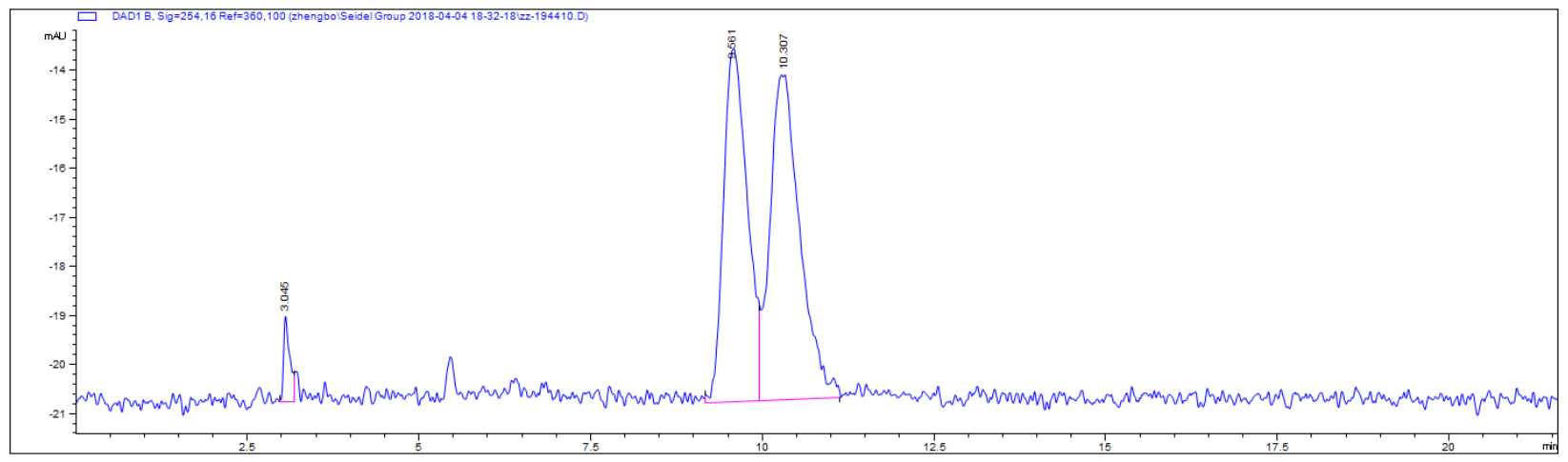

\begin{tabular}{|l|l|l|}
\hline Peak & Retention time (min) & Area \% \\
\hline 1 & 9.561 & 49.141 \\
\hline 2 & 10.307 & 50.859 \\
\hline
\end{tabular}


HPLC Profile of $\mathbf{2 r}$

Conditions: Daicel Chiralcel OB-H, $n$-hexane $/ i-\mathrm{PrOH}=90 / 10$, Flow rate $=1 \mathrm{~mL} / \mathrm{min}, \mathrm{UV}=230 \mathrm{~nm}, \mathrm{t}_{\mathrm{R}}=17.6 \mathrm{~min}$ (major) and $t_{R}=21.3$ min.
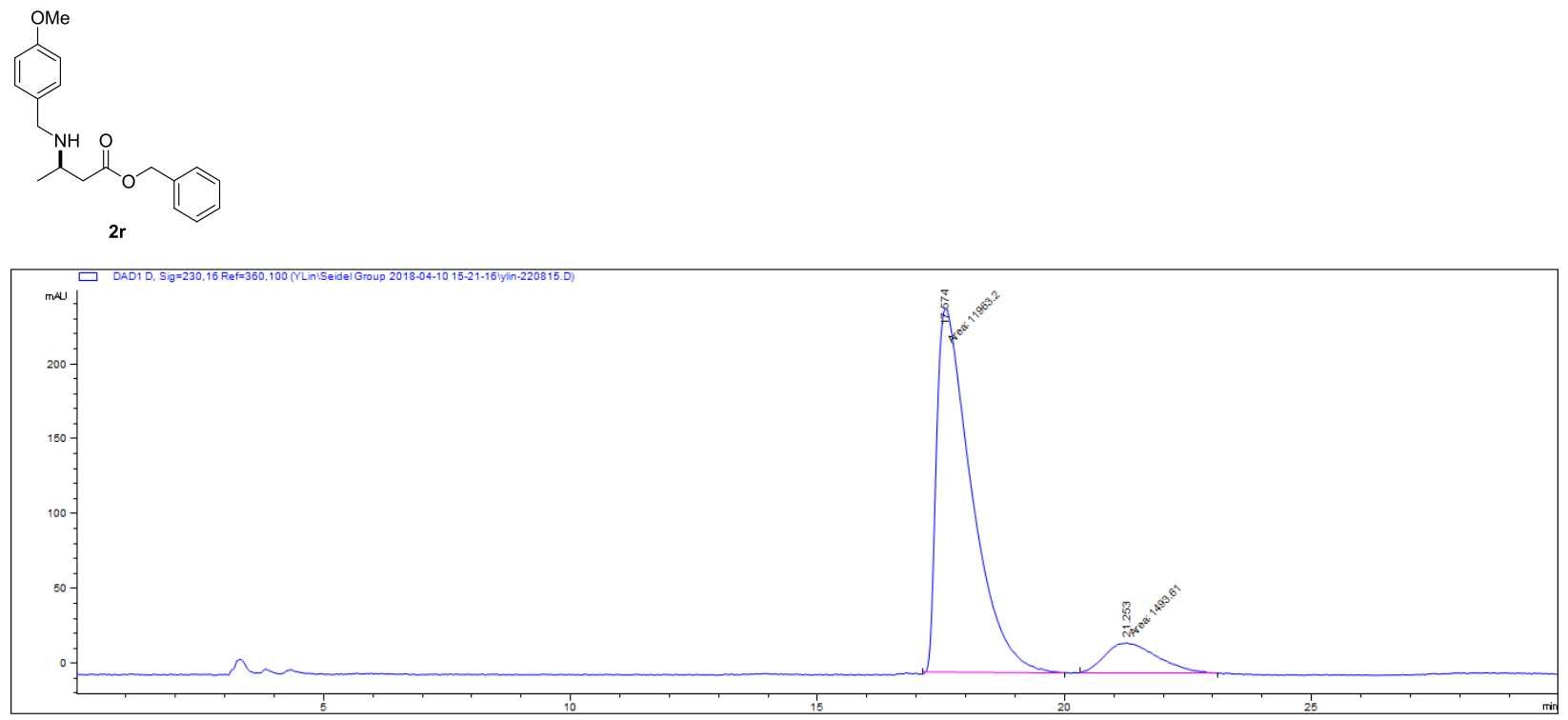

\begin{tabular}{|l|l|l|}
\hline Peak & Retention time $(\mathrm{min})$ & Area \% \\
\hline 1 & 17.574 & 88.901 \\
\hline 2 & 21.253 & 11.099 \\
\hline
\end{tabular}

\section{Racemic sample}

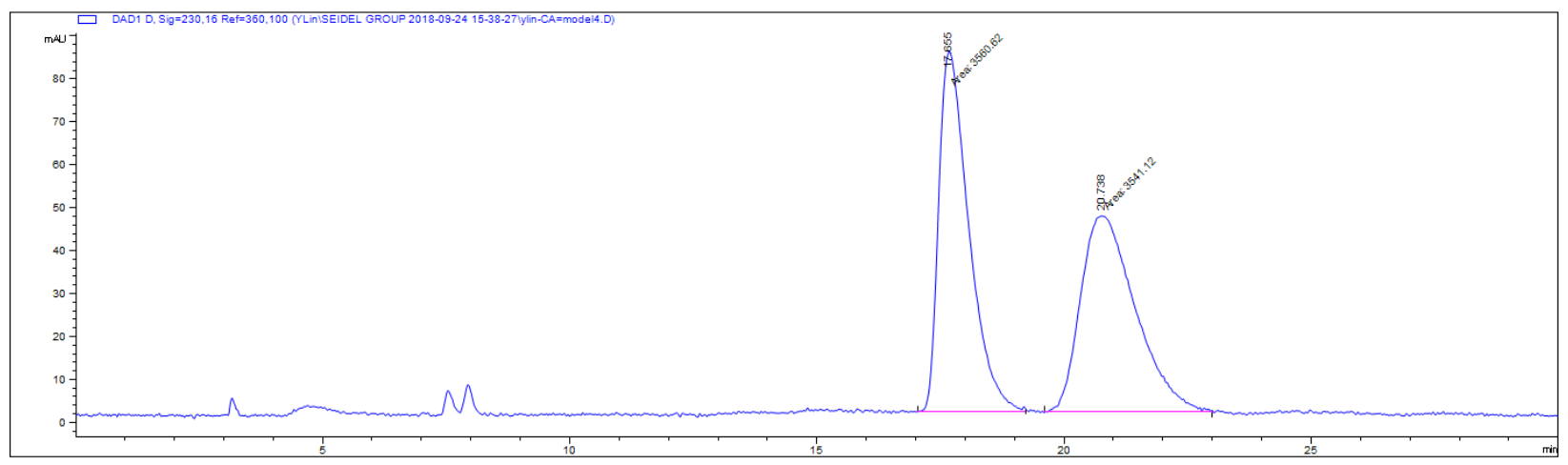

\begin{tabular}{|l|l|l|}
\hline Peak & Retention time (min) & Area \% \\
\hline 1 & 17.655 & 50.137 \\
\hline 2 & 20.738 & 49.863 \\
\hline
\end{tabular}


HPLC Profile of $\mathbf{2 s}$

Conditions: Daicel Chiralcel OD-H, $n$-hexane $/ i$-PrOH $=90 / 10$, Flow rate $=1 \mathrm{~mL} / \mathrm{min}, \mathrm{UV}=280 \mathrm{~nm}, \mathrm{t}_{\mathrm{R}}=7.2 \mathrm{~min}$ (major) and $t_{R}=8.7$ min.

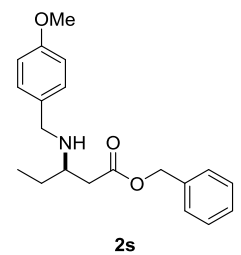

2s

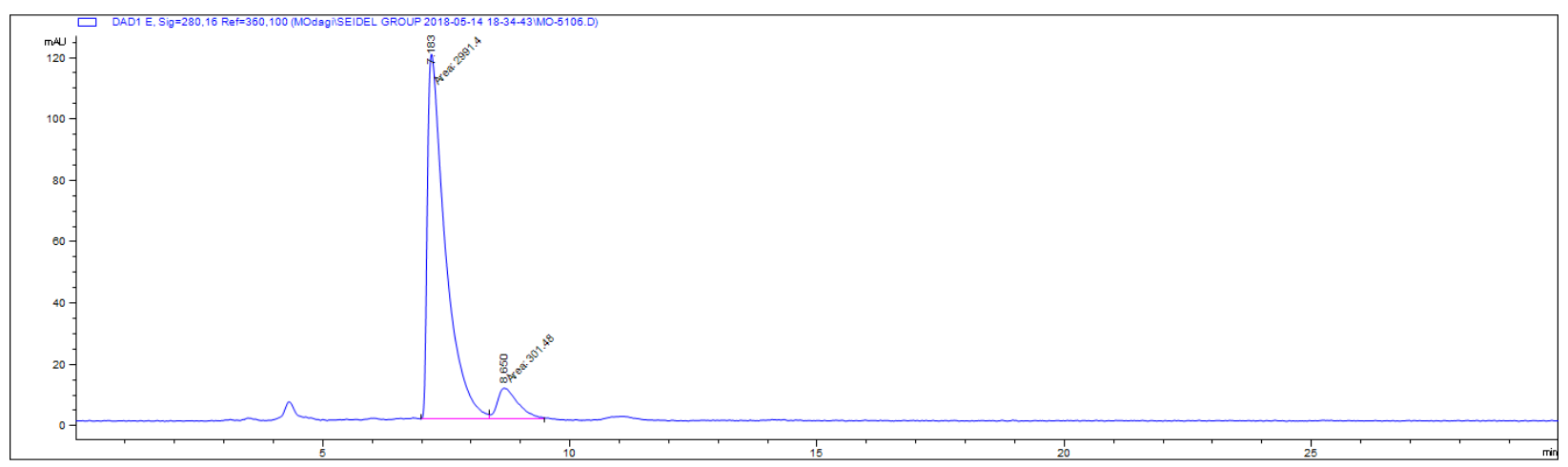

\begin{tabular}{|l|l|l|}
\hline Peak & Retention time $(\mathrm{min})$ & Area \% \\
\hline 1 & 7.183 & 90.844 \\
\hline 2 & 8.650 & 9.156 \\
\hline
\end{tabular}

Racemic sample

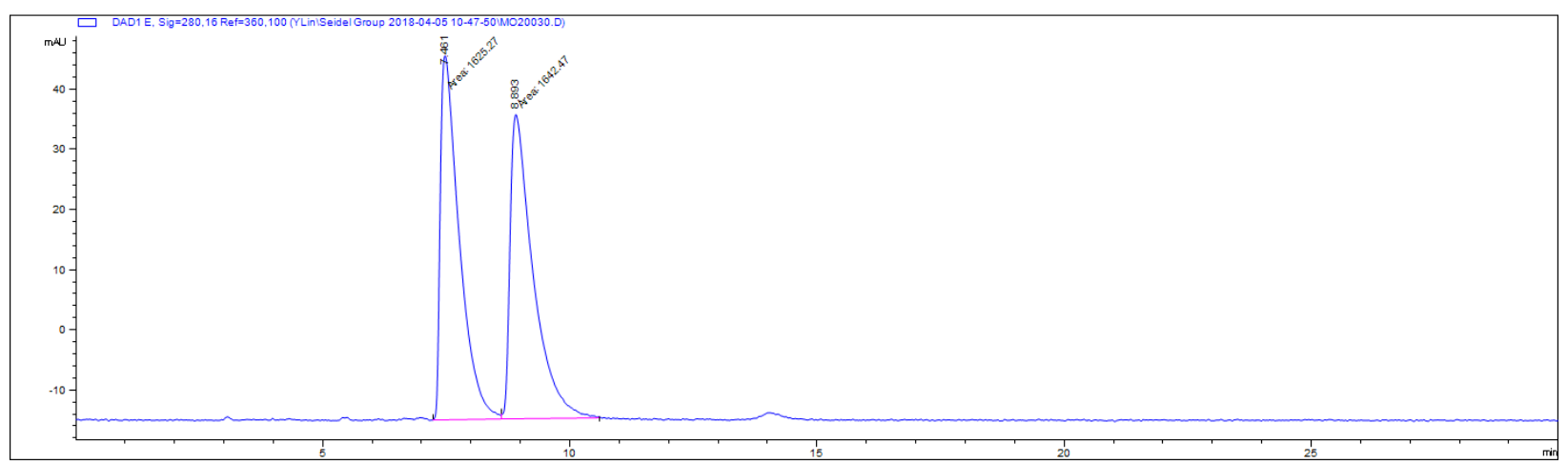

\begin{tabular}{|l|l|l|}
\hline Peak & Retention time $(\mathrm{min})$ & Area \% \\
\hline 1 & 7.461 & 49.737 \\
\hline 2 & 8.893 & 50.263 \\
\hline
\end{tabular}




\section{HPLC Profile of $\mathbf{2 t}$}

Conditions: Daicel Chiralcel OB-H, $n$-hexane $/ i-\mathrm{PrOH}=99 / 1$, Flow rate $=1 \mathrm{~mL} / \mathrm{min}, \mathrm{UV}=230 \mathrm{~nm}, \mathrm{t}_{\mathrm{R}}=9.1 \mathrm{~min}$ and $t_{R}=11.9 \min$ (major).
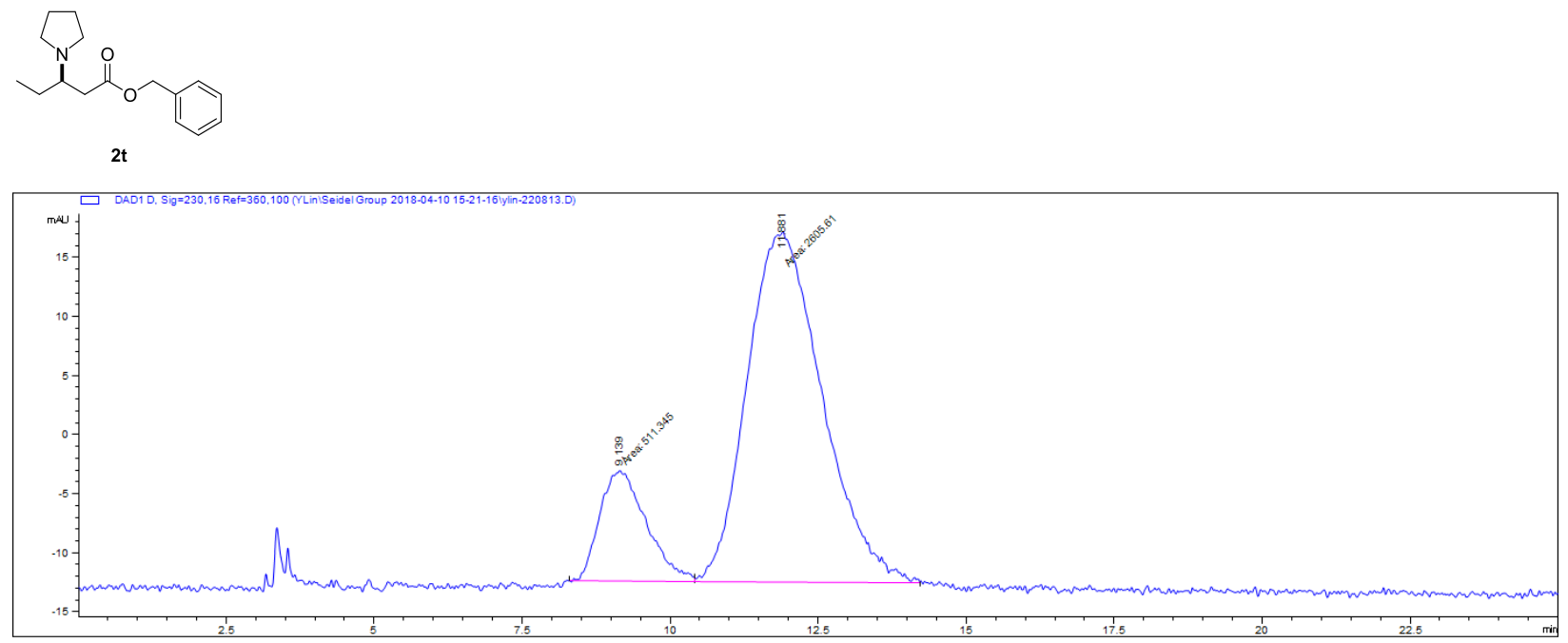

\begin{tabular}{|l|l|l|}
\hline Peak & Retention time $(\mathrm{min})$ & Area \% \\
\hline 1 & 9.139 & 16.405 \\
\hline 2 & 11.881 & 83.595 \\
\hline
\end{tabular}

\section{Racemic sample}

\begin{tabular}{|l|l|l|}
\hline \\
\hline Peak
\end{tabular}


HPLC Profile of $\mathbf{4 a}$

Conditions: Daicel Chiralpak AD-H, $n$-hexane $/ i-\mathrm{PrOH}=99 / 1$, Flow rate $=1 \mathrm{~mL} / \mathrm{min}, \mathrm{UV}=230 \mathrm{~nm}, \mathrm{t}_{\mathrm{R}}=7.5 \mathrm{~min}$ (major) and $t_{R}=8.9$ min.

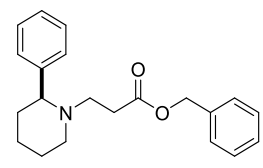

4a

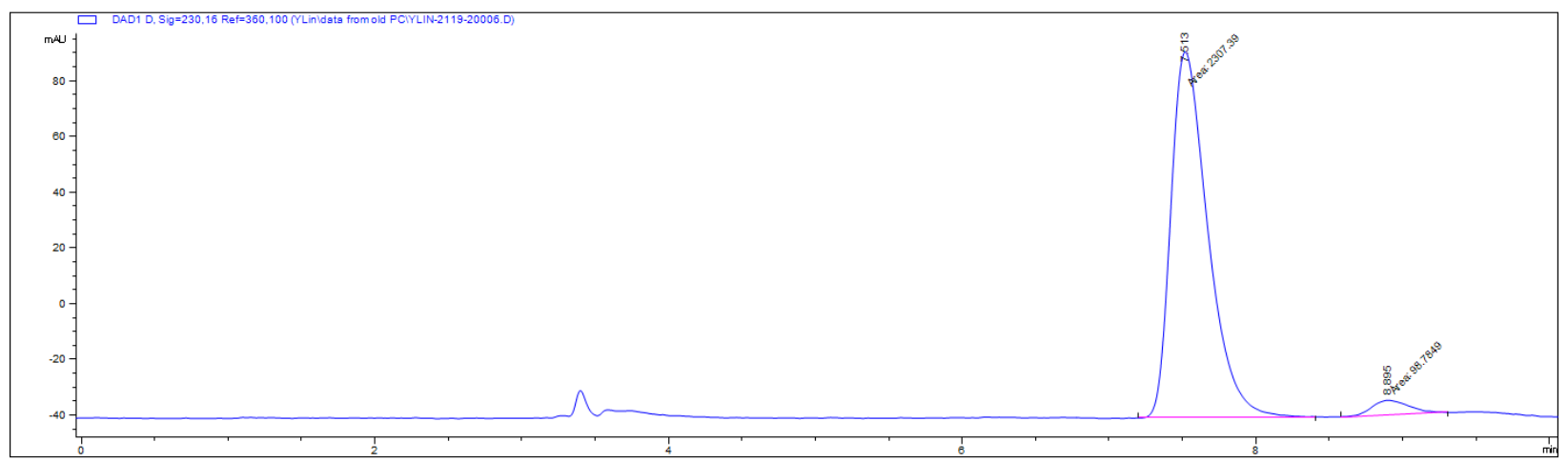

\begin{tabular}{|l|l|l|}
\hline Peak & Retention time $(\mathrm{min})$ & Area \% \\
\hline 1 & 7.513 & 95.895 \\
\hline 2 & 8.895 & 4.105 \\
\hline
\end{tabular}

Racemic sample

\begin{tabular}{|l|l|l|}
\hline \\
\hline
\end{tabular}


HPLC Profile of $\mathbf{5 a}$

Conditions: Daicel Chiralcel OJ-H, $n$-hexane $/ i-\mathrm{PrOH}=99 / 1$, Flow rate $=0.5 \mathrm{~mL} / \mathrm{min}, \mathrm{UV}=230 \mathrm{~nm}, \mathrm{t}_{\mathrm{R}}=12.5 \mathrm{~min}$ (major) and $t_{R}=14.0$ min.

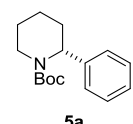

5a

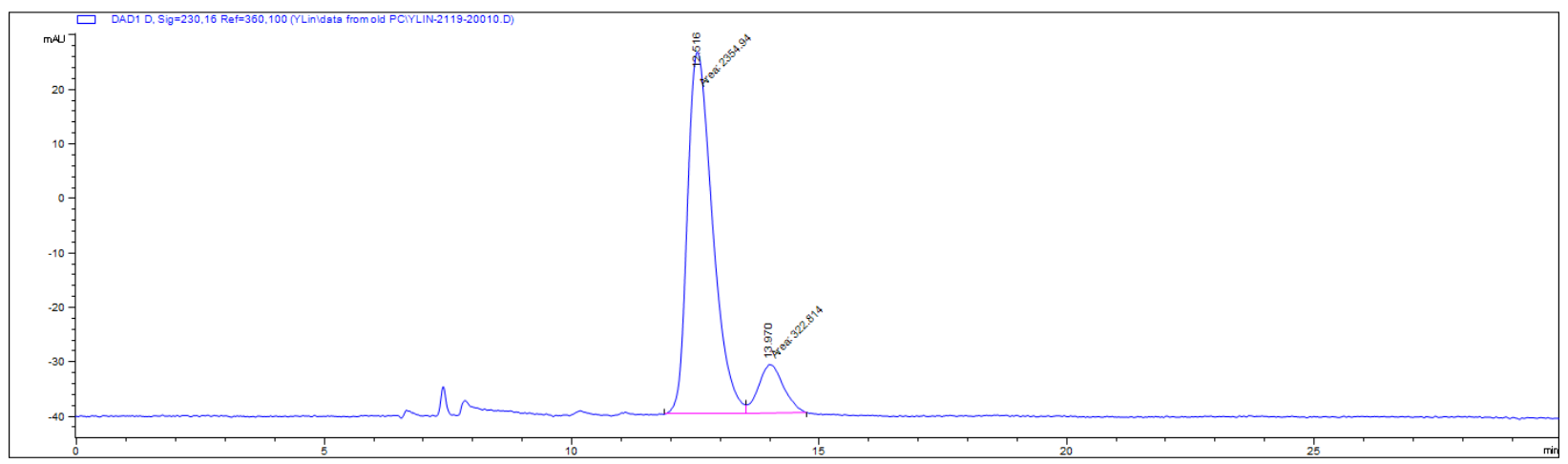

\begin{tabular}{|l|l|l|}
\hline Peak & Retention time $(\mathrm{min})$ & Area \% \\
\hline 1 & 12.516 & 87.945 \\
\hline 2 & 13.970 & 12.055 \\
\hline
\end{tabular}

\section{Racemic sample}

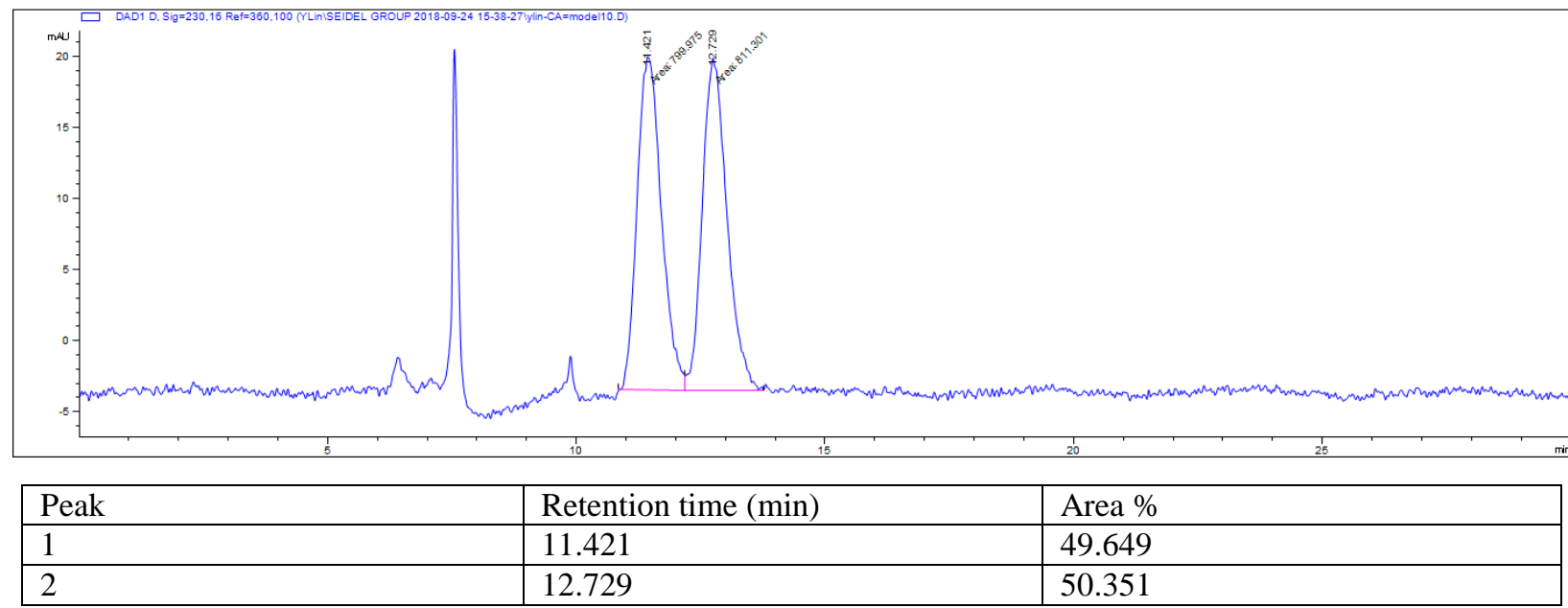


HPLC Profile of $\mathbf{4 b}$

Conditions: Daicel Chiralcel OJ-H, $n$-hexane $/ i-\mathrm{PrOH}=90 / 10$, Flow rate $=1 \mathrm{~mL} / \mathrm{min}, \mathrm{UV}=280 \mathrm{~nm}, \mathrm{t}_{\mathrm{R}}=12.0 \mathrm{~min}$ (major) and $t_{R}=15.5$ min.

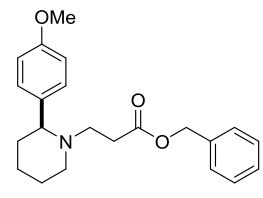

4b

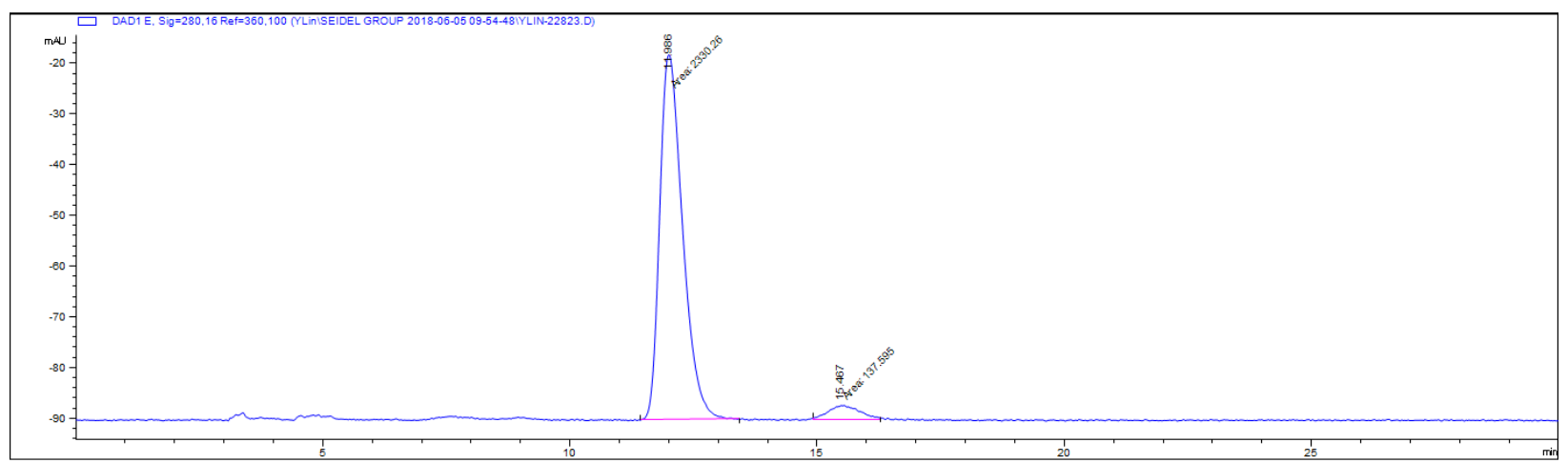

\begin{tabular}{|l|l|l|}
\hline Peak & Retention time $(\min )$ & Area \% \\
\hline 1 & 11.986 & 94.425 \\
\hline 2 & 15.467 & 5.575 \\
\hline
\end{tabular}

Racemic sample

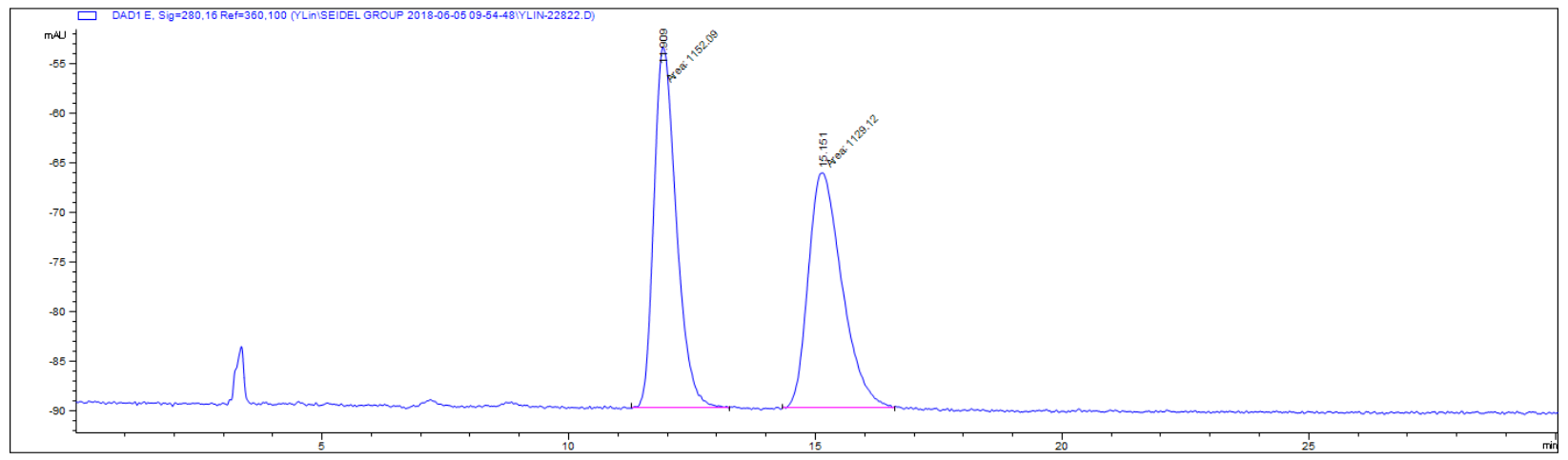

\begin{tabular}{|l|l|l|}
\hline Peak & Retention time $(\mathrm{min})$ & Area \% \\
\hline 1 & 11.909 & 50.503 \\
\hline 2 & 15.151 & 49.497 \\
\hline
\end{tabular}




\section{SFC Profile of $\mathbf{5 b}$}

Conditions: Daicel Chiralcel OJ-H, column temperature $=40{ }^{\circ} \mathrm{C}, \mathrm{CO}_{2} / \mathrm{MeOH}=90 / 10$, Flow rate $=2 \mathrm{~mL} / \mathrm{min}, \mathrm{UV}=$ $280 \mathrm{~nm}, \mathrm{t}_{\mathrm{R}}=2.1 \mathrm{~min}$ (major) and $\mathrm{t}_{\mathrm{R}}=2.4 \mathrm{~min}$.
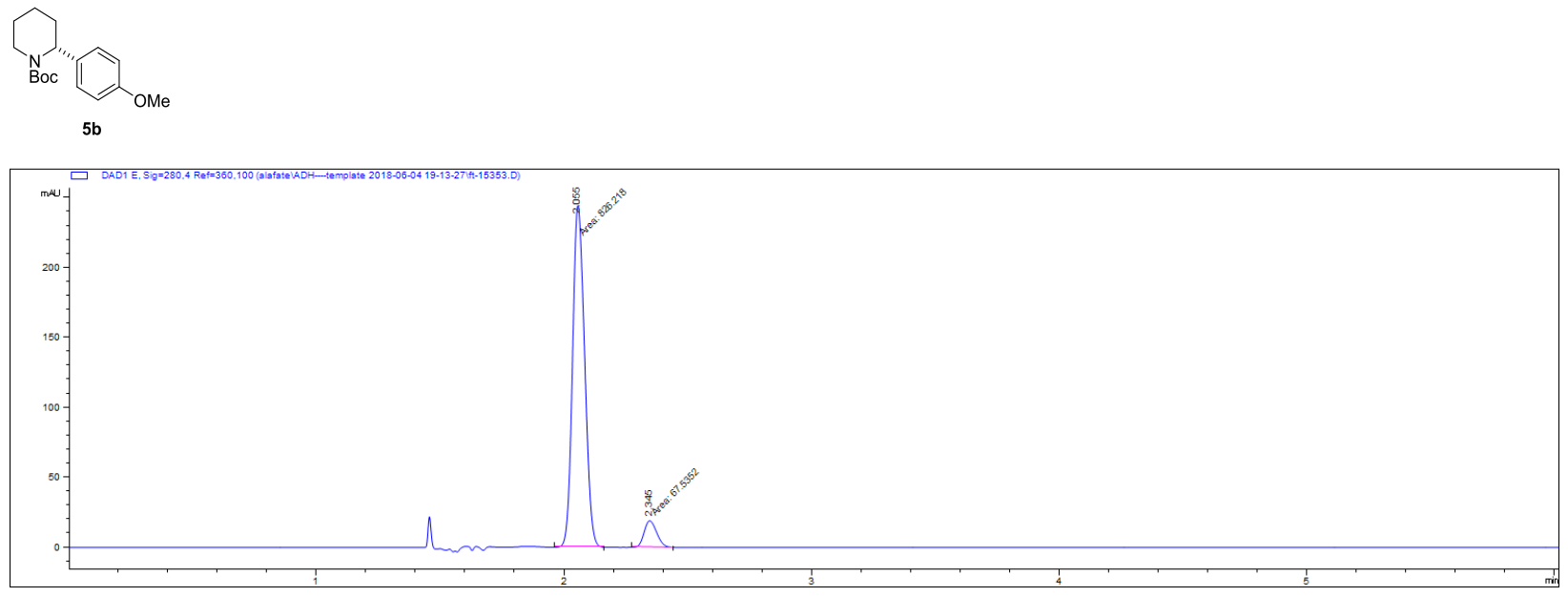

\begin{tabular}{|l|l|l|}
\hline Peak & Retention time $(\mathrm{min})$ & Area \% \\
\hline 1 & 2.055 & 92.444 \\
\hline 2 & 2.345 & 7.556 \\
\hline
\end{tabular}

Racemic sample

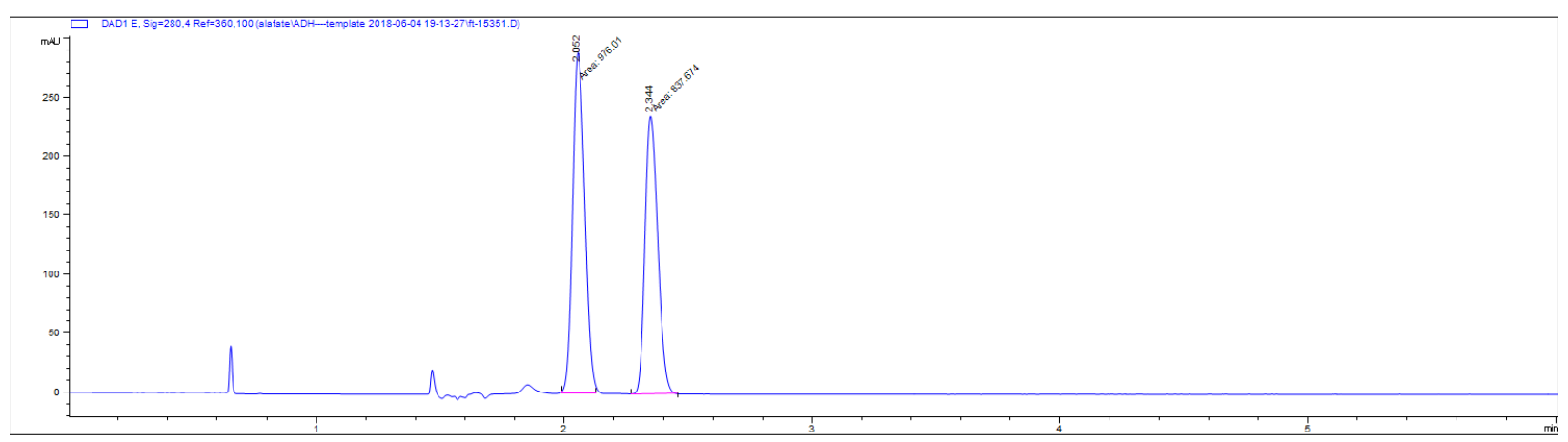

\begin{tabular}{|l|l|l|}
\hline Peak & Retention time $(\mathrm{min})$ & Area \% \\
\hline 1 & 2.052 & 53.814 \\
\hline 2 & 2.344 & 46.186 \\
\hline
\end{tabular}




\section{SFC Profile of $\mathbf{4 c}$}

Conditions: Daicel Chiralcel OD-H, column temperature $=40{ }^{\circ} \mathrm{C}, \mathrm{CO}_{2} / \mathrm{MeOH}=90 / 10$, Flow rate $=1 \mathrm{~mL} / \mathrm{min}$, UV $=210 \mathrm{~nm}, \mathrm{t}_{\mathrm{R}}=13.6 \min$ (major) and $\mathrm{t}_{\mathrm{R}}=14.4 \mathrm{~min}$.

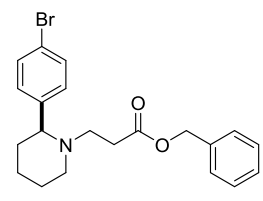

$4 \mathrm{c}$

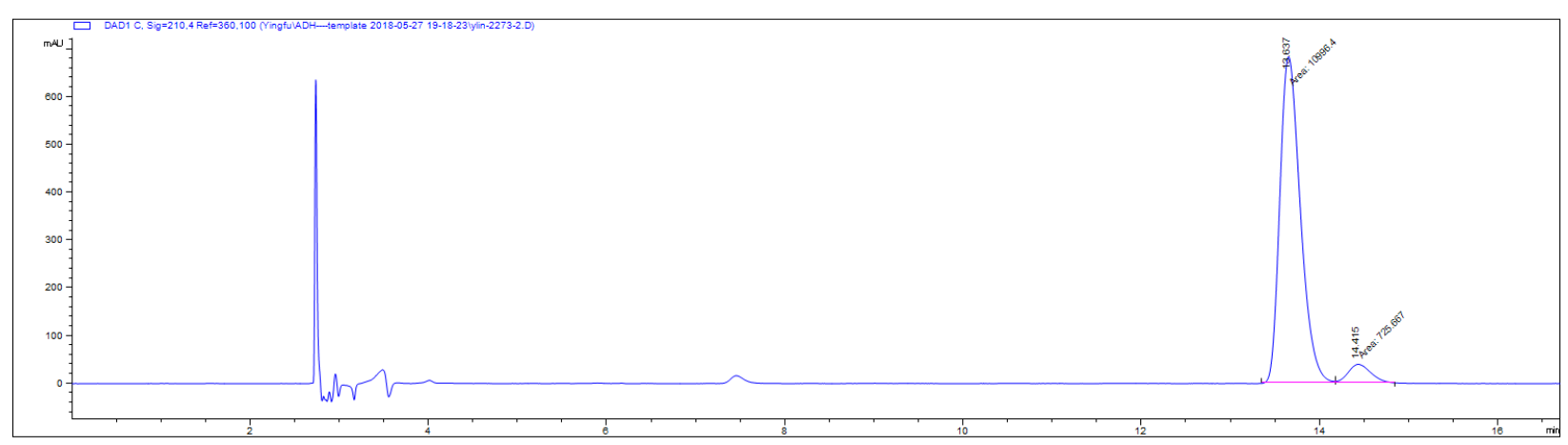

\begin{tabular}{|l|l|l|}
\hline Peak & Retention time (min) & Area \% \\
\hline 1 & 13.637 & 93.809 \\
\hline 2 & 14.415 & 6.191 \\
\hline
\end{tabular}

Racemic sample

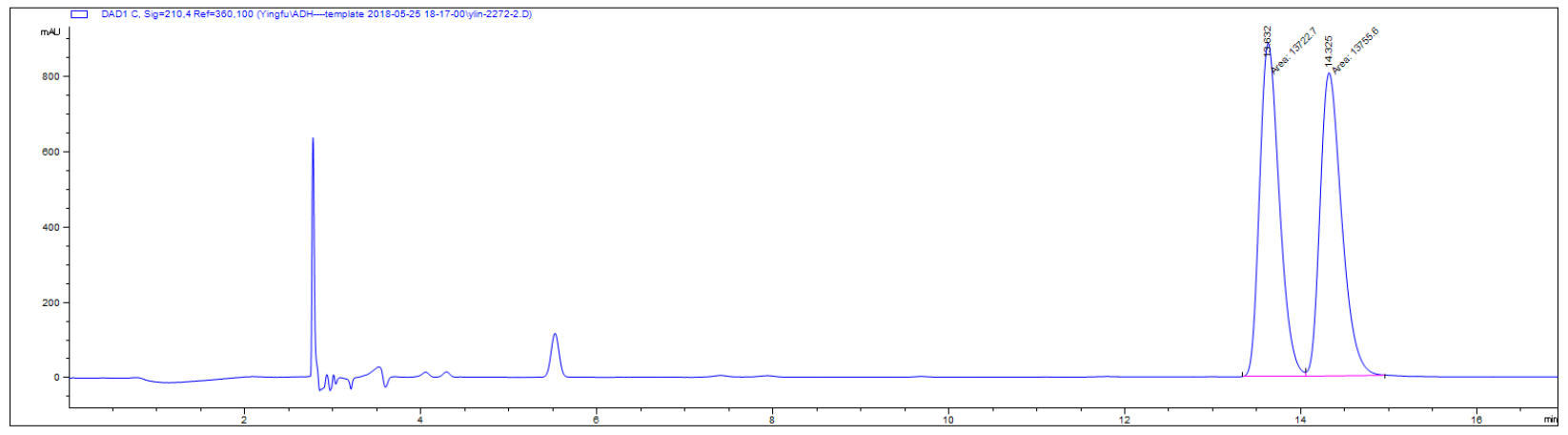

\begin{tabular}{|l|l|l|}
\hline Peak & Retention time $(\mathrm{min})$ & Area \% \\
\hline 1 & 13.632 & 49.940 \\
\hline 2 & 14.325 & 50.060 \\
\hline
\end{tabular}




\section{SFC Profile of 5c}

Conditions: Daicel Chiralcel OJ-H, column temperature $=40{ }^{\circ} \mathrm{C}, \mathrm{CO}_{2} / \mathrm{MeOH}=90 / 10$, Flow rate $=2 \mathrm{~mL} / \mathrm{min}, \mathrm{UV}=$ $210 \mathrm{~nm}, \mathrm{t}_{\mathrm{R}}=2.2 \min$ (major) and $\mathrm{t}_{\mathrm{R}}=2.4 \mathrm{~min}$.
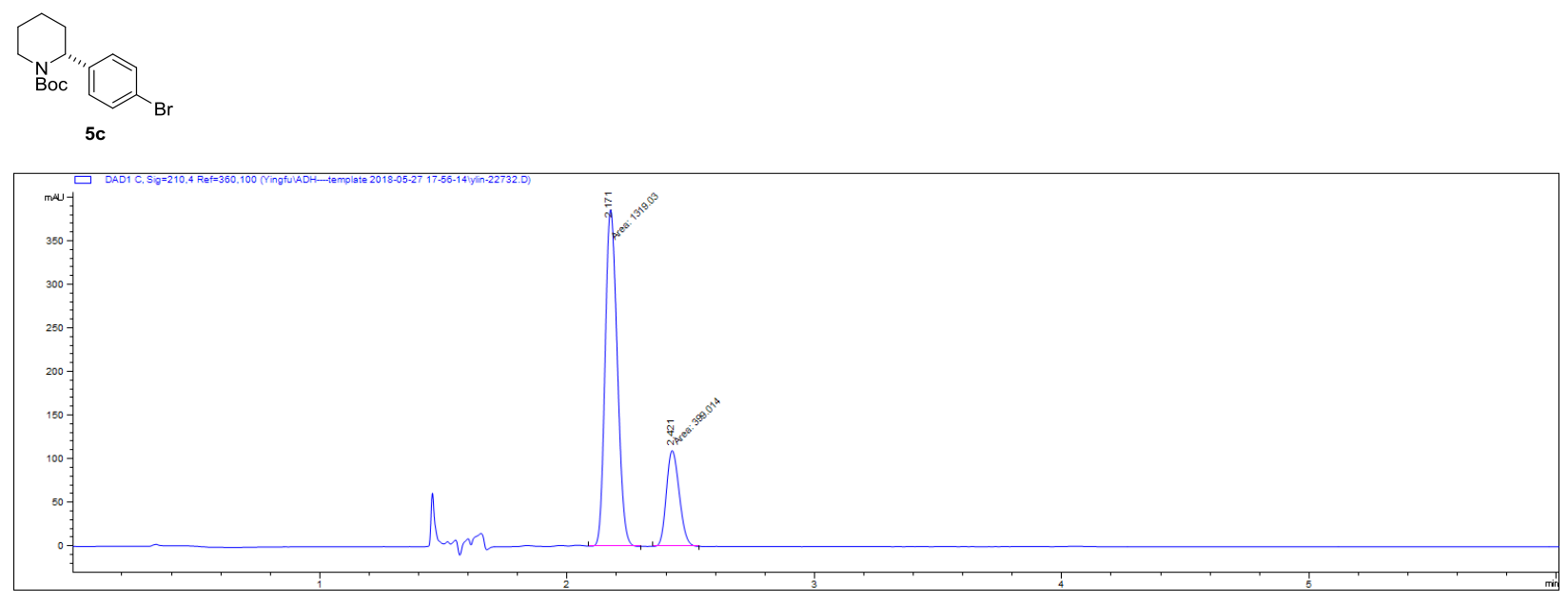

\begin{tabular}{|l|l|l|}
\hline Peak & Retention time (min) & Area \% \\
\hline 1 & 2.171 & 76.775 \\
\hline 2 & 2.421 & 23.225 \\
\hline
\end{tabular}

Racemic sample

\begin{tabular}{|l|l|l|}
\hline \begin{tabular}{|l|l|}
\hline \\
\hline
\end{tabular} \\
\hline Peak
\end{tabular}




\section{SFC Profile of $\mathbf{4 d}$}

Conditions: Daicel Chiralcel OD-H, column temperature $=40^{\circ} \mathrm{C}, \mathrm{CO}_{2} / \mathrm{MeOH}=95 / 5$, Flow rate $=2 \mathrm{~mL} / \mathrm{min}$, UV $=$ $210 \mathrm{~nm}, \mathrm{t}_{\mathrm{R}}=7.7 \mathrm{~min}$ (major) and $\mathrm{t}_{\mathrm{R}}=8.1 \mathrm{~min}$.

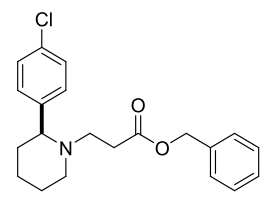

$4 d$

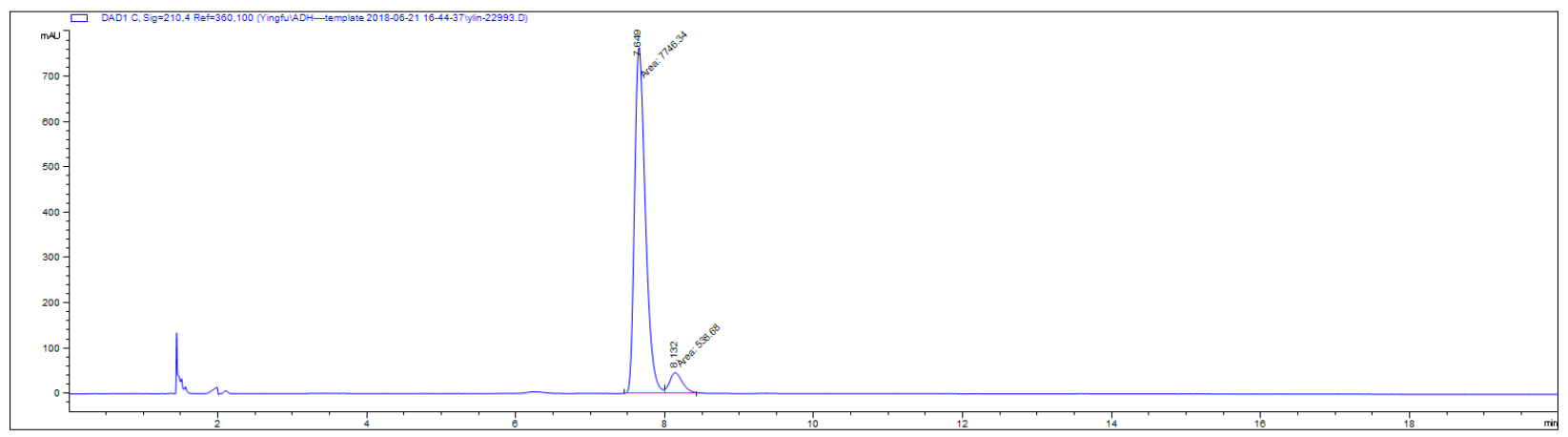

\begin{tabular}{|l|l|l|}
\hline Peak & Retention time $(\mathrm{min})$ & Area \% \\
\hline 1 & 7.649 & 93.498 \\
\hline 2 & 8.132 & 6.502 \\
\hline
\end{tabular}

Racemic sample

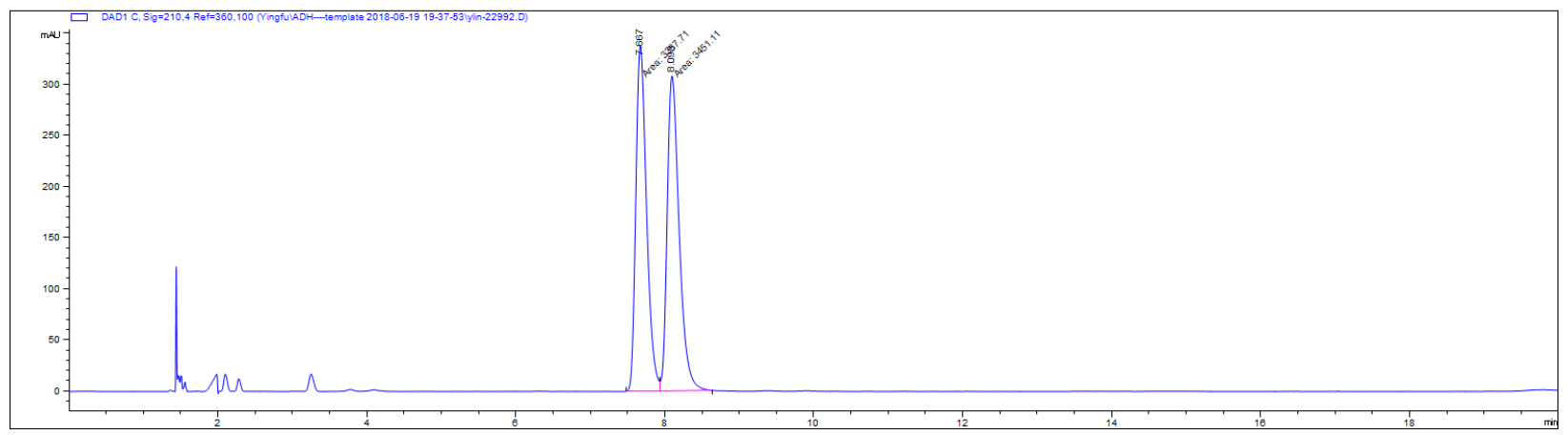

\begin{tabular}{|l|l|l|}
\hline Peak & Retention time (min) & Area \% \\
\hline 1 & 7.667 & 49.536 \\
\hline 2 & 8.098 & 50.464 \\
\hline
\end{tabular}




\section{SFC Profile of 5d}

Conditions: Daicel Chiralcel OJ-H, column temperature $=40^{\circ} \mathrm{C}, \mathrm{CO}_{2} / \mathrm{MeOH}=95 / 5$, Flow rate $=2 \mathrm{~mL} / \mathrm{min}, \mathrm{UV}=$ $210 \mathrm{~nm}, \mathrm{t}_{\mathrm{R}}=2.3 \min$ (major) and $\mathrm{t}_{\mathrm{R}}=2.6 \mathrm{~min}$
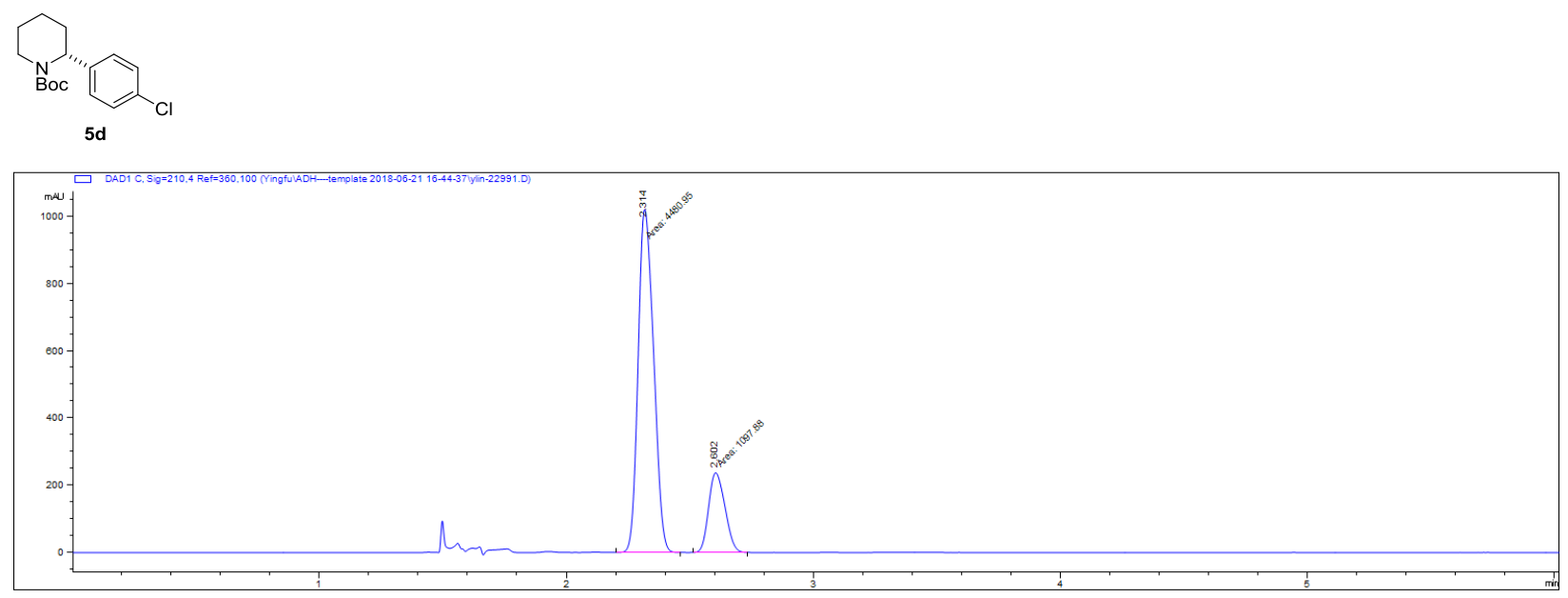

\begin{tabular}{|l|l|l|}
\hline Peak & Retention time (min) & Area \% \\
\hline 1 & 2.314 & 80.321 \\
\hline 2 & 2.602 & 19.679 \\
\hline
\end{tabular}

Racemic sample

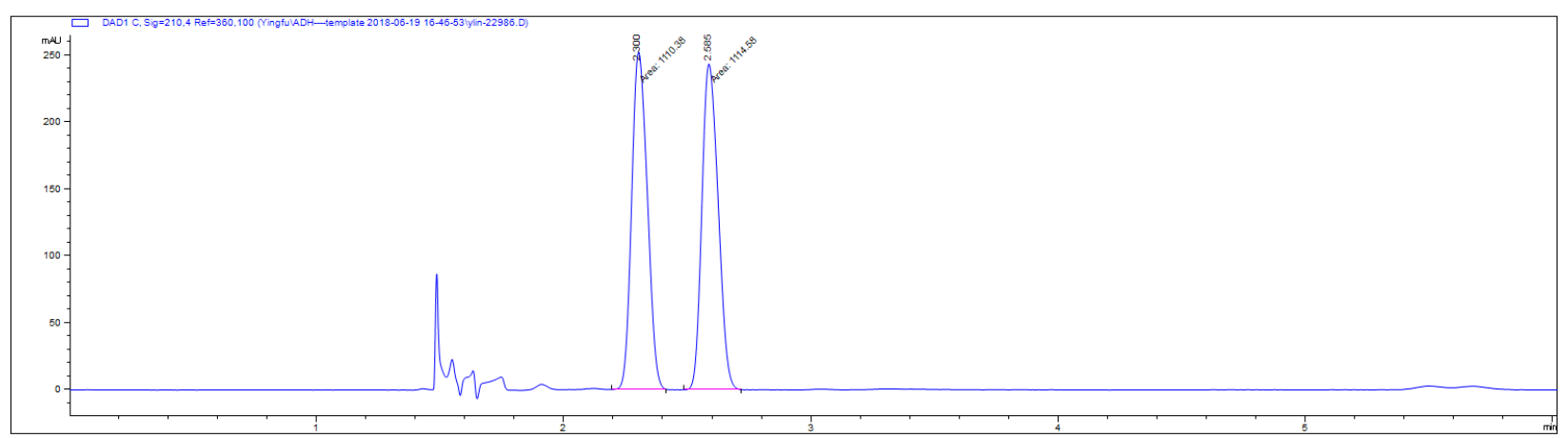

\begin{tabular}{|l|l|l|}
\hline Peak & Retention time $(\mathrm{min})$ & Area \% \\
\hline 1 & 2.300 & 49.906 \\
\hline 2 & 2.585 & 50.094 \\
\hline
\end{tabular}




\section{SFC Profile of $\mathbf{4 e}$}

Conditions: Daicel Chiralcel OD-H, column temperature $=40^{\circ} \mathrm{C}, \mathrm{CO}_{2} / \mathrm{MeOH}=95 / 5$, Flow rate $=2 \mathrm{~mL} / \mathrm{min}$, UV $=$ $230 \mathrm{~nm}, \mathrm{t}_{\mathrm{R}}=8.0 \min$ (major) and $\mathrm{t}_{\mathrm{R}}=8.7 \mathrm{~min}$.
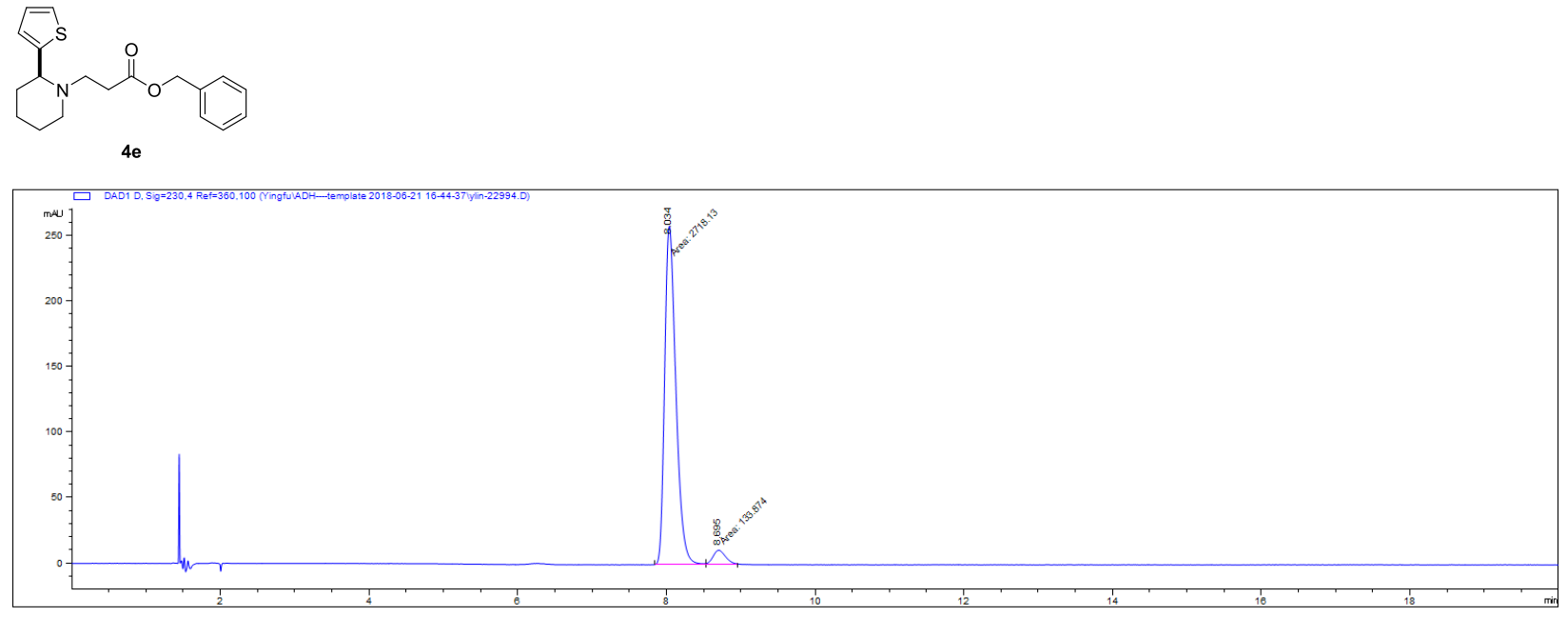

\begin{tabular}{|l|l|l|}
\hline Peak & Retention time $(\mathrm{min})$ & Area \% \\
\hline 1 & 8.034 & 95.306 \\
\hline 2 & 8.695 & 4.694 \\
\hline
\end{tabular}

\section{Racemic sample}

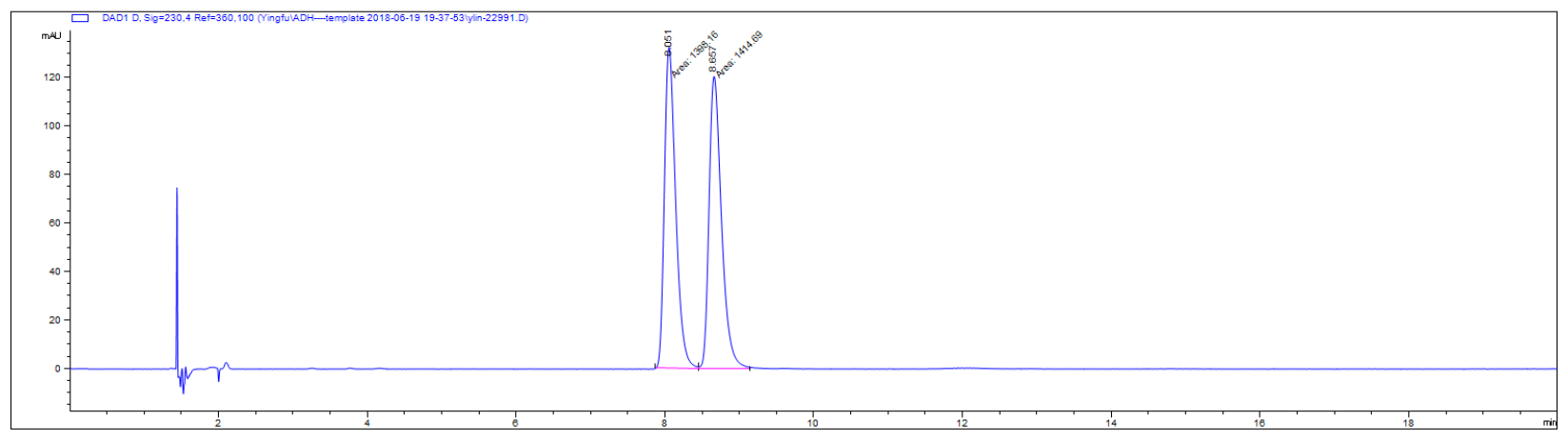

\begin{tabular}{|l|l|l|}
\hline Peak & Retention time $(\mathrm{min})$ & Area \% \\
\hline 1 & 8.051 & 49.706 \\
\hline 2 & 8.657 & 50.294 \\
\hline
\end{tabular}




\section{SFC Profile of 5e}

Conditions: Daicel Chiralcel OJ-H, column temperature $=40^{\circ} \mathrm{C}, \mathrm{CO}_{2} / \mathrm{MeOH}=95 / 5$, Flow rate $=2 \mathrm{~mL} / \mathrm{min}, \mathrm{UV}=$ $230 \mathrm{~nm}, \mathrm{t}_{\mathrm{R}}=2.2 \min$ (major) and $\mathrm{t}_{\mathrm{R}}=2.4 \mathrm{~min}$.
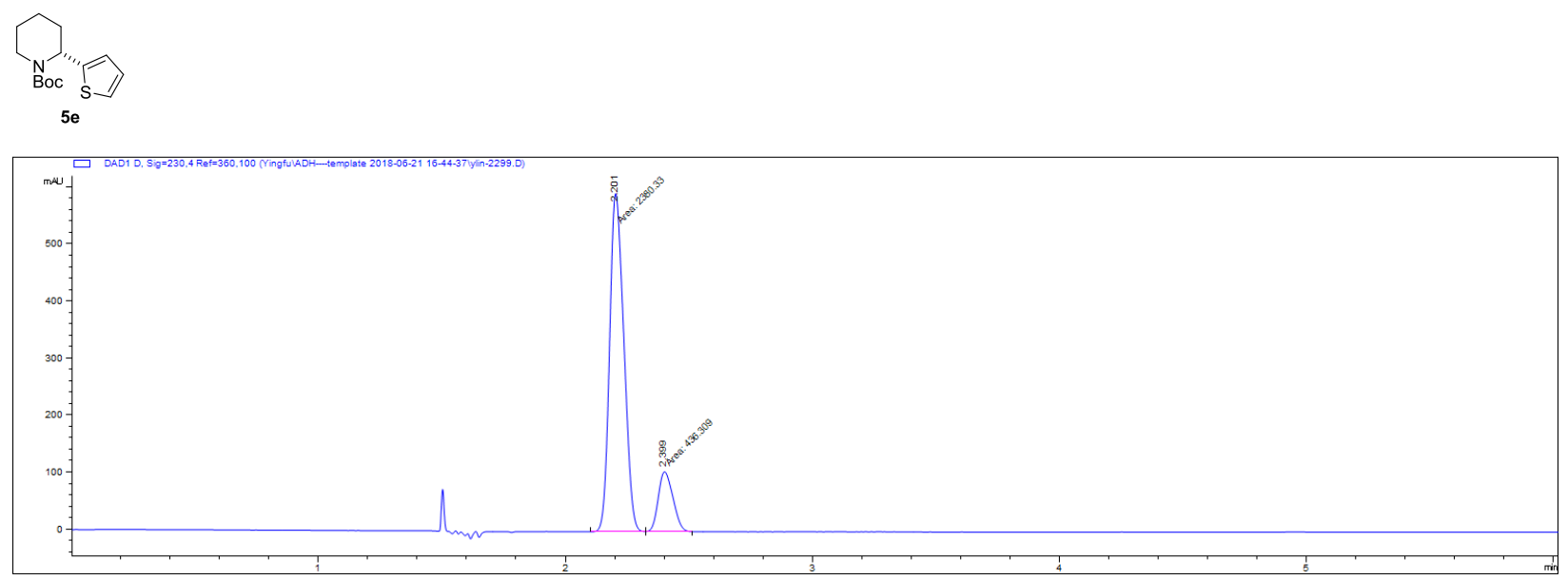

\begin{tabular}{|l|l|l|}
\hline Peak & Retention time $(\min )$ & Area \% \\
\hline 1 & 2.201 & 84.510 \\
\hline 2 & 2.399 & 15.490 \\
\hline
\end{tabular}

\section{Racemic sample}

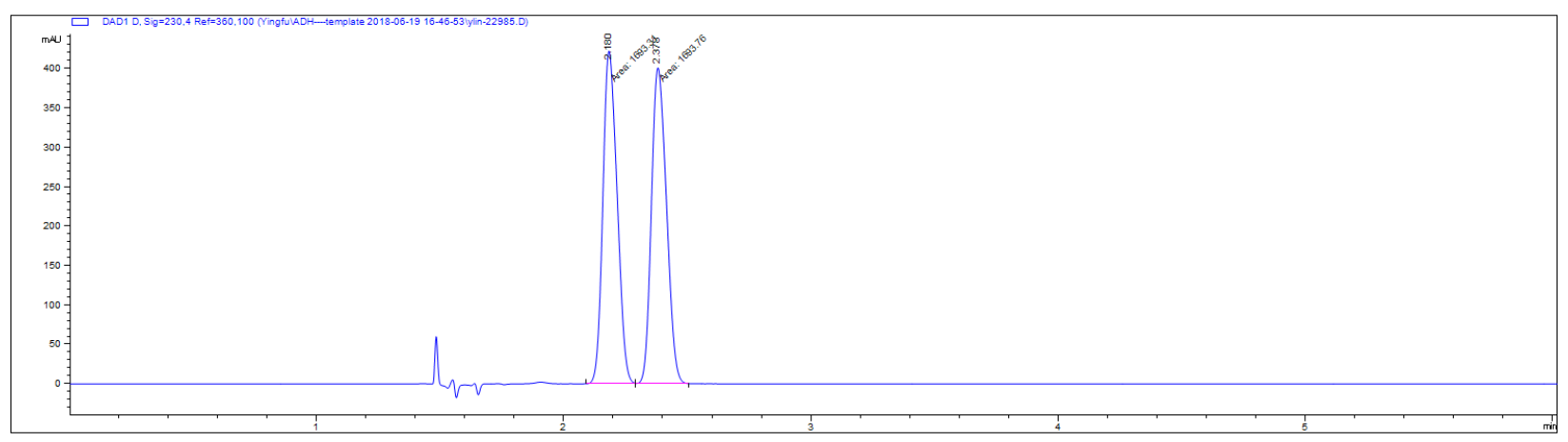

\begin{tabular}{|l|l|l|}
\hline Peak & Retention time $(\mathrm{min})$ & Area \% \\
\hline 1 & 2.180 & 49.993 \\
\hline 2 & 2.378 & 50.007 \\
\hline
\end{tabular}




\section{SFC Profile of $\mathbf{4 f}$}

Conditions: Daicel Chiralcel OD-H, column temperature $=40{ }^{\circ} \mathrm{C}, \mathrm{CO}_{2} / \mathrm{MeOH}=90 / 10$, Flow rate $=1 \mathrm{~mL} / \mathrm{min}$, UV $=210 \mathrm{~nm}, \mathrm{t}_{\mathrm{R}}=9.9 \min$ (major) and $\mathrm{t}_{\mathrm{R}}=10.5 \mathrm{~min}$.
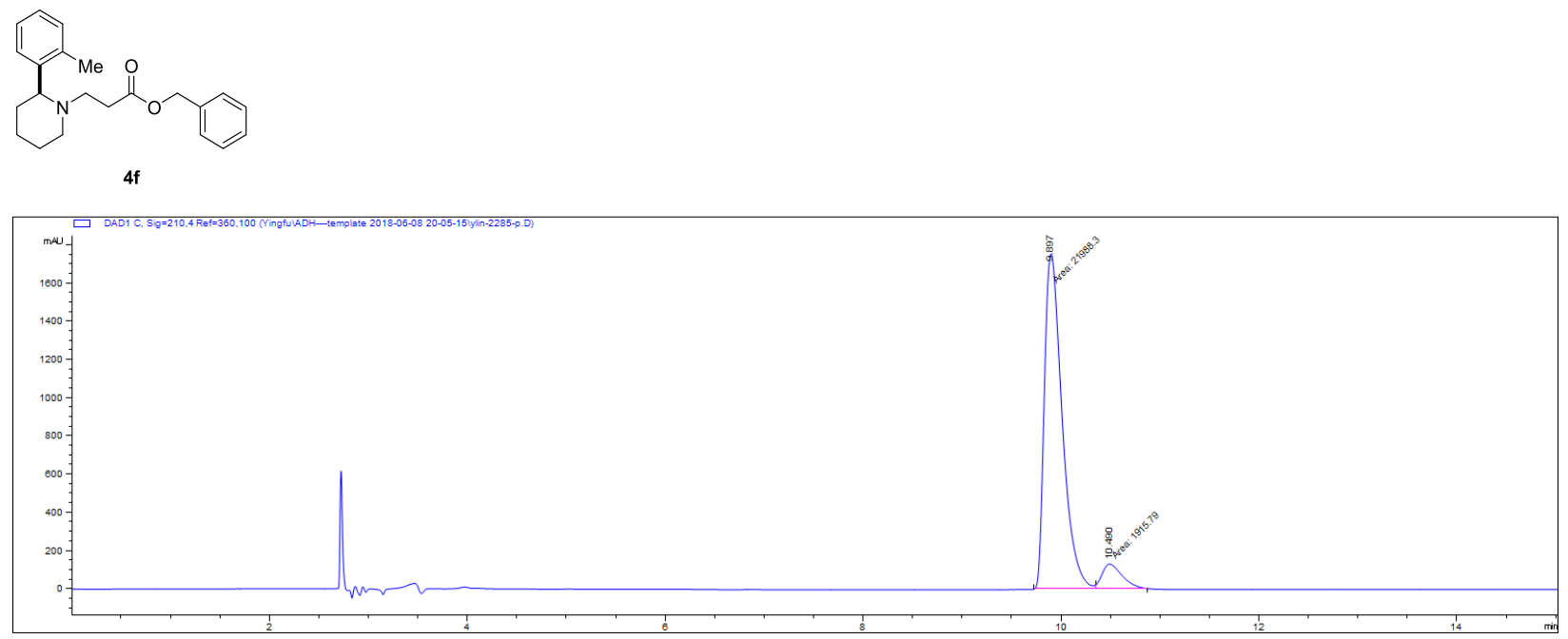

\begin{tabular}{|l|l|l|}
\hline Peak & Retention time $(\mathrm{min})$ & Area \% \\
\hline 1 & 9.897 & 91.896 \\
\hline 2 & 10.490 & 8.014 \\
\hline
\end{tabular}

Racemic sample

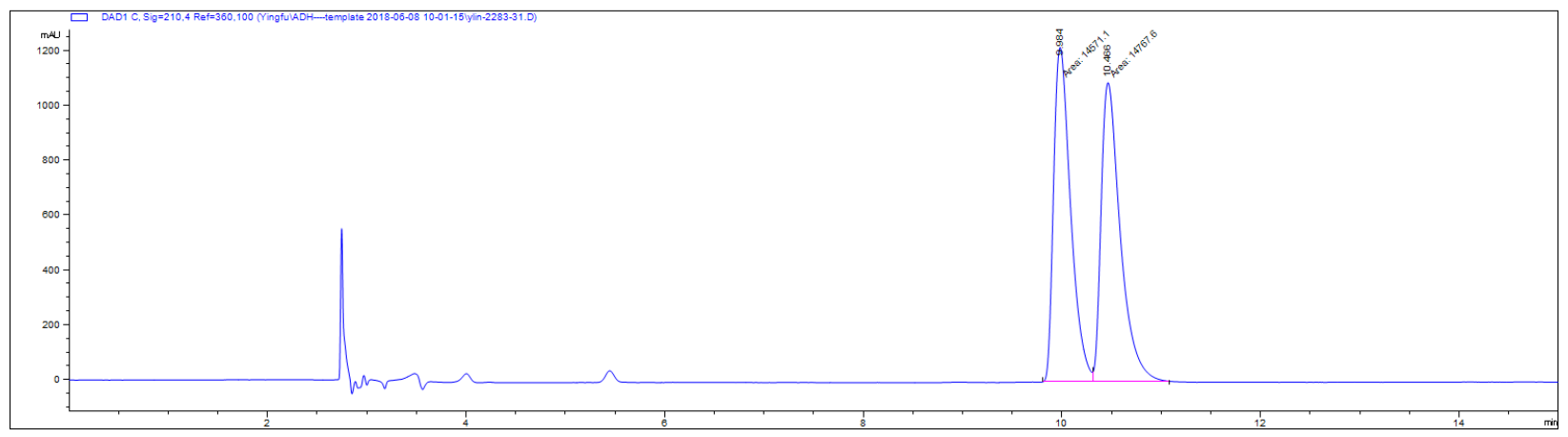

\begin{tabular}{|l|l|l|}
\hline Peak & Retention time $(\mathrm{min})$ & Area \% \\
\hline 1 & 9.984 & 49.665 \\
\hline 2 & 10.466 & 50.335 \\
\hline
\end{tabular}




\section{SFC Profile of $\mathbf{5 f}$}

Conditions: Daicel Chiralcel OD-H, column temperature $=40{ }^{\circ} \mathrm{C}, \mathrm{CO}_{2} / \mathrm{MeOH}=98 / 2$, Flow rate $=2 \mathrm{~mL} / \mathrm{min}, \mathrm{UV}=$ $210 \mathrm{~nm}, \mathrm{t}_{\mathrm{R}}=4.6 \min$ (major) and $\mathrm{t}_{\mathrm{R}}=4.9 \mathrm{~min}$.
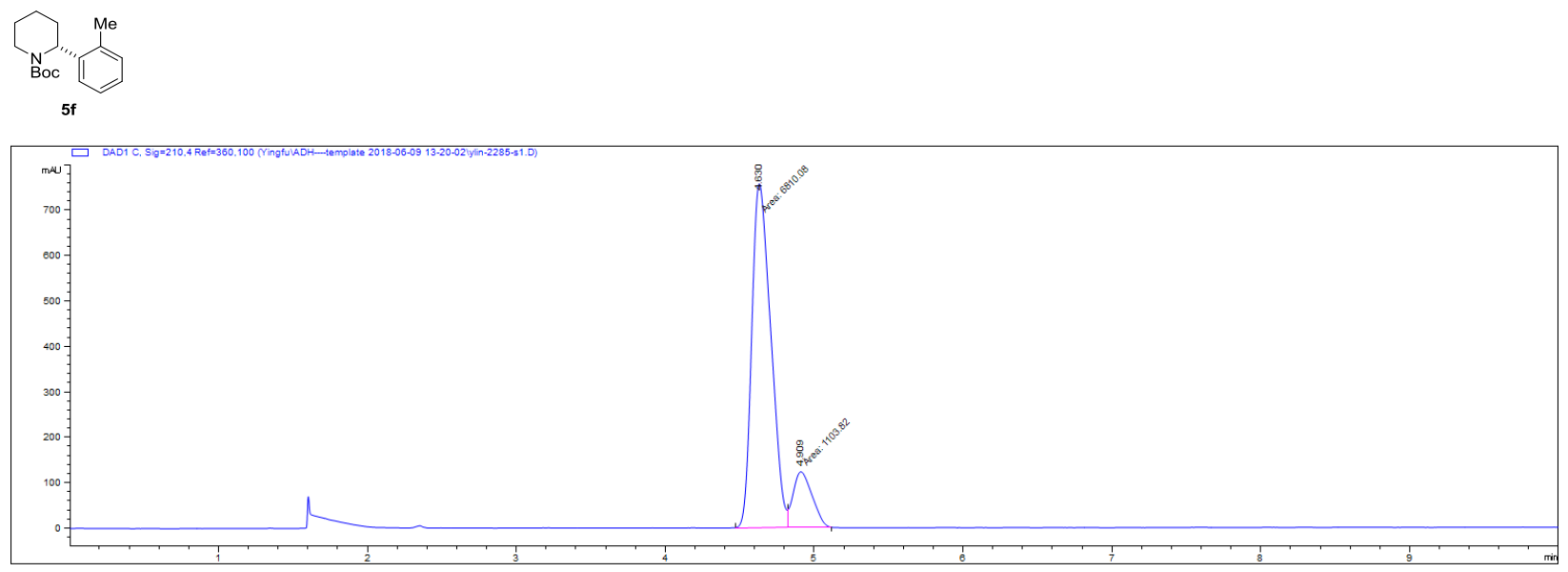

\begin{tabular}{|l|l|l|}
\hline Peak & Retention time (min) & Area \% \\
\hline 1 & 4.630 & 86.052 \\
\hline 2 & 4.909 & 13.948 \\
\hline
\end{tabular}

\section{Racemic sample}

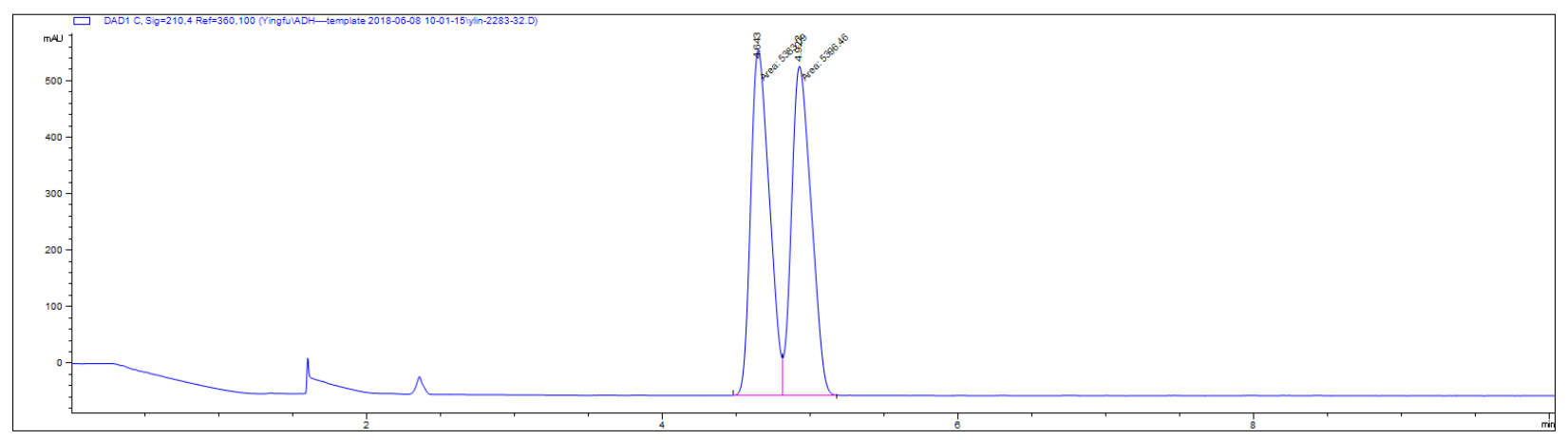

\begin{tabular}{|l|l|l|}
\hline Peak & Retention time (min) & Area \% \\
\hline 1 & 4.643 & 49.938 \\
\hline 2 & 4.922 & 50.062 \\
\hline
\end{tabular}




\section{SFC Profile of $\mathbf{4 g}$}

Conditions: Daicel Chiralcel OD-H, column temperature $=40{ }^{\circ} \mathrm{C}, \mathrm{CO}_{2} / \mathrm{MeOH}=90 / 10$, Flow rate $=2 \mathrm{~mL} / \mathrm{min}$, UV $=210 \mathrm{~nm}, \mathrm{t}_{\mathrm{R}}=9.7 \min$ (major) and $\mathrm{t}_{\mathrm{R}}=10.6 \mathrm{~min}$.

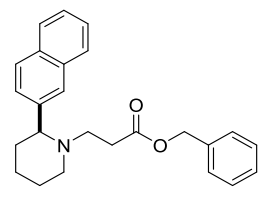

$\mathbf{4 g}$

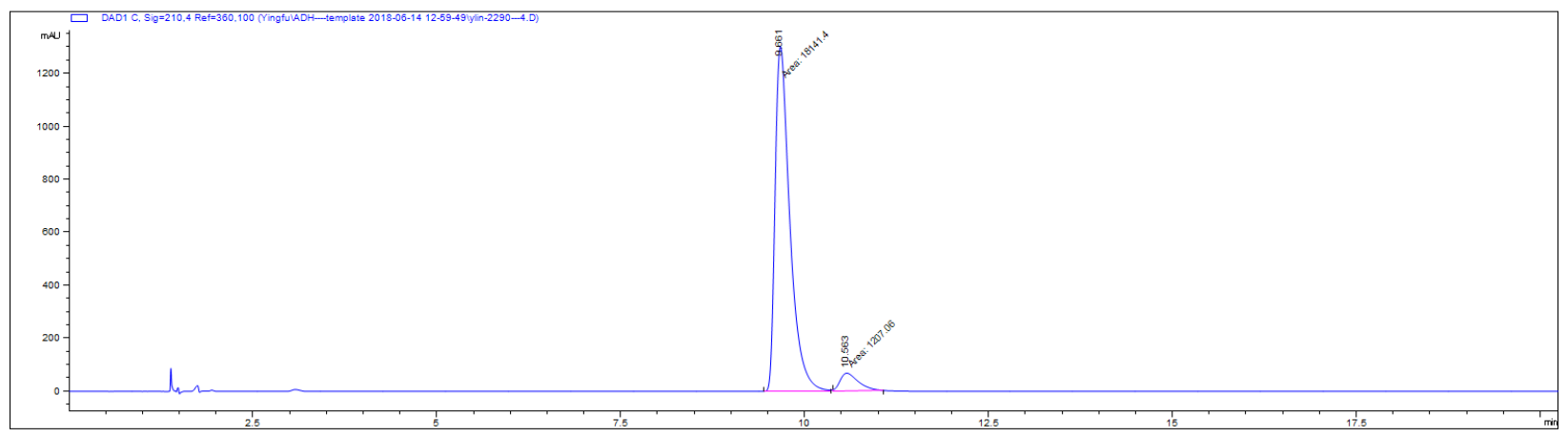

\begin{tabular}{|l|l|l|}
\hline Peak & Retention time $(\min )$ & Area \% \\
\hline 1 & 9.661 & 93.761 \\
\hline 2 & 10.563 & 6.239 \\
\hline
\end{tabular}

Racemic sample

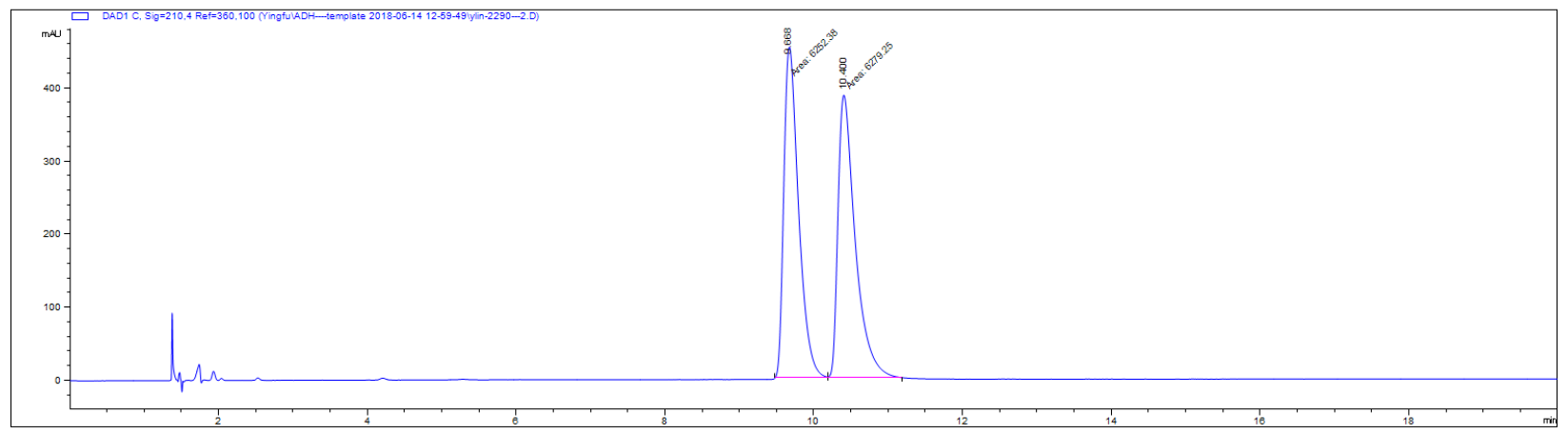

\begin{tabular}{|l|l|l|}
\hline Peak & Retention time $(\mathrm{min})$ & Area \% \\
\hline 1 & 9.668 & 49.893 \\
\hline 2 & 10.400 & 50.107 \\
\hline
\end{tabular}




\section{SFC Profile of 5g}

Conditions: Daicel Chiralcel OD-H, column temperature $=40{ }^{\circ} \mathrm{C}, \mathrm{CO}_{2} / \mathrm{MeOH}=90 / 10$, Flow rate $=2 \mathrm{~mL} / \mathrm{min}$, UV $=210 \mathrm{~nm}, \mathrm{t}_{\mathrm{R}}=4.0 \mathrm{~min}$ (major) and $\mathrm{t}_{\mathrm{R}}=4.9 \mathrm{~min}$.
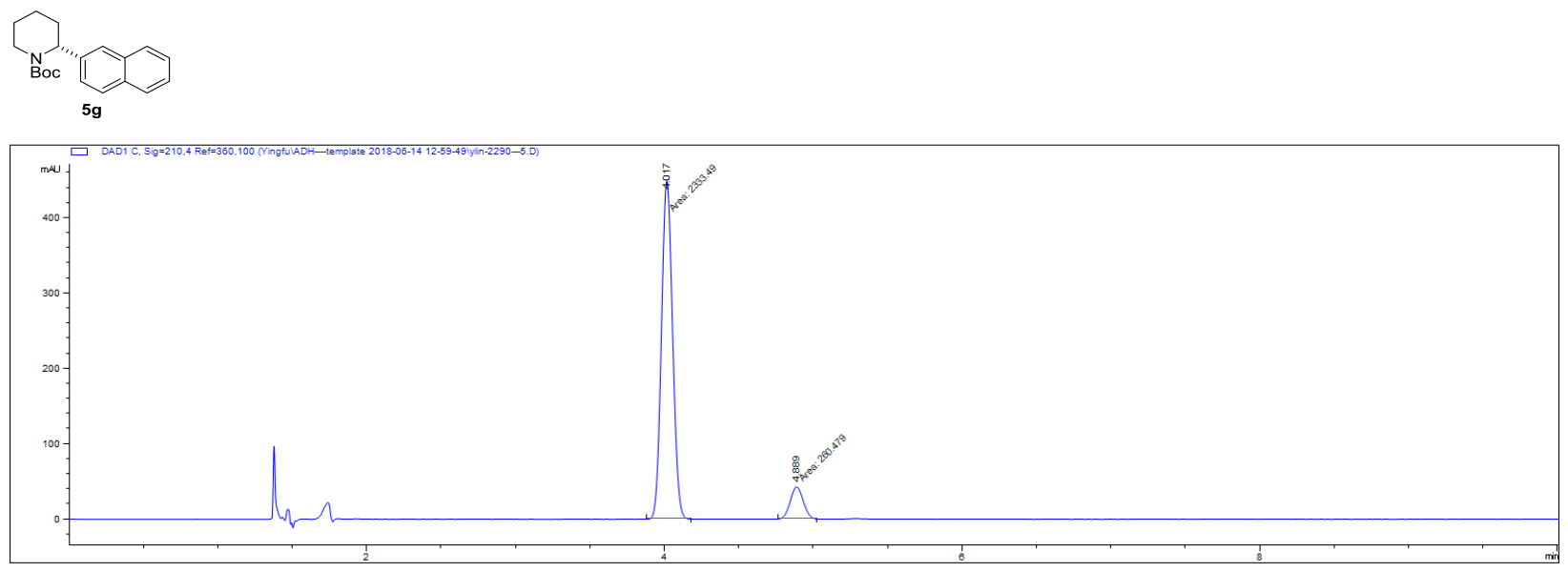

\begin{tabular}{|l|l|l|}
\hline Peak & Retention time $(\min )$ & Area \% \\
\hline 1 & 4.017 & 89.958 \\
\hline 2 & 4.889 & 10.042 \\
\hline
\end{tabular}

\section{Racemic sample}

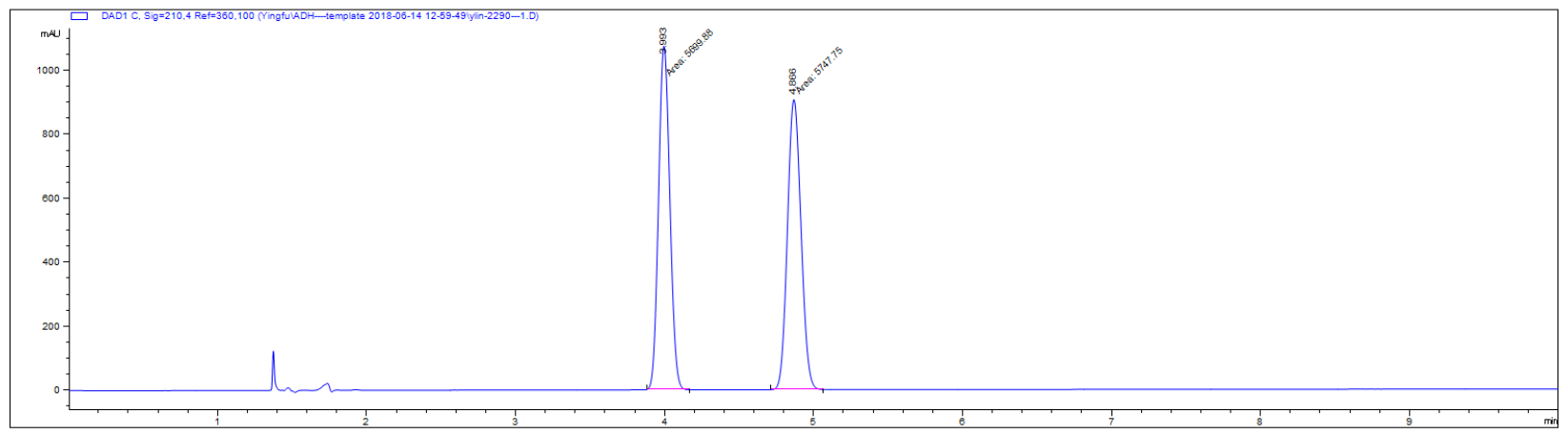

\begin{tabular}{|l|l|l|}
\hline Peak & Retention time $(\min )$ & Area \% \\
\hline 1 & 3.993 & 49.791 \\
\hline 2 & 4.866 & 50.209 \\
\hline
\end{tabular}




\section{SFC Profile of $\mathbf{4 h}$}

Conditions: Daicel Chiralcel OD-H, column temperature $=40{ }^{\circ} \mathrm{C}, \mathrm{CO}_{2} / \mathrm{MeOH}=90 / 10$, Flow rate $=2 \mathrm{~mL} / \mathrm{min}$, UV $=210 \mathrm{~nm}, \mathrm{t}_{\mathrm{R}}=5.1 \mathrm{~min}$ (major) and $\mathrm{t}_{\mathrm{R}}=5.6 \mathrm{~min}$.

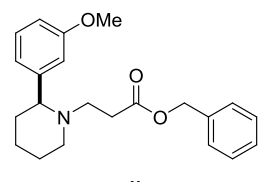

$4 h$

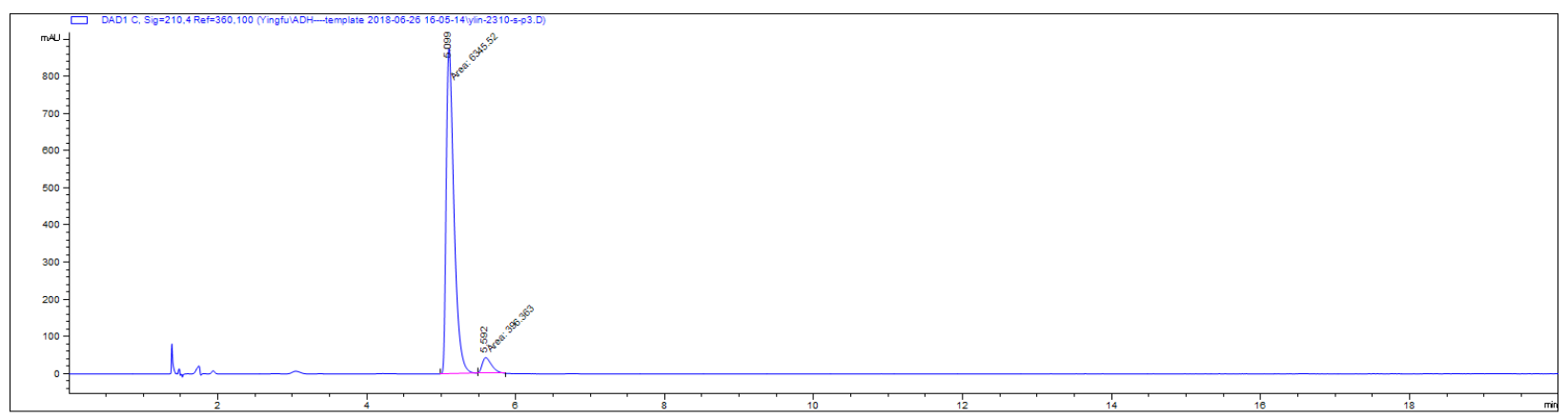

\begin{tabular}{|l|l|l|}
\hline Peak & Retention time (min) & Area \% \\
\hline 1 & 5.099 & 94.261 \\
\hline 2 & 5.592 & 5.739 \\
\hline
\end{tabular}

Racemic sample

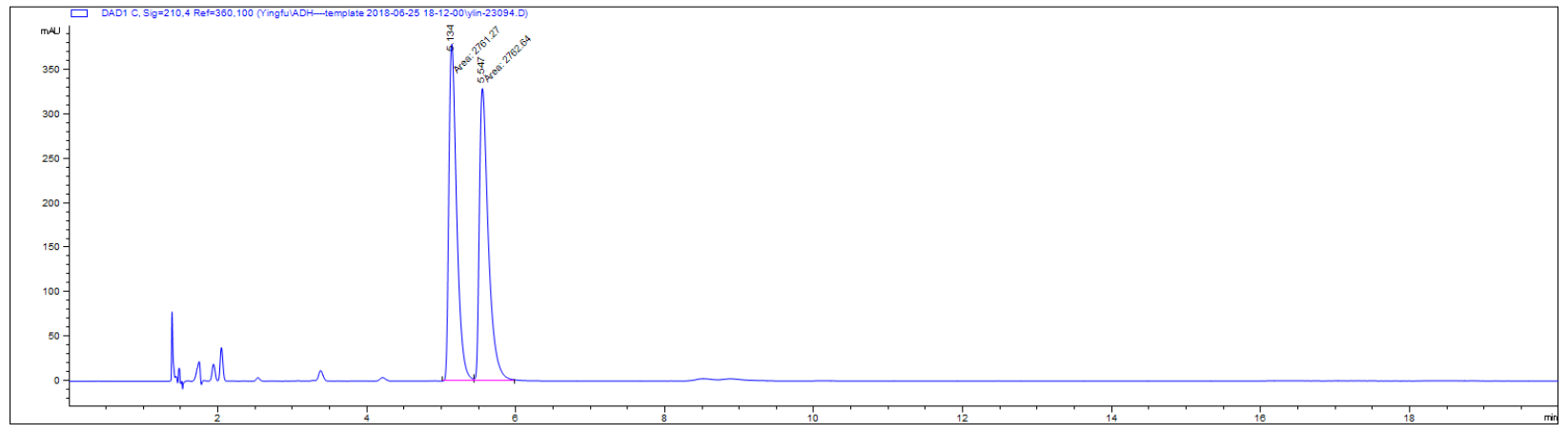

\begin{tabular}{|l|l|l|}
\hline Peak & Retention time $(\mathrm{min})$ & Area \% \\
\hline 1 & 5.134 & 49.988 \\
\hline 2 & 5.547 & 50.012 \\
\hline
\end{tabular}




\section{SFC Profile of $\mathbf{5 h}$}

Conditions: Daicel Chiralcel OJ-H, column temperature $=40{ }^{\circ} \mathrm{C}, \mathrm{CO}_{2} / \mathrm{MeOH}=98 / 2$, Flow rate $=2 \mathrm{~mL} / \mathrm{min}$, UV $=$ $210 \mathrm{~nm}, \mathrm{t}_{\mathrm{R}}=2.6 \min$ (major) and $\mathrm{t}_{\mathrm{R}}=3.0 \mathrm{~min}$.
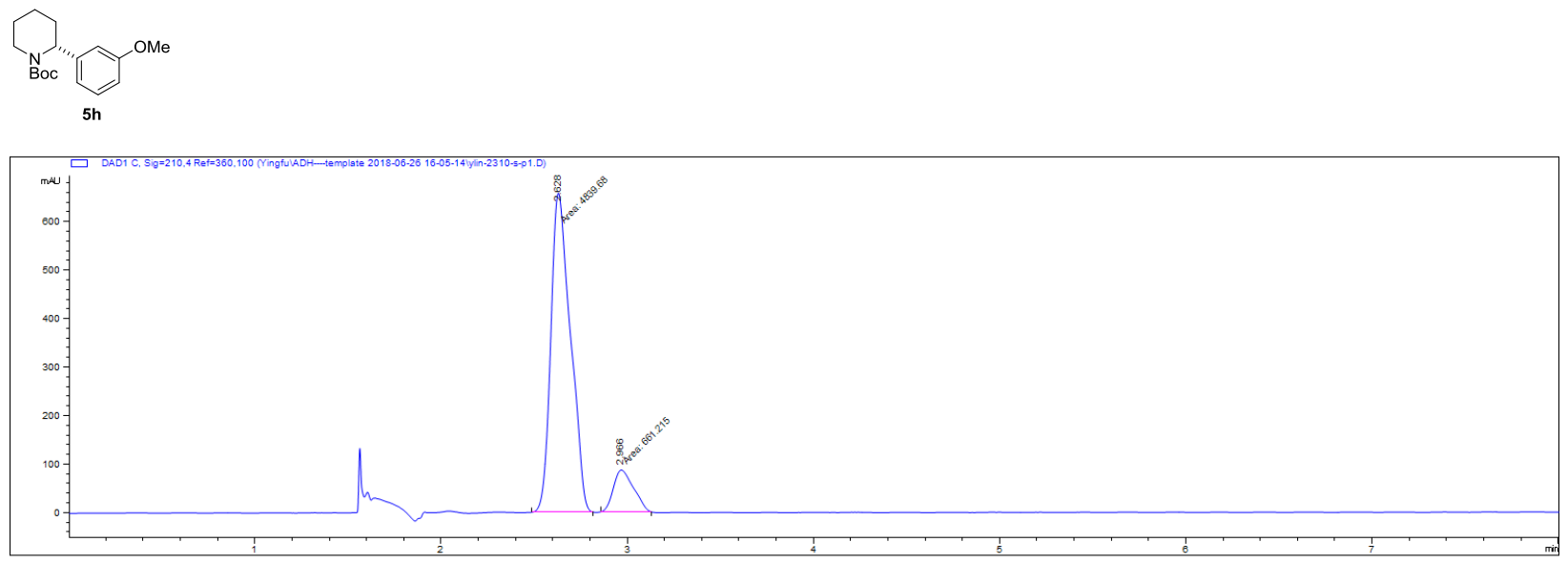

\begin{tabular}{|l|l|l|}
\hline Peak & Retention time $(\mathrm{min})$ & Area \% \\
\hline 1 & 2.628 & 87.980 \\
\hline 2 & 2.966 & 12.020 \\
\hline
\end{tabular}

\section{Racemic sample}

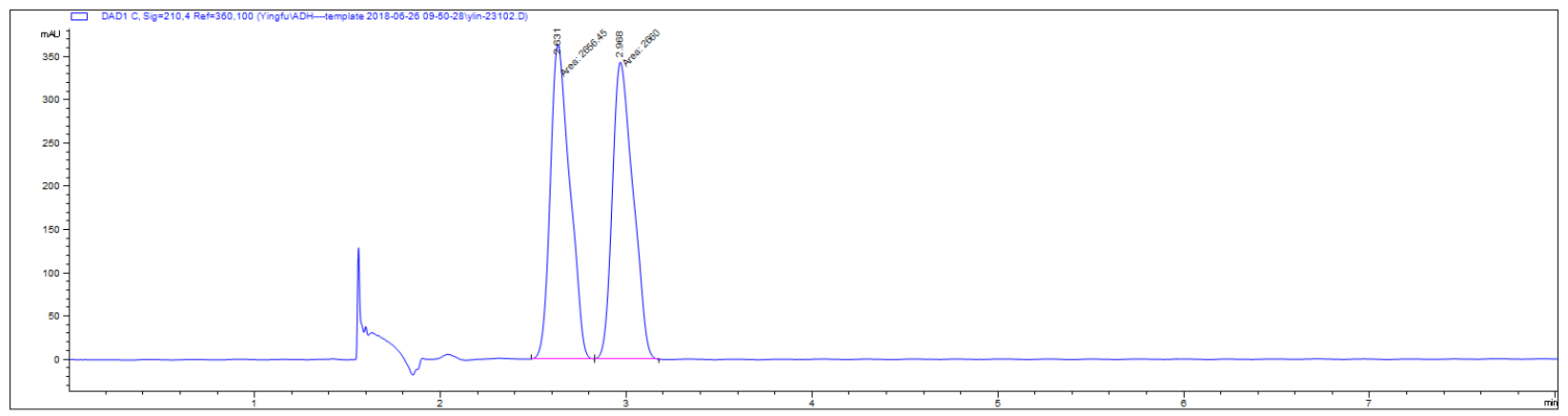

\begin{tabular}{|l|l|l|}
\hline Peak & Retention time $(\mathrm{min})$ & Area \% \\
\hline 1 & 2.631 & 49.967 \\
\hline 2 & 2.968 & 50.033 \\
\hline
\end{tabular}




\section{SFC Profile of 4i}

Conditions: Daicel Chiralcel OD-H, column temperature $=40{ }^{\circ} \mathrm{C}, \mathrm{CO}_{2} / \mathrm{MeOH}=90 / 10$, Flow rate $=2 \mathrm{~mL} / \mathrm{min}$, UV $=210 \mathrm{~nm}, \mathrm{t}_{\mathrm{R}}=4.6 \min$ (major) and $\mathrm{t}_{\mathrm{R}}=5.9 \mathrm{~min}$.
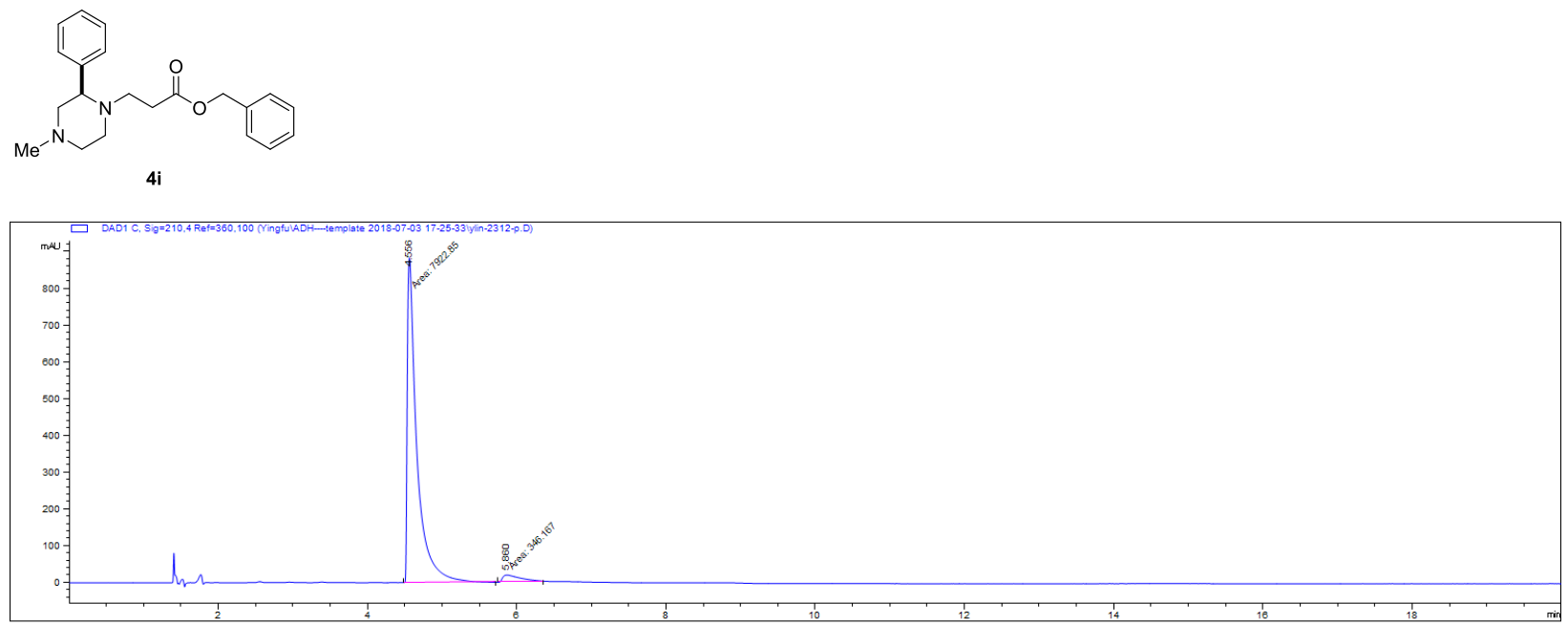

\begin{tabular}{|l|l|l|}
\hline Peak & Retention time $(\mathrm{min})$ & Area \% \\
\hline 1 & 4.556 & 95.814 \\
\hline 2 & 5.860 & 4.186 \\
\hline
\end{tabular}

Racemic sample

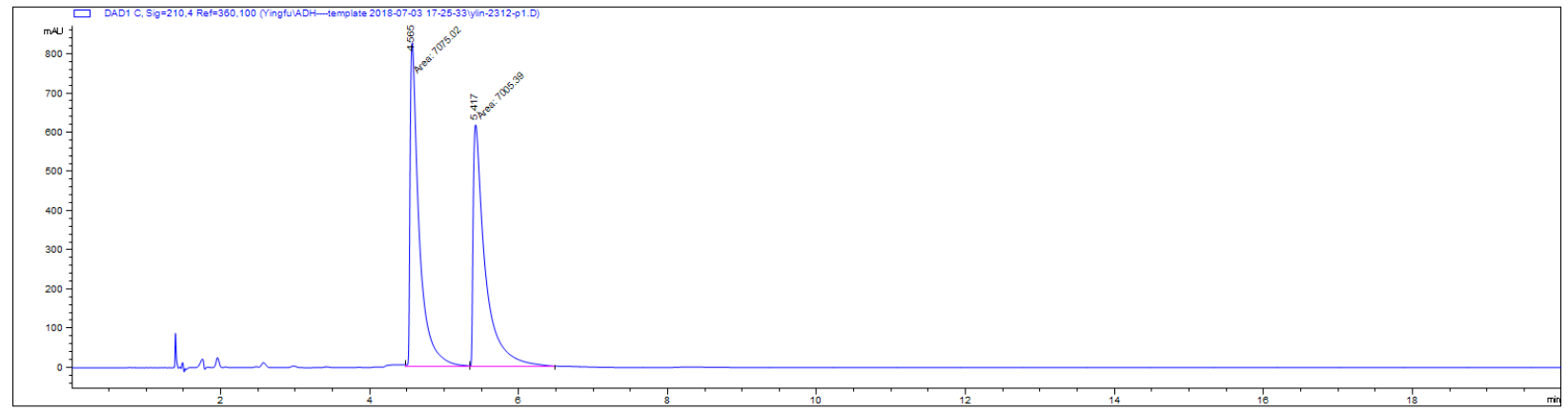

\begin{tabular}{|l|l|l|}
\hline Peak & Retention time $(\mathrm{min})$ & Area \% \\
\hline 1 & 4.565 & 50.247 \\
\hline 2 & 5.417 & 49.753 \\
\hline
\end{tabular}


SFC Profile of $\mathbf{5 i}$

Conditions: Daicel Chiralcel OD-H, column temperature $=40{ }^{\circ} \mathrm{C}, \mathrm{CO}_{2} / \mathrm{MeOH}=97 / 3$, Flow rate $=2 \mathrm{~mL} / \mathrm{min}, \mathrm{UV}=$ $210 \mathrm{~nm}, \mathrm{t}_{\mathrm{R}}=3.4$ min and $\mathrm{t}_{\mathrm{R}}=3.7 \min$ (major).
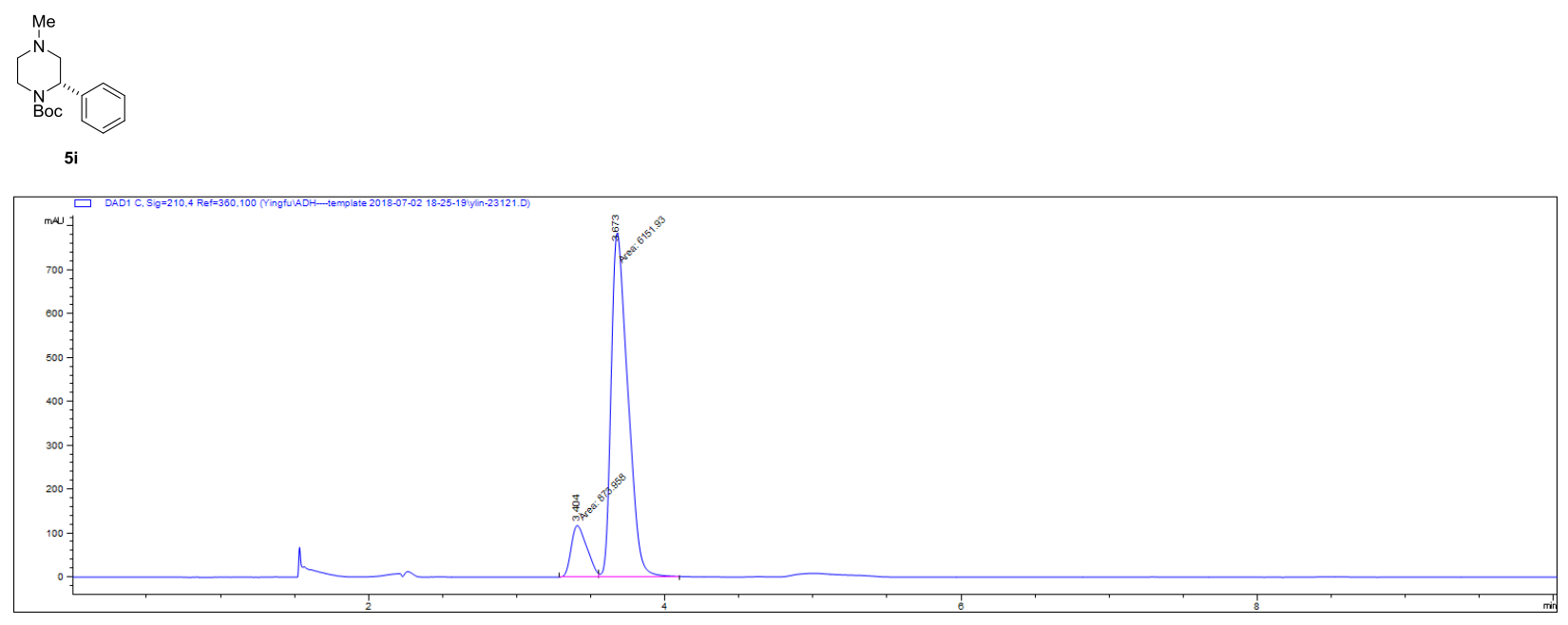

\begin{tabular}{|l|l|l|}
\hline Peak & Retention time $(\min )$ & Area \% \\
\hline 1 & 3.404 & 12.439 \\
\hline 2 & 3.673 & 87.561 \\
\hline
\end{tabular}

\section{Racemic sample}

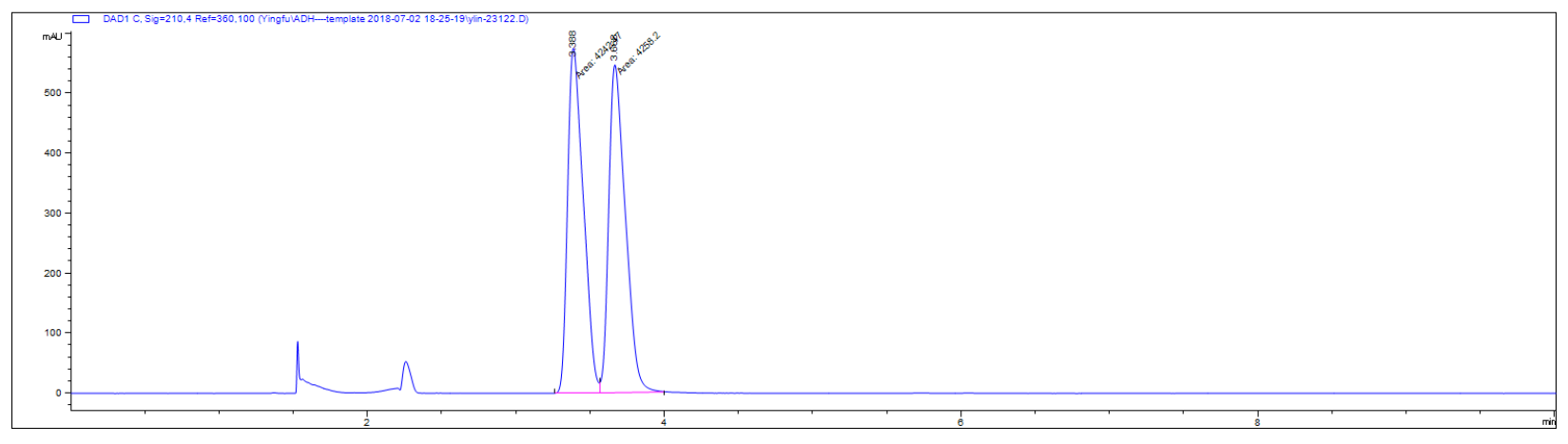

\begin{tabular}{|l|l|l|}
\hline Peak & Retention time $(\mathrm{min})$ & Area \% \\
\hline 1 & 3.388 & 49.906 \\
\hline 2 & 3.668 & 50.094 \\
\hline
\end{tabular}




\section{HPLC Profile of $\mathbf{4 j}$}

Conditions: REGIS $(S, S)$-Whelk-O1, $n$-hexane $/ i-\mathrm{PrOH}=90 / 10$, Flow rate $=0.1 \mathrm{~mL} / \mathrm{min}, \mathrm{UV}=230 \mathrm{~nm}, \mathrm{t}_{\mathrm{R}}=111.1$ $\min$ (major) and $t_{R}=117.5$ min.
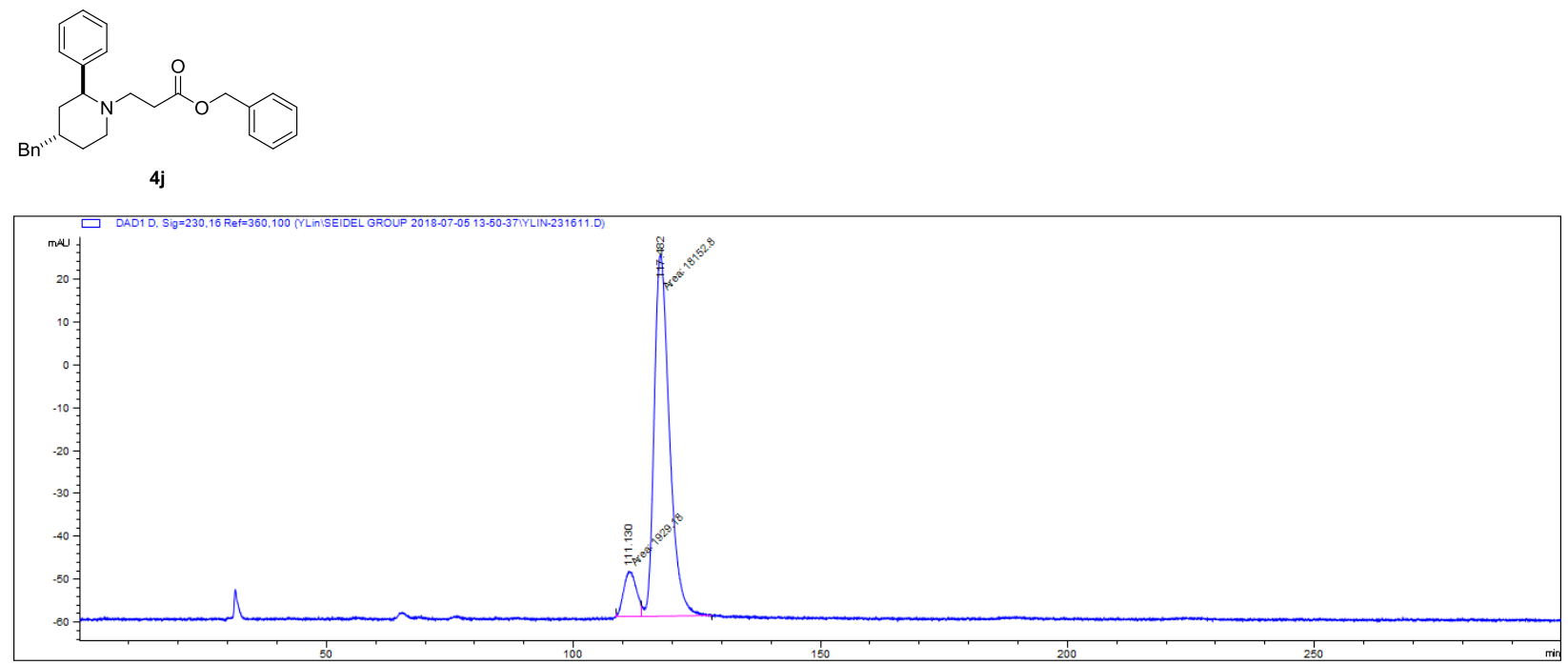

\begin{tabular}{|l|l|l|}
\hline Peak & Retention time $(\mathrm{min})$ & Area \% \\
\hline 1 & 111.130 & 9.607 \\
\hline 2 & 117.482 & 90.393 \\
\hline
\end{tabular}

Racemic sample

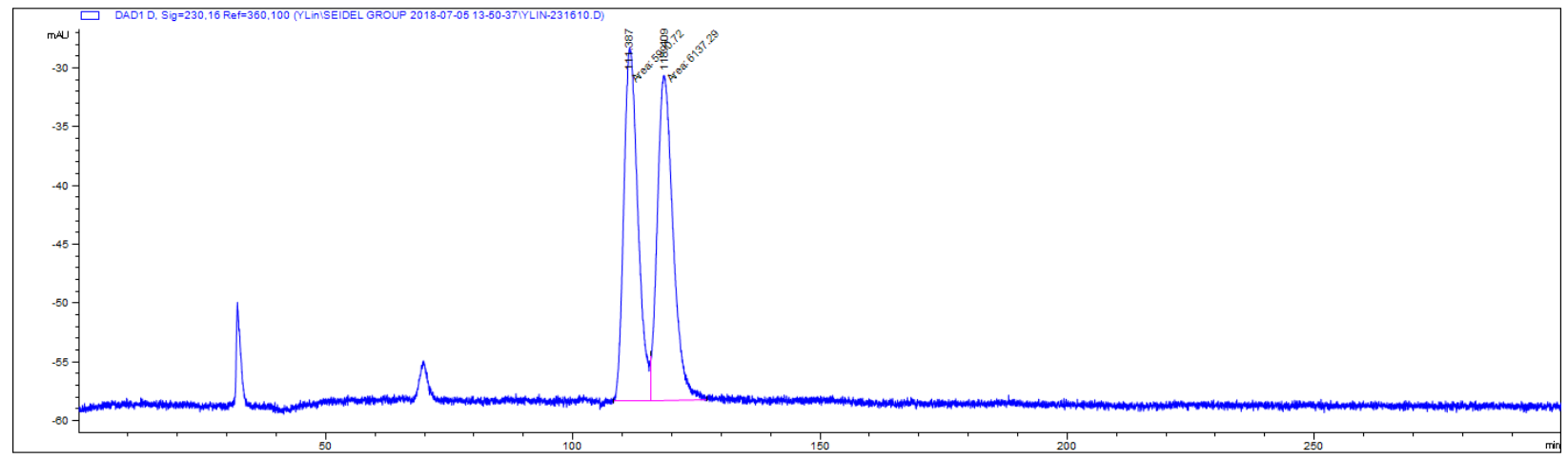

\begin{tabular}{|l|l|l|}
\hline Peak & Retention time $(\mathrm{min})$ & Area \% \\
\hline 1 & 111.387 & 49.396 \\
\hline 2 & 118.409 & 50.604 \\
\hline
\end{tabular}




\section{SFC Profile of $\mathbf{5 j}$}

Conditions: Daicel Chiralcel OJ-H, column temperature $=40{ }^{\circ} \mathrm{C}, \mathrm{CO}_{2} / \mathrm{MeOH}=90 / 10$, Flow rate $=2 \mathrm{~mL} / \mathrm{min}, \mathrm{UV}=$ $210 \mathrm{~nm}, \mathrm{t}_{\mathrm{R}}=2.4 \min$ (major) and $\mathrm{t}_{\mathrm{R}}=2.8 \mathrm{~min}$.
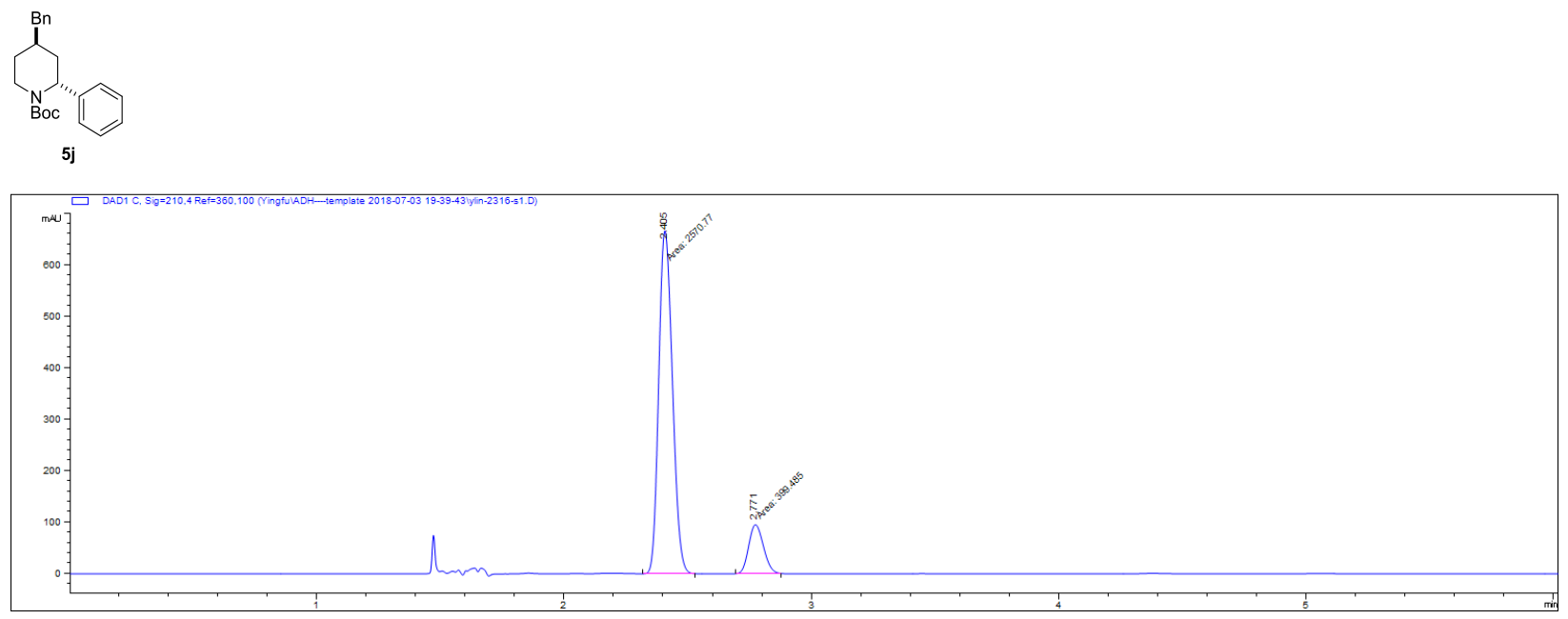

\begin{tabular}{|l|l|l|}
\hline Peak & Retention time $(\min )$ & Area \% \\
\hline 1 & 2.405 & 86.550 \\
\hline 2 & 2.771 & 13.450 \\
\hline
\end{tabular}

\section{Racemic sample}

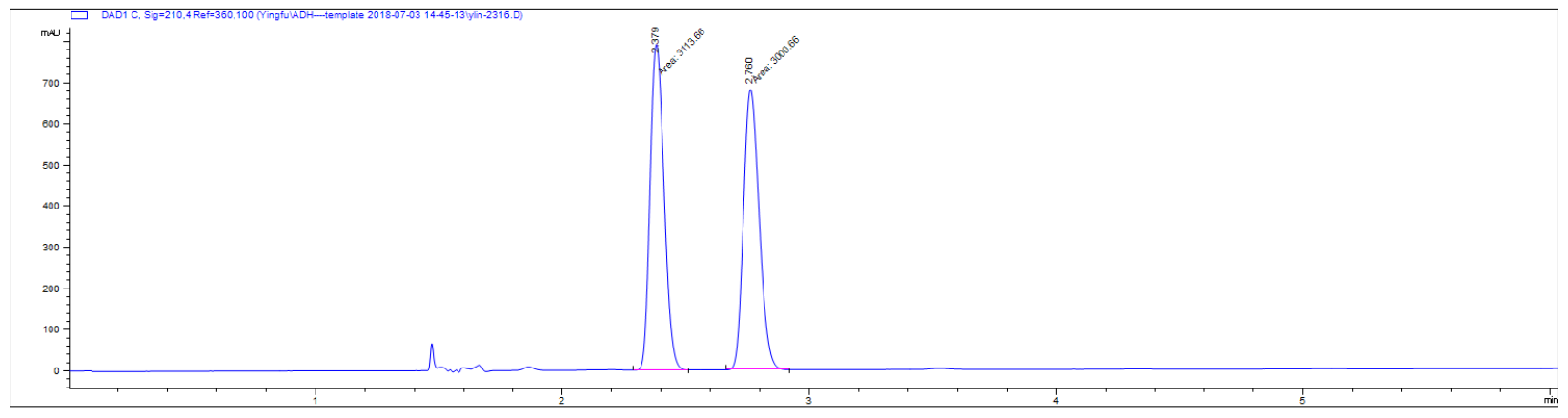

\begin{tabular}{|l|l|l|}
\hline Peak & Retention time $(\mathrm{min})$ & Area \% \\
\hline 1 & 2.379 & 50.924 \\
\hline 2 & 2.760 & 49.076 \\
\hline
\end{tabular}




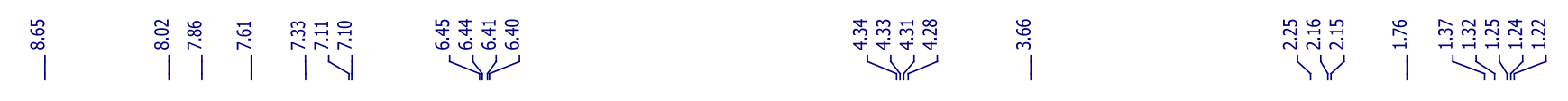

${ }^{1} \mathrm{H}$-NMR of $1 \mathrm{~g}$ in $\mathrm{CDCl}_{3}$
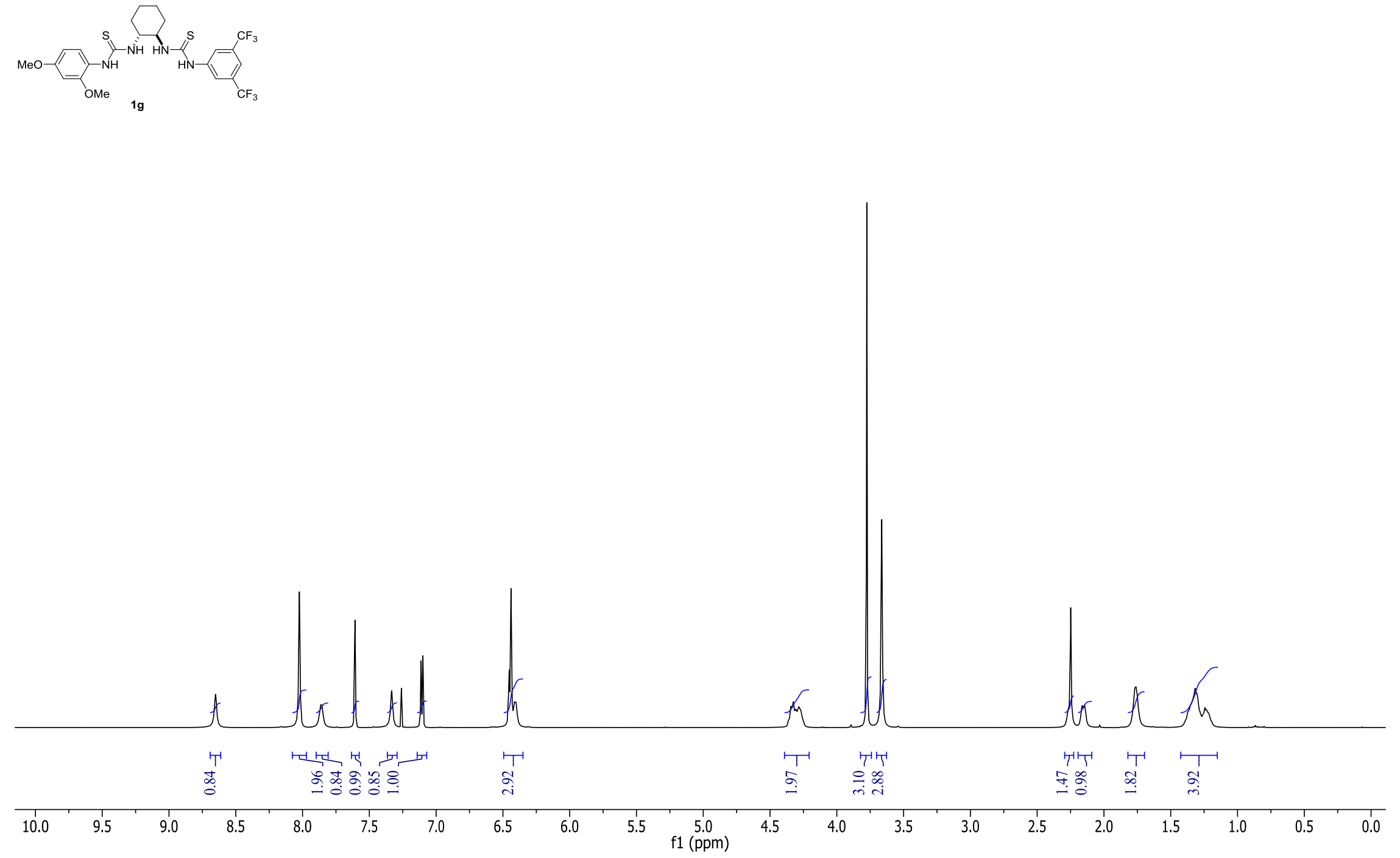


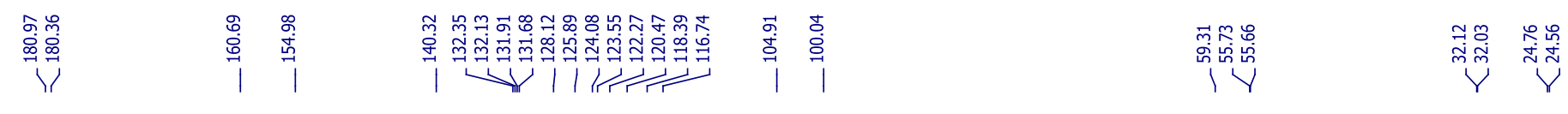

${ }^{13} \mathrm{C}-\mathrm{NMR}$ of $\mathbf{1 g}$ in $\mathrm{CDCl}_{3}$
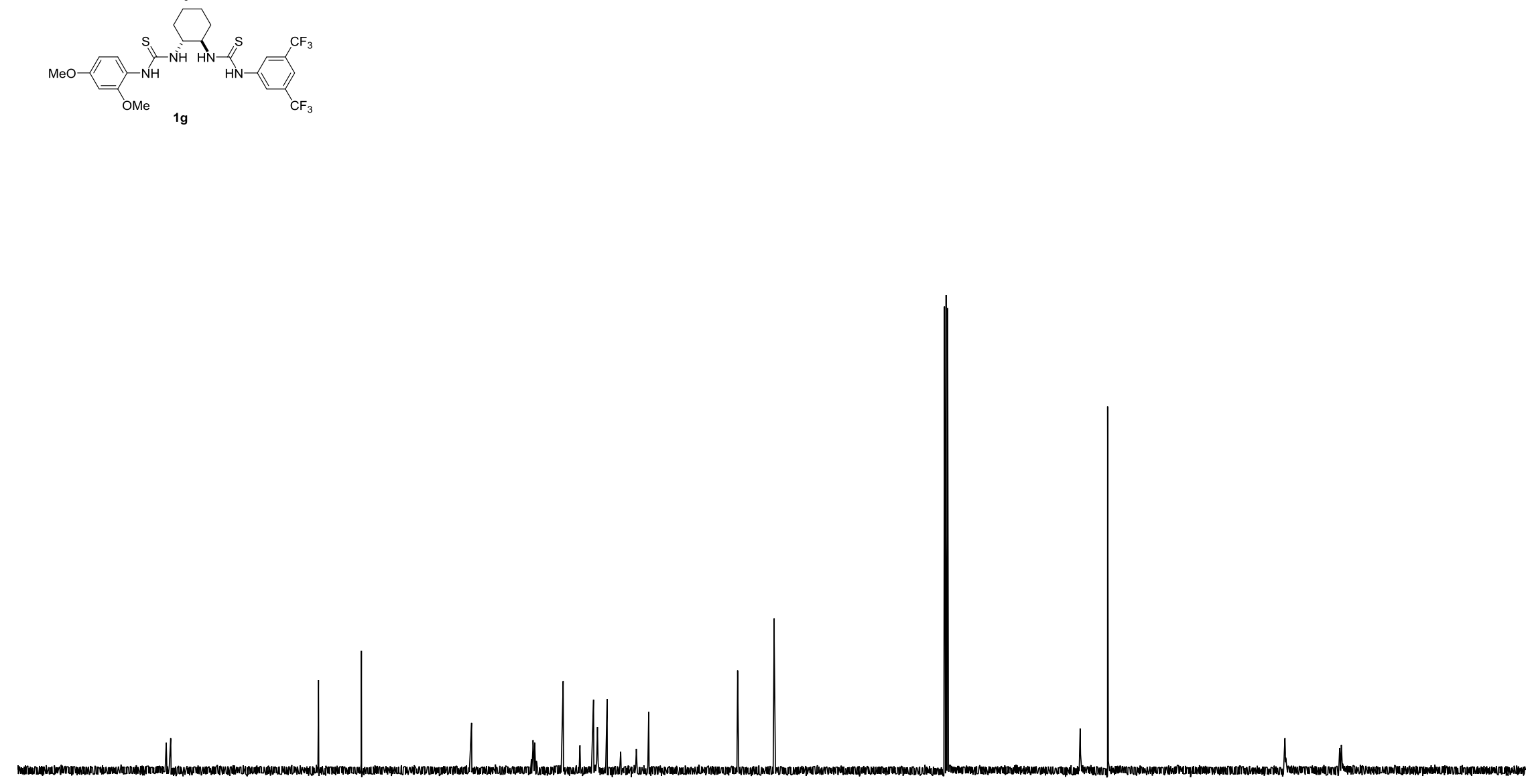


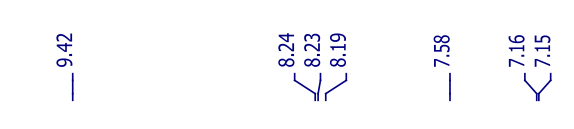

\section{${ }^{1} \mathrm{H}-\mathrm{NMR}$ of $1 \mathrm{~h}$ in $\mathrm{CDCl}_{3}$}

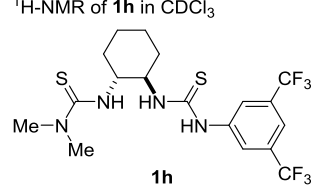

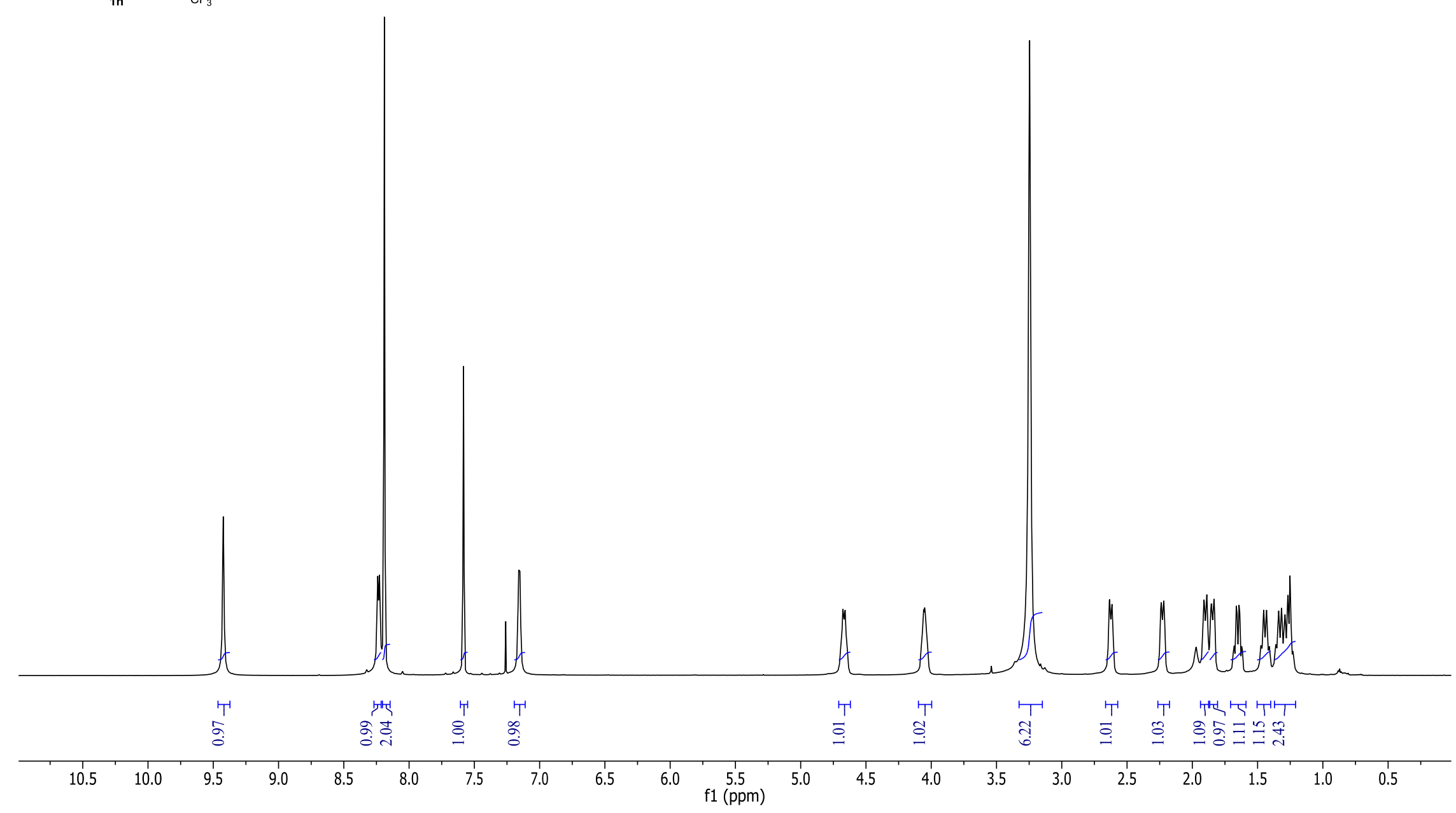


${ }^{13} \mathrm{C}-\mathrm{NMR}$ of $1 \mathrm{~h}$ in $\mathrm{CDCl}_{3}$
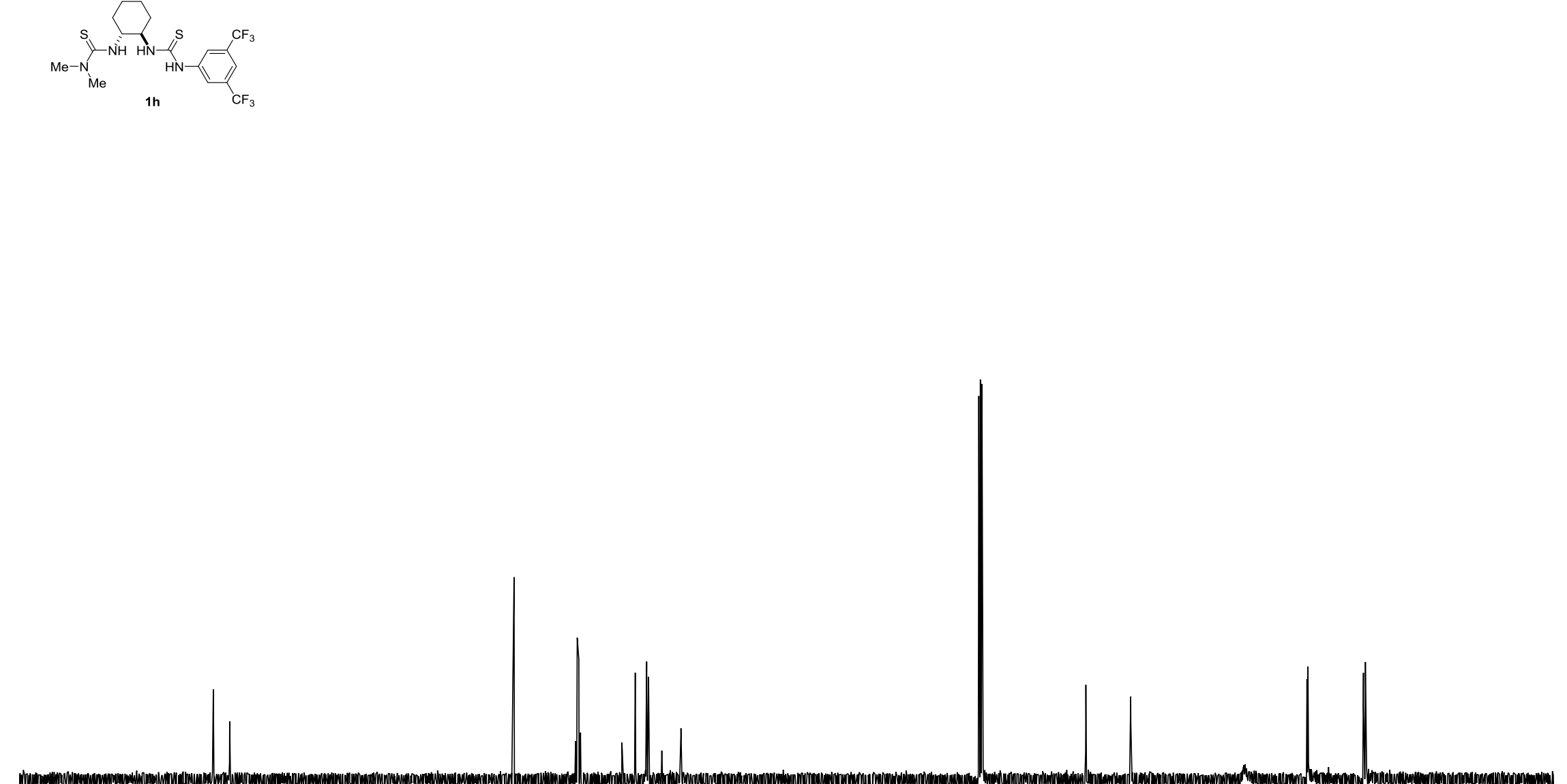

싱
蕬落

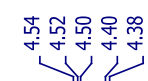

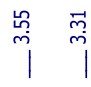

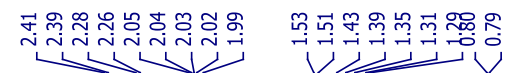

${ }^{1} \mathrm{H}-\mathrm{NMR}$ of $1 \mathrm{i}$ in $\mathrm{CDCl}_{3}$

(i)

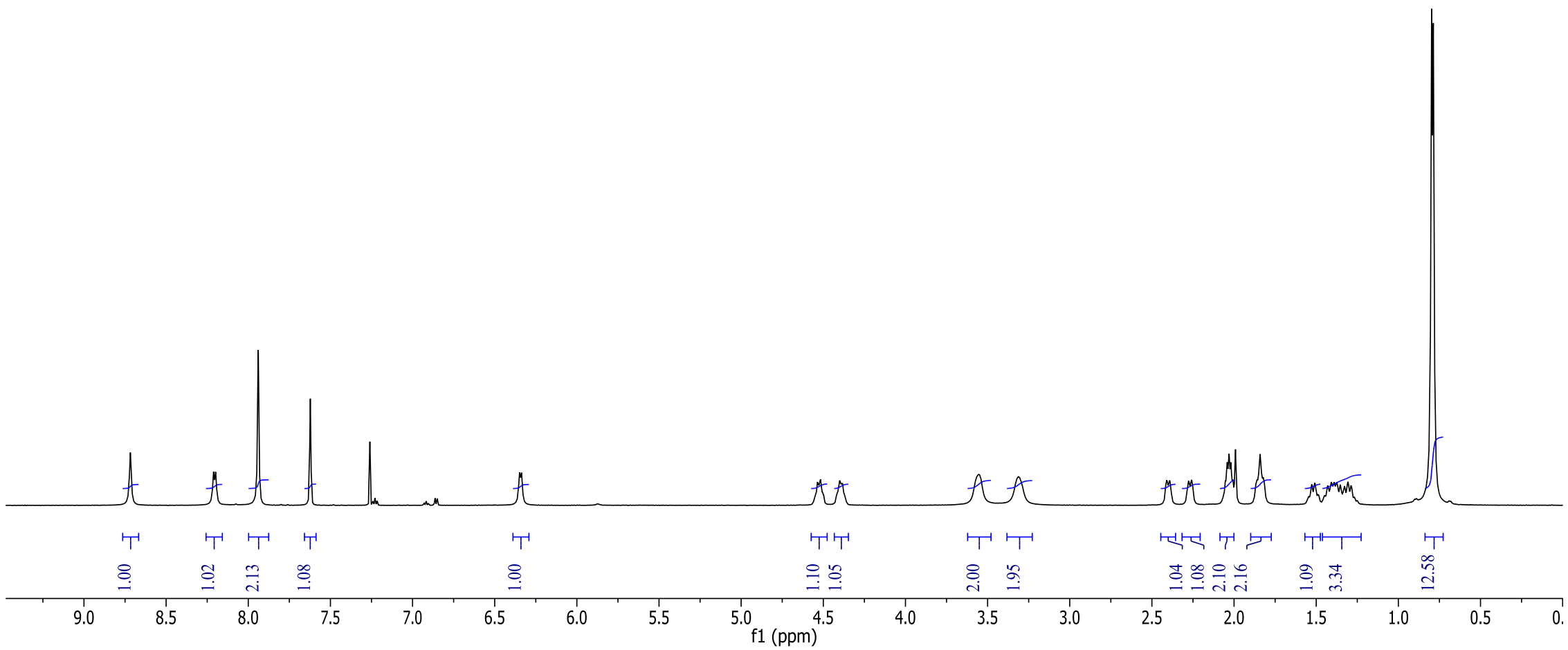




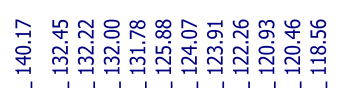

1 प vivit

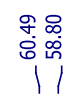

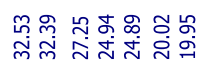

Y TYY

${ }^{13} \mathrm{C}-\mathrm{NMR}$ of $1 \mathrm{i}$ in $\mathrm{CDCl}_{3}$

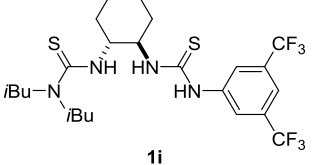

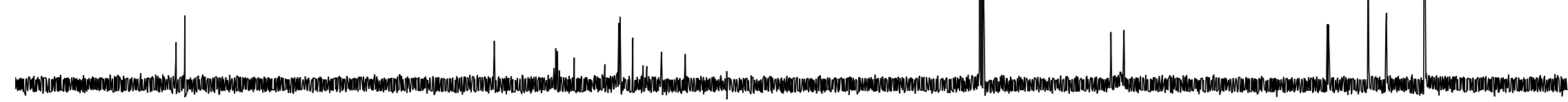




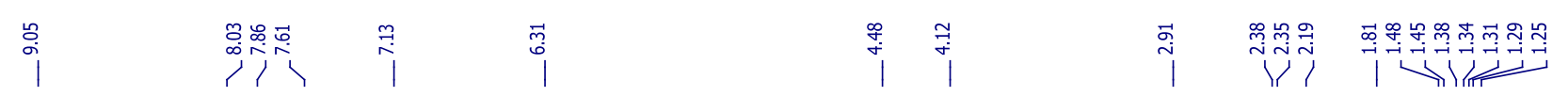

${ }^{1} \mathrm{H}-\mathrm{NMR}$ of $1 \mathbf{k}$ in $\mathrm{CDCl}_{3}$

(N)

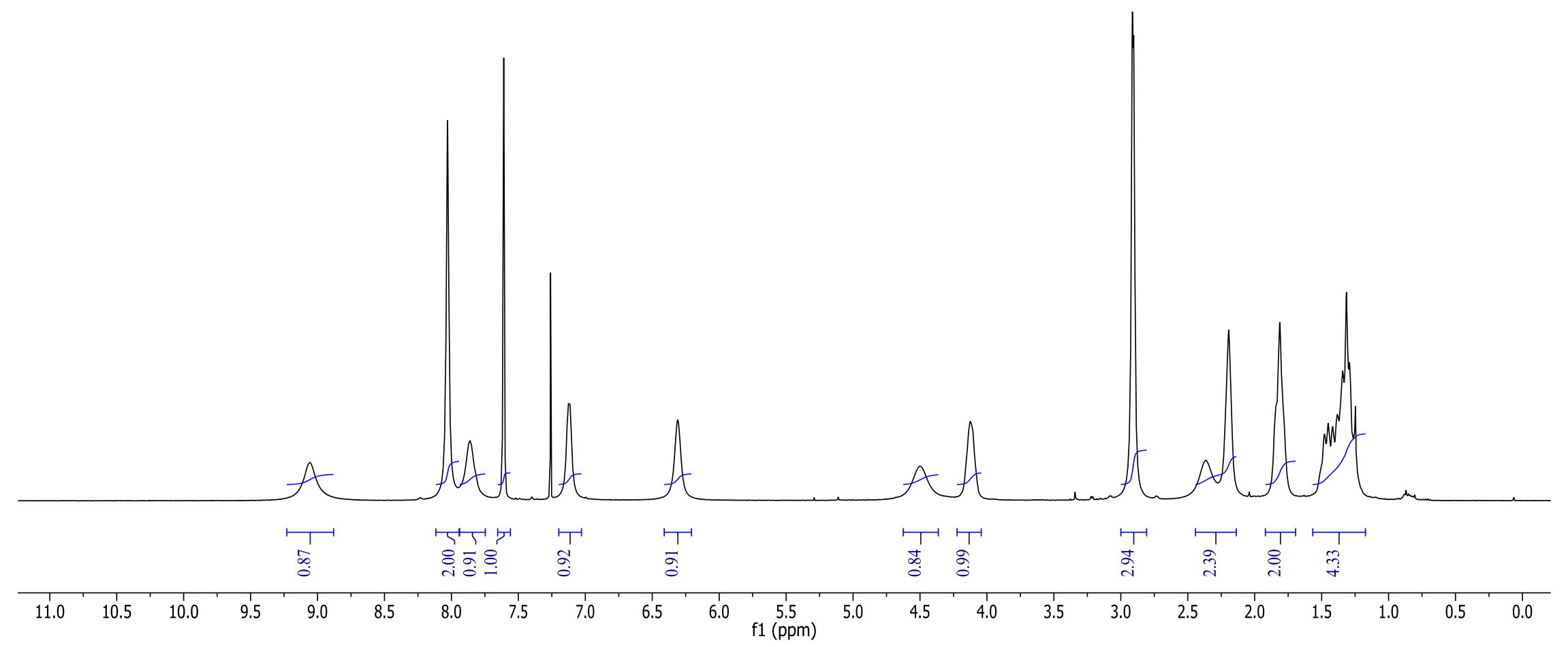




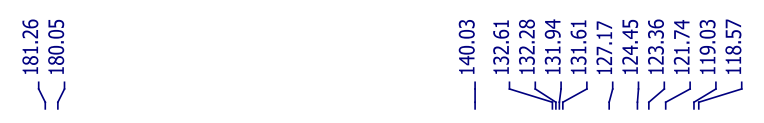

${ }^{13} \mathrm{C}-\mathrm{NMR}$ of $\mathbf{1} \mathbf{k}$ in $\mathrm{CDCl}_{3}$

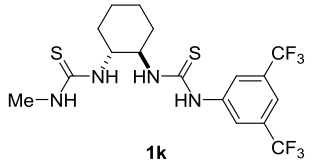

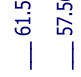

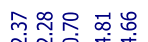

in $^{m}$

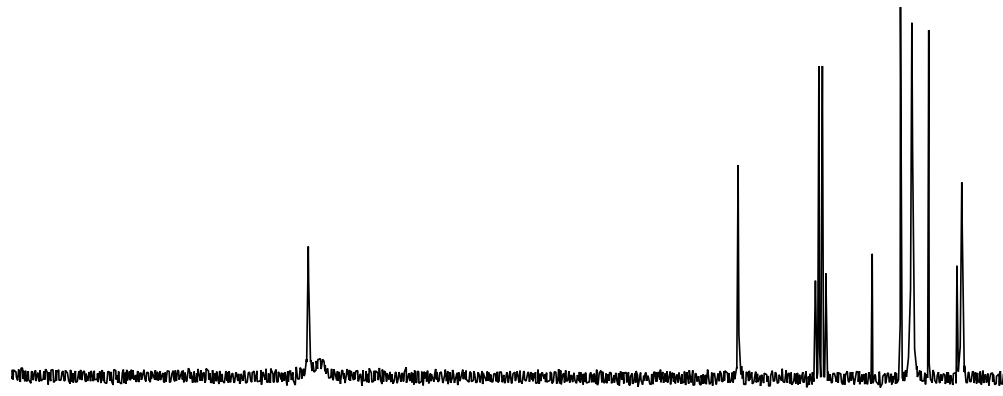


H-NMR of $\mathbf{S} 1$ in $\mathrm{CDCl}_{3}$
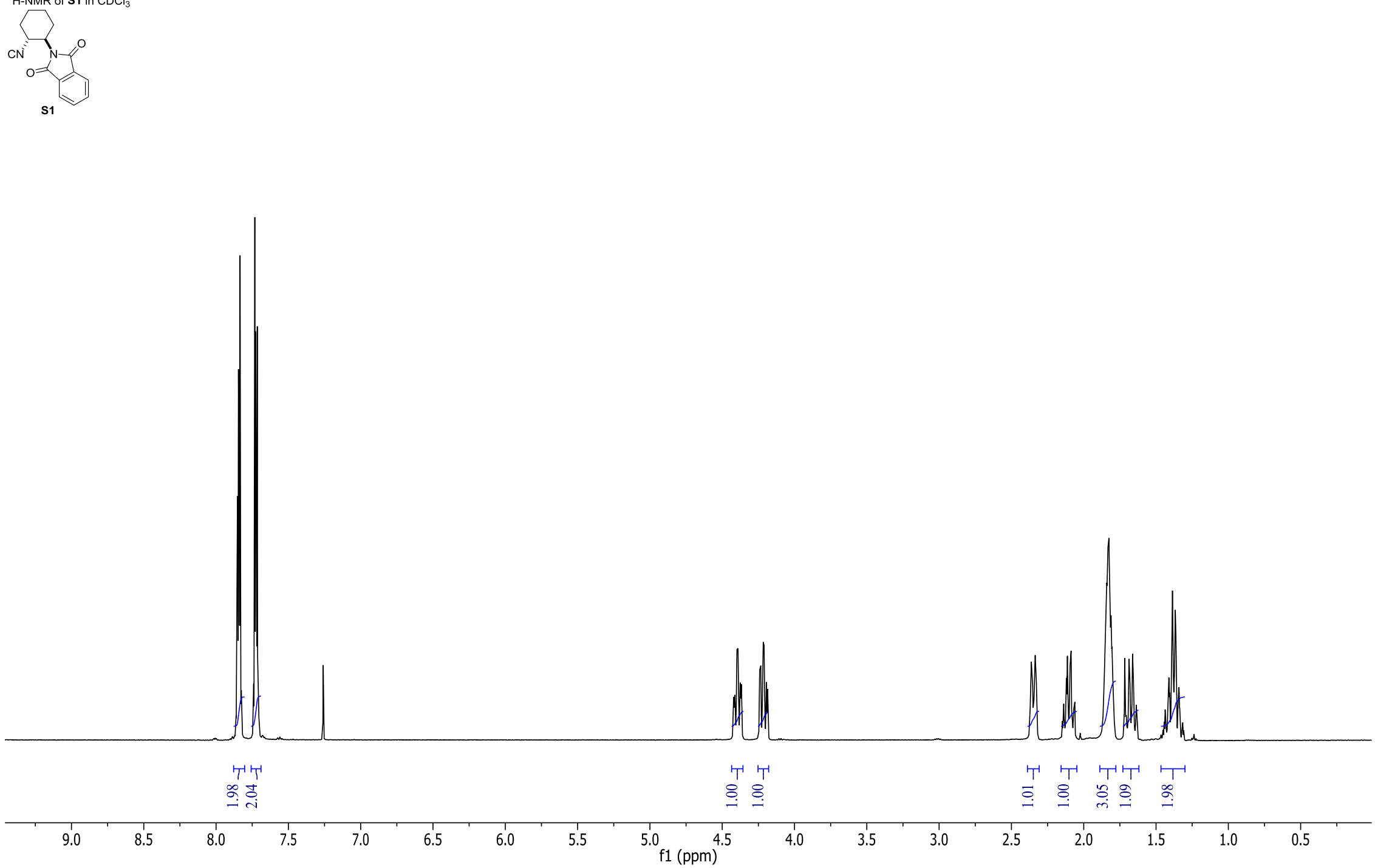


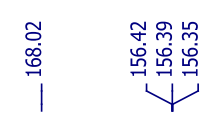
|
$\sqrt{\text { ma }}$
|

${ }^{13} \mathrm{C}-\mathrm{NMR}$ of $\mathbf{S} 1$ in $\mathrm{CDCl}_{3}$

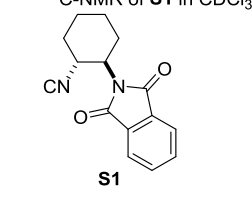




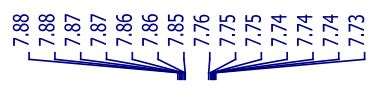

'H-NMR of $\mathbf{S} 2$ in $\mathrm{CDCl}_{3}$
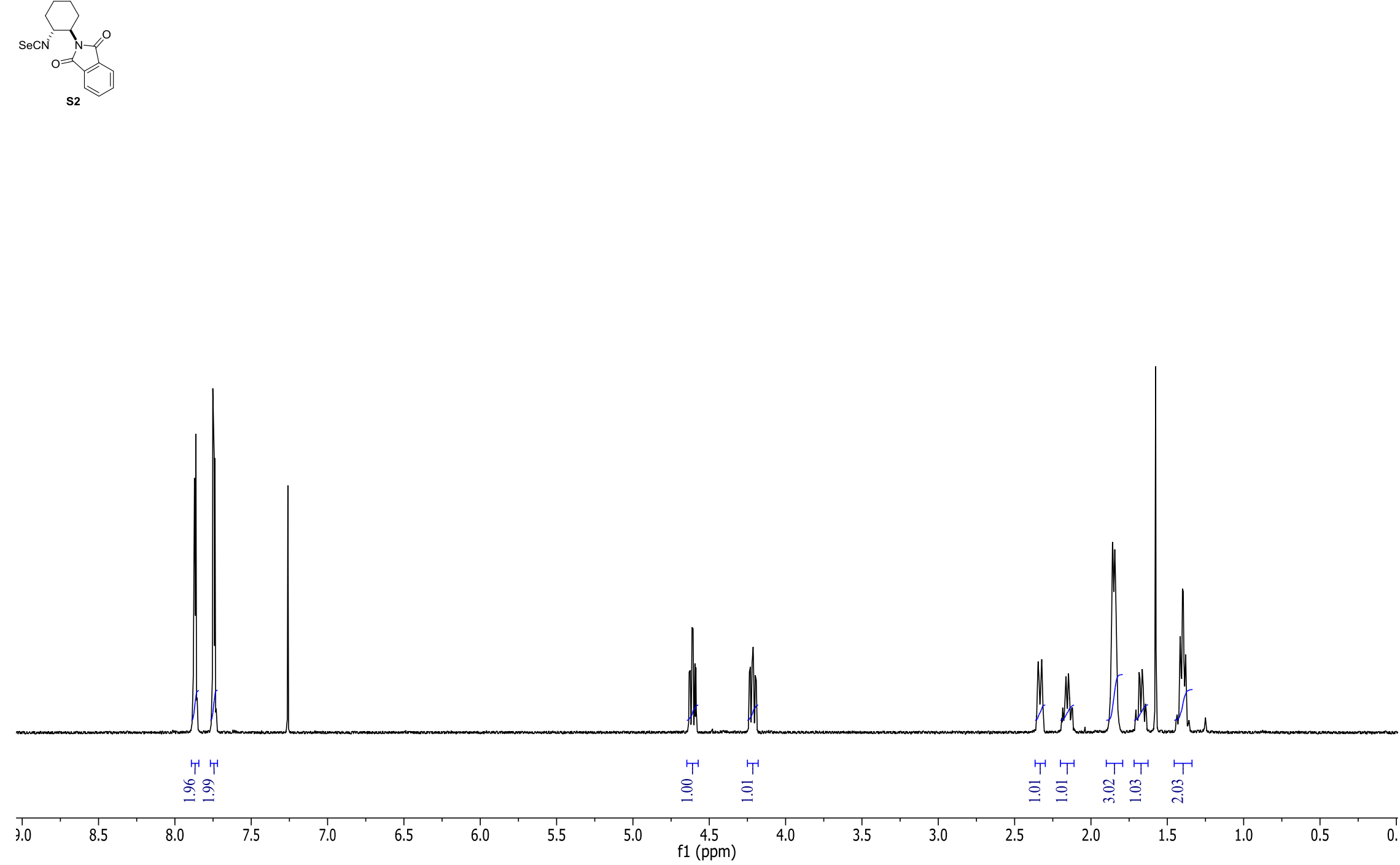


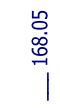
|
|

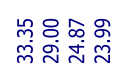

${ }^{13} \mathrm{C}-\mathrm{NMR}$ of $\mathbf{S} 2$ in $\mathrm{CDCl}_{3}$

(2)

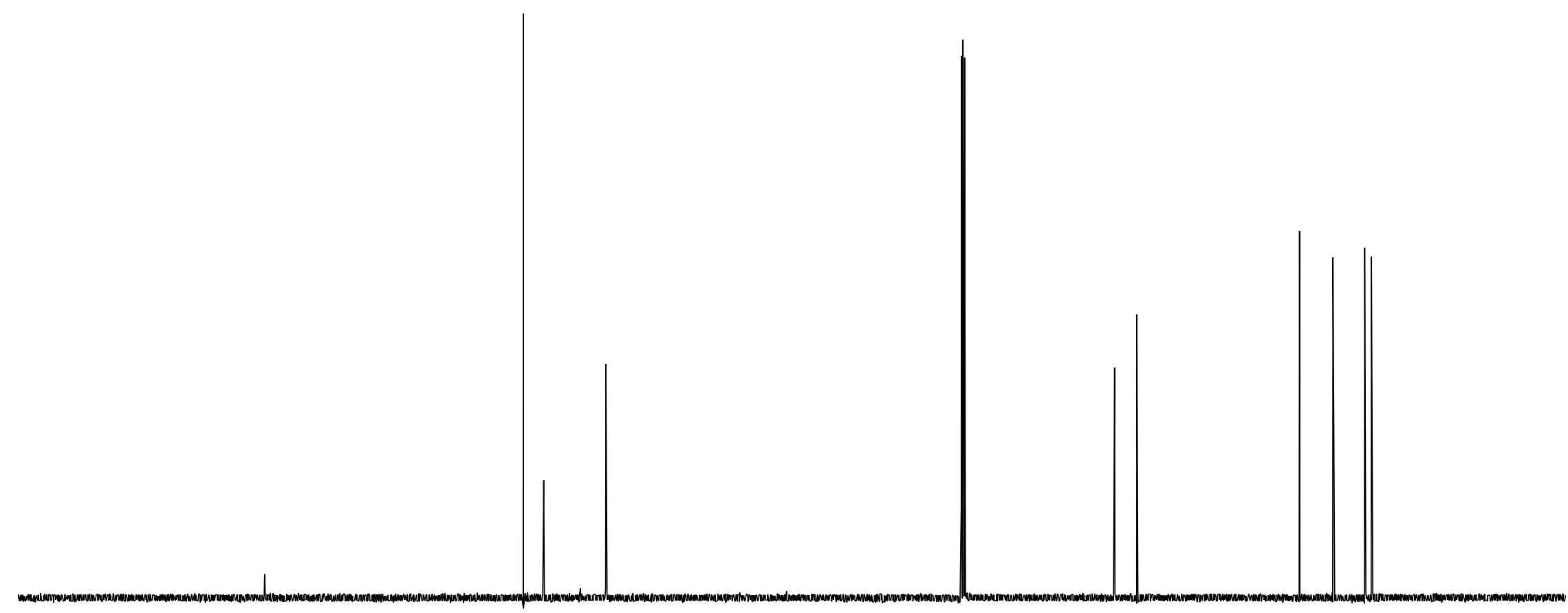

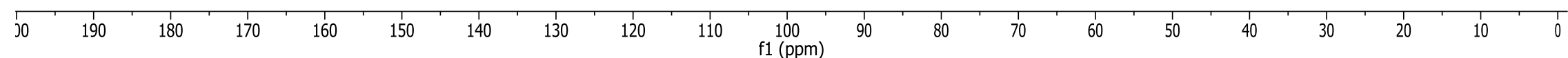


${ }^{77} \mathrm{Se}-\mathrm{NMR}$ of $\mathbf{S 2}$ in

s2

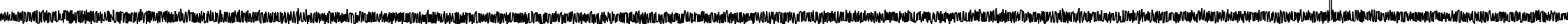

$450 \quad 400$

$350 \quad 300$

250

200

150

100

f1 (ppm)

S-244 


$$
\text { | }
$$

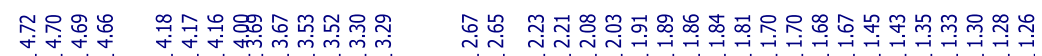

H-NMR of $1 \mathbf{n}$ in $\mathrm{CDCl}_{3}$

in

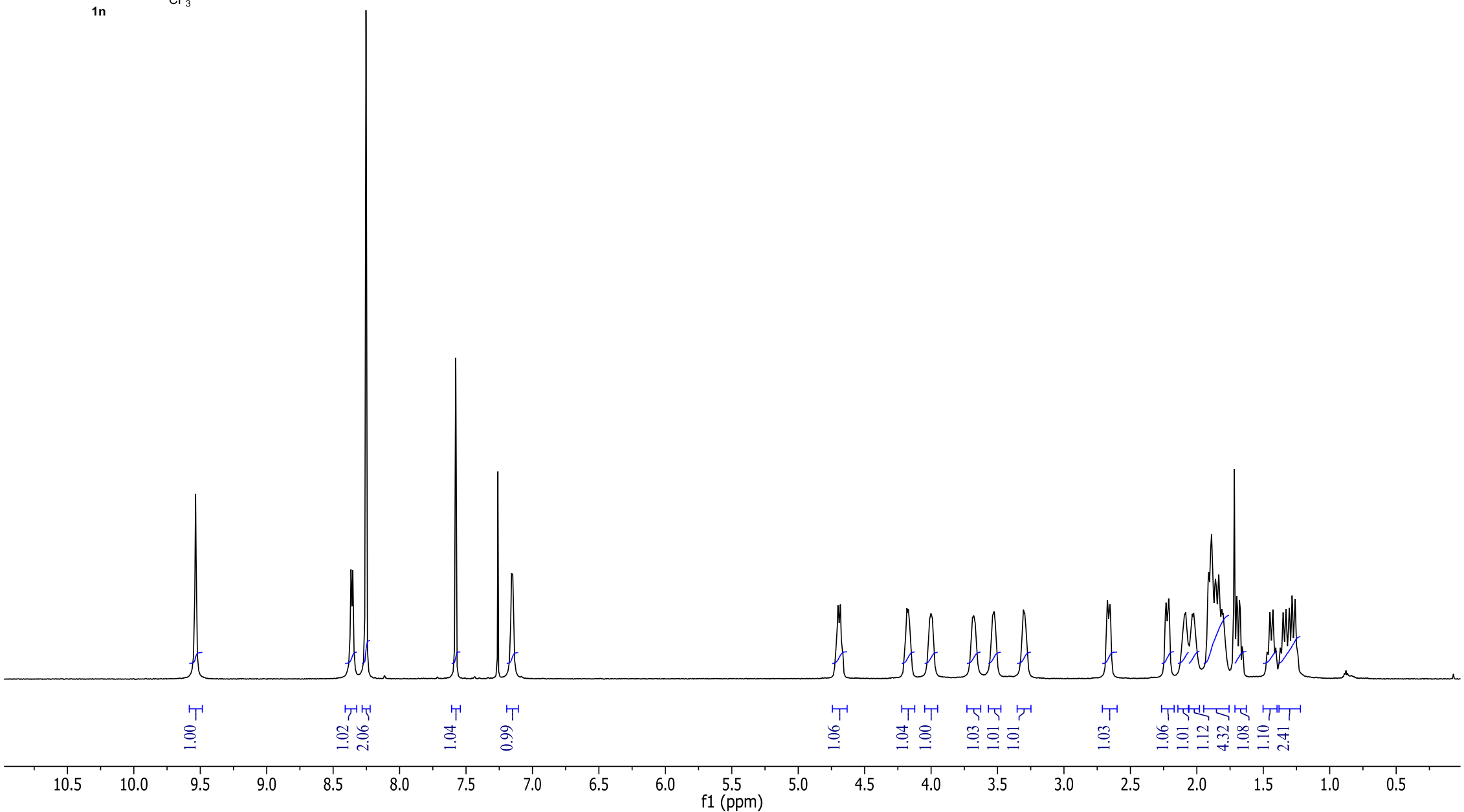




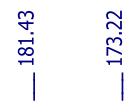

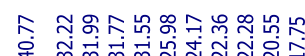
身

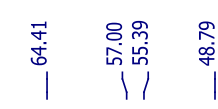

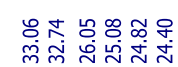

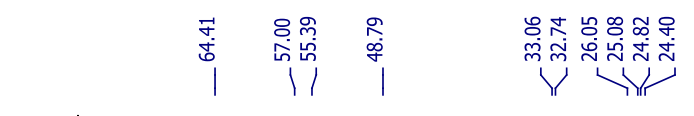

${ }^{13} \mathrm{C}$-NMR of $\mathbf{1} \mathbf{n}$ in $\mathrm{CDCl}_{3}$

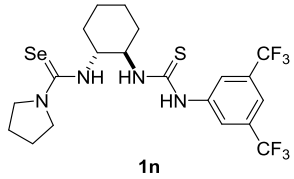

$$
\sum_{C F_{3}}
$$


${ }^{77} \mathrm{Se}-\mathrm{NMR}$ of $1 \mathrm{n}$ in $\mathrm{CDCl}_{3}$

$\sum_{1 \mathrm{~N}}^{\mathrm{N}}$

.

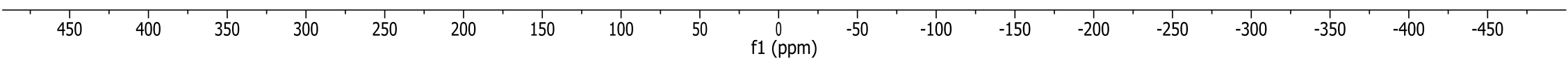




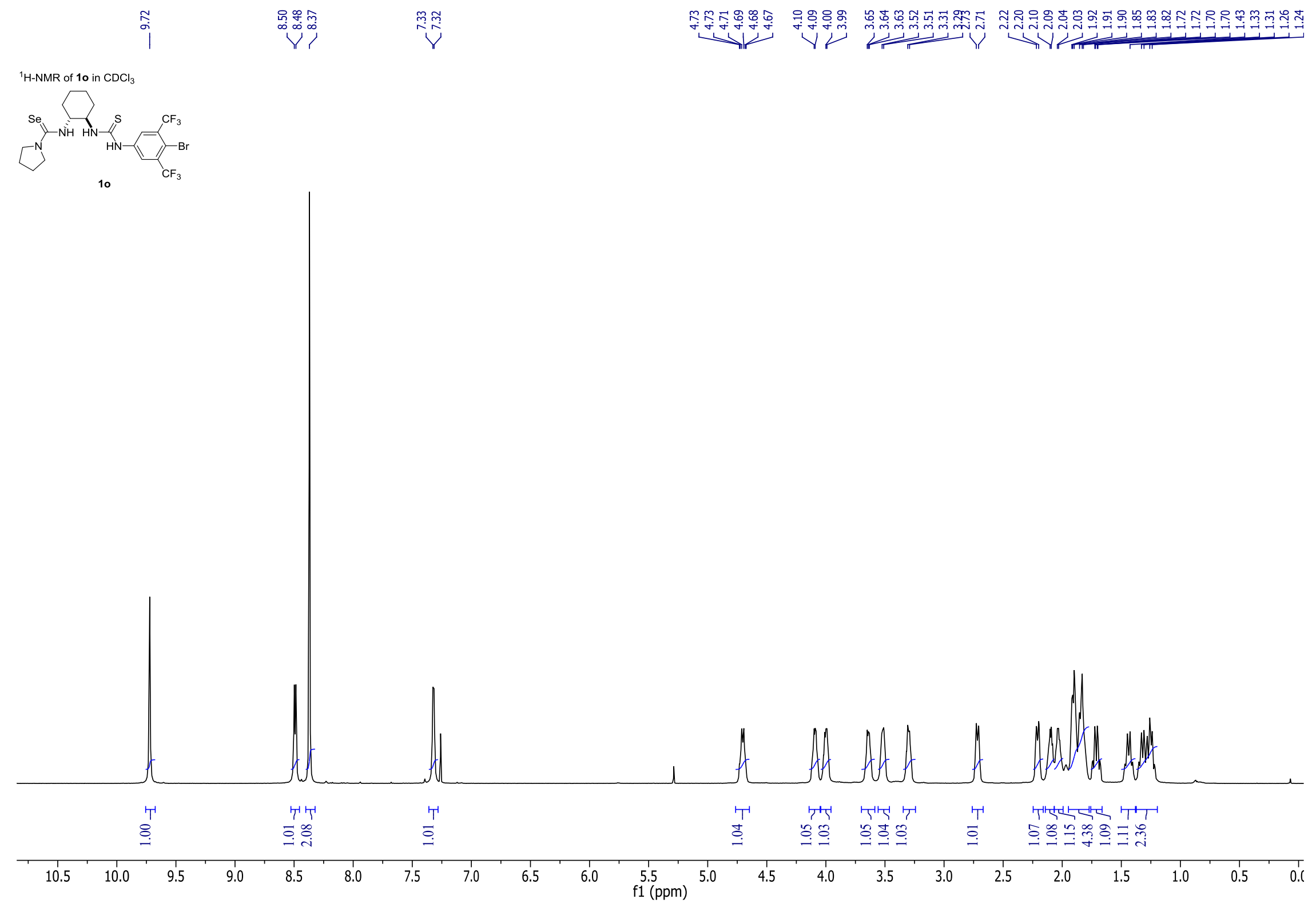




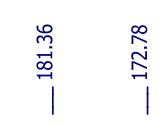
|

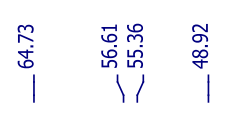

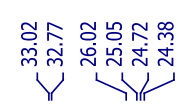

${ }^{13} \mathrm{C}$-NMR of 10 in $\mathrm{CDCl}_{3}$

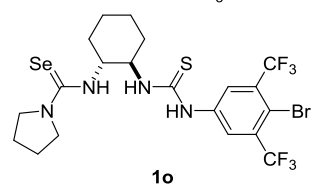

10

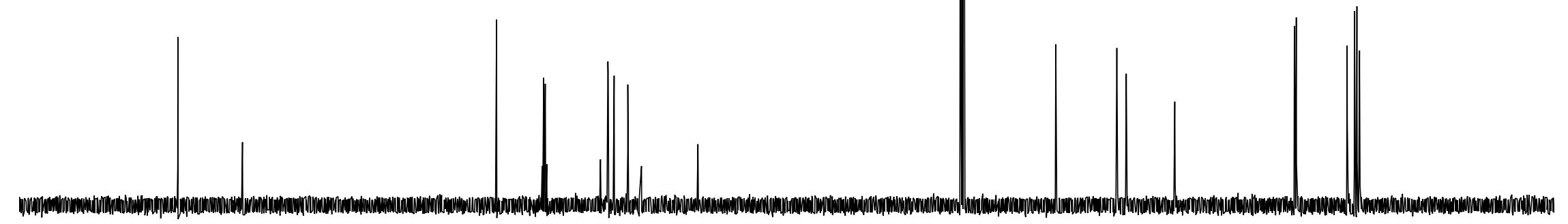


${ }^{77} \mathrm{Se}-\mathrm{NMR}$ of 10 in $\mathrm{CDCl}_{3}$

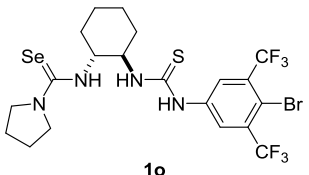

10

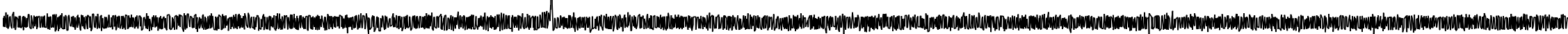




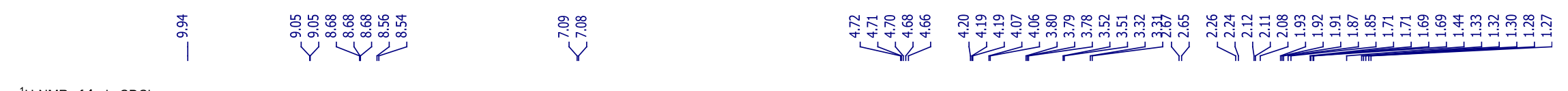

${ }^{1} \mathrm{H}$-NMR of $1 \mathrm{P}$ in $\mathrm{CDCl}$
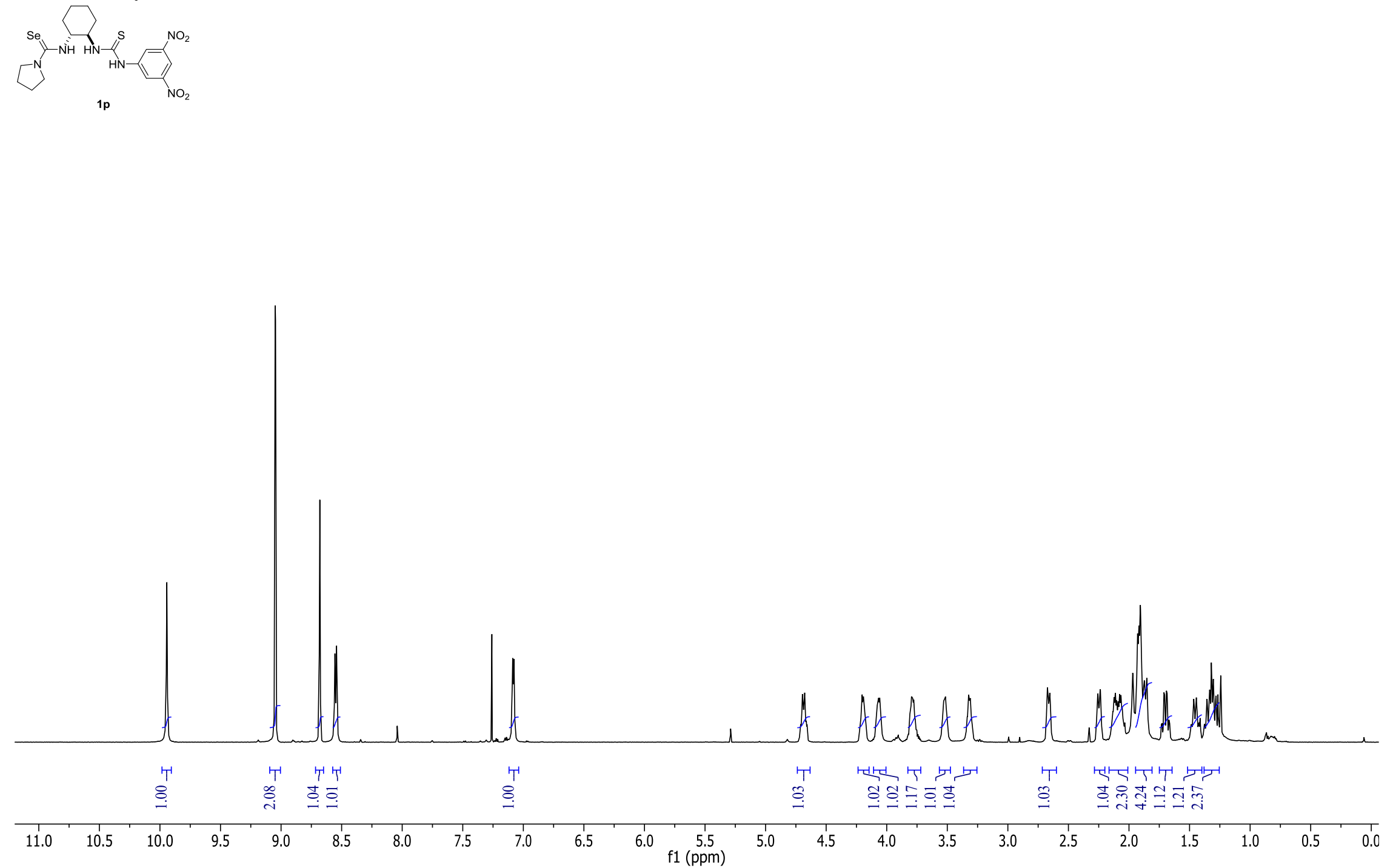

|

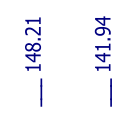

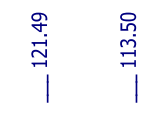
m

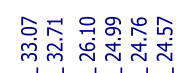

${ }^{13} \mathrm{C}-\mathrm{NMR}$ of $1 \mathrm{p}$ in $\mathrm{CDCl}_{3}$

(P)

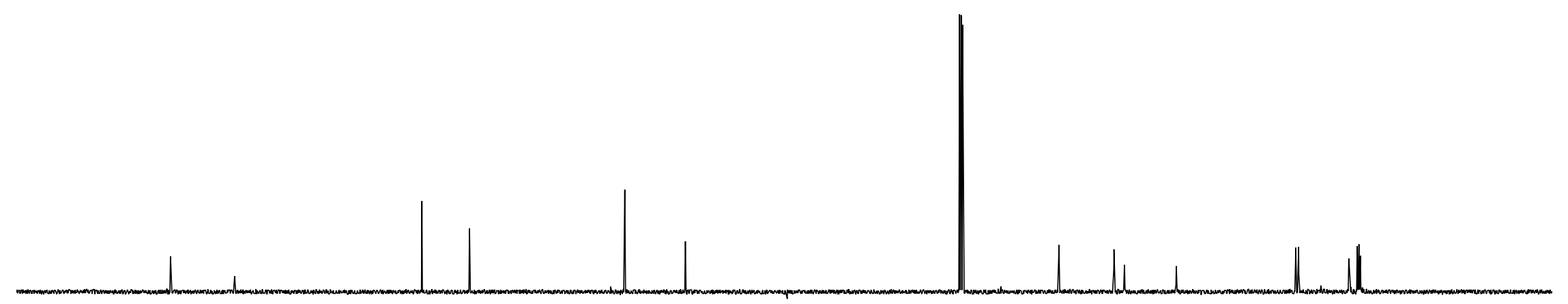

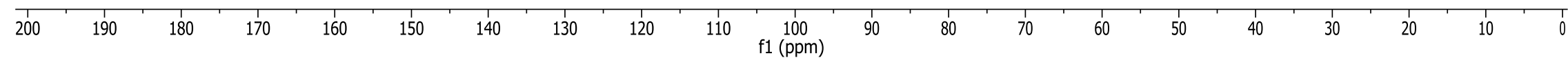


${ }^{77} \mathrm{Se}$-NMR of $1 \mathrm{p}$ in $\mathrm{CDCl}_{3}$

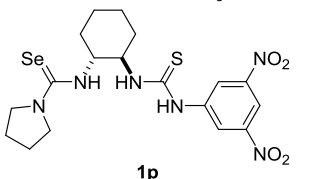

.

$\begin{array}{lll}450 & 400 & 350\end{array}$ 


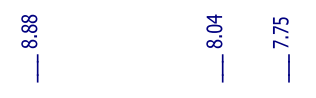

${ }^{1} \mathrm{H}$-NMR of $\mathrm{S} 5$ in $\mathrm{CDCl}_{3}$
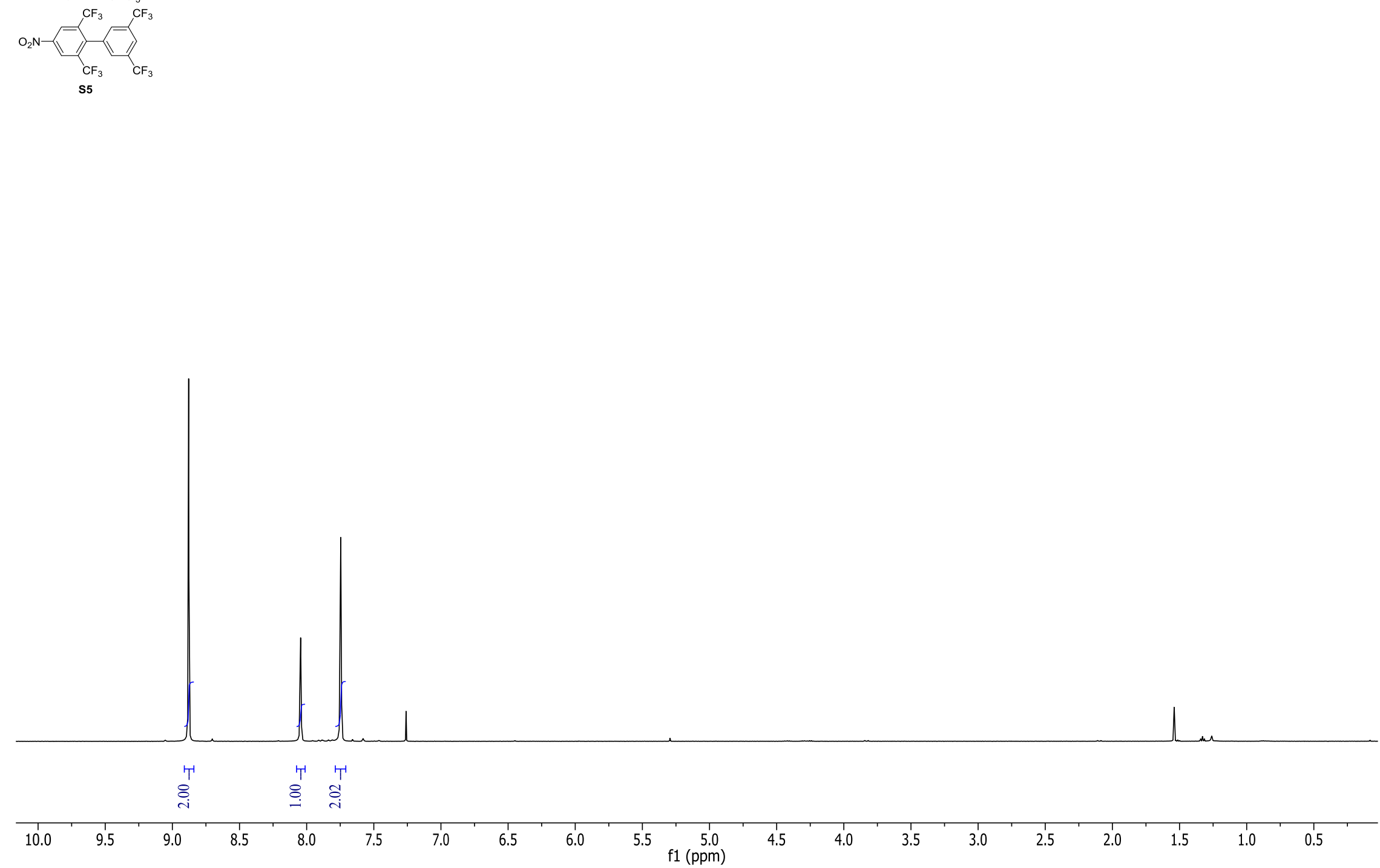


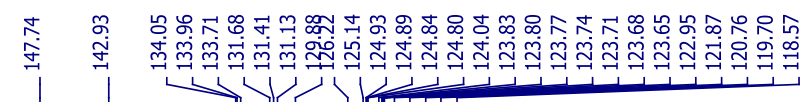

${ }^{13} \mathrm{C}-\mathrm{NMR}$ of $\mathbf{S} 5$ in $\mathrm{CDCl}_{3}$

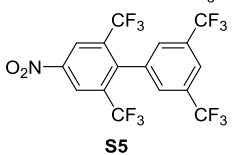

S5

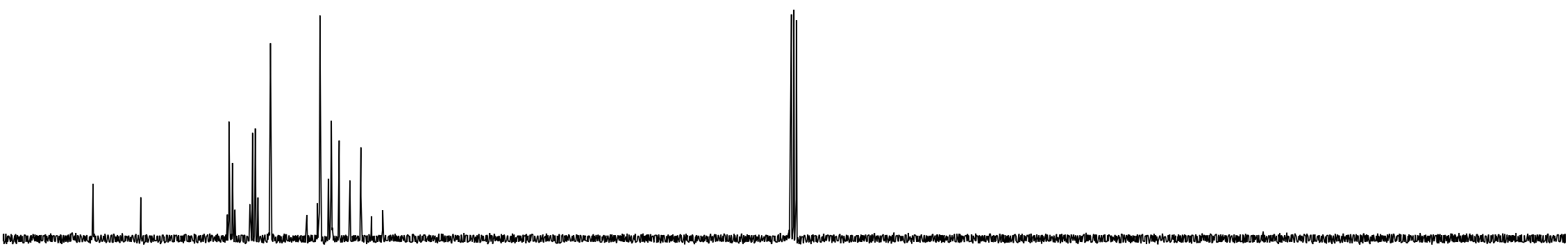

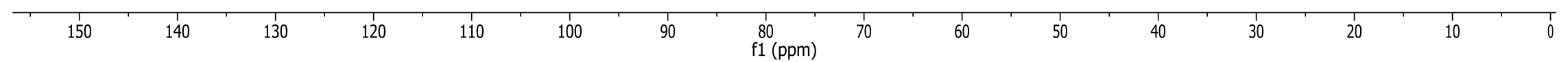


${ }^{19} \mathrm{~F}-\mathrm{NMR}$ of $\mathbf{S} 5$ in $\mathrm{CDCl}_{3}$

S5

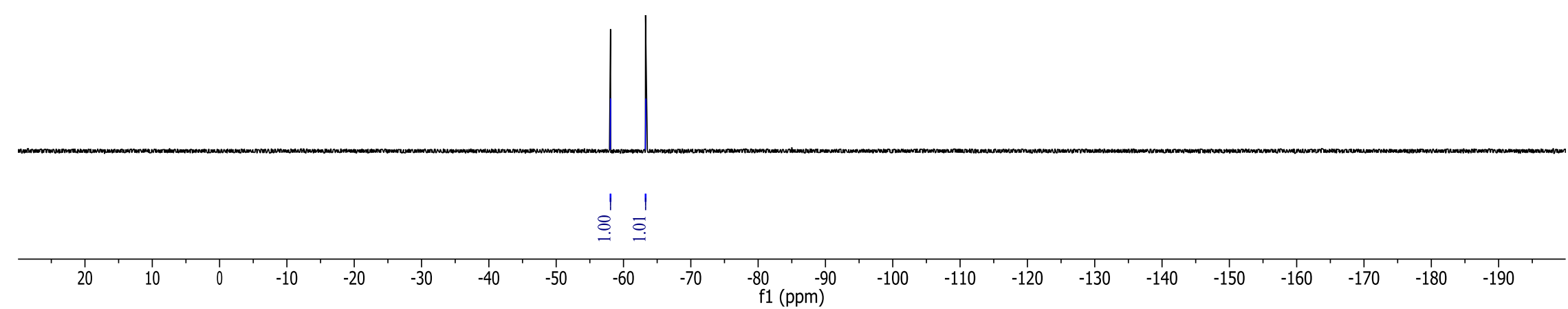


${ }^{14} \mathrm{~N}-\mathrm{NMR}$ of $\mathbf{S} 5 \mathrm{in} \mathrm{CDCl}_{3}$

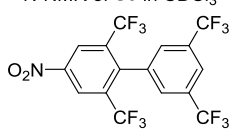

S5

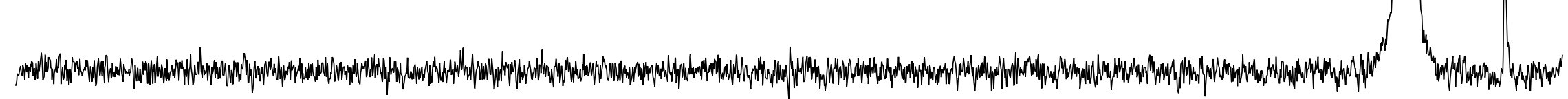

$$
00
$$

650

600

550

500

450

400

350

300
f1
$(\mathrm{ppm})$

250

200

150

100

50

$-50$ 


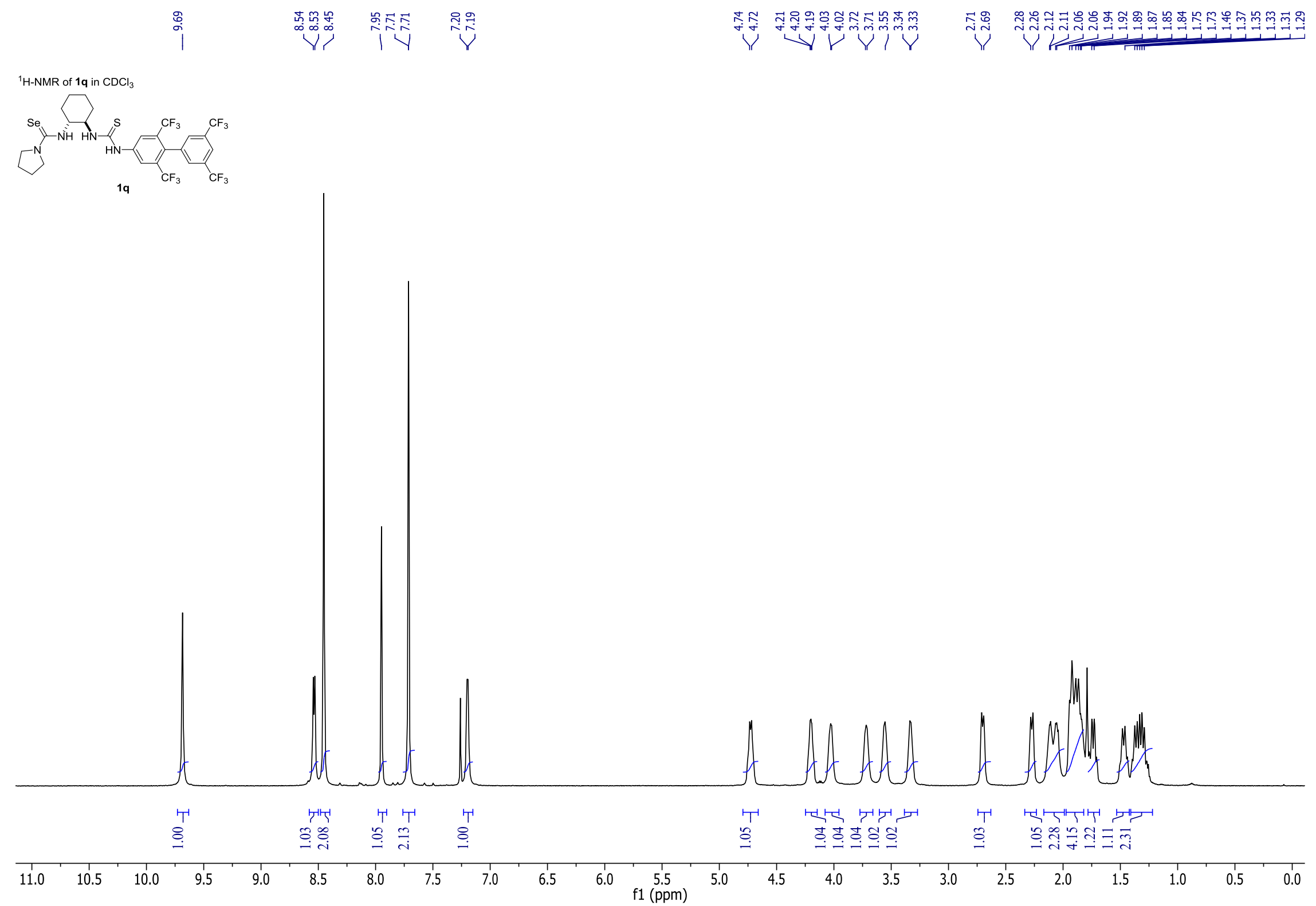




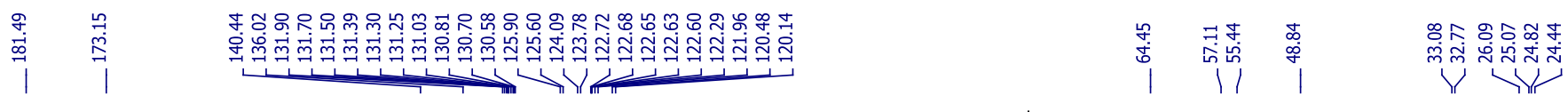

${ }^{13} \mathrm{C}-\mathrm{NMR}$ of $1 \mathrm{q}$ in $\mathrm{CDCl}_{3}$
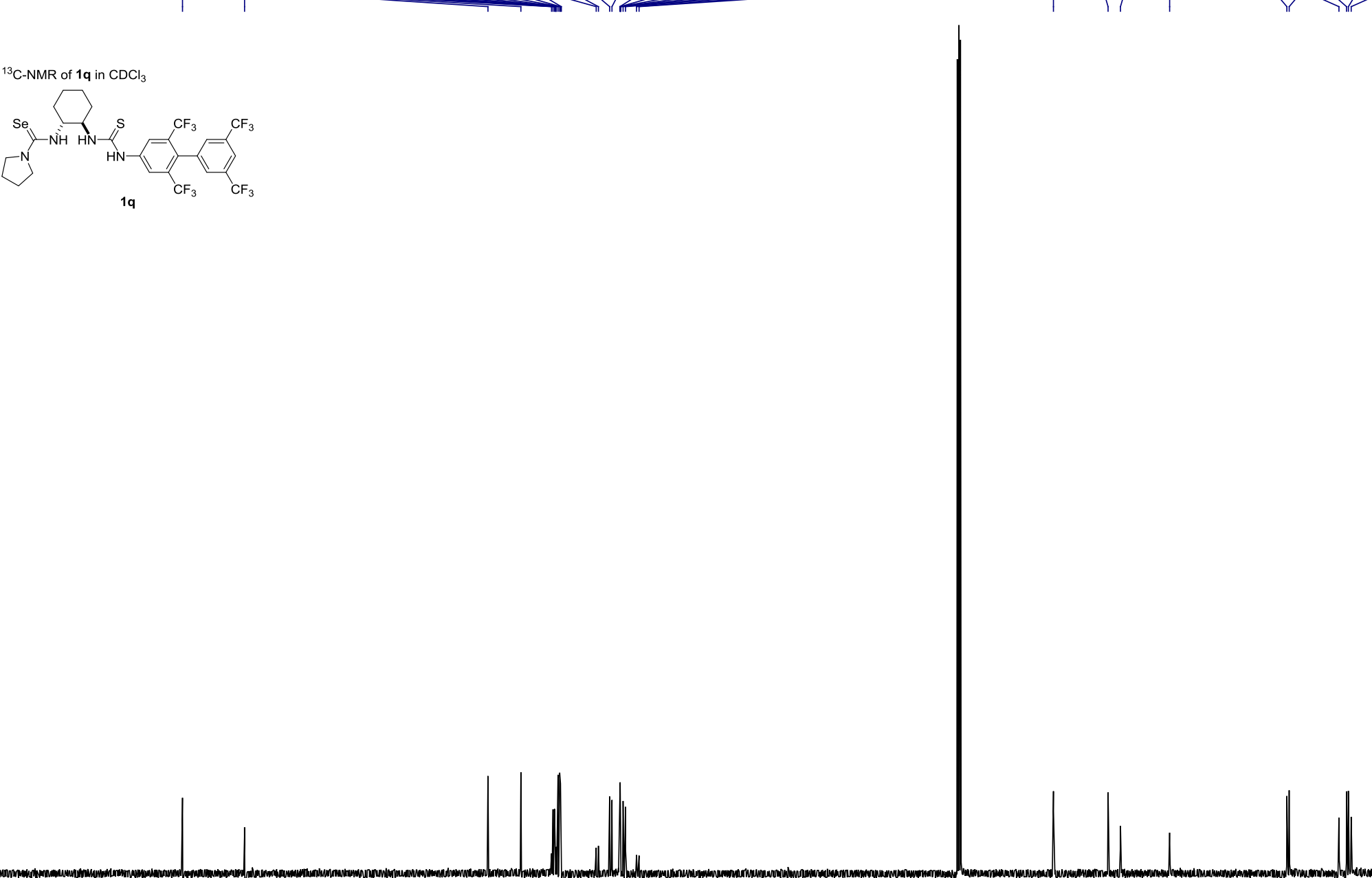
${ }^{77} \mathrm{Se}-\mathrm{NMR}$ of $1 \mathrm{q}$ in $\mathrm{CDCl}_{3}$

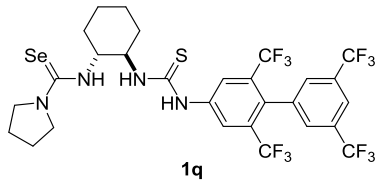

$\int_{\text {mand }}$

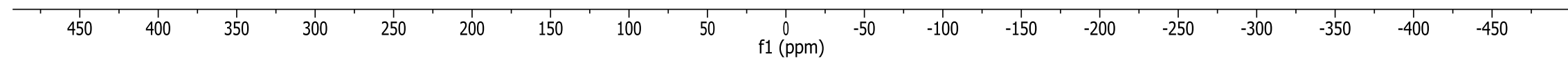




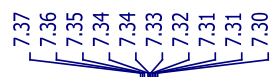

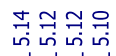

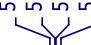

${ }^{1} \mathrm{H}-\mathrm{NMR}$ of $\mathbf{2 a}$ in $\mathrm{CDCl}_{3}$

$\underbrace{}_{2 a}$

2a

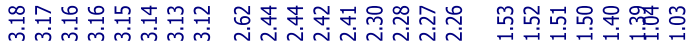

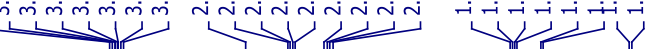

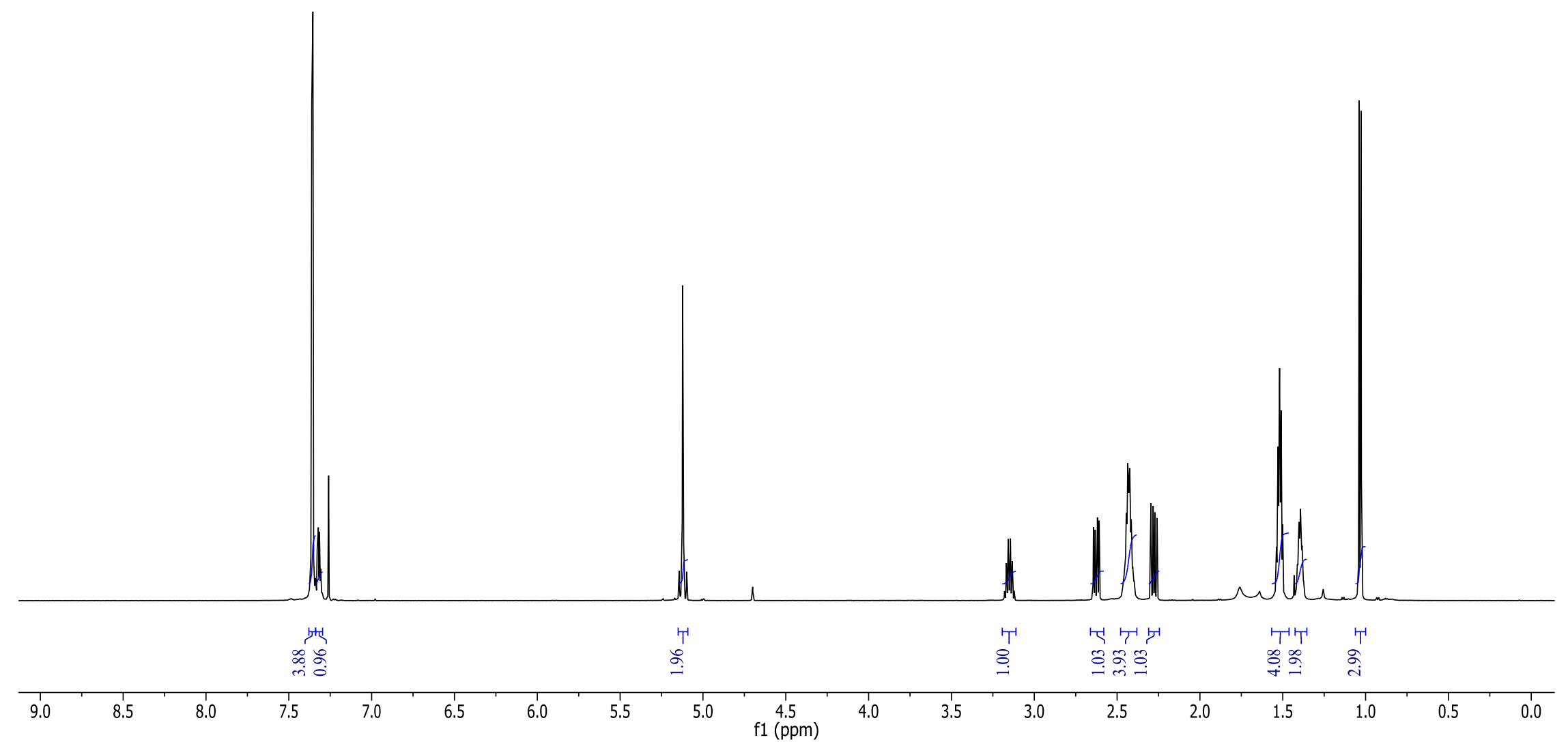




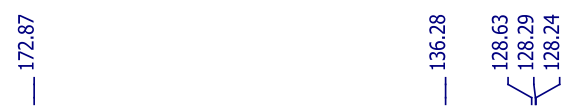

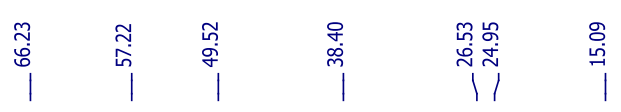

${ }^{13} \mathrm{C}-\mathrm{NMR}$ of $2 \mathrm{a}$ in $\mathrm{CDCl}_{3}$

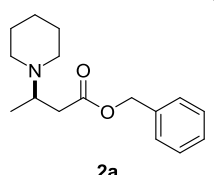

2a

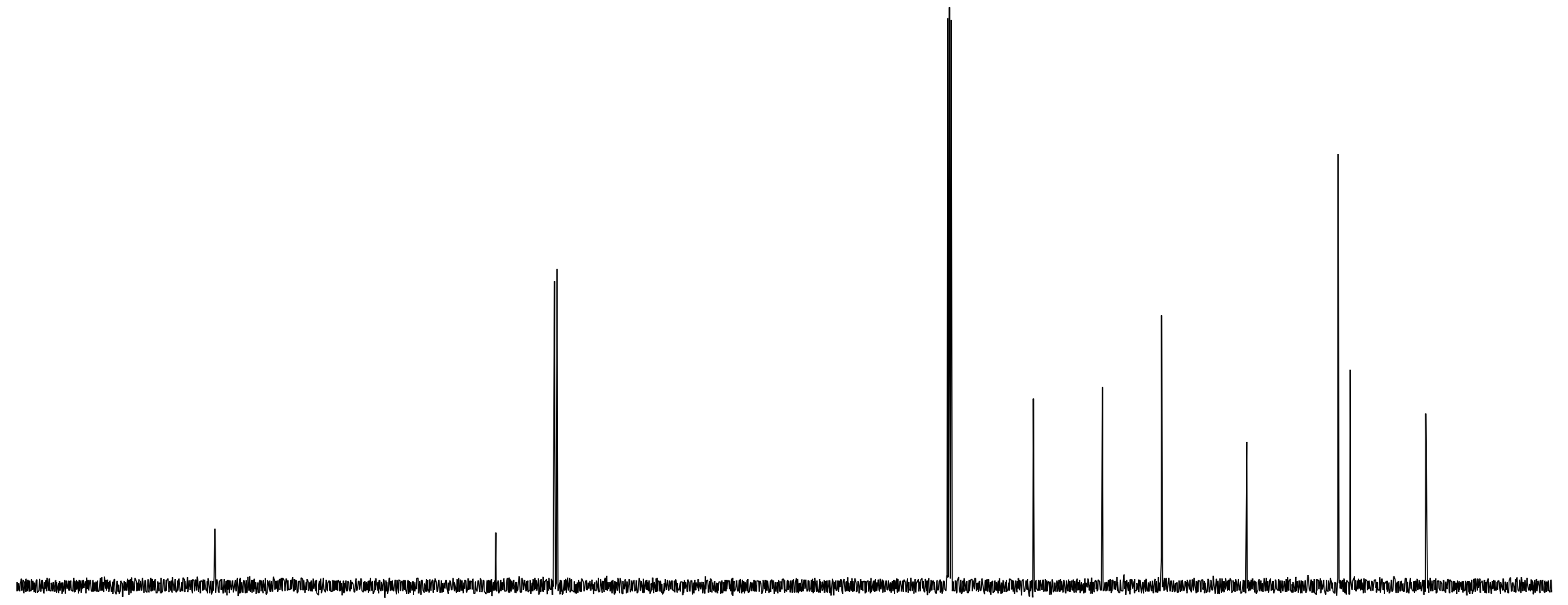

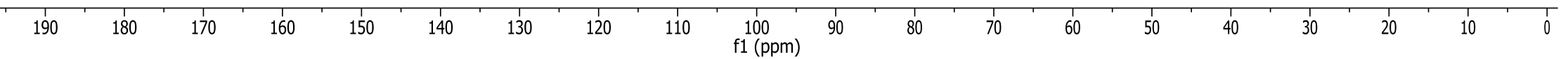


${ }^{1} \mathrm{H}-\mathrm{NMR}$ of $\mathbf{2 b}$ in $\mathrm{CDCl}_{3}$

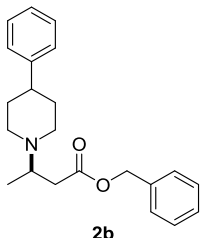

2b

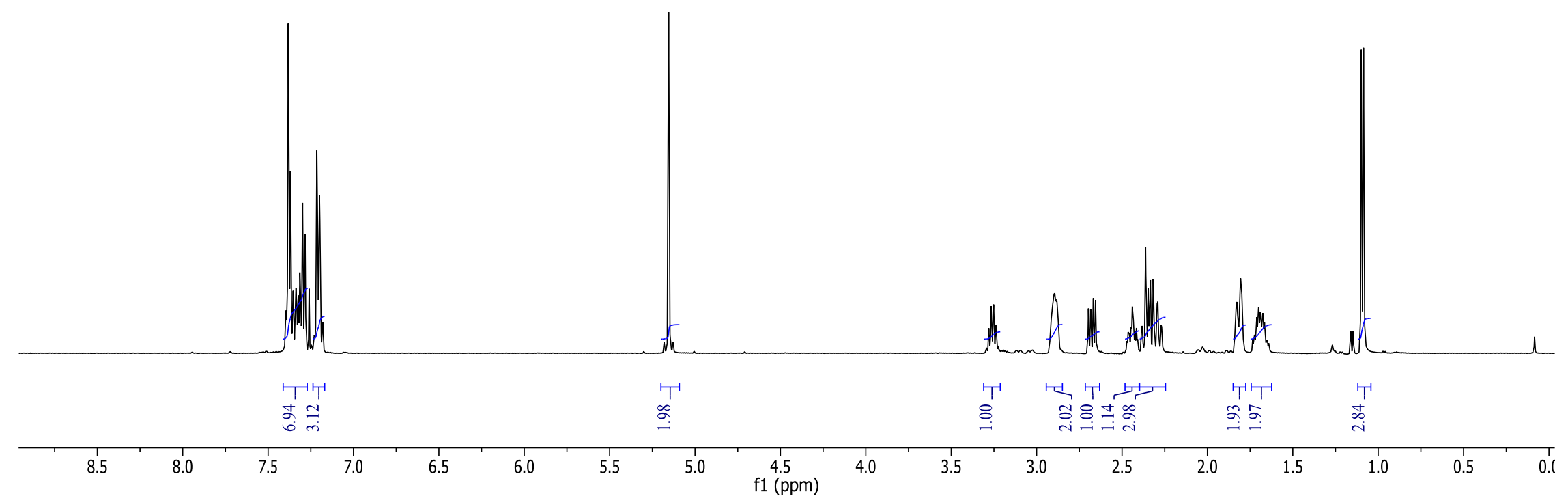




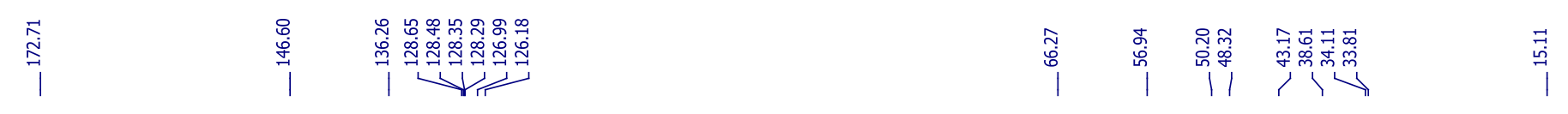

${ }^{13} \mathrm{C}-\mathrm{NMR}$ of $\mathbf{2 b}$ in $\mathrm{CDCl}_{3}$

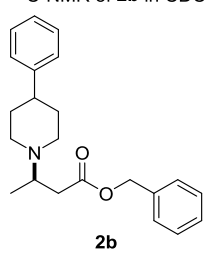

2b
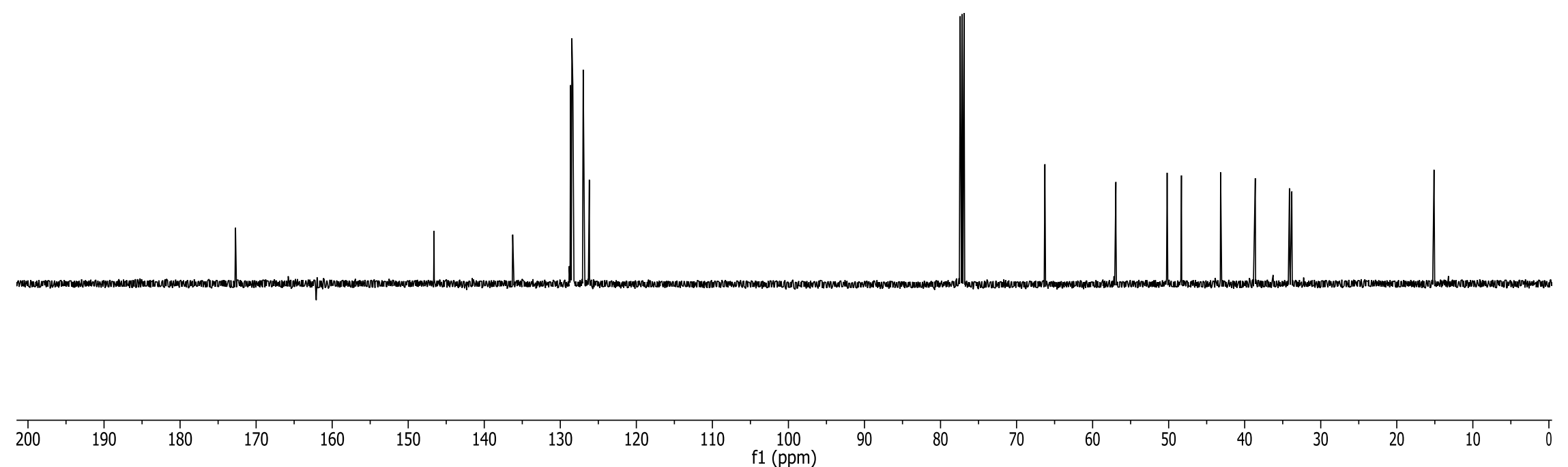


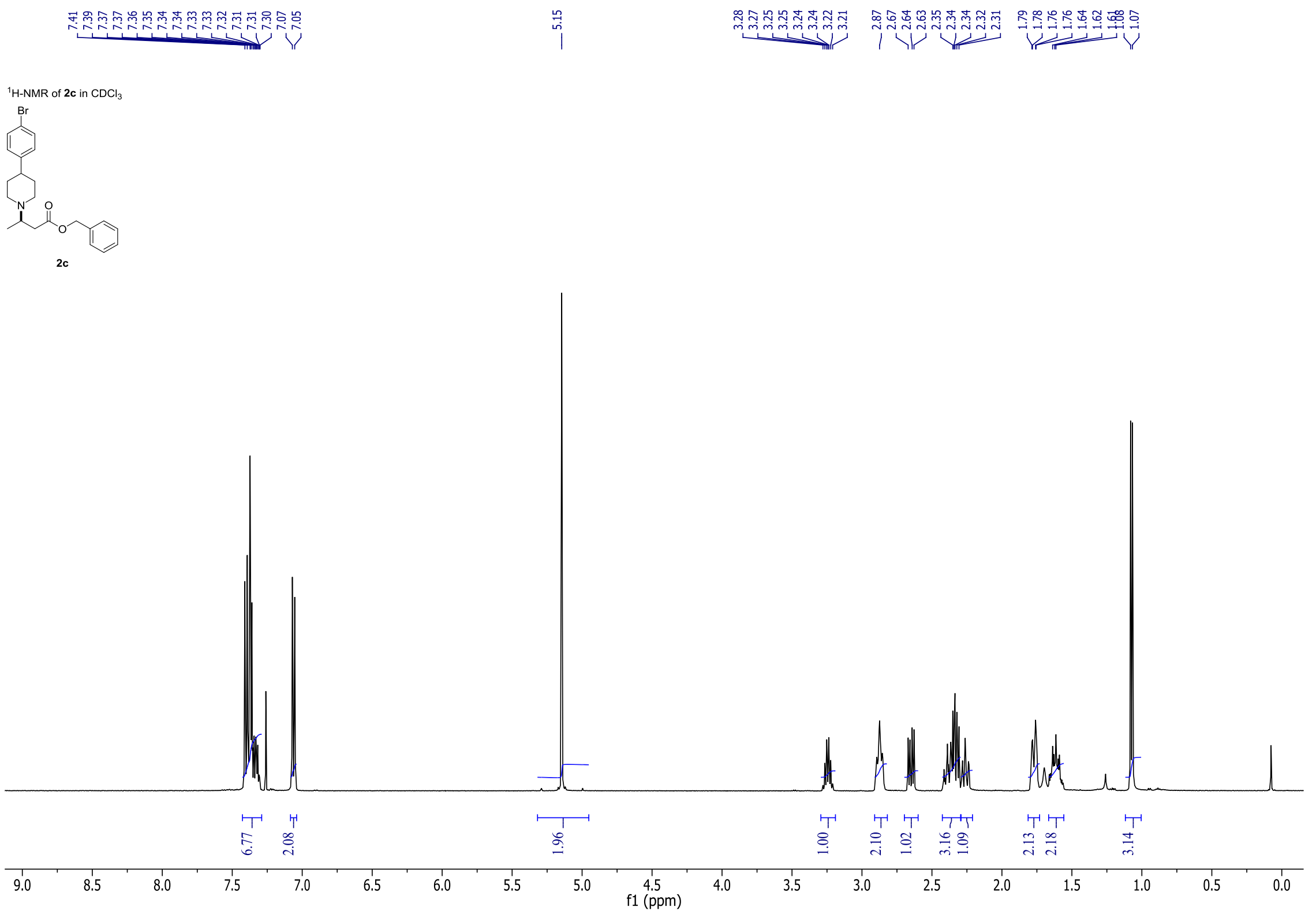




旁
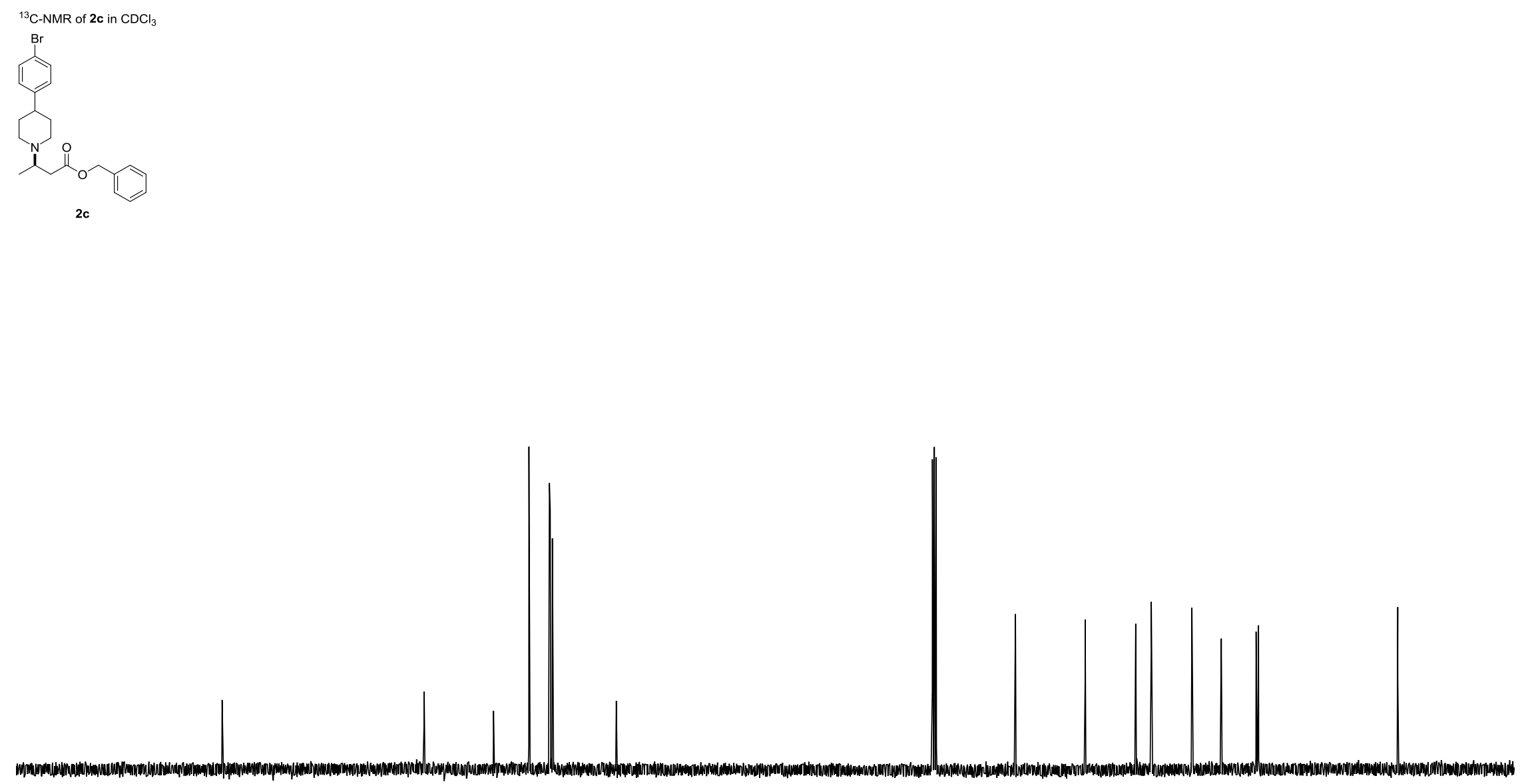
1H-NMR of $\mathbf{2 d}$ in $\mathrm{CDC}$
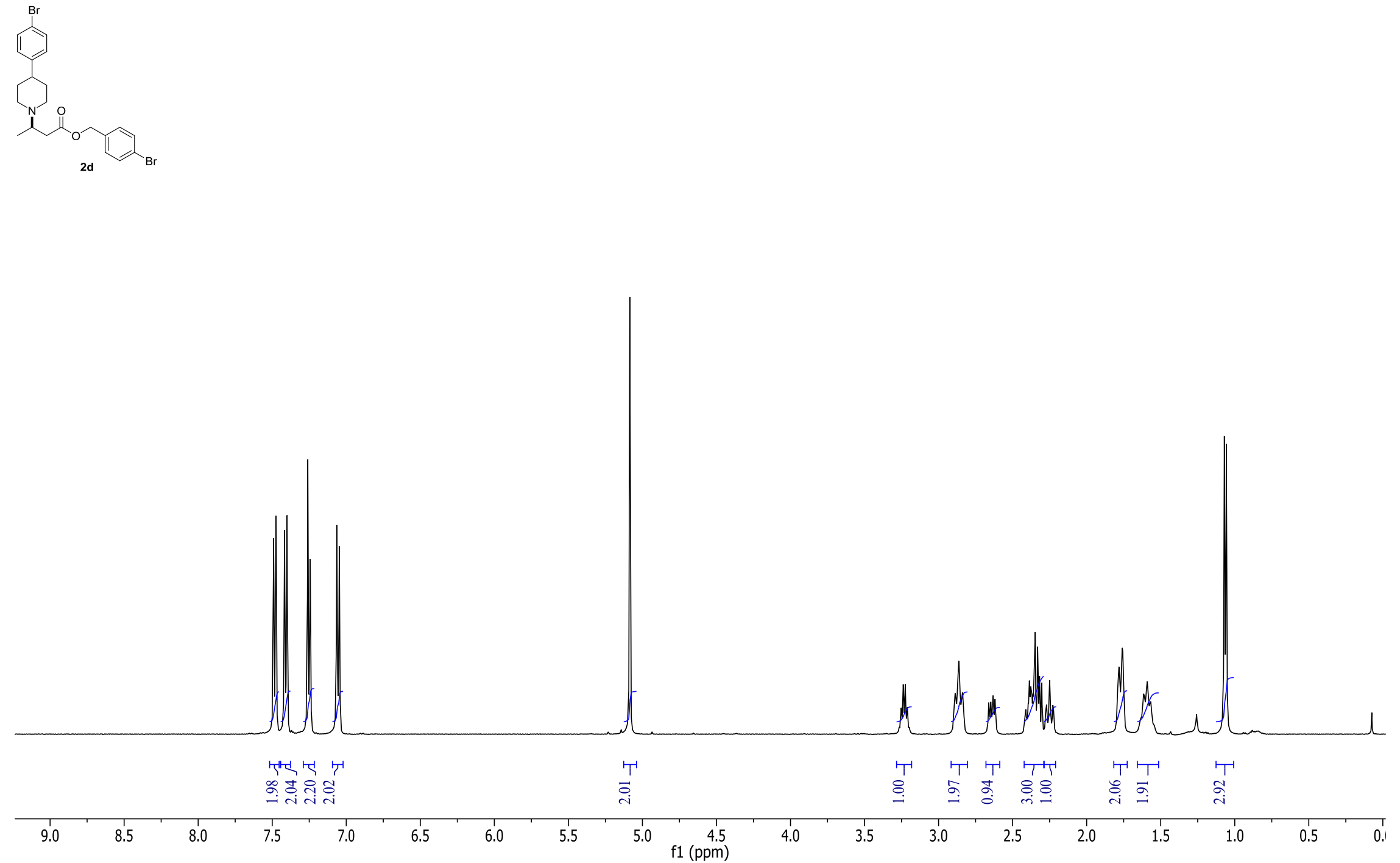


草
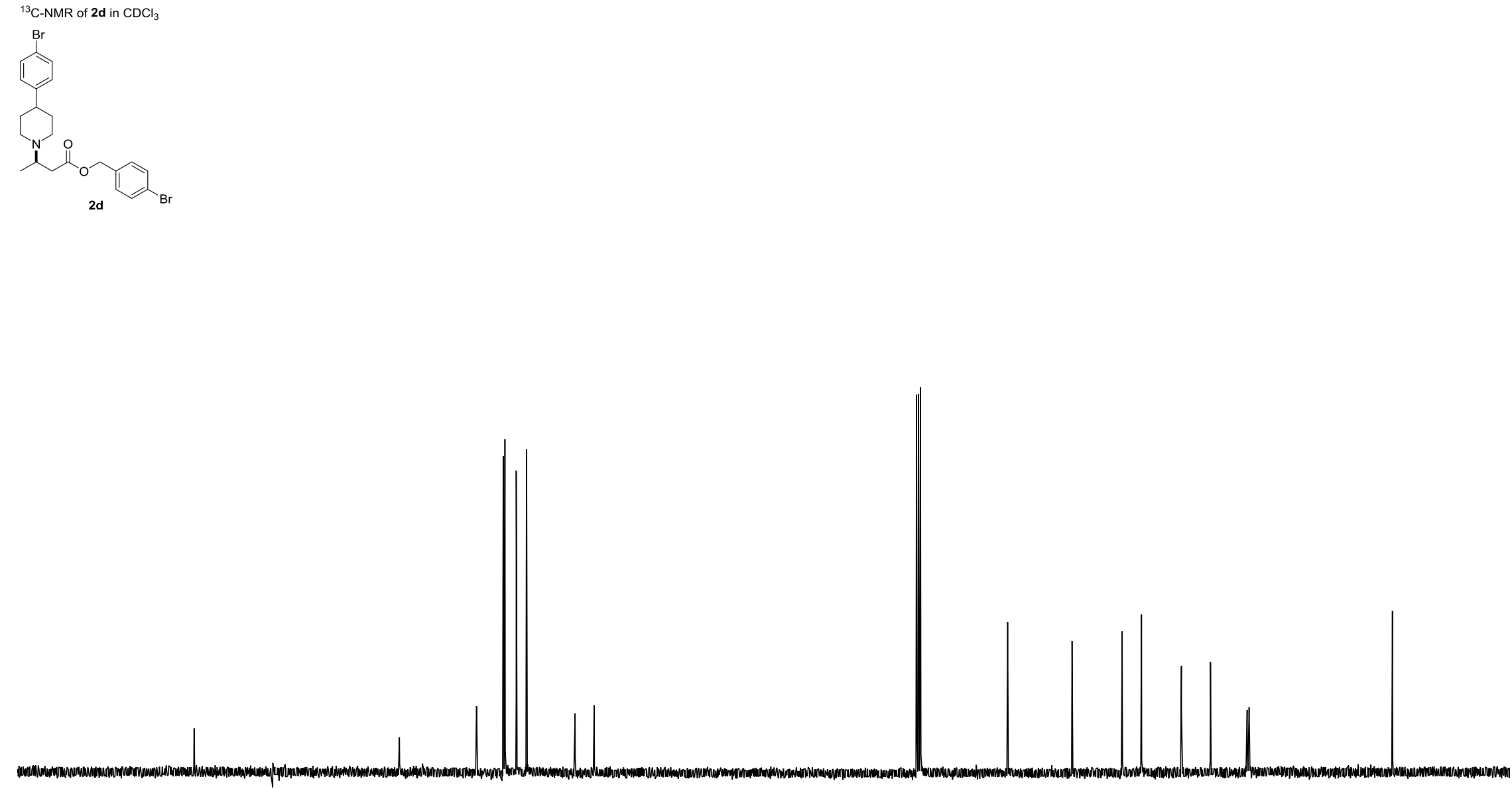
${ }^{1} \mathrm{H}-\mathrm{NMR}$ of $2 \mathrm{e}$ in $\mathrm{CDCl}_{3}$<smiles>CC(CC(=O)OCc1ccccc1)N1CCC(Br)CC1</smiles>

2e

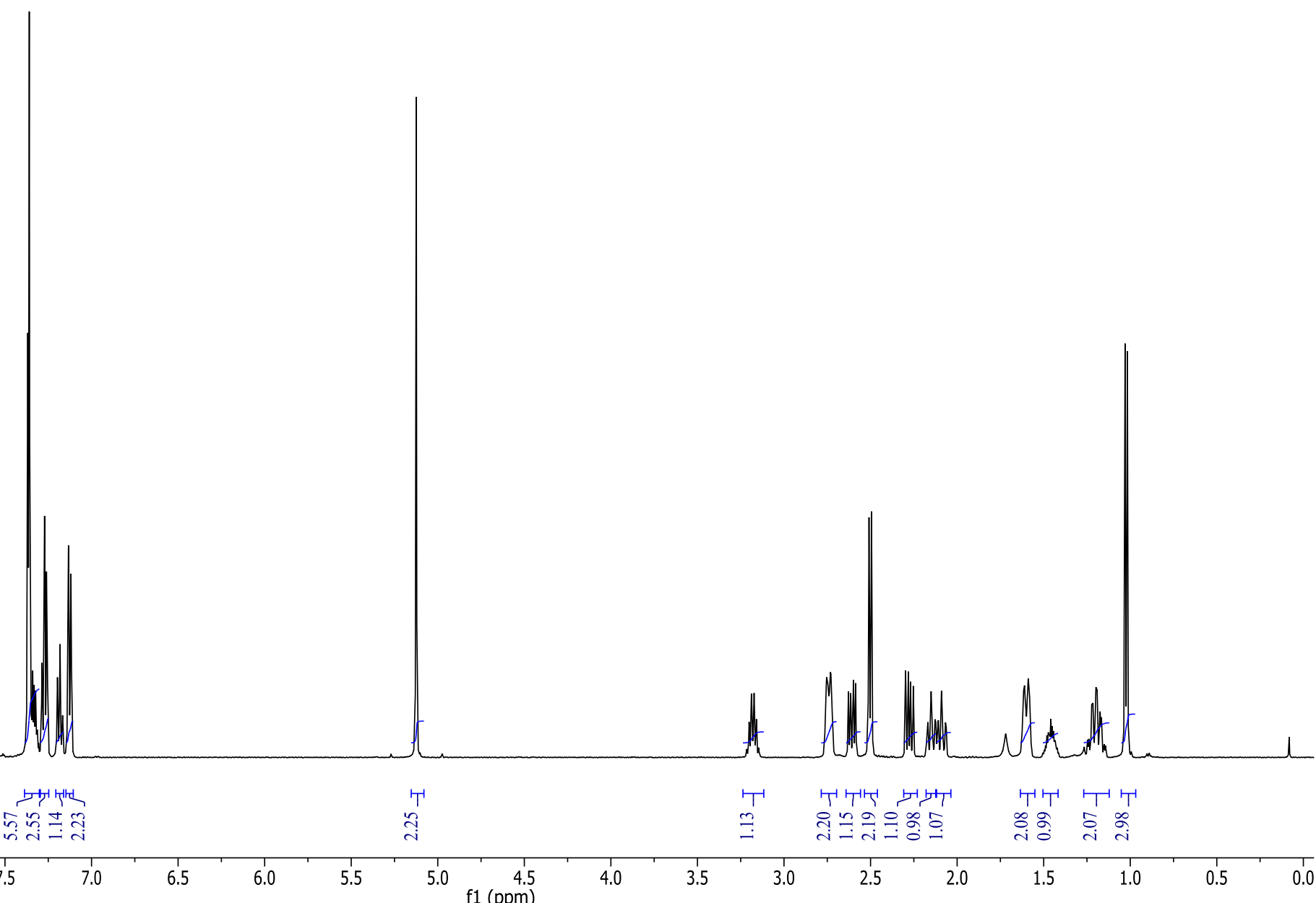




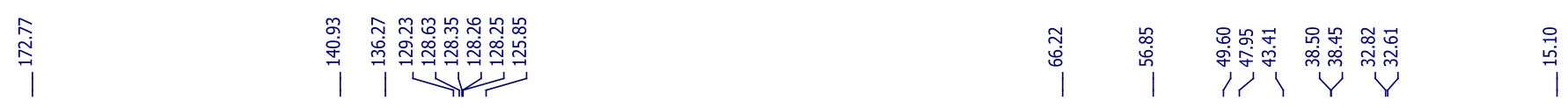

${ }^{13} \mathrm{C}-\mathrm{NMR}$ of $2 \mathrm{e}$ in $\mathrm{CDCl}_{3}$
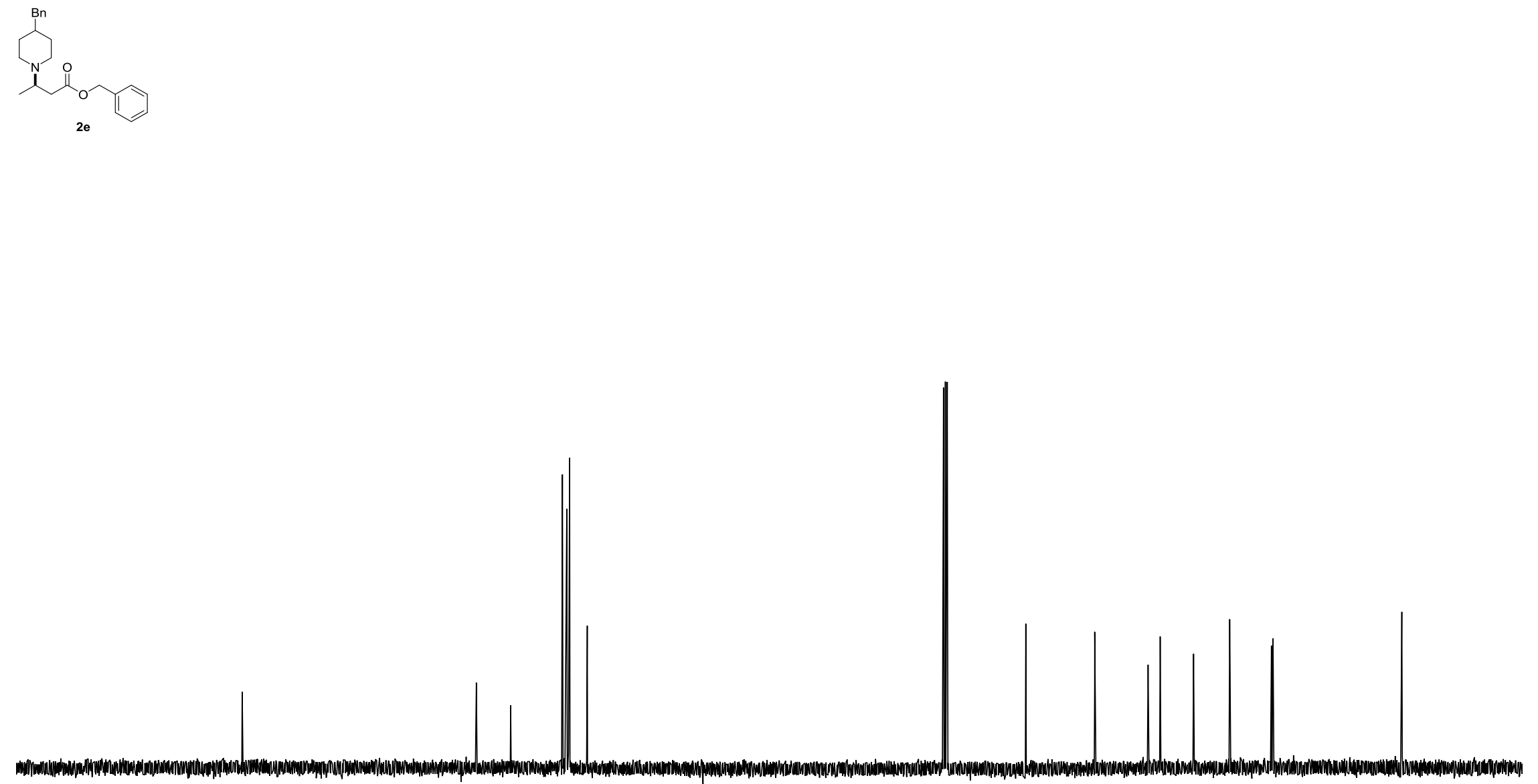

$200+190+$

$180 \quad 170$

160

150

140

130

120

$110 \quad 100$

$90 \quad 80$

70
60

$50 \quad 40$

$30,20,10,10$

S-270 


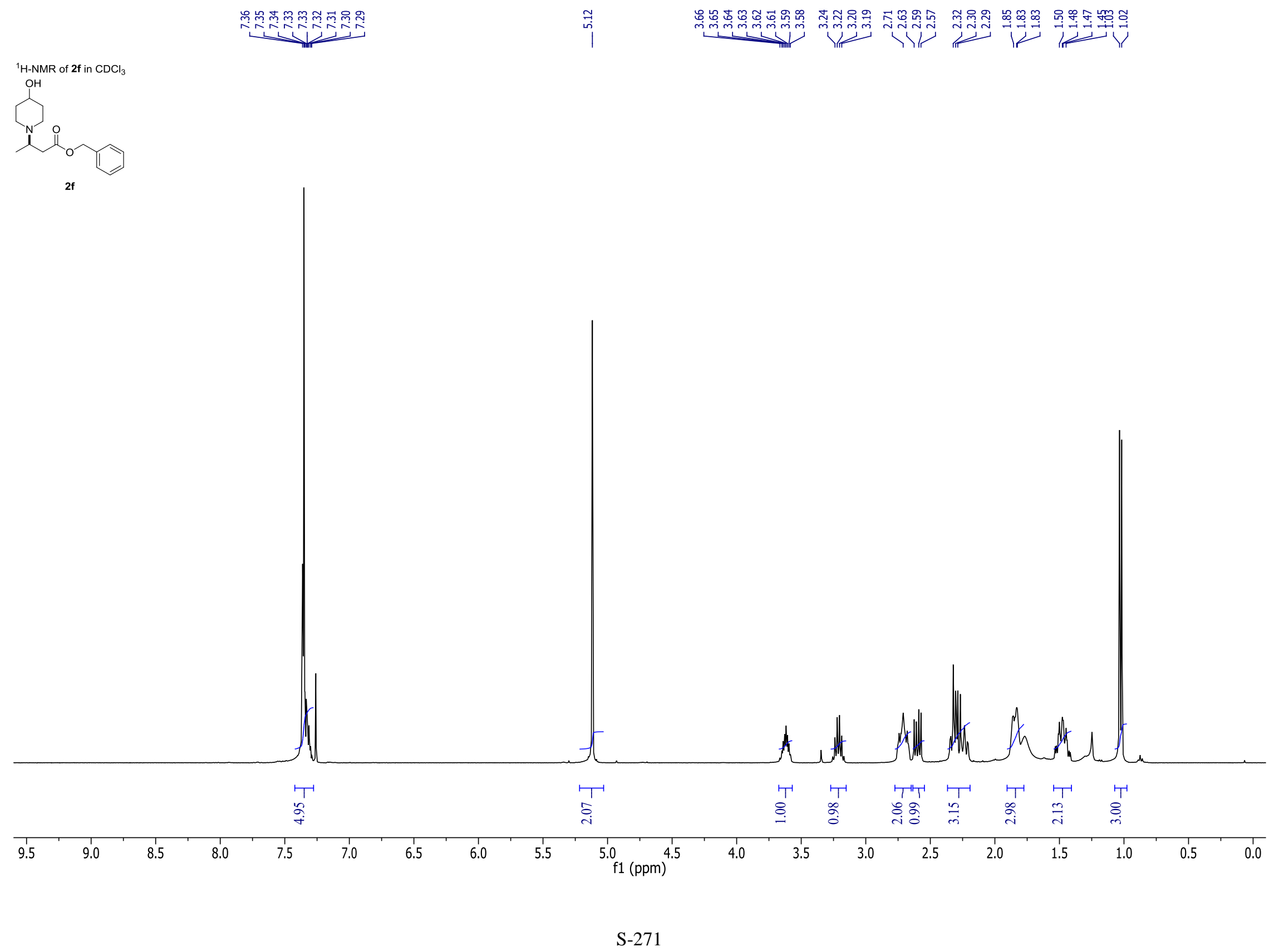




|

${ }^{13} \mathrm{C}-\mathrm{NMR}$ of $2 \mathrm{fin} \mathrm{CDCl}_{3}$
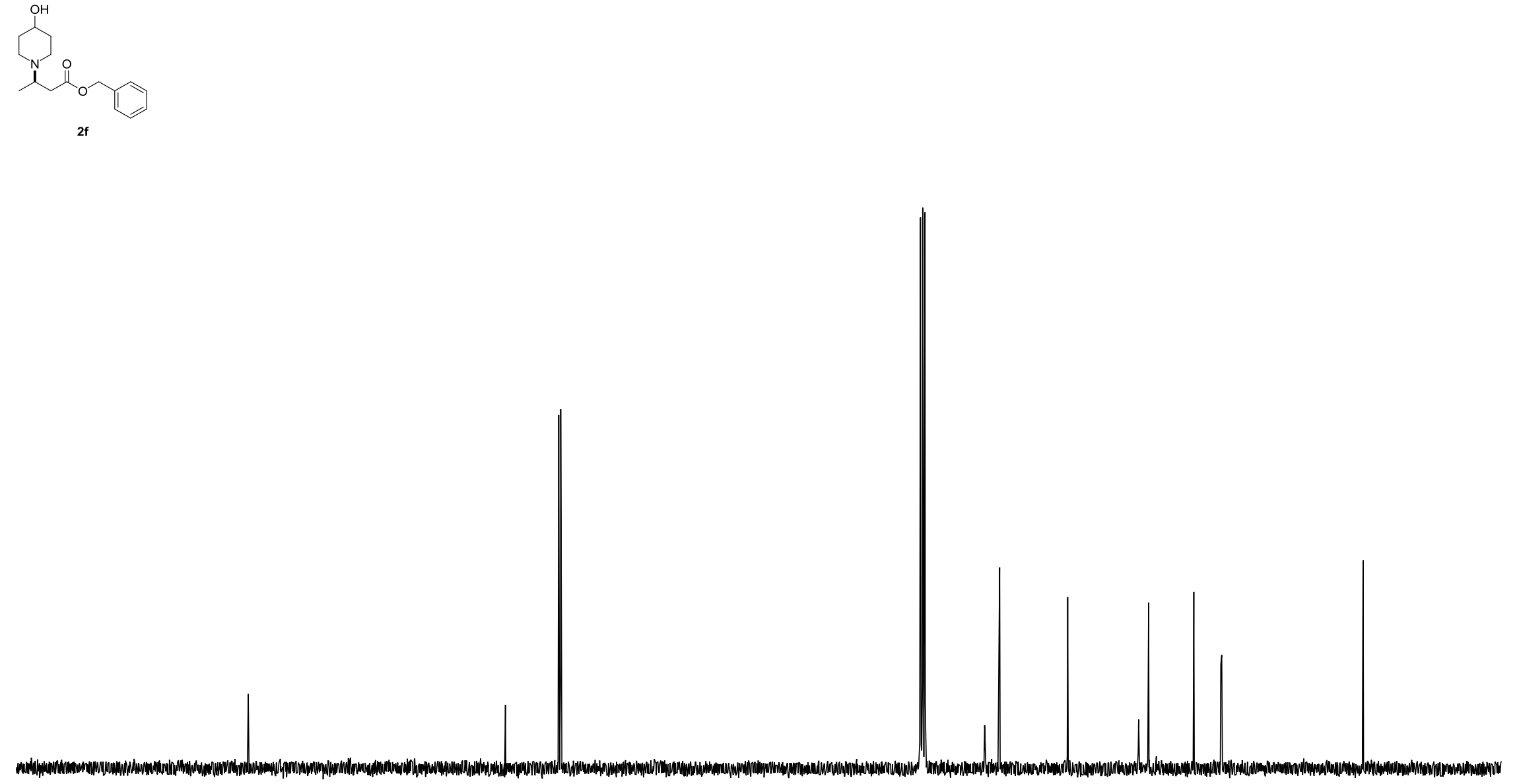

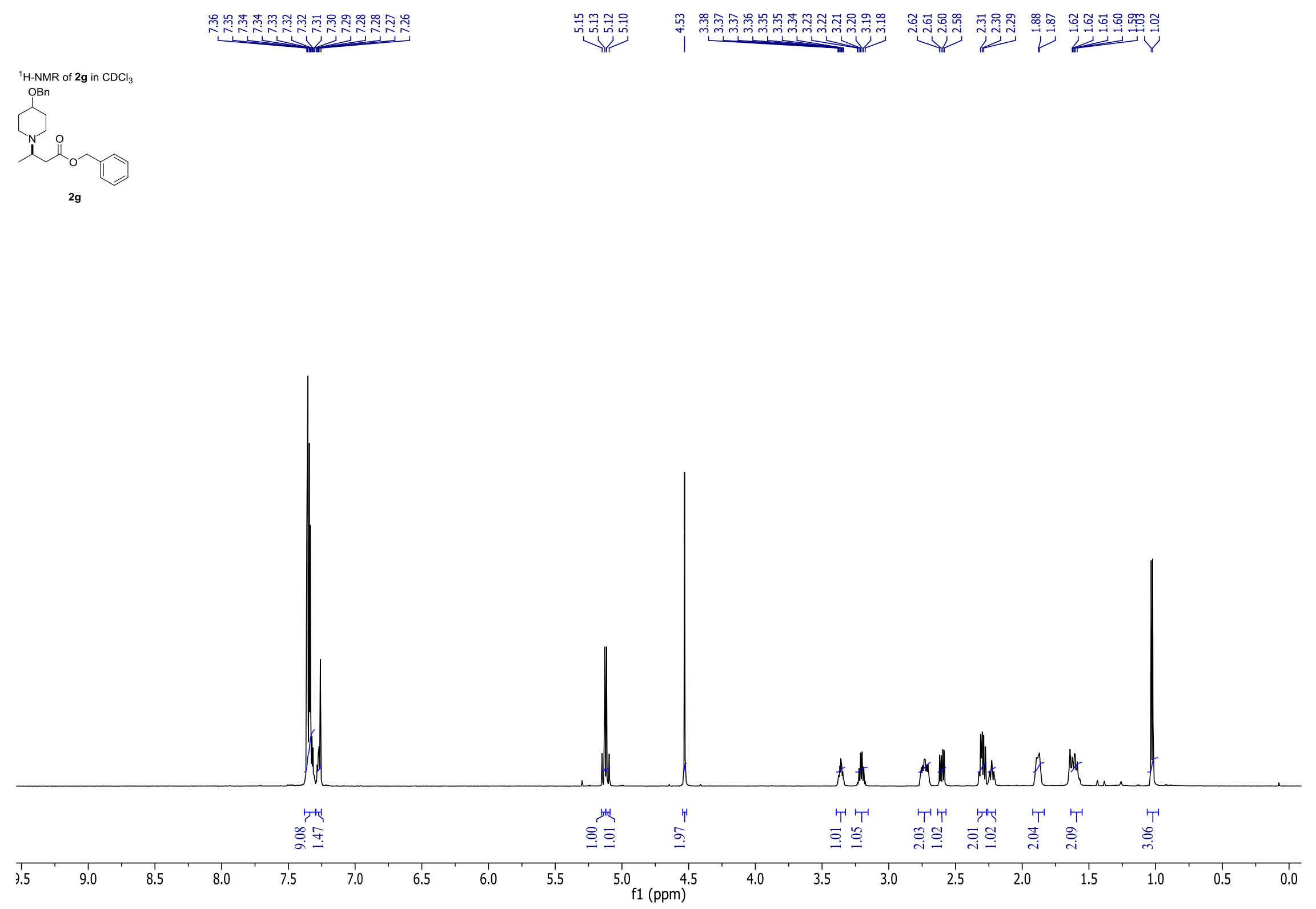


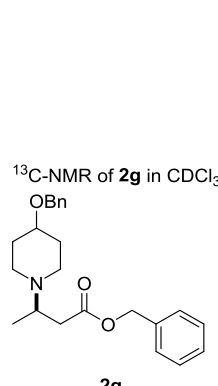

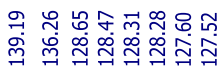

1,1

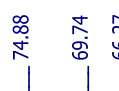

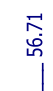

总荨 总

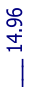

2g

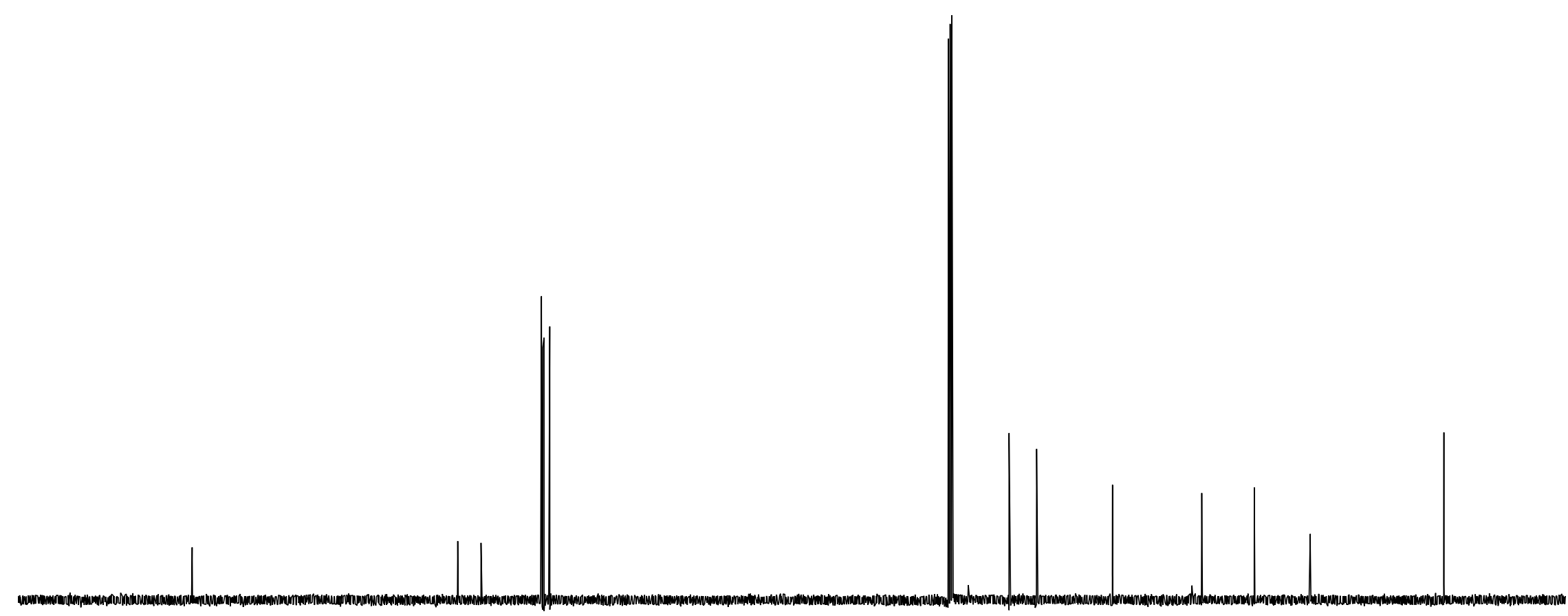




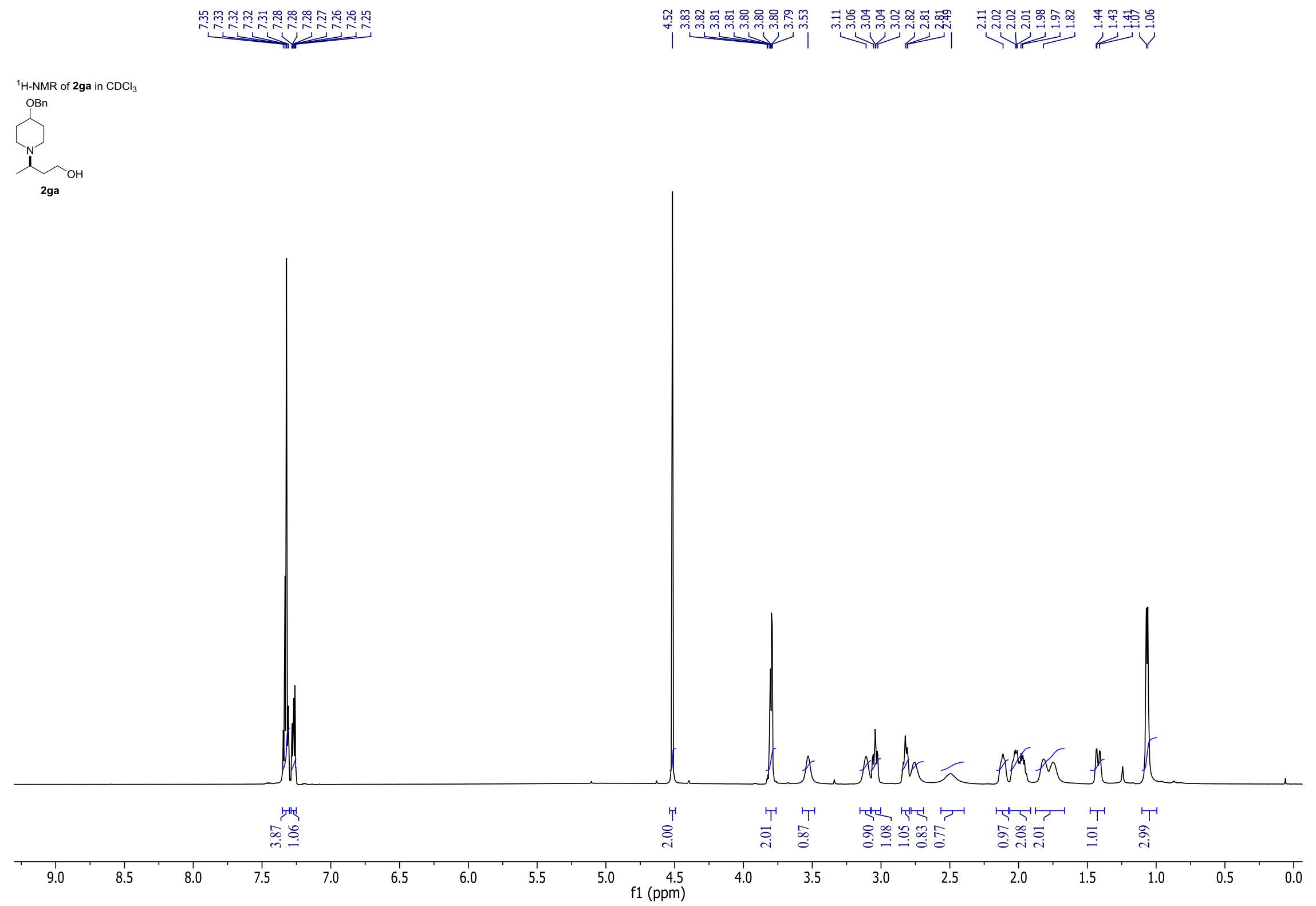



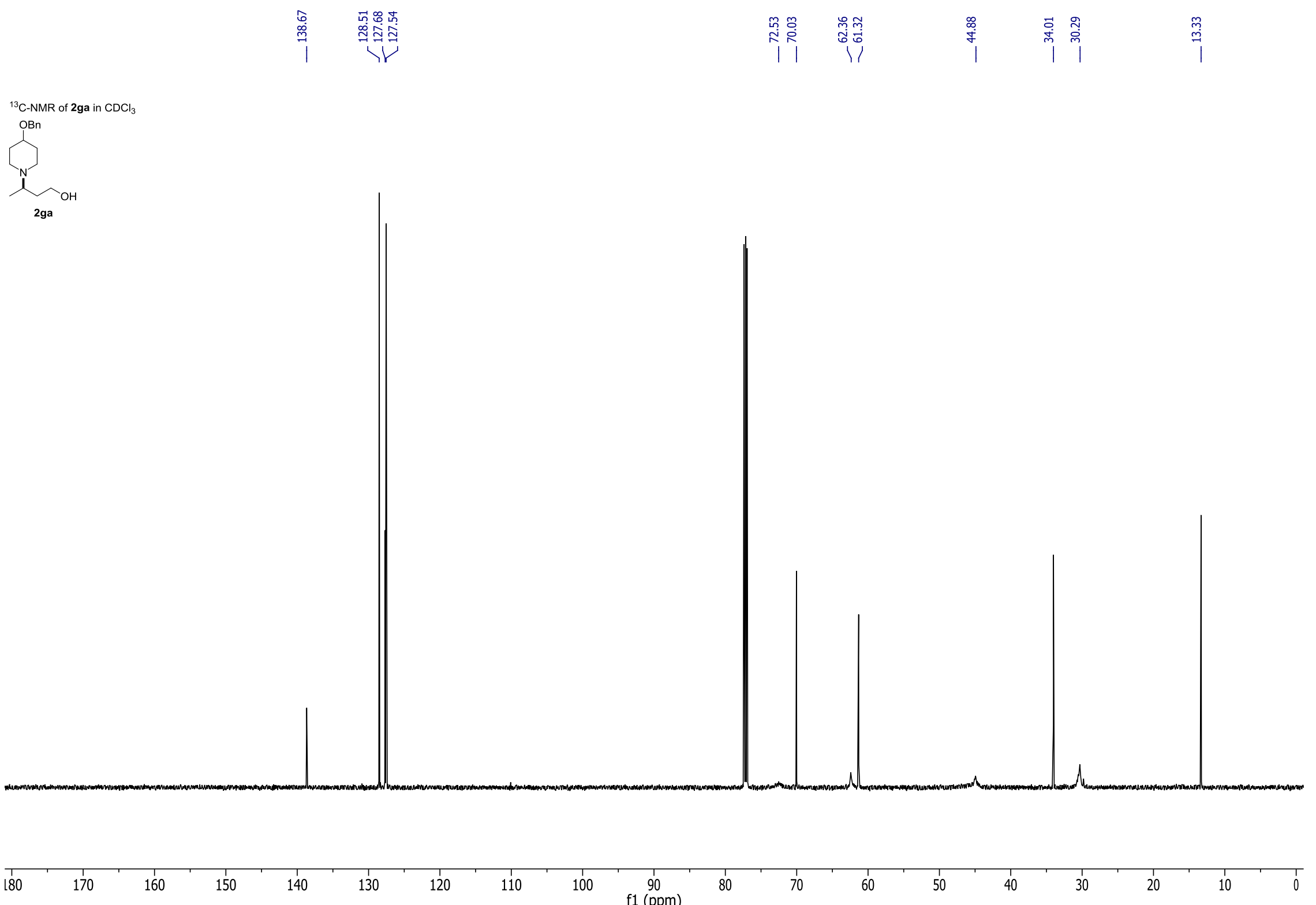
'H-NMR of $2 \mathrm{~h}$ in $\mathrm{CDCl}$

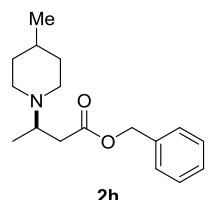

2h

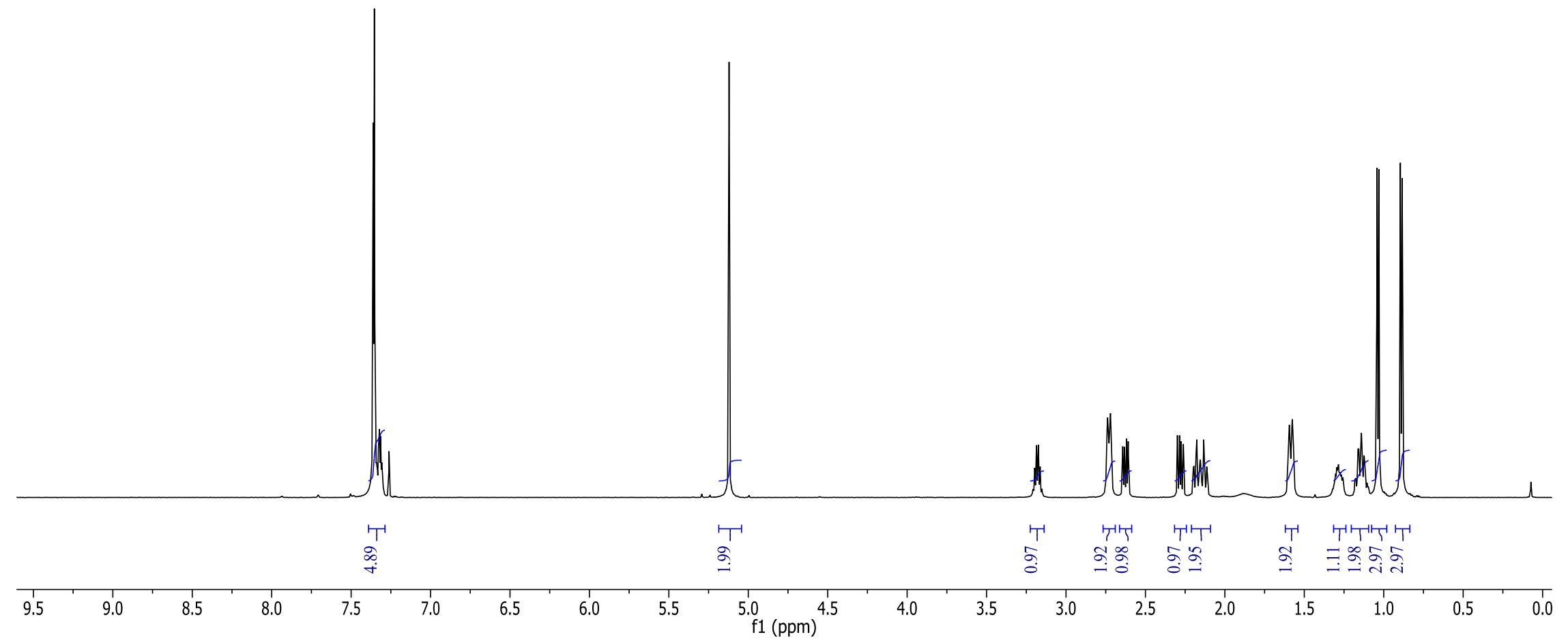


${ }^{13} \mathrm{C}-\mathrm{NMR}$ of $\mathbf{2 h}$ in $\mathrm{CDCl}_{3}$

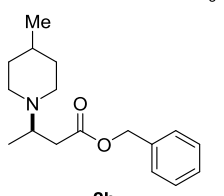

2h

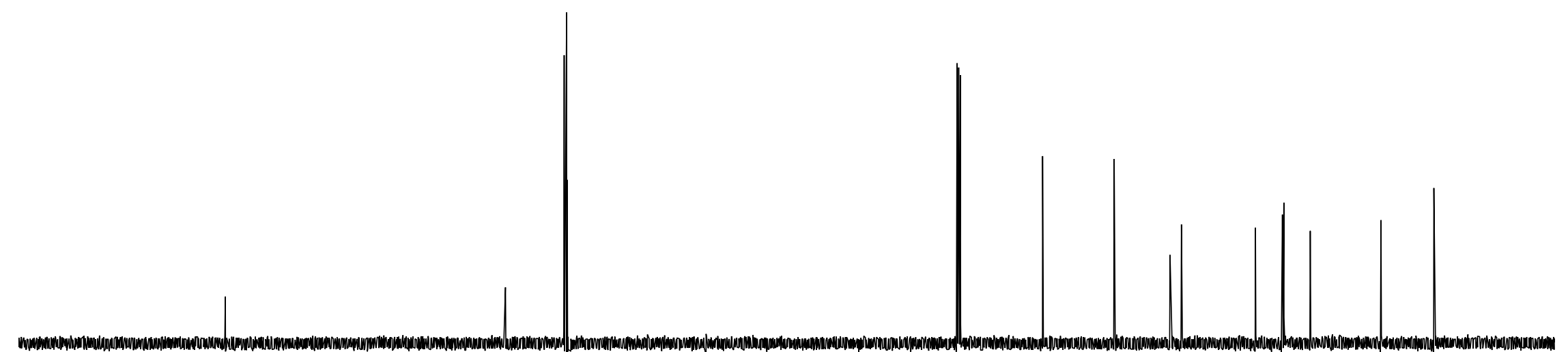




\section{罳翼}

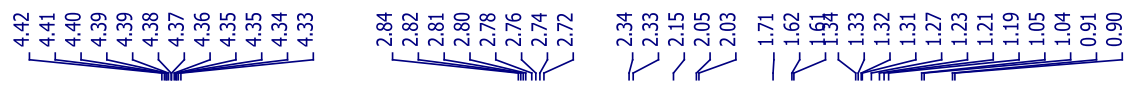

${ }^{1} \mathrm{H}-\mathrm{NMR}$ of 2 ha in $\mathrm{CDCl}_{3}$
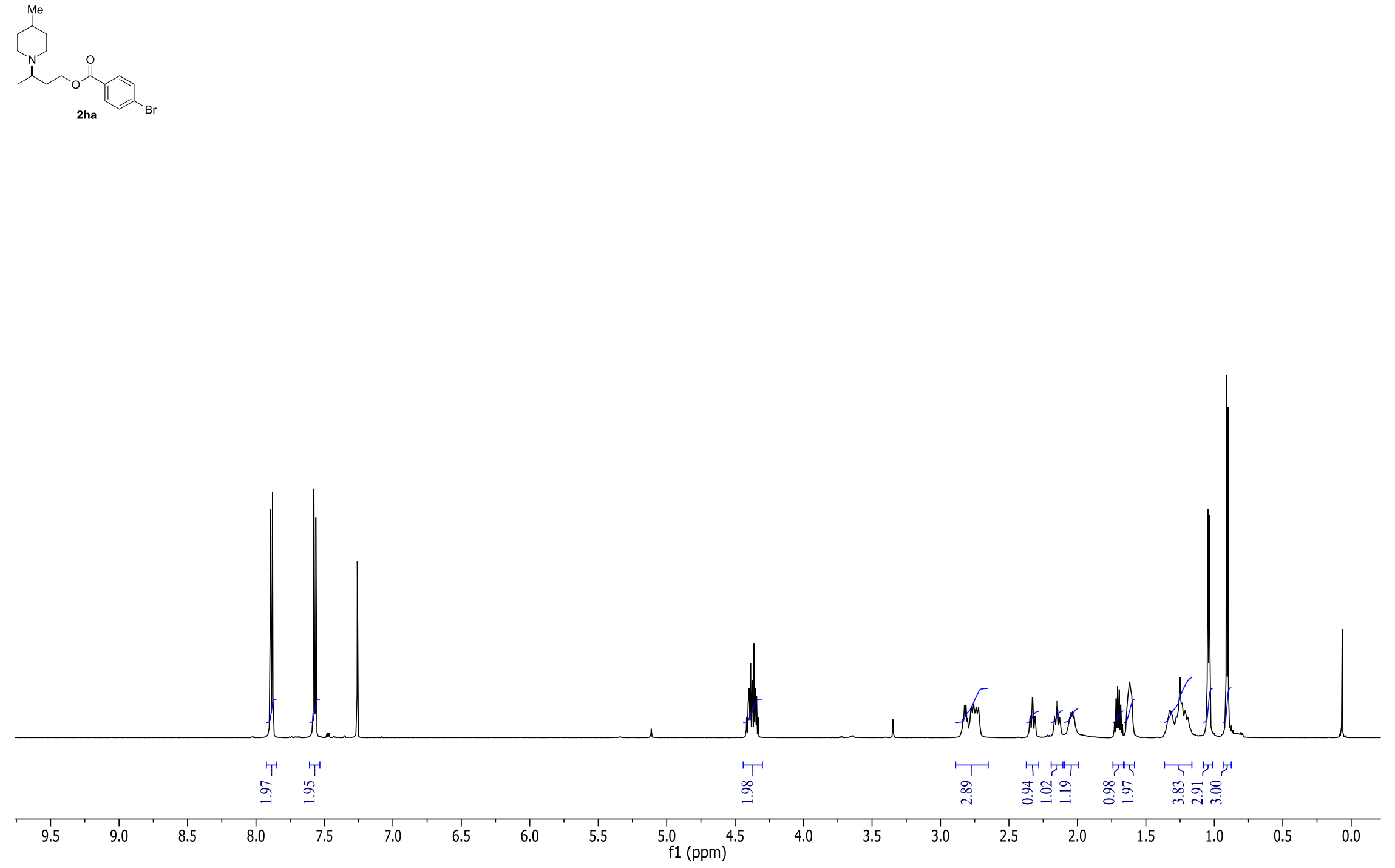
${ }^{13} \mathrm{C}-\mathrm{NMR}$ of $2 \mathrm{ha}$ in $\mathrm{CDCl}_{3}$

$\mathrm{Me}$

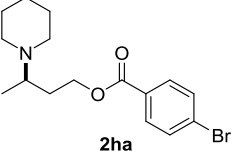

2ha

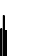
I

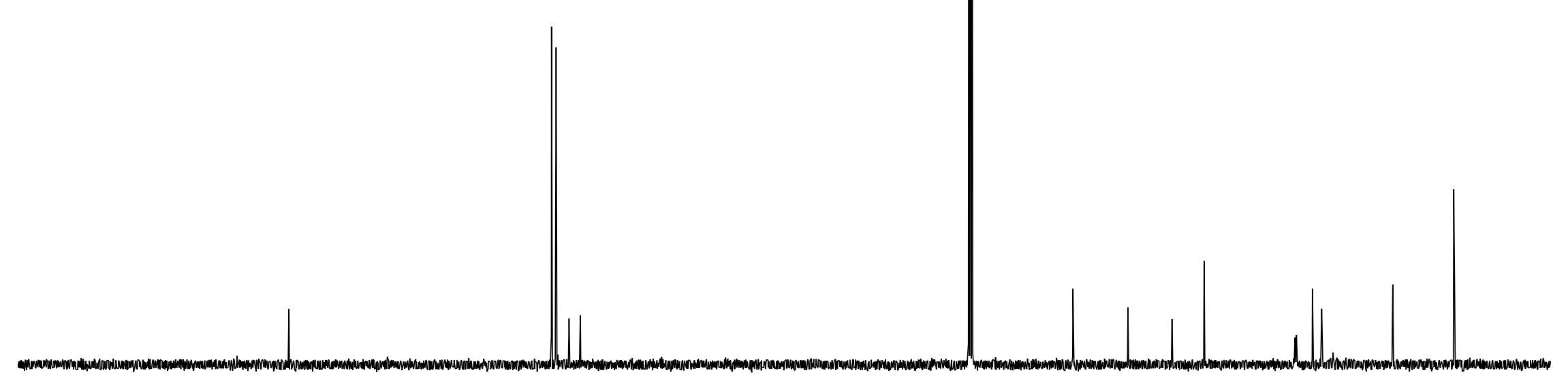


'H-NMR of $2 \mathrm{i}$ in $\mathrm{CDCl}_{3}$

$\mathrm{Me}_{-{ }^{-}-\mathrm{Ph}}$

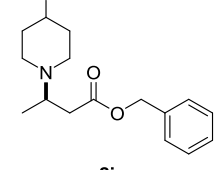

2i

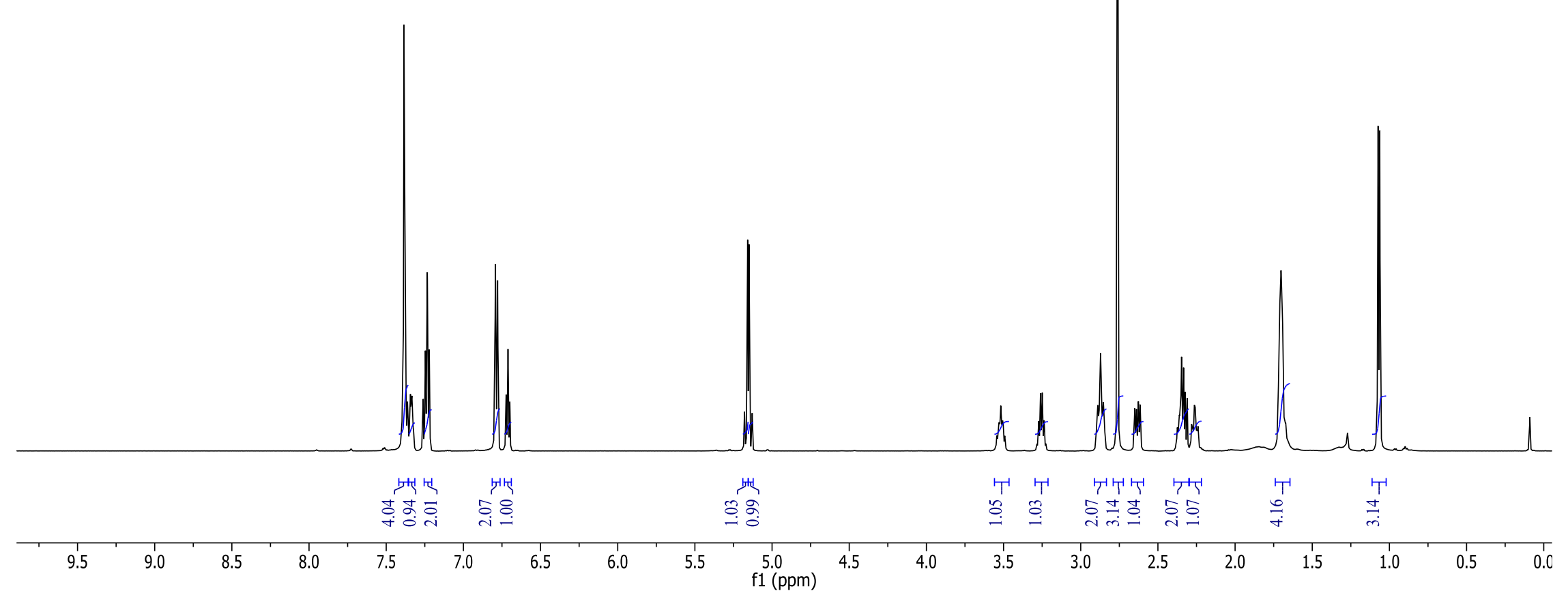




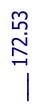

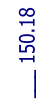

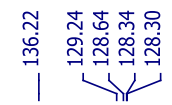

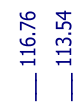

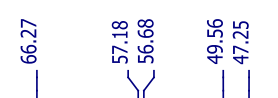

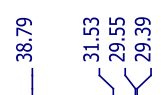
$\underset{\substack{8 \\ \hdashline}}{8}$

${ }^{13} \mathrm{C}-\mathrm{NMR}$ of $2 \mathrm{i}$ in $\mathrm{CDCl}$

$\mathrm{Me}{ }^{-}{ }^{-P h}$
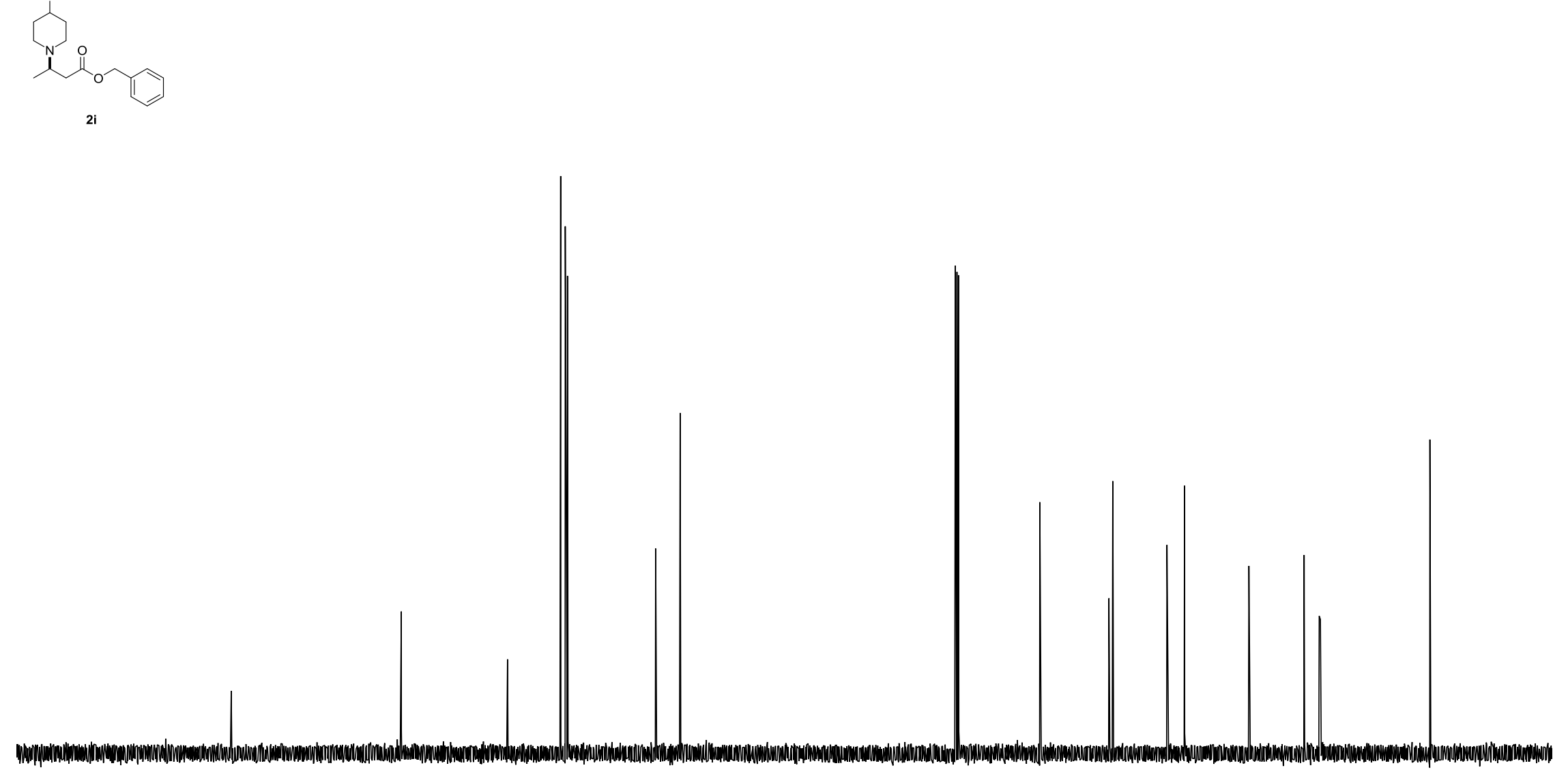

.00

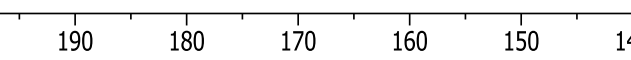

120

100

90

80

70

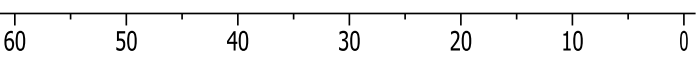

S-282 


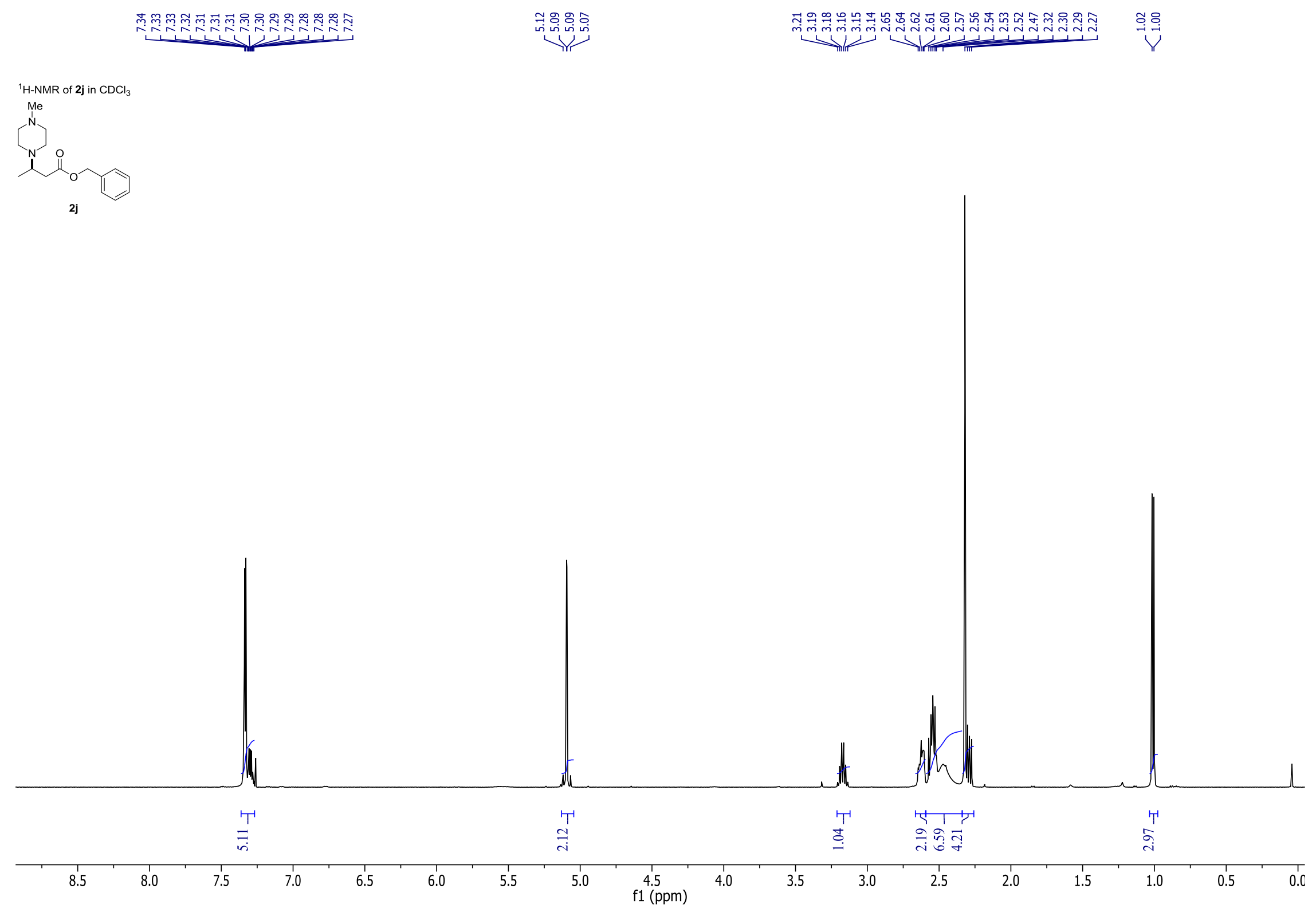

S-283 

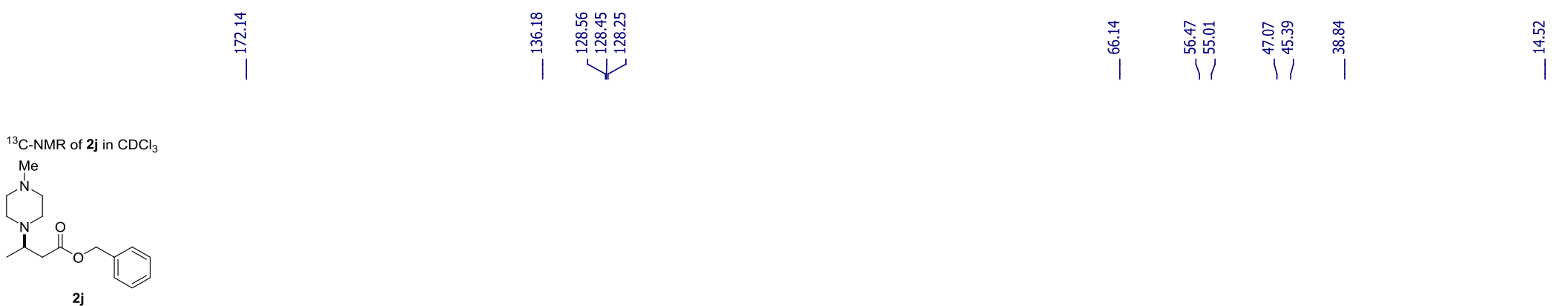

2j

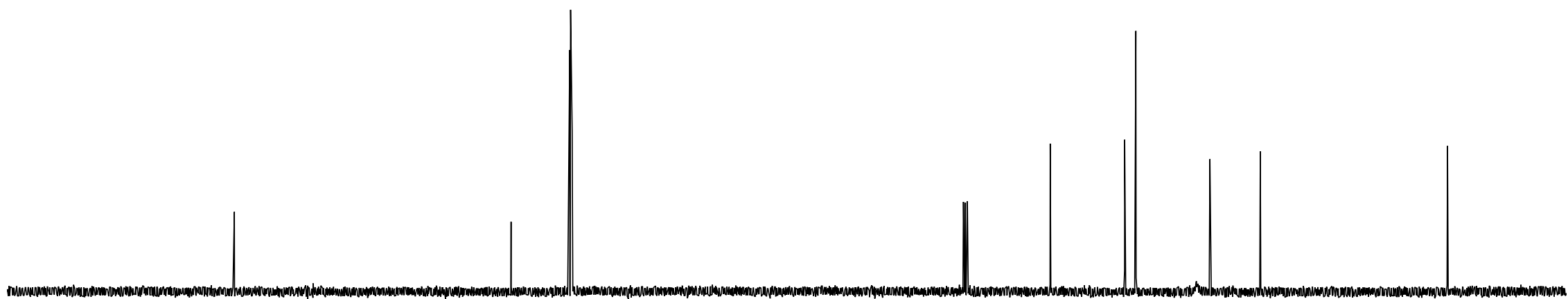



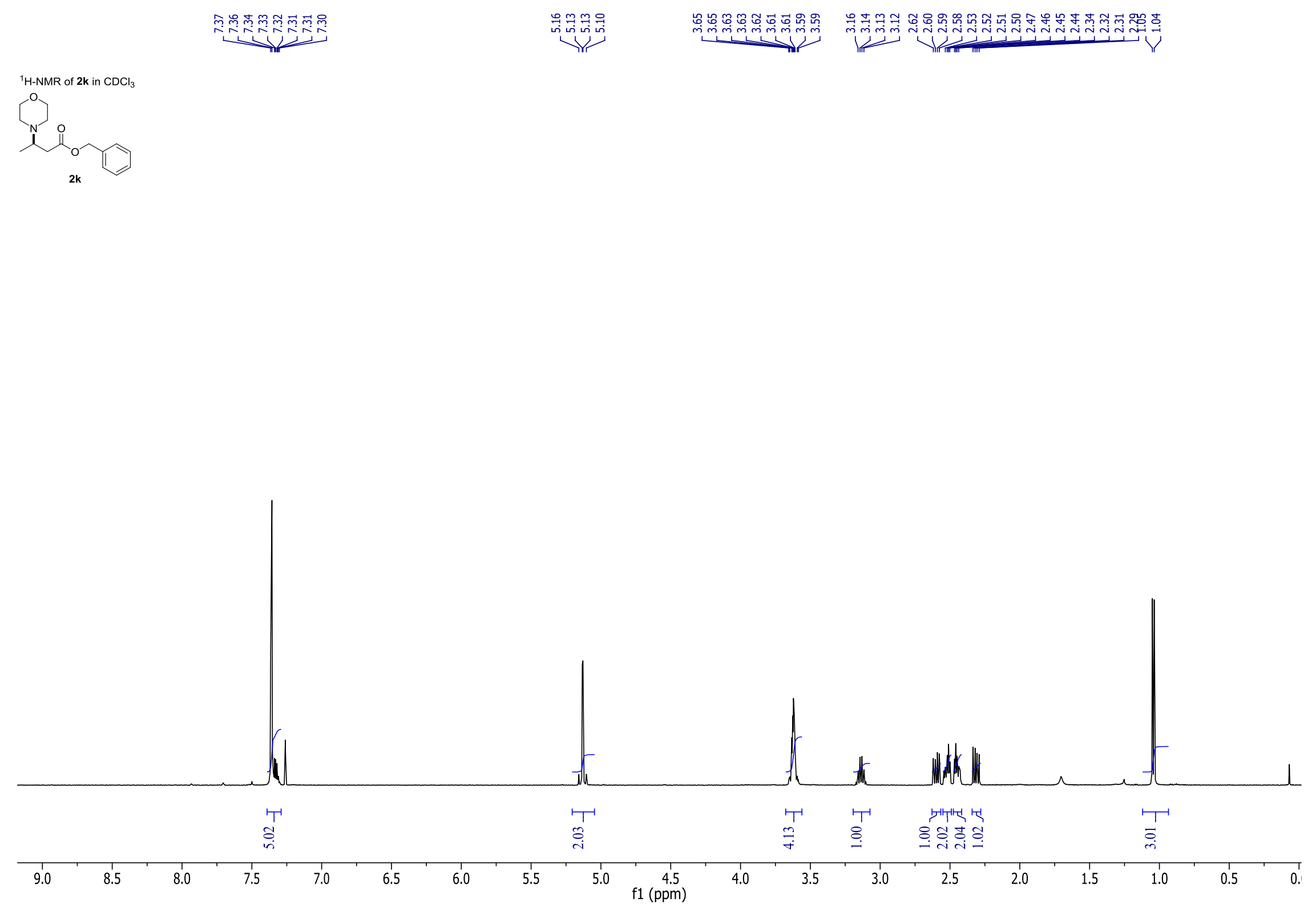
${ }^{13} \mathrm{C}-\mathrm{NMR}$ of $\mathbf{2 k}$ in $\mathrm{CDCl}_{3}$

$\overbrace{2 k}^{O}$

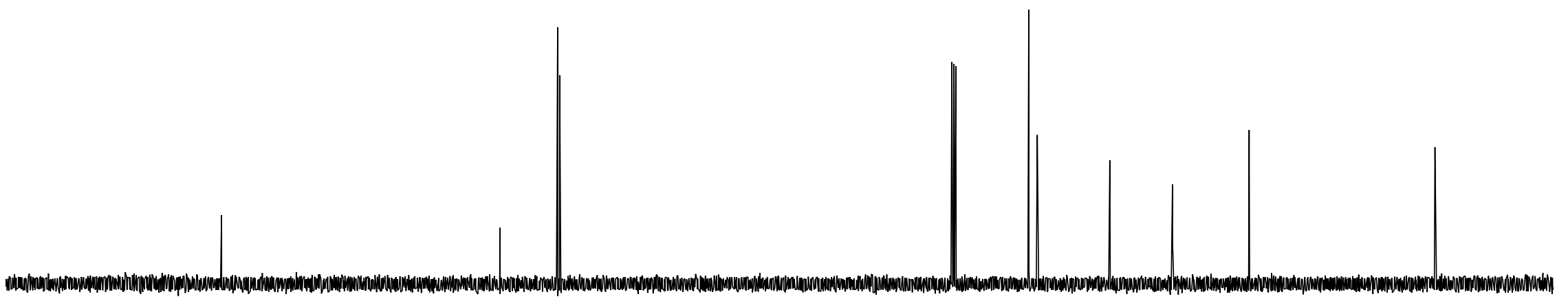

00

190

180

170

$50 \quad 140$

130

120

110

100

100 


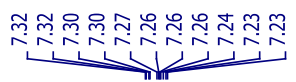

บำ

岤|

$\stackrel{y}{y}$
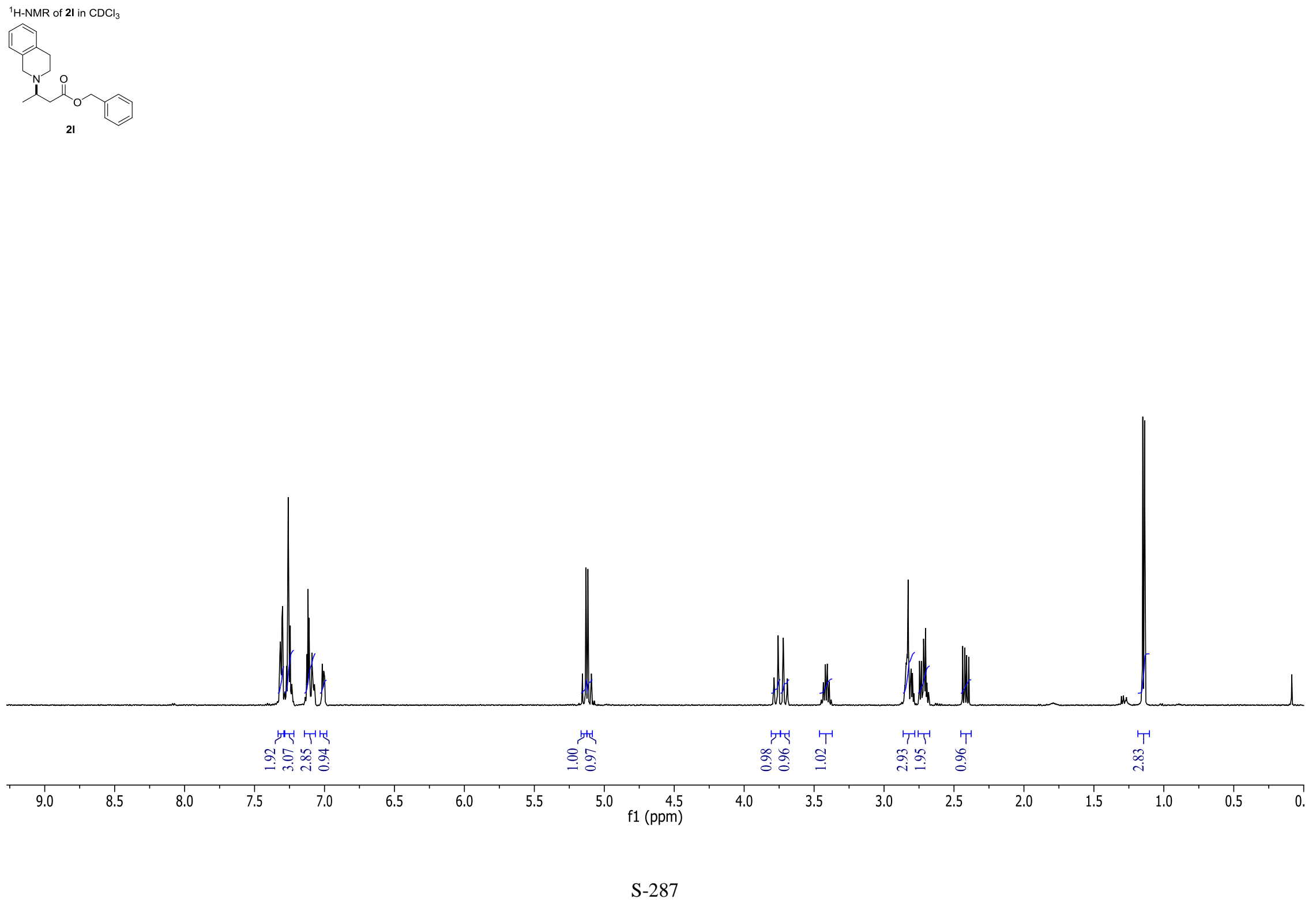
${ }^{13} \mathrm{C}-\mathrm{NMR}$ of $2 \mathrm{lin} \mathrm{CDCl}_{3}$
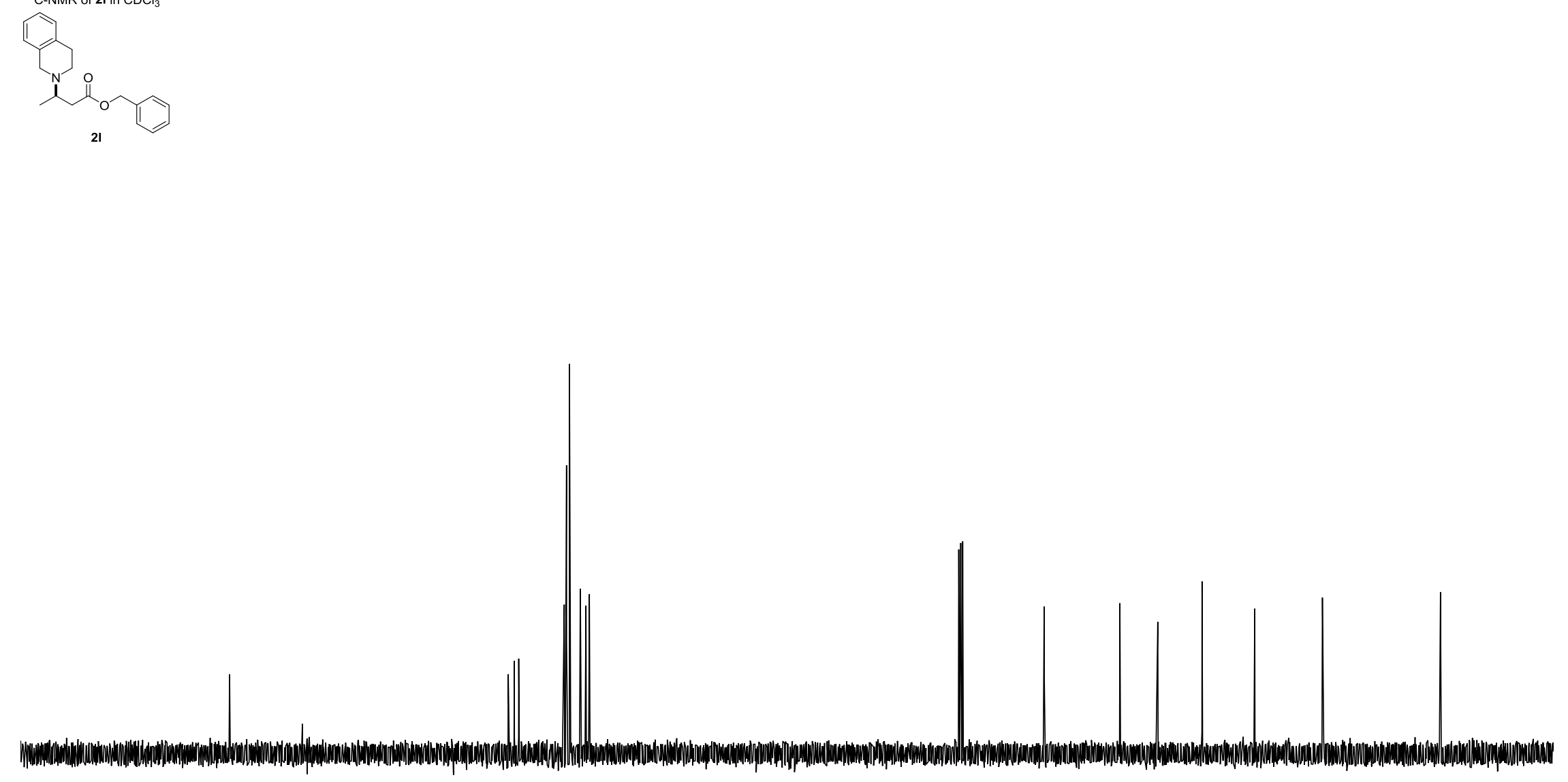

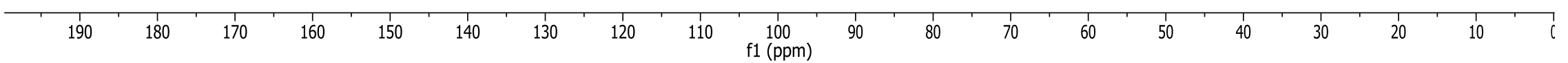




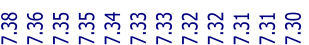

(1)

${ }^{1} \mathrm{H}-\mathrm{NMR}$ of $2 \mathrm{~m}$ in $\mathrm{CDCl}_{3}$

$\overbrace{2 m}$

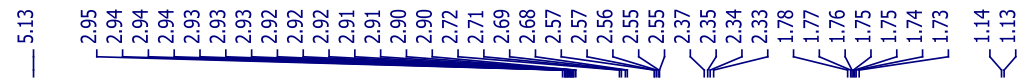

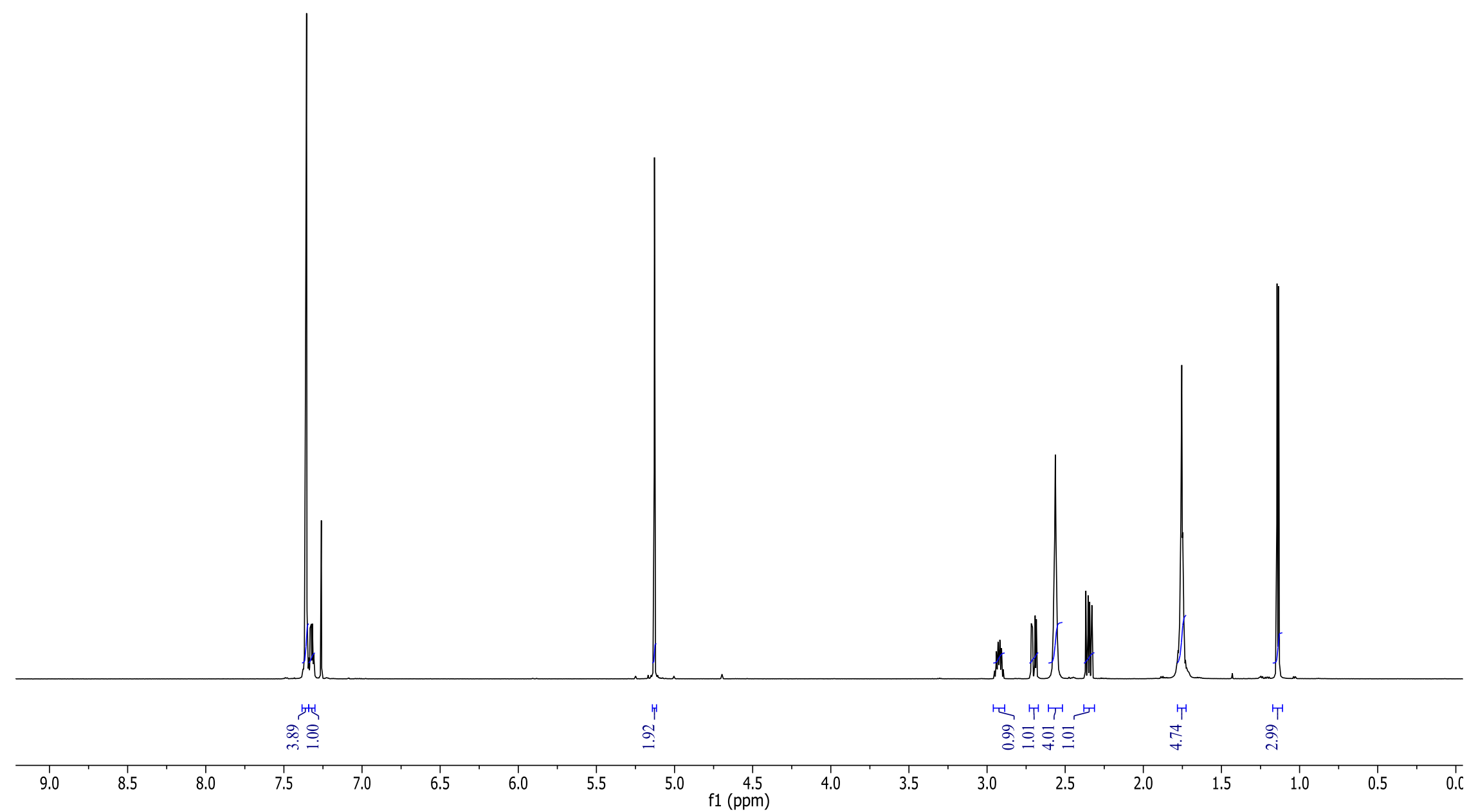


${ }^{13} \mathrm{C}-\mathrm{NMR}$ of $2 \mathrm{~m}$ in $\mathrm{CDCl}_{3}$

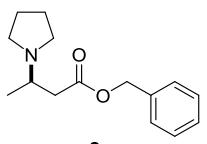

$2 \mathrm{~m}$

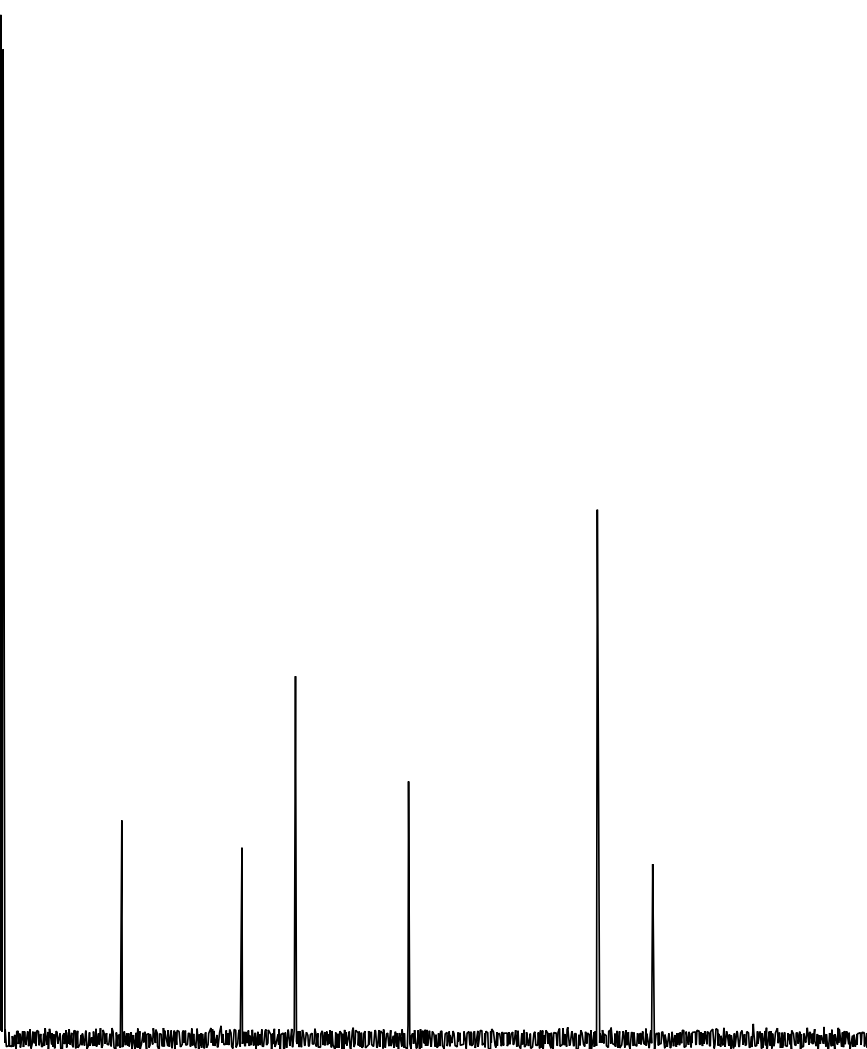



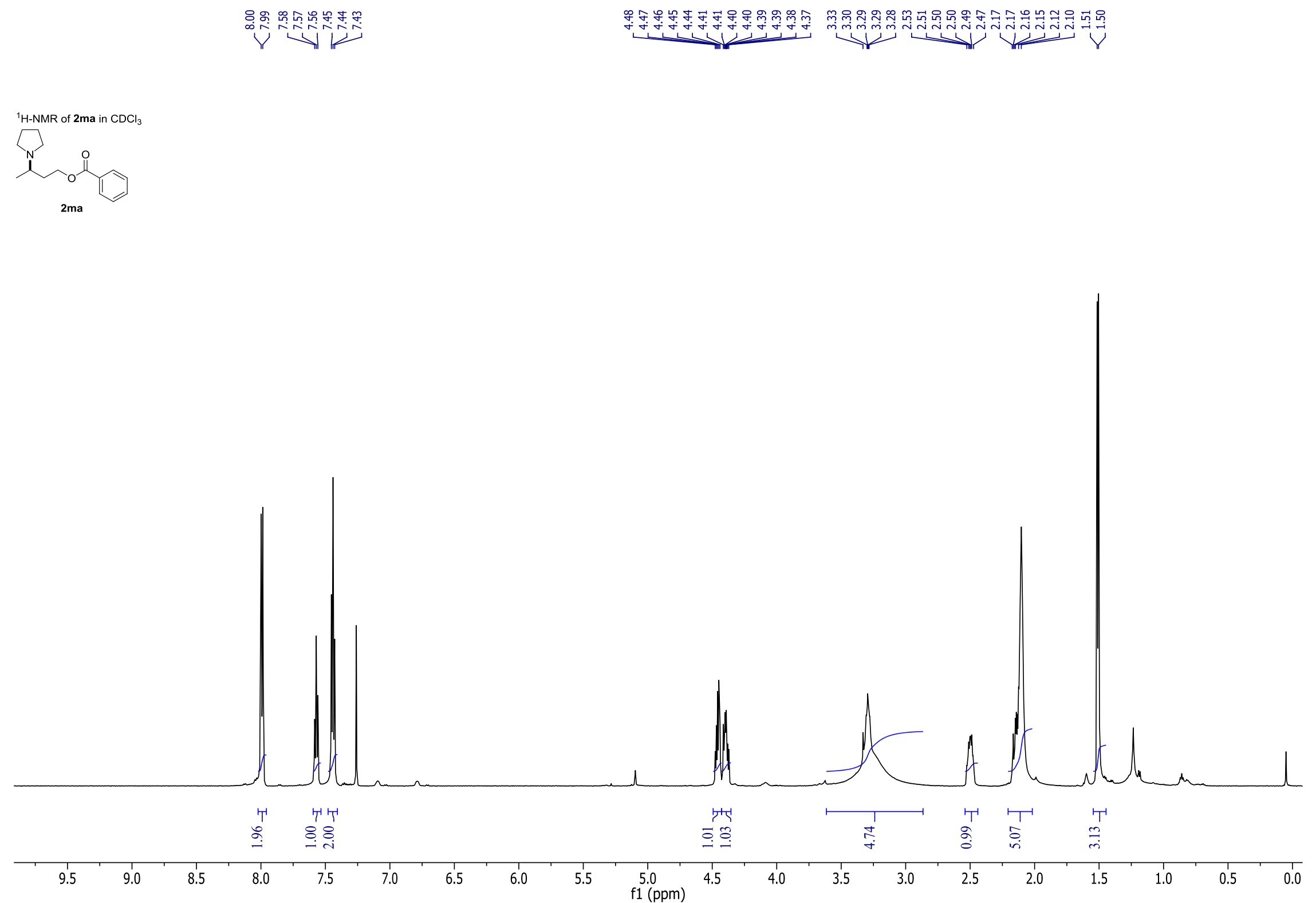
${ }^{13} \mathrm{C}-\mathrm{NMR}$ of $2 \mathrm{ma}$ in $\mathrm{CDCl}_{3}$
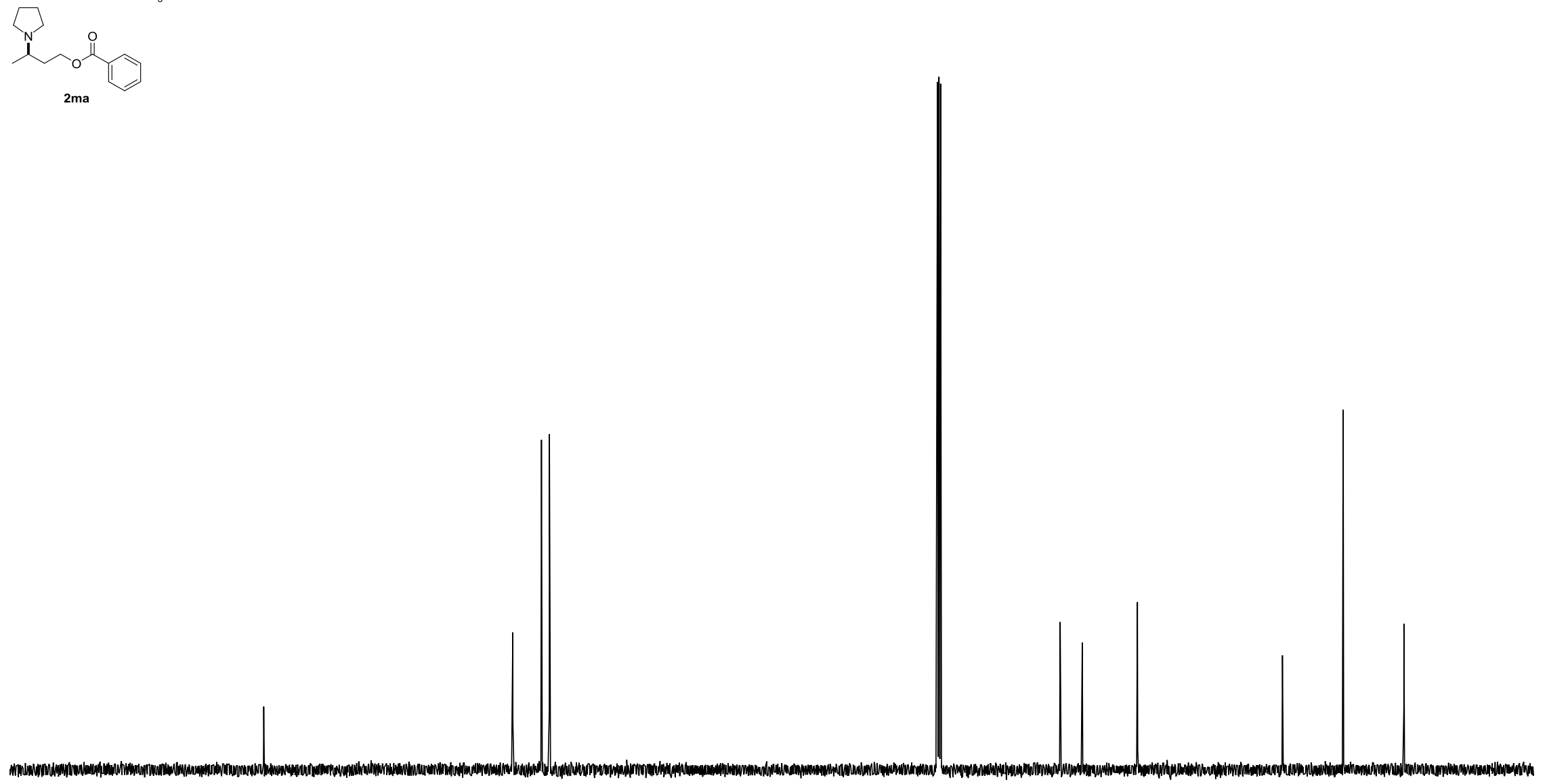
${ }^{1} \mathrm{H}-\mathrm{NMR}$ of $\mathbf{2 n}$ in $\mathrm{CDCl}_{3}$<smiles>CC(CC(=O)OCc1ccccc1)N1CCC2CCCC2C1</smiles>

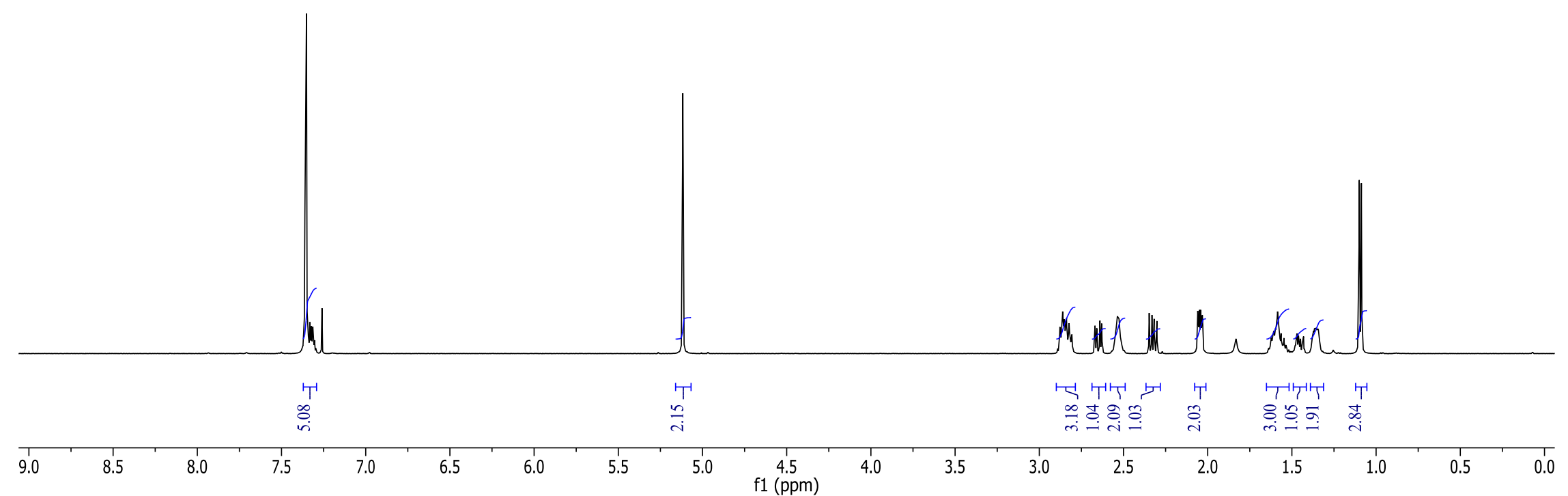


${ }^{13} \mathrm{C}-\mathrm{NMR}$ of $\mathbf{2 n}$ in $\mathrm{CDCl}_{3}$

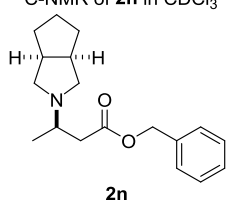

2n

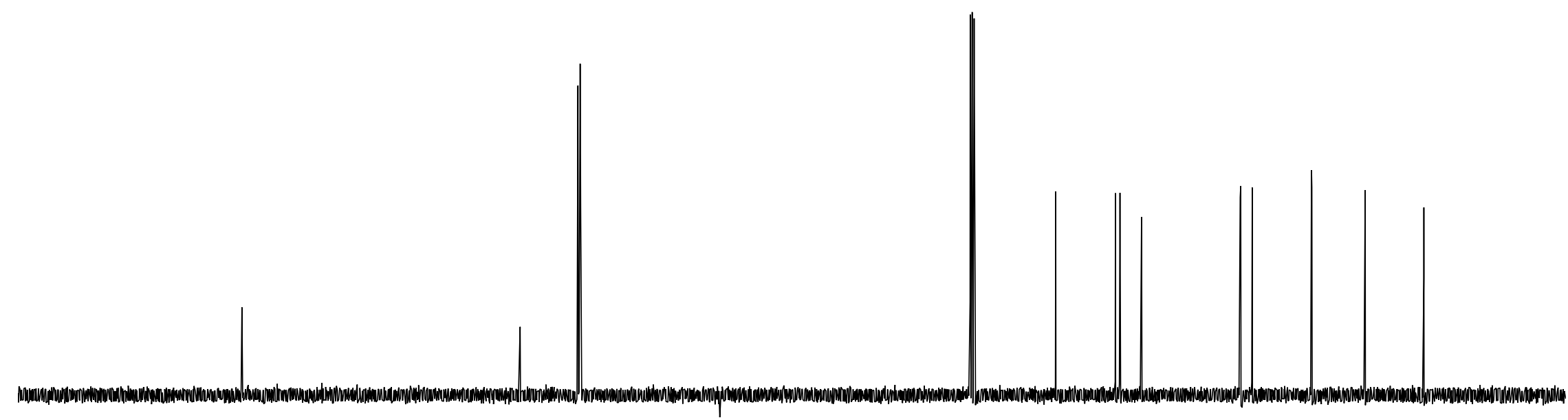



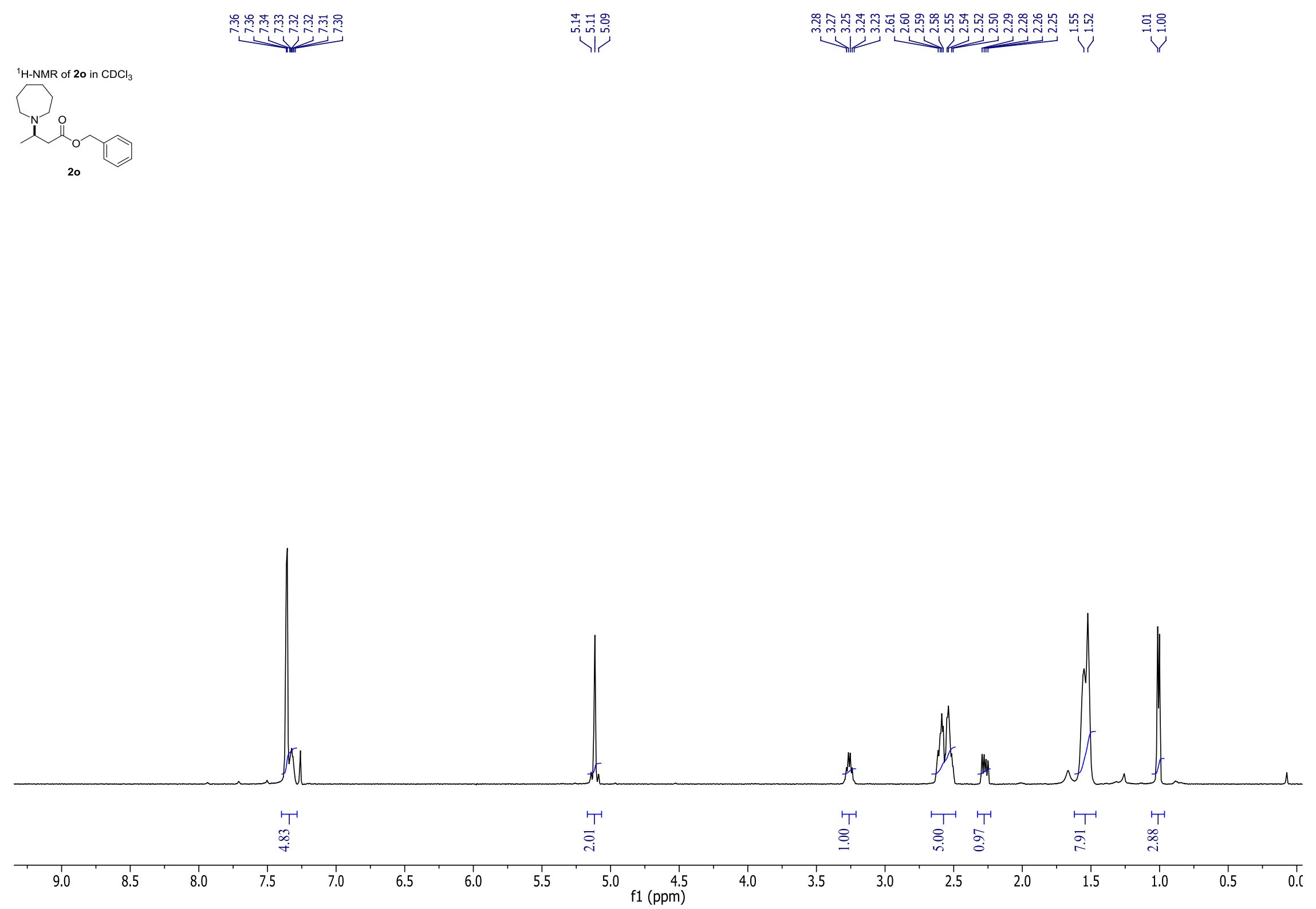

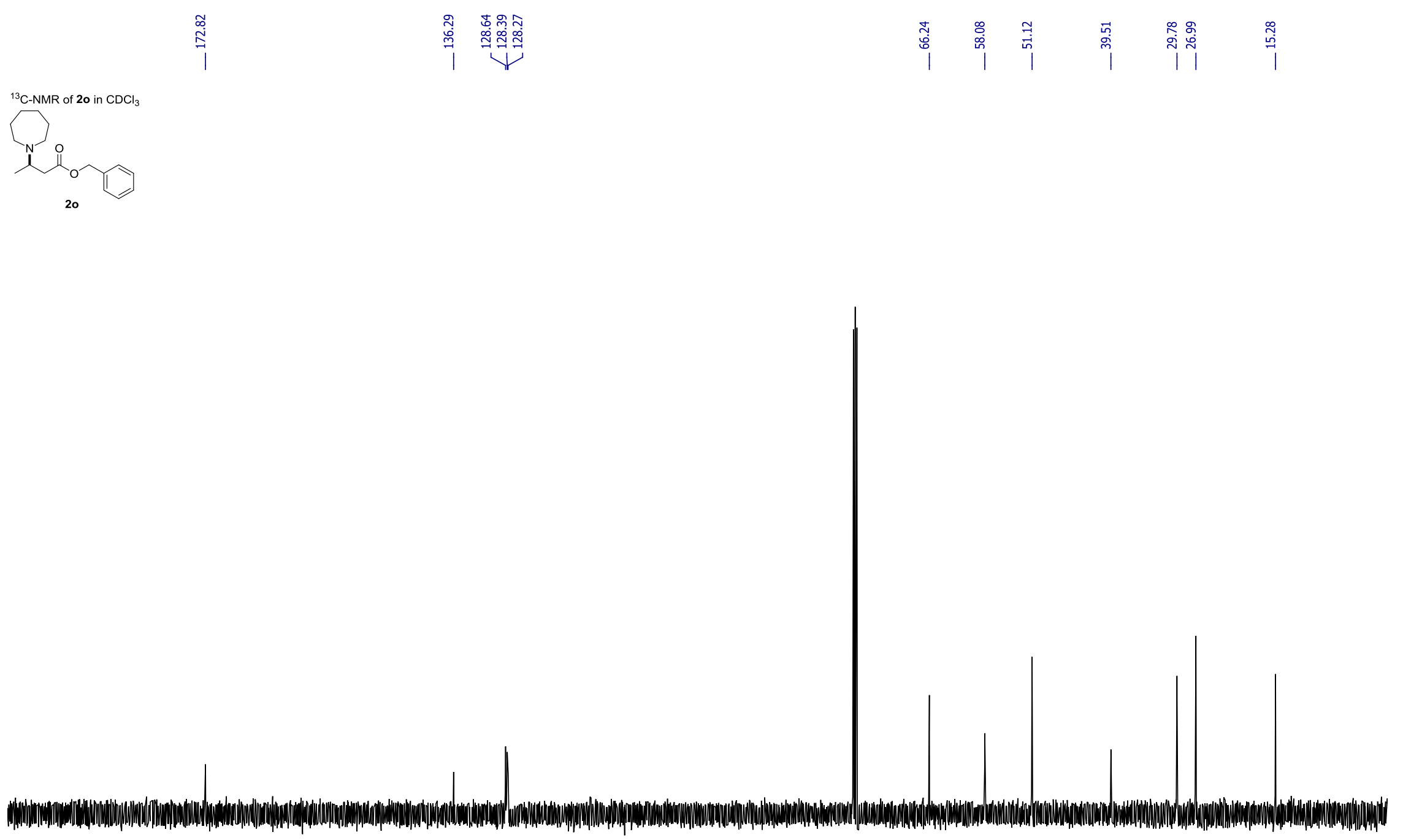

200

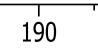

180

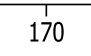

160

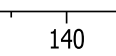

130

120

100
f1
$(\mathrm{ppm})$

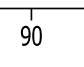

80

70

60

40

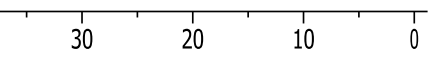

S-296 

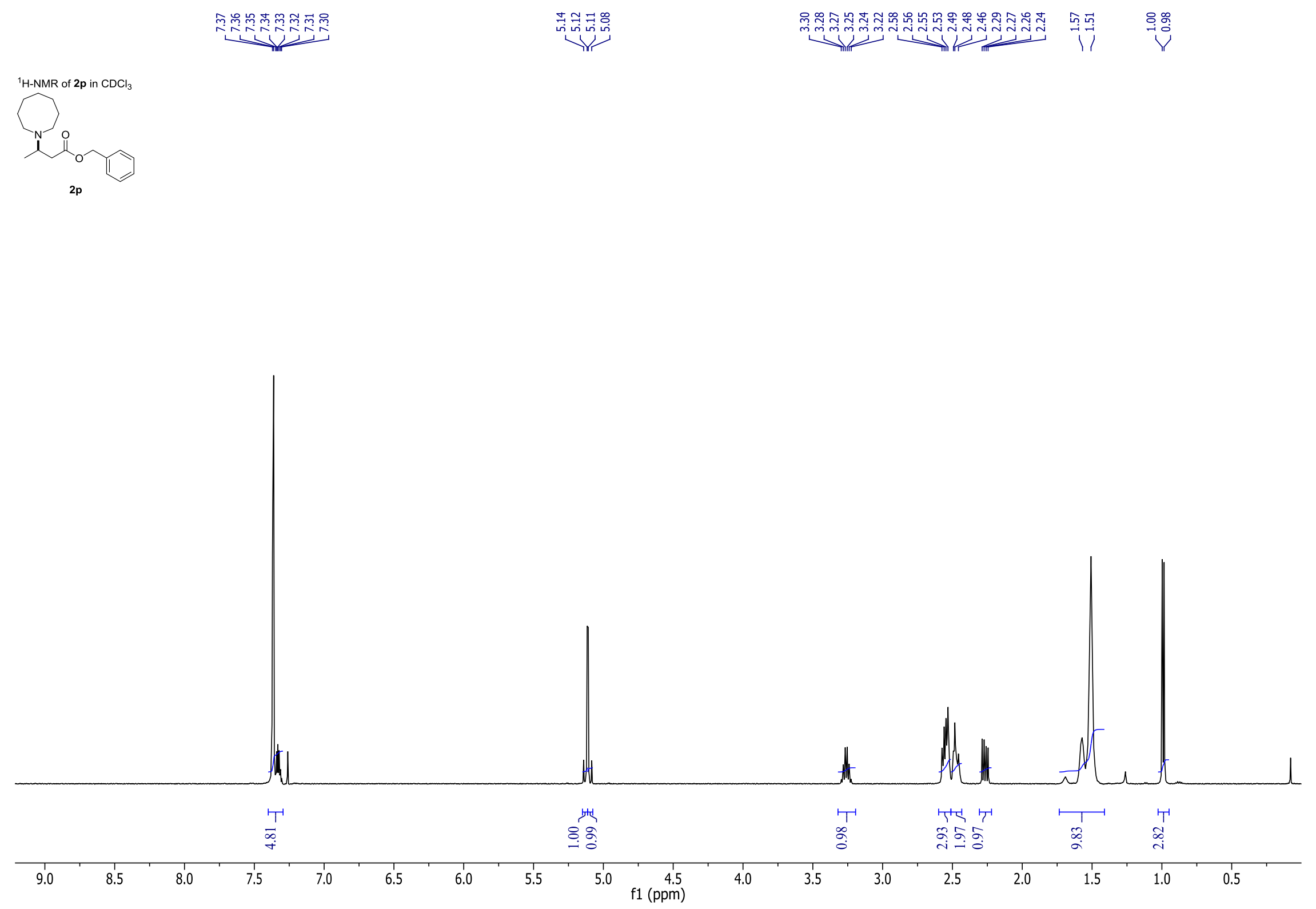


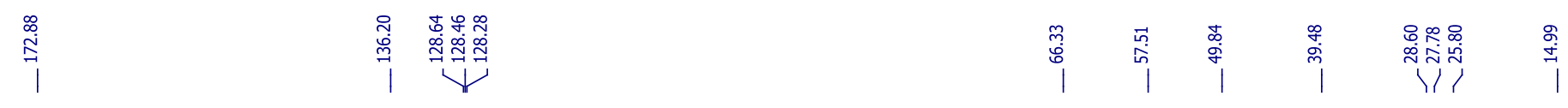

${ }^{13} \mathrm{C}-\mathrm{NMR}$ of $2 \mathrm{p}$ in $\mathrm{CDCl}_{3}$

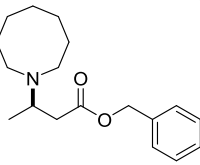

$2 p$

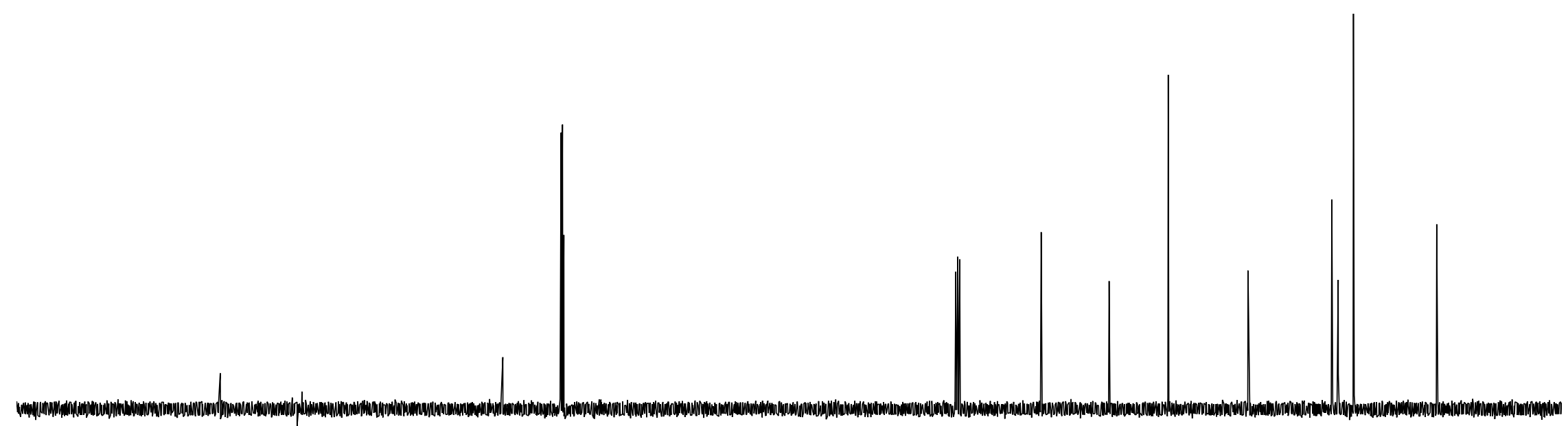




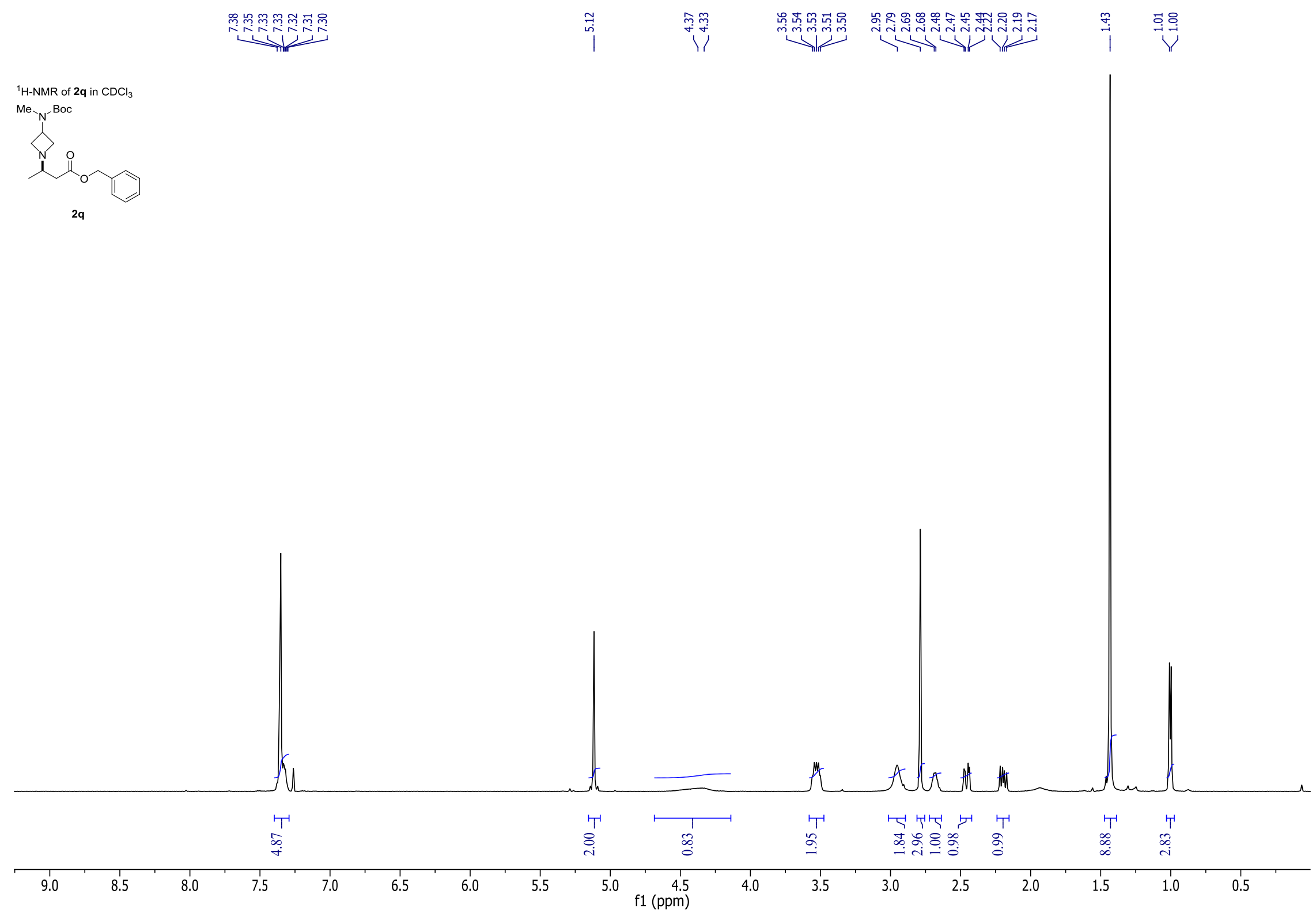




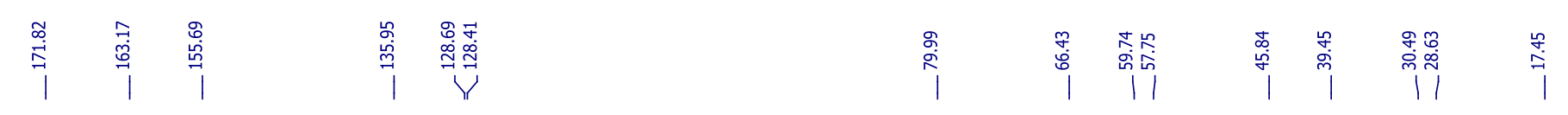

${ }^{13} \mathrm{C}-\mathrm{NMR}$ of $2 \mathrm{q}$ in $\mathrm{CDCl}_{3}$

$\mathrm{Me}{ }^{-}{ }^{-B o c}$

$\hat{\lambda}^{N}$

$2 q$

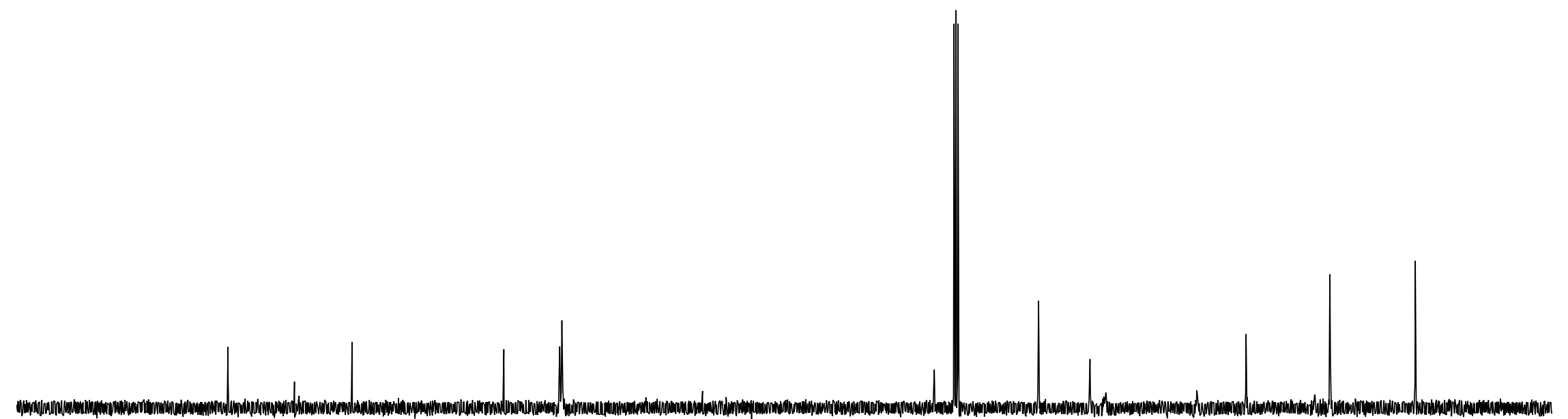

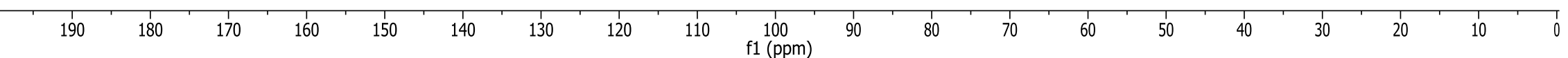




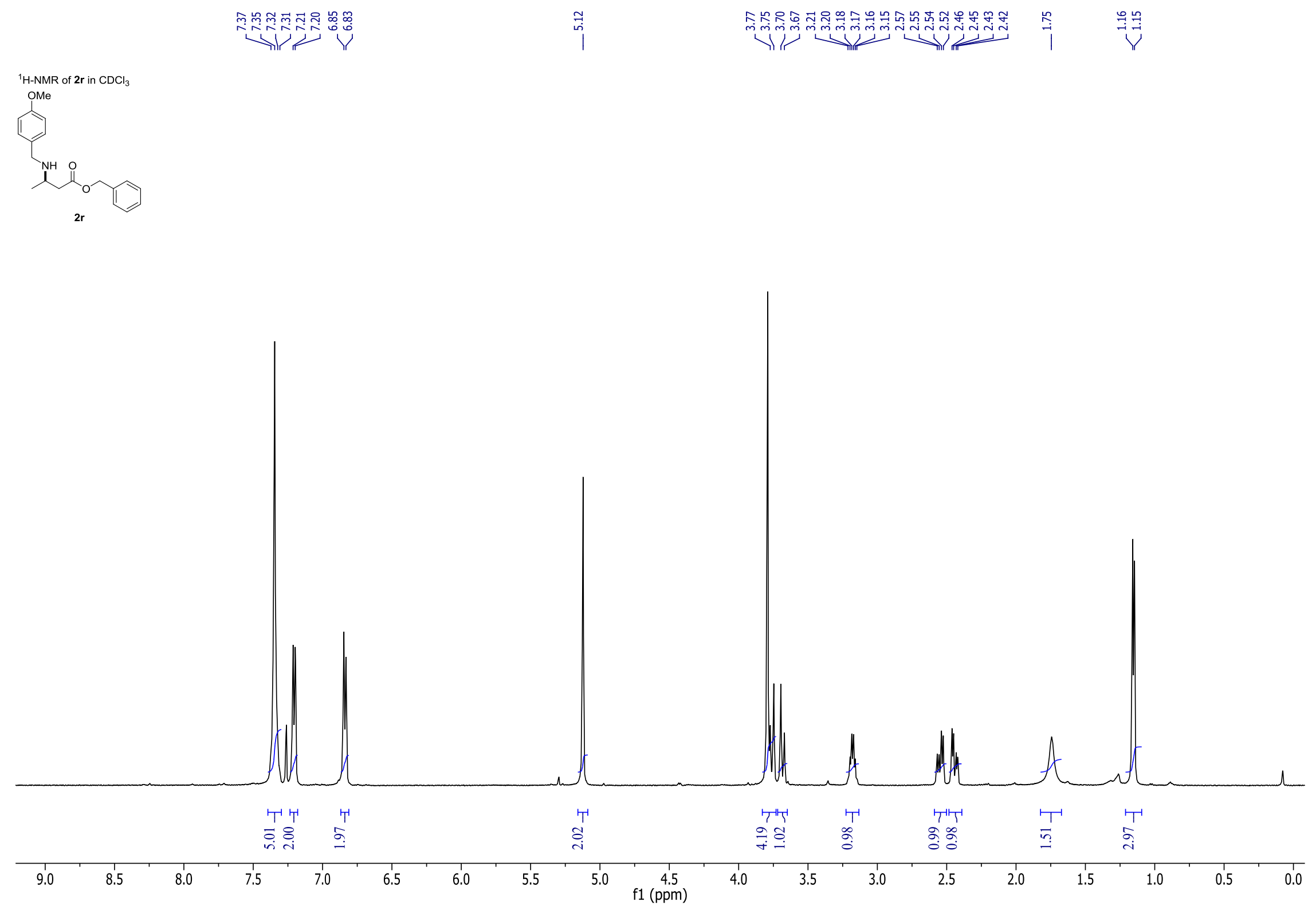




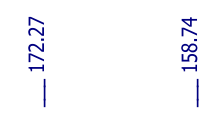

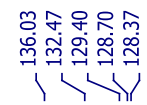

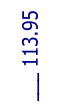

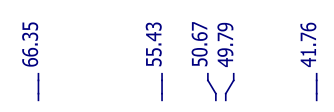
$\stackrel{8}{\stackrel{8}{8}}$

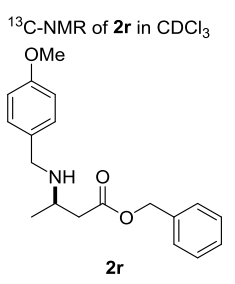

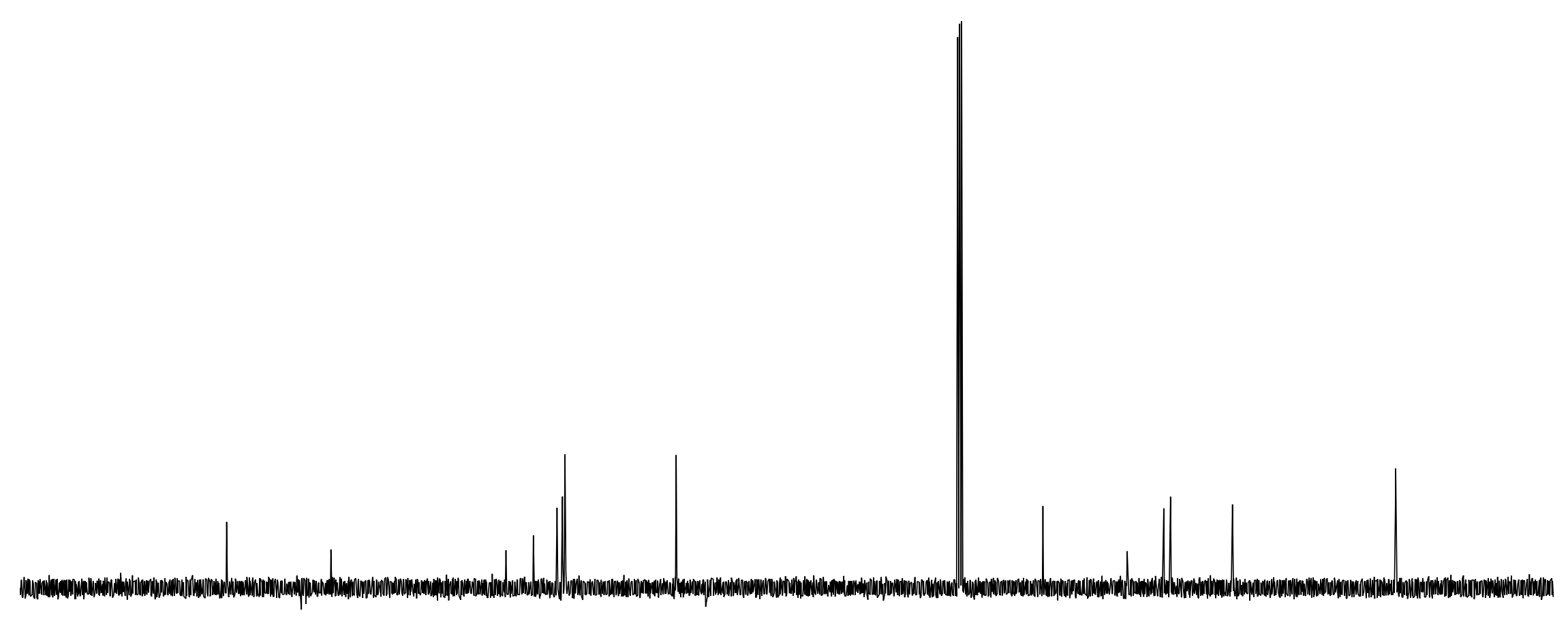

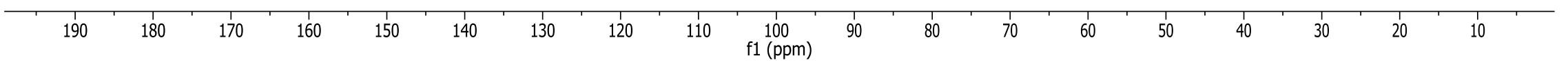




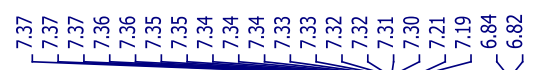

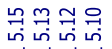

$\underbrace{\underbrace{2}_{0}}$

${ }^{1} \mathrm{H}-\mathrm{NMR}$ of $2 \mathrm{~s}$ in $\mathrm{CDCl}_{3}$

OMe
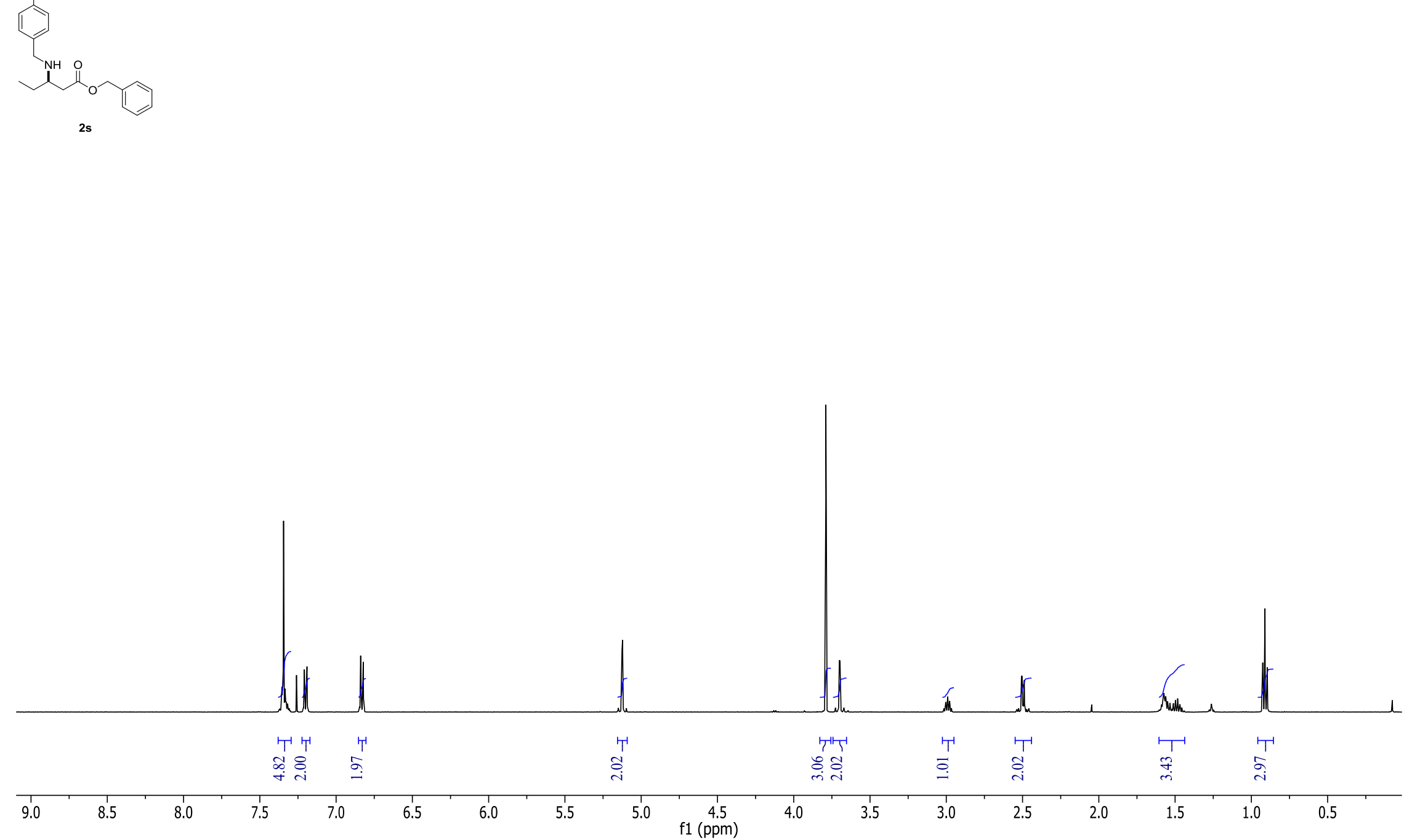

S-303 
|

荡

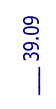

$\stackrel{\substack{0 \\ \text { I }}}{\mid}$

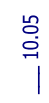

${ }^{13} \mathrm{C}-\mathrm{NMR}$ of $2 \mathrm{~s}$ in $\mathrm{CDCl}_{3}$

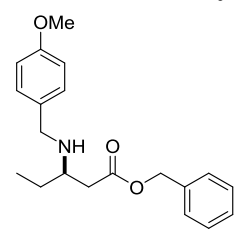

$2 s$
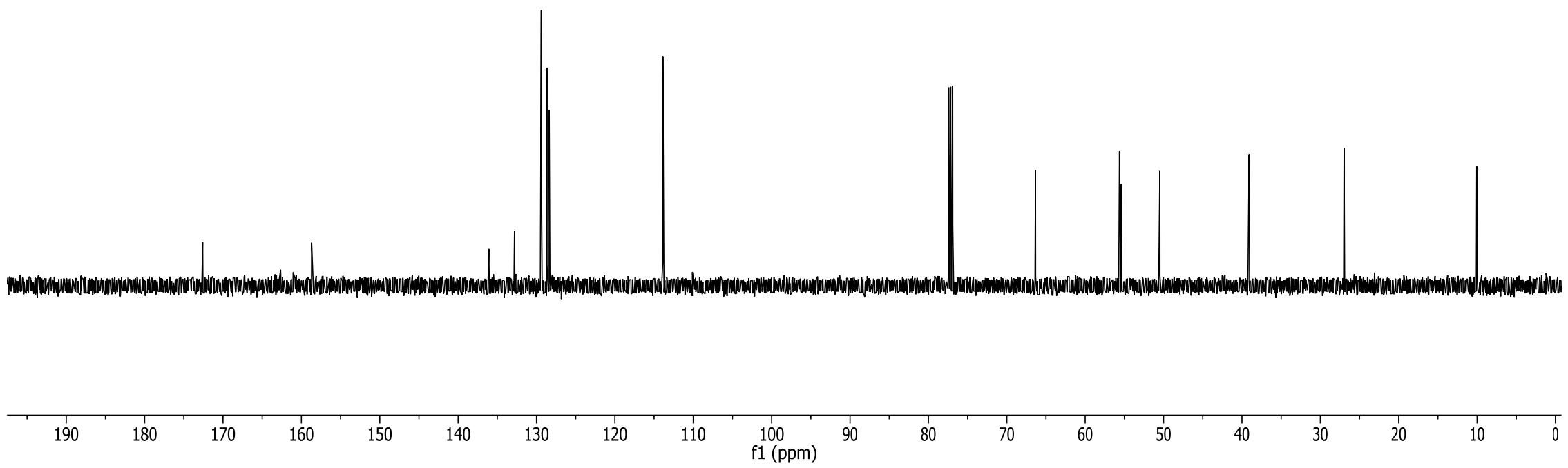

S-304 


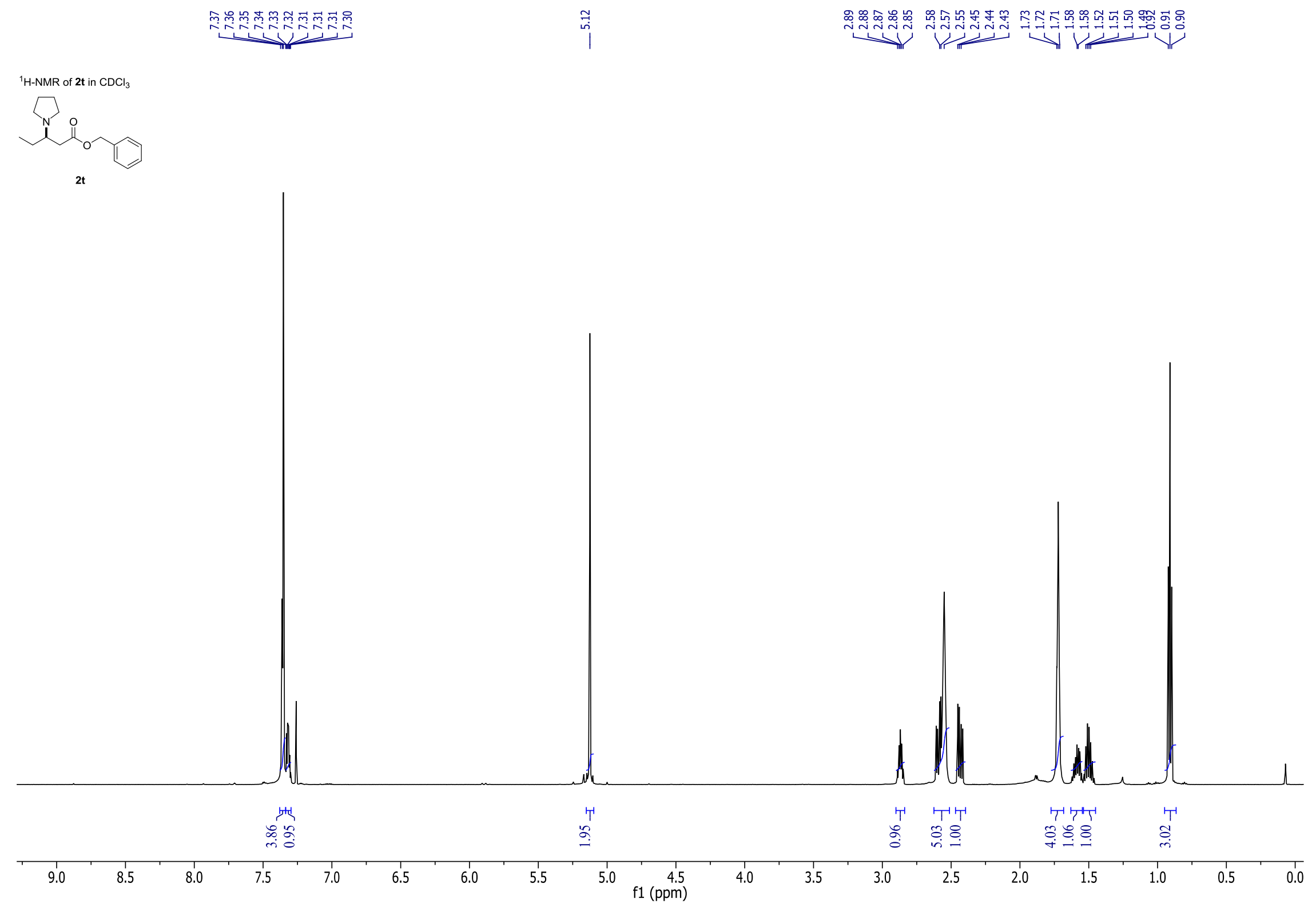




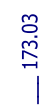

$\sqrt{\substack{\sim \\ 0}}$

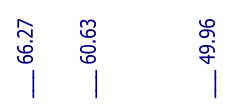

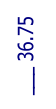

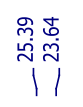

$\stackrel{\text { I }}{\stackrel{7}{0}}$

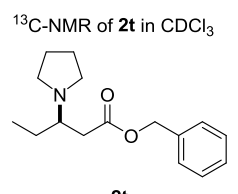

$2 t$
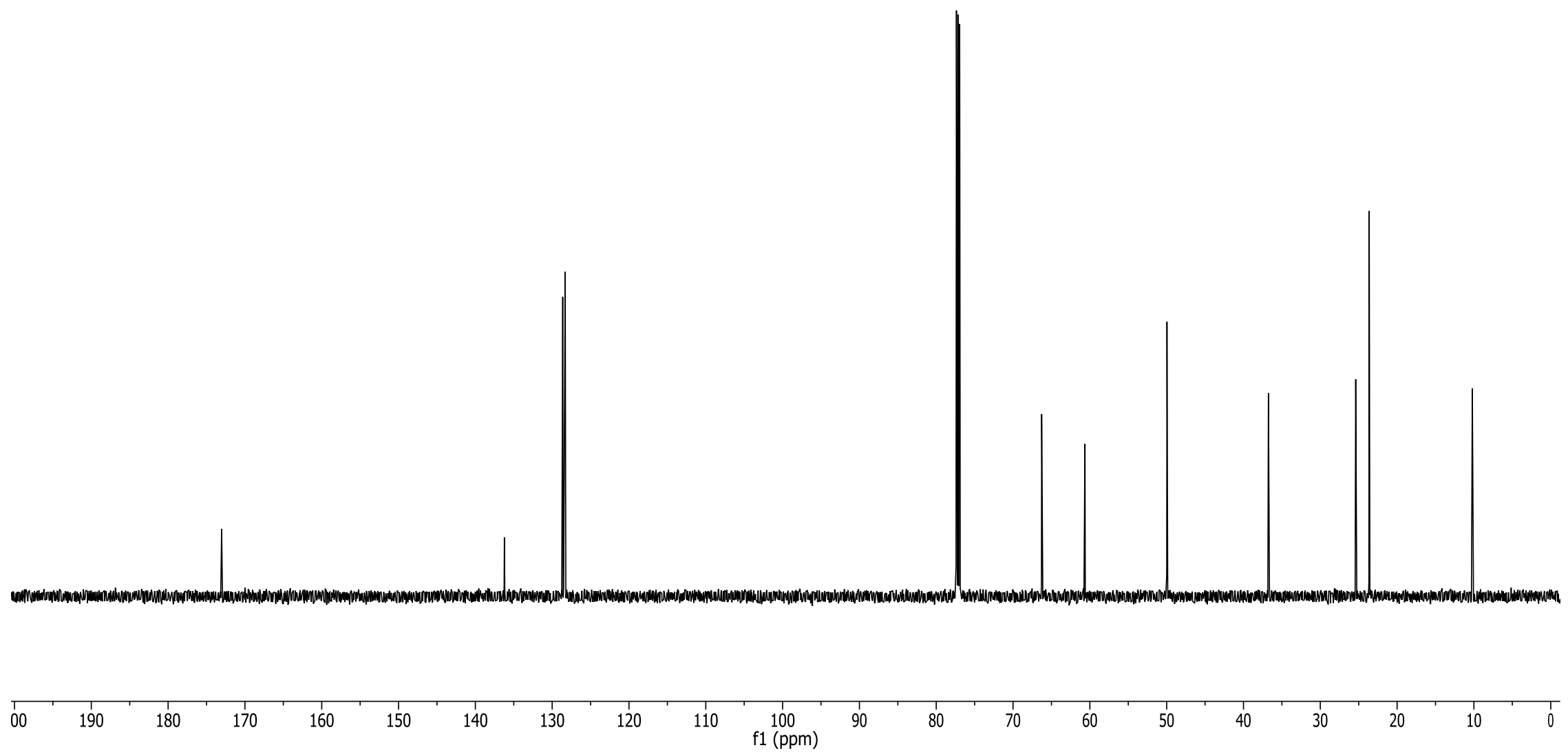

S-306 


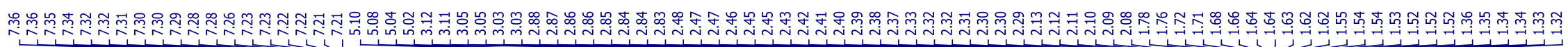

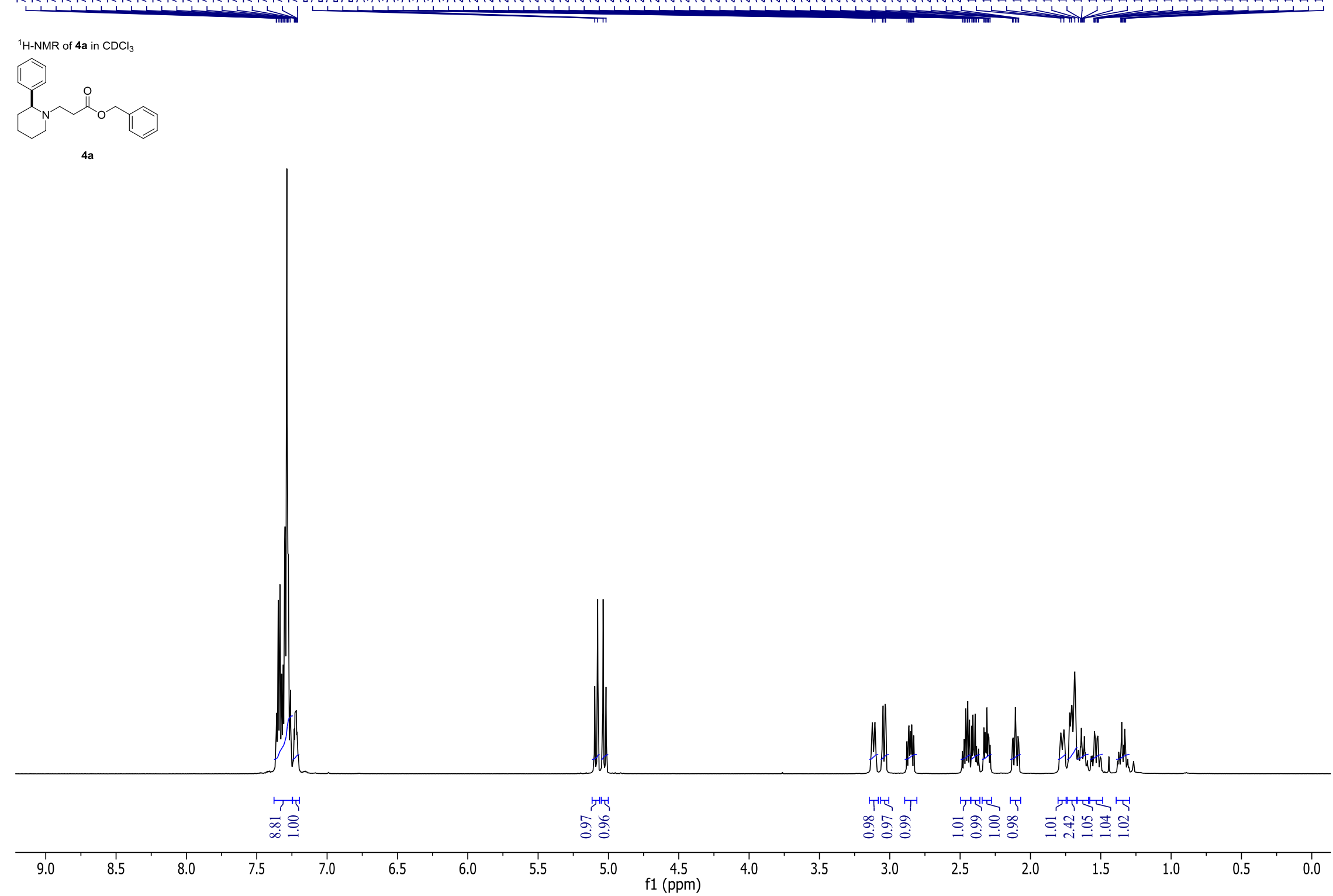


|

영

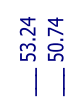

|⿱宀⿻心㇒山

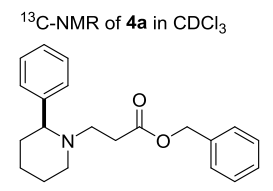

$4 a$
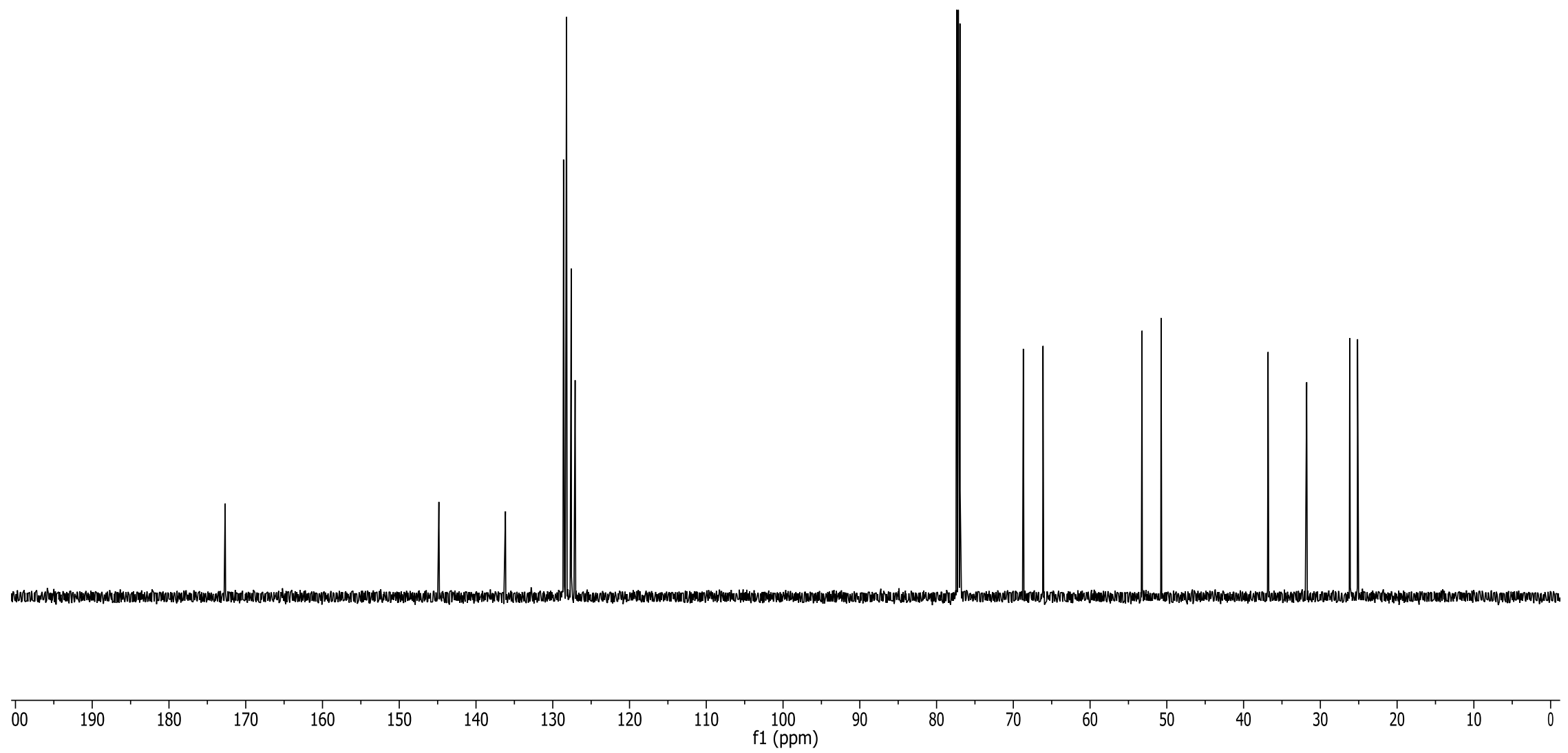

S-308 


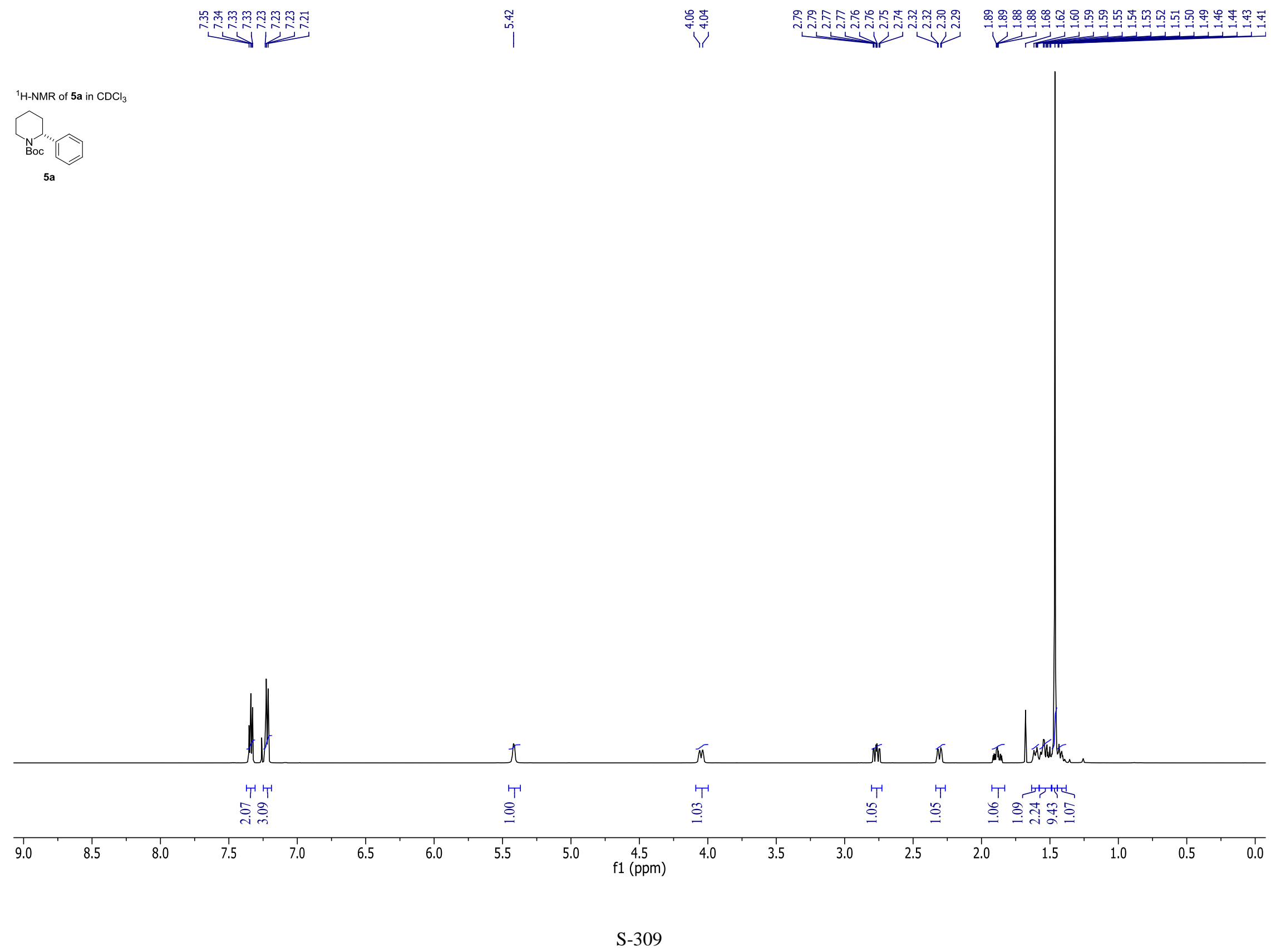




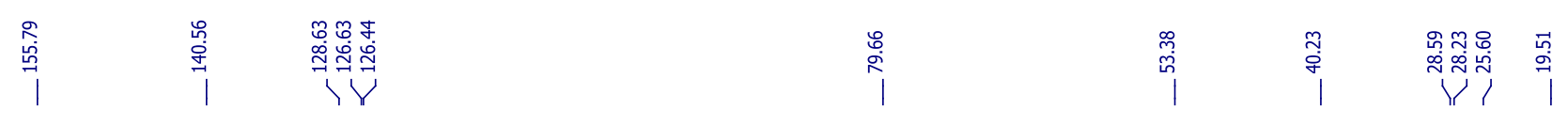

${ }^{13} \mathrm{C}-\mathrm{NMR}$ of $5 \mathbf{a}$ in $\mathrm{CDCl}_{3}$

Boc , I

$5 a$
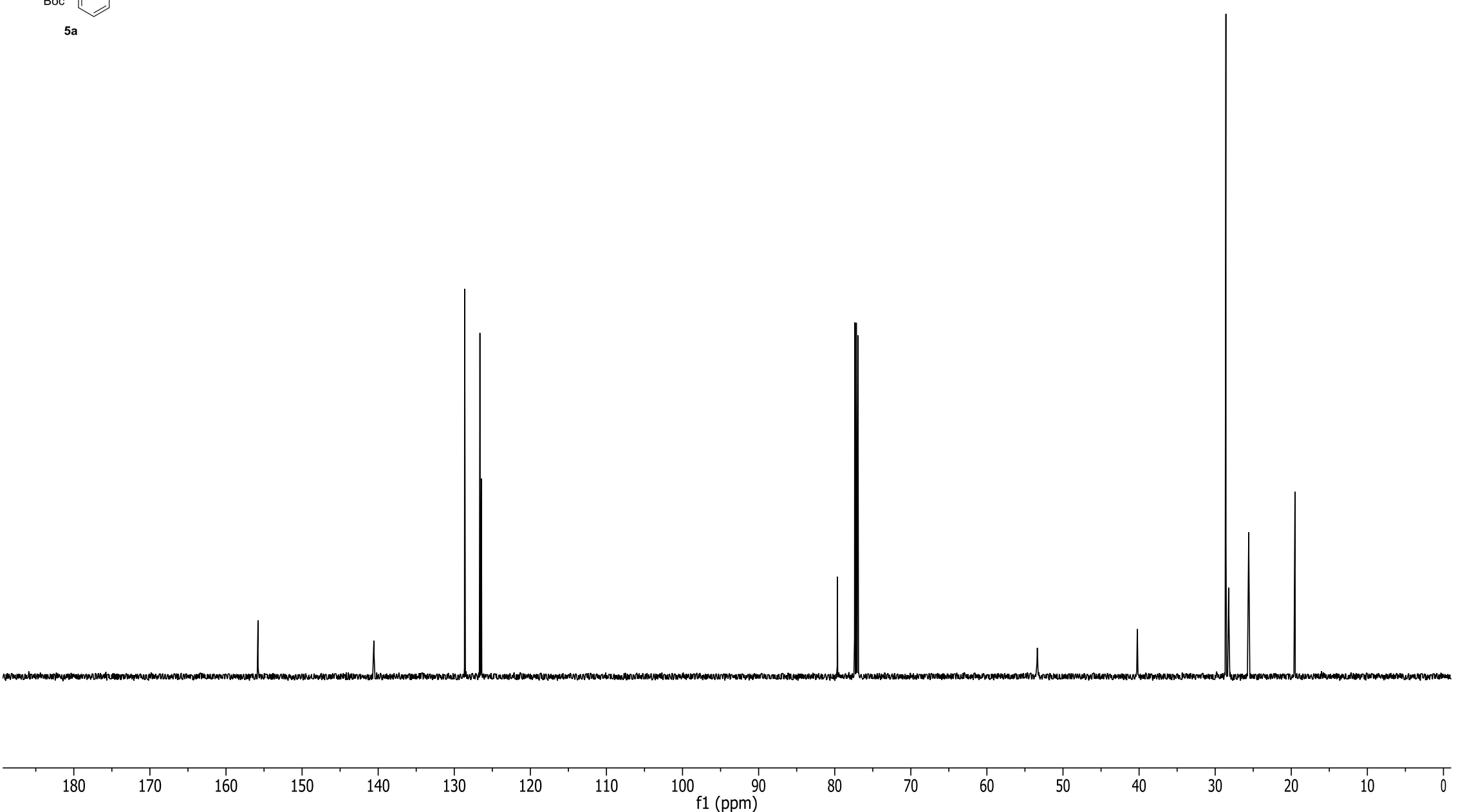

S-310 

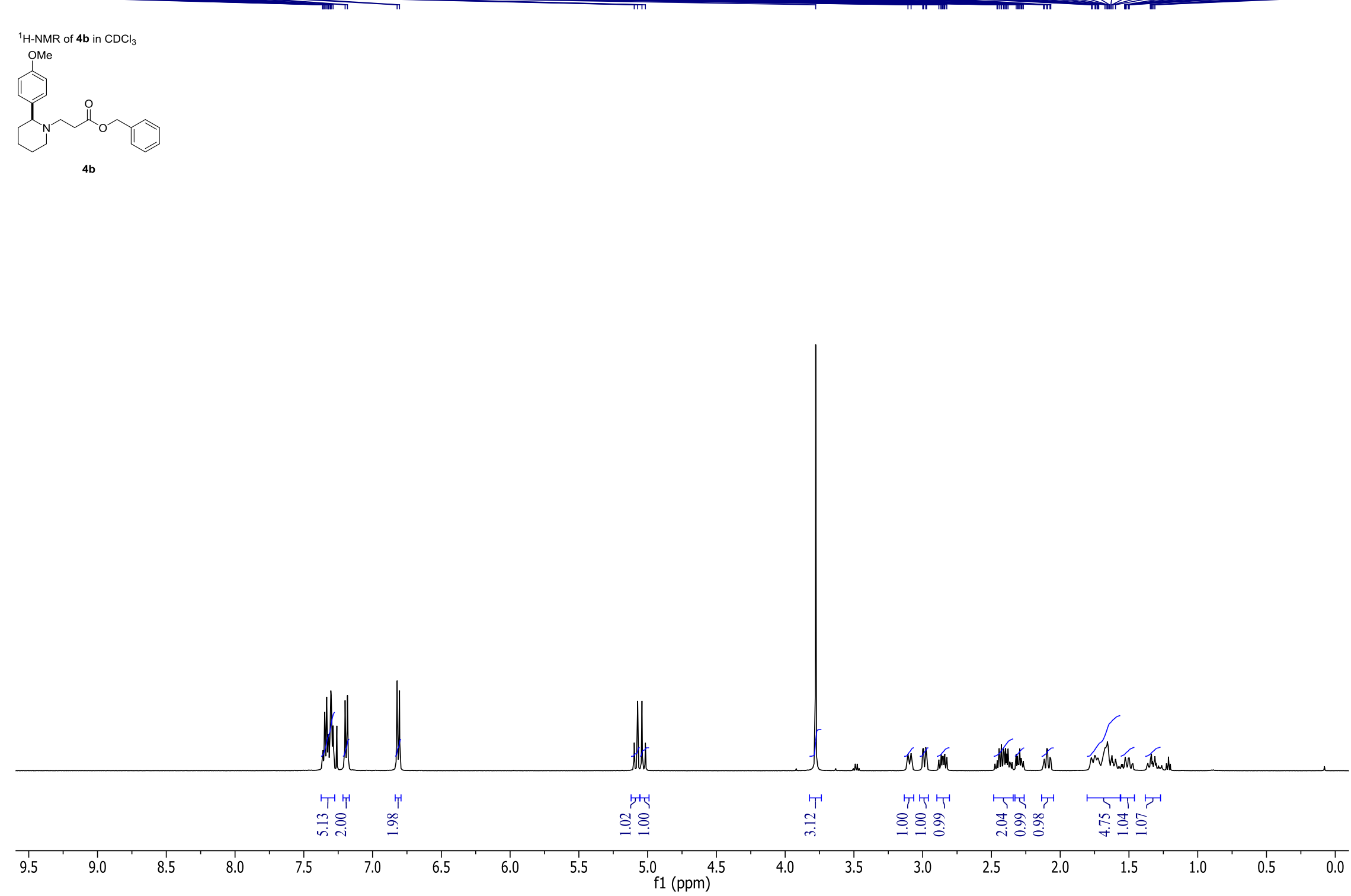


尊

${ }^{13} \mathrm{C}-\mathrm{NMR}$ of $4 \mathbf{b}$ in $\mathrm{CDCl}_{3}$

(1)

$4 b$

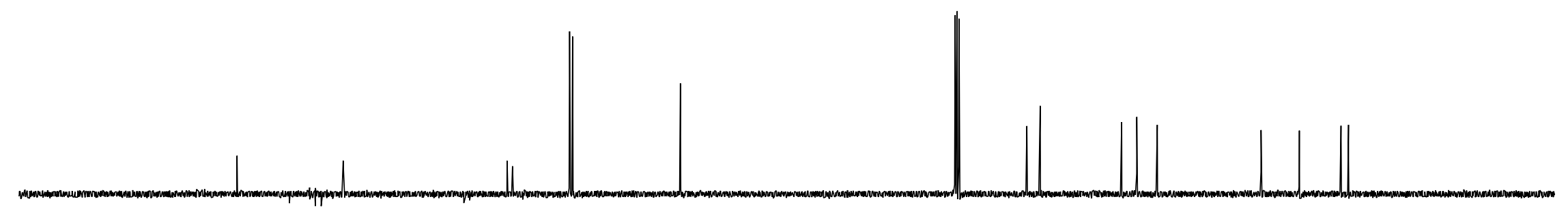




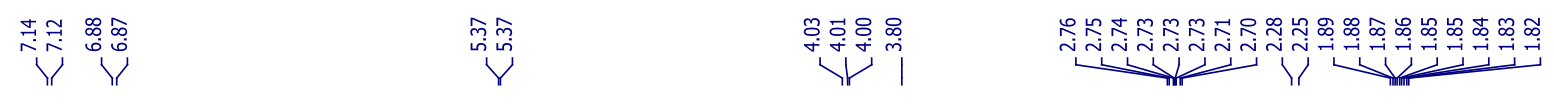

${ }^{1} \mathrm{H}-\mathrm{NMR}$ of $\mathbf{5 b}$ in $\mathrm{CDCl}_{3}$

Bbc

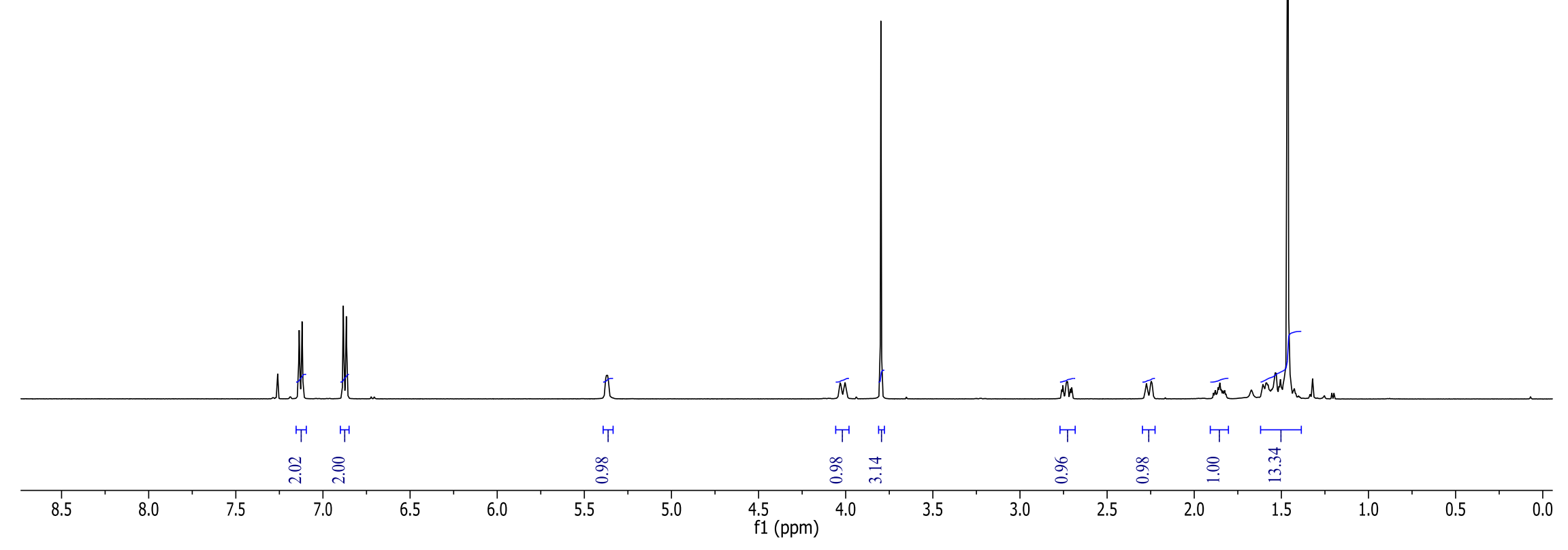

S-313 


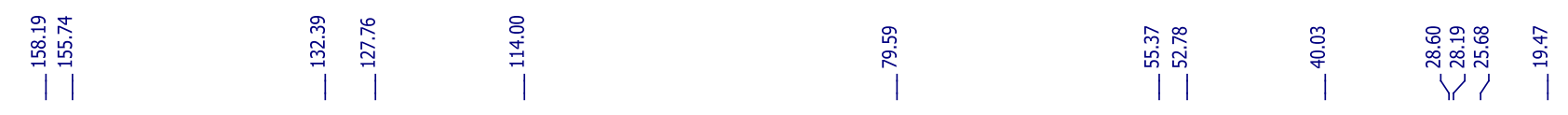
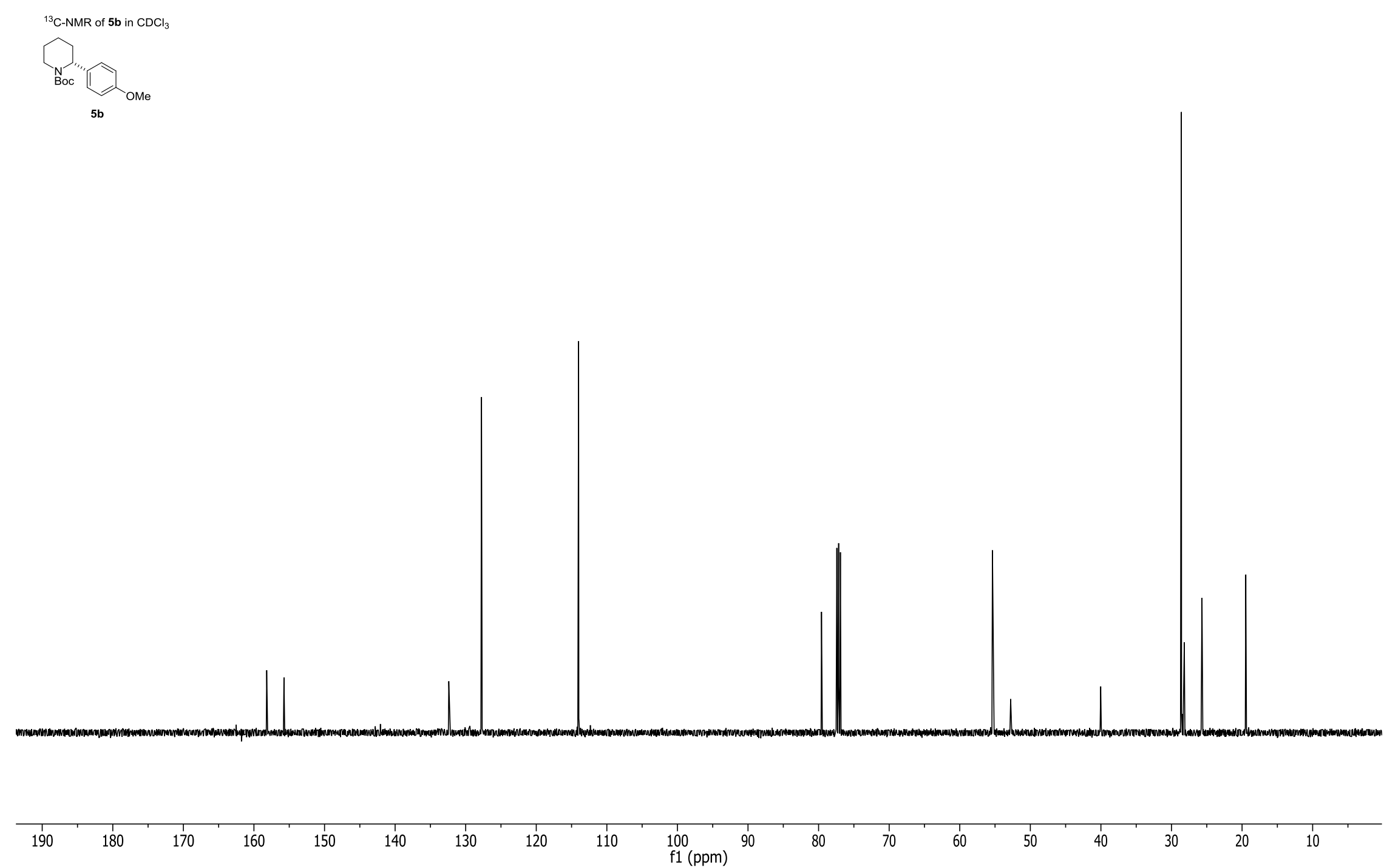


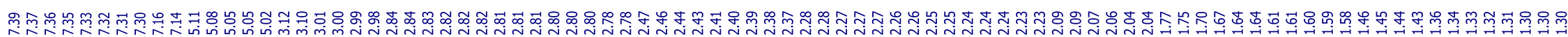
${ }^{1} \mathrm{H}-\mathrm{NMR}$ of $4 \mathrm{c}$ in $\mathrm{CDCl}_{3}$
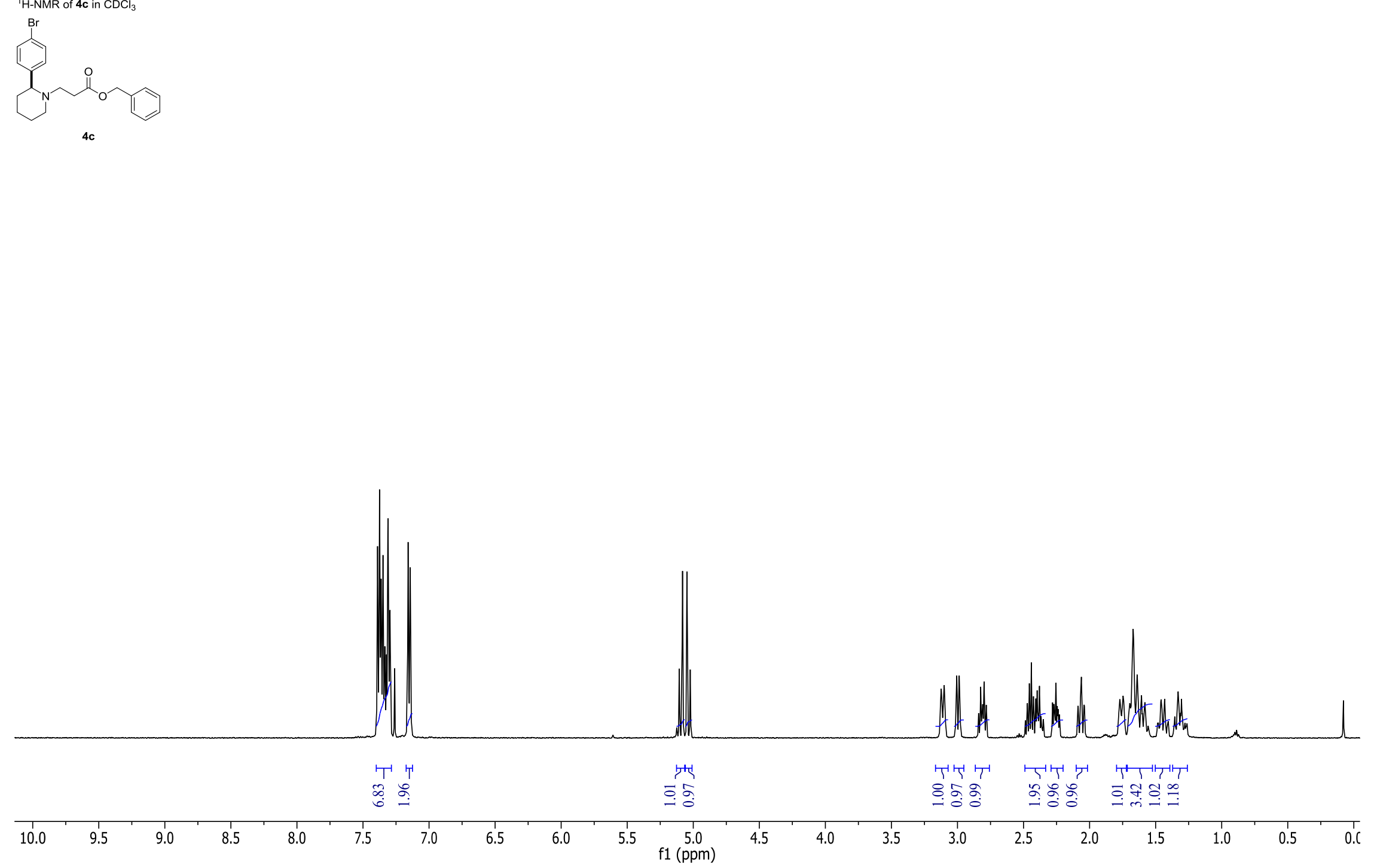


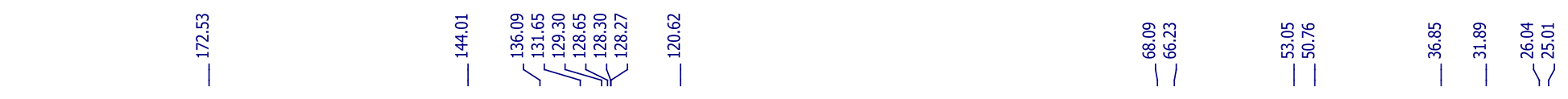
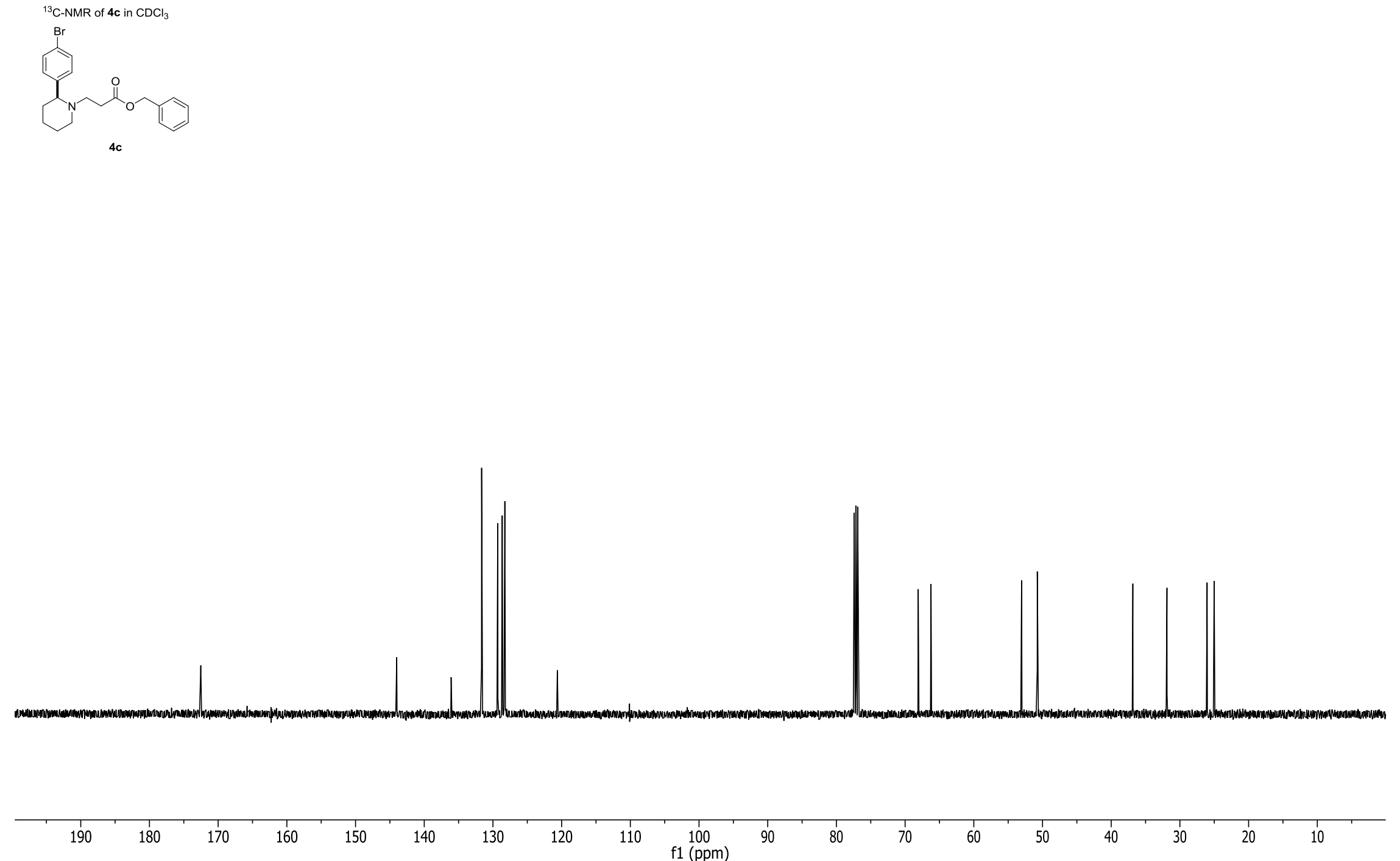


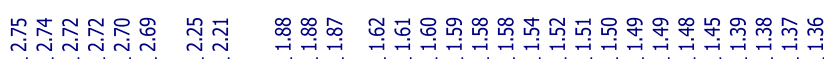

${ }^{1} \mathrm{H}-\mathrm{NMR}$ of $5 \mathrm{C}$ in $\mathrm{CDCl}_{3}$

Bc

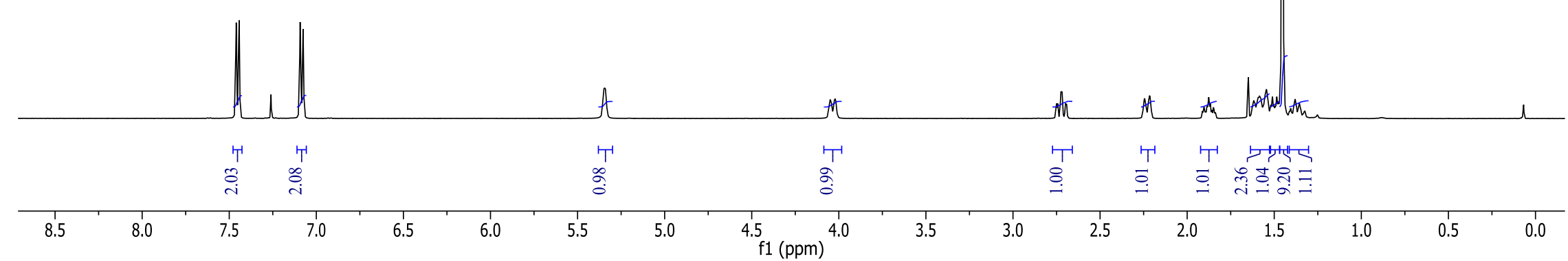




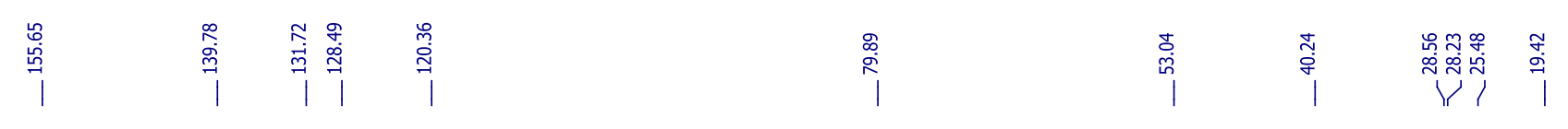

${ }^{13} \mathrm{C}-\mathrm{NMR}$ of $5 \mathrm{C}$ in $\mathrm{CDCl}_{3}$

Boc

$5 c$

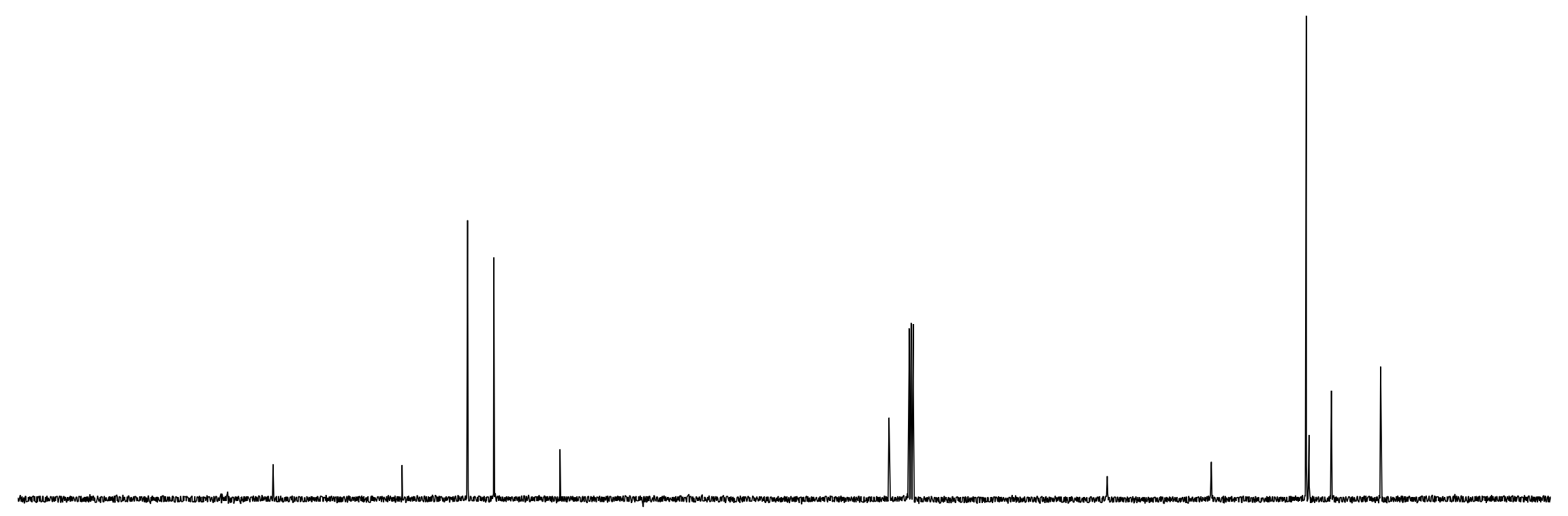

180

170

160

150

140

130

$20 \quad 110$

$100 \quad 90$

80

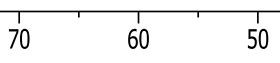

40

30

$20 \quad 10 \quad 0$

S-318 


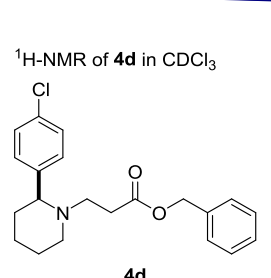

$4 d$

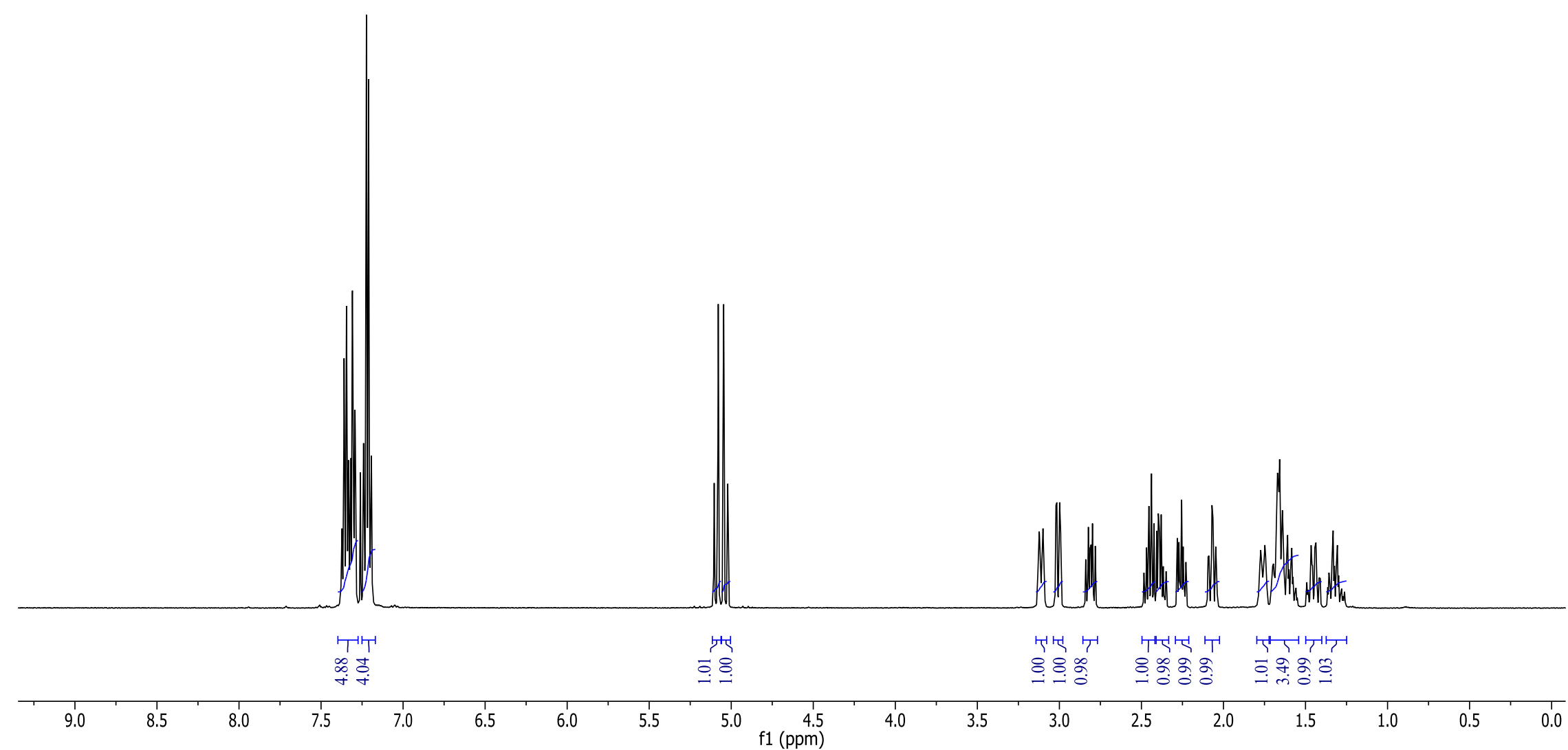




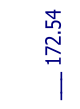

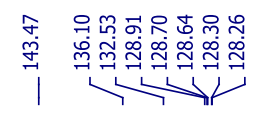
赵

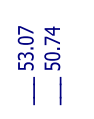
i

${ }^{13} \mathrm{C}-\mathrm{NMR}$ of $4 \mathbf{d}$ in $\mathrm{CDCl}_{3}$

(1)

$4 d$

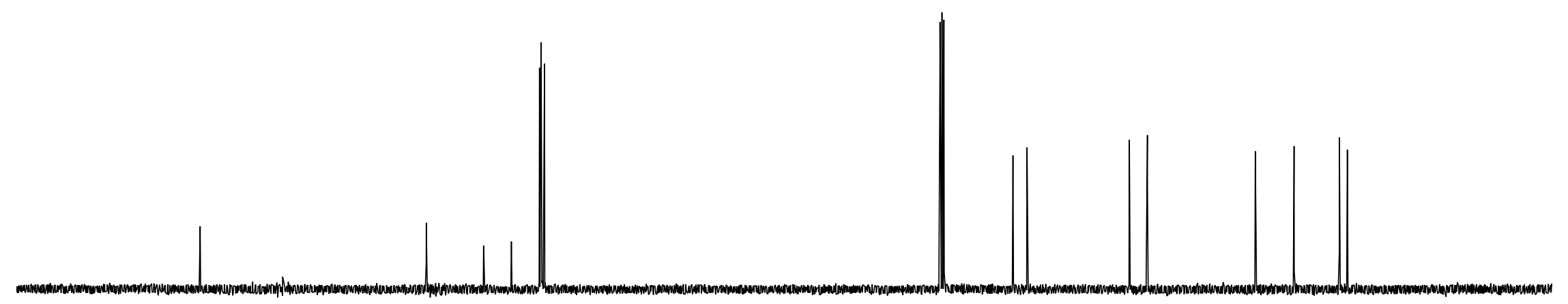




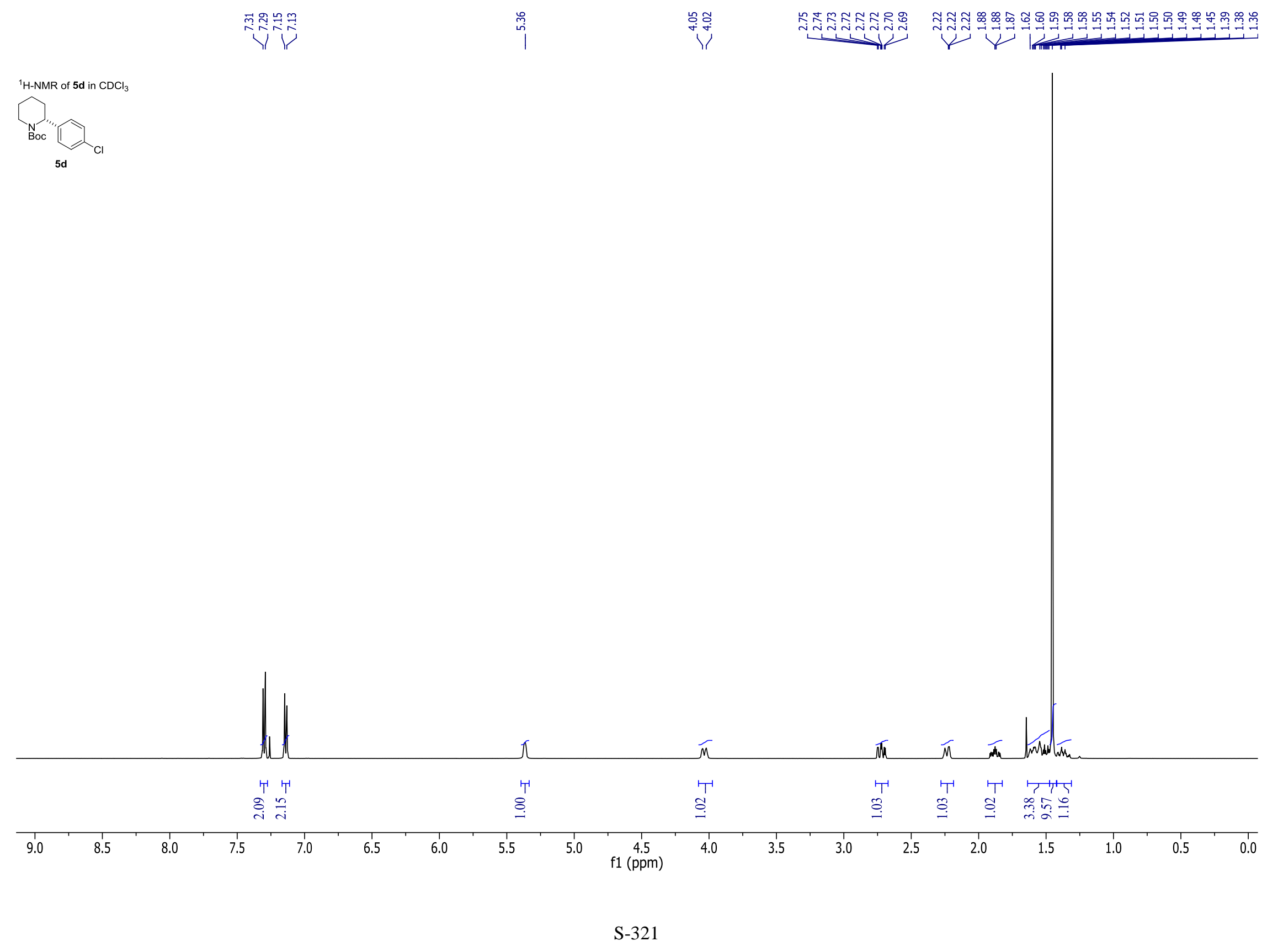




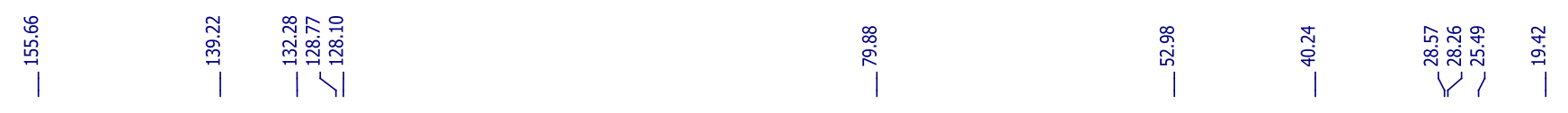

${ }^{13} \mathrm{C}-\mathrm{NMR}$ of $5 \mathrm{~d}$ in $\mathrm{CDCl}_{3}$

Boc

$5 d$

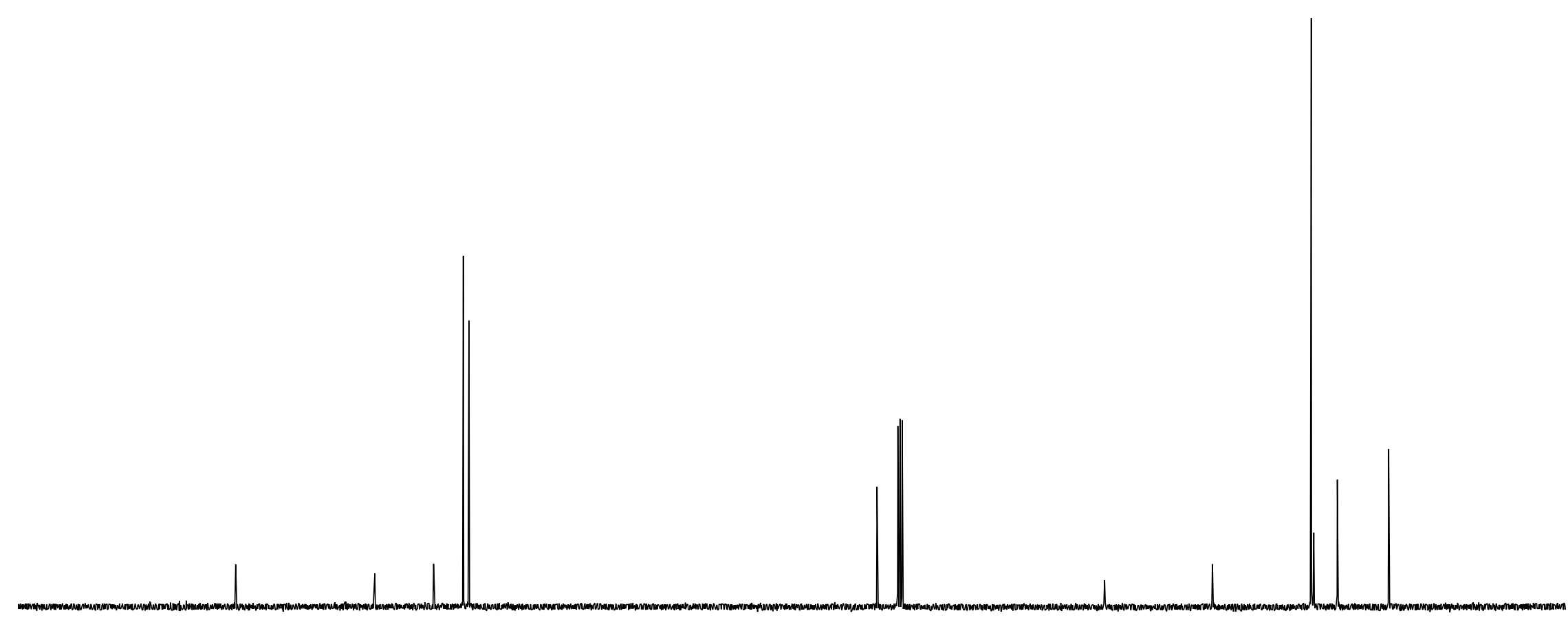

780

170

160

150

$140 \quad 130$

120

100

$\stackrel{90}{90}$

S-322 


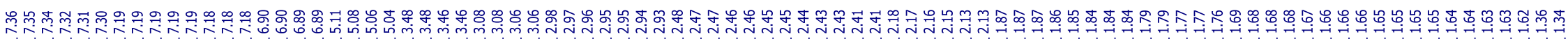
iin?

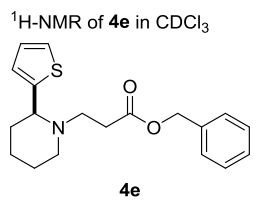

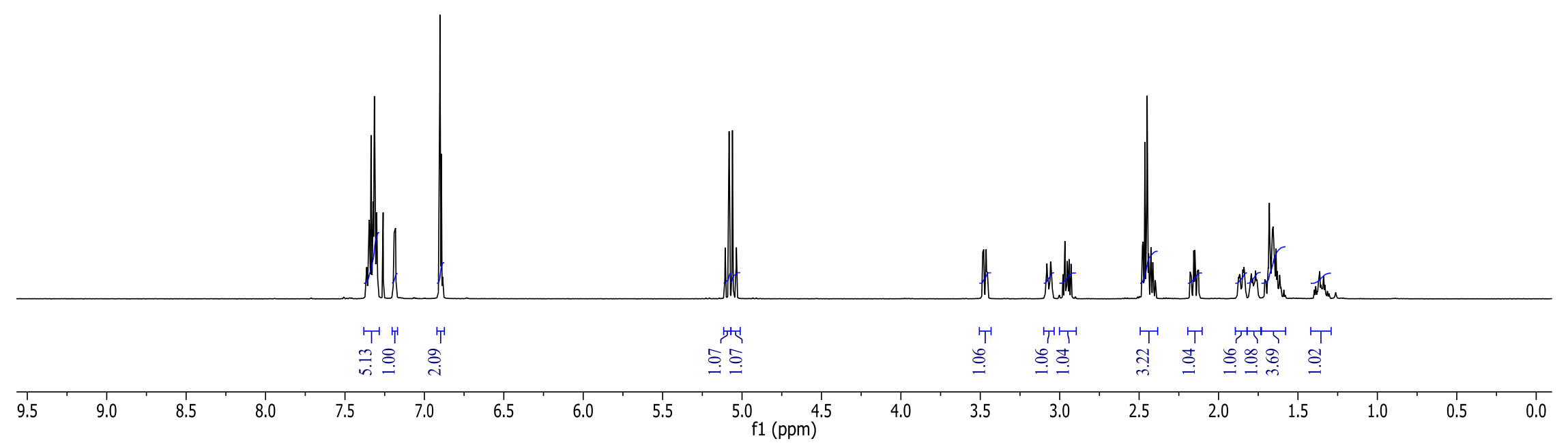




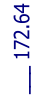

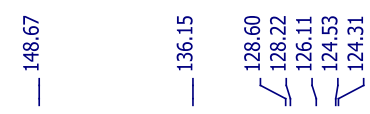
잉요
m

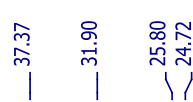

${ }^{13} \mathrm{C}-\mathrm{NMR}$ of $4 \mathrm{e}$ in $\mathrm{CDCl}_{3}$

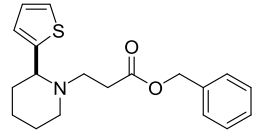

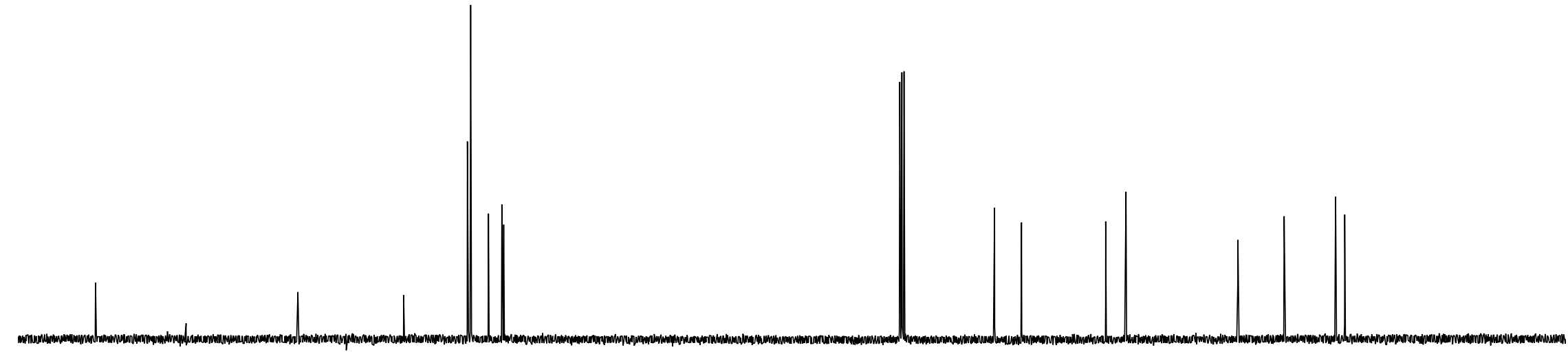




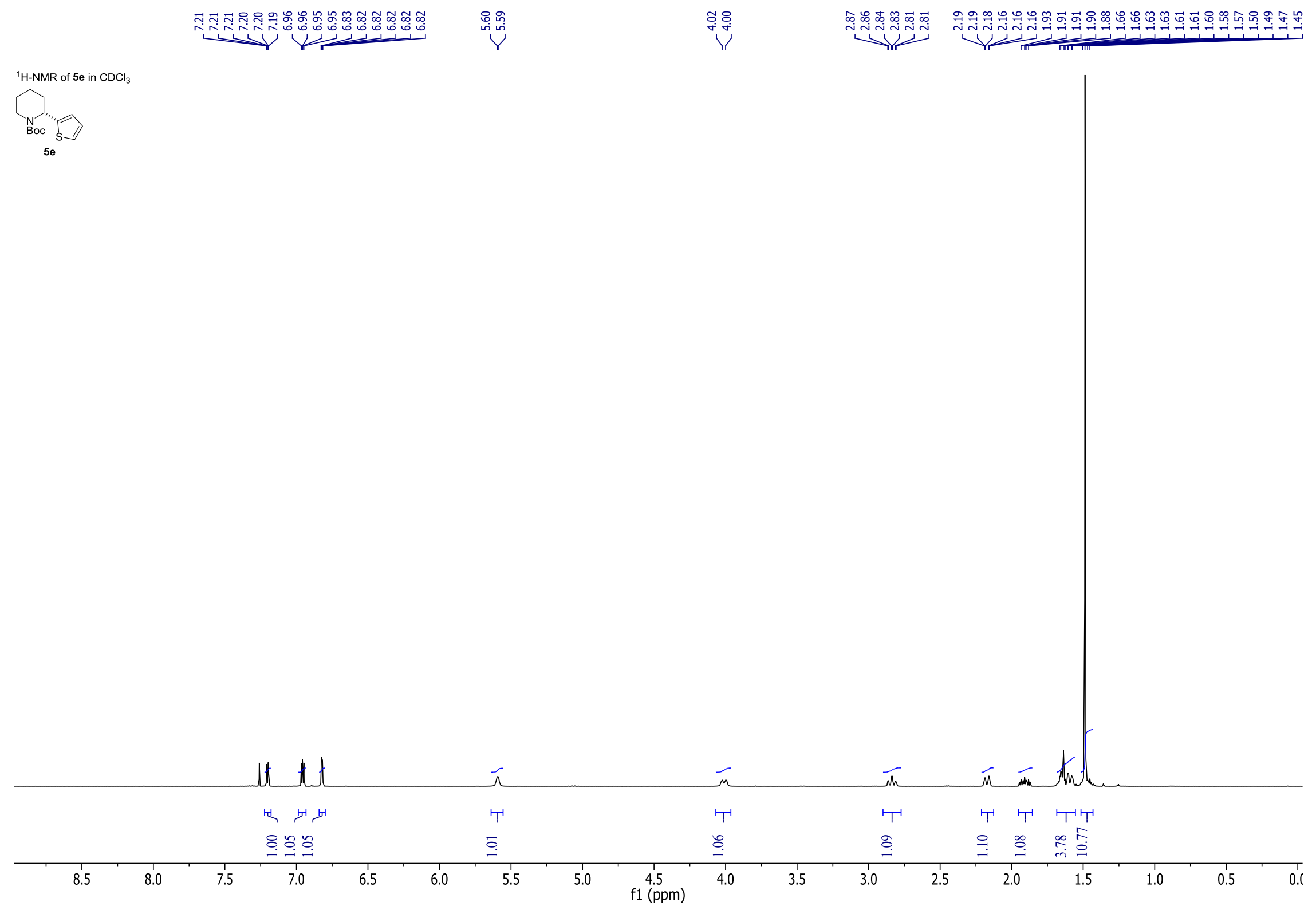

S-325 


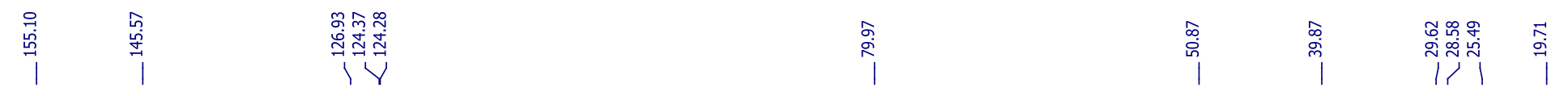

${ }^{13} \mathrm{C}-\mathrm{NMR}$ of $5 \mathrm{e}$ in $\mathrm{CDCl}_{3}$

$\overbrace{B \text { Boc }}>$

5

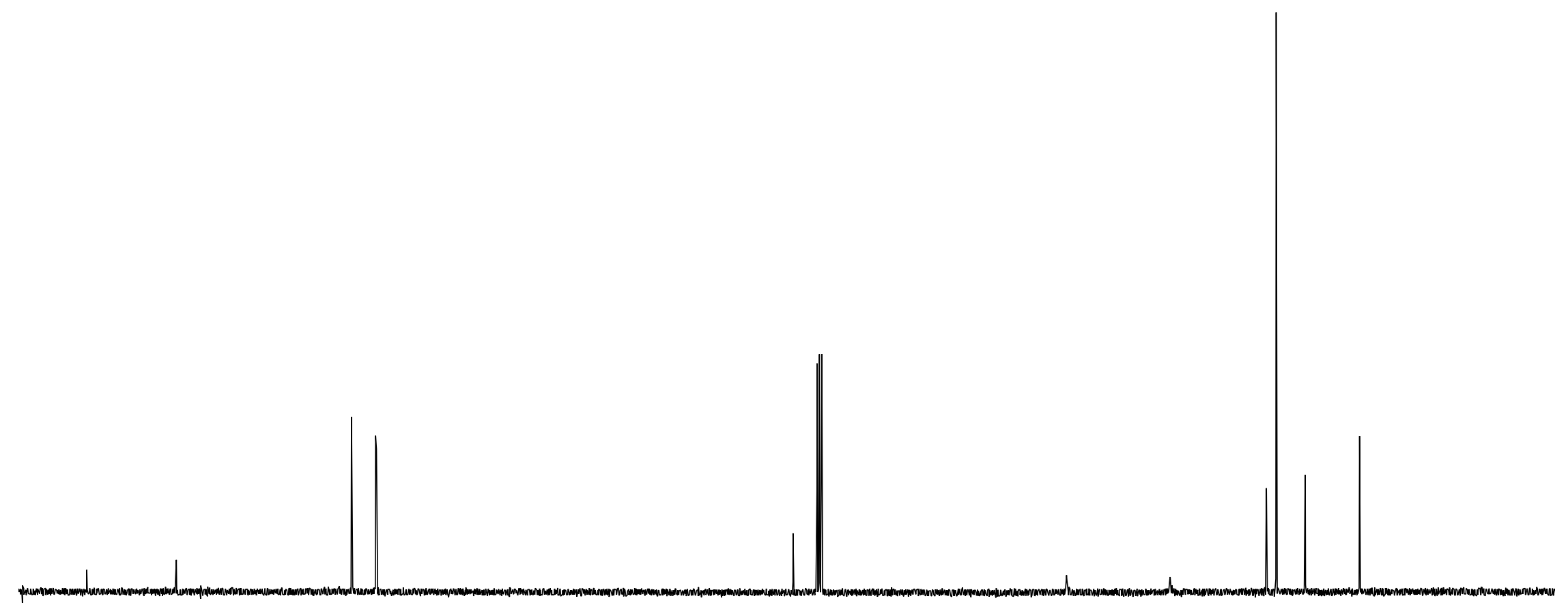

$\underset{160}{1} 150$

140

130

120

110

100

90

80
f1 $(\mathrm{ppm})$ 
${ }^{1} \mathrm{H}-\mathrm{NMR}$ of $4 \mathrm{f} \mathrm{in} \mathrm{CDCl}_{3}$

(f)

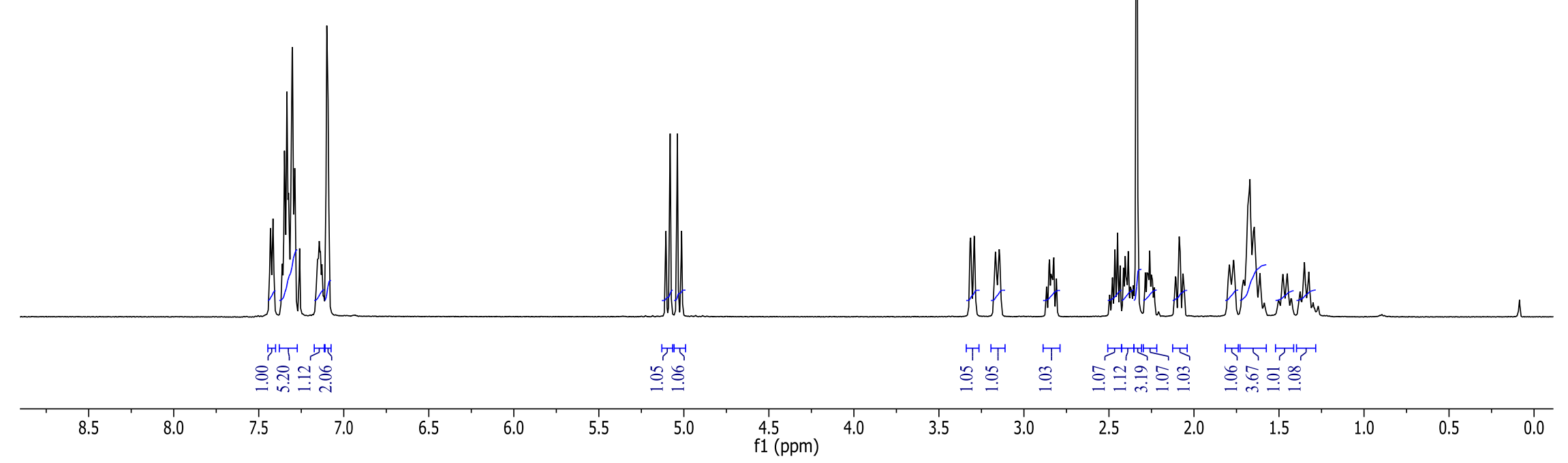




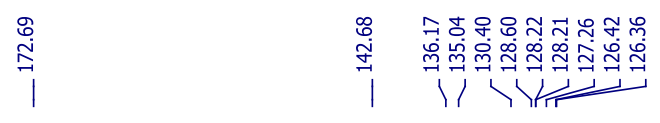

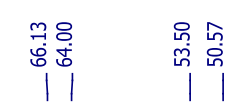

|

${ }^{13} \mathrm{C}-\mathrm{NMR}$ of $4 \mathrm{f}$ in $\mathrm{CDCl}_{3}$

serso

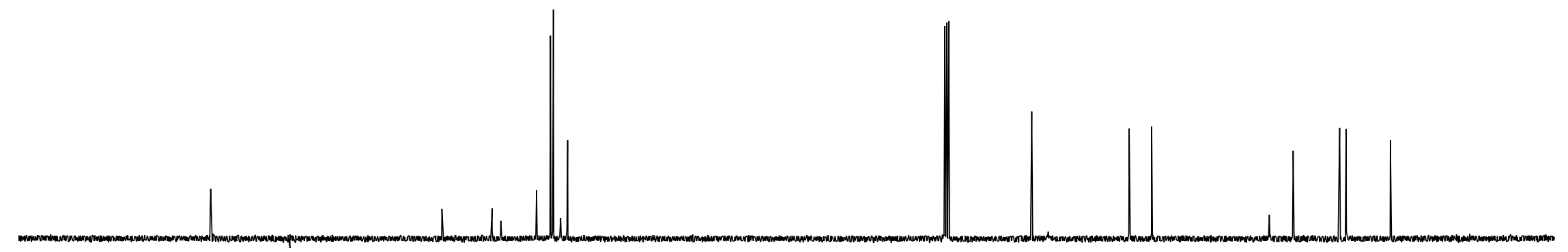

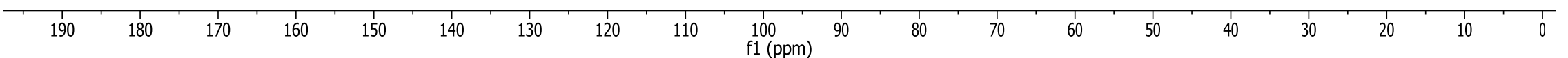




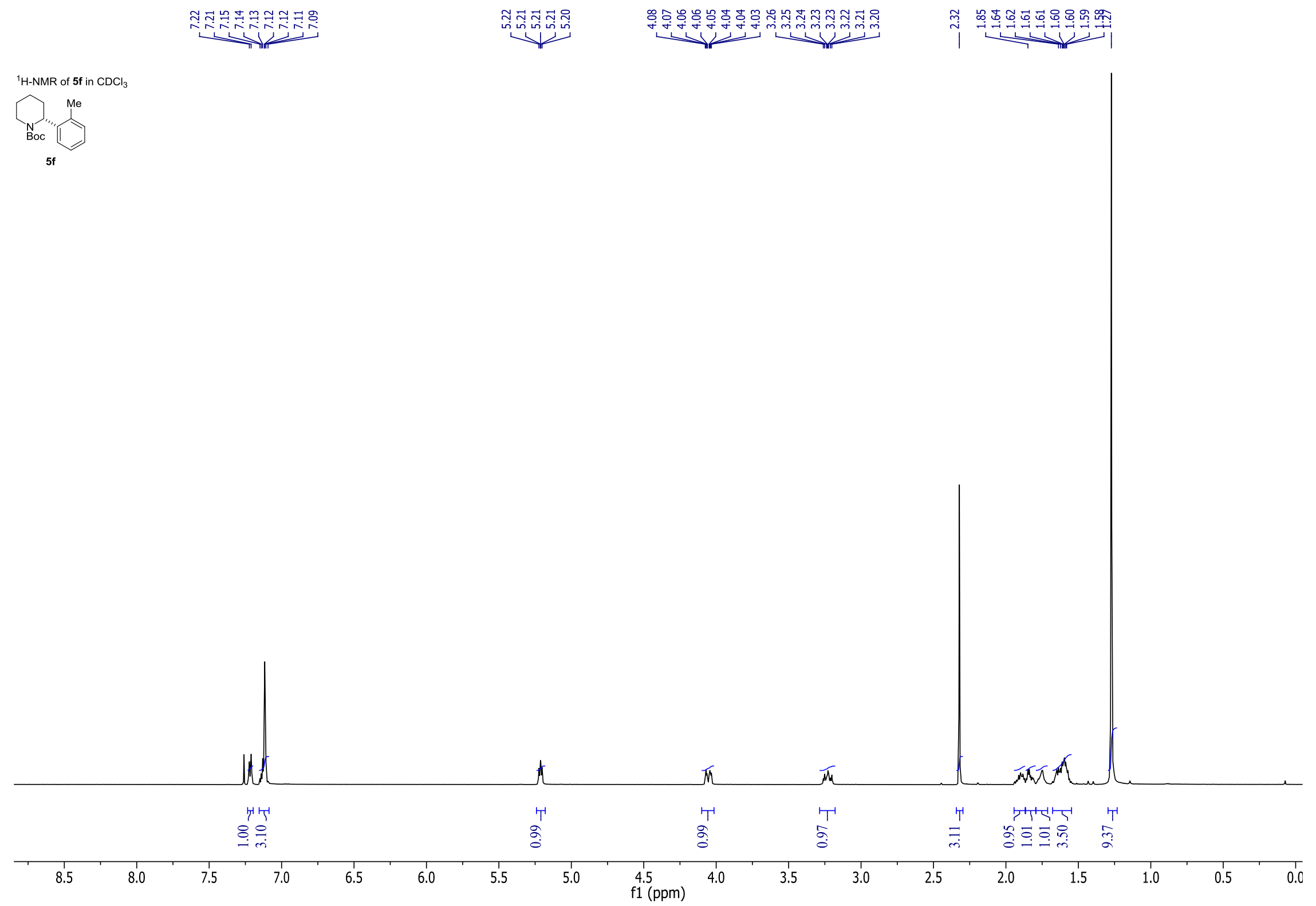

S-329 
${ }^{13} \mathrm{C}-\mathrm{NMR}$ of $5 \mathrm{fin} \mathrm{CDCl}_{3}$

Boc

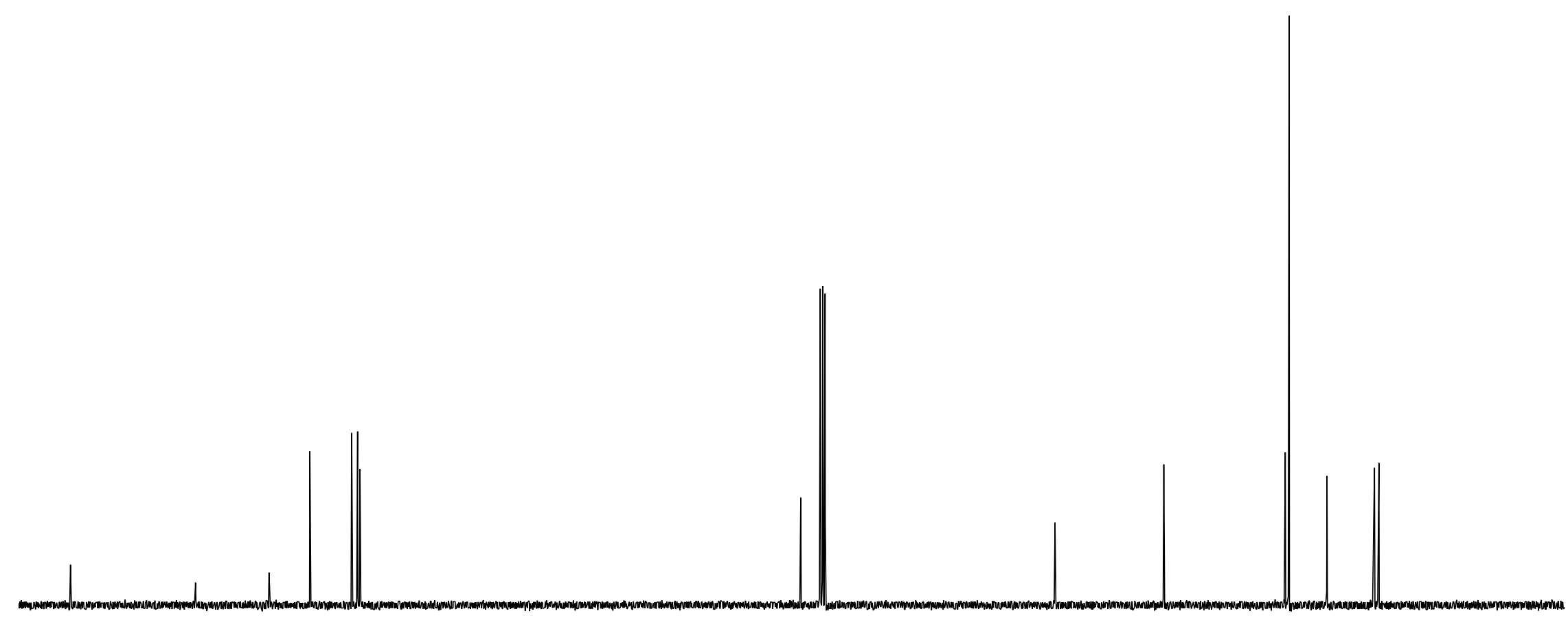

$$
\frac{7}{160}
$$




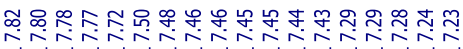 \\ पil}

${ }^{1} \mathrm{H}-\mathrm{NMR}$ of $4 \mathrm{~g}$ in $\mathrm{CDCl}_{3}$

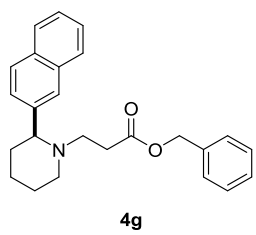

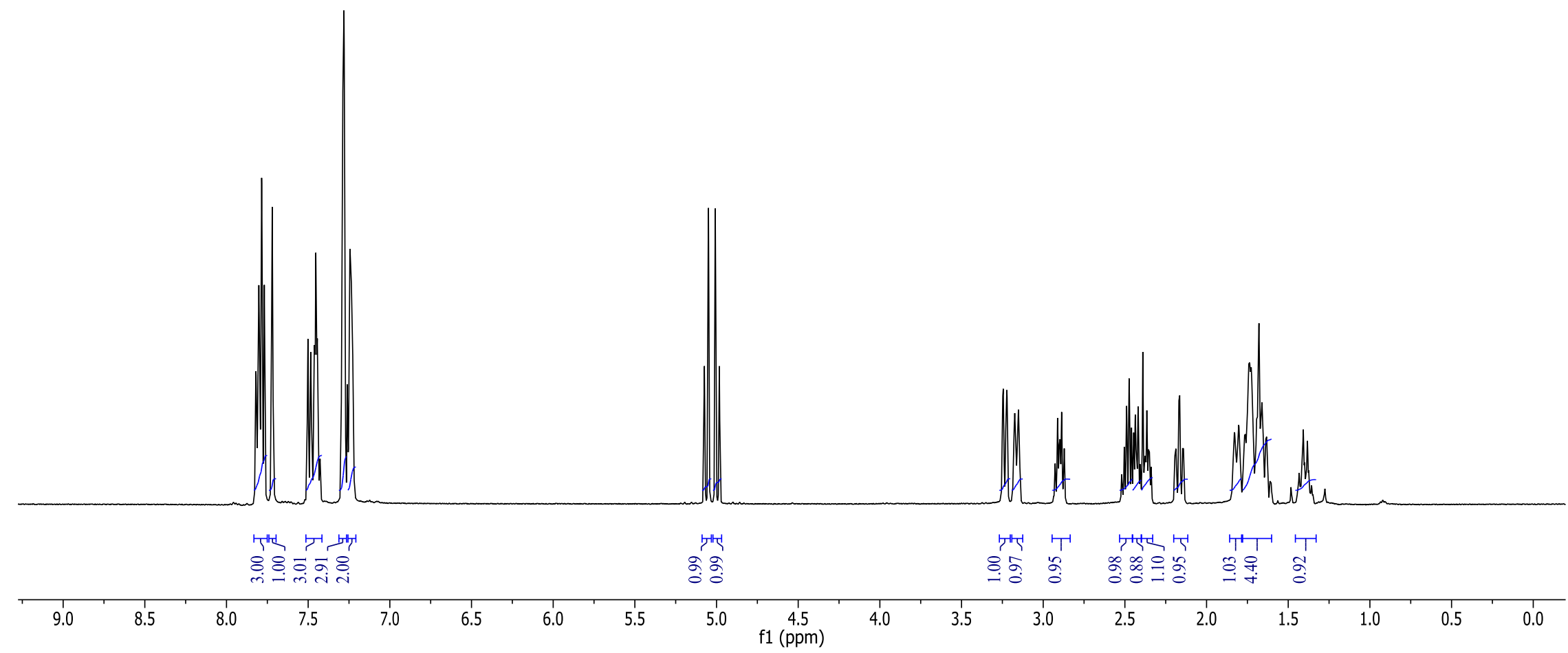



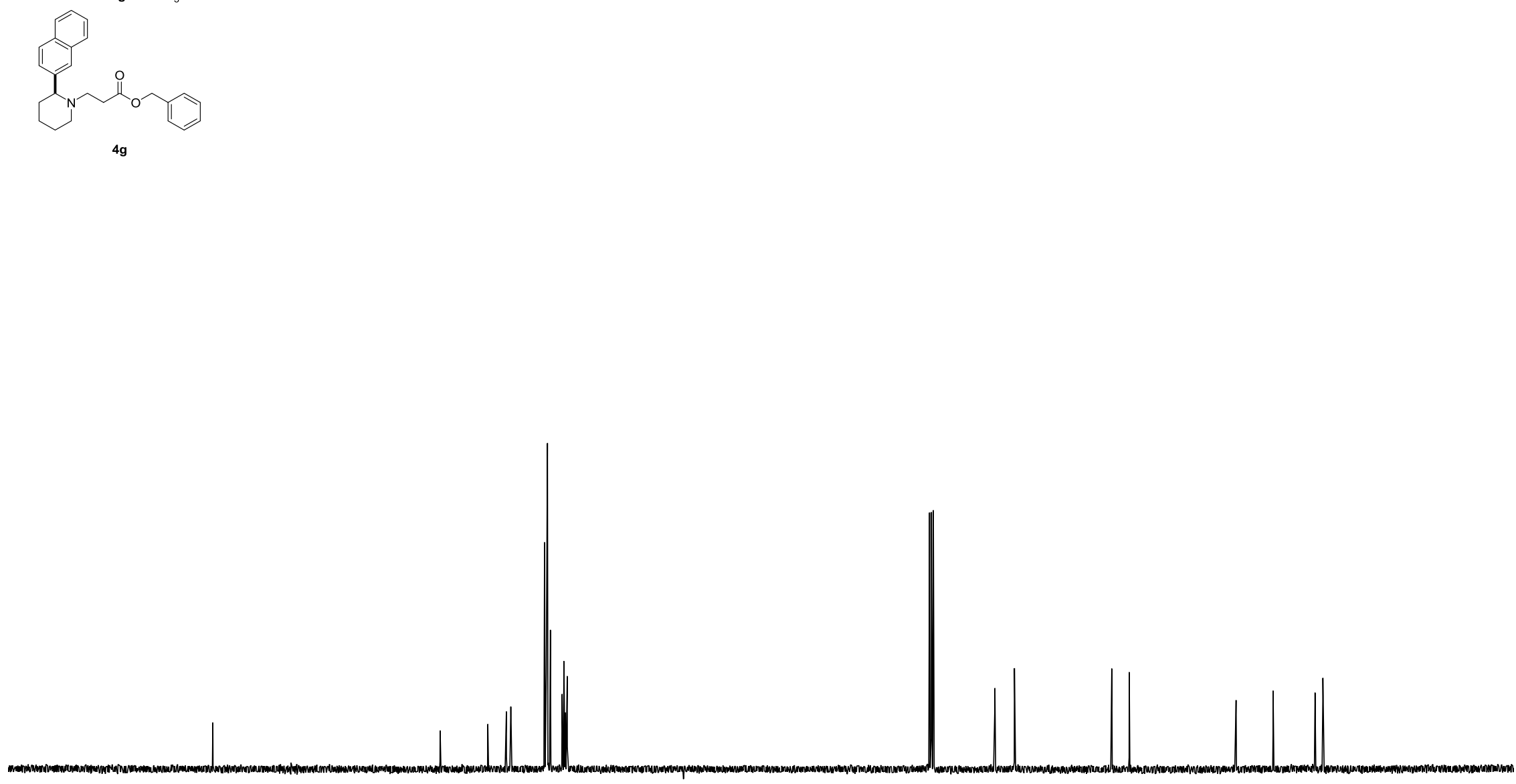


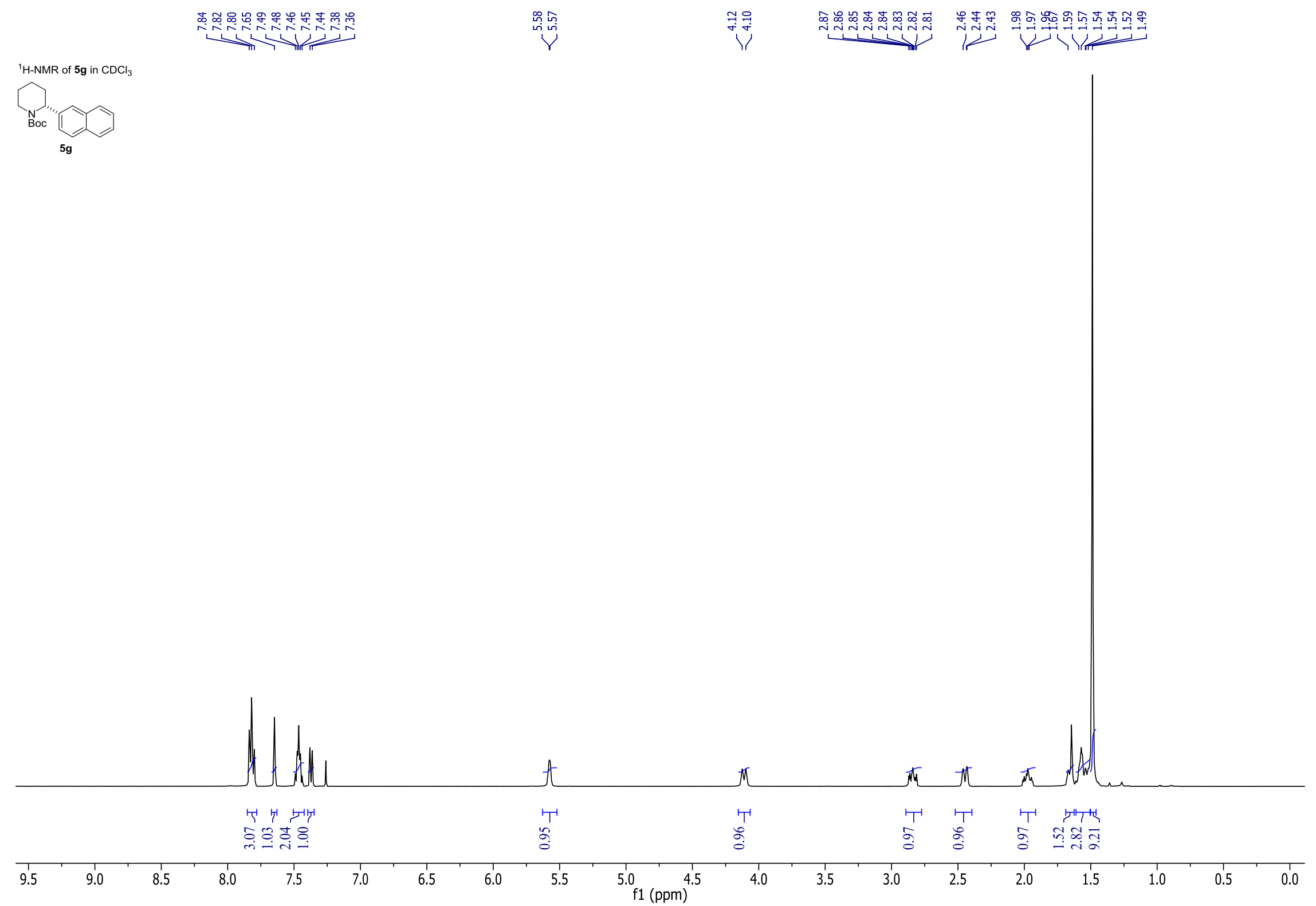



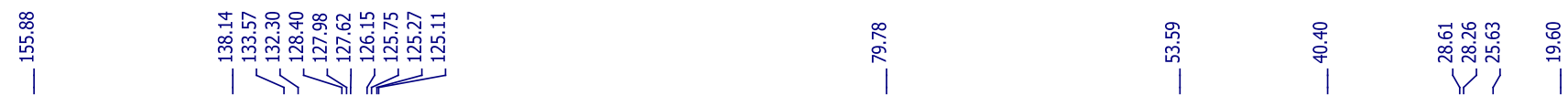

${ }^{13} \mathrm{C}-\mathrm{NMR}$ of $5 \mathrm{~g}$ in $\mathrm{CDCl}_{3}$

Boc

$5 \mathrm{~g}$

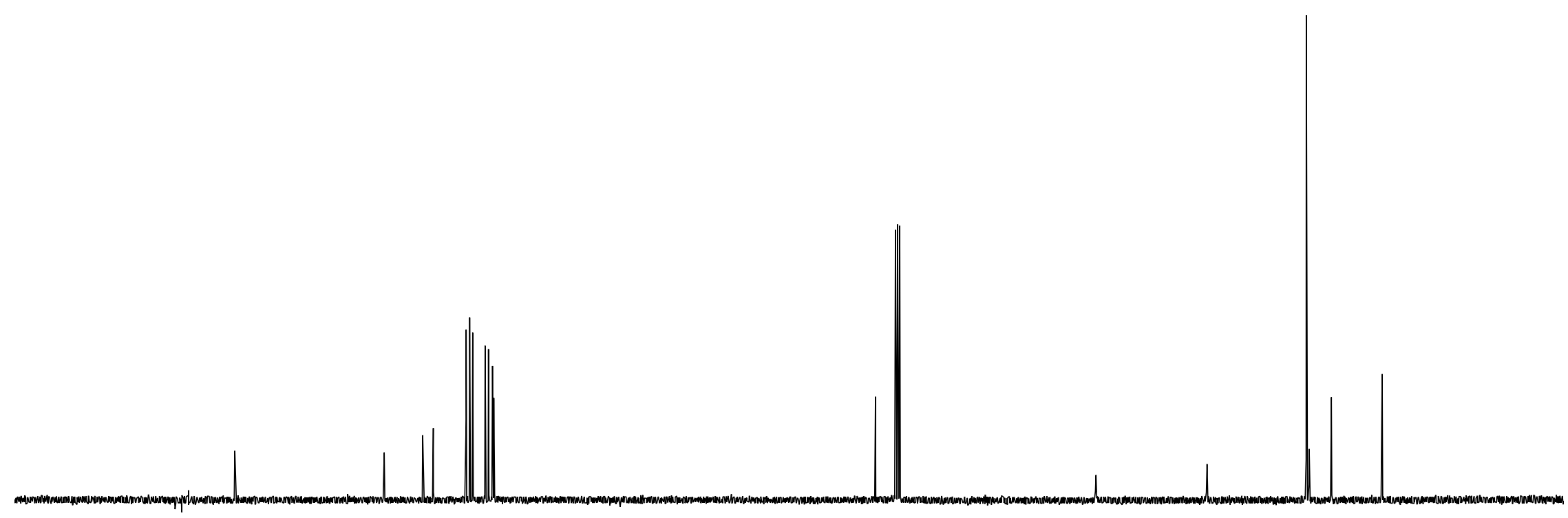


${ }^{1} \mathrm{H}-\mathrm{NMR}$ of $4 \mathrm{~h}$ in $\mathrm{CDC}$
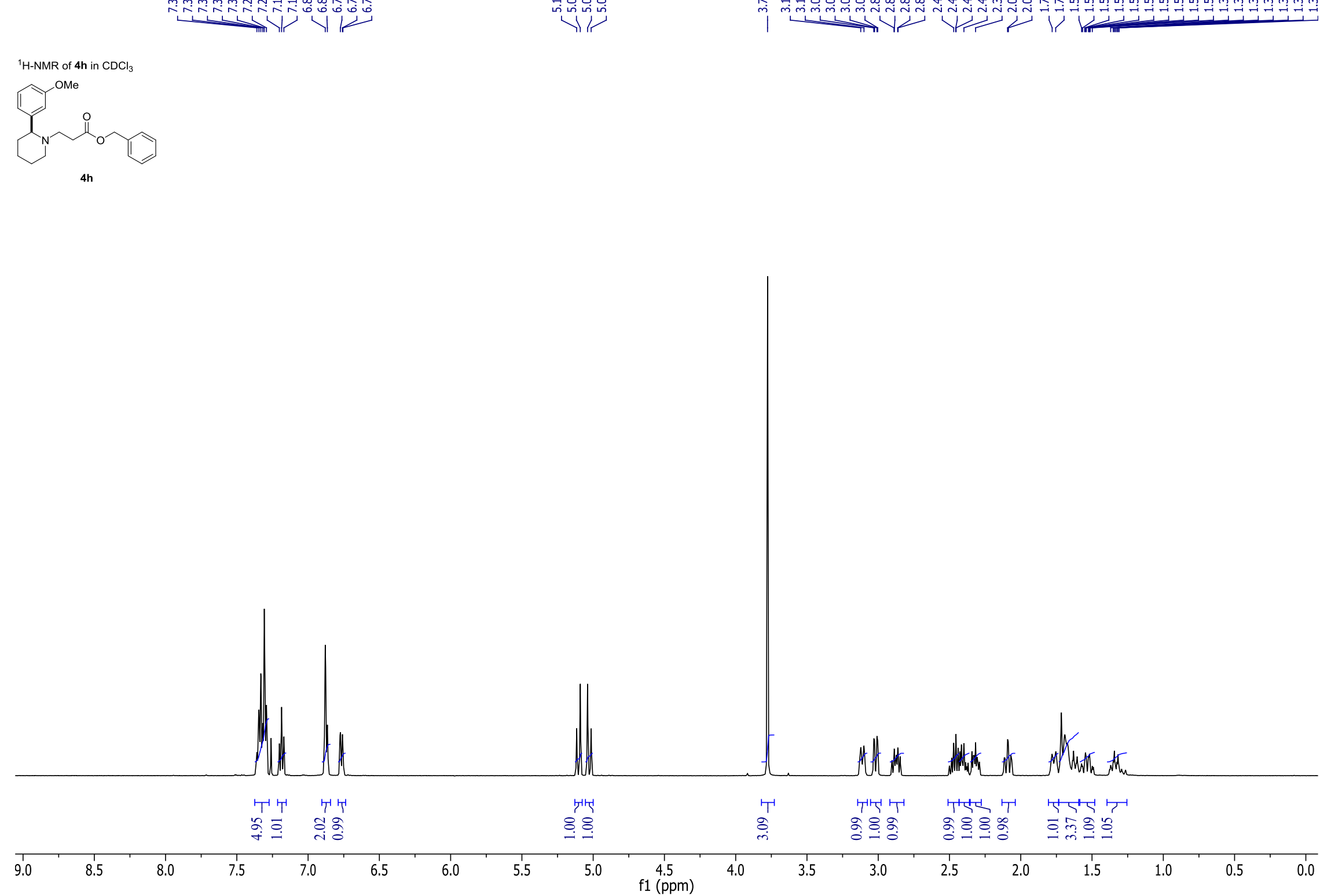


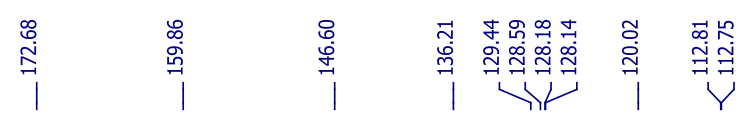

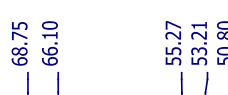
|

${ }^{13} \mathrm{C}-\mathrm{NMR}$ of $4 \mathrm{~h}$ in $\mathrm{CDCl}_{3}$

(Ome

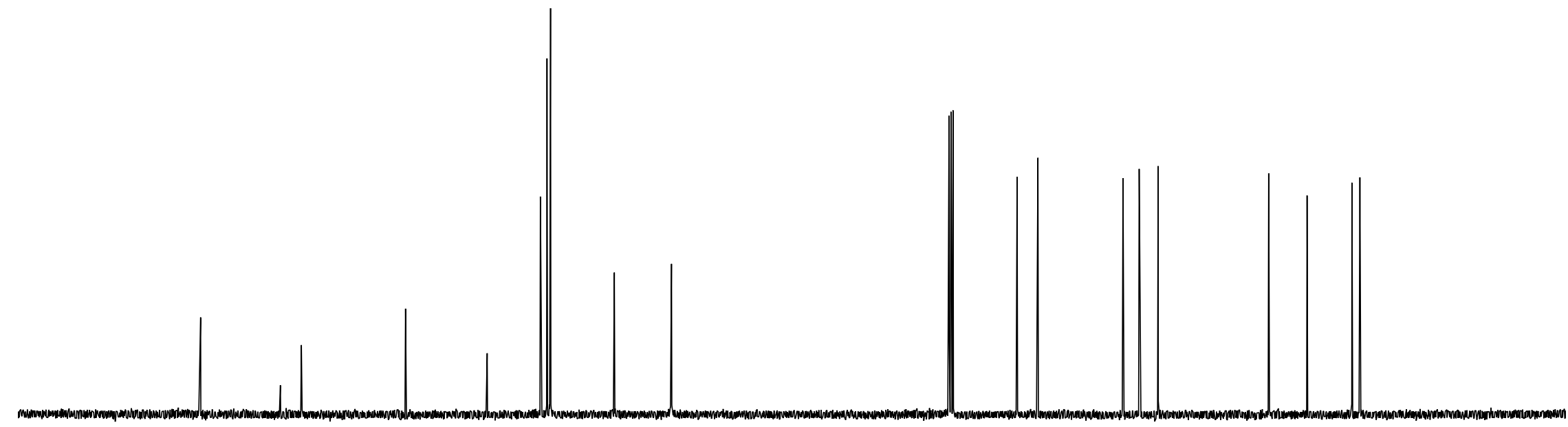




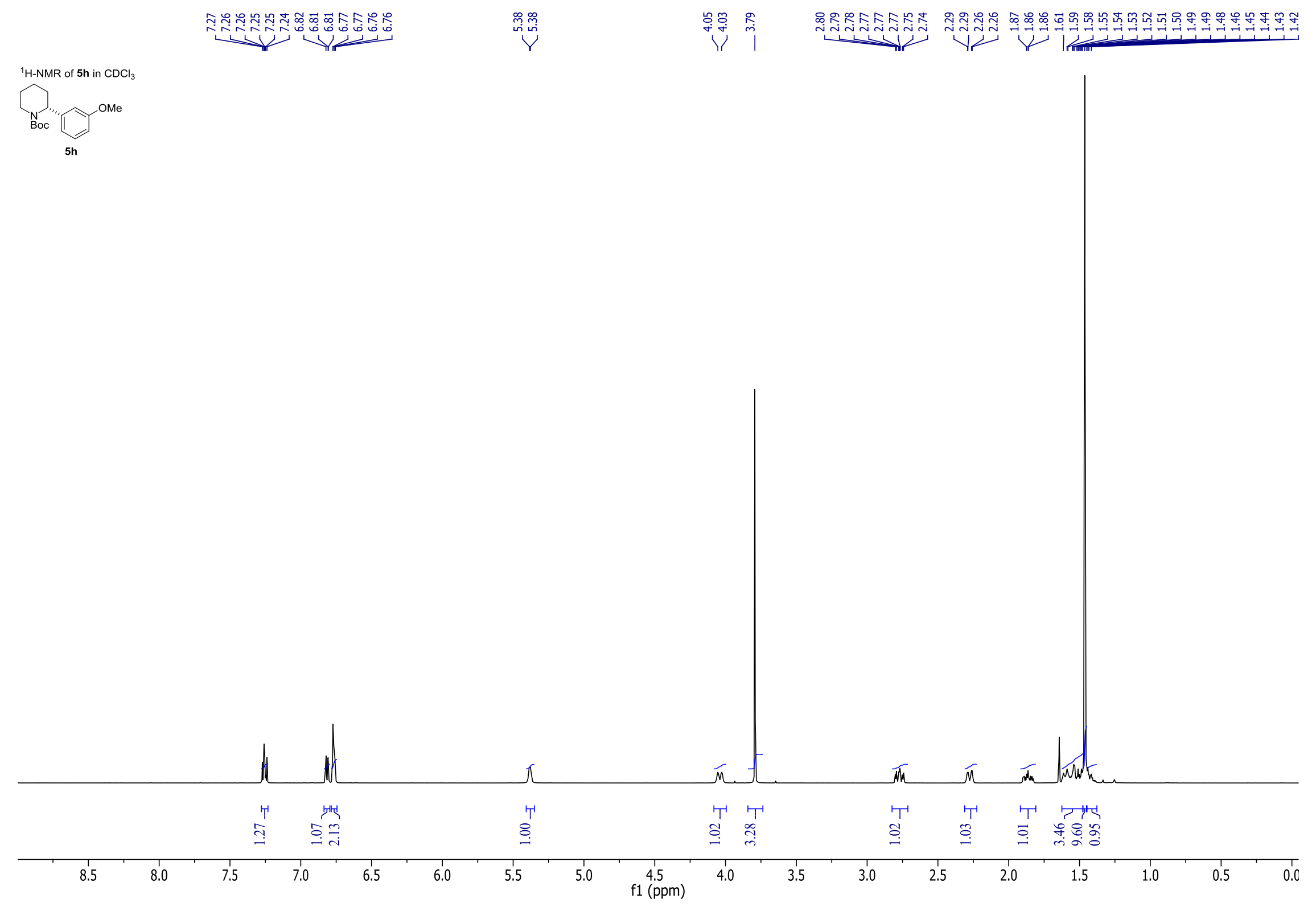

S-337 

|

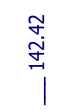

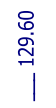
|

)
|

${ }^{13} \mathrm{C}-\mathrm{NMR}$ of $5 \mathrm{~h}$ in $\mathrm{CDCl}_{3}$

Boc "OMe

$5 \mathrm{~h}$

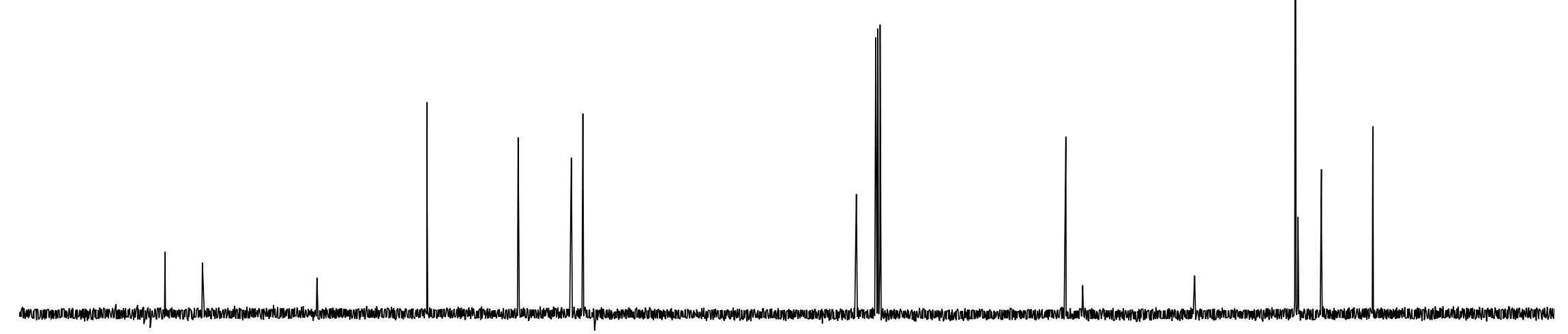

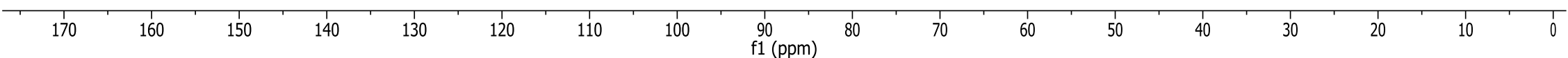




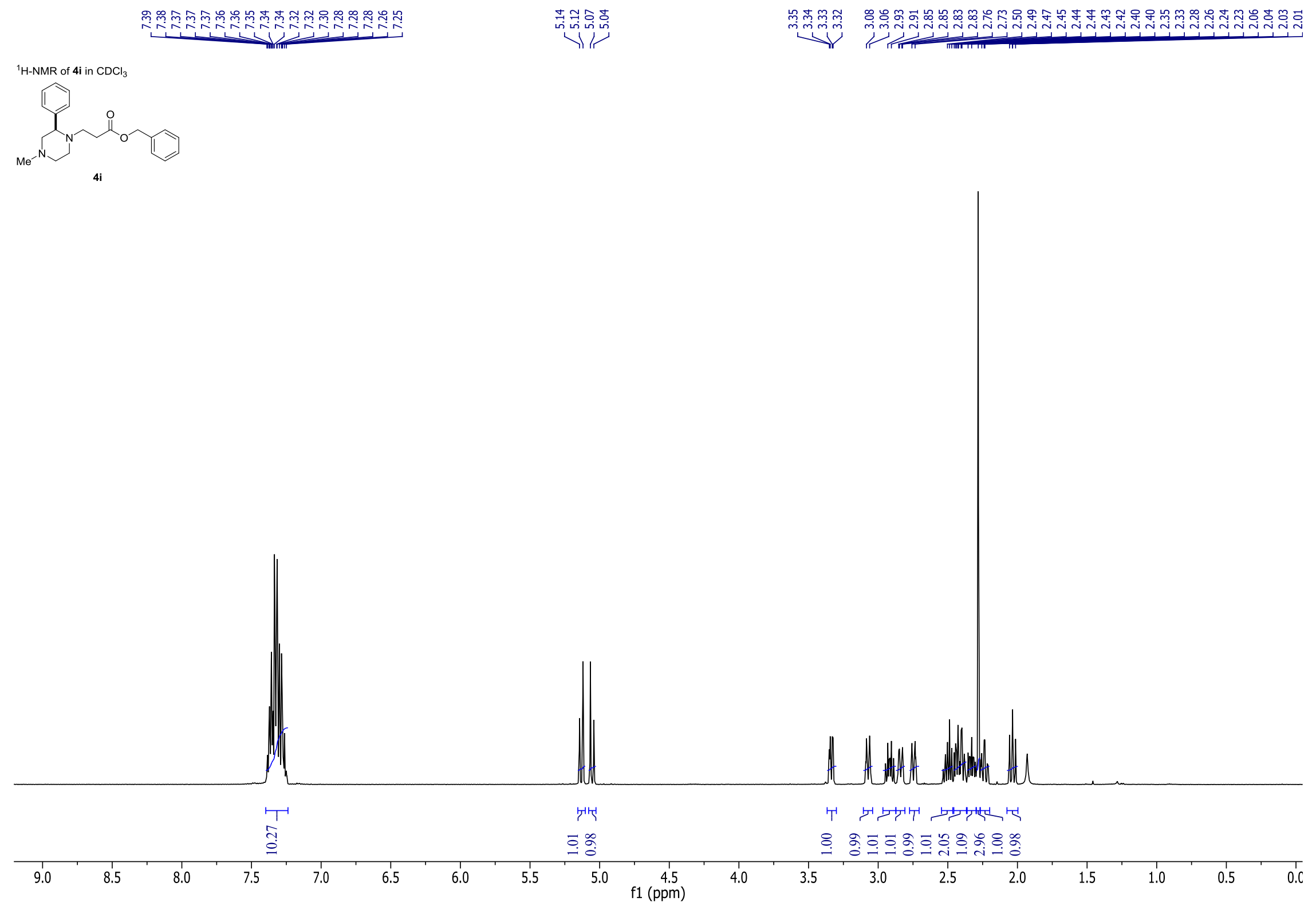

S-339 


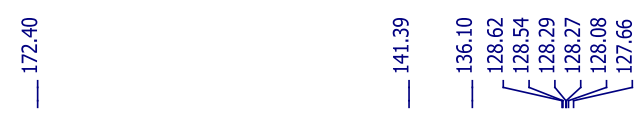

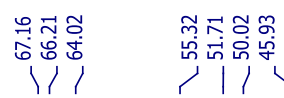

${ }^{13} \mathrm{C}-\mathrm{NMR}$ of $4 \mathrm{i}$ in $\mathrm{CDCl}_{3}$
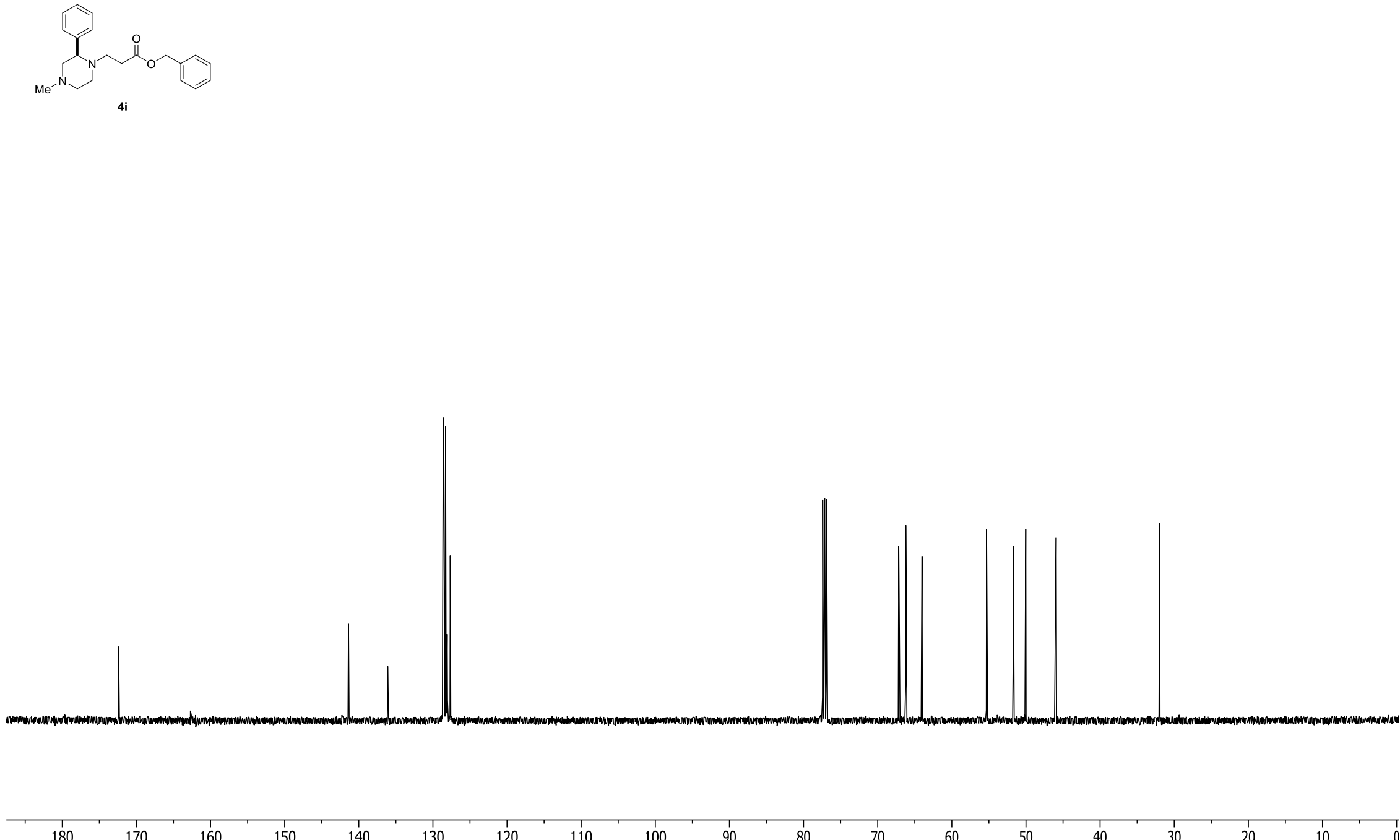

$100 \quad 90$

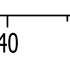

30 


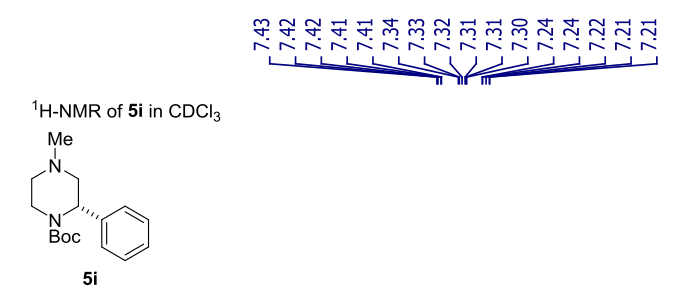

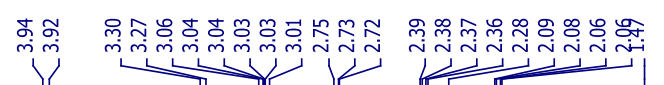

${ }^{1} \mathrm{H}-\mathrm{NMR}$ of $5 \mathbf{i}$ in $\mathrm{CDCl}_{3}$

Boc "I

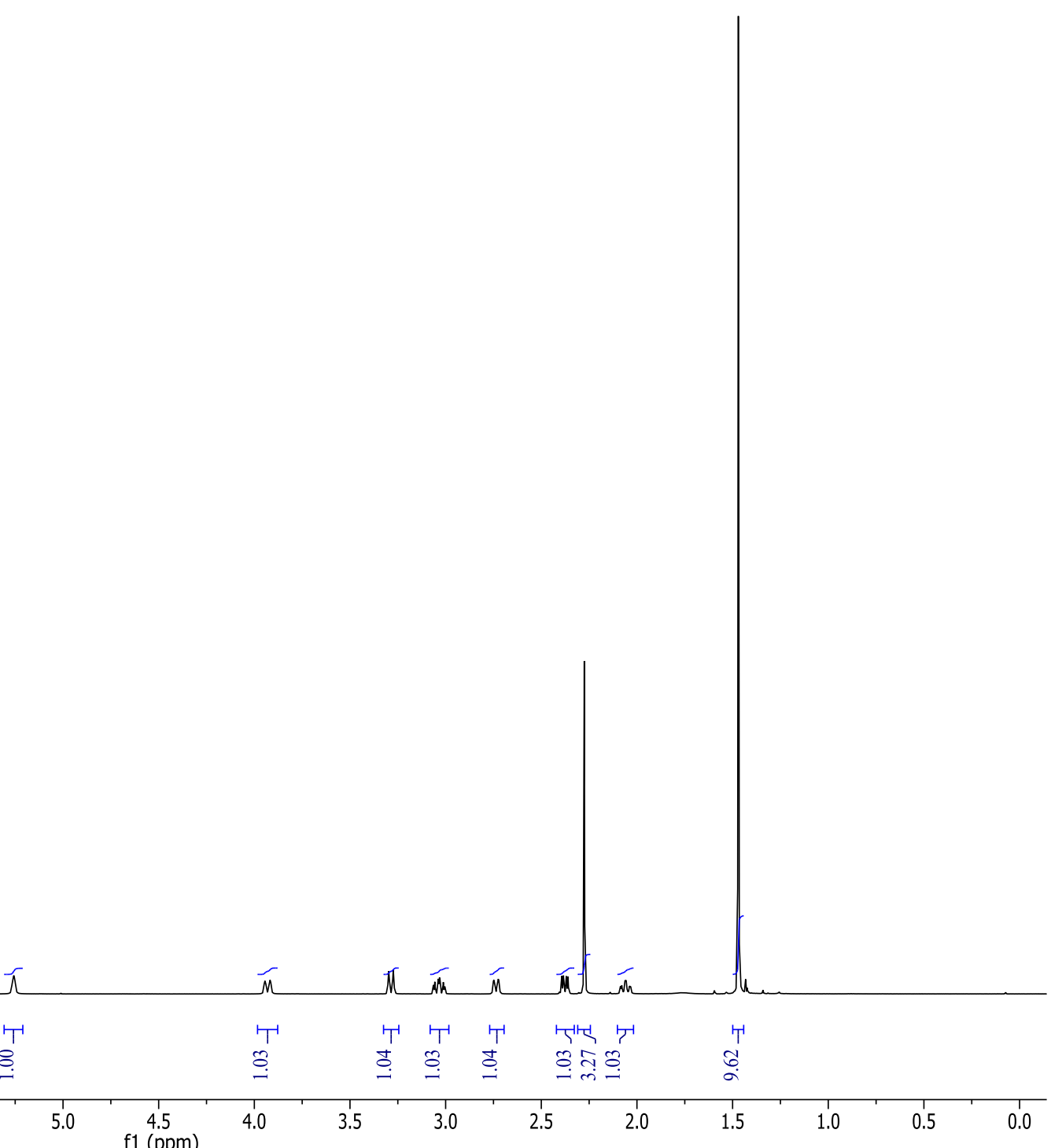


${ }^{13} \mathrm{C}-\mathrm{NMR}$ of $5 \mathrm{i}$ in $\mathrm{CDCl}_{3}$

Moc

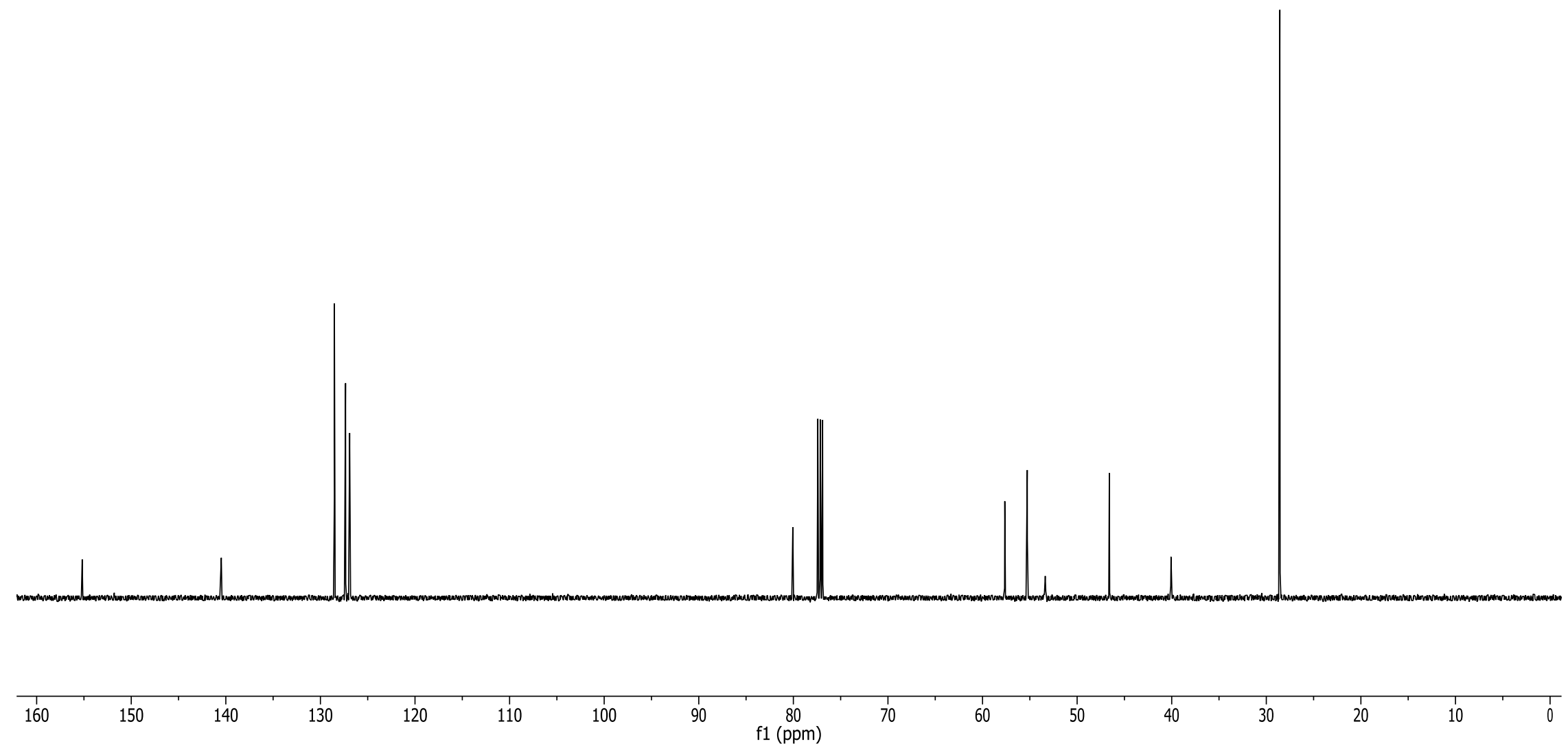


${ }^{1} \mathrm{H}-\mathrm{NMR}$ of $( \pm)-3 \mathrm{j}$ in $\mathrm{CDCl}_{3}$

$\sum_{( \pm)-3 \mathrm{j}}^{\mathrm{Bn}}$

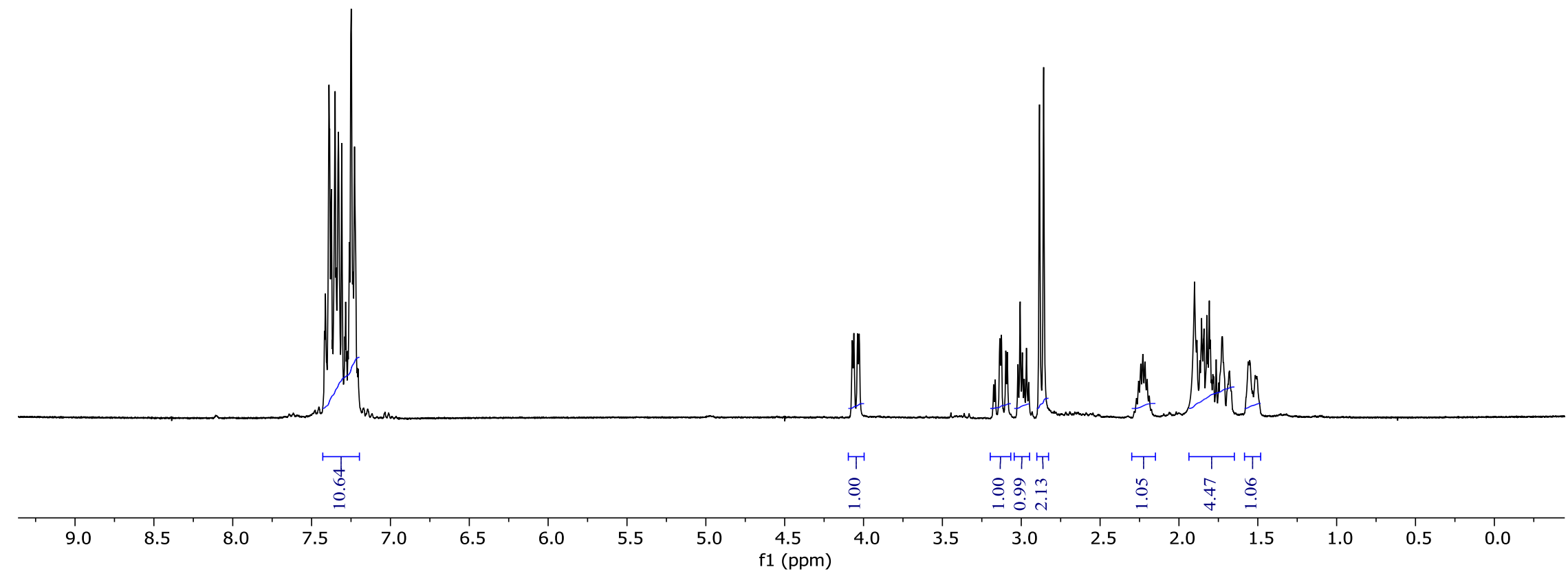




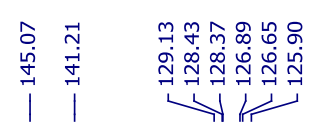

|

${ }^{13} \mathrm{C}-\mathrm{NMR}$ of $( \pm)$

(士)-3j
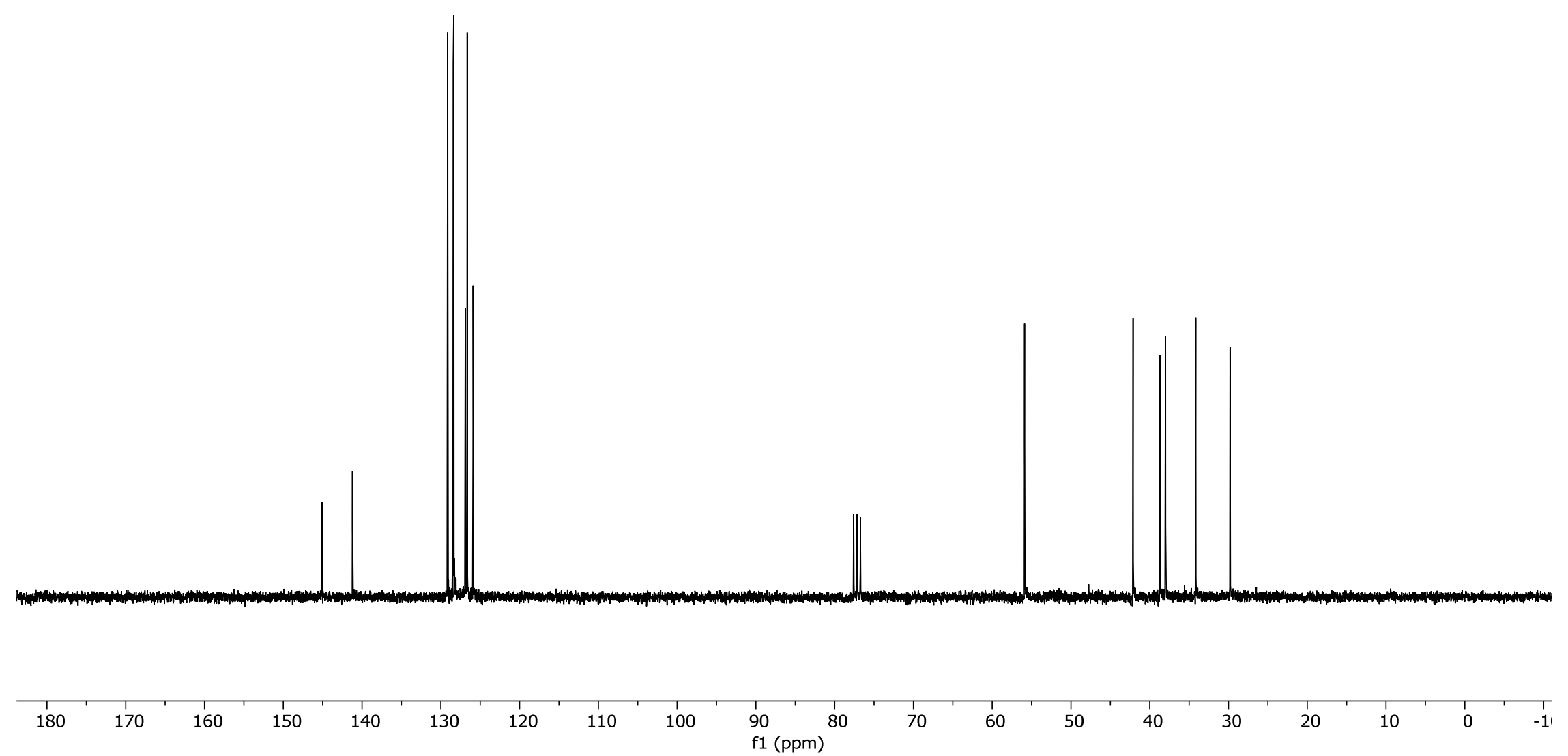

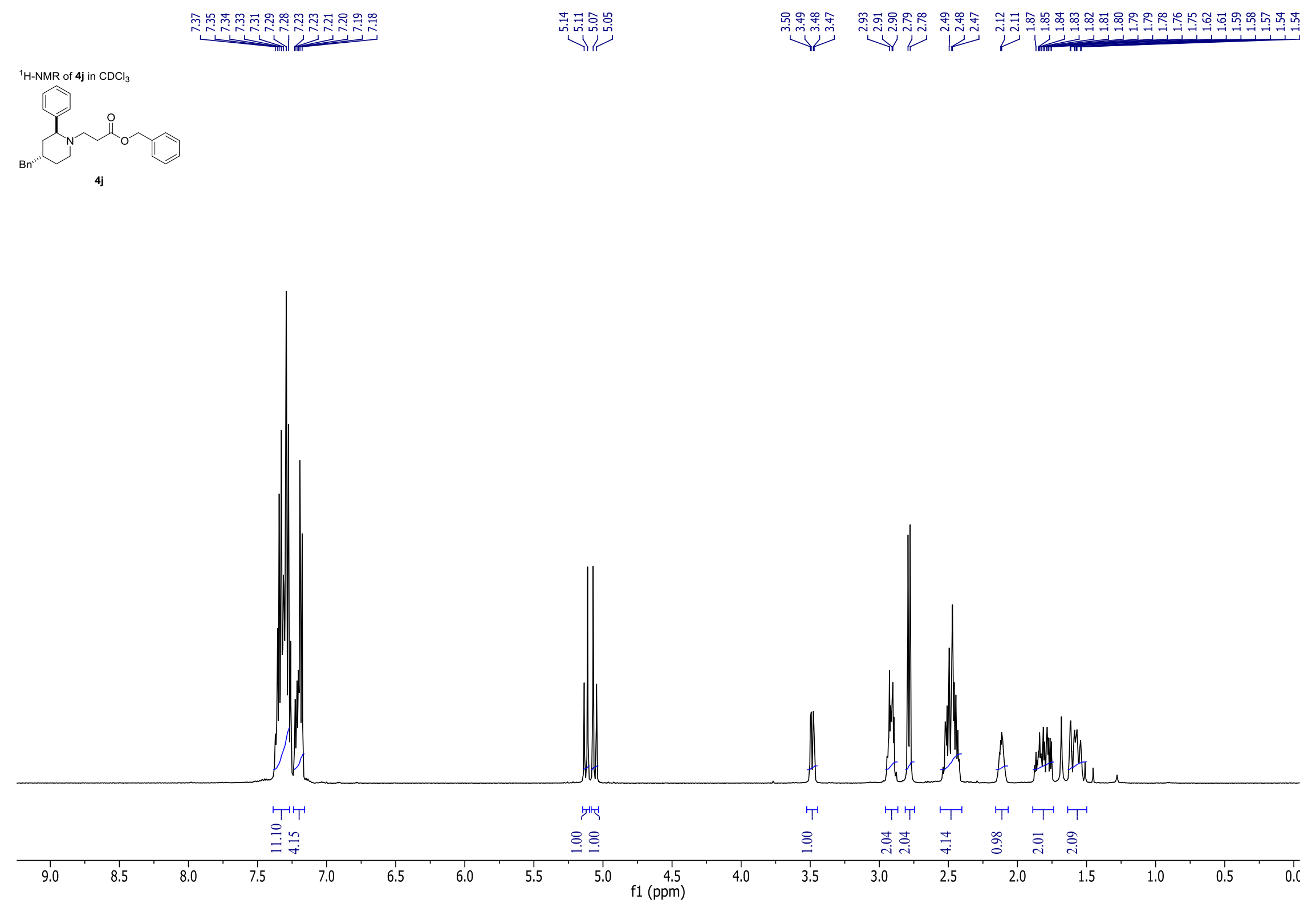


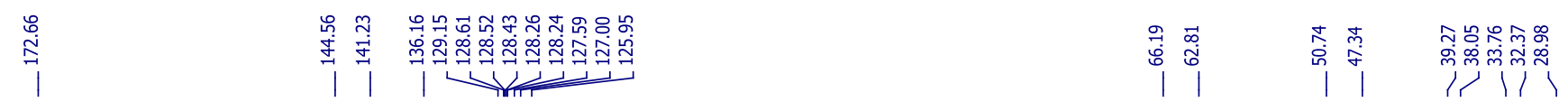

${ }^{13} \mathrm{C}-\mathrm{NMR}$ of $4 \mathrm{j}$ in $\mathrm{CDCl}_{3}$

(1)

$4 \mathrm{j}$

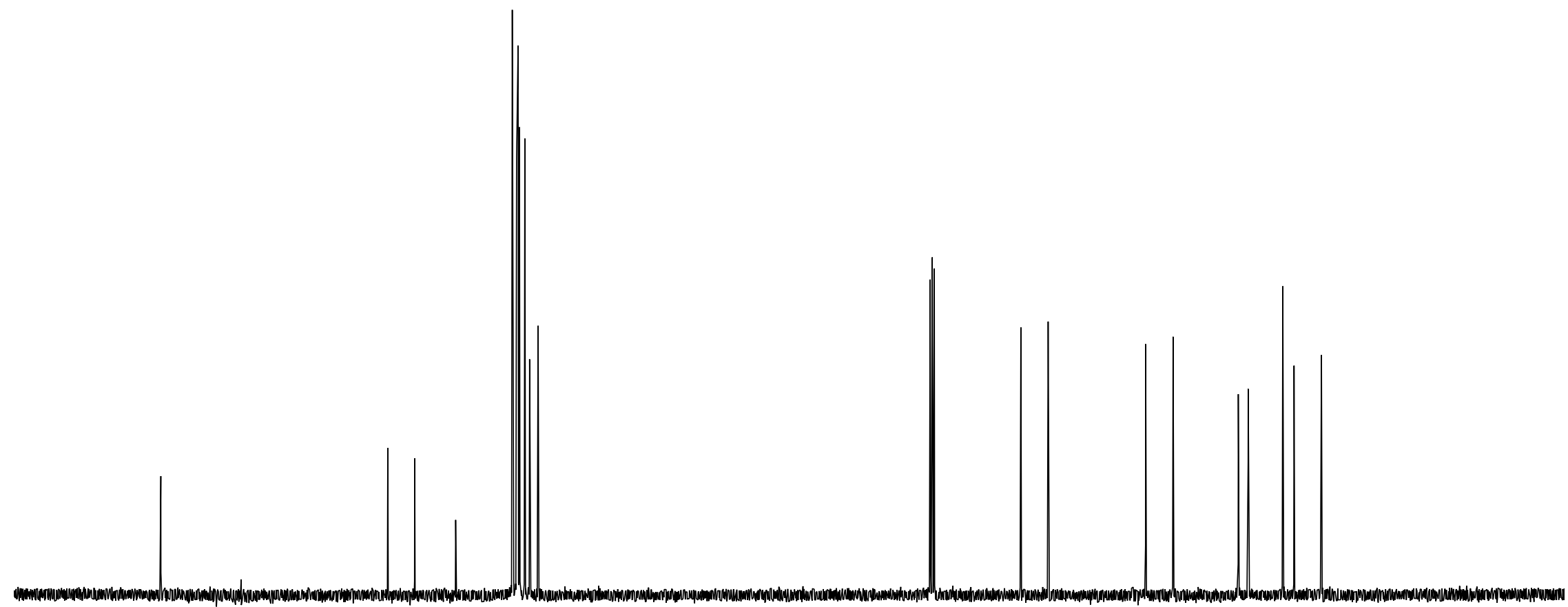

.90 


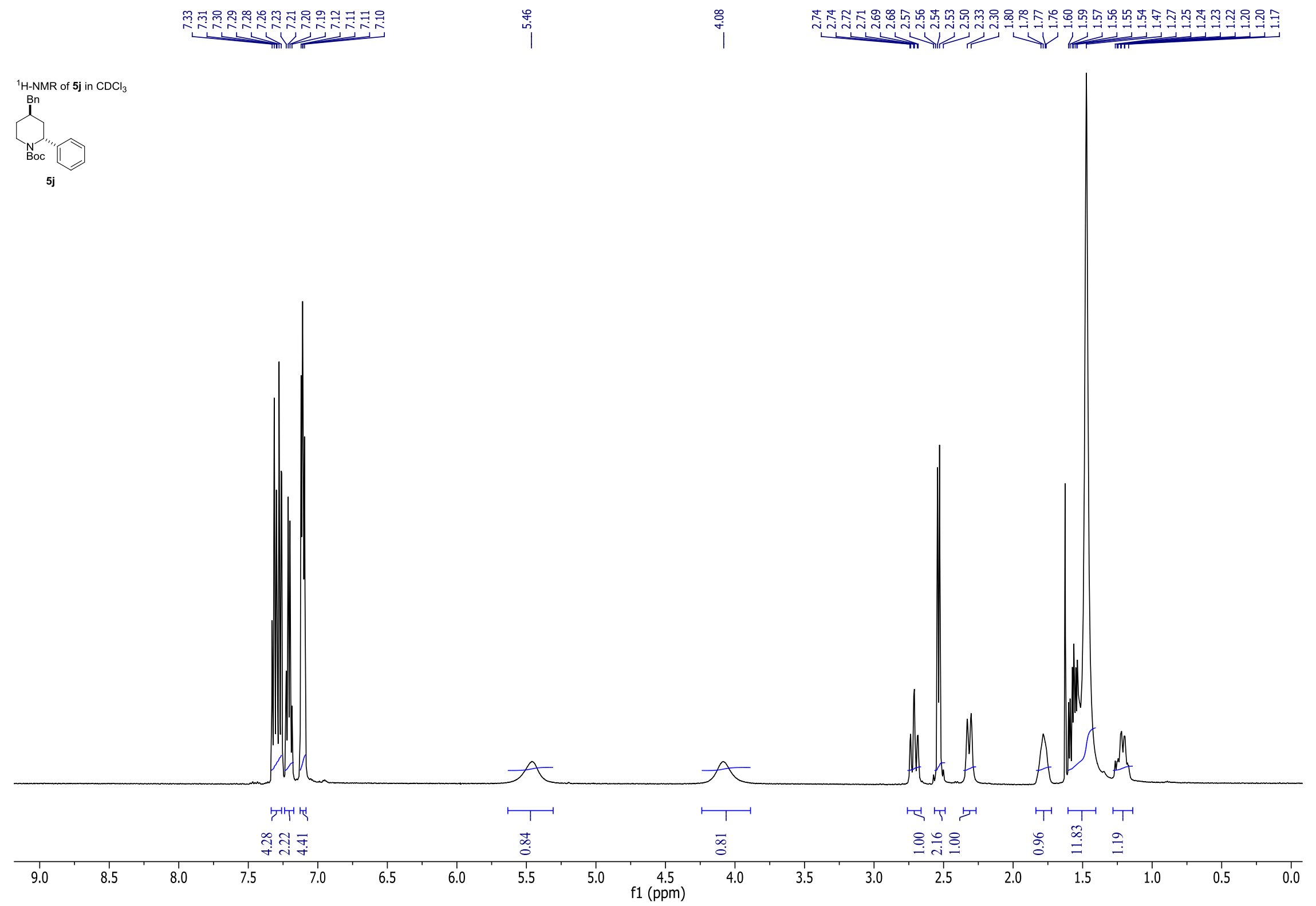

S-347 


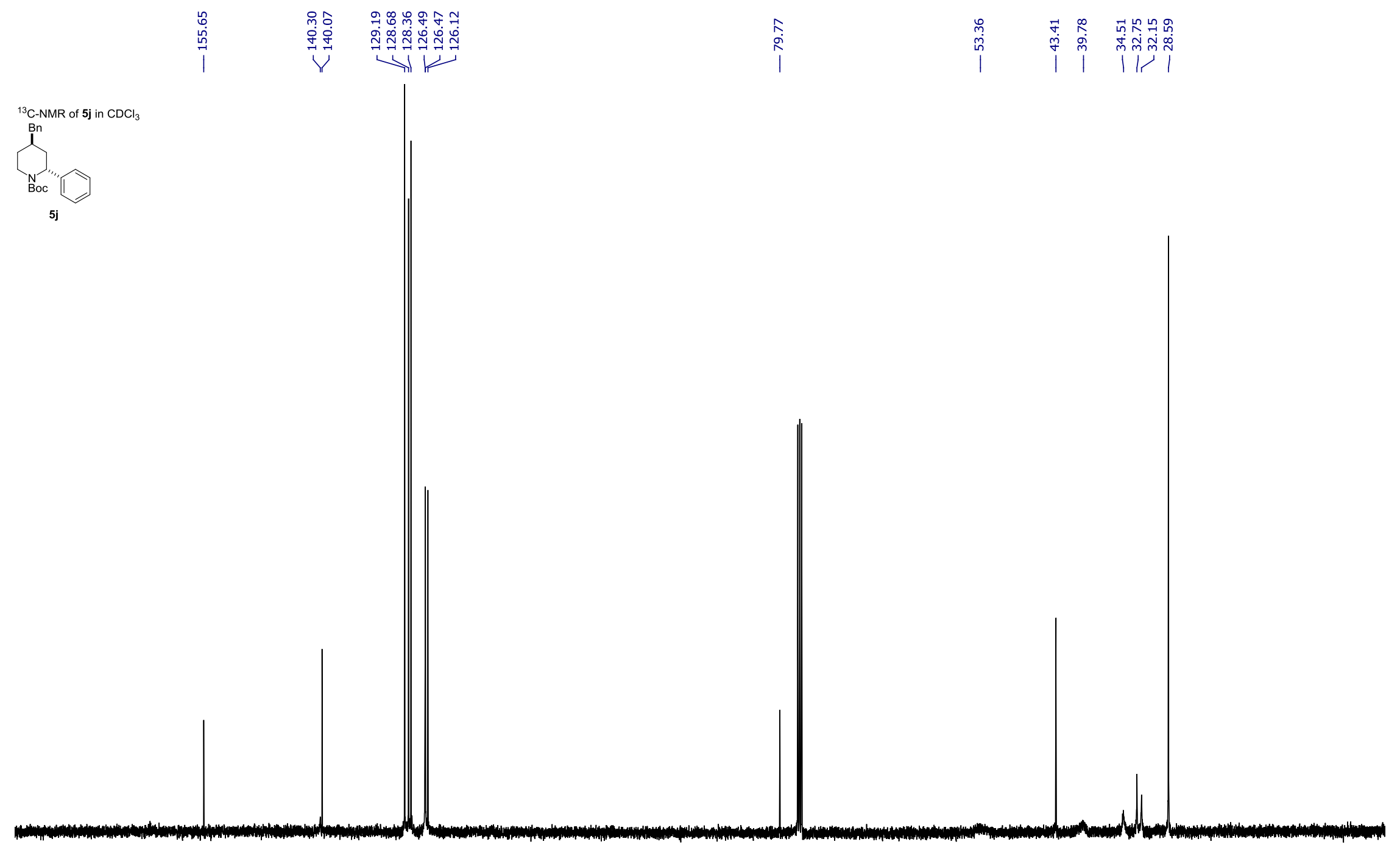

80
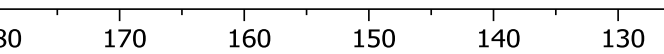

120

110

100

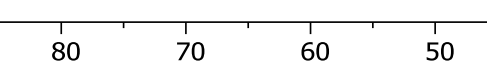

40

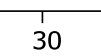


${ }^{1} \mathrm{H}-\mathrm{NMR}$ of $2 \mathrm{a}$ in toluene- $\mathrm{d}_{8}$

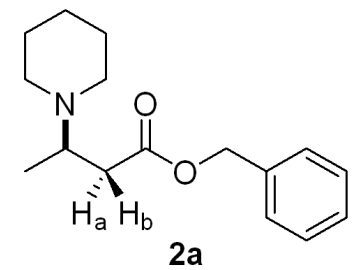

$2 a$

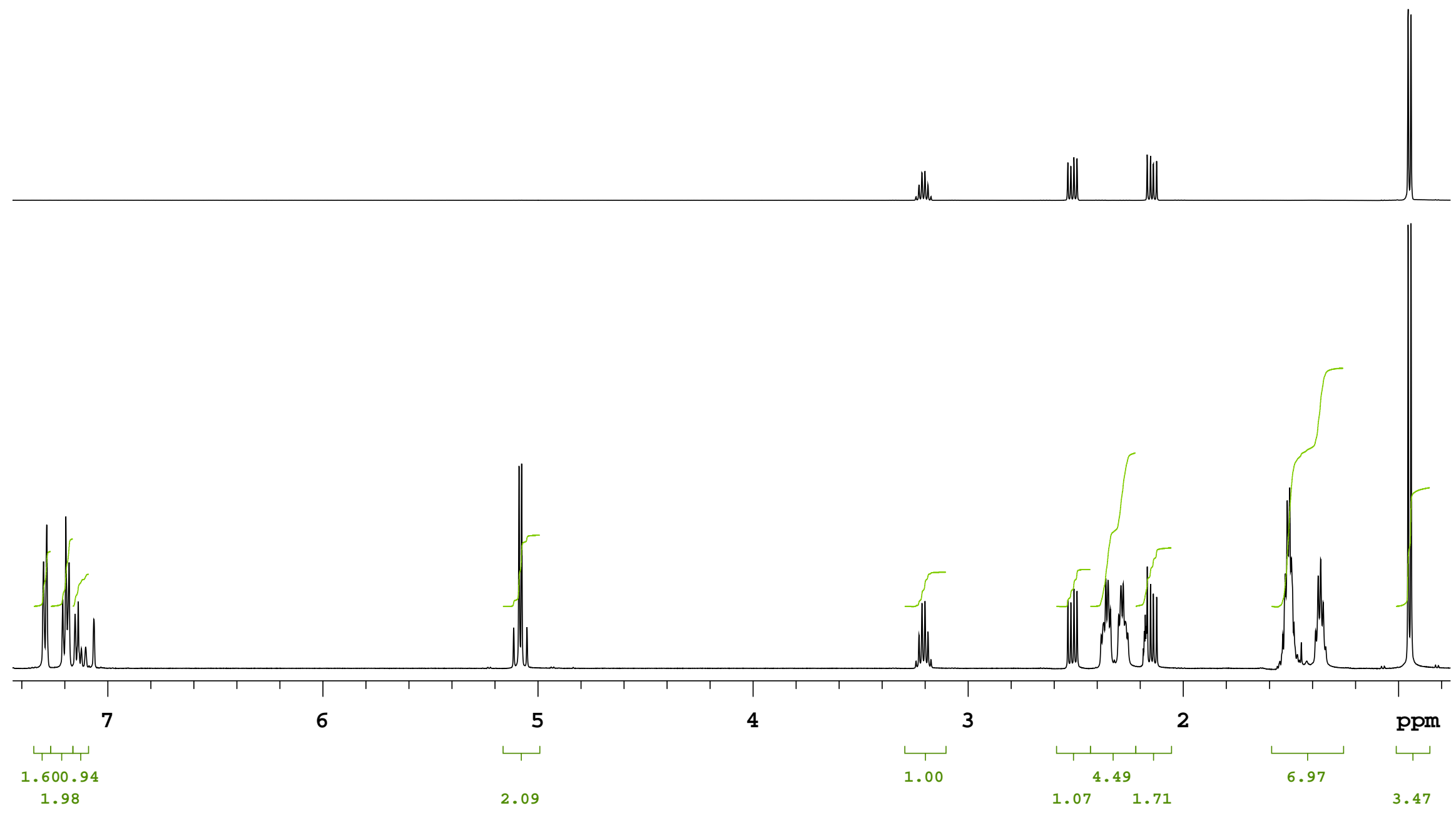


HMBC-NMR of $\mathbf{2} \mathbf{a}$ in toluene- $\mathrm{d}_{8}$

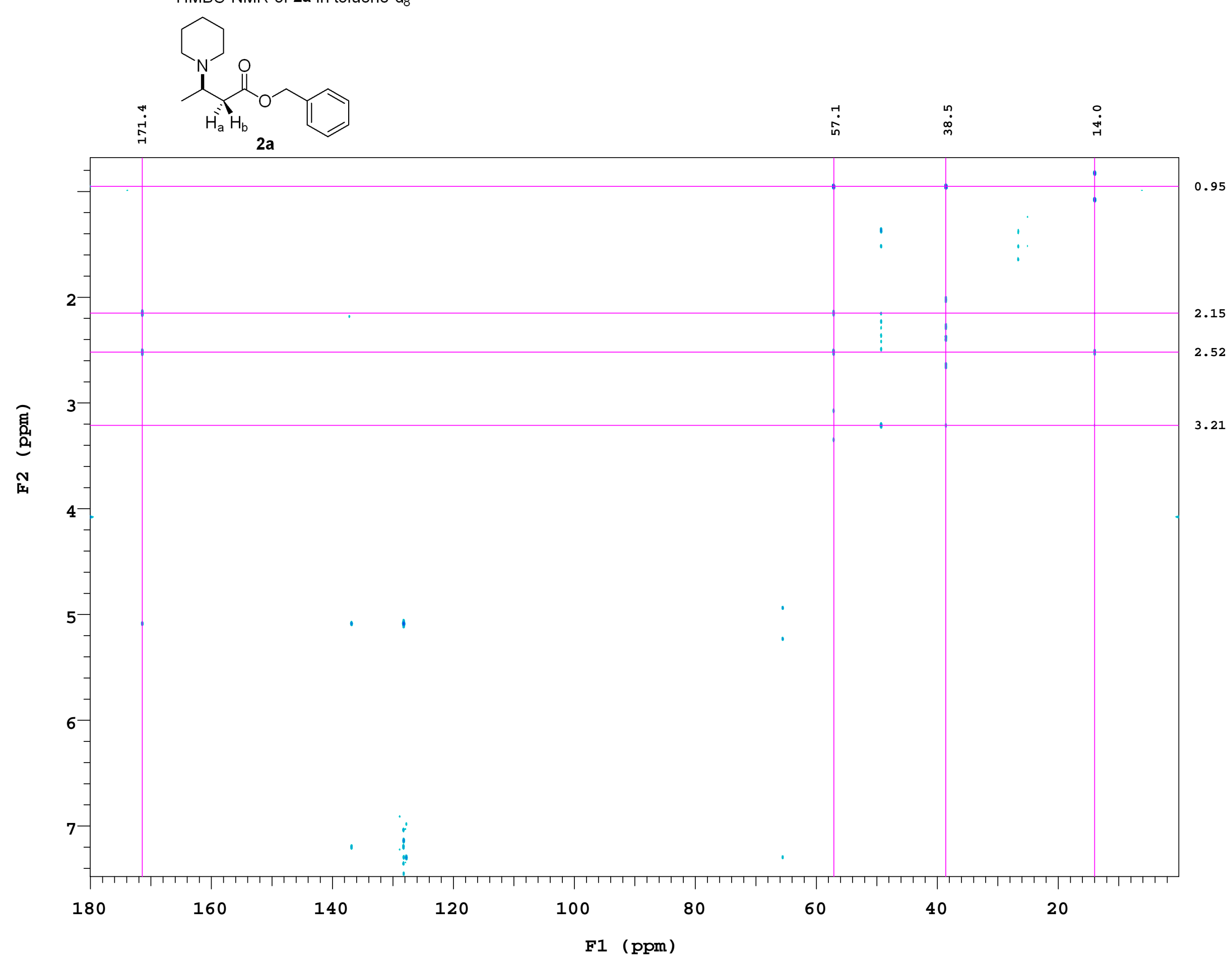


HSQC-NMR of $2 \mathbf{a}$ in toluene- $d_{8}$

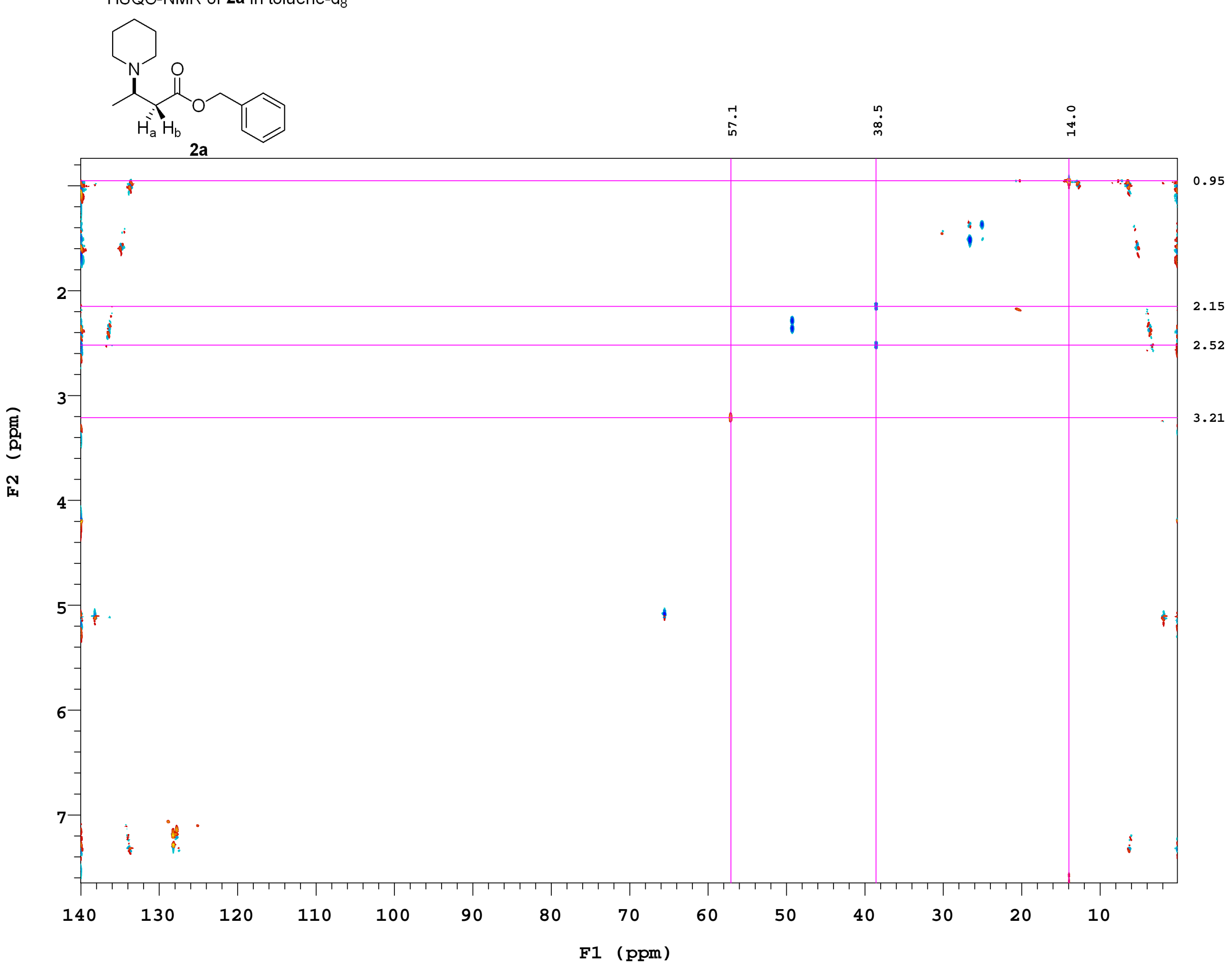


EXSIDE $(\delta=2.15)$ of $\mathbf{2 a}$ in toluene- $d_{8}$
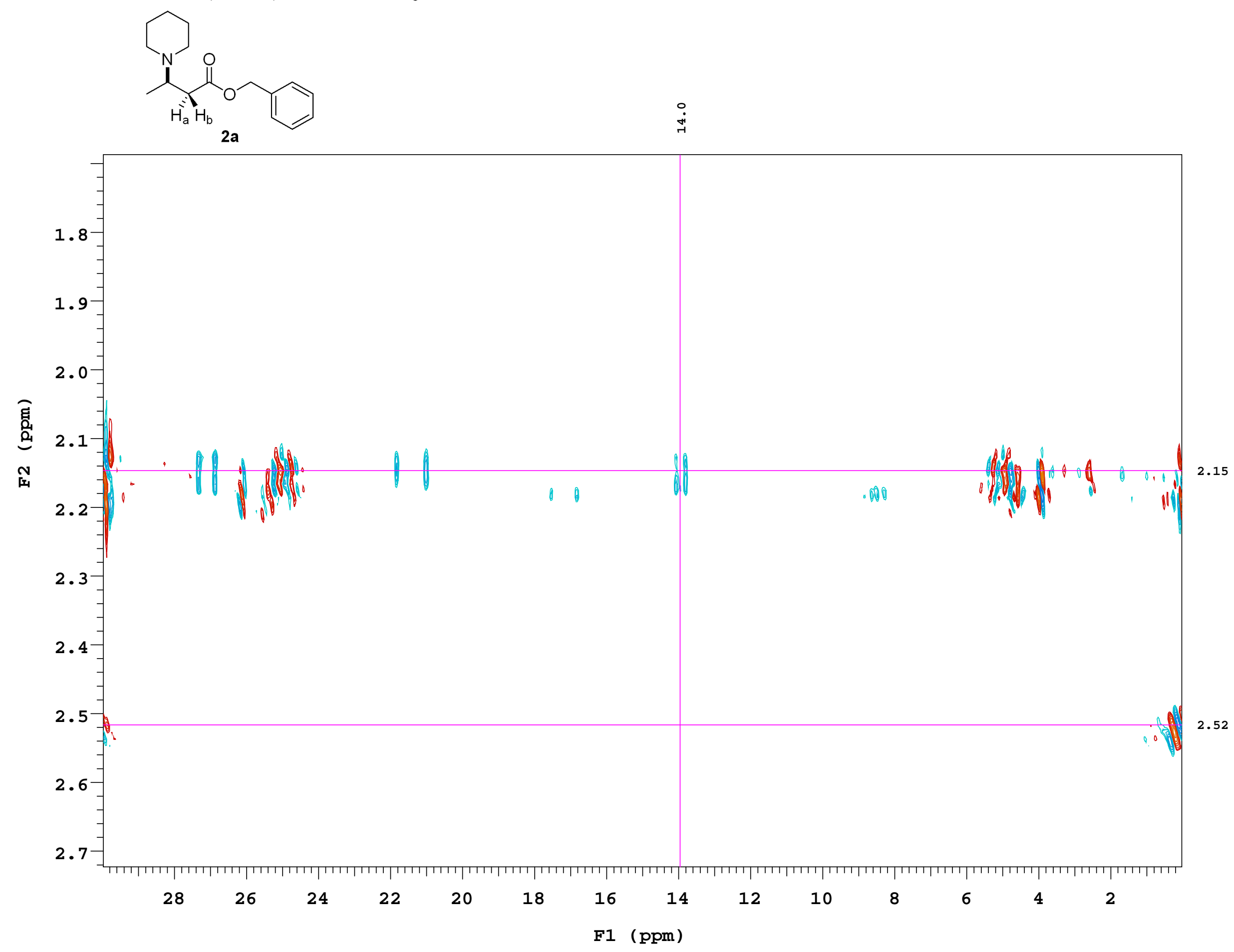
EXSIDE $(\delta=2.52)$ of $\mathbf{2} \mathbf{a}$ in toluene- $d_{8}$

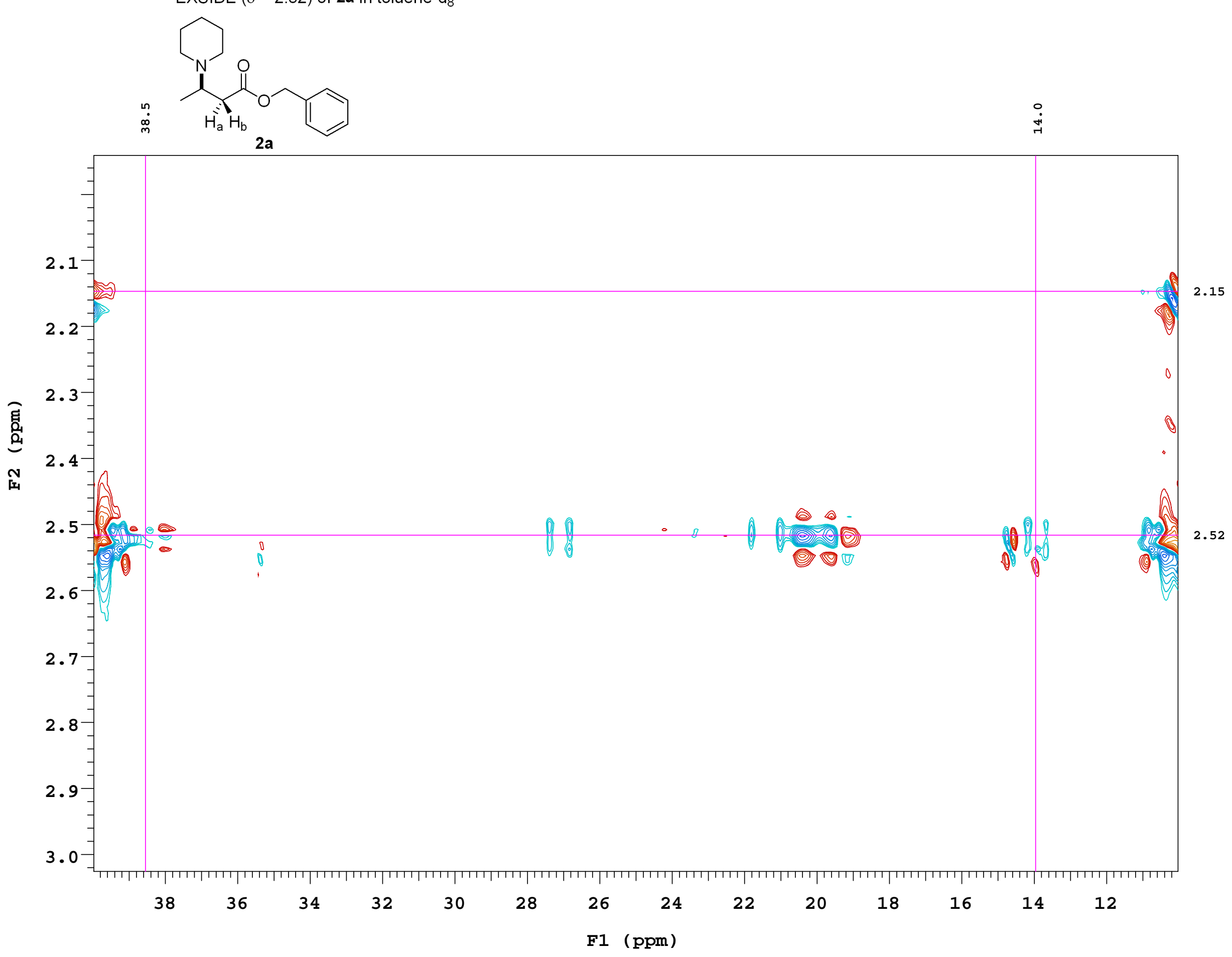


EXSIDE $(\delta=3.21)$ of $\mathbf{2} \mathbf{a}$ in toluene- $\mathrm{d}_{8}$

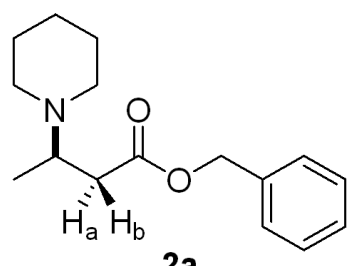

2a
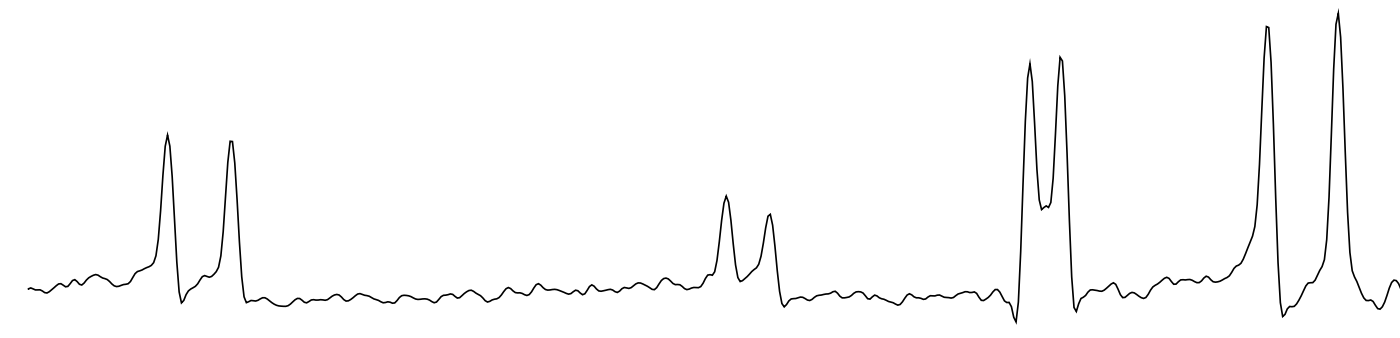

ppm 